\title{
Hanford Site Climatological Data Summary 1999 With Historical Data
}

\author{
D. J. Hoitink \\ K. W. Burk \\ J. V. Ramsdell
}

April 2000

Prepared for

the U.S. Department of Energy

under Contract DE-AC06-76RLO 1830

Pacific Northwest National Laboratory

Richland, Washington 99352 
$\begin{array}{ccc}\because & \therefore \\ & \therefore & \therefore\end{array}$ 


\section{DISCLAIMER}

This report was prepared as an account of work sponsored by an agency of the United States Government. Neither the United States Government nor any agency thereof, nor any of their employees, make any warranty, express or implied, or assumes any legal liability or responsibility for the accuracy, completeness, or usefulness of any information, apparatus, product, or process disclosed, or represents that its use would not infringe privately owned rights. Reference herein to any specific commercial product, process, or service by trade name, trademark, manufacturer, or otherwise does not necessarily constitute or imply its endorsement, recommendation, or favoring by the United States Government or any agency thereof. The views and opinions of authors expressed herein do not necessarily state or reflect those of the United States Government or any agency thereof. 


\section{DISCLAIMER}

Portions of this document may be illegible in electronic image products. Images are produced from the best available original document. 


\section{Summary}

This document presents the climatological data measured at the U.S. Department of Energy's Hanford Site for calendar year 1999. Pacific Northwest National Laboratory ${ }^{1}$ operates the Hanford Meteorology Station and the Hanford Meteorological Monitoring Network from which these data were collected. The information contained herein includes updated historical climatologies for temperature, precipitation, normal and extreme values of temperature and precipitation, and other miscellaneous meteorological parameters. Further, the data are adjunct to and update Hoitink et al. (1999), and Hoitink and Burk (1994, 1995, 1996, 1997, 1998); however, Appendix B-Wind Climatology (1994) is excluded.

1999 was warmer than normal at the Hanford Meteorology Station with an average temperature of $54.4^{\circ} \mathrm{F}, 1.1^{\circ} \mathrm{F}$ above normal $\left(53.3^{\circ} \mathrm{F}\right)$. The hottest temperature was $105^{\circ} \mathrm{F}$ on July 28 , while the coldest was $18^{\circ} \mathrm{F}$ on January 3. The maximum temperature of $64^{\circ} \mathrm{F}$ on August 30 was the lowest maximum temperature ever recorded in August, while the maximum temperature of $76^{\circ} \mathrm{F}$ on November 13 was the highest maximum temperature ever recorded in November. For the 12-month period, 6 months were warmer than normal and 6 were cooler than normal

1999 was the fourth driest year on record. Precipitation totaled 3.75 inches, $60 \%$ of normal (6.26 inches); snowfall totaled 0.6 inch, the least calendar year snowfall on record (compared to the normal of 13.8 inches).

1999 was the windiest year on record with an average wind speed of $8.8 \mathrm{mph}, 1.1 \mathrm{mph}$ above normal $(7.7 \mathrm{mph}$ ). There were 48 days with peak gusts $\geq 40 \mathrm{mph}$, compared to a yearly average of $26 \mathrm{mph}$. The peak gust during the year was $65 \mathrm{mph}$ on February 6.

The heating-degree days for $1998-1999$ were 4,802 ( $8 \%$ below the 5,231 normal). Cooling-degree days for 1999 were 891 (10\% below the 994 normal).

For inquiries regarding this report, contact Mr. D. J. Hoitink at Pacific Northwest National Laboratory, P.O. Box 999, Richland, Washington 99352, or by electronic mail at dana.j.hoitink@pnl.gov.

\footnotetext{
${ }^{1}$ Pacific Northwest National Laboratory is operated by Battelle for the U.S. Department of Energy.
} 


\section{Acknowledgments}

The authors wish to acknowledge Dana Ward, the Department of Energy Richland Operations Office Technical Monitor for the Meteorological and Climatological Services Project, and Roger Dirkes, Manager of the Public Safety and Resource Protection Program (PNNL), for their continued strong support. 


\section{Notes on Units of Measure}

This document mainly uses English units (e.g., miles per hour [mph], inches [in.], degrees Fahrenheit $\left.\left[{ }^{\circ} \mathrm{F}\right]\right)$ when presenting all information. This decision to use English units was based on the fact that English units are still the standard in National Oceanic and Atmospheric Administration (specifically, the National Climatic Data Center and National Weather Service) reporting and publications.

Throughout this document the term "normal" is used to indicate climatological normal, defined as an average value over a period of years of any meteorological element such as temperature, pressure, and rainfall. The accepted convention uses a 30-year time period, ending with the first year of each new decade (such as 1951-1980, 1961-1990, 1971-2000). The current time period used for climatological normals is $1961-1990$.

Some useful conversions between English units and metric equivalents are:

$$
\begin{aligned}
& 1 \text { foot }(\mathrm{ft})=0.3048 \text { meter }(\mathrm{m}) \\
& 1 \text { mile }(\mathrm{mi})=1.609 \text { kilometers }(\mathrm{km}) \\
& 1 \text { inch }(\mathrm{in} .)=2.54 \text { centimeters }(\mathrm{cm}) \\
& 1 \text { mile per hour }(\mathrm{mph})=0.447 \text { meter } / \mathrm{second}(\mathrm{m} / \mathrm{s}) \\
& \text { degrees Fahrenheit }\left({ }^{\circ} \mathrm{F}\right)=\left(9 / 5 \times{ }^{\circ} \mathrm{C}\right)+32 \\
& \text { degrees Celsius }\left({ }^{\circ} \mathrm{C}\right)=5 / 9 \times\left({ }^{\circ} \mathrm{F}-32\right) \\
& 1 \text { langley }=1 \mathrm{gm}-\mathrm{cal} / \mathrm{cm}^{2}
\end{aligned}
$$





\section{Contents}

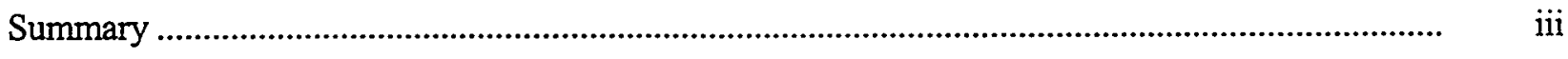

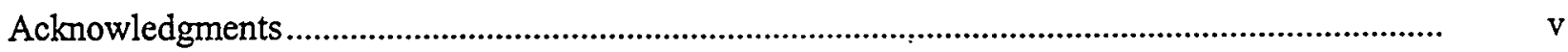

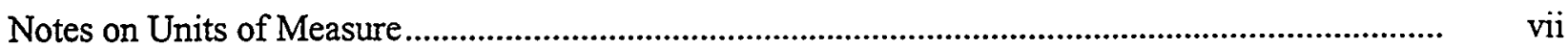

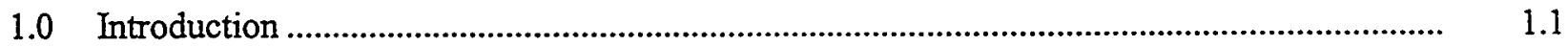

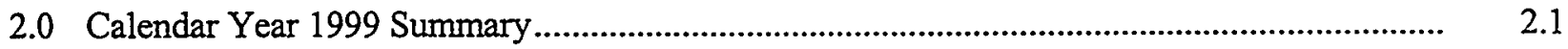

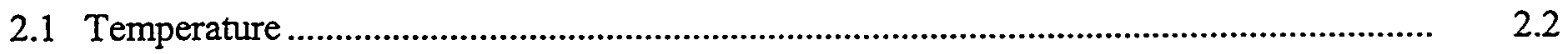

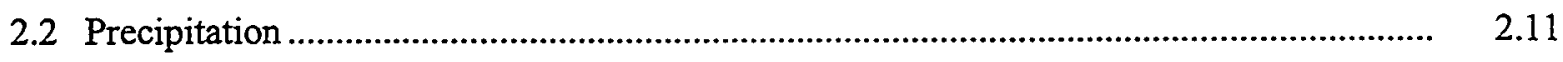

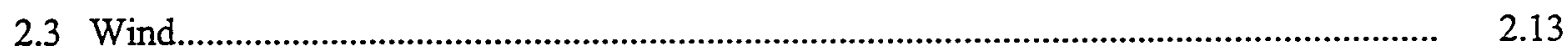

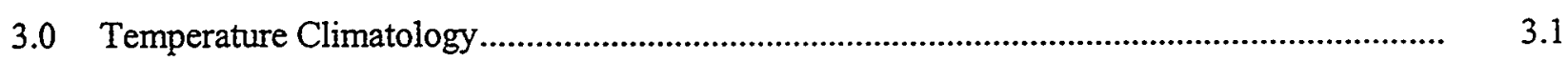

3.1 Monthly, Seasonal, and Annual Average....................................................................

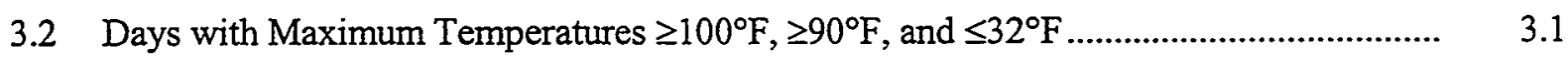

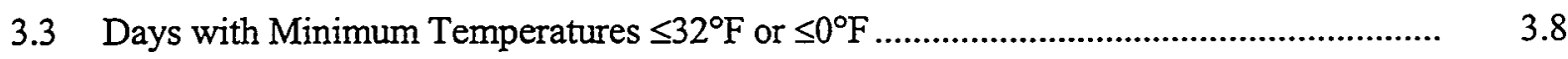

3.4 Monthly Extremes Daily Maximum and Minimum Temperatures.................................. $\quad 3.13$

3.5 Daily Temperature Distributions ................................................................................ $\quad 3.13$

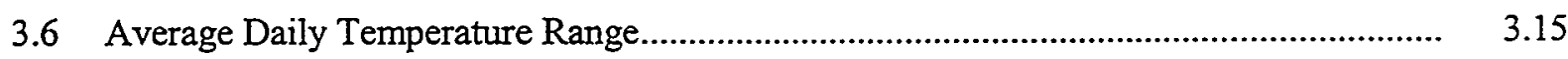

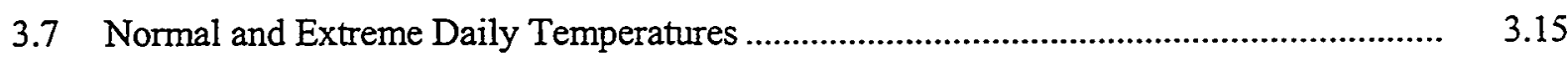

3.8 Subsurface Soil Temperatures ................................................................................ 3.25

3.9 Heating- and Cooling-Degree Days ......................................................................... 3.25

4.0 Precipitation Climatology ............................................................................................

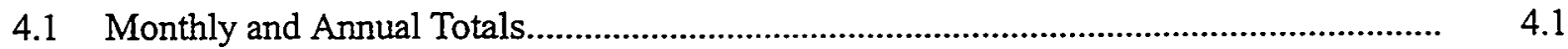

4.2 Total Monthly Precipitation Distributions ................................................................. 4.1

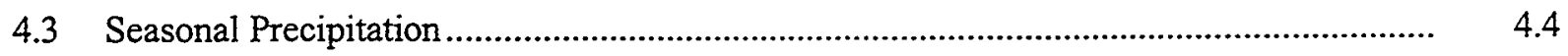

4.4 Average Number of Days with Specified Amounts of Precipitation ............................. 4.4 
4.5 Total Time with Precipitation Observed ..............................................................................

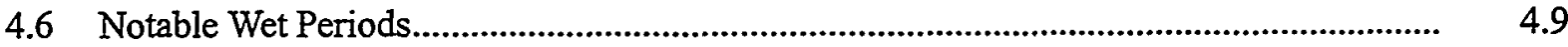

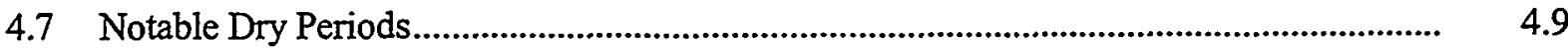

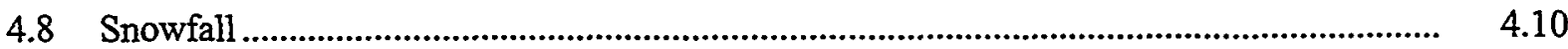

4.9 Normal and Maximum Daily Precipitation.............................................................. 4.15

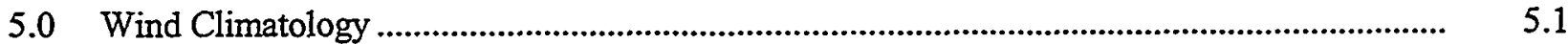

5.1 Monthly and Annual Prevailing Wind Directions, Average Speeds, and Peak Gusts ....... $\quad 5.1$

5.2 Days with Peak Gusts Above or Below Specific Thresholds ......................................... 5.1

5.3 Frequency of Monthly and Annual Wind Direction and Speed at 50-Foot Level ............. $\quad 5.3$

5.4 Composite Wind Roses and Joint Frequency Distributions for the Hanford Meteorological Monitoring Network ........................................................................ 5.4

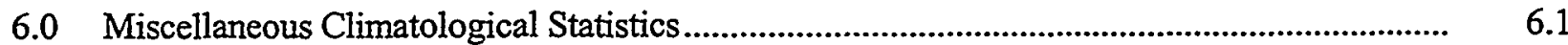

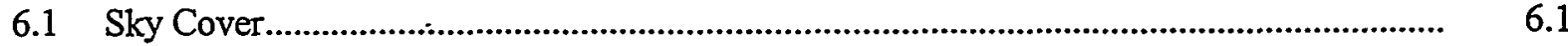

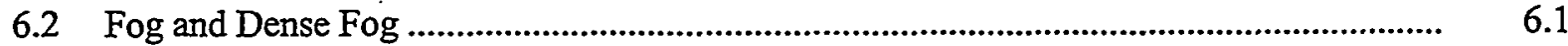

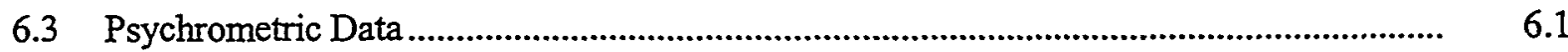

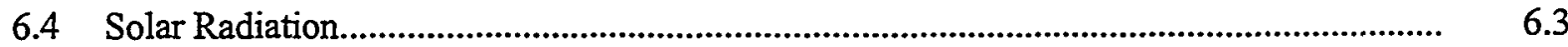

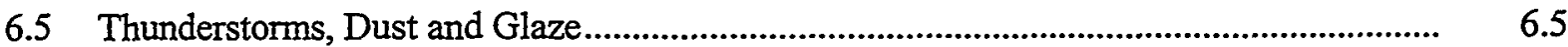

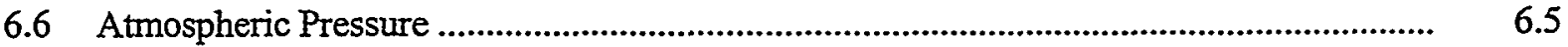

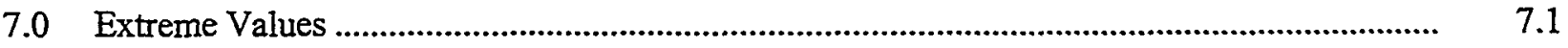

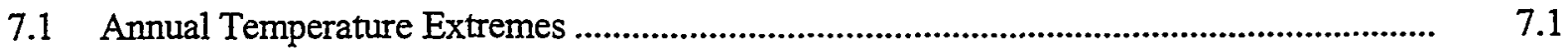

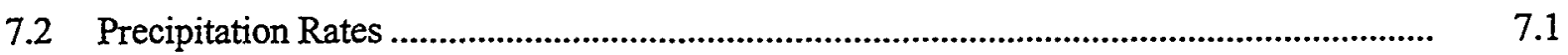

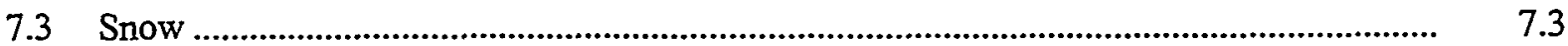

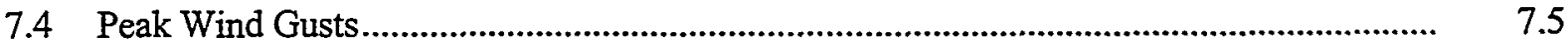

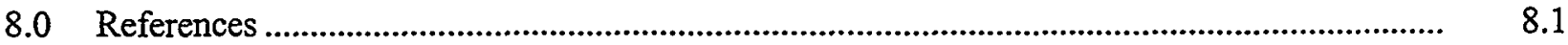

Appendix - 1999 Wind Climatology …...................................................................................... A.1 


\section{Figures}

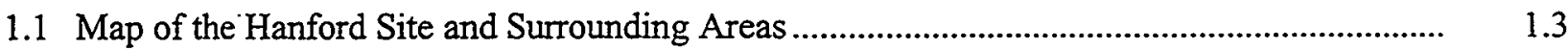

2.11999 Observed Daily Temperatures from the Hanford Meteorology Station ........................... 2.12

2.2 1999 Hanford Meteorological Monitoring Network Wind Roses at 10-Meter Level ............... $\quad 2.15$

2.3 1999 Hanford Meteorological Monitoring Network Wind Roses at 60-Meter Level ............... $\quad 2.16$

3.1 Graphical Presentation of Daily Maximum Temperatures......................................................... 3.14

3.2 Climatological Statistics of Daily Maximum Temperatures at the Hanford Meteorology Station.

3.3 Climatological Statistics of Daily Minimum Temperatures at the Hanford Meteorology Station.

4.1 Graphical Representation of Total Monthly Precipitation

4.2 Climatological Statistics of Total Monthly Precipitation

4.3 Climatological Statistics of Precipitation Accumulation

5.1 Hanford Meteorological Monitoring Network Wind Roses at 10-Meter Level, 1982 Through 1999

5.2 Hanford Meteorological Monitoring Network Wind Roses at 60-Meter Level, 1986 Through 1999

7.1 Probability of an Annual Maximum Temperature Exceeding a Given Value

7.2 Probability of an Annual Minimum Temperature Being Less Than a Given Value

7.3 Probability of Precipitation Rate Exceeding Given Values by Duration

7.4 Probability of Exceeding a Given Seasonal Snowfall

7.5 Probability of Exceeding a Given Snowfall in a Single Storm

7.6 Probability of Exceeding a Given Snow Depth

7.7 Probabilities of Peak Wind Gusts Exceeding Given Values. 


\section{Tables}

1.1 Station Numbers, Names, and Codes for the Hanford Meteorological Monitoring Network

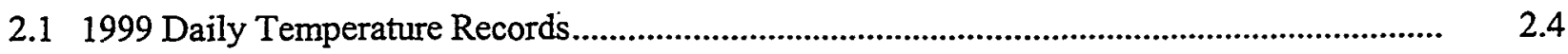

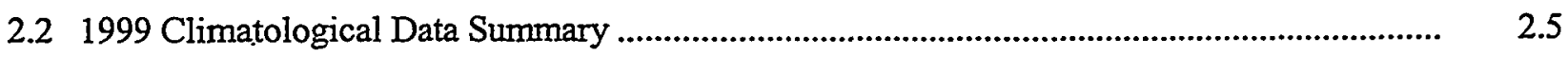

2.31999 Monthly and Seasonal Temperature and Precipitation................................................... 2.7

2.4 1999 Monthly and Annual Average Temperatures from the Hanford Meteorological Monitoring Network......................................................................................................... 2.8

2.5 1999 Monthly and Annual Average Precipitation from the Hanford Meteorological Monitoring Network........................................................................................................

2.6 1999 Monthly and Annual Average Wind Speed from the Hanford Meteorological Monitoring Network..................................................................................................... 2.10

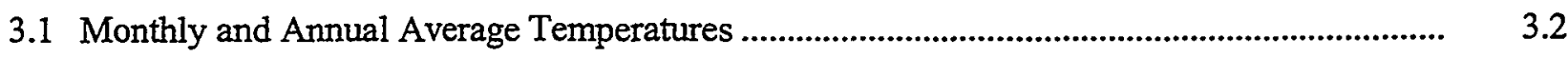

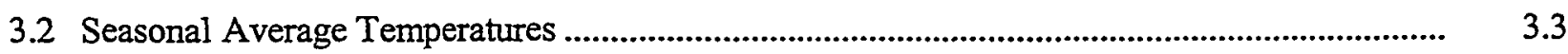

3.3 Monthly and Seasonal Number of Days with Maximum Temperatures Above or Below Certain Thresholds ....................................................................................................

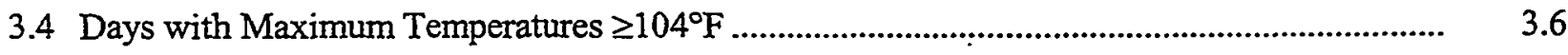

3.5 Record of Annual First and Last Dates with Maximum Temperatures $\geq 90^{\circ} \mathrm{F}$ and Minimum Temperatures $\leq 32^{\circ} \mathrm{F}$

3.6 Monthly and Annual Maximum Temperatures.....

3.7 Monthly and Seasonal Number of Days with Minimum Temperatures Below Certain Thresholds

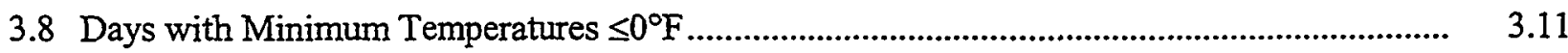

3.9 Monthly and Annual Minimum Temperatures................................................................ 3.12

3.10 Monthly Normal Temperatures and Monthly Extremes of Maximum and Minimum Temperatures 
3.12 Normal and Extreme Daily Maximum and Minimum Temperatures

3.13 Subsurface Soil Temperatures at Depths of $0.5,15$, and 36 Inches

3.14 Monthly and Seasonal Heating-Degree Days

3.15 Monthly and Annual Cooling-Degree Days

4.1 Monthly and Annual Precipitation

4.2 Seasonal Precipitation

4.3 Average Number of Days with Precipitation of Specified Amount

4.4 Monthly and Annual Averages and Extremes in Total Time with Precipitation Observed: July 1946 Through June 1971, July 1974 Through December 1999

4.5 Total Duration of Precipitation by Month and Year

4.6 Monthly and Seasonal Snowfall, Including First and Last Dates of Both Trace and Measurable Snowfalls

4.7 Snowfall - Greatest Amount from a Single Storm

4.8 Miscellaneous Snowfall Statistics, 1946 Through 1999

4.9 Maximum Precipitation

4.10 Normal and Maximum Daily Precipitation

5.1 Monthly and Annual Prevailing Wind Directions, Average Speeds, and Peak Gusts at 50-Foot Level, 1945 Through 1999

5.2 Number of Days with Peak Gusts Above or Below Specific Thresholds at 50-Foot Level, 1945 Through 1999

5.3 Frequency of Monthly and Annual Wind Direction and Speed at 50-Foot Level, 1955 Through 1999

5.4 Joint Frequency Distributions for Hanford Meteorological Monitoring Network Wind Stations at 10-Meter Level, 1982 Through 1999

5.5 Joint Frequency Distributions for Hanford Meteorological Monitoring Network Wind Stations at 60-Meter Level, 1986 Through 1999

6.1 Average Sky Cover, 1946 Through 1999, and Number of Days Clear, Partly Cloudy, and Cloudy, 1954 Through 1999 
6.2 Monthly and Annual Number of Days with Fog and Dense Fog, 1945 Through 1999 6.3

6.3 Monthly Averages and Extremes of Psychrometric Data, 1950 Through 1999

6.4

6.4 Average and Extreme Solar Radiation Daily Values, 1953 Through 1999

6.4

6.5 Average Number of Days of Various Meteorological Phenomena, 1945 Through 1999

6.5

6.6 Average and Extreme Station and Sea-Level Pressure Data, 1955 Through 1999.

6.7

7.1 Return Periods for Annual Maximum and Minimum Temperatures.

7.1

7.2 Precipitation Rates for 1 to 24 Hours Duration and Return Periods from 2 to 1,000 Years ......

7.3 Precipitation Amounts for 1 to 24 Hours Duration and Return Periods from 2 to 1,000 Years.

7.4 Snowfall Extremes for Return Periods from 2 to 1,000 Years.

7.5 Peak Wind Gusts for Return Periods from 2 to 10,000 Years. 


\subsection{Introduction}

The U.S. Department of Energy's Hanford Site lies within the semiarid shrub-steppe Pasco Basin of the Columbia Plateau in southeastern Washington state. The Hanford Site occupies an area of 560 square miles north of the confluence of the Snake and Yakima Rivers with the Columbia River. The Columbia River flows through the northern part of the Hanford Site and, turning south, forms part of the site's eastern boundary. The Yakima River runs along part of the southern boundary and joins the Columbia River below the city of Richland, which bounds the Hanford Site on the southeast. Rattlesnake Mountain, Yakima Ridge, and Umtanum Ridge form the southwestern and western boundaries. The Saddle Mountains form the northern boundary of the Hanford Site.

The Cascade Range, beyond Yakima to the west, greatly influences the climate of the Hanford Site area by means of its rain shadow effect. The regional temperatures, precipitation, and winds are greatly affected by the presence of mountain barriers. The Rocky Mountains and ranges in southern British Columbia are effective in protecting the inland basin from the more severe cold polar air masses moving southward across Canada and winter storms associated with them.

This document presents the calendar year 1999 climatological data summary for the Hanford Meteorology Station and additional climatologies for temperature, wind, precipitation, and other meteorological parameters for the Hanford Meteorology Station and the automated stations of the Hanford Meteorological Monitoring Network. Climatological normal and extreme values for temperature and precipitation are also presented. Currently, 30 monitoring stations are located within and near the U.S. Department of Energy's Hanford Site (Table 1.1, Figure 1.1). A detailed description of each of the monitoring stations, including photographs of the topography surrounding each site, is provided in Glantz and Islam (1988). A description of instrumentation and calibration is provided in PNNL et al. (1997).

Operation of the Hanford Meteorology Station is a function of the Meteorological and Climatological Services Project funded by the U.S. Department of Energy. This project, managed by the Pacific Northwest National Laboratory, is responsible for providing the U.S. Department of Energy and Hanford Site contractors ongoing meteorological and climatological services support, primarily for emergency response activities, Hanford Site work scheduling, and general site safety. Detailed, real-time meteorological data are needed in the event of a release of hazardous material to the atmosphere from any of the Hanford Site facilities. These data can be used to model atmospheric dispersion and to estimate the environmental impact of the release. Meteorological data and weather forecasts are also necessary to ensure that operations and activities on the Hanford Site are conducted safely, particularly where specific weather conditions might affect those operations or activities. The climatological database is also used in environmental studies, environmental impact reports, facility design, and planning operations.

During the period April 1912 through March 1943, cooperative observers for the U.S. Weather Bureau (now the National Weather Service) recorded daily maximum and minimum temperatures and precipitation, including measurements of unmelted snow at the Old Hanford Townsite $\sim 10$ miles eastnortheast of the present Hanford Meteorology Station. From late 1943 until mid 1944, the U.S. Weather 
Table 1.1. Station Numbers, Names, and Codes for the Hanford Meteorological Monitoring. Network

\begin{tabular}{|c|c|c|c|c|}
\hline $\begin{array}{l}\text { Station } \\
\text { Number }\end{array}$ & Station Name. & $\begin{array}{l}\text { Station } \\
\text { Code }\end{array}$ & $\begin{array}{c}\text { Station } \\
\text { Elevation }(\mathrm{ft})\end{array}$ & $\begin{array}{l}\text { Period of } \\
\text { Operation }\end{array}$ \\
\hline 1 & Prosser Barricade & PROS & 480 & $01 / 82$ - Present \\
\hline 2 & Emergency Operations Center & EOC & 1,240 & $01 / 82$ - Present \\
\hline 3 & Army Loop Road & ARMY & 565 & $01 / 82$ - Present \\
\hline 4 & Rattlesnake Springs & RSPG & 680 & $01 / 82$ - Present \\
\hline 5 & Edna & EDNA & 410 & 01/82 - Present \\
\hline 6 & 200 East & $200 \mathrm{E}$ & 680 & $01 / 82$ - Present \\
\hline 7 & 200 West & $200 \mathrm{~W}$ & 650 & 01/82 - Present \\
\hline $8 \mathrm{~B}$ & Beverly & BVLY & 555 & 08/91 - Present \\
\hline $8 \mathrm{~W}$ & Wahluke (no longer active) & WAHL & 855 & $01 / 82-07 / 91$ \\
\hline 9 & Fast Flux Test Facility & FFTF & 570 & 01/82 - Present \\
\hline 10 & Yakima Barricade & YAKB & 795 & 01/82 - Present \\
\hline 11 & 300 Area & $300 \mathrm{~A}$ & 390 & $01 / 82$ - Present \\
\hline 12 & Wye Barricade & WYEB & 550 & 01/82 - Present \\
\hline 13 & $100-N$ & $100 \mathrm{~N}$ & 460 & $01 / 82$ - Present \\
\hline 14 & WNP-2 & WPPS & 450 & 01/82 - Present \\
\hline 15 & Franklin County & FRNK & 875 & $01 / 82$ - Present \\
\hline 16 & Gable Mountain & GABL & 1,085 & 01/82 - Present \\
\hline 17 & Ringold & RING & 620 & $01 / 82$ - Present \\
\hline 18 & Richland Airport & RICH & 390 & 01/82 - Present \\
\hline $19 \mathrm{P}$ & Plutonium Finishing Plant-200W & PFP & 675 & 02/94 - Present \\
\hline 19S & Sagehill (no longer active) & SAGE & 985 & $03 / 82-01 / 93$ \\
\hline 20 & Rattlesnake Mountain & RMTN & 3,560 & $01 / 82$ - Present \\
\hline 21 & Hanford Meteorology Station & HMS & 733 & $01 / 82$ - Present \\
\hline 22 & Pasco Airport & PASC & 410 & 10/87 - Present \\
\hline 23 & Gable West & GABW & 490 & $03 / 86$ - Present \\
\hline 24 & $100-\mathrm{F}$ & $100 \mathrm{~F}$ & 410 & $03 / 86$ - Present \\
\hline 25 & Vernita Bridge & VERN & 430 & $02 / 88$ - Present \\
\hline 26 & Benton City & BENT & 1,055 & 02/95 - Present \\
\hline 27 & Tri-City Vocational Skills Center & VSTA & 505 & 02/91 - Present \\
\hline 28 & Roosevelt, WA & SURF & 360 & 09/94 - Present \\
\hline 29 & $100-K$ & $100 \mathrm{~K}$ & 450 & 03/96 - Present \\
\hline 30 & HAMMER & HAMR & 500 & $01 / 98$ - Present \\
\hline
\end{tabular}

Bureau recorded some meteorological operations in Richland. Then, in 1944 as part of the Manhattan Project, the Hanford Meteorology Station was established. Hourly observations began on December 7 , 1944.

The Hanford Meteorology Station and its 410-foot instrument tower are located near the center of the Hanford Site between the 200 West and 200 East Areas (see Figure 1.1). Hourly observations of wind direction, wind speed, and air temperature are made at multiple levels on the 410-foot tower. Throughout this document, wind measurements from the Hanford Meteorology Station are reported from the 50-foot level and temperature measurements are reported from the 3-foot level. A variety of other meteorological 


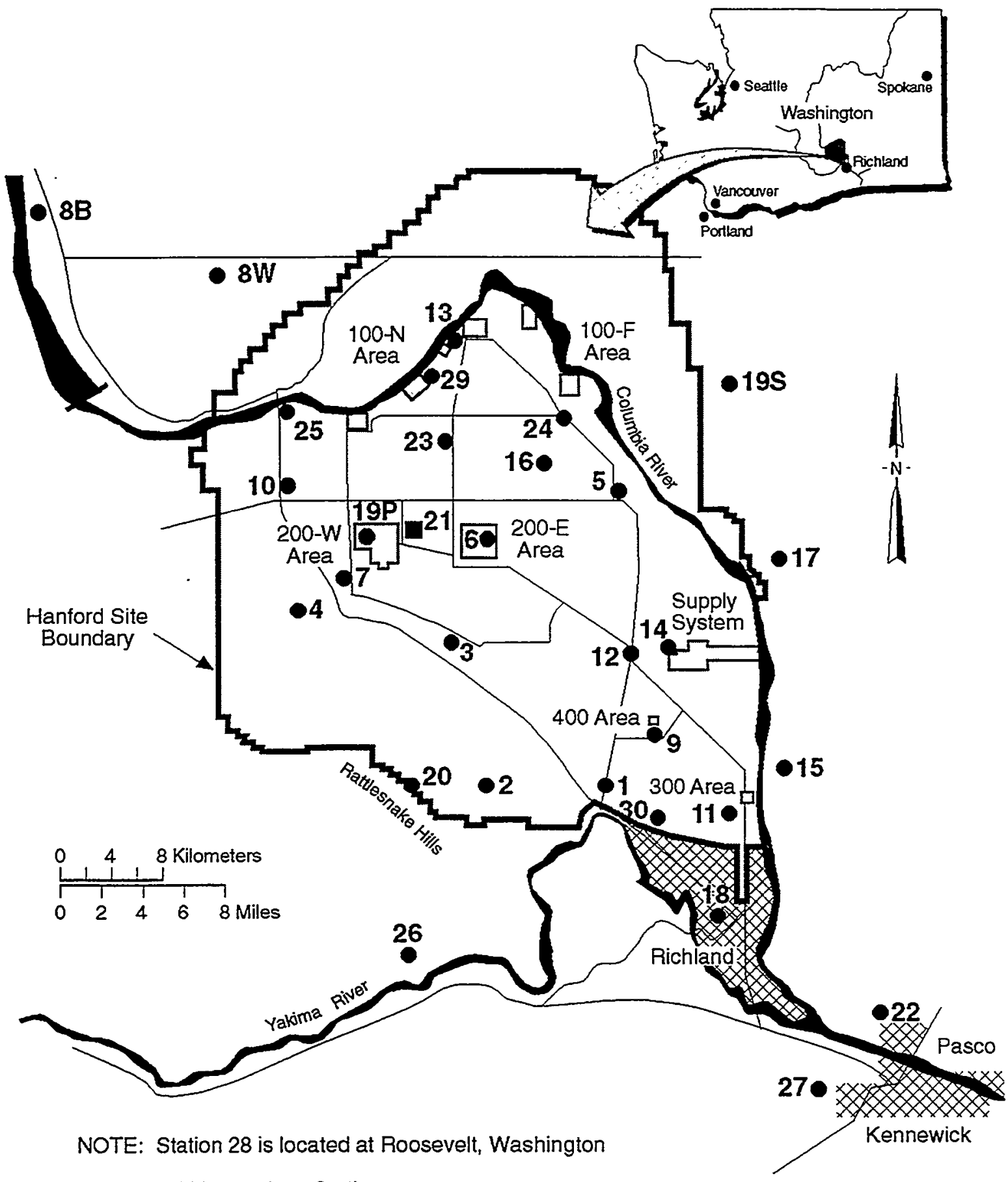

Hanford Meteorology Station

- Hanford Meteorological Monitoring Network Station

G00010107

Figure 1.1. Map of the Hanford Site and Surrounding Areas 
parameters are also measured or observed, including current weather, dew point temperature, relative humidity, precipitation, atmospheric pressure, cloud cover, visibility, and solar radiation. Several climatological summaries of data collected at the Hanford Meteorology Station, at the Old Hanford Townsite, and Richland monitoring locations were published over the past 30 years (Jenne and Kerns 1959; Stone et al. 1972, 1983; Hoitink and Burk 1994, 1995, 1996, 1997, 1998; Hoitink et al. 1999).

This document is composed of the following information. The 1999 calendar year summary of climatological data for the Hanford Site is contained in Section 2.0. Temperature, precipitation, wind, and miscellaneous climatological statistics are contained in Sections 3.0 through 6.0, respectively. Section 7.0 contains information on extreme value analysis. Section 8.0 lists the references cited in the document, and Section 9.0 provides a bibliography of database, computer code, and other pertinent reports. The appendix gives the station-specific wind roses and joint frequency distributions for 1999. 


\subsection{Calendar Year 1999 Summary}

This section summarizes climatology for calendar year 1999. More detailed information can be found in Section 3.0 Temperature Climatology, Section 4.0 Precipitation Climatology, and Section 5.0 Wind Climatology.

The 1998-99 winter season (December, January, and February) was much warmer than normal, averaging $37.7^{\circ} \mathrm{F}, 4.1^{\circ}$ above normal $\left(33.6^{\circ} \mathrm{F}\right)$, and tied the winter of $1997-98$ as the sixth warmest winter on record. The warmest was $40.6^{\circ} \mathrm{F}$ in $1966-67$, and the coldest was $24.2^{\circ} \mathrm{F}$ in $1948-49$. The coldest temperature during the winter was $-1{ }^{\circ} \mathrm{F}$ on December 21. Precipitation for the 1998-99 winter season was below normal, totaling 2.03 inches, $83 \%$ of normal ( 2.44 inches). The wettest winter, in 1996-97, received 5.45 inches; while the driest, $1946-47$, received only 0.70 inch.

Spring 1999 (March, April, and May) was cooler than normal, averaging $51.7^{\circ} \mathrm{F}, 1.5^{\circ}$ below normal $\left(53.2^{\circ} \mathrm{F}\right)$. The warmest spring, in 1992 , averaged $58.2^{\circ} \mathrm{F}$, while the coolest (1955) averaged $48^{\circ} \mathrm{F}$. Spring 1999 was the sixth driest on record with precipitation totaling 0.40 inch, $29 \%$ of normal (1.40 inches). The wettest spring, in 1995, received 3.28 inches, while the driest (1968) received only 0.09 inch.

The summer of 1999 (June, July, and August) was cooler than normal, averaging $72.5^{\circ} \mathrm{F}, 1.2^{\circ}$ below normal $\left(73.7^{\circ} \mathrm{F}\right)$. The hottest summer, in 1958 , averaged $78.2^{\circ} \mathrm{F}$, while the coolest, in 1980 , averaged $70.2^{\circ} \mathrm{F}$. The highest temperature was $105^{\circ} \mathrm{F}$ on July 28 . Precipitation for the summer season totaled 0.95 inch, $115 \%$ of normal ( 0.83 inch). The wettest summer, in 1950 , received 2.99 inches, while the driest, in 1973, received only 0.03 inch.

Autumn 1999 (September, October, and November) was warmer and drier than normal. The average temperature was $54.2^{\circ} \mathrm{F}, 1.3^{\circ} \mathrm{F}$ above normal $\left(52.9^{\circ} \mathrm{F}\right)$. The warmest autumn $(1990)$ averaged $57.1^{\circ} \mathrm{F}$, while the coolest (1985) averaged $44.5^{\circ} \mathrm{F}$. Precipitation totaled $0.74 \mathrm{inch}, 46 \%$ of normal ( $1.60 \mathrm{inches}$ ). The wettest autumn (1973) received 4.79 inches, while the driest (1976) received only 0.04 inch.

The atmospheric pressure on Christmas Day reached 30.91 inches of mercury, the highest pressure since 31.12 inches on January 1,1979 (the highest on record). 
The following are some additional statistics for 1999:

\begin{tabular}{|c|c|c|c|c|}
\hline \multirow[b]{2}{*}{ Category } & \multirow{2}{*}{$\begin{array}{l}\text { Number } \\
\text { of Days }\end{array}$} & \multirow[b]{2}{*}{ Normal } & \multicolumn{2}{|c|}{ Record } \\
\hline & & & Maximum & Minimum \\
\hline Maximum temperatures $\geq 100^{\circ} \mathrm{F}$ & 7 & 13 & 28 & 1 \\
\hline Maximum temperatures $\geq 90^{\circ} \mathrm{F}$ & 49 & 52 & 79 & 29 \\
\hline Maximum temperatures $\leq 32^{\circ} \mathrm{F}$ & 7 & 24 & 58 & 2 \\
\hline Minimum temperatures $\geq 70^{\circ} \mathrm{F}$ & 9 & 7 & 21 & 0 \\
\hline Minimum temperatures $\leq 32^{\circ} \mathrm{F}$ & 95 & 107 & 139 & 77 \\
\hline Minimum temperatures $\leq 0^{\circ} \mathrm{F}$ & 0 & 3 & 18 & 0 \\
\hline Thunderstorms & 10 & 10 & 23 & 3 \\
\hline Fog (visibility $\leq 6 \mathrm{mi}$ ) & 27 & 45 & 76 & 22 \\
\hline Dense fog (visibility $\leq 0.25 \mathrm{mi}$ ) & 15 & 24 & 42 & 9 \\
\hline Peak wind gusts $\leq 12 \mathrm{mph}$ & 34 & 50 & 87 & 28 \\
\hline Peak wind gusts $\geq 25 \mathrm{mph}$ & 192 & 155 & 192 & 123 \\
\hline Peak wind gusts $\geq 40 \mathrm{mph}$. & 48 & 26 & 57 & 10 \\
\hline Peak wind gusts $\geq 50 \mathrm{mph}$ & 10 & 5 & 18 & 0 \\
\hline
\end{tabular}

\subsection{Temperature}

Calendar year 1999 was warmer than normal at the Hanford Meteorology Station. The average temperature was $54.4^{\circ} \mathrm{F}, 1.1^{\circ}$ above normal $\left(53.3^{\circ} \mathrm{F}\right)$. The warmest years on record are 1992 and 1998 , which averaged $56.4^{\circ} \mathrm{F}$, while the coldest year on record is 1985 , which averaged $49.6^{\circ} \mathrm{F}$. The hottest temperature of 1999 was $105^{\circ} \mathrm{F}$ on July 28 , while the coldest was $18^{\circ} \mathrm{F}$ on January 3.

The year was evenly divided with 6 months warmer than normal, and 6 months cooler than normal. Four months were $3^{\circ}$ or more above normal, with January warmer by $7^{\circ}$. January was the fifth warmest on record, November the third warmest, and December the fifth warmest. Only one month (May) was more than $3^{\circ}$ cooler than normal, and that by $3.4^{\circ}$. January 1999 was much warmer than normal, averaging $38.3^{\circ} \mathrm{F}, 7^{\circ}$ above normal $\left(31.3^{\circ} \mathrm{F}\right)$, and was the fifth warmest January on record. The warmest January occurred in 1953 and averaged $42.5^{\circ} \mathrm{F}$, while the coldest was $12.1^{\circ} \mathrm{F}$ in 1950 .

January 1999 was much warmer than normal, averaging $38.3^{\circ} \mathrm{F}, 7^{\circ}$ above normal $\left(31.3^{\circ} \mathrm{F}\right)$, and was the fifth warmest January on record. The warmest January occurred in 1953 and averaged $42.5^{\circ} \mathrm{F}$, while the coldest was $12.1^{\circ} \mathrm{F}$ in 1950 .

February 1999 was warmer than normal, averaging $41.7^{\circ} \mathrm{F}, 3.7^{\circ}$ above normal $\left(38^{\circ} \mathrm{F}\right)$. The warmest February occurred in 1958 and averaged $44.5^{\circ} \mathrm{F}$, while the coldest was $25.6^{\circ} \mathrm{F}$ in 1956.

March 1999 was slightly warmer than normal, averaging $46.3^{\circ} \mathrm{F}, 0.7^{\circ}$ above normal $\left(45.6^{\circ} \mathrm{F}\right)$. The warmest March occurred in 1992 and averaged $51.5^{\circ} \mathrm{F}$, while the coldest was $39.4^{\circ} \mathrm{F}$ in 1955 .

April 1999 was cooler than normal, averaging $50.9^{\circ} \mathrm{F}, 1.8^{\circ}$ below normal $\left(52.7^{\circ} \mathrm{F}\right)$. The warmest April occurred in 1994 and averaged $58.2^{\circ} \mathrm{F}$, while the coldest was $47.5^{\circ} \mathrm{F}$ in 1955 . 
May 1999 was cooler than normal, averaging $57.9^{\circ} \mathrm{F}, 3.4^{\circ}$ below normal $\left(61.3^{\circ} \mathrm{F}\right)$. The first three weeks of the month averaged $7.2^{\circ}$ below normal, with the first above normal day for the month not occurring until May 22. The warmest May occurred in 1947 and averaged $68.7^{\circ} \mathrm{F}$, while the coolest was $56^{\circ} \mathrm{F}$ in 1984.

June 1999 was cooler than normal, averaging $67.4^{\circ} \mathrm{F}, 2.3^{\circ}$ below normal $\left(69.7^{\circ} \mathrm{F}\right)$. The warmest June occurred in 1992 and averaged $76.8^{\circ} \mathrm{F}$, while the coolest was $63.0^{\circ} \mathrm{F}$ in 1953.

July 1999 was cooler than normal, averaging $73.8^{\circ} \mathrm{F}, 2.4^{\circ}$ below normal $\left(76.2^{\circ} \mathrm{F}\right)$. This was the fourth consecutive month with a below normal average temperature following a period (August 1997 through March 1999) when the monthly average temperature was above normal for 19 out of 20 months. The warmest July occurred in 1985 and averaged $82.2^{\circ} \mathrm{F}$, while the coolest was $70.5^{\circ} \mathrm{F}$ in 1993 .

August 1999 was slightly warmer than normal, averaging $76.2^{\circ} \mathrm{F}, 1.1^{\circ}$ above normal $\left(75.1^{\circ} \mathrm{F}\right)$. The warmest August occurred in 1967 and averaged $81.5^{\circ} \mathrm{F}$, while the coolest was $69.8^{\circ} \mathrm{F}$ in 1964 .

September 1999 was slightly cooler than normal, averaging $65.0^{\circ} \mathrm{F}, 0.7^{\circ}$ below normal $\left(65.7^{\circ} \mathrm{F}\right)$. The warmest September occurred in 1990 and averaged $72.4^{\circ} \mathrm{F}$, while the coolest was $58.8^{\circ} \mathrm{F}$ in 1985 . For calendar year 1999 there were 49 days with maximum temperatures $\geq 90^{\circ} \mathrm{F}$ compared to a normal of 52 days, and 7 days with maximum temperatures $\geq 100^{\circ} \mathrm{F}$ compared to a normal of 13 days.

October 1999 was cooler than normal, averaging $51.8^{\circ} \mathrm{F}, 1.1^{\circ}$ below normal $\left(52.9^{\circ} \mathrm{F}\right)$. The warmest October occurred in 1988 and averaged $59.6^{\circ} \mathrm{F}$, while the coolest was $47.9^{\circ} \mathrm{F}$ in 1984 . The first official (measured at the Hanford Meteorology Station) freezing temperature (minimum temperature $\leq 32^{\circ} \mathrm{F}$ ) occurred on October 17. The average date for the first freezing temperature is October 20, with the earliest occurring on September 25, 1972, and the latest on November 12, 1962.

November 1999 was the third warmest November on record, averaging $45.8^{\circ} \mathrm{F}, 5.6^{\circ}$ above normal $\left(40.2^{\circ} \mathrm{F}\right)$. The average temperature for every day after November 5 was normal or above, with the period from November 8 through 17 averaging $11.4^{\circ}$ above normal. The maximum temperature of $76^{\circ} \mathrm{F}$ on November 13 was the warmest temperature ever recorded in November, breaking the record of $75^{\circ} \mathrm{F}$ recorded just the day before, and also on November 3, 1975. The warmest November occurred in 1990 and averaged $46.5^{\circ} \mathrm{F}$, while the coolest was $24.8^{\circ} \mathrm{F}$ in 1985 .

December 1999 was the fifth warmest December on record, averaging $37.7^{\circ} \mathrm{F}, 6.3^{\circ}$ above normal $\left(31.4^{\circ} \mathrm{F}\right)$. The period from December $11-19$ averaged $14.1^{\circ}$ above normal. The warmest December occurred in 1957 and averaged $38.5^{\circ} \mathrm{F}$, while the coolest was $21.0^{\circ} \mathrm{F}$ in 1985.

Table 2.1 lists the daily temperature records for 1999 along with the previous record and year of occurrence. Table 2.2 lists the monthly and annual totals for numerous meteorological parameters for 1999. Table 2.3 lists the 1999 monthly and seasonal temperature and precipitation compared to normals and extremes. Tables 2.4, 2.5, and 2.6 list the 1999 monthly and annual average temperature, precipitation, and wind speed, respectively, from the Hanford Meteorological Monitoring Network. 
Table 2.1. 1999 Daily Temperature Records (previous record and year of occurrence in parentheses)

\begin{tabular}{|c|c|c|c|c|}
\hline \multirow[b]{2}{*}{ Date } & \multicolumn{2}{|c|}{ Maximum } & \multicolumn{2}{|c|}{ Minimum } \\
\hline & High & Low & High & Low \\
\hline $\operatorname{Jan} 14$ & $62(60,1961)$ & & & \\
\hline $\operatorname{Jan} 28$ & & & $42(41,1953)$ & \\
\hline $\operatorname{Jan} 29$ & & & $47(44,1992)$ & \\
\hline Feb 6 & & & $44(37,1961)$ & \\
\hline Feb 22 & & & $42^{(a)}(42,1958)$ & \\
\hline May 7 & & $59(61,1990)$ & & \\
\hline May 8 & & $56^{(\mathrm{a})}(56,1962)$ & & \\
\hline May 9 & & & & $34^{(\mathrm{a})}\left(34,1996^{[\mathrm{b}]}\right)$ \\
\hline May 24 & $97(96,1962)$ & & & \\
\hline $\operatorname{Jun} 2$ & & $65^{(\mathrm{a})}(65,1980)$ & & \\
\hline Jun 6 & & & & $38(46,1962)$ \\
\hline $\operatorname{Jun} 7$ & & & & $42(44,1961)$ \\
\hline Jun 9 & & & & $42(44,1985)$ \\
\hline Jun 13 & $\cdot$ & & $70(67,1989)$ & \\
\hline Jun 15 & $102^{(a)}(102,1961)$ & & & \\
\hline Jul 3 & & & & $45(46,1990)$ \\
\hline Jul 5 & & & & $47^{(a)}(47,1980)$ \\
\hline Jul 26 & & & & $53(54,1948)$ \\
\hline Aug 3 & & & $75^{(a)}(75,1994)$ & \\
\hline Aug 30 & & $64^{(\mathrm{c})}(68,1951)$ & & \\
\hline Aug 31 & & $72^{(2)}(72,1971)$ & & $43(45,1965)$ \\
\hline Sep 1 & & & & $43(44,1980)$ \\
\hline Oct 3 & & & & $39^{(\mathrm{a})}\left(33,1973^{[\mathrm{b}]}\right)$ \\
\hline Oct 13 & $81(79,1945)$ & & & \\
\hline Oct 27 & & $43^{(\mathrm{a})}\left(43,1993^{[\mathrm{b}]}\right)$ & & \\
\hline Nov 12 & $75(65,1991)$ & & & \\
\hline Nov 13 & $76^{(d)}(64,1987)$ & & & \\
\hline $\operatorname{Dec} 12$ & $57^{(2)}(57,1988)$ & & & \\
\hline Dec 15 & & & $49(41,1956)$ & \\
\hline Dec 16 & $56(56,1956)$ & & $44(43,1973)$ & \\
\hline Dec 18 & $56^{(2)}(56,1956)$ & & $42(38,1994)$ & \\
\hline
\end{tabular}

\footnotetext{
(a) Ties record.

(b) Most recent of several occurrences.

(c) All-time low maximum for month of August, previous record $67^{\circ} \mathrm{F}$ on August 20, 1959.

(d) All-time high maximum for month of November, previous record $75^{\circ} \mathrm{F}$ on November 12,1999 and November 3, 1975 .
} 
Table 2.2. 1999 Climatological Data Summary

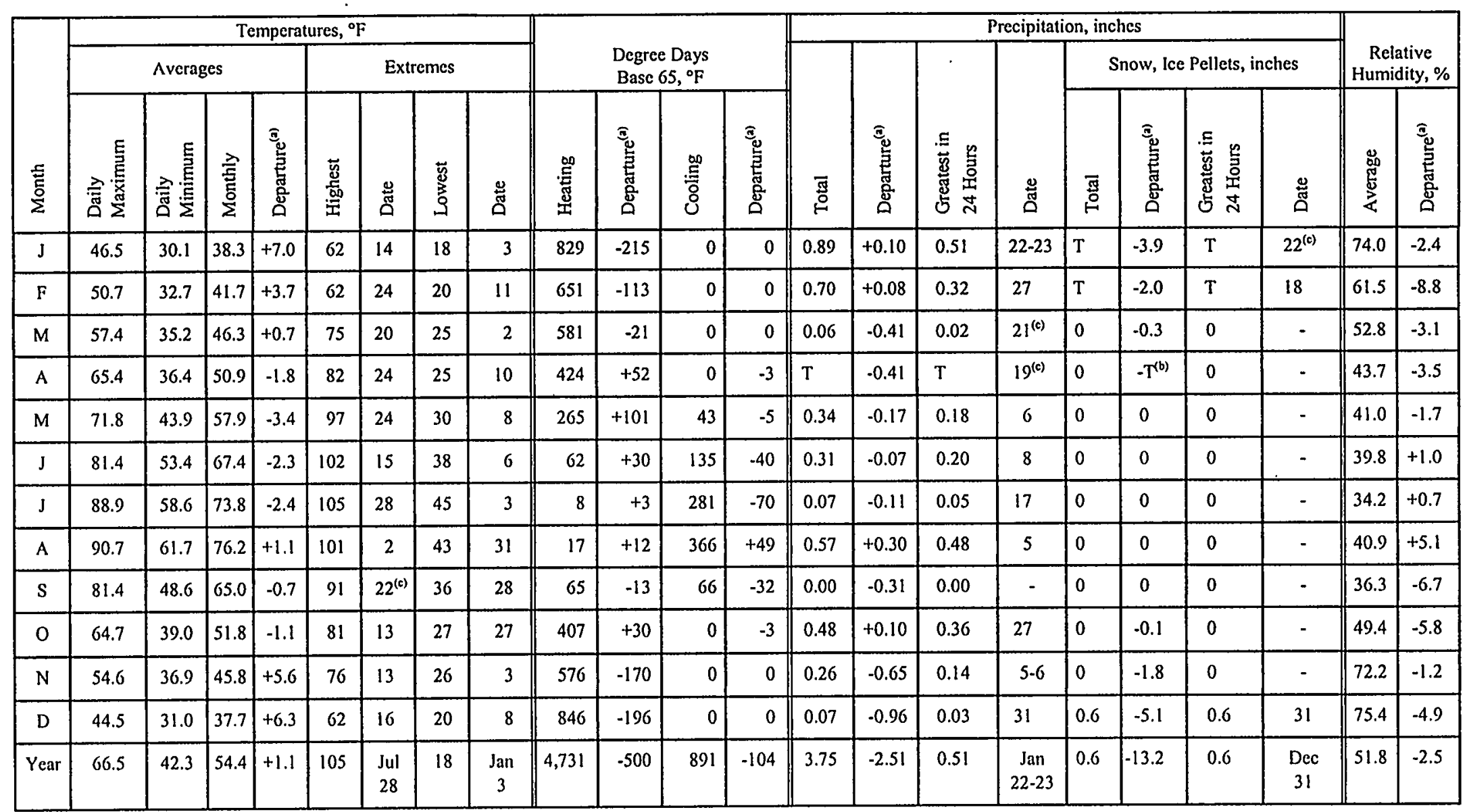




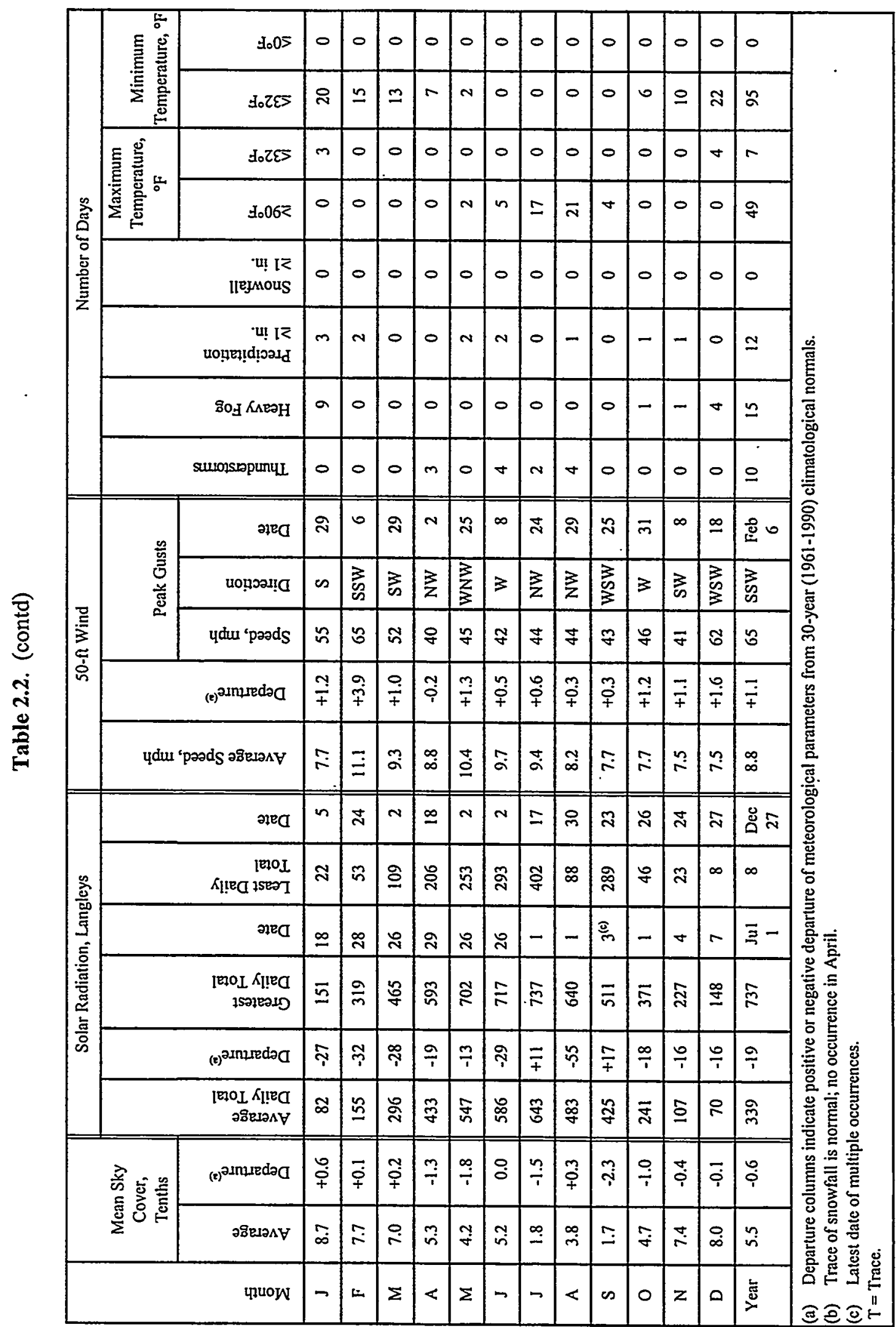




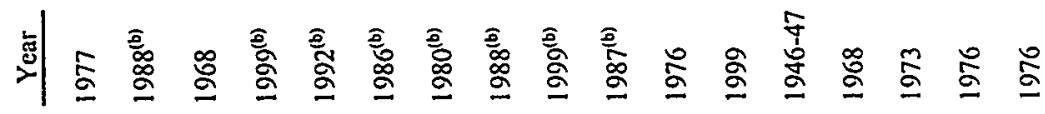
总离递向|

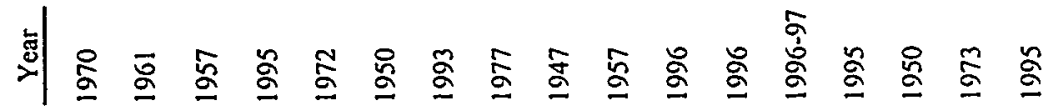

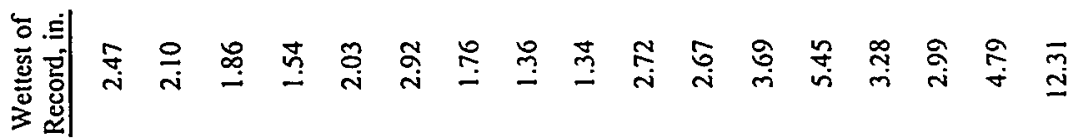

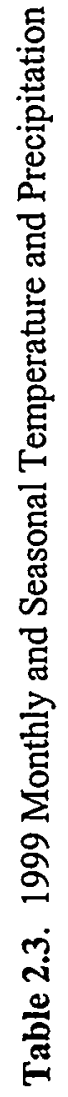

$$
\text { 㣊| }
$$

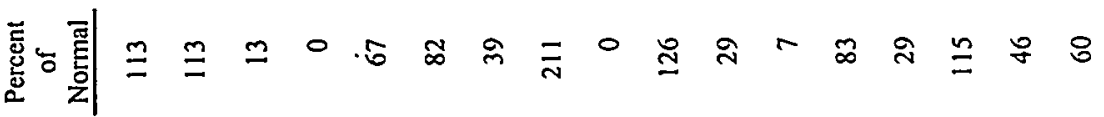

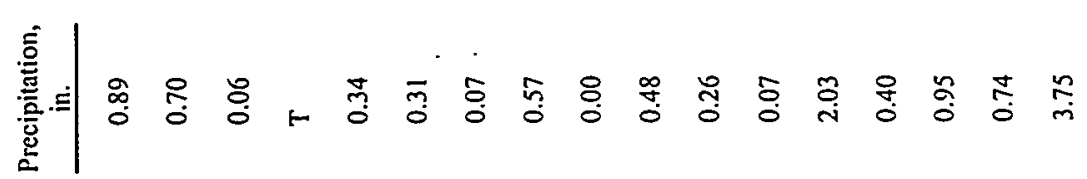

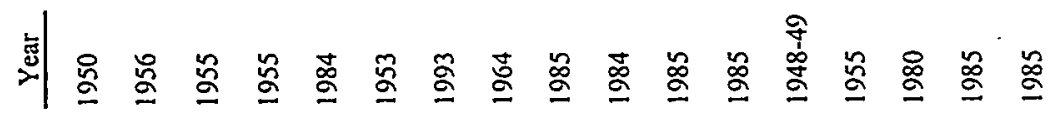
产密范| ๖|

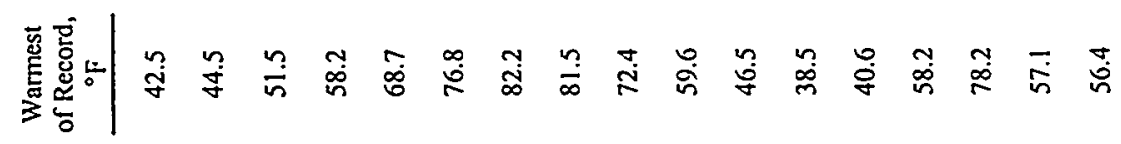

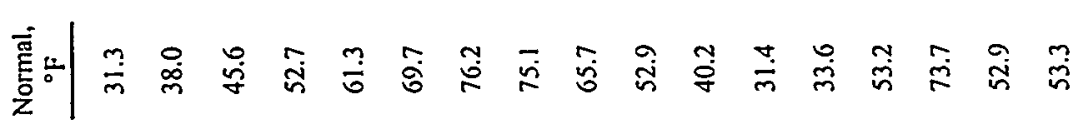
罤

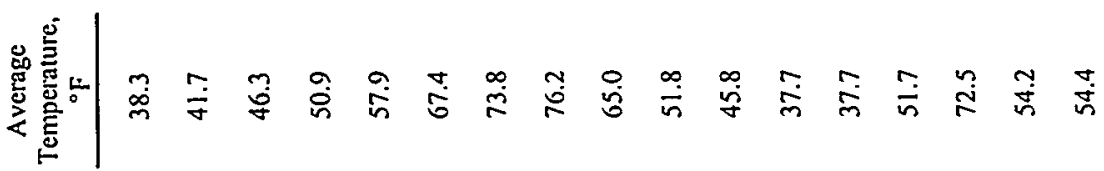

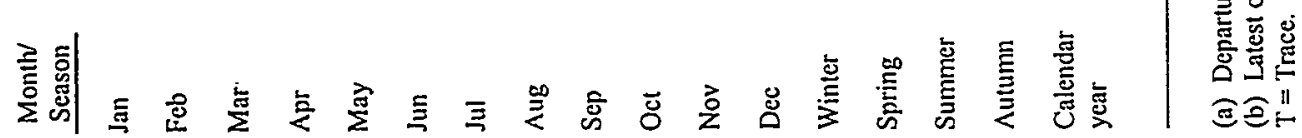


Table 2.4. 1999 Monthly and Annual Average Temperatures $\left({ }^{\circ} \mathrm{F}\right)$ from the Hanford Meteorological Monitoring Network

\begin{tabular}{|c|c|c|c|c|c|c|c|c|c|c|c|c|c|}
\hline Station & Jan & Feb & Mar & Apr & May & Jun & Jul & Aug & Sep & Oct & Nov & Dec & Annual \\
\hline 1 PROS & 38.3 & 41.8 & 45.5 & 50.7 & 58.0 & 68.0 & 74.3 & 76.5 & 62.6 & 50.7 & 45.6 & 38.4 & 54.3 \\
\hline 2 EOC & 39.8 & 41.4 & 45.5 & 51.7 & 57.3 & 66.5 & 73.7 & 76.4 & 65.9 & 53.3 & 47.0 & 38.2 & 54.8 \\
\hline 3 ARMY & 38.1 & 42.5 & 46.0 & 51.7 & 58.8 & 68.3 & 75.2 & 77.4 & 64.5 & 51.8 & 47.3 & 38.8 & 55.2 \\
\hline 4 RSPG & 39.3 & 42.8 & 45.6 & 51.3 & 58.1 & 67.5 & 74.2 & 76.8 & 63.6 & 51.0 & 45.7 & 38.7 & 54.6 \\
\hline 5 EDNA & 41.1 & 45.5 & 47.2 & 52.8 & 59.2 & 68.3 & 74.4 & 76.4 & 63.1 & 51.1 & 45.5 & 38.2 & 55.3 \\
\hline $6200 \mathrm{E}$ & 38.7 & 42.3 & 46.7 & 53.1 & 59.7 & 68.9 & 75.9 & 77.9 & 66.4 & 53.4 & 47.0 & 39.2 & 55.9 \\
\hline $7200 \mathrm{~W}$ & 37.3 & 41.1 & 45.0 & 50.3 & 58.0 & 67.8 & 74.6 & 76.6 & 62.5 & 50.1 & 45.3 & 38.1 & 54.0 \\
\hline $8 \mathrm{BVLY}$ & 38.6 & 41.5 & 46.4 & 52.7 & 59.0 & 67.7 & 74.0 & 76.0 & 64.3 & 52.4 & 46.7 & 39.0 & 54.9 \\
\hline 9 FFIF & 39.1 & 42.1 & 45.9 & 51.7 & 58.5 & 67.9 & 74.5 & 76.7 & 64.6 & 52.4 & 46.8 & 38.8 & 55.0 \\
\hline 10 YAKB & 37.8 & 41.1 & 45.9 & 52.1 & 58.6 & 68.0 & 75.0 & 77.2 & 65.8 & 52.5 & 46.2 & 38.3 & 55.0 \\
\hline $11300 \mathrm{~A}$. & 39.6 & 42.6 & 45.7 & 51.4 & 58.4 & 67.8 & 73.7 & 75.8 & 63.0 & 51.5 & 47.0 & 39.9 & 54.7 \\
\hline 12 WYEB & 38.3 & 41.9 & 45.7 & 51.9 & 58.7 & 68.2 & 75.0 & 77.0 & 64.4 & 52.1 & 46.0 & 38.5 & 54.9 \\
\hline $13100 \mathrm{~N}$ & 37.5 & 40.9 & 45.4 & 51.6 & 58.3 & 67.8 & 73.9 & 75.9 & 64.0 & 52.0 & 45.6 & 38.4 & 54.3 \\
\hline 14 WPPS & 38.3 & 41.8 & 45.2 & 50.7 & 58.3 & 68.4 & 74.9 & 77.0 & 63.4 & 51.7 & 46.1 & 38.7 & 54.6 \\
\hline 15 FRNK & 38.8 & 41.2 & 45.0 & 50.6 & 56.9 & 65.1 & 70.7 & 72.2 & 61.8 & 51.0 & 45.6 & 38.2 & 53.2 \\
\hline $16 \mathrm{GABL}$ & 39.1 & 41.3 & 45.7 & 52.3 & 58.0 & 66.7 & 74.2 & 76.7 & 66.9 & 53.8 & 46.6 & 38.2 & 55.1 \\
\hline 17 RING & 38.2 & 41.5 & 45.6 & 50.3 & 57.4 & 64.9 & 70.7 & 72.8 & 60.8 & 50.3 & 45.3 & 38.5 & 53.0 \\
\hline $18 \mathrm{RICH}$ & 40.8 & 43.4 & 46.5 & 52.3 & 59.0 & 68.0 & 74.0 & 76.5 & 64.0 & 52.7 & 47.5 & 40.2 & 55.5 \\
\hline 19 PFP & 37.9 & 41.6 & 45.8 & 51.7 & 58.5 & 67.8 & 74.9 & 77.1 & 65.0 & 52.1 & 46.0 & 38.4 & 54.8 \\
\hline 20 RMTN & 33.5 & 31.6 & 36.0 & 42.4 & 47.6 & 56.8 & 64.8 & 68.1 & 59.0 & 47.0 & 41.3 & 30.6 & 46.7 \\
\hline 21 HMS & 38.3 & 41.7 & 46.3 & 50.9 & 57.9 & 67.4 & 73.8 & 76.2 & 65.0 & 51.8 & 45.8 & 37.7 & 54.4 \\
\hline 22 PASC & 40.2 & 43.4 & 46.4 & 52.2 & 59.5 & 68.6 & 74.3 & 76.8 & 63.3 & 52.0 & 46.8 & 39.9 & 55.4 \\
\hline $23 \mathrm{GABW}$ & 36.7 & 40.7 & 44.8 & 50.5 & 58.0 & 68.0 & 74.4 & 76.1 & 62.5 & 50.4 & 45.0 & 37.5 & 53.8 \\
\hline $24100 F$ & 37.6 & 41.4 & 45.3 & 51.2 & 58.5 & 68.2 & 74.4 & 76.4 & 63.3 & 51.1 & 45.3 & 38.3 & 54.3 \\
\hline 25 VERN & 38.4 & 41.9 & 46.6 & 53.0 & 59.6 & 68.3 & 74.8 & 77.1 & 65.7 & 53.3 & 47.4 & 39.5 & 55.5 \\
\hline 26 BENT & 38.3 & 40.5 & 44.8 & 51.0 & 56.7 & 66.3 & 72.4 & 74.4 & 63.9 & 51.6 & 45.4 & 37.4 & 53.6 \\
\hline 27 VSTA & 41.6 & 44.0 & 47.0 & 53.0 & 59.4 & 68.2 & 74.4 & 76.5 & 64.4 & 52.9 & 48.1 & 40.5 & 55.9 \\
\hline 28 SURF & 40.6 & 42.2 & 45.1 & 51.5 & 57.3 & 66.3 & 73.4 & 75.9 & 64.1 & 52.2 & 46.6 & 40.3 & 54.6 \\
\hline $29100 \mathrm{~K}$ & 37.4 & 41.3 & 45.8 & 51.9 & 58.9 & 68.4 & 74.8 & 76.5 & 64.4 & 52.1 & 45.9 & 38.4 & 54.7 \\
\hline 30 HAMR & 40.8 & 42.8 & 46.2 & 51.8 & 58.7 & 67.7 & 74.0 & 76.7 & 63.8 & 51.9 & 47.8 & 39.6 & 55.4 \\
\hline
\end{tabular}


Table 2.5. 1999 Monthly and Annual Average Precipitation (inches) from the Hanford Meteorological Monitoring Network ${ }^{(a)}$

\begin{tabular}{|c|c|c|c|c|c|c|c|c|c|c|c|c|c|}
\hline Station & Jan & Feb & Mar & Apr & May & Jun & Jul & Aug & Sep & Oct & Nov & Dec & Annual \\
\hline I PROS & 0.74 & 0.74 & 0.07 & 0.06 & 0.39 & 0.03 & 0.01 & 0.11 & 0.00 & 0.33 & 0.39 & 0.35 & 3.22 \\
\hline $2 \mathrm{EOC}$ & 1.17 & 1.65 & 0.14 & 0.11 & 0.46 & 0.13 & 0.00 & 0.31 & 0.00 & 0.46 & 0.81 & 0.64 & 5.88 \\
\hline 3 ARMY & 0.66 & 0.52 & 0.02 & 0.01 & 0.27 & 0.04 & 0.01 & 0.17 & 0.00 & 0.45 & 0.39 & 0.15 & 2.69 \\
\hline $4 \mathrm{RSPG}$ & 0.58 & 0.51 & 0.09 & 0.21 & 0.22 & 0.08 & 0.11 & 0.12 & 0.02 & 0.41 & 0.40 & 0.23 & 2.98 \\
\hline $6200 E$ & 0.77 & 0.52 & 0.03 & 0.01 & 0.42 & 0.06 & 0.03 & 0.34 & 0.00 & 0.36 & 0.18 & 0.02 & 2.74 \\
\hline $7200 \mathrm{~W}$ & 0.59 & 0.35 & 0.07 & 0.02 & 0.24 & 0.48 & 0.10 & 0.08 & 0.00 & 0.44 & 0.34 & 0.16 & 2.87 \\
\hline $8 \mathrm{BVLY}$ & 1.03 & 0.47 & 0.02 & 0.05 & 0.34 & 0.12 & 0.01 & 0.24 & 0.02 & 0.32 & 0.31 & 0.04 & 2.97 \\
\hline $9 \mathrm{FFTF}$ & 0.52 & 0.33 & 0.03 & 0.02 & 0.19 & 0.06 & 0.02 & 0.12 & 0.00 & 0.24 & 0.14 & 0.23 & 1.90 \\
\hline 10 YAKB & 0.48 & 0.30 & 0.02 & 0.02 & 0.21 & 0.38 & 0.42 & 0.13 & 0.00 & 0.33 & 0.17 & 0.11 & 2.57 \\
\hline $11300 \mathrm{~A}$ & 0.57 & 0.50 & 0.06 & 0.17 & 0.34 & 0.02 & 0.00 & 0.30 & 0.00 & 0.35 & 0.23 & 0.25 & 2.79 \\
\hline 12 WYEB & 0.66 & 0.58 & 0.00 & 0.04 & 0.34 & 0.02 & 0.00 & 0.24 & 0.00 & 0.36 & 0.35 & 0.26 & 2.85 \\
\hline $13100 \mathrm{~N}$ & 0.82 & 0.66 & 0.00 & 0.01 & 0.34 & 0.07 & 0.04 & 0.21 & 0.00 & 0.26 & 0.21 & 0.15 & 2.77 \\
\hline 14 WPPS & 0.76 & 0.66 & 0.01 & 0.10 & 0.38 & 0.02 & 0.02 & 0.46 & 0.00 & 0.46 & 0.38 & 0.32 & 3.57 \\
\hline 17 RING & 0.65 & 0.80 & 0.02 & 0.09 & 0.55 & 0.29 & 0.05 & 0.55 & 0.00 & 0.45 & 0.41 & 0.42 & 4.28 \\
\hline $18 \mathrm{RICH}$ & 0.58 & 0.47 & 0.04 & 0.19 & 0.27 & 0.03 & 0.00 & 0.05 & 0.00 & 0.38 & 0.42 & 0.23 & 2.66 \\
\hline 20 RMTN & 0.71 & 0.63 & 0.09 & 0.10 & 0.53 & 0.08 & 0.02 & 0.14 & 0.01 & 0.31 & 0.32 & 0.30 & 3.24 \\
\hline $21 \mathrm{HMS}$ & 0.89 & 0.70 & 0.06 & 0.00 & 0.34 & 0.31 & 0.07 & 0.57 & 0.00 & 0.48 & 0.26 & 0.07 & 3.75 \\
\hline 22 PASC & 0.63 & 0.65 & 0.12 & 0.20 & 0.44 & 0.01 & 0.19 & 0.20 & 0.00 & 0.44 & 0.35 & 0.24 & 3.47 \\
\hline $24100 \mathrm{~F}$ & 0.78 & 0.69 & 0.02 & 0.02 & 0.40 & 0.08 & 0.02 & 0.18 & 0.01 & 0.36 & 0.40 & 0.28 & 3.24 \\
\hline 26 BENT & 0.84 & 0.83 & 0.08 & 0.09 & 0.37 & 0.05 & 0.00 & 0.09 & 0.00 & 0.56 & 0.48 & 0.29 & 3.68 \\
\hline 27 VSTA & 0.60 & 0.54 & 0.04 & 0.08 & 0.22 & 0.00 & 0.14 & 0.13 & 0.00 & 0.44 & 0.24 & 0.22 & 2.65 \\
\hline 28 SURF & 1.41 & 1.12 & 0.22 & 0.02 & 0.45 & 0.00 & 0.00 & 0.36 & 0.00 & 0.58 & 1.21 & 0.45 & 5.82 \\
\hline $29100 \mathrm{~K}$ & 0.87 & 0.61 & 0.04 & 0.05 & 0.35 & 0.16 & 0.07 & 0.28 & 0.25 & 0.33 & 0.31 & 0.17 & 3.49 \\
\hline
\end{tabular}

(a) Stations $5,15,16,19,23$, and 25 are solar powered; therefore, insufficient power is available to operate the heated tippingbucket precipitation gauges. 
Table 2.6. 1999 Monthly and Annual Average Wind Speed ( $\mathrm{mph}$ ) from the Hanford Meteorological Monitoring Network

\begin{tabular}{|c|c|c|c|c|c|c|c|c|c|c|c|c|c|}
\hline Station & Jan & Feb & Mar & Apr & May & Jun & Jul & Aug & Sep & Oct & Nov & Dec & Annual \\
\hline 1 PROS & 7.3 & 10.7 & 9.1 & 7.6 & 8.6 & 8.8 & 7.7 & 7.1 & 6.1 & 7.0 & 7.2 & 8.1 & 7.9 \\
\hline $2 \mathrm{EOC}$ & 10.8 & 14.9 & 11.3 & 9.8 & 10.3 & 8.5 & 8.3 & 8.0 & 8.4 & 8.8 & 10.6 & 11.4 & 10.1 \\
\hline 3 ARMY & 6.4 & 10.8 & 8.3 & 7.5 & 8.6 & 7.8 & 7.6 & 6.3 & 6.3 & 6.2 & 6.4 & 7.5 & 7.5 \\
\hline 4 RSPG & 7.0 & 10.0 & 8.7 & 8.4 & 9.2 & 7.5 & 7.8 & 6.7 & 8.0 & 7.8 & 6.7 & 7.5 & 7.9 \\
\hline 5 EDNA & 6.0 & 8.3 & 7.2 & 6.8 & 8.3 & 8.1 & 7.5 & 6.5 & 5.8 & 5.8 & 5.8 & 6.1 & 6.8 \\
\hline $6200 \mathrm{E}$ & 6.4 & 10.1 & 8.8 & 8.6 & 10.1 & 9.5 & 9.0 & 7.5 & 7.3 & 7.2 & 6.6 & 6.9 & 8.2 \\
\hline $7200 w$ & 5.8 & 9.1 & 7.3 & 6.7 & 8.5 & 7.6 & 7.3 & 6.0 & 5.6 & 5.6 & 5.5 & 6.2 & 6.7 \\
\hline $8 \mathrm{BVLY}$ & 6.0 & 8.5 & 7.5 & 7.7 & 8.3 & 8.7 & 7.9 & 6.5 & 5.8 & 5.5 & 6.2 & 5.5 & 7.0 \\
\hline 9 FFTF & 7.9 & 11.3 & 9.7 & 8.3 & 9.4 & 9.0 & 7.9 & 7.5 & 6.9 & 7.6 & 7.8 & 8.6 & 8.5 \\
\hline 10 YAKB & 7.0 & 9.9 & 8.9 & 8.6 & 10.3 & 9.6 & 9.4 & 7.6 & 7.7 & 7.3 & 6.9 & 6.9 & 8.3 \\
\hline $11300 \mathrm{~A}$ & 7.9 & 10.9 & 9.1 & 7.9 & 8.8 & 8.9 & 8.2 & 7.1 & 6.3 & 6.8 & 7.2 & 8.5 & 8.1 \\
\hline 12 WYEB & 7.4 & 10.1 & 8.4 & 7.7 & 9.0 & 8.4 & 7.8 & 7.0 & 6.6 & 7.2 & 7.1 & 7.6 & 7.8 \\
\hline $13100 \mathrm{~N}$ & 5.2 & 7.6 & 6.5 & 7.2 & 8.0 & 8.3 & 7.8 & 5.8 & 5.6 & 5.4 & 5.0 & 5.7 & 6.5 \\
\hline 14 WPPS & 7.2 & 9.8 & 8.3 & 7.1 & 8.4 & 8.0 & 7.4 & 6.8 & 5.7 & 6.3 & 6.8 & 7.5 & 7.4 \\
\hline 15 FRNK & 8.0 & 10.0 & 8.3 & 6.9 & 7.0 & 7.1 & 6.2 & 6.1 & 5.5 & 6.1 & 6.7 & 7.9 & 7.1 \\
\hline $16 \mathrm{GABL}$ & 10.9 & 15.1 & 12.4 & 12.6 & 13.9 & 13.4 & 12.3 & 11.0 & 10.4 & 10.5 & 10.1 & 10.7 & 11.9 \\
\hline 17 RNG & 6.5 & 8.6 & 7.7 & 7.0 & 7.7 & 7.0 & 6.0 & 5.5 & 5.7 & 5.7 & 5.5 & 6.8 & 6.6 \\
\hline $18 \mathrm{RICH}$ & 7.0 & 9.5 & 7.9 & 6.8 & 7.9 & 7.6 & 6.5 & 6.1 & 5.1 & 5.4 & 6.2 & 7.4 & 6.9 \\
\hline 19 PFP & 4.8 & 7.6 & 6.0 & 5.5 & 6.7 & 5.9 & 5.8 & 4.7 & 4.3 & 4.3 & 4.1 & 4.8 & 5.4 \\
\hline $20 \mathrm{RMTN}$ & 21.7 & 27.6 & 20.6 & 20.8 & 19.2 & 16.1 & 14.7 & 14.3 & 16.3 & 17.9 & 21.8 & 24.1 & 19.5 \\
\hline $21 \mathrm{HMS}$ & 7.7 & 11.1 & 9.3 & 8.8 & 10.4 & 9.7 & 9.4 & 8.2 & 7.7 & 7.7 & 7.5 & 7.5 & 8.8 \\
\hline 22 PASC & 5.9 & 8.2 & 7.0 & 5.8 & 6.7 & 6.8 & 5.4 & 5.7 & 4.2 & 4.7 & 5.2 & 6.4 & 6.0 \\
\hline $23 \mathrm{GABW}$ & 5.6 & 8.5 & 7.2 & 7.2 & 9.0 & 8.7 & 8.2 & 6.7 & 5.9 & 6.2 & 5.6 & 6.0 & 7.1 \\
\hline $24100 \mathrm{~F}$ & 5.7 & 8.1 & 6.8 & 6.8 & 8.3 & 8.2 & 7.2 & 6.3 & 5.5 & 5.6 & 5.3 & 5.9 & 6.6 \\
\hline 25 VERN & 6.4 & 9.0 & 8.4 & 8.8 & 10.1 & 10.3 & 9.6 & 7.8 & 7.1 & 7.0 & 6.9 & 6.7 & 8.2 \\
\hline 26 BENT & 7.2 & 8.6 & 7.6 & 8.2 & 7.8 & 6.7 & 6.6 & 5.9 & 6.7 & 6.1 & 5.9 & 7.1 & 7.0 \\
\hline 27 VSTA & 7.0 & 9.3 & 7.8 & 6.5 & 7.6 & 7.6 & 6.0 & 7.4 & 4.6 & 5.4 & 6.1 & 7.5 & 6.9 \\
\hline 28 SURF & 8.7 & 10.0 & 9.8 & 11.3 & 12.1 & 15.4 & 12.2 & 11.5 & 8.2 & 8.2 & 7.1 & 8.0 & 10.1 \\
\hline $29100 \mathrm{~K}$ & 5.1 & 7.9 & 6.6 & 7.0 & 8.5 & 8.6 & 7.7 & 6.2 & 5.5 & 5.5 & 5.0 & 5.7 & 6.6 \\
\hline $30 \mathrm{HAMR}$ & 6.9 & 9.8 & 8.4 & 7.5 & 8.2 & 8.1 & 7.2 & 6.8 & 6.0 & 6.4 & 6.6 & 7.5 & 7.4 \\
\hline
\end{tabular}


Figure 2.1 depicts the 1999 observed daily maximum and minimum temperatures and the normal maximum, minimum, and mean daily temperatures for the Hanford Meteorology Station.

\subsection{Precipitation}

1999 was the fourth driest year on record. Precipitation totaled 3.75 inches, $60 \%$ of normal (6.26 inches). The wettest year was 1995 with 12.31 inches; the driest was 1976 with only 2.99 inches. Calendar year snowfall totaled 0.6 inch, least on record. The previous low was 0.9 inch in 1958 , with an annual normal snowfall of 13.8 inches. The greatest calendar year snowfall was 57.5 inches (1996).

January 1999 had a total of 0.89 inch of precipitation, $113 \%$ of normal $(0.79$ inch). The wettest January, in 1970 , received 2.47 inches, while the driest, in 1977, received 0.08 inch. There was only a trace of snowfall recorded in January 1999, compared to a January normal of 3.9 inches. The snowiest January received 23.4 inches (1950), while January 1994 received no snowfall.

Precipitation for February 1999 totaled 0.70 inch, $113 \%$ of normal ( 0.62 inch). The wettest February, in 1961 , received 2.10 inches, while the driest, in 1988 , received only a trace. There was only a trace of snowfall recorded in February 1999, compared to a February normal of 2.0 inches. The snowiest February received 17.0 inches (1989), while February 1991 received no snowfall.

Precipitation for March 1999 totaled only 0.06 inch, $13 \%$ of normal ( 0.47 inch). The wettest March, in 1957, received 1.86 inches, while the driest, in 1968, received only 0.02 inch. No snowfall was recorded in March 1999, compared to a March normal of 0.3 inch. The snowiest March received 4.2 inches (1951), while numerous March's have received no snowfall.

There was only a trace of precipitation in April 1999, $0 \%$ of normal ( 0.41 inch), tying four prior Aprils (1986, 1977, 1973, and 1956) as the driest on record. The wettest April, in 1995, received 1.54 inches. No snowfall was recorded in April 1999, compared to an April normal of a trace. The snowiest April received 1.0 inch (1982), while numerous Aprils have received no snowfall.

May 1999 had a total of 0.34 inch of precipitation, $67 \%$ of normal ( $0.51 \mathrm{inch})$. The wettest May, in 1972, received 2.03 inches, while the driest, in 1992 and earlier years, received only a trace.

June 1999 had a total of 0.31 inch of precipitation, $82 \%$ of normal ( $0.38 \mathrm{inch})$. The wettest June, in 1950 , received 2.92 inches, while the driest, in 1986 and earlier years, received only a trace.

Precipitation for July 1999 totaled 0.07 inch, $39 \%$ of normal ( 0.18 inch). The wettest July, in 1993, received 1.76 inches, while the driest, in 1980 and earlier years, received only a trace.

Precipitation for August 1999 totaled 0.57 inch, $211 \%$ of normal (0.27 inch). Most of the precipitation ( $0.48 \mathrm{inch}$ ) was received during a thunderstorm on August 5, with 0.40 inch falling in a 35-minute period. The wettest August, in 1977, received 1.36 inches, while the driest, in 1988, received no precipitation.

No precipitation was recorded during September, tying the record for the driest September on record. September 1991 was also totally dry. There have only been 4 months since recordkeeping began at the 


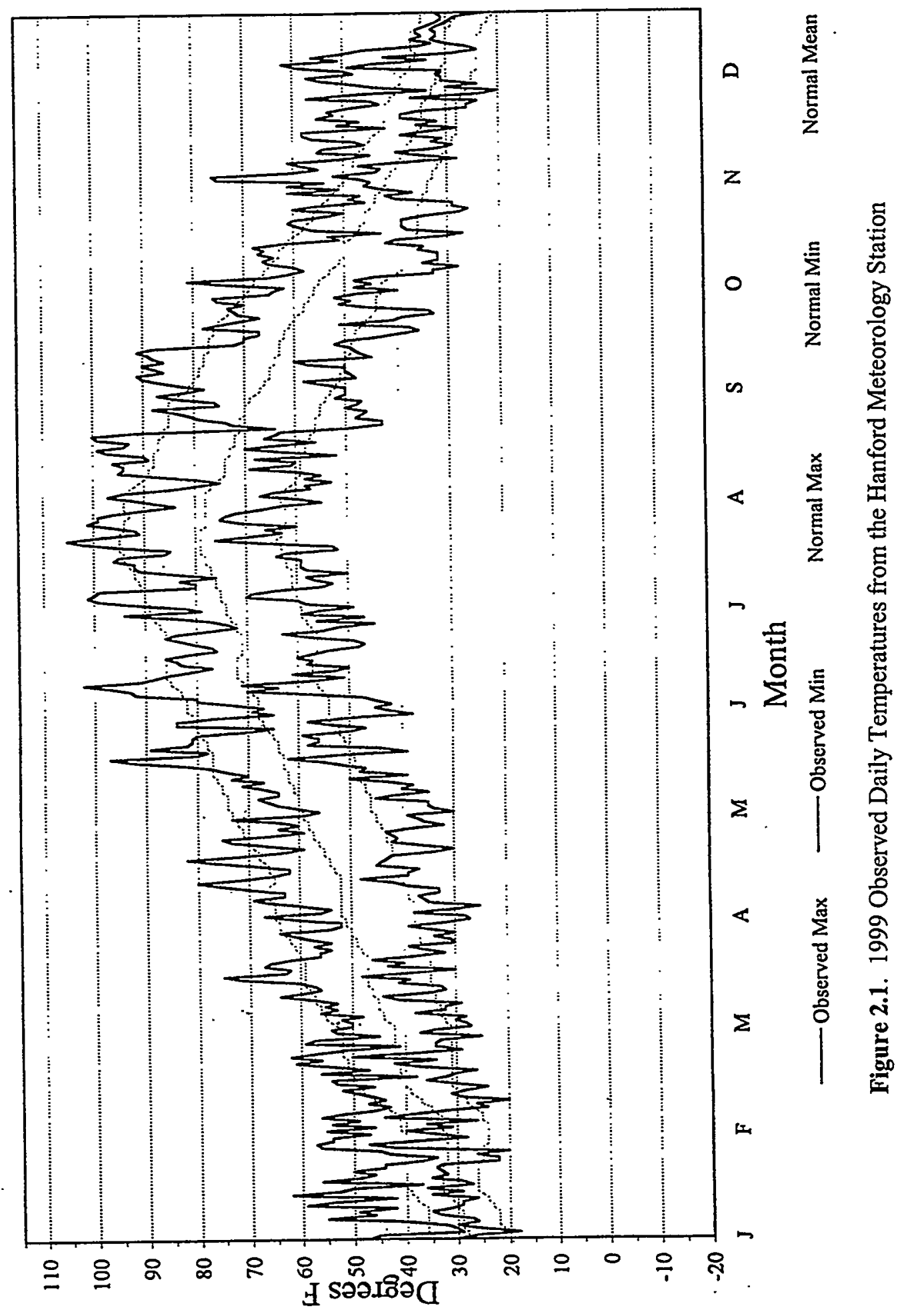


Hanford Meteorology Station that have received no precipitation-August 1955 and 1988, and September 1991 and 1999. The normal rainfall amount for September is 0.31 inch. The wettest September, in 1947, received 1.34 inches.

Precipitation for October 1999 totaled 0.48 inch, $126 \%$ of normal ( 0.38 inch). The wettest October, in 1957, received 2.72 inches, while the October with the least precipitation (1987 and earlier years) received only a trace.

November 1999 had a total of 0.26 inch of precipitation, $29 \%$ of normal ( 0.91 inch). The wettest November, in 1996, received 2.67 inches, while November 1976 received only a trace. No snowfall was recorded during November 1999, compared to a normal of 1.8 inches. The snowiest November (1985) received 18.3 inches, while many Novembers have been without snow.

December 1999 had a total of 0.07 inch of precipitation, 7\% of normal (1.03 inches) and was the driest December on record. The previous driest December was 1976 with 0.11 inch. The wettest December, in 1996, received 3.69 inches. Snowfall for December 1999 totaled 0.6 inch, compared to a normal of 5.7 inches. The snowiest December (1996) received 22.6 inches, while December 1962 received only a trace. Snowfall for the $1999-2000$ winter season (through December 1999) is 0.6 inch, compared to a normal through December of 7.6 inches.

\subsection{Wind}

1999 was the windiest year on record, averaging 8.8 miles per hour ( $\mathrm{mph}$ ), $1.1 \mathrm{mph}$ above normal (7.7 $\mathrm{mph}$ ). The previous windiest year was 1983, which averaged $8.4 \mathrm{mph}$. The peak gust for the year was $65 \mathrm{mph}$ on February 6.

The average wind speed for January 1999 was $7.7 \mathrm{mph}$ compared to a January normal of $6.5 \mathrm{mph}$. The peak wind gust for the month was $55 \mathrm{mph}$ recorded on January 29. The record wind gust for January was $80 \mathrm{mph}$ in 1972. The windiest January averaged $10.3 \mathrm{mph}$ (1972); while the January with the lightest winds (1985) averaged $2.9 \mathrm{mph}$.

February 1999 was the windiest February on record, and tied April 1972 as the windiest month ever recorded at the Hanford Meteorology Station. The average wind speed for the month was $11.1 \mathrm{mph}$, compared to a February normal of $7.2 \mathrm{mph}$. The previous windiest February was $10.8 \mathrm{mph}$ in 1976 . The February with the lightest winds was $4.6 \mathrm{mph}$ in 1963 . The peak gust for the month was $65 \mathrm{mph}$ on February 6, tying the record gust for February. A 65-mph gust was also recorded in February 1971. There were 10 days in February 1999 with gusts of $40 \mathrm{mph}$ or greater; this tied February 1976 for most wind gusts $\geq 40 \mathrm{mph}$. The most for any month was 11 in January 1990 .

March 1999 was windier than normal, with an average wind speed of $9.3 \mathrm{mph}, 1.0 \mathrm{mph}$ above normal (8.3 mph). The windiest March on record averaged $10.7 \mathrm{mph}$ (1977), while the March with the lightest winds (1958) averaged $5.9 \mathrm{mph}$. The peak gust for the month was $52 \mathrm{mph}$ on March 29. The record wind gust for March was $70 \mathrm{mph}$ in 1956.

The average wind speed for April 1999 was nearly normal, with an average speed of $8.8 \mathrm{mph}$, $0.2 \mathrm{mph}$ below normal ( $9.0 \mathrm{mph}$ ). The windiest April on record averaged $11.1 \mathrm{mph}$ (1972), while the 
April with the lightest winds (1989) averaged $7.4 \mathrm{mph}$. The peak gust for the month was $40 \mathrm{mph}$ on April 2. The record wind gust for April was $73 \mathrm{mph}$ in 1972.

The average wind speed for May 1999 was $10.4 \mathrm{mph}, 1.3 \mathrm{mph}$ above normal (9.1 mph). The windiest May on record averaged $10.7 \mathrm{mph}$ (1983), while the May with the lightest winds (1957) averaged $5.8 \mathrm{mph}$. The peak gust for the month was $45 \mathrm{mph}$ on May 25. The record wind gust for May was $71 \mathrm{mph}$ in 1948.

The average wind speed for June 1999 was $9.7 \mathrm{mph}, 0.5 \mathrm{mph}$ above normal ( $9.2 \mathrm{mph}$ ). The windiest June on record averaged $10.7 \mathrm{mph}$ (1983), while the June with the lightest winds (1950) averaged $7.7 \mathrm{mph}$. The peak gust for the month was $42 \mathrm{mph}$ on June 8 . The record wind gust for June was $72 \mathrm{mph}$ in 1957.

The average wind speed for July 1999 was $9.4 \mathrm{mph}, 0.6 \mathrm{mph}$ above normal ( $8.8 \mathrm{mph}$ ). The windiest July on record averaged $10.7 \mathrm{mph}$ (1983), while the July with the lightest winds (1955) averaged $6.8 \mathrm{mph}$. The peak gust for the month was $44 \mathrm{mph}$ on July 24. The record wind gust for July was $69 \mathrm{mph}$ in 1979.

The average wind speed for August 1999 was $8.2 \mathrm{mph}, 0.3 \mathrm{mph}$ above normal ( $7.9 \mathrm{mph})$. The windiest August on record averaged $9.5 \mathrm{mph}$ (1996), while the August with the lightest winds (1956) averaged $6.0 \mathrm{mph}$. The peak gust for the month was $44 \mathrm{mph}$ on August 29. The record wind gust for August was $66 \mathrm{mph}$ in 1961.

The average wind speed for September 1999 was $7.7 \mathrm{mph}, 0.3 \mathrm{mph}$ above normal (7.4 mph). The windiest September on record averaged $9.2 \mathrm{mph}$ (1961), while the September with the lightest winds (1957) averaged $5.4 \mathrm{mph}$. The peak gust for the month was $42 \mathrm{mph}$ on September 25 . The record wind gust for September was $65 \mathrm{mph}$ in 1953.

The average wind speed for October 1999 was $7.7 \mathrm{mph}, 1.2 \mathrm{mph}$ above normal ( $6.5 \mathrm{mph}$ ). The windiest October on record averaged $9.1 \mathrm{mph}$ (1946), while the October with the lightest winds (1952) averaged $4.4 \mathrm{mph}$. The peak gust for the month was $46 \mathrm{mph}$ on October 31 . The record wind gust for October was $72 \mathrm{mph}$ in 1997.

The average wind speed for November 1999 was $7.5 \mathrm{mph}, 1.1 \mathrm{mph}$ above normal ( $6.4 \mathrm{mph})$. The windiest November on record averaged $10.0 \mathrm{mph}$ (1990), while the November with the lightest winds (1956) averaged $2.9 \mathrm{mph}$. The peak gust for the month was $41 \mathrm{mph}$ on November 8 . The record wind gust for November was $67 \mathrm{mph}$ in 1993.

The average wind speed for December 1999 was $7.5 \mathrm{mph}, 1.6 \mathrm{mph}$ above normal (5.9 $\mathrm{mph}$ ). The windiest December on record averaged $8.3 \mathrm{mph}$ (1968), while the December with the lightest winds (1985) averaged $3.3 \mathrm{mph}$. The peak gust for the month was $62 \mathrm{mph}$ on December 18 . The record wind gust for December was $71 \mathrm{mph}$ in 1955.

Figures 2.2 and 2.3 give a composite of the wind roses (at the 10- and 60-meter levels, respectively) from the Hanford Meteorological Monitoring Network for 1999. The appendix gives the individual 1999 wind roses from the Hanford Meteorological Monitoring Network stations. 


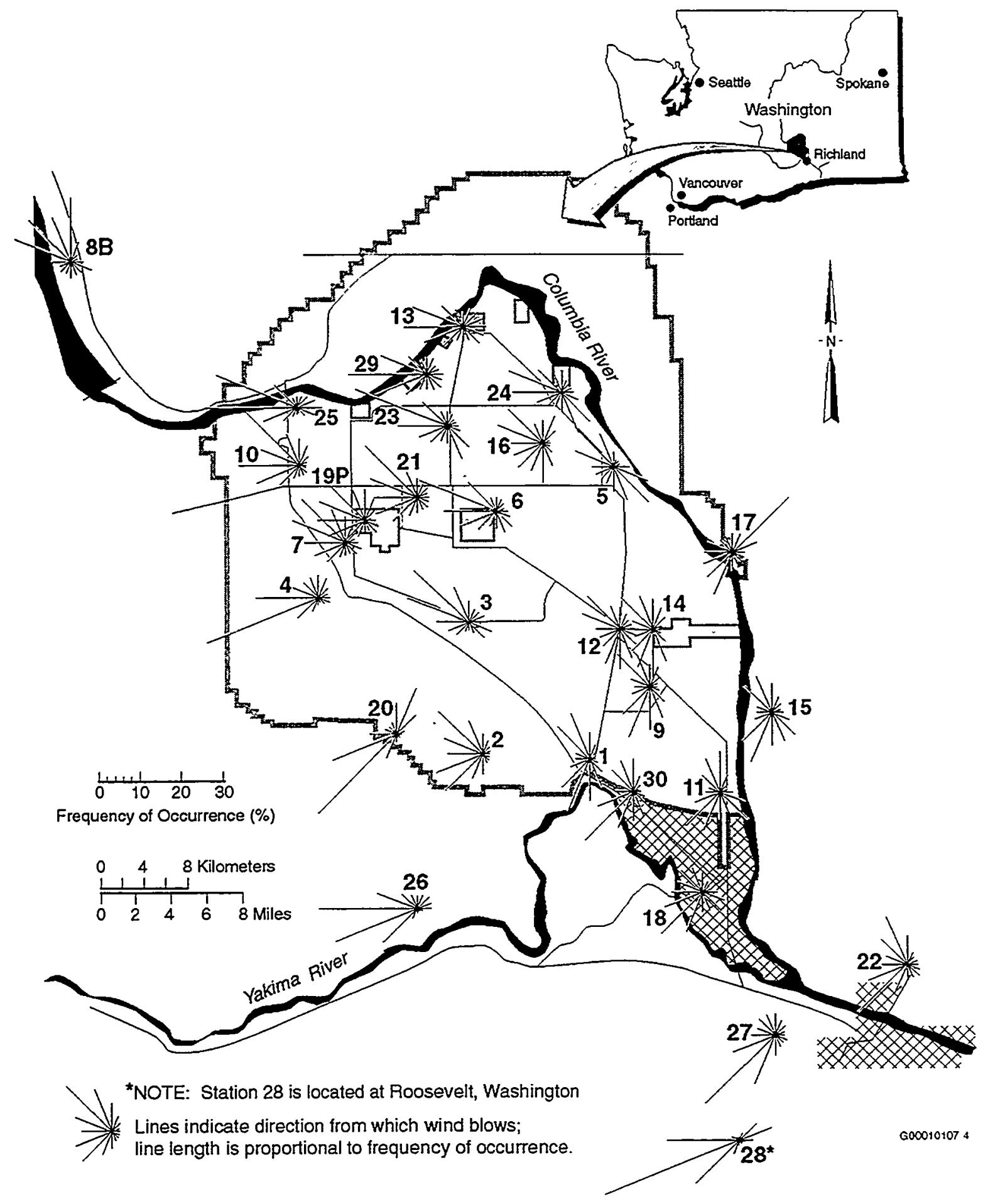

Figure 2.2. 1999 Hanford Meteorological Monitoring Network Wind Roses at 10-Meter Level (see Appendix for station-specific wind rose) 


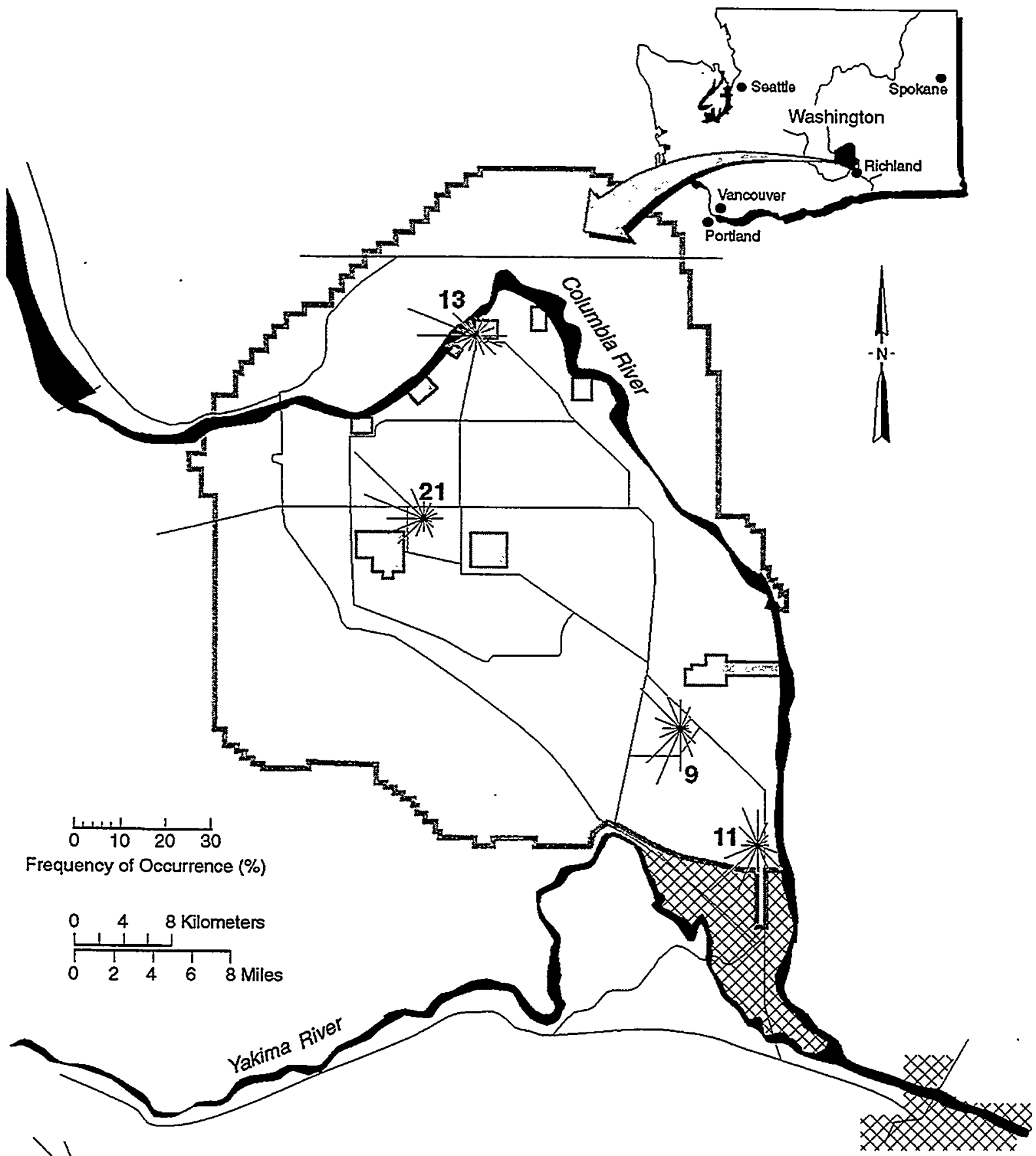

Lines indicate direction from which wind blows; line length is proportional to frequency of occurrence.

G00010107 2

Figure 2.3. 1999 Hanford Meteorological Monitoring Network Wind Roses at 60-Meter Level (see Appendix for station-specific wind rose) 


\subsection{Temperature Climatology}

\subsection{Monthly, Seasonal, and Annual Average}

Monthly, seasonal, and annual average temperatures, computed from observed daily maximum and minimum temperatures for the period 1945 through 1999, are presented in Tables 3.1 and 3.2. In these tables, the highest and lowest values, representing the warmest and coldest month, season, or year, are noted. Averages are based on the entire period of record, and climatological normal temperatures are based on the period 1961 through 1990.

As indicated in Table 3.1, much wider ranges and variabilities in temperatures are found during the late autumn and winter months (November through February) than during the rest of the year. The range of average monthly temperatures for January is from $12.1^{\circ} \mathrm{F}(1950)$ to $42.5^{\circ} \mathrm{F}(1953), 30.4^{\circ} \mathrm{F}$; for November $21.7^{\circ} \mathrm{F}$; February $18.9^{\circ} \mathrm{F}$; and December $17.5^{\circ} \mathrm{F}$; whereas for the rest of the year, the monthly range is from a low of $10.5^{\circ} \mathrm{F}$ in April to a high of $13.8^{\circ} \mathrm{F}$ in June. The coldest month recorded was January $1950\left(12.1^{\circ} \mathrm{F}\right)$; the hottest month recorded was July $1985\left(82.2^{\circ} \mathrm{F}\right)$. As shown in Table 3.2, the seasonal range is from $8^{\circ} \mathrm{F}$ during the summer (June, July, and August) to $16.4^{\circ} \mathrm{F}$ in winter (December, January, and February). The coldest season was the winter of $1948-1949\left(24.2^{\circ} \mathrm{F}\right)$; the hottest was the summer of $1958\left(78.2^{\circ} \mathrm{F}\right)$.

\subsection{Days with Maximum Temperatures $\geq 100^{\circ} \mathrm{F}, \geq 90^{\circ} \mathrm{F}$, and $\leq 32^{\circ} \mathrm{F}$}

Table 3.3 contains the number of days each year with maximum temperatures in the categories $\geq 100^{\circ} \mathrm{F}, \geq 90^{\circ} \mathrm{F}$, and $\leq 32^{\circ} \mathrm{F}$.

Maximum temperatures $\geq 100^{\circ} \mathrm{F}$ occurred as early as May 5 (1966) and as late as September 6 (1955). The annual number of days with maximum temperatures in this category ranged from 1 to 28 (1954 and 1958 , respectively). The greatest number of consecutive days with maximum temperatures $\geq 100^{\circ} \mathrm{F}$ is 11 and occurred 3 times: July 22 through August 1, 1962; August 10 through 20, 1967; and August 6 through 16, 1981.

One particularly notable period of above normal temperatures occurred July 15 through August 13, 1971. This 30-day period included 27 days with maximum temperatures $\geq 100^{\circ} \mathrm{F}$ in 3 separate periods of 9 consecutive days each. The lowest maximum temperature during the 30 -day period was $98^{\circ} \mathrm{F}$; the highest was $112^{\circ} \mathrm{F}$. The average maximum temperature during this period was $104.7^{\circ} \mathrm{F}$.

Table 3.4 lists the dates of all occurrences of maximum temperatures $\geq 104^{\circ} \mathrm{F}$. 
Table 3.1. Monthly and Annual Average Temperatures $\left({ }^{\circ} \mathrm{F}\right)$

\begin{tabular}{|c|c|c|c|c|c|c|c|c|c|c|c|c|c|}
\hline Year & Jan & Feb & Mar & Apr & May & Jun & Jul & Aug & Sep & Oct & Nov & Dec & Annual \\
\hline 1945 & 33.9 & 38.6 & 42.1 & 50.3 & 61.7 & 67.5 & 78.0 & 77.5 & 64.6 & 56.4 & 40.6 & 32.7 & 53.7 \\
\hline $\begin{array}{l}1946 \\
1947 \\
1948 \\
1949 \\
1950\end{array}$ & $\begin{array}{l}34.4 \\
27.4 \\
32.0 \\
13.9 \\
12.1^{(0)}\end{array}$ & $\begin{array}{l}39.6 \\
40.0 \\
31.8 \\
31.8 \\
30.7\end{array}$ & $\begin{array}{l}45.5 \\
49.6 \\
42.1 \\
45.2 \\
42.3\end{array}$ & $\begin{array}{l}53.7 \\
56.1 \\
49.4 \\
55.5 \\
49.9\end{array}$ & $\begin{array}{l}64.2 \\
68.7^{(2)} \\
58.3 \\
67.0 \\
59.0\end{array}$ & $\begin{array}{l}66.9 \\
67.8 \\
72.4 \\
69.3 \\
66.5\end{array}$ & $\begin{array}{l}76.1 \\
75.3 \\
72.8 \\
74.9 \\
75.4\end{array}$ & $\begin{array}{l}76.6 \\
71.8 \\
71.8 \\
74.8 \\
76.4\end{array}$ & $\begin{array}{l}63.5 \\
65.4 \\
64.4 \\
68.3 \\
67.5\end{array}$ & $\begin{array}{l}49.5 \\
53.4 \\
51.0 \\
50.2 \\
51.1\end{array}$ & $\begin{array}{l}35.8 \\
41.2 \\
40.8 \\
45.2 \\
40.7\end{array}$ & $\begin{array}{l}34.8 \\
33.1 \\
26.9 \\
35.1 \\
36.2\end{array}$ & $\begin{array}{l}53.4 \\
54.2 \\
51.1 \\
52.6 \\
50.6\end{array}$ \\
\hline $\begin{array}{l}1951 \\
1952 \\
1953 \\
1954 \\
1955\end{array}$ & $\begin{array}{l}33.0 \\
25.2 \\
42.5^{(2)} \\
28.9 \\
30.0\end{array}$ & $\begin{array}{l}36.9 \\
36.7 \\
41.2 \\
39.3 \\
35.3\end{array}$ & $\begin{array}{l}40.1 \\
44.1 \\
46.2 \\
41.5 \\
39.4^{(2)}\end{array}$ & $\begin{array}{l}54.1 \\
55.2 \\
51.0 \\
51.4 \\
\mathbf{4 7 . 5 ^ { ( 2 ) }}\end{array}$ & $\begin{array}{l}61.1 \\
62.7 \\
58.0 \\
62.9 \\
57.0\end{array}$ & $\begin{array}{l}69.4 \\
67.1 \\
63.0^{(2)} \\
65.5 \\
70.2\end{array}$ & $\begin{array}{l}76.7 \\
77.0 \\
75.8 \\
73.9 \\
73.0\end{array}$ & $\begin{array}{l}74.2 \\
74.0 \\
74.0 \\
71.4 \\
75.5\end{array}$ & $\begin{array}{l}66.8 \\
69.0 \\
67.8 \\
65.1 \\
66.4\end{array}$ & $\begin{array}{l}51.5 \\
59.0 \\
55.4 \\
51.4 \\
53.3\end{array}$ & $\begin{array}{l}39.5 \\
34.0 \\
43.4 \\
46.0 \\
31.3\end{array}$ & $\begin{array}{l}27.4 \\
34.8 \\
37.6 \\
34.0 \\
29.4\end{array}$ & $\begin{array}{l}52.6 \\
53.2 \\
54.7 \\
52.6 \\
50.7\end{array}$ \\
\hline $\begin{array}{l}1956 \\
1957 \\
1958 \\
1959 \\
1960\end{array}$ & $\begin{array}{l}31.8 \\
16.5 \\
37.1 \\
32.0 \\
23.3\end{array}$ & $\begin{array}{l}25.6^{(2)} \\
34.1 \\
44.5^{(2)} \\
35.5 \\
37.4\end{array}$ & $\begin{array}{l}43.8 \\
44.0 \\
43.5 \\
45.1 \\
45.1\end{array}$ & $\begin{array}{l}56.2 \\
55.2 \\
51.3 \\
54.2 \\
52.6\end{array}$ & $\begin{array}{l}65.3 \\
65.9 \\
68.1 \\
57.5 \\
58.5\end{array}$ & $\begin{array}{l}65.7 \\
70.8 \\
73.9 \\
68.6 \\
70.1\end{array}$ & $\begin{array}{l}78.9 \\
74.3 \\
81.2 \\
77.7 \\
81.8\end{array}$ & $\begin{array}{l}75.3 \\
72.9 \\
79.4 \\
71: 8 \\
71.4\end{array}$ & $\begin{array}{l}67.3 \\
69.0 \\
65.6 \\
62.6 \\
67.7\end{array}$ & $\begin{array}{l}52.1 \\
50.7 \\
54.4 \\
53.4 \\
54.5\end{array}$ & $\begin{array}{l}36.6 \\
40.4 \\
40.6 \\
36.5 \\
41.2\end{array}$ & $\begin{array}{l}34.6 \\
38.5^{(2)} \\
35.2 \\
33.1 \\
29.0\end{array}$ & $\begin{array}{l}52.8 \\
52.7 \\
56.2 \\
52.3 \\
52.7\end{array}$ \\
\hline $\begin{array}{l}1961 \\
1962 \\
1963 \\
1964 \\
1965\end{array}$ & $\begin{array}{l}35.0 \\
29.8 \\
25.4 \\
35.6 \\
32.3\end{array}$ & $\begin{array}{l}43.7 \\
36.6 \\
38.3 \\
38.1 \\
40.5\end{array}$ & $\begin{array}{l}46.1 \\
42.6 \\
46.4 \\
43.8 \\
42.9\end{array}$ & $\begin{array}{l}52.3 \\
55.6 \\
49.8 \\
50.2 \\
54.8\end{array}$ & $\begin{array}{l}60.0 \\
56.9 \\
61.7 \\
59.7 \\
60.5\end{array}$ & $\begin{array}{l}74.0 \\
68.3 \\
69.4 \\
67.7 \\
69.3\end{array}$ & $\begin{array}{l}79.4 \\
76.0 \\
72.4 \\
74.5 \\
76.5\end{array}$ & $\begin{array}{l}80.2 \\
71.9 \\
75.7 \\
69.8^{(2)} \\
74.7\end{array}$ & $\begin{array}{l}63.8 \\
67.1 \\
71.1 \\
63.0 \\
62.4\end{array}$ & $\begin{array}{l}51.6 \\
52.6 \\
56.0 \\
53.3 \\
57.1\end{array}$ & $\begin{array}{l}35.3 \\
43.2 \\
42.8 \\
38.2 \\
43.1\end{array}$ & $\begin{array}{l}33.7 \\
36.8 \\
30.2 \\
25.5 \\
33.0\end{array}$ & $\begin{array}{l}54.6 \\
53.1 \\
53.3 \\
51.6 \\
53.9\end{array}$ \\
\hline $\begin{array}{l}1966 \\
1967 \\
1968 \\
1969 \\
1970\end{array}$ & $\begin{array}{l}34.0 \\
39.8 \\
35.7 \\
19.8 \\
30.7\end{array}$ & $\begin{array}{l}39.9 \\
43.7 \\
41.8 \\
31.7 \\
40.6\end{array}$ & $\begin{array}{l}45.4 \\
44.3 \\
49.0 \\
45.8 \\
45.0\end{array}$ & $\begin{array}{l}54.6 \\
47.6 \\
51.3 \\
52.2 \\
49.0\end{array}$ & $\begin{array}{l}63.2 \\
60.5 \\
62.4 \\
64.6 \\
61.5\end{array}$ & $\begin{array}{l}66.9 \\
72.5 \\
69.8 \\
75.1 \\
73.6\end{array}$ & $\begin{array}{l}73.3 \\
78.6 \\
79.7 \\
76.0 \\
78.6\end{array}$ & $\begin{array}{l}75.6 \\
81.5^{(2)} \\
71.5 \\
72.8 \\
76.3\end{array}$ & $\begin{array}{l}68.8 \\
71.8 \\
66.8 \\
67.4 \\
61.8\end{array}$ & & & $\begin{array}{l}38.2 \\
33.1 \\
30.6 \\
34.6 \\
30.8\end{array}$ & $\begin{array}{l}54.8 \\
55.8 \\
54.2 \\
52.6 \\
53.2\end{array}$ \\
\hline $\begin{array}{l}1971 \\
1972 \\
1973 \\
1974 \\
1975\end{array}$ & $\begin{array}{l}35.8 \\
30.5 \\
29.1 \\
29.4 \\
32.5\end{array}$ & $\begin{array}{l}39.1 \\
34.8 \\
38.5 \\
40.9 \\
33.7\end{array}$ & $\begin{array}{l}40.7 \\
47.0 \\
47.4 \\
45.2 \\
42.5\end{array}$ & $\begin{array}{l}52.0 \\
49.6 \\
53.6 \\
52.9 \\
48.2\end{array}$ & $\begin{array}{l}64.0 \\
64.3 \\
63.1 \\
57.9 \\
60.2\end{array}$ & $\begin{array}{l}65.3 \\
69.7 \\
68.7 \\
72.6 \\
67.2\end{array}$ & $\begin{array}{l}78.7 \\
76.2 \\
78.2 \\
74.5 \\
79.5\end{array}$ & $\begin{array}{l}80.5 \\
77.6 \\
73.9 \\
75.5 \\
71.0\end{array}$ & $\begin{array}{l}61.5 \\
61.4 \\
65.7 \\
68.0 \\
68.0\end{array}$ & $\begin{array}{l}51.7 \\
52.3 \\
52.4 \\
52.5 \\
52.5\end{array}$ & & & $\begin{array}{l}53.4 \\
52.6 \\
53.9 \\
53.9 \\
52.4\end{array}$ \\
\hline $\begin{array}{l}1976 \\
1977 \\
1978 \\
1979 \\
1980\end{array}$ & $\begin{array}{l}32.0 \\
25.2 \\
32.5 \\
13.9 \\
23.7\end{array}$ & $\begin{array}{l}37.6 \\
40.5 \\
37.9 \\
34.2 \\
34.6\end{array}$ & $\begin{array}{l}41.4 \\
45.4 \\
47.5 \\
46.5 \\
44.5\end{array}$ & $\begin{array}{l}50.8 \\
57.3 \\
51.9 \\
52.8 \\
55.2\end{array}$ & $\begin{array}{l}60.5 \\
56.9 \\
58.6 \\
64.1 \\
61.4\end{array}$ & $\begin{array}{l}65.6 \\
72.6 \\
70.3 \\
70.8 \\
64.7\end{array}$ & $\begin{array}{l}75.1 \\
73.7 \\
75.7 \\
77.2 \\
74.7\end{array}$ & $\begin{array}{l}70.8 \\
79.2 \\
72.7 \\
74.6 \\
71.2\end{array}$ & $\begin{array}{l}69.0 \\
61.5 \\
63.8 \\
69.2 \\
66.0\end{array}$ & $\begin{array}{l}52.4 \\
52.0 \\
52.2 \\
56.5 \\
52.6\end{array}$ & & & $\begin{array}{l}52.2 \\
53.1 \\
51.9 \\
52.5 \\
52.2\end{array}$ \\
\hline $\begin{array}{l}1981 \\
1982 \\
1983 \\
1984 \\
1985\end{array}$ & $\begin{array}{l}38.0 \\
29.8 \\
37.5 \\
31.6 \\
25.0\end{array}$ & $\begin{array}{l}39.7 \\
38.1 \\
40.9 \\
38.7 \\
29.9\end{array}$ & $\begin{array}{l}48.7 \\
45.9 \\
48.5 \\
47.2 \\
44.0\end{array}$ & $\begin{array}{l}54.0 \\
49.4 \\
51.1 \\
50.5 \\
55.5\end{array}$ & $\begin{array}{l}60.5 \\
60.4 \\
63.8 \\
56.0^{(2)} \\
63.2\end{array}$ & $\begin{array}{l}66.0 \\
73.1 \\
65.4 \\
65.7 \\
70.2\end{array}$ & $\begin{array}{l}73.9 \\
74.9 \\
71.3 \\
76.1 \\
82.2^{(2)}\end{array}$ & $\begin{array}{l}79.0 \\
75.8 \\
74.4 \\
74.0 \\
70.5\end{array}$ & $\begin{array}{l}66.3 \\
65.4 \\
61.7 \\
62.1 \\
58.8^{(\text {()) }}\end{array}$ & $\begin{array}{l}52.0 \\
51.4 \\
52.6 \\
47.9^{(2)} \\
49.8\end{array}$ & $\begin{array}{l}42.7 \\
36.9 \\
43.6 \\
39.4 \\
24.8^{(2)}\end{array}$ & $\begin{array}{l}32.8 \\
32.0 \\
21.2 \\
23.6 \\
21.0^{(2)}\end{array}$ & $\begin{array}{l}54.5 \\
52.8 \\
52.7 \\
51.1 \\
49.6^{(2)}\end{array}$ \\
\hline $\begin{array}{l}1986 \\
1987 \\
1988 \\
1989 \\
1990\end{array}$ & $\begin{array}{l}34.0 \\
30.7 \\
31.9 \\
37.2 \\
40.4\end{array}$ & $\begin{array}{l}39.1 \\
40.1 \\
41.0 \\
27.3 \\
37.6\end{array}$ & $\begin{array}{l}48.6 \\
48.3 \\
45.9 \\
43.8 \\
48.0\end{array}$ & $\begin{array}{l}50.9 \\
58.0 \\
55.2 \\
56.6 \\
57.9\end{array}$ & $\begin{array}{l}62.3 \\
66.2 \\
61.1 \\
61.5 \\
60.7\end{array}$ & $\begin{array}{l}73.0 \\
73.4 \\
69.2 \\
72.0 \\
70.1\end{array}$ & $\begin{array}{l}70.6 \\
74.3 \\
77.3 \\
75.5 \\
80.8\end{array}$ & $\begin{array}{l}79.2 \\
76.6 \\
75.2 \\
73.4 \\
76.8\end{array}$ & $\begin{array}{l}62.2 \\
69.9 \\
65.6 \\
67.4 \\
72.4^{(\text {a) }}\end{array}$ & $\begin{array}{l}54.7 \\
55.5 \\
59.6^{(2)} \\
54.0 \\
52.3\end{array}$ & $\begin{array}{l}42.3 \\
43.6 \\
44.2 \\
44.3 \\
46.5^{(2)}\end{array}$ & $\begin{array}{l}32.4 \\
31.5 \\
31.8 \\
33.3 \\
24.1\end{array}$ & $\begin{array}{l}54.1 \\
55.7 \\
54.8 \\
53.9 \\
55.6\end{array}$ \\
\hline $\begin{array}{l}1991 \\
1992 \\
1993 \\
1994 \\
1995\end{array}$ & $\begin{array}{l}28.7 \\
37.5 \\
24.8 \\
38.6 \\
34.2\end{array}$ & $\begin{array}{l}44.5 \\
42.6 \\
30.8 \\
36.0 \\
43.1\end{array}$ & 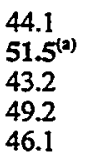 & 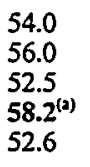 & $\begin{array}{l}60.4 \\
67.2 \\
66.5 \\
64.9 \\
64.5\end{array}$ & $\begin{array}{l}65.6 \\
76.8^{(2)} \\
68.4 \\
69.8 \\
68.1\end{array}$ & $\begin{array}{l}78.0 \\
76.6 \\
70.5^{(2)} \\
81.0 \\
77.1\end{array}$ & $\begin{array}{l}78.9 \\
76.9 \\
73.1 \\
76.6 \\
72.0\end{array}$ & $\begin{array}{l}69.7 \\
64.5 \\
66.4 \\
70.5 \\
69.9\end{array}$ & $\begin{array}{l}52.9 \\
55.7 \\
55.4 \\
54.4 \\
52.1\end{array}$ & $\begin{array}{l}41.3 \\
41.2 \\
34.6 \\
39.6 \\
44.1\end{array}$ & $\begin{array}{l}37.8 \\
30.0 \\
35.4 \\
35.1 \\
32.6\end{array}$ & $\begin{array}{l}54.7 \\
56.4^{(2)} \\
51.8 \\
56.2 \\
54.7\end{array}$ \\
\hline $\begin{array}{l}1996 \\
1997 \\
1998 \\
1999\end{array}$ & $\begin{array}{l}28.8 \\
33.6 \\
36.2 \\
38.3\end{array}$ & $\begin{array}{l}32.8 \\
40.2 \\
42.2 \\
41.7\end{array}$ & $\begin{array}{l}44.8 \\
47.4 \\
48.4 \\
46.3\end{array}$ & $\begin{array}{l}55.0 \\
51.8 \\
54.4 \\
50.9\end{array}$ & $\begin{array}{l}58.1 \\
65.0 \\
62.4 \\
57.9\end{array}$ & $\begin{array}{l}69.0 \\
68.5 \\
71.0 \\
67.4\end{array}$ & $\begin{array}{l}79.5 \\
75.3 \\
82.0 \\
73.8\end{array}$ & $\begin{array}{l}75.6 \\
78.0 \\
77.9 \\
76.2\end{array}$ & $\begin{array}{l}64.4 \\
66.8 \\
71.0 \\
65.0\end{array}$ & $\begin{array}{l}52.4 \\
53.2 \\
52.4 \\
51.8\end{array}$ & $\begin{array}{l}38.4 \\
43.2 \\
45.6 \\
45.8\end{array}$ & $\begin{array}{l}29.8 \\
34.7 \\
33.0 \\
37.7\end{array}$ & $\begin{array}{l}52.4 \\
54.8 \\
56.4^{(\mathfrak{)})} \\
54.4\end{array}$ \\
\hline $\begin{array}{l}\text { Average } \\
\text { Normal }\end{array}$ & $\begin{array}{l}30.8 \\
31.3\end{array}$ & $\begin{array}{l}37.7 \\
38.0\end{array}$ & $\begin{array}{l}45.3 \\
45.6\end{array}$ & $\begin{array}{l}52.9 \\
52.7\end{array}$ & $\begin{array}{l}61.8 \\
61.3\end{array}$ & $\begin{array}{l}69.3 \\
69.7\end{array}$ & $\begin{array}{l}76.4 \\
76.2\end{array}$ & $\begin{array}{l}75.0 \\
75.1\end{array}$ & $\begin{array}{l}66.2 \\
65.7\end{array}$ & $\begin{array}{l}53.0 \\
52.9\end{array}$ & $\begin{array}{l}40.2 \\
40.2\end{array}$ & $\begin{array}{l}32.4 \\
31.4\end{array}$ & $\begin{array}{l}53.4 \\
53.3\end{array}$ \\
\hline
\end{tabular}

(a) Highest and lowest averages. 
Table 3.2. Seasonal Average Temperatures ( $\left.{ }^{\circ} \mathrm{F}\right)$

\begin{tabular}{|c|c|c|c|c|}
\hline Year & $\begin{array}{c}\text { Winter } \\
\text { Dec-Feb } \\
\end{array}$ & $\begin{array}{c}\text { Spring } \\
\text { Mar-May } \\
\end{array}$ & $\begin{array}{l}\text { Summet } \\
\text { Jun-Aug } \\
\end{array}$ & $\begin{array}{l}\text { Autumn } \\
\text { Sep-Nov }\end{array}$ \\
\hline 1945 & - & 51.4 & 74.3 & 53.9 \\
\hline $\begin{array}{l}1946 \\
1947 \\
1948 \\
1949 \\
1950\end{array}$ & $\begin{array}{l}35.6 \\
34.1 \\
32.3 \\
24.2^{(2)} \\
26.0\end{array}$ & $\begin{array}{l}54.5 \\
58.1 \\
49.9 \\
55.9 \\
50.4\end{array}$ & $\begin{array}{l}73.2 \\
71.6 \\
72.3 \\
73.0 \\
72.8\end{array}$ & $\begin{array}{l}49.6 \\
53.3 \\
52.1 \\
54.6 \\
53.1\end{array}$ \\
\hline $\begin{array}{l}1951 \\
1952 \\
1953 \\
1954 \\
1955\end{array}$ & $\begin{array}{l}35.4 \\
29.8 \\
39.5 \\
35.3 \\
33.1\end{array}$ & $\begin{array}{l}51.8 \\
54.0 \\
51.7 \\
51.9 \\
\mathbf{4 8 . 0}^{(\mathbf{2})}\end{array}$ & $\begin{array}{l}73.4 \\
72.7 \\
70.9 \\
70.3 \\
72.9\end{array}$ & $\begin{array}{l}52.6 \\
54.0 \\
55.5 \\
54.2 \\
50.3\end{array}$ \\
\hline $\begin{array}{l}1956 \\
1957 \\
1958 \\
1959 \\
1960\end{array}$ & $\begin{array}{l}28.9 \\
28.4 \\
40.0 \\
34.2 \\
31.3\end{array}$ & $\begin{array}{l}55.1 \\
55.0 \\
54.3 \\
52.3 \\
52.1\end{array}$ & $\begin{array}{l}73.3 \\
72.7 \\
78.2^{(2)} \\
72.7 \\
74.4\end{array}$ & $\begin{array}{l}52.0 \\
53.4 \\
53.5 \\
50.8 \\
54.5\end{array}$ \\
\hline $\begin{array}{l}1961 \\
1962 \\
1963 \\
1964 \\
1965\end{array}$ & $\begin{array}{l}35.9 \\
33.4 \\
33.5 \\
34.6 \\
32.8\end{array}$ & $\begin{array}{l}52.8 \\
51.7 \\
52.6 \\
51.2 \\
52.7\end{array}$ & $\begin{array}{l}77.9 \\
72.1 \\
72.5 \\
70.7 \\
73.5\end{array}$ & $\begin{array}{l}50.2 \\
54.3 \\
56.6 \\
51.5 \\
54.2\end{array}$ \\
\hline $\begin{array}{l}1966 \\
1967 \\
1968 \\
1969 \\
1970\end{array}$ & $\begin{array}{l}35.6 \\
40.6^{(2)} \\
36.9 \\
27.4 \\
35.3\end{array}$ & $\begin{array}{l}54.4 \\
50.8 \\
54.2 \\
54.2 \\
51.8\end{array}$ & $\begin{array}{l}71.9 \\
77.5 \\
73.7 \\
74.6 \\
76.2\end{array}$ & $\begin{array}{l}55.3 \\
56.1 \\
52.9 \\
52.9 \\
50.8\end{array}$ \\
\hline $\begin{array}{l}1971 \\
1972 \\
1973 \\
1974 \\
1975\end{array}$ & $\begin{array}{l}35.2 \\
32.0 \\
31.6 \\
36.1 \\
34.1\end{array}$ & $\begin{array}{l}52.2 \\
53.6 \\
54.7 \\
52.0 \\
50.3\end{array}$ & $\begin{array}{l}74.8 \\
74.5 \\
73.6 \\
74.2 \\
72.6\end{array}$ & $\begin{array}{l}51.2 \\
51.2 \\
52.2 \\
54.0 \\
53.3\end{array}$ \\
\hline $\begin{array}{l}1976 \\
1977 \\
1978 \\
1979 \\
1980\end{array}$ & $\begin{array}{l}34.7 \\
32.1 \\
34.7 \\
25.2 \\
31.6\end{array}$ & $\begin{array}{l}50.9 \\
53.2 \\
52.7 \\
54.5 \\
53.7\end{array}$ & $\begin{array}{l}70.5 \\
75.2 \\
72.9 \\
74.2 \\
\mathbf{7 0 . 2}^{(2)}\end{array}$ & $\begin{array}{l}54.0 \\
50.8 \\
49.4 \\
53.3 \\
53.2\end{array}$ \\
\hline $\begin{array}{l}1981 \\
1982 \\
1983 \\
1984 \\
1985\end{array}$ & $\begin{array}{l}38.1 \\
33.6 \\
36.8 \\
30.5 \\
26.2\end{array}$ & $\begin{array}{l}54.4 \\
51.9 \\
54.5 \\
51.2 \\
54.2\end{array}$ & $\begin{array}{l}73.0 \\
74.6 \\
70.4 \\
71.9 \\
74.3\end{array}$ & $\begin{array}{l}53.7 \\
51.2 \\
52.6 \\
\mathbf{4 9 . 8} \\
\mathbf{4 4 . 5 ^ { ( 2 ) }}\end{array}$ \\
\hline $\begin{array}{l}1986 \\
1987 \\
1988 \\
1989 \\
1990\end{array}$ & $\begin{array}{l}31.4 \\
34.4 \\
34.8 \\
32.1 \\
37.1\end{array}$ & $\begin{array}{l}53.9 \\
57.5 \\
54.1 \\
54.0 \\
55.5\end{array}$ & $\begin{array}{l}74.3 \\
74.8 \\
73.9 \\
73.6 \\
75.9\end{array}$ & $\begin{array}{l}53.1 \\
56.3 \\
56.5 \\
55.2 \\
\mathbf{5 7 . 1}^{(2)}\end{array}$ \\
\hline $\begin{array}{l}1991 \\
1992 \\
1993 \\
1994 \\
1995\end{array}$ & $\begin{array}{l}32.4 \\
39.3 \\
28.5 \\
36.7 \\
37.5\end{array}$ & 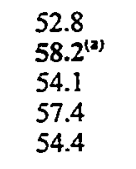 & $\begin{array}{l}74.2 \\
76.8 \\
70.7 \\
75.8 \\
72.4\end{array}$ & $\begin{array}{l}54.6 \\
53.8 \\
52.1 \\
54.8 \\
55.4\end{array}$ \\
\hline $\begin{array}{l}1996 \\
1997 \\
1998 \\
1999\end{array}$ & $\begin{array}{l}31.4 \\
34.5 \\
37.7 \\
37.7\end{array}$ & $\begin{array}{l}52.6 \\
54.7 \\
55.1 \\
51.7\end{array}$ & $\begin{array}{l}74.7 \\
73.9 \\
77.0 \\
72.5\end{array}$ & $\begin{array}{l}51.7 \\
54.4 \\
56.3 \\
54.2\end{array}$ \\
\hline $\begin{array}{l}\text { Average } \\
\text { Normal }\end{array}$ & $\begin{array}{l}33.6 \\
33.6\end{array}$ & $\begin{array}{l}53.3 \\
53.2\end{array}$ & $\begin{array}{l}73.6 \\
73.7\end{array}$ & $\begin{array}{l}53.1 \\
52.9\end{array}$ \\
\hline
\end{tabular}

(a) Highest and lowest averages. 


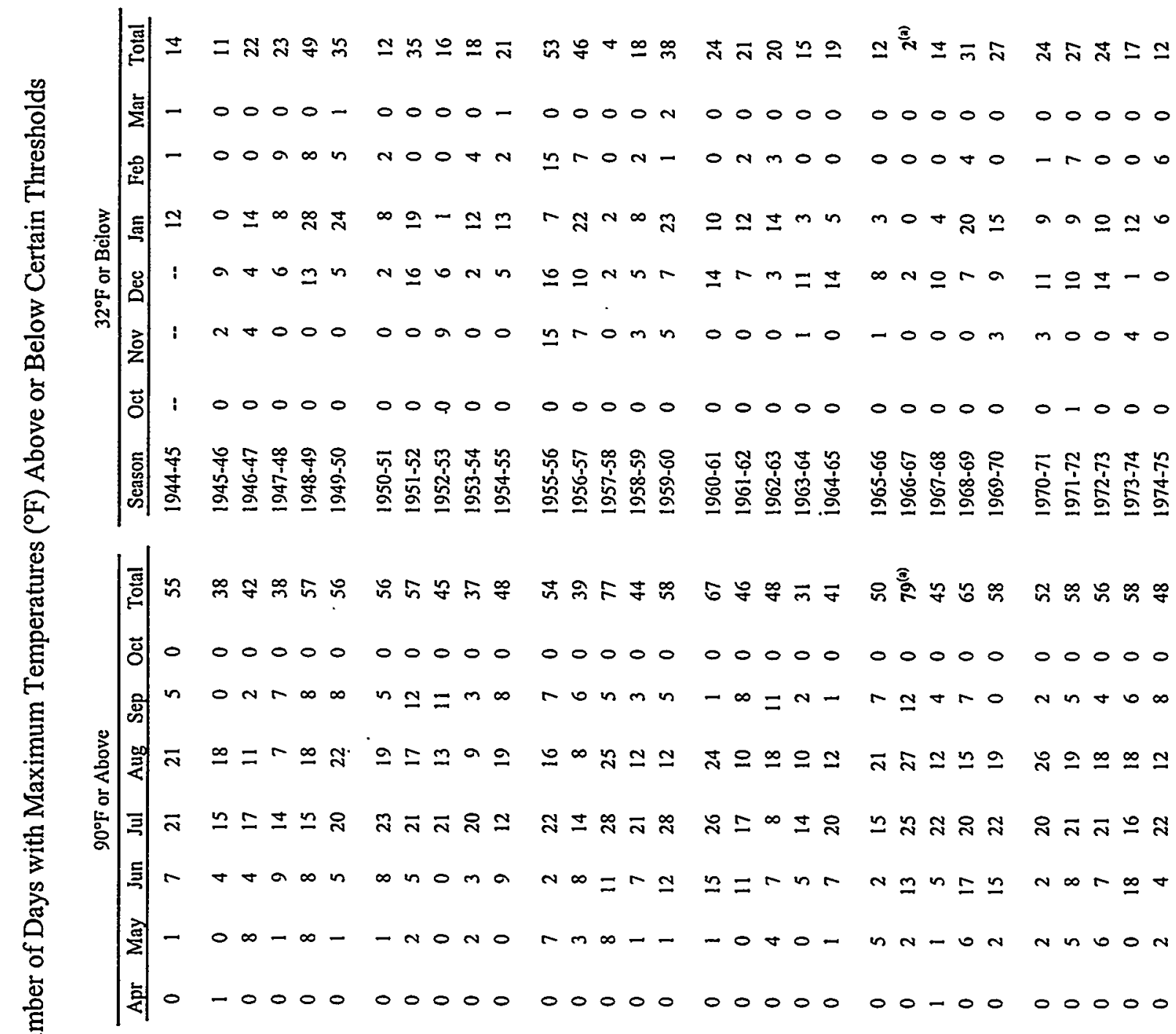

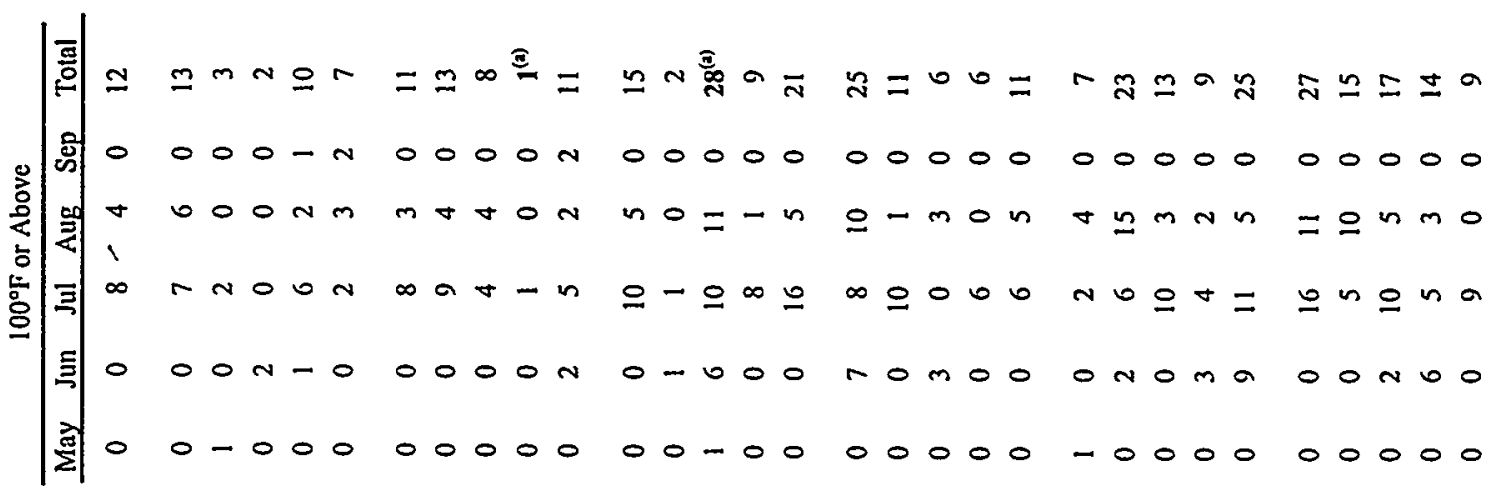

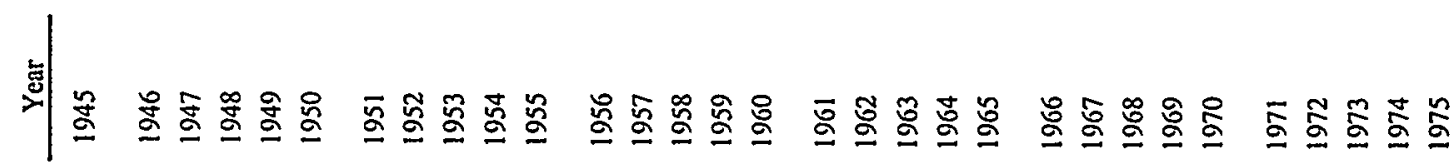




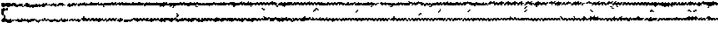

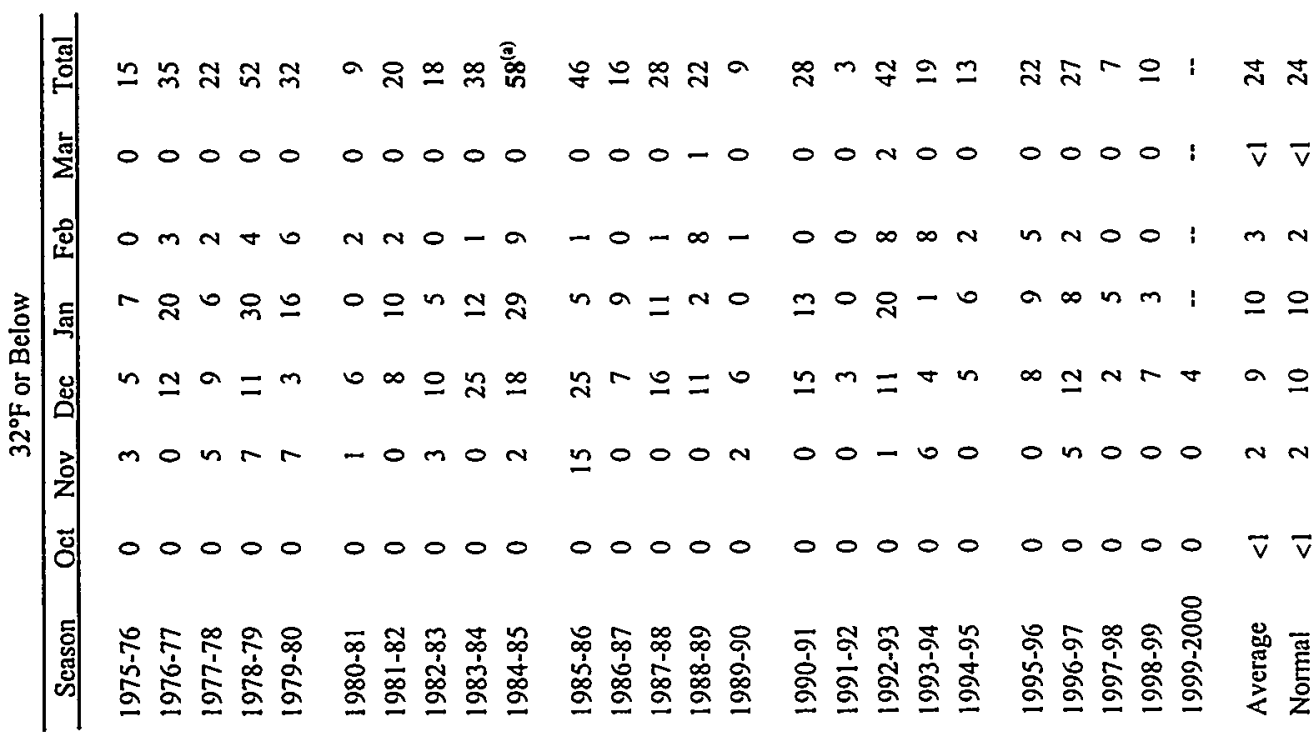

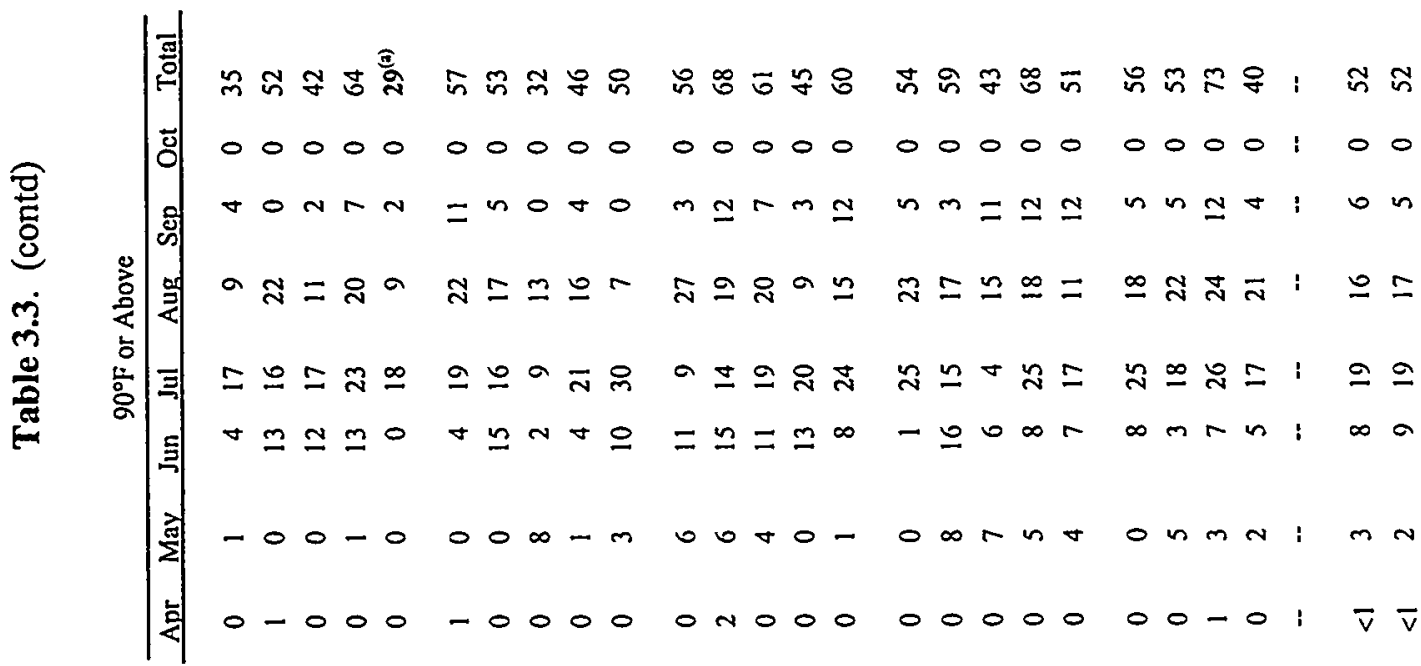

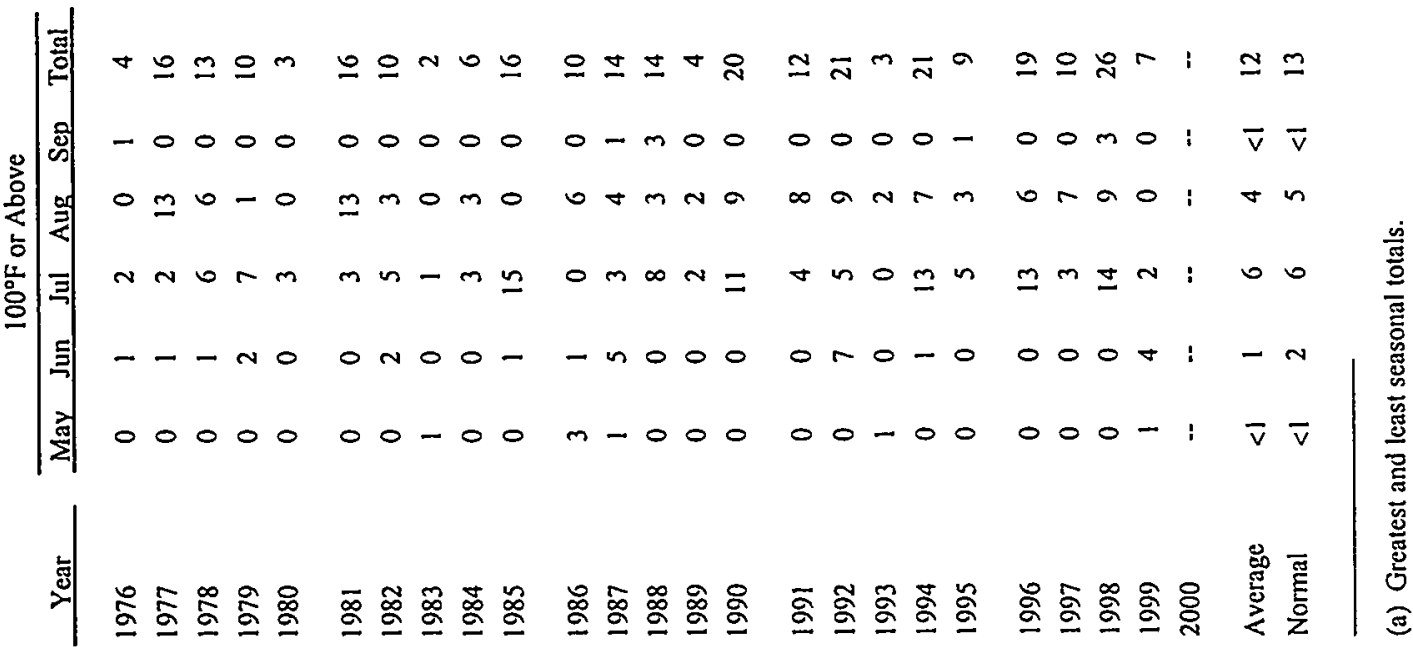


Table 3.4. Days with Maximum Temperatures $\geq 104^{\circ} \mathrm{F}$

\begin{tabular}{|c|c|c|c|c|c|c|c|}
\hline $\begin{array}{c}\text { Temperature, } \\
{ }^{\circ} \mathrm{F} \\
\end{array}$ & & & & (s) of Occu & ence & & \\
\hline 113 & $08 / 04 / 61$ & & & & & & \\
\hline 112 & $07 / 27 / 98$ & $08 / 09 / 71$ & & & - & & $\cdot$ \\
\hline $11 !$ & $07 / 22 / 94$ & $06 / 23 / 92$ & $07 / 31 / 71$ & & & & \\
\hline 110 & $\begin{array}{l}08 / 04 / 98 \\
07 / 17 / 60\end{array}$ & $07 / 12 / 90$ & $07 / 20 / 79$ & $07 / 09 / 75$ & $08 / 08 / 72$ & $07 / 06 / 68$ & $07 / 18 / 60$ \\
\hline 109 & $\begin{array}{l}08 / 10 / 96 \\
08 / 07 / 72\end{array}$ & $\begin{array}{l}07 / 24 / 94 \\
08 / 10 / 71\end{array}$ & $\begin{array}{l}07 / 23 / 94 \\
08 / 01 / 71\end{array}$ & $07 / 21 / 94$ & $08 / 14 / 92$ & $07 / 11 / 90$ & $07 / 19 / 79$ \\
\hline 108 & $\begin{array}{l}07 / 28 / 98 \\
07 / 27 / 75 \\
07 / 08 / 68 \\
06 / 17 / 61\end{array}$ & $\begin{array}{l}07 / 26 / 98 \\
07 / 05 / 75 \\
07 / 04 / 68\end{array}$ & $\begin{array}{l}07 / 26 / 96 \\
08 / 12 / 71 \\
08 / 18 / 67\end{array}$ & $\begin{array}{l}07 / 15 / 96 \\
08 / 11 / 71 \\
08 / 17 / 67\end{array}$ & $\begin{array}{l}06 / 24 / 92 \\
07 / 27 / 71 \\
08 / 16 / 67\end{array}$ & $\begin{array}{l}08 / 05 / 90 \\
07 / 19 / 71 \\
07 / 31 / 65\end{array}$ & $\begin{array}{l}07 / 18 / 79 \\
07 / 28 / 68 \\
07 / 13 / 61\end{array}$ \\
\hline 107 & $\begin{array}{l}08 / 13 / 92 \\
08 / 08 / 81 \\
08 / 03 / 61\end{array}$ & $\begin{array}{l}08 / 01 / 92 \\
07 / 17 / 79 \\
07 / 22 / 59\end{array}$ & $\begin{array}{l}07 / 31 / 92 \\
08 / 18 / 77 \\
07 / 20 / 59\end{array}$ & $\begin{array}{l}06 / 25 / 92 \\
08 / 08 / 71 \\
07 / 19 / 59\end{array}$ & $\begin{array}{l}07 / 14 / 87 \\
07 / 30 / 71 \\
07 / 28 / 58\end{array}$ & $\begin{array}{l}07 / 29 / 82 \\
07 / 28 / 71 \\
07 / 14 / 55\end{array}$ & $\begin{array}{l}07 / 28 / 82 \\
07 / 05 / 68\end{array}$ \\
\hline 106 & $\begin{array}{l}08 / 14 / 98 \\
08 / 18 / 92 \\
07 / 22 / 80 \\
07 / 15 / 73 \\
06 / 16 / 61\end{array}$ & $\begin{array}{l}08 / 05 / 98 \\
07 / 18 / 92 \\
08 / 09 / 78 \\
08 / 06 / 72 \\
06 / 22 / 58\end{array}$ & $\begin{array}{l}08 / 14 / 97 \\
06 / 22 / 92 \\
07 / 23 / 78 \\
07 / 20 / 71 \\
07 / 19 / 56\end{array}$ & $\begin{array}{l}08 / 04 / 97 \\
09 / 01 / 87 \\
08 / 17 / 77 \\
07 / 04 / 70 \\
07 / 09 / 52\end{array}$ & $\begin{array}{l}07 / 27 / 96 \\
06 / 30 / 87 \\
08 / 13 / 77 \\
08 / 01 / 65\end{array}$ & $\begin{array}{l}07 / 14 / 96 \\
07 / 09 / 85 \\
07 / 10 / 75 \\
07 / 12 / 64\end{array}$ & $\begin{array}{l}08 / 02 / 94 \\
07 / 25 / 84 \\
07 / 29 / 73 \\
07 / 24 / 62\end{array}$ \\
\hline 105 & $\begin{array}{l}07 / 28 / 99 \\
07 / 24 / 96 \\
08 / 09 / 87 \\
08 / 04 / 78 \\
07 / 16 / 70 \\
08 / 19 / 67 \\
07 / 07 / 60\end{array}$ & $\begin{array}{l}08 / 13 / 98 \\
07 / 19 / 95 \\
07 / 20 / 85 \\
08 / 03 / 78 \\
07 / 08 / 70 \\
08 / 15 / 67 \\
07 / 13 / 55\end{array}$ & $\begin{array}{l}07 / 22 / 98 \\
07 / 17 / 92 \\
07 / 27 / 82 \\
07 / 04 / 75 \\
07 / 27 / 68 \\
08 / 13 / 67 \\
08 / 04 / 52\end{array}$ & $\begin{array}{l}08 / 06 / 97 \\
07 / 03 / 91 \\
07 / 26 / 82 \\
07 / 21 / 71 \\
07 / 07 / 68 \\
07 / 03 / 67 \\
07 / 30 / 52\end{array}$ & $\begin{array}{l}08 / 05 / 97 \\
07 / 22 / 90 \\
08 / 16 / 81 \\
07 / 18 / 71 \\
07 / 03 / 68 \\
08 / 02 / 61 \\
07 / 10 / 52\end{array}$ & $\begin{array}{l}08 / 25 / 96 \\
07 / 15 / 90 \\
08 / 13 / 81 \\
08 / 23 / 70 \\
08 / 30 / 67 \\
08 / 11 / 60 \\
07 / 20 / 46\end{array}$ & $\begin{array}{l}07 / 28 / 96 \\
07 / 26 / 88 \\
08 / 12 / 81 \\
07 / 19 / 70 \\
08 / 20 / 67 \\
07 / 19 / 60 \\
08 / 16 / 45\end{array}$ \\
\hline 104 & $\begin{array}{l}08 / 03 / 98 \\
07 / 18 / 95 \\
05 / 31 / 86 \\
08 / 11 / 81 \\
08 / 20 / 77 \\
06 / 22 / 73 \\
07 / 03 / 70 \\
07 / 25 / 62 \\
08 / 25 / 58 \\
07 / 23 / 56 \\
07 / 31 / 49 \\
07 / 21 / 46\end{array}$ & $\begin{array}{l}07 / 25 / 98 \\
07 / 20 / 94 \\
05 / 30 / 86 \\
08 / 10 / 81 \\
08 / 12 / 77 \\
08 / 28 / 72 \\
06 / 23 / 70 \\
07 / 23 / 62 \\
08 / 24 / 58 \\
07 / 21 / 56 \\
07 / 30 / 49 \\
07 / 11 / 45\end{array}$ & $\begin{array}{l}07 / 17 / 98 \\
07 / 17 / 94 \\
07 / 29 / 85 \\
08 / 07 / 81 \\
07 / 30 / 74 \\
08 / 09 / 72 \\
06 / 21 / 70 \\
08 / 14 / 61 \\
08 / 11 / 58 \\
07 / 22 / 55 \\
07 / 15 / 49 \\
07 / 10 / 45\end{array}$ & $\begin{array}{l}08 / 09 / 96 \\
07 / 10 / 90 \\
07 / 21 / 85 \\
07 / 27 / 81 \\
07 / 28 / 74 \\
08 / 13 / 71 \\
08 / 31 / 67 \\
06 / 18 / 61 \\
07 / 17 / 58 \\
08 / 15 / 53 \\
06 / 29 / 48\end{array}$ & $\begin{array}{l}07 / 25 / 96 \\
07 / 25 / 88 \\
07 / 08 / 85 \\
07 / 04 / 81 \\
08 / 01 / 73 \\
08 / 07 / 71 \\
08 / 11 / 67 \\
08 / 10 / 60 \\
07 / 11 / 58 \\
07 / 23 / 51 \\
08 / 22 / 46\end{array}$ & $\begin{array}{l}07 / 23 / 96 \\
07 / 21 / 88 \\
07 / 04 / 85 \\
08 / 08 / 78 \\
07 / 27 / 73 \\
07 / 16 / 71 \\
07 / 12 / 67 \\
07 / 16 / 60 \\
08 / 22 / 56 \\
07 / 17 / 51 \\
08 / 21 / 46\end{array}$ & $\begin{array}{l}07 / 13 / 96 \\
07 / 20 / 88 \\
08 / 07 / 82 \\
07 / 25 / 78 \\
07 / 19 / 73 \\
07 / 09 / 70 \\
07 / 30 / 65 \\
07 / 18 / 59 \\
07 / 24 / 56 \\
08 / 01 / 49 \\
07 / 28 / 46\end{array}$ \\
\hline
\end{tabular}

Maximum temperatures $\geq 90^{\circ} \mathrm{F}$ occur an average of 52 times per year and vary from a low of 29 times in 1980 to a high of 79 times in 1967. The earliest occurrences varied from April 24 (1977) through July 2 (1953), with an average annual date of May 21 (Table 3.5). The latest annual occurrence of maximum temperatures $\geq 90^{\circ} \mathrm{F}$ varied from August 17 (1983) through September 29 (1993 and earlier years). The average date for the period 1946 through 1999 is September 15. The longest period of consecutive maximum temperatures $\geq 90^{\circ} \mathrm{F}$ is 32 days from July 13 through August 13, 1971.

The average seasonal number of days with maximum temperatures $\leq 32^{\circ} \mathrm{F}$ is 24 . The earliest seasonal occurrence of a day with a maximum temperature $\leq 32^{\circ} \mathrm{F}$ was October 30 (1971) and the latest was March 11 (1950). The number of winter days with maximum temperatures $\leq 32^{\circ} \mathrm{F}$ varied from 
Table 3.5. Record of Annual First and Last Dates with Maximum Temperatures $\geq 90^{\circ} \mathrm{F}$ and Minimum Temperatures $\leq 32^{\circ} \mathrm{F}$

\begin{tabular}{|c|c|c|c|c|c|}
\hline \multirow[b]{2}{*}{ Year } & \multicolumn{2}{|c|}{ Maximum Temperature $\geq 90^{\circ} \mathrm{F}$} & \multicolumn{2}{|c|}{ Minimum Temperature $\leq 32^{\circ} \mathrm{F}$} & \multirow[b]{2}{*}{ Growing Days ${ }^{(2)}$} \\
\hline & First in Spring & Last in Summer & Last in Spring & First in Autumm & \\
\hline 1945 & May 30 & Sep 14 & Apr 10 & Oct 18 & 190 \\
\hline $\begin{array}{l}1946 \\
1947 \\
1948 \\
1949 \\
1950\end{array}$ & $\begin{array}{l}\text { Apr } 25 \\
\text { May 06 } \\
\text { May 26 } \\
\text { May 08 } \\
\text { May 26 }\end{array}$ & $\begin{array}{l}\text { Aug } 24 \\
\text { Sep 12 } \\
\text { Sep } 13 \\
\text { Sep } 27 \\
\text { Sep 23 }\end{array}$ & $\begin{array}{l}\text { Apr } 07 \\
\text { Apr 07 } \\
\text { May 02 } \\
\text { May 03 } \\
\text { Apr 27 }\end{array}$ & $\begin{array}{l}\text { Oct } 11 \\
\text { Nov } 04 \\
\text { Oct } 17 \\
\text { Oct } 08 \\
\text { Nov } 08\end{array}$ & $\begin{array}{l}186 \\
210 \\
167 \\
157 \\
194\end{array}$ \\
\hline $\begin{array}{l}1951 \\
1952 \\
1953 \\
1954 \\
1955\end{array}$ & $\begin{array}{l}\text { May } 22 \\
\text { May 24 } \\
\text { Jul 02 } \\
\text { May } 17 \\
\text { Jun 06 }\end{array}$ & $\begin{array}{l}\text { Sep } 19 \\
\text { Sep } 26 \\
\text { Sep } 15 \\
\text { Sep } 10 \\
\text { Sep } 10\end{array}$ & $\begin{array}{l}\text { Apr 21 } \\
\text { Apr 29 } \\
\text { Apr 15 } \\
\text { May 01 } \\
\text { May 14 }\end{array}$ & $\begin{array}{l}\text { Oct } 15 \\
\text { Nov } 01 \\
\text { Oct } 24 \\
\text { Oct } 01 \\
\text { Oct } 31\end{array}$ & $\begin{array}{l}176 \\
185 \\
191 \\
152 \\
169\end{array}$ \\
\hline $\begin{array}{l}1956 \\
1957 \\
1958 \\
1959 \\
1960\end{array}$ & $\begin{array}{l}\text { May } 16 \\
\text { May } 29 \\
\text { May } 18 \\
\text { May 13 } \\
\text { May 10 }\end{array}$ & $\begin{array}{l}\text { Sep } 19 \\
\text { Sep } 15 \\
\text { Sep } 10 \\
\text { Sep } 13 \\
\text { Sep } 18\end{array}$ & $\begin{array}{l}\text { Apr 06 } \\
\text { Mar 26 } \\
\text { Mar 19(0) } \\
\text { May 05 } \\
\text { Apr 21 }\end{array}$ & $\begin{array}{l}\text { Oct } 22 \\
\text { Oct 22 } \\
\text { Oct 21 } \\
\text { Oct } 30 \\
\text { Oct } 11\end{array}$ & $\begin{array}{l}198 \\
209 \\
215 \\
177 \\
172\end{array}$ \\
\hline $\begin{array}{l}1961 \\
1962 \\
1963 \\
1964 \\
1965\end{array}$ & $\begin{array}{l}\text { May 25 } \\
\text { Jun 08 } \\
\text { May 20 } \\
\text { Jun 23 } \\
\text { May 28 }\end{array}$ & $\begin{array}{l}\text { Sep 04 } \\
\text { Sep 26 } \\
\text { Sep 29 } \\
\text { Sep 24 } \\
\text { Sep 01 }\end{array}$ & $\begin{array}{l}\text { Apr } 19 \\
\text { May 04 } \\
\text { Apr } 16 \\
\text { Apr } 19 \\
\text { May 05 }\end{array}$ & $\begin{array}{l}\text { Oct } 20 \\
\text { Nov } 12^{(\mathfrak{b})} \\
\text { Oct } 25 \\
\text { Oct } 16 \\
\text { Oct } 16\end{array}$ & $\begin{array}{l}183 \\
191 \\
191 \\
179 \\
163\end{array}$ \\
\hline $\begin{array}{l}1966 \\
1967 \\
1968 \\
1969 \\
1970\end{array}$ & $\begin{array}{l}\text { May } 03 \\
\text { May } 20 \\
\text { Apr } 29 \\
\text { May } 09 \\
\text { May } 16\end{array}$ & $\begin{array}{l}\text { Sep } 22 \\
\text { Sep } 28 \\
\text { Sep 09 } \\
\text { Sep 12 } \\
\text { Aug } 31\end{array}$ & $\begin{array}{l}\text { Apr 19 } \\
\text { Apr 28 } \\
\text { Apr } 22 \\
\text { Apr 26 } \\
\text { May 11 }\end{array}$ & $\begin{array}{l}\text { Oct } 14 \\
\text { Oct } 26 \\
\text { Oct } 21 \\
\text { Oct } 13 \\
\text { Oct } 07\end{array}$ & $\begin{array}{l}177 \\
180 \\
181 \\
169 \\
148\end{array}$ \\
\hline $\begin{array}{l}1971 \\
1972 \\
1973 \\
1974 \\
1975\end{array}$ & $\begin{array}{l}\text { May } 11 \\
\text { May } 13 \\
\text { May } 13 \\
\text { Jun } 10 \\
\text { May } 30\end{array}$ & $\begin{array}{l}\text { Sep } 10 \\
\text { Sep } 16 \\
\text { Sep } 11 \\
\text { Sep } 25 \\
\text { Sep } 15\end{array}$ & $\begin{array}{l}\text { Apr } 22 \\
\text { Apr } 30 \\
\text { Apr } 08 \\
\text { May } 16^{(0)} \\
\text { Apr } 29\end{array}$ & $\begin{array}{l}\text { Oct } 16 \\
\text { Sep 25 } \\
\text { Oct } 04 \\
\text { Oct } 06 \\
\text { Oct } 23\end{array}$ & $\begin{array}{l}176 \\
147 \\
178 \\
142 \\
176\end{array}$ \\
\hline $\begin{array}{l}1976 \\
1977 \\
1978 \\
1979 \\
1980\end{array}$ & $\begin{array}{l}\text { May 16 } \\
\text { Apr 24(b) } \\
\text { Jun 02 } \\
\text { May 22 } \\
\text { Jun 01 }\end{array}$ & $\begin{array}{l}\text { Sep 29 } \\
\text { Aug 22 } \\
\text { Sep 03 } \\
\text { Sep 20 } \\
\text { Sep 06 }\end{array}$ & $\begin{array}{l}\text { Apr } 23 \\
\text { Apr } 14 \\
\text { Apr } 23 \\
\text { Apr } 19 \\
\text { Apr } 11\end{array}$ & $\begin{array}{l}\text { Oct } 19 \\
\text { Oct } 27 \\
\text { Oct } 07 \\
\text { Oct } 31 \\
\text { Oct } 22\end{array}$ & $\begin{array}{l}178 \\
195 \\
166 \\
194 \\
193\end{array}$ \\
\hline $\begin{array}{l}1981 \\
1982 \\
1983 \\
1984 \\
1985\end{array}$ & $\begin{array}{l}\text { Apr } 30 \\
\text { Jun 10 } \\
\text { May 23 } \\
\text { May } 29 \\
\text { May } 18\end{array}$ & $\begin{array}{l}\text { Sep } 18 \\
\text { Sep } 08 \\
\text { Aug } 17^{(b)} \\
\text { Sep } 18 \\
\text { Aug } 29\end{array}$ & $\begin{array}{l}\text { Apr 13 } \\
\text { Apr 21 } \\
\text { Apr 16 } \\
\text { Apr 13 } \\
\text { Apr 21 }\end{array}$ & $\begin{array}{l}\text { Oct } 14 \\
\text { Oct } 18 \\
\text { Oct } 11 \\
\text { Oct } 14 \\
\text { Oct } 07\end{array}$ & $\begin{array}{l}183 \\
179 \\
177 \\
183 \\
168\end{array}$ \\
\hline $\begin{array}{l}1986 \\
1987 \\
1988 \\
1989 \\
1990\end{array}$ & $\begin{array}{l}\text { May } 25 \\
\text { Apr } 27 \\
\text { May } 11 \\
\text { Jun 01 } \\
\text { May 05 }\end{array}$ & $\begin{array}{l}\text { Sep } 04 \\
\text { Sep 23 } \\
\text { Sep } 14 \\
\text { Sep 24 } \\
\text { Sep 29 }\end{array}$ & $\begin{array}{l}\text { Apr } 30 \\
\text { Apr 20 } \\
\text { Apr 09 } \\
\text { Mar } 30 \\
\text { Mar } 27\end{array}$ & $\begin{array}{l}\text { Nov } 09 \\
\text { Oct } 16 \\
\text { Oct } 27 \\
\text { Oct } 29 \\
\text { Oct } 17\end{array}$ & $\begin{array}{l}192 \\
178 \\
200 \\
212 \\
203\end{array}$ \\
\hline $\begin{array}{l}1991 \\
1992 \\
1993 \\
1994 \\
1995\end{array}$ & $\begin{array}{l}\text { Jun } 10 \\
\text { May 04 } \\
\text { May 10 } \\
\text { May 07 } \\
\text { May 28 }\end{array}$ & $\begin{array}{l}\text { Sep } 26 \\
\text { Sep } 03 \\
\text { Sep } 29^{(0, e)} \\
\text { Sep } 28 \\
\text { Sep 17 }\end{array}$ & $\begin{array}{l}\text { Apr 08 } \\
\text { Apr 08 } \\
\text { Apr 06 } \\
\text { Mar 26 } \\
\text { Apr } 15\end{array}$ & $\begin{array}{l}\text { Oct } 22 \\
\text { Oct } 15 \\
\text { Oct } 20 \\
\text { Oct } 29 \\
\text { Oct } 29\end{array}$ & $\begin{array}{l}196 \\
189 \\
196 \\
216 \\
196\end{array}$ \\
\hline $\begin{array}{l}1996 \\
1997 \\
1998 \\
1999\end{array}$ & $\begin{array}{l}\text { Jun } 02 \\
\text { May } 12 \\
\text { Apr } 30 \\
\text { May } 23\end{array}$ & $\begin{array}{l}\text { Sep } 15 \\
\text { Sep } 25 \\
\text { Sep } 17 \\
\text { Sep } 22\end{array}$ & $\begin{array}{l}\text { May } 08 \\
\text { May 02 } \\
\text { Apr } 13 \\
\text { May } 8\end{array}$ & $\begin{array}{l}\text { Oct } 17 \\
\text { Oct } 08 \\
\text { Oct } 19 \\
\text { Oct } 17\end{array}$ & $\begin{array}{l}161 \\
158 \\
188 \\
163\end{array}$ \\
\hline $\begin{array}{l}\text { Average } \\
\text { Normal }\end{array}$ & $\begin{array}{l}\text { May } 21 \\
\text { May 21 }\end{array}$ & $\begin{array}{l}\text { Sep } 15 \\
\text { Sep } 14\end{array}$ & $\begin{array}{l}\text { Apr } 20 \\
\text { Apr } 21\end{array}$ & $\begin{array}{l}\text { Oct } 19 \\
\text { Oct } 18\end{array}$ & $\begin{array}{l}181 \\
179\end{array}$ \\
\hline
\end{tabular}

(a) Days between last freezing temperature in spring and first freezing temperature in autumn.

(b) Earliest and latest dates.

(c) Also in previous years. 
2 to 58 days (winters of 1966-1967 and 1984-1985, respectively). The greatest consecutive number of days with maximum temperatures $\leq 32^{\circ} \mathrm{F}$ is 29 days, from December 30, 1984, through January 27, 1985. During the period December 27, 1978, through February 4, 1979 (40 days), only 1 maximum temperature greater than $32^{\circ} \mathrm{F}$ occurred. The average maximum temperature for that period was $21^{\circ} \mathrm{F}$.

Table 3.6 lists the monthly and annual maximum temperatures. Only 6 days were recorded when the daily maximum temperature was $\leq 0^{\circ} \mathrm{F}$. These were:

\begin{tabular}{lc}
\multicolumn{1}{c}{ Maximum Date } & Temperature \\
January 31, 1950 & $-2^{\circ} \mathrm{F}$ \\
February 1, 1950 & $-3^{\circ} \mathrm{F}$ \\
February 2, 1950 & $-3^{\circ} \mathrm{F}$ \\
January 27, 1957 & $0^{\circ} \mathrm{F}$ \\
December 29, 1968 & $-2^{\circ} \mathrm{F}$ \\
December 30, 1968 & $-2^{\circ} \mathrm{F}$
\end{tabular}

\subsection{Days with Minimum Temperatures $\leq 32^{\circ} \mathrm{F}$ or $\leq 0^{\circ} \mathrm{F}$}

The monthly and seasonal number of days with minimum temperatures at or below $32^{\circ} \mathrm{F}$ or $0^{\circ} \mathrm{F}$ are listed in Table 3.7.

The seasonal average number of days with minimum temperatures $\leq 32^{\circ} \mathrm{F}$ is 106 ; however, the number ranges from 70 to 143 days (winters of 1991-1992 and 1984-1985, respectively). The greatest consecutive number of days with minimum temperatures of $\leq 32^{\circ} \mathrm{F}$ is 93 , from November 9,1978 , through February 9, 1979.

The first autumn temperature $\leq 32^{\circ} \mathrm{F}$ occurred as early as September 25 (1972) and as late as November 12 (1962). The average date is October 19 (see Table 3.5). The last date in spring for minimum temperatures $\leq 32^{\circ} \mathrm{F}$ varied from March 19 (1958) to May $16(1974)$, with an average date of April 20. The average number of days between last freezing temperature in the spring and first freezing temperature in the autumn is 181 days.

On average, 3 days per winter season have a minimum temperature $\leq 0^{\circ} \mathrm{F}$; however, nearly half of all winters have no minimum temperatures in this category (see Table 3.7). The most of those days in any season was 18 (winter of 1949-1950) and the least of those days was 0 (as recently as the winter of 19971998). The greatest number of consecutive days with minimum temperatures $\leq 0^{\circ} \mathrm{F}$ is 11 days, from January 25 through February 4, 1950. During this same period, 4 consecutive days had minimum temperatures $\leq-20^{\circ} \mathrm{F}$. Table 3.8 lists all days with minimum temperatures $\leq 0^{\circ} \mathrm{F}$. Table 3.9 lists monthly and annual minimum temperatures. 
Table 3.6. Monthly and Annual Maximum Temperatures $\left({ }^{\circ} \mathrm{F}\right)$

\begin{tabular}{|c|c|c|c|c|c|c|c|c|c|c|c|c|c|}
\hline Year & Jan & Feb & Mar & $\mathrm{Apr}$ & May & Jun & Jul & Aug & Sep & Oct & Nov & $\underline{\text { Dec }}$ & Annual \\
\hline 1945 & 61 & 65 & 72 & 76 & 90 & 98 & 104 & 105 & 93 & 84 & 72 & 57 & 105 \\
\hline $\begin{array}{l}1946 \\
1947 \\
1948 \\
1949 \\
1950\end{array}$ & $\begin{array}{l}57 \\
57 \\
60 \\
48 \\
50\end{array}$ & $\begin{array}{l}60 \\
68 \\
64 \\
56 \\
63\end{array}$ & $\begin{array}{l}76 \\
76 \\
73 \\
64 \\
64\end{array}$ & $\begin{array}{l}91 \\
88 \\
76 \\
84 \\
78\end{array}$ & $\begin{array}{r}89 \\
101 \\
91 \\
98 \\
90\end{array}$ & $\begin{array}{r}98 \\
97 \\
104 \\
102 \\
99\end{array}$ & $\begin{array}{r}105 \\
103 \\
98 \\
104 \\
102\end{array}$ & $\begin{array}{r}104 \\
98 \\
97 \\
104 \\
103\end{array}$ & $\begin{array}{r}89 \\
94 \\
98 \\
100 \\
102\end{array}$ & $\begin{array}{l}75 \\
83 \\
78 \\
74 \\
76\end{array}$ & $\begin{array}{l}64 \\
63 \\
57 \\
65 \\
62\end{array}$ & $\begin{array}{l}64 \\
55 \\
52 \\
60 \\
55\end{array}$ & $\begin{array}{l}105 \\
103 \\
104 \\
104 \\
103\end{array}$ \\
\hline $\begin{array}{l}1951 \\
1952 \\
1953 \\
1954 \\
1955\end{array}$ & $\begin{array}{l}55 \\
50 \\
63 \\
59 \\
50\end{array}$ & $\begin{array}{l}65 \\
55 \\
65 \\
63 \\
58\end{array}$ & $\begin{array}{l}65 \\
70 \\
69 \\
65 \\
63\end{array}$ & $\begin{array}{l}82 \\
89 \\
78 \\
83 \\
77\end{array}$ & $\begin{array}{l}94 \\
92 \\
88 \\
98 \\
86\end{array}$ & $\begin{array}{r}97 \\
94 \\
86 \\
94 \\
102\end{array}$ & $\begin{array}{l}104 \\
106 \\
103 \\
100 \\
107\end{array}$ & $\begin{array}{r}101 \\
105 \\
104 \\
99 \\
101\end{array}$ & $\begin{array}{r}97 \\
97 \\
97 \\
92 \\
101\end{array}$ & $\begin{array}{l}79 \\
85 \\
81 \\
73 \\
75\end{array}$ & $\begin{array}{l}60 \\
62 \\
65 \\
62 \\
66\end{array}$ & $\begin{array}{l}58 \\
54 \\
59 \\
54 \\
56\end{array}$ & $\begin{array}{l}104 \\
106 \\
104 \\
100 \\
107\end{array}$ \\
\hline $\begin{array}{l}1956 \\
1957 \\
1958 \\
1959 \\
1960\end{array}$ & $\begin{array}{l}59 \\
48 \\
60 \\
59 \\
55\end{array}$ & $\begin{array}{l}56 \\
65 \\
63 \\
60 \\
55\end{array}$ & $\begin{array}{l}64 \\
66 \\
63 \\
65 \\
83\end{array}$ & $\begin{array}{l}85 \\
89 \\
78 \\
79 \\
82\end{array}$ & $\begin{array}{r}96 \\
97 \\
101 \\
91 \\
90\end{array}$ & $\begin{array}{r}95 \\
100 \\
106 \\
97 \\
96\end{array}$ & $\begin{array}{l}106 \\
102 \\
107 \\
107 \\
110\end{array}$ & $\begin{array}{r}104 \\
96 \\
104 \\
103 \\
105\end{array}$ & $\begin{array}{l}94 \\
98 \\
97 \\
92 \\
94\end{array}$ & $\begin{array}{l}79 \\
73 \\
89 \\
77 \\
82\end{array}$ & $\begin{array}{l}64 \\
60 \\
67 \\
70 \\
63\end{array}$ & $\begin{array}{l}59 \\
59 \\
60 \\
64 \\
52\end{array}$ & $\begin{array}{l}106 \\
102 \\
107 \\
107 \\
110\end{array}$ \\
\hline $\begin{array}{l}1961 \\
1962 \\
1963 \\
1964 \\
1965\end{array}$ & $\begin{array}{l}60 \\
63 \\
56 \\
57 \\
60\end{array}$ & $\begin{array}{l}64 \\
60 \\
64 \\
60 \\
67\end{array}$ & $\begin{array}{l}68 \\
70 \\
70 \\
74 \\
71\end{array}$ & $\begin{array}{l}75 \\
85 \\
72 \\
73 \\
82\end{array}$ & $\begin{array}{l}94 \\
81 \\
93 \\
88 \\
91\end{array}$ & $\begin{array}{r}108 \\
98 \\
102 \\
95 \\
96\end{array}$ & $\begin{array}{r}108 \\
106 \\
96 \\
106 \\
108\end{array}$ & $\begin{array}{r}113 \\
100 \\
101 \\
97 \\
106\end{array}$ & $\begin{array}{l}90 \\
97 \\
98 \\
90 \\
91\end{array}$ & $\begin{array}{l}81 \\
76 \\
83 \\
80 \\
84\end{array}$ & $\begin{array}{l}58 \\
67 \\
61 \\
60 \\
64\end{array}$ & $\begin{array}{l}56 \\
56 \\
57 \\
57 \\
56\end{array}$ & $\begin{array}{l}113 \\
106 \\
102 \\
106 \\
108\end{array}$ \\
\hline $\begin{array}{l}1966 \\
1967 \\
1968 \\
1969 \\
1970\end{array}$ & $\begin{array}{l}56 \\
62 \\
66 \\
44 \\
56\end{array}$ & $\begin{array}{l}59 \\
67 \\
64 \\
46 \\
60\end{array}$ & $\begin{array}{l}78 \\
65 \\
68 \\
74 \\
67\end{array}$ & $\begin{array}{l}81 \\
71 \\
90 \\
80 \\
71\end{array}$ & $\begin{array}{r}100 \\
92 \\
90 \\
95 \\
92\end{array}$ & $\begin{array}{r}95 \\
101 \\
99 \\
103 \\
104\end{array}$ & $\begin{array}{l}100 \\
105 \\
110 \\
101 \\
106\end{array}$ & $\begin{array}{l}102 \\
108 \\
102 \\
102 \\
105\end{array}$ & $\begin{array}{l}99 \\
98 \\
97 \\
96 \\
89\end{array}$ & $\begin{array}{l}82 \\
78 \\
73 \\
74 \\
86\end{array}$ & $\begin{array}{l}64 \\
65 \\
60 \\
63 \\
63\end{array}$ & $\begin{array}{l}56 \\
62 \\
59 \\
54 \\
58\end{array}$ & $\begin{array}{l}102 \\
108 \\
110 \\
103 \\
106\end{array}$ \\
\hline $\begin{array}{l}1971 \\
1972 \\
1973 \\
1974 \\
1975\end{array}$ & $\begin{array}{l}72 \\
59 \\
51 \\
61 \\
56\end{array}$ & $\begin{array}{l}66 \\
68 \\
61 \\
59 \\
58\end{array}$ & $\begin{array}{l}65 \\
76 \\
68 \\
69 \\
65\end{array}$ & $\begin{array}{l}76 \\
78 \\
80 \\
77 \\
75\end{array}$ & $\begin{array}{l}92 \\
96 \\
98 \\
86 \\
90\end{array}$ & $\begin{array}{r}99 \\
98 \\
104 \\
103 \\
95\end{array}$ & $\begin{array}{l}111 \\
103 \\
106 \\
104 \\
110\end{array}$ & $\begin{array}{r}112 \\
110 \\
104 \\
103 \\
98\end{array}$ & $\begin{array}{l}91 \\
95 \\
98 \\
92 \\
96\end{array}$ & $\begin{array}{l}85 \\
83 \\
76 \\
80 \\
82\end{array}$ & $\begin{array}{l}64 \\
58 \\
62 \\
64 \\
75\end{array}$ & $\begin{array}{l}50 \\
65 \\
58 \\
60 \\
62\end{array}$ & $\begin{array}{l}112 \\
110 \\
106 \\
104 \\
110\end{array}$ \\
\hline $\begin{array}{l}1976 \\
1977 \\
1978 \\
1979 \\
1980\end{array}$ & $\begin{array}{l}59 \\
61 \\
51 \\
37 \\
51\end{array}$ & $\begin{array}{l}59 \\
70 \\
57 \\
62 \\
59\end{array}$ & $\begin{array}{l}69 \\
73 \\
74 \\
76 \\
68\end{array}$ & $\begin{array}{l}80 \\
94 \\
76 \\
83 \\
87\end{array}$ & $\begin{array}{l}90 \\
82 \\
87 \\
94 \\
87\end{array}$ & $\begin{array}{r}100 \\
100 \\
101 \\
102 \\
88\end{array}$ & $\begin{array}{l}102 \\
101 \\
106 \\
110 \\
106\end{array}$ & $\begin{array}{r}98 \\
107 \\
106 \\
101 \\
98\end{array}$ & $\begin{array}{r}102 \\
87 \\
90 \\
96 \\
95\end{array}$ & $\begin{array}{l}84 \\
75 \\
81 \\
84 \\
89\end{array}$ & $\begin{array}{l}71 \\
68 \\
69 \\
59 \\
65\end{array}$ & $\begin{array}{l}57 \\
64 \\
54 \\
59 \\
69\end{array}$ & $\begin{array}{l}102 \\
107 \\
106 \\
110 \\
106\end{array}$ \\
\hline $\begin{array}{l}1981 \\
1982 \\
1983 \\
1984 \\
1985\end{array}$ & $\begin{array}{l}55 \\
57 \\
61 \\
60 \\
36\end{array}$ & $\begin{array}{l}66 \\
68 \\
62 \\
62 \\
60\end{array}$ & $\begin{array}{l}70 \\
71 \\
64 \\
67 \\
68\end{array}$ & $\begin{array}{l}91 \\
81 \\
77 \\
79 \\
82\end{array}$ & $\begin{array}{r}89 \\
88 \\
103 \\
94 \\
95\end{array}$ & $\begin{array}{r}96 \\
102 \\
92 \\
96 \\
102\end{array}$ & $\begin{array}{l}104 \\
107 \\
100 \\
106 \\
106\end{array}$ & $\begin{array}{r}107 \\
104 \\
99 \\
103 \\
97\end{array}$ & $\begin{array}{l}99 \\
94 \\
87 \\
92 \\
86\end{array}$ & $\begin{array}{l}83 \\
75 \\
78 \\
81 \\
74\end{array}$ & $\begin{array}{l}65 \\
63 \\
67 \\
61 \\
66\end{array}$ & $\begin{array}{l}58 \\
62 \\
46 \\
52 \\
39\end{array}$ & $\begin{array}{l}107 \\
107 \\
103 \\
106 \\
106\end{array}$ \\
\hline $\begin{array}{l}1986 \\
1987 \\
1988 \\
1989 \\
1990\end{array}$ & $\begin{array}{l}57 \\
55 \\
54 \\
67 \\
60\end{array}$ & $\begin{array}{l}72 \\
60 \\
71 \\
53 \\
64\end{array}$ & $\begin{array}{l}74 \\
70 \\
71 \\
67 \\
76\end{array}$ & $\begin{array}{l}84 \\
93 \\
83 \\
80 \\
81\end{array}$ & $\begin{array}{r}104 \\
102 \\
94 \\
88 \\
94\end{array}$ & $\begin{array}{r}103 \\
106 \\
99 \\
97 \\
96\end{array}$ & $\begin{array}{r}99 \\
107 \\
105 \\
101 \\
110\end{array}$ & $\begin{array}{l}103 \\
105 \\
102 \\
103 \\
108\end{array}$ & $\begin{array}{r}95 \\
106 \\
102 \\
94 \\
98\end{array}$ & $\begin{array}{l}84 \\
87 \\
88 \\
80 \\
80\end{array}$ & $\begin{array}{l}63 \\
66 \\
69 \\
73 \\
68\end{array}$ & $\begin{array}{l}52 \\
59 \\
57 \\
58 \\
57\end{array}$ & $\begin{array}{l}104 \\
107 \\
105 \\
103 \\
110\end{array}$ \\
\hline $\begin{array}{l}1991 \\
1992 \\
1993 \\
1994 \\
1995\end{array}$ & $\begin{array}{l}59 \\
60 \\
56 \\
61 \\
67\end{array}$ & $\begin{array}{l}66 \\
62 \\
52 \\
63 \\
68\end{array}$ & $\begin{array}{l}69 \\
78 \\
66 \\
79 \\
69\end{array}$ & $\begin{array}{l}82 \\
85 \\
73 \\
88 \\
80\end{array}$ & $\begin{array}{r}83 \\
98 \\
100 \\
95 \\
95\end{array}$ & $\begin{array}{r}93 \\
111 \\
98 \\
101 \\
98\end{array}$ & $\begin{array}{r}105 \\
107 \\
96 \\
111 \\
105\end{array}$ & $\begin{array}{l}103 \\
109 \\
100 \\
106 \\
102\end{array}$ & $\begin{array}{r}95 \\
91 \\
98 \\
94 \\
101\end{array}$ & $\begin{array}{l}88 \\
87 \\
86 \\
84 \\
74\end{array}$ & $\begin{array}{l}65 \\
62 \\
65 \\
62 \\
69\end{array}$ & $\begin{array}{l}59 \\
53 \\
67 \\
64 \\
57\end{array}$ & $\begin{array}{l}105 \\
111 \\
100 \\
111 \\
105\end{array}$ \\
\hline $\begin{array}{l}1996 \\
1997 \\
1998 \\
1999\end{array}$ & $\begin{array}{l}58 \\
57 \\
57 \\
62\end{array}$ & $\begin{array}{l}63 \\
64 \\
58 \\
62\end{array}$ & $\begin{array}{l}68 \\
76 \\
72 \\
75\end{array}$ & $\begin{array}{l}82 \\
75 \\
92 \\
82\end{array}$ & $\begin{array}{l}86 \\
94 \\
93 \\
97\end{array}$ & $\begin{array}{r}98 \\
98 \\
99 \\
102\end{array}$ & $\begin{array}{l}108 \\
101 \\
112 \\
105\end{array}$ & $\begin{array}{l}109 \\
106 \\
110 \\
101\end{array}$ & $\begin{array}{r}94 \\
95 \\
103 \\
91\end{array}$ & $\begin{array}{l}86 \\
77 \\
84 \\
81\end{array}$ & $\begin{array}{l}66 \\
63 \\
67 \\
76\end{array}$ & $\begin{array}{l}52 \\
52 \\
60 \\
62\end{array}$ & $\begin{array}{l}109 \\
106 \\
112 \\
105\end{array}$ \\
\hline
\end{tabular}


Table 3.7. Monthly and Seasonal Number of Days with Minimum Temperatures $\left({ }^{\circ} \mathrm{F}\right)$ Below Certain Thresholds

Minimum Temperature $\leq 32^{\circ} \mathrm{F}$

Minimum Temperature $\leq 0^{\circ} \mathrm{F}$

\begin{tabular}{|c|c|c|c|c|c|c|c|c|c|c|c|c|c|c|c|}
\hline \multirow[b]{2}{*}{ Season } & \multicolumn{10}{|c|}{ Minimum Temperature $\leq 32^{\circ} \mathrm{F}$} & \multicolumn{5}{|c|}{ Minimum Temperature $\leq 0^{\circ} \mathrm{F}$} \\
\hline & Sep & Oct & Nov & Dec & Jan & Feb & Mar & Apr & May & Total & Nov & Dec & $\operatorname{Jan}$ & Feb & Total \\
\hline $1944-1945$ & - & - & - & - & 27 & 18 & 12 & 6 & 0 & 63 & - & - & 0 & 0 & 0 \\
\hline $\begin{array}{l}1945-1946 \\
1946-1947 \\
1947-1948 \\
1948-1949 \\
1949-1950\end{array}$ & $\begin{array}{l}0 \\
0 \\
0 \\
0 \\
0\end{array}$ & $\begin{array}{r}5 \\
8 \\
0 \\
8 \\
10\end{array}$ & $\begin{array}{r}14 \\
23 \\
11 \\
15 \\
4\end{array}$ & $\begin{array}{l}25 \\
24 \\
26 \\
30 \\
25\end{array}$ & $\begin{array}{l}27 \\
27 \\
25 \\
31 \\
30\end{array}$ & $\begin{array}{l}20 \\
19 \\
24 \\
25 \\
22\end{array}$ & $\begin{array}{r}10 \\
6 \\
21 \\
11 \\
18\end{array}$ & $\begin{array}{l}2 \\
1 \\
7 \\
4 \\
4\end{array}$ & $\begin{array}{l}0 \\
0 \\
1 \\
1 \\
0\end{array}$ & $\begin{array}{l}103 \\
108 \\
115 \\
125 \\
113\end{array}$ & $\begin{array}{l}0 \\
0 \\
0 \\
0 \\
0\end{array}$ & $\begin{array}{l}0 \\
0 \\
0 \\
2 \\
0\end{array}$ & $\begin{array}{r}0 \\
1 \\
0 \\
9 \\
14\end{array}$ & $\begin{array}{l}0 \\
0 \\
0 \\
0 \\
4\end{array}$ & $\begin{array}{c}0 \\
1 \\
0 \\
11 \\
18^{(2)}\end{array}$ \\
\hline $\begin{array}{l}1950-1951 \\
1951-1952 \\
1952-1953 \\
1953-1954 \\
1954-1955\end{array}$ & $\begin{array}{l}0 \\
0 \\
0 \\
0 \\
0\end{array}$ & $\begin{array}{l}0 \\
6 \\
0 \\
1 \\
6\end{array}$ & $\begin{array}{r}13 \\
19 \\
25 \\
14 \\
6\end{array}$ & $\begin{array}{l}19 \\
26 \\
19 \\
22 \\
26\end{array}$ & $\begin{array}{r}26 \\
31 \\
9 \\
23 \\
30\end{array}$ & $\begin{array}{l}25 \\
24 \\
15 \\
16 \\
25\end{array}$ & $\begin{array}{l}21 \\
20 \\
12 \\
19 \\
22\end{array}$ & $\begin{array}{r}2 \\
6 \\
4 \\
4 \\
10\end{array}$ & $\begin{array}{l}0 \\
0 \\
0 \\
1 \\
1\end{array}$ & $\begin{array}{r}106 \\
132 \\
84 \\
100 \\
126\end{array}$ & $\begin{array}{l}0 \\
0 \\
0 \\
0 \\
0\end{array}$ & $\begin{array}{l}0 \\
0 \\
0 \\
0 \\
0\end{array}$ & $\begin{array}{l}0 \\
0 \\
0 \\
2 \\
0\end{array}$ & $\begin{array}{l}0 \\
0 \\
0 \\
0 \\
0\end{array}$ & $\begin{array}{l}0 \\
0 \\
0 \\
2 \\
0\end{array}$ \\
\hline $\begin{array}{l}1955-1956 \\
1956-1957 \\
1957-1958 \\
1958-1959 \\
1959-1960\end{array}$ & $\begin{array}{l}0 \\
0 \\
0 \\
0 \\
0\end{array}$ & $\begin{array}{l}1 \\
3 \\
2 \\
4 \\
2\end{array}$ & $\begin{array}{l}22 \\
18 \\
17 \\
14 \\
24\end{array}$ & $\begin{array}{l}28 \\
21 \\
16 \\
24 \\
26\end{array}$ & $\begin{array}{l}25 \\
31 \\
19 \\
25 \\
31\end{array}$ & $\begin{array}{r}26 \\
23 \\
5 \\
24 \\
21\end{array}$ & $\begin{array}{l}14 \\
11 \\
16 \\
14 \\
10\end{array}$ & $\begin{array}{l}2 \\
0 \\
0 \\
2 \\
4\end{array}$ & $\begin{array}{l}0 \\
0 \\
0 \\
1 \\
0\end{array}$ & $\begin{array}{r}118 \\
107 \\
75 \\
108 \\
118\end{array}$ & $\begin{array}{l}1 \\
0 \\
0 \\
0 \\
0\end{array}$ & $\begin{array}{l}0 \\
0 \\
0 \\
0 \\
0\end{array}$ & $\begin{array}{r}2 \\
12 \\
0 \\
2 \\
1\end{array}$ & $\begin{array}{l}3 \\
1 \\
0 \\
0 \\
0\end{array}$ & $\begin{array}{r}6 \\
13 \\
0 \\
2 \\
1\end{array}$ \\
\hline $\begin{array}{l}1960-1961 \\
1961-1962 \\
1962-1963 \\
1963-1964 \\
1964-1965\end{array}$ & $\begin{array}{l}0 \\
0 \\
0 \\
0 \\
0\end{array}$ & $\begin{array}{l}4 \\
7 \\
0 \\
5 \\
5\end{array}$ & $\begin{array}{r}15 \\
28 \\
13 \\
8 \\
13\end{array}$ & $\begin{array}{l}29 \\
26 . \\
17 \\
31 \\
29\end{array}$ & $\begin{array}{l}23 \\
27 \\
27 \\
26 \\
25\end{array}$ & $\begin{array}{l}10 \\
17 \\
17 \\
26 \\
18\end{array}$ & $\begin{array}{l}7 \\
19 \\
11 \\
16 \\
19\end{array}$ & $\begin{array}{l}5 \\
0 \\
2 \\
4 \\
1\end{array}$ & $\begin{array}{l}0 \\
1 \\
0 \\
0 \\
1\end{array}$ & $\begin{array}{r}93 \\
125 \\
87 \\
116 \\
111\end{array}$ & $\begin{array}{l}0 \\
0 \\
0 \\
0 \\
0\end{array}$ & $\begin{array}{l}0 \\
0 \\
0 \\
0 \\
2\end{array}$ & $\begin{array}{l}0 \\
1 \\
2 \\
0 \\
0\end{array}$ & $\begin{array}{l}0 \\
0 \\
0 \\
0 \\
0\end{array}$ & $\begin{array}{l}0 \\
1 \\
2 \\
0 \\
2\end{array}$ \\
\hline $\begin{array}{l}1965-1966 \\
1966-1967 \\
1967-1968 \\
1968-1969 \\
1969-1970\end{array}$ & $\begin{array}{l}0 \\
0 \\
0 \\
0 \\
0\end{array}$ & $\begin{array}{l}1 \\
3 \\
1 \\
4 \\
5\end{array}$ & $\begin{array}{r}8 \\
11 \\
17 \\
8 \\
19\end{array}$ & $\begin{array}{r}25 \\
18 \\
25 \\
24 \\
21\end{array}$ & $\begin{array}{l}26 \\
20 \\
23 \\
30 \\
28\end{array}$ & $\begin{array}{l}22 \\
17 \\
13 \\
25 \\
13\end{array}$ & $\begin{array}{r}13 \\
18 \\
6 \\
15 \\
16\end{array}$ & $\begin{array}{l}3 \\
9 \\
5 \\
1 \\
7\end{array}$ & $\begin{array}{l}0 \\
0 \\
0 \\
0 \\
1\end{array}$ & $\begin{array}{r}98 \\
96 \\
90 \\
107 \\
110\end{array}$ & $\begin{array}{l}0 \\
0 \\
0 \\
0 \\
0\end{array}$ & $\begin{array}{l}0 \\
0 \\
0 \\
4 \\
0\end{array}$ & $\begin{array}{l}0 \\
0 \\
0 \\
5 \\
0\end{array}$ & $\begin{array}{l}0 \\
0 \\
0 \\
1 \\
0\end{array}$ & $\begin{array}{r}0 \\
0 \\
0 \\
10 \\
0\end{array}$ \\
\hline $\begin{array}{l}1970-1971 \\
1971-1972 \\
1972-1973 \\
1973-1974 \\
1974-1975\end{array}$ & $\begin{array}{l}0 \\
0 \\
3 \\
0 \\
0\end{array}$ & $\begin{array}{l}8 \\
9 \\
6 \\
4 \\
4\end{array}$ & $\begin{array}{l}14 \\
18 \\
13 \\
14 \\
12\end{array}$ & $\begin{array}{l}28 \\
27 \\
23 \\
16 \\
26\end{array}$ & $\begin{array}{l}24 \\
25 \\
30 \\
19 \\
29\end{array}$ & $\begin{array}{l}19 \\
23 \\
23 \\
15 \\
24\end{array}$ & $\begin{array}{l}20 \\
13 \\
10 \\
12 \\
17\end{array}$ & $\begin{array}{l}7 \\
6 \\
4 \\
0 \\
7\end{array}$ & $\begin{array}{l}0 \\
0 \\
0 \\
1 \\
0\end{array}$ & $\begin{array}{r}120 \\
121 \\
112 \\
81 \\
119\end{array}$ & $\begin{array}{l}0 \\
0 \\
0 \\
0 \\
0\end{array}$ & $\begin{array}{l}0 \\
0 \\
7 \\
0 \\
0\end{array}$ & $\begin{array}{l}0 \\
3 \\
1 \\
8 \\
0\end{array}$ & $\begin{array}{l}0 \\
1 \\
0 \\
0 \\
0\end{array}$ & $\begin{array}{l}0 \\
4 \\
8 \\
8 \\
0\end{array}$ \\
\hline $\begin{array}{l}1975-1976 \\
1976-1977 \\
1977-1978 \\
1978-1979 \\
1979-1980\end{array}$ & $\begin{array}{l}0 \\
0 \\
0 \\
0 \\
0\end{array}$ & $\begin{array}{l}2 \\
8 \\
3 \\
7 \\
1\end{array}$ & $\begin{array}{l}23 \\
17 \\
18 \\
26 \\
23\end{array}$ & $\begin{array}{l}28 \\
30 \\
25 \\
31 \\
22\end{array}$ & $\begin{array}{l}30 \\
30 \\
22 \\
31 \\
31\end{array}$ & $\begin{array}{l}22 \\
19 \\
17 \\
21 \\
22\end{array}$ & $\begin{array}{l}19 \\
14 \\
11 \\
13 \\
13\end{array}$ & $\begin{array}{l}6 \\
1 \\
4 \\
2 \\
3\end{array}$ & $\begin{array}{l}0 \\
0 \\
0 \\
0 \\
0\end{array}$ & $\begin{array}{l}130 \\
119 \\
100 \\
131 \\
115\end{array}$ & $\begin{array}{l}0 \\
0 \\
0 \\
0 \\
0\end{array}$ & $\begin{array}{l}0 \\
0 \\
1 \\
3 \\
0\end{array}$ & $\begin{array}{l}0 \\
0 \\
2 \\
8 \\
1\end{array}$ & $\begin{array}{l}0 \\
0 \\
0 \\
2 \\
0\end{array}$ & $\begin{array}{r}0 \\
0 \\
3 \\
13 \\
1\end{array}$ \\
\hline $\begin{array}{l}1980-1981 \\
1981-1982 \\
1982-1983 \\
1983-1984 \\
1984-1985\end{array}$ & $\begin{array}{l}0 \\
0 \\
0 \\
0 \\
0\end{array}$ & $\begin{array}{r}4 \\
5 \\
4 \\
3 \\
14\end{array}$ & $\begin{array}{l}16 \\
13 \\
21 \\
11 \\
20\end{array}$ & $\begin{array}{l}16 \\
23 \\
26 \\
31 \\
31\end{array}$ & $\begin{array}{l}17 \\
27 \\
20 \\
26 \\
31\end{array}$ & $\begin{array}{l}17 \\
17 \\
13 \\
17 \\
25\end{array}$ & $\begin{array}{r}11 \\
12 \\
4 \\
5 \\
20\end{array}$ & $\begin{array}{r}6 \\
12 \\
9 \\
2 \\
2\end{array}$ & $\begin{array}{l}0 \\
0 \\
0 \\
0 \\
0\end{array}$ & $\begin{array}{c}87 \\
109 \\
97 \\
95 \\
143^{(2)}\end{array}$ & $\begin{array}{l}0 \\
0 \\
0 \\
0 \\
0\end{array}$ & $\begin{array}{l}0 \\
0 \\
0 \\
4 \\
4\end{array}$ & $\begin{array}{l}0 \\
2 \\
0 \\
0 \\
0\end{array}$ & $\begin{array}{l}0 \\
0 \\
0 \\
0 \\
3\end{array}$ & $\begin{array}{l}0 \\
2 \\
0 \\
4 \\
7\end{array}$ \\
\hline $\begin{array}{l}1985-1986 \\
1986-1987 \\
1987-1988 \\
1988-1989 \\
1989-1990\end{array}$ & $\begin{array}{l}0 \\
0 \\
0 \\
0 \\
0\end{array}$ & $\begin{array}{l}7 \\
0 \\
3 \\
1 \\
2\end{array}$ & $\begin{array}{l}23 \\
11 \\
11 \\
12 \\
11\end{array}$ & $\begin{array}{l}31 \\
29 \\
25 \\
23 \\
25\end{array}$ & $\begin{array}{l}23 \\
25 \\
29 \\
24 \\
18\end{array}$ & $\begin{array}{l}17 \\
17 \\
22 \\
25 \\
20\end{array}$ & $\begin{array}{r}8 \\
9 \\
13 \\
11 \\
11\end{array}$ & $\begin{array}{l}4 \\
2 \\
2 \\
0 \\
0\end{array}$ & $\begin{array}{l}0 \\
0 \\
0 \\
0 \\
0\end{array}$ & $\begin{array}{r}113 \\
93 \\
105 \\
96 \\
87\end{array}$ & $\begin{array}{l}5 \\
0 \\
0 \\
0 \\
0\end{array}$ & $\begin{array}{l}1 \\
0 \\
0 \\
0 \\
0\end{array}$ & $\begin{array}{l}0 \\
0 \\
0 \\
0 \\
0\end{array}$ & $\begin{array}{l}0 \\
0 \\
0 \\
4 \\
0\end{array}$ & $\begin{array}{l}6 \\
0 \\
0 \\
4 \\
0\end{array}$ \\
\hline $\begin{array}{c}1990-1991 \\
1991-1992 \\
1992-1993 \\
1993-1994 \\
1994-1995\end{array}$ & $\begin{array}{l}0 \\
0 \\
0 \\
0 \\
0 \\
0\end{array}$ & $\begin{array}{l}2 \\
6 \\
1 \\
5 \\
2\end{array}$ & $\begin{array}{r}11 \\
8 \\
9 \\
26 \\
20\end{array}$ & $\begin{array}{l}27 \\
18 \\
29 \\
22 \\
24\end{array}$ & $\begin{array}{l}27 \\
22 \\
27 \\
21 \\
20\end{array}$ & $\begin{array}{l}14 \\
11 \\
23 \\
21 \\
11\end{array}$ & $\begin{array}{r}14 \\
3 \\
10 \\
12 \\
13\end{array}$ & $\begin{array}{l}2 \\
2 \\
1 \\
0 \\
2\end{array}$ & $\begin{array}{l}0 \\
0 \\
0 \\
0 \\
0\end{array}$ & $\begin{array}{c}97 \\
70^{(2)} \\
100 \\
107 \\
92\end{array}$ & $\begin{array}{l}0 \\
0 \\
0 \\
1 \\
0\end{array}$ & $\begin{array}{l}8 \\
0 \\
0 \\
0 \\
0\end{array}$ & $\begin{array}{l}0 \\
0 \\
2 \\
0 \\
0\end{array}$ & $\begin{array}{l}0 \\
0 \\
0 \\
0 \\
0\end{array}$ & $\begin{array}{l}8 \\
0 \\
2 \\
1 \\
0\end{array}$ \\
\hline $\begin{array}{l}1995-1996 \\
1996-1997 \\
1997-1998 \\
1998-1999\end{array}$ & $\begin{array}{l}0 \\
0 \\
0 \\
0\end{array}$ & $\begin{array}{l}3 \\
7 \\
4 \\
5\end{array}$ & $\begin{array}{l}12 \\
19 \\
12 \\
10\end{array}$ & $\begin{array}{l}25 \\
26 \\
25 \\
23\end{array}$ & $\begin{array}{l}28 \\
24 \\
21 \\
20\end{array}$ & $\begin{array}{l}22 \\
18 \\
14 \\
15\end{array}$ & $\begin{array}{r}13 \\
10 \\
9 \\
13\end{array}$ & $\begin{array}{l}5 \\
7 \\
3 \\
7\end{array}$ & $\begin{array}{l}1 \\
1 \\
0 \\
2\end{array}$ & $\begin{array}{r}109 \\
112 \\
88 \\
95\end{array}$ & $\begin{array}{l}0 \\
0 \\
0 \\
0\end{array}$ & $\begin{array}{l}0 \\
1 \\
0 \\
1\end{array}$ & $\begin{array}{l}2 \\
0 \\
0 \\
0\end{array}$ & $\begin{array}{l}3 \\
0 \\
0 \\
0\end{array}$ & $\begin{array}{l}5 \\
1 \\
0^{(2,0)} \\
1\end{array}$ \\
\hline $\begin{array}{l}\text { Average } \\
\text { Normal }\end{array}$ & $\begin{array}{l}<1 \\
<1\end{array}$ & $\begin{array}{l}4 \\
4\end{array}$ & $\begin{array}{l}15 \\
15\end{array}$ & $\begin{array}{l}24 \\
25\end{array}$ & $\begin{array}{l}26 \\
26\end{array}$ & $\begin{array}{l}19 \\
19\end{array}$ & $\begin{array}{l}13 \\
13\end{array}$ & $\begin{array}{l}4 \\
4\end{array}$ & $\begin{array}{l}<1 \\
<1\end{array}$ & $\begin{array}{l}106 \\
107\end{array}$ & $\begin{array}{l}<1 \\
<1\end{array}$ & $\begin{array}{l}1 \\
1\end{array}$ & $\begin{array}{l}1 \\
1\end{array}$ & $\begin{array}{l}<1 \\
<1\end{array}$ & $\begin{array}{l}3 \\
3\end{array}$ \\
\hline
\end{tabular}

(a) Greatest and least seasonal totals.

(b) Most recent of numerous occurrences. 
Table 3.8. Days with Minimum Temperatures $\leq 0^{\circ} \mathrm{F}$

\begin{tabular}{|c|c|c|c|c|c|c|}
\hline $\begin{array}{c}\text { Temperature } \\
\left({ }^{\circ} \mathrm{F}\right)\end{array}$ & & & Date(s)o & ccurrence & & \\
\hline-23 & $02 / 03 / 50$ & $02 / 01 / 50$ & & & & \\
\hline-22 & $01 / 26 / 57$ & & & & & \\
\hline-21 & $01 / 27 / 57$ & $02 / 02 / 50$ & $01 / 31 / 50$ & & & \\
\hline-18 & $02 / 01 / 96$ & $01 / 31 / 96$ & $01 / 29 / 50$ & & & \\
\hline-15 & $02 / 03 / 96$ & & & & & \\
\hline-14 & $02 / 02 / 96$ & $12 / 30 / 68$ & $01 / 29 / 57$ & $01 / 28 / 57$ & & \\
\hline-13 & $11 / 23 / 85$ & $12 / 22 / 83$ & $01 / 09 / 74$ & $12 / 16 / 64$ & $01 / 30 / 50$ & \\
\hline-12 & $12 / 22 / 90$ & $11 / 24 / 85$ & $02 / 01 / 79$ & $12 / 17 / 64$ & $01 / 25 / 57$ & \\
\hline-11 & $01 / 30 / 96$ & $01 / 01 / 79$ & $01 / 17 / 50$ & $01 / 14 / 50$ & $01 / 25 / 49$ & \\
\hline-10 & $\begin{array}{l}12 / 29 / 90 \\
02 / 02 / 56\end{array}$ & $\begin{array}{l}12 / 21 / 90 \\
02 / 01 / 56\end{array}$ & $02 / 02 / 79$ & $12 / 30 / 78$ & $01 / 06 / 74$ & $12 / 29 / 68$ \\
\hline-9 & $12 / 23 / 83$ & $01 / 06 / 79$ & $12 / 31 / 78$ & $01 / 02 / 78$ & $01 / 08 / 74$ & \\
\hline-8 & $\begin{array}{l}12 / 01 / 85 \\
01 / 16 / 50\end{array}$ & $01 / 06 / 82$ & $01 / 07 / 74$ & $12 / 10 / 72$ & $01 / 23 / 69$ & $01 / 30 / 57$ \\
\hline-7 & $01 / 07 / 79$ & $01 / 31 / 56$ & $01 / 28 / 50$ & $01 / 05 / 50$ & & \\
\hline-6 & $\begin{array}{l}12 / 28 / 96 \\
01 / 29 / 69 \\
01 / 11 / 49\end{array}$ & $\begin{array}{l}11 / 22 / 85 \\
01 / 28 / 69\end{array}$ & $\begin{array}{l}01 / 31 / 79 \\
01 / 18 / 57\end{array}$ & $\begin{array}{l}01 / 05 / 74 \\
01 / 20 / 54\end{array}$ & $\begin{array}{l}12 / 13 / 72 \\
01 / 04 / 50\end{array}$ & $\begin{array}{l}12 / 08 / 72 \\
01 / 24 / 49\end{array}$ \\
\hline-5 & $\begin{array}{l}02 / 05 / 89 \\
01 / 15 / 50\end{array}$ & $02 / 04 / 85$ & $01 / 01 / 78$ & $1 / 10 / 74$ & $12 / 12 / 72$ & $12 / 09 / 72$ \\
\hline-4 & $\begin{array}{l}01 / 13 / 93 \\
01 / 11 / 74 \\
01 / 12 / 49\end{array}$ & $\begin{array}{l}12 / 23 / 90 \\
12 / 11 / 72\end{array}$ & $\begin{array}{l}02 / 04 / 89 \\
01 / 28 / 72\end{array}$ & $\begin{array}{l}12 / 19 / 84 \\
01 / 12 / 63\end{array}$ & $\begin{array}{l}12 / 21 / 83 \\
01 / 28 / 49\end{array}$ & $\begin{array}{l}01 / 27 / 79 \\
01 / 13 / 49\end{array}$ \\
\hline-3 & $\begin{array}{l}02 / 06 / 89 \\
12 / 29 / 78 \\
01 / 11 / 63\end{array}$ & $\begin{array}{l}11 / 25 / 85 \\
12 / 31 / 77 \\
01 / 17 / 57\end{array}$ & $\begin{array}{l}02 / 03 / 85 \\
01 / 31 / 69\end{array}$ & $\begin{array}{l}12 / 18 / 84 \\
01 / 30 / 69\end{array}$ & $\begin{array}{l}01 / 10 / 80 \\
12 / 31 / 68\end{array}$ & $\begin{array}{l}01 / 08 / 79 \\
12 / 28 / 68\end{array}$ \\
\hline-2 & $\begin{array}{l}12 / 31 / 90 \\
01 / 04 / 74 \\
01 / 10 / 49\end{array}$ & $\begin{array}{l}12 / 30 / 90 \\
12 / 14 / 72 \\
12 / 27 / 48\end{array}$ & $\begin{array}{l}12 / 20 / 90 \\
01 / 22 / 62\end{array}$ & $\begin{array}{l}12 / 21 / 84 \\
01 / 31 / 57\end{array}$ & $\begin{array}{l}12 / 20 / 84 \\
01 / 19 / 57\end{array}$ & $\begin{array}{l}01 / 05 / 79 \\
01 / 20 / 49\end{array}$ \\
\hline-1 & $\begin{array}{l}12 / 21 / 98 \\
02 / 01 / 69 \\
01 / 30 / 56\end{array}$ & $\begin{array}{l}11 / 24 / 93 \\
01 / 18 / 60 \\
11 / 14 / 55\end{array}$ & $\begin{array}{l}11 / 26 / 85 \\
01 / 04 / 59 \\
02 / 04 / 50\end{array}$ & $\begin{array}{l}01 / 08 / 73 \\
02 / 02 / 57 \\
01 / 25 / 50\end{array}$ & $\begin{array}{l}02 / 03 / 72 \\
01 / 16 / 57 \\
01 / 13 / 50\end{array}$ & $\begin{array}{l}01 / 26 / 72 \\
02 / 03 / 56\end{array}$ \\
\hline 0 & $\begin{array}{l}01 / 11 / 93 \\
01 / 28 / 79 \\
01 / 26 / 50\end{array}$ & $\begin{array}{l}12 / 24 / 90 \\
01 / 27 / 72 \\
01 / 04 / 49\end{array}$ & $\begin{array}{l}02 / 02 / 89 \\
01 / 03 / 59 \\
12 / 26 / 48\end{array}$ & $\begin{array}{l}02 / 06 / 85 \\
01 / 24 / 57 \\
01 / 15 / 47\end{array}$ & $\begin{array}{l}12 / 27 / 83 \\
01 / 21 / 54\end{array}$ & $\begin{array}{l}01 / 07 / 82 \\
01 / 27 / 50\end{array}$ \\
\hline
\end{tabular}


Table 3.9. Monthly and Annual Minimum Temperatures $\left({ }^{\circ} \mathrm{F}\right)$

\begin{tabular}{|c|c|c|c|c|c|c|c|c|c|c|c|c|c|}
\hline Year & Jan & Feb & Mar & Apr & May & Jun & $\mathrm{Jul}$ & Aug & Sep & Oct & Nov & $\underline{\text { Dec }}$ & Annual \\
\hline 1945 & 21 & 14 & 10 & 28 & 38 & 46 & 53 & 47 & 35 & 26 & 16 & 13 & 10 \\
\hline 1946 & 18 & 18 & 25 & 30 & 33 & 44 & 50 & 49 & 35 & 21 & 16 & 6 & 6 \\
\hline 1947 & 0 & 11 & 23 & 32 & 42 & 45 & 53 & 50 & 40 & 34 & 22 & 16 & 0 \\
\hline 1948 & 14 & 1 & 13 & 28 & 32 & 51 & 49 & 47 & 34 & 22 & 20 & -2 & -2 \\
\hline 1949 & -11 & 3 & 27 & 30 & 31 & 42 & 49 & 47 & 38 & 23 & 28 & 10 & -11 \\
\hline 1950 & -21 & -23 & 20 & 27 & 38 & 44 & 49 & 51 & 38 & 34 & 21 & 22 & -23 \\
\hline 1951 & 6 & 18 & 22 & 26 & 37 & 41 & 51 & 4 & 33 & 27 & 23 & 4 & 4 \\
\hline 1952 & 5 & 16 & 24 & 27 & 37 & 42 & 49 & 46 & 42 & 34 & 7 & 17 & 5 \\
\hline 1953 & 24 & 20 & 23 & 27 & 36 & 40 & 52 & 51 & 37 & 30 & 24 & 20 & 20 \\
\hline 1954 & -6 & 17 & 18 & 26 & 28 & 41 & 45 & 48 & 36 & 26 & 23 & 14 & -6 \\
\hline 1955 & 18 & 15 & 6 & 26 & 31 & 42 & 43 & 48 & 37 & 32 & -1 & 9 & -1 \\
\hline 1956 & -7 & -10 & 15 & 28 & 38 & 40 & 54 & 49 & 39 & 31 & 15 & 2 & -10 \\
\hline 1957 & -22 & -1 & 28 & 34 & 48 & 48 & 51 & 52 & 36 & 32 & 20 & 23 & -22 \\
\hline 1958 & 16 & 29 & 23 & 34 & 38 & 47 & 49 & 53 & 34 & 30 & 9 & 21 & 9 \\
\hline 1959 & -1 & 19 & 25 & 30 & 30 & 41 & 49 & 49 & 41 & 26 & 6 & 14 & -1 \\
\hline 1960 & -1 & 10 & 13 & 30 & 33 & 46 & 52 & 41 & 40 & 30 & 22 & 14 & -1 \\
\hline 1961 & 16 & 27 & 25 & 31 & 38 & 44 & 50 & 56 & 36 & 26 & 10 & 3 & 3 \\
\hline 1962 & -2 & 7 & 15 & 33 & 31 & 37 & 42 & 49 & 40 & 34 & 16 & 16 & -2 \\
\hline 1963 & -4 & 8 & 22 & 28 & 36 & 45 & 49 & 49 & 45 & 23 & 17 & 7 & -4 \\
\hline 1964 & 15 & 19 & 15 & 30 & 35 & 45 & 50 & 44 & 39 & 30 & 20 & -13 & -13 \\
\hline 1965 & 10 & 18 & 14 & 32 & 32 & 48 & 50 & 42 & 33 & 30 & 26 & 10 & 10 \\
\hline 1966 & 17 & 19 & 19 & 26 & 37 & 38 & 48 & 50 & 43 & 29 & 22 & 22 & 17 \\
\hline 1967 & 23 & 20 & 20 & 27 & 34 & 47 & 52 & 56 & 43 & 30 & 1.7 & 6 & 6 \\
\hline 1968 & 10 & 15 & 25 & 23 & 33 & 42 & 51 & 47 & 39 & 30 & 23 & -14 & -14 \\
\hline 1969 & -8 & -1 & 22 & 31 & 38 & 52 & 53 & 45 & 41 & 29 & 19 & 19 & -8 \\
\hline 1970 & 8 & 21 & 24 & 26 & 30 & 46 & 50 & 52 & 34 & 23 & 11 & 8 & 8 \\
\hline 1971 & 8 & 15 & 15 & 27 & 36 & 44 & 44 & 51 & 38 & 13 & 21 & 5 & 5 \\
\hline 1972 & -4 & -1 & 24 & 26 & 36 & 45 & 50 & 49 & 30 & 20 & 24 & -8 & -8 \\
\hline 1973 & -1 & 21 & 26 & 27 & 34 & 45 & 46 & 46 & 43 & 31 & 16 & 14 & -1 \\
\hline 1974 & -13 & 23 & 21 & 33 & 32 & 41 & 48 & 48 & 40 & 29 & 24 & 17 & -13 \\
\hline 1975 & 14 & 10 & 19 & 21 & 33 & 38 & 53 & 46 & 44 & 26 & 15 & 14 & 10 \\
\hline 1976 & 16 & 10 & 11 & 25 & 35 & 37 & 47 & 44 & 42 & 28 & 13 & 12 & 10 \\
\hline 1977 & 4 & 21 & 24 & 31 & 34 & 39 & 49 & 48 & 36 & 28 & 9 & -3 & -3 \\
\hline 1978 & -9 & 17 & 25 & 30 & 37 & 44 & 50 & 47 & 41 & 21 & 7 & -10 & -10 \\
\hline 1979 & -11 & -12 & 20 & 29 & 38 & 45 & 39 & 53 & 42 & 32 & 13 & 19 & -12 \\
\hline 1980 & -3 & 19 & 25 & 28 & 38 & 40 & 47 & 42 & 41 & 30 & 18 & 9 & -3 \\
\hline 1981 & 23 & 8 & 24 & 24 & 35 & 40 & 45 & 48 & 34 & 27 & 19 & 8 & 8 \\
\hline 1982 & -8 & 9 & 24 & 24 & 33 & 47 & 45 & 51 & 41 & 26 & 18 & 13 & -8 \\
\hline 1983 & 12 & 15 & 29 & 27 & 37 & 40 & 49 & 50 & 35 & 29 & 22 & -13 & -13 \\
\hline 1984 & 10 & 24 & 25 & 30 & 33 & 37 & 51 & 47 & 36 & 12 & 25 & -4 & -4 \\
\hline 1985 & 5 & -5 & 21 & 26 & 33 & 44 & 56 & 46 & 33 & 26 & -13 & -8 & -13 \\
\hline 1986 & 12 & 15 & 29 & 28 & 37 & 43 & 48 & 54 & 38 & 33 & 16 & 18 & 12 \\
\hline 1987 & 9 & 18 & 24 & 30 & 38 & 43 & 49 & 51 & 41 & 31 & 17 & 9 & 9 \\
\hline 1988 & 14 & 9 & 24 & 31 & 35 & 42 & 47 & 52 & 38 & 32 & 28 & 8 & 8 \\
\hline 1989 & 15 & -5 & 14 & 35 & 39 & 46 & 49 & 52 & 44 & 27 & 21 & 19 & -5 \\
\hline 1990 & 22 & 9 & 24 & 37 & 39 & 47 & 46 & 52 & 48 & 31 & 28 & -12 & -12 \\
\hline 1991 & 5 & 26 & 22 & 31 & 38 & 44 & 55 & 47 & 42 & 23 & 23 & 20 & 5 \\
\hline 1992 & 19 & 22 & 32 & 27 & 37 & 49 & 54 & 43 & 40 & 30 & 17 & 12 & 12 \\
\hline 1993 & -4 & 3 & 17 & 32 & 35 & 46 & 50 & 43 & 37 & 29 & -1 & 21 & -4 \\
\hline 1994 & 20 & 5 & 19 & 35 & 36 & 44 & 50 & 53 & 47 & 30 & 19 & 8 & 5 \\
\hline 1995 & 8 & 8 & 21 & 28 & 39 & 47 & 52 & 45 & 42 & 16 & 17 & 16 & 8 \\
\hline 1996 & -18 & -18 & 18 & 30 & 29 & 45 & 49 & 48 & 34 & 34 & 17 & -6 & -18 \\
\hline 1997 & 8 & 20 & 28 & 25 & 30 & 46 & 49 & 52 & 44 & 29 & 23 & 19 & 8 \\
\hline 1998 & 7 & 22 & 23 & 29 & 39 & 47 & 58 & 50 & 43 & 25 & 27 & -1 & -1 \\
\hline 1999 & 18 & 20 & 25 & 25 & 30 & 38 & 45 & 43 & 36 & 27 & 26 & 20 & 18 \\
\hline
\end{tabular}




\subsection{Monthly Extremes of Daily Maximum and Minimum Temperatures}

Monthly extremes of daily maximum and minimum temperatures are presented in Table 3.10. Note that ranges are comparable in the winter and in the summer. February temperatures ranged from $72^{\circ} \mathrm{F}$ to $23^{\circ} \mathrm{F}$, a range of $95^{\circ} \mathrm{F}$. July temperatures ranged from $112^{\circ} \mathrm{F}$ to $39^{\circ} \mathrm{F}$, a range of $83^{\circ} \mathrm{F}$.

\subsection{Daily Temperature Distributions}

Daily temperatures are generally described relative to a long-term average temperature or to record high or low temperatures. For example, the daily maximum temperature may be described as above average or near the record high for the day. However, this type of description does not provide information about whether the temperature is in the range of temperatures that is typical for the day. Figure 3.1 shows an example of a new way of displaying climatological temperature information that places temperatures in better context. The figure shows the record low and high daily maximum temperatures for November 12 at the Hanford Meteorology Station based on climatological records from 1947 through 1999. Between the record low and high temperatures, the figure has a bar that shows the range of daily maximum temperatures that have occurred 70 percent of the time. This range can be interpreted as the range of typical daily maximum temperatures for the date. On this bar is a solid square that indicates the median daily maximum temperature. The median temperature is the daily maximum temperature that is exceeded 50 percent of the time. For temperatures at the Hanford Meteorology Station, the median temperature is generally very close to the long-term average temperature. The following information can be drawn from the figure:

- The median (as well as the average) daily maximum temperature is about $52^{\circ} \mathrm{F}$.

- The record high temperature for the date is $75^{\circ} \mathrm{F}$.

- The record low maximum temperature is $20^{\circ} \mathrm{F}$.

- About 70 percent of the time ( 14 out of 20 years), the daily maximum temperature will be between $42^{\circ} \mathrm{F}$ and $60^{\circ} \mathrm{F}$.

- About 15 percent of the time ( 3 out of 20 years), the daily maximum temperature will be above $60^{\circ} \mathrm{F}$.

- About 35 percent of the time (7 out of 20 years), the daily maximum temperature will be between $52^{\circ} \mathrm{F}$ and $60^{\circ} \mathrm{F}$.

- About 35 percent of the time ( 7 out of 20 years), the daily maximum temperature will be between $42^{\circ} \mathrm{F}$ and $52^{\circ} \mathrm{F}$.

- About 15 percent of the time ( 3 out of 20 years), the daily maximum temperature will be below $42^{\circ} \mathrm{F}$. 
This method of presenting climatological data is appropriate for daily minimum temperatures and other meteorological parameters as well.

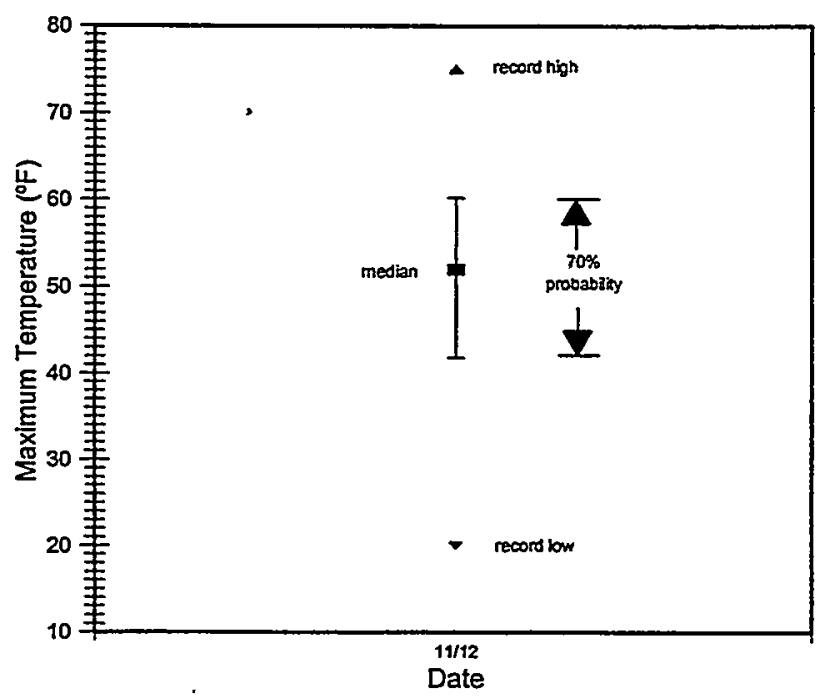

Figure 3.1. Graphical Presentation of Daily Maximum Temperatures

Figures 3.2 and 3.3 show the climatological statistics of the daily maximum and daily minimum temperatures, respectively, for the Hanford Meteorology Station. The statistics are for days taken at approximately weekly intervals.

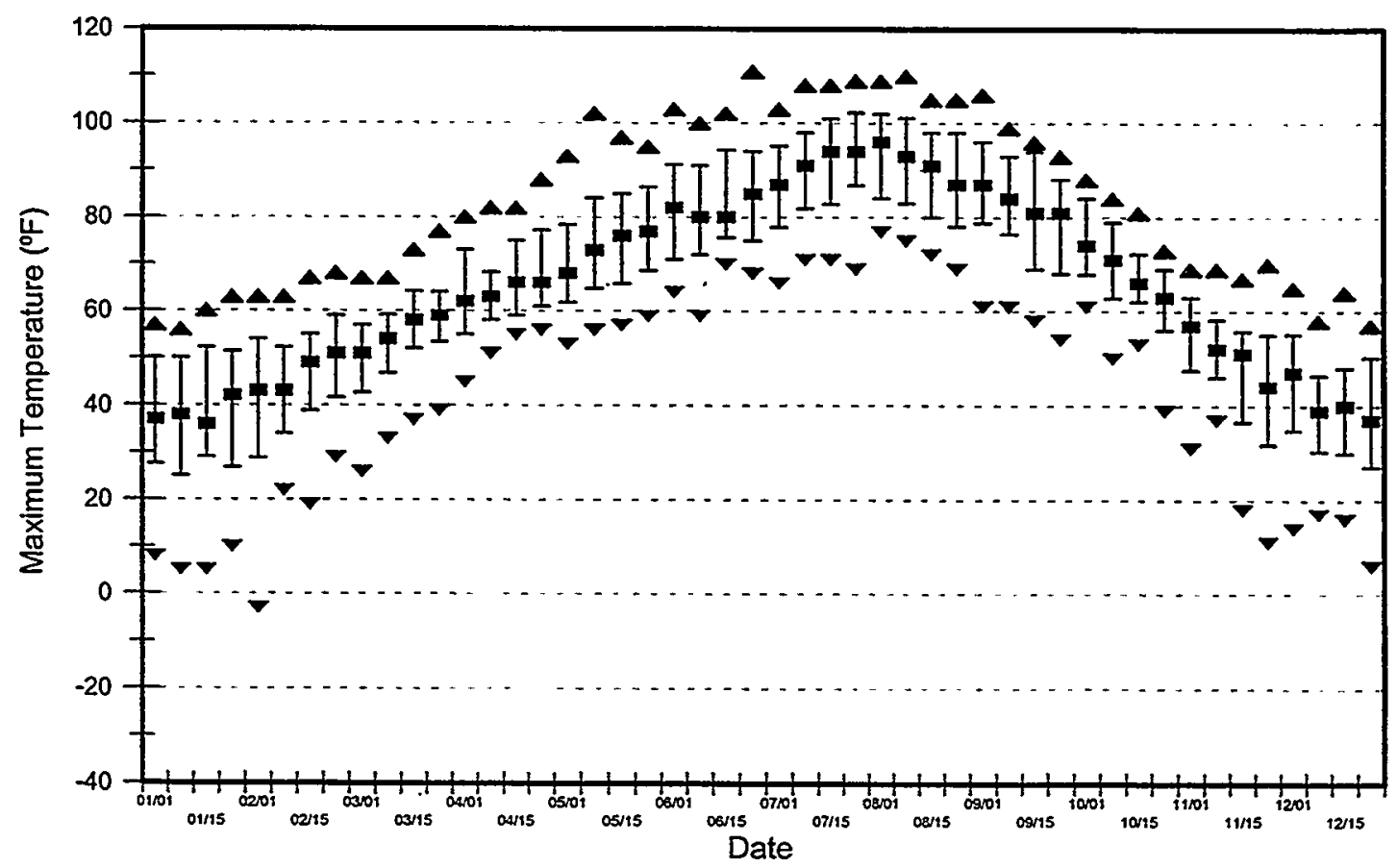

Figure 3.2. Climatological Statistics of Daily Maximum Temperatures at the Hanford Meteorology Station 


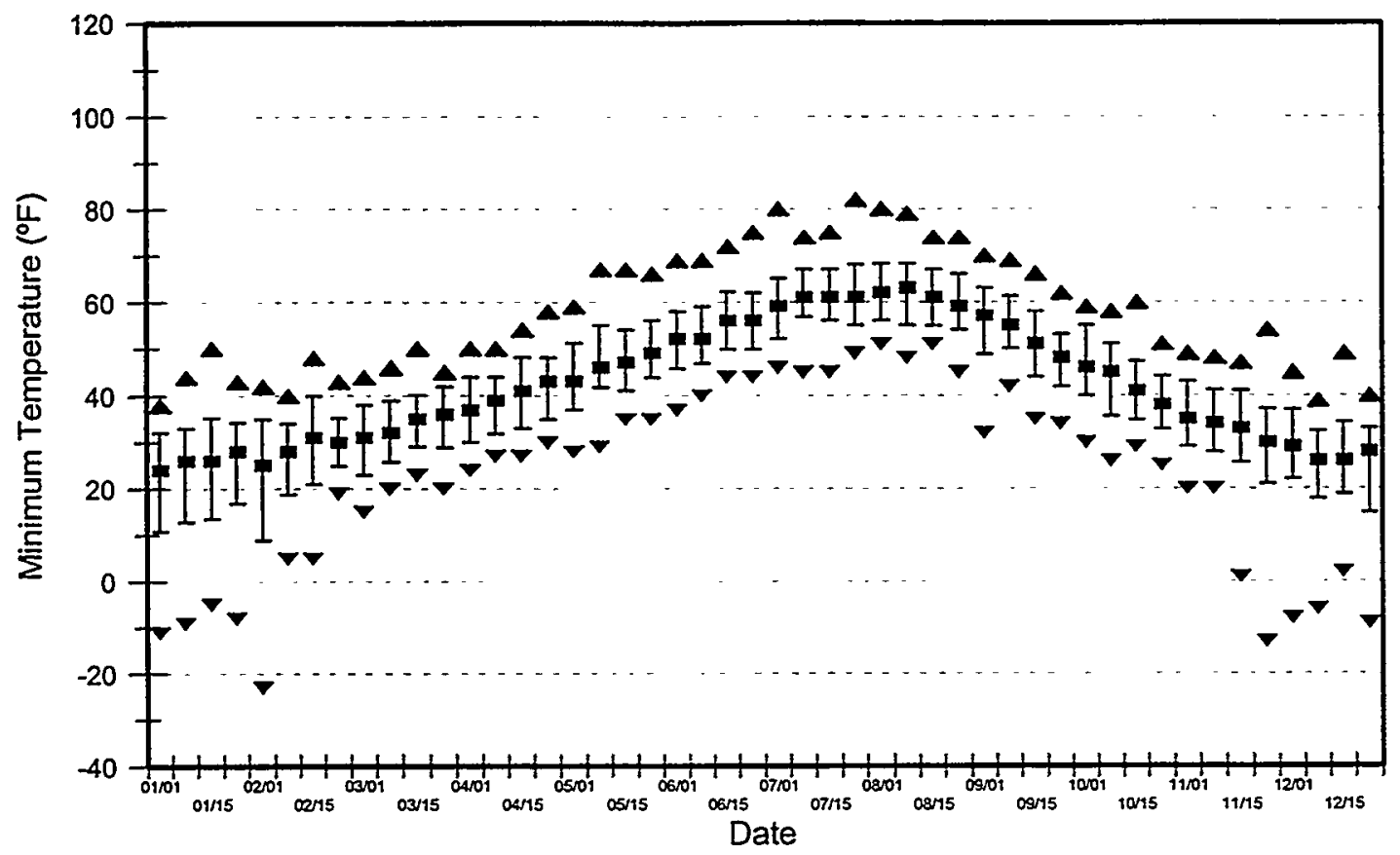

Figure 3.3. Climatological Statistics of Daily Minimum Temperatures at the Hanford Meteorology Station

\subsection{Average Daily Temperature Range}

Table 3.11 represents the average daily temperature range by month and year for the period 1945 through 1999. This statistic is compiled by determining each daily temperature range (the difference between the maximum and minimum temperature), totaling for every day of the month, and dividing by the number of days in the month. As can be seen from the table, the average daily temperature ranges for July and August $\left(>30^{\circ} \mathrm{F}\right)$ are more than double the ranges for December and January $\left(<15^{\circ} \mathrm{F}\right)$. The lowest average daily temperature range was $8^{\circ} \mathrm{F}$ in December 1985; the greatest was $34.5^{\circ} \mathrm{F}$ in August 1967. The greatest range for any single day was $48^{\circ} \mathrm{F}$ on August 14,1995 (high of $93^{\circ} \mathrm{F}$, low of $45^{\circ} \mathrm{F}$ ) and also on May 11,1946 (high of $86^{\circ} \mathrm{F}$, low of $38^{\circ} \mathrm{F}$ ). The least range for any single day was $1^{\circ} \mathrm{F}$ as recently as December 6,1997 (high of $30^{\circ} \mathrm{F}$, low of $29^{\circ} \mathrm{F}$ ).

\subsection{Normal and Extreme Daily Temperatures}

Table 3.12 lists the normal and extreme daily maximum and minimum temperatures. Climatological normals are computed every 10 years and are based on a 30-year period, ending with the first year of each new decade. The current normal period is 1961 through 1990; the next will be 1971 through 2000. The normal temperatures in Table 3.12 are computed using a 7-day running mean, centered about each day.

Four possible temperature extremes are presented for each day, a record high and low maximum and a record high and low minimum. These daily records, plus the year of occurrence for the period 1945 through 1999, are also indicated in Table 3.12. 


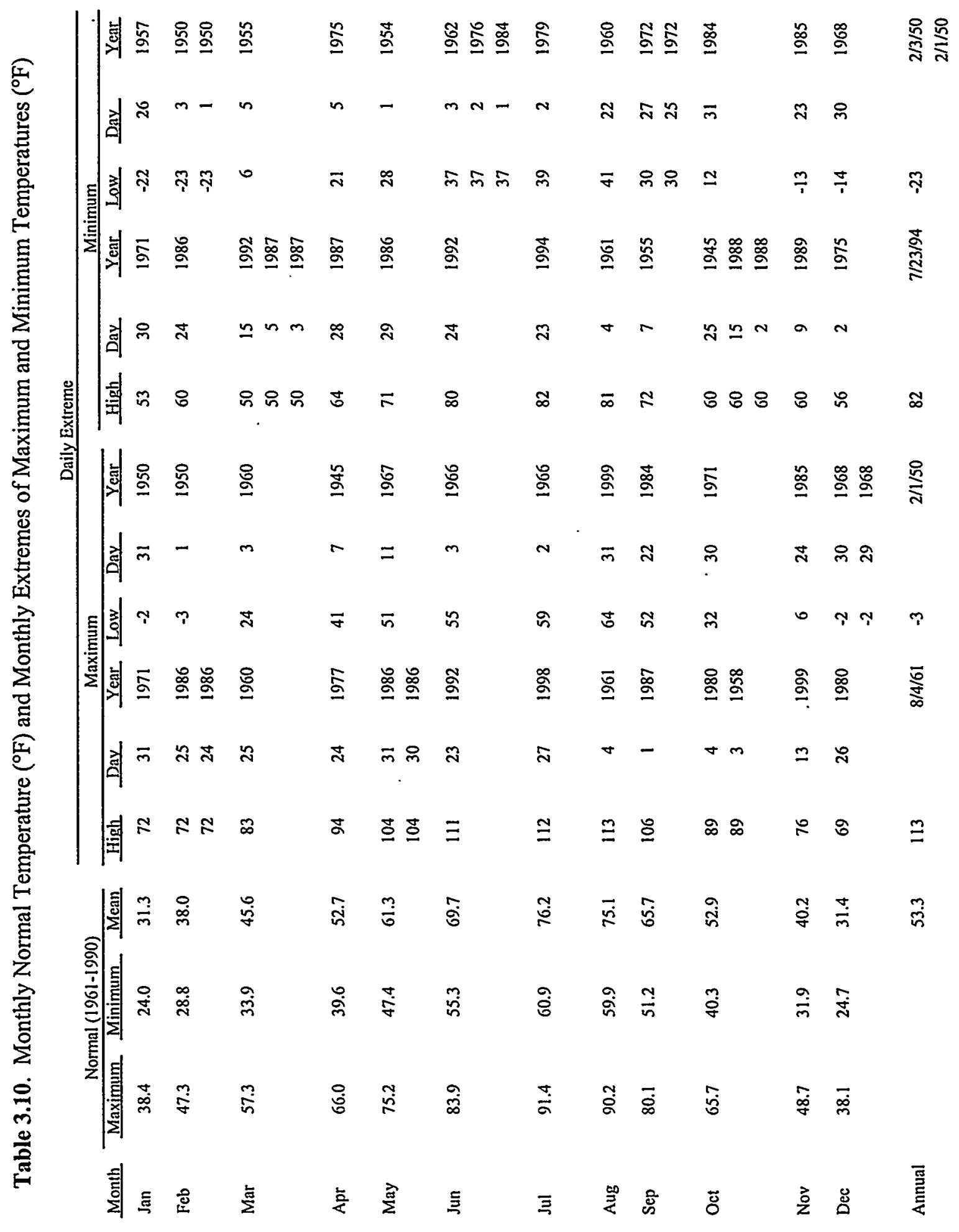


Table 3.11. Average Daily Temperature $\left({ }^{\circ} \mathrm{F}\right)$ Range

\begin{tabular}{|c|c|c|c|c|c|c|c|c|c|c|c|c|c|}
\hline Year & Jan & Feb & Mar & Apr & May & Jun & Jul & Aug & Sep & Oct & Nov & Dec & Annual \\
\hline $\begin{array}{l}1945 \\
1946 \\
1947 \\
1948 \\
1949 \\
1950\end{array}$ & $\begin{array}{l}10.5 \\
17.3 \\
18.4 \\
15.0 \\
18.8^{(2)} \\
16.0\end{array}$ & $\begin{array}{l}17.4 \\
21.0 \\
22.2 \\
17.2 \\
19.3 \\
15.6\end{array}$ & $\begin{array}{l}19.5 \\
21.8 \\
25.5 \\
23.0 \\
20.6 \\
20.0\end{array}$ & $\begin{array}{l}25.1 \\
26.1 \\
27.7 \\
23.2 \\
30.5 \\
25.3\end{array}$ & $\begin{array}{l}25.0 \\
29.6 \\
29.6 \\
22.6^{(2)} \\
28.2 \\
29.6\end{array}$ & $\begin{array}{l}26.1 \\
25.6 \\
25.3^{(2)} \\
26.4 \\
30.2 \\
25.7\end{array}$ & $\begin{array}{l}31.0 \\
30.4 \\
29.3 \\
29.1 \\
30.5 \\
32.3\end{array}$ & $\begin{array}{l}29.7 \\
29.4 \\
28.8 \\
28.0 \\
30.4 \\
31.6\end{array}$ & $\begin{array}{l}26.9 \\
26.8 \\
27.3 \\
28.7 \\
27.2 \\
32.4\end{array}$ & $\begin{array}{l}27.4 \\
23.6 \\
18.1^{(2)} \\
26.5 \\
26.6 \\
18.2\end{array}$ & $\begin{array}{l}16.3 \\
17.7 \\
15.4 \\
17.8 \\
16.8 \\
14.7\end{array}$ & $\begin{array}{r}11.4 \\
15.8 \\
11.1 \\
15.9 \\
16.7 \\
9.7\end{array}$ & $\begin{array}{l}22.2 \\
23.8 \\
23.2 \\
22.8 \\
24.6 \\
22.6\end{array}$ \\
\hline $\begin{array}{l}1951 \\
1952 \\
1953 \\
1954 \\
1955\end{array}$ & $\begin{array}{c}13.7 \\
12.6 \\
15.6 \\
14.3 \\
9.2^{(2)}\end{array}$ & $\begin{array}{l}18.4 \\
17.0 \\
19.8 \\
13.8 \\
18.9\end{array}$ & $\begin{array}{l}20.8 \\
22.8 \\
24.4 \\
23.7 \\
21.8\end{array}$ & $\begin{array}{l}30.3 \\
30.3 \\
24.0 \\
26.3 \\
24.9\end{array}$ & $\begin{array}{l}30.2 \\
28.1 \\
27.9 \\
28.1 \\
25.3\end{array}$ & $\begin{array}{l}28.9 \\
27.3 \\
26.4 \\
26.8 \\
29.4\end{array}$ & $\begin{array}{l}33.8^{(2)} \\
32.6 \\
32.8 \\
31.4 \\
27.9\end{array}$ & $\begin{array}{l}31.5 \\
32.2 \\
29.1 \\
27.6 \\
31.7\end{array}$ & $\begin{array}{l}30.9 \\
32.8 \\
32.5 \\
26.2 \\
27.5\end{array}$ & $\begin{array}{l}23.3 \\
29.0 \\
27.8 \\
24.5 \\
22.7\end{array}$ & $\begin{array}{l}17.4 \\
16.0 \\
20.3 \\
15.8 \\
16.1\end{array}$ & $\begin{array}{l}13.7 \\
9.4 \\
17.7^{(2)} \\
13.2 \\
12.3\end{array}$ & $\begin{array}{l}24.4 \\
24.2 \\
24.9 \\
22.6 \\
22.3\end{array}$ \\
\hline $\begin{array}{l}1956 \\
1957 \\
1958 \\
1959 \\
1960\end{array}$ & $\begin{array}{l}12.9 \\
15.2 \\
13.3 \\
14.1 \\
14.6\end{array}$ & $\begin{array}{l}15.0 \\
18.3 \\
15.0 \\
16.2 \\
19.3\end{array}$ & $\begin{array}{l}20.9 \\
18.4^{(2)} \\
22.0 \\
24.3 \\
23.2\end{array}$ & $\begin{array}{l}28.0 \\
24.7 \\
23.9 \\
26.9 \\
25.7\end{array}$ & $\begin{array}{l}26.6 \\
24.8 \\
29.6 \\
26.5 \\
26.5\end{array}$ & $\begin{array}{l}26.9 \\
27.7 \\
27.3 \\
27.1 \\
31.2\end{array}$ & $\begin{array}{l}30.8 \\
28.7 \\
30.5 \\
31.1 \\
32.8\end{array}$ & $\begin{array}{l}28.8 \\
27.0 \\
33.1 \\
29.9 \\
28.1\end{array}$ & $\begin{array}{l}30.6 \\
29.8 \\
27.0 \\
23.8 \\
28.3\end{array}$ & $\begin{array}{l}22.1 \\
18.4 \\
27.8 \\
24.4 \\
25.7\end{array}$ & $\begin{array}{l}13.9 \\
19.1 \\
17.8 \\
21.9 \\
18.7\end{array}$ & $\begin{array}{l}13.4 \\
14.7 \\
10.2 \\
13.4 \\
10.9\end{array}$ & $\begin{array}{l}22.5 \\
22.2 \\
23.1 \\
23.3 \\
23.8\end{array}$ \\
\hline $\begin{array}{l}1961 \\
1962 \\
1963 \\
1964 \\
1965\end{array}$ & $\begin{array}{l}12.5 \\
18.1 \\
17.2 \\
16.0 \\
12.3\end{array}$ & $\begin{array}{l}17.2 \\
17.4 \\
16.8 \\
24.1^{(a)} \\
20.3\end{array}$ & $\begin{array}{l}20.0 \\
22.0 \\
23.3 \\
23.7 \\
25.6\end{array}$ & $\begin{array}{l}25.3 \\
28.9 \\
21.4^{(2)} \\
27.1 \\
26.4\end{array}$ & $\begin{array}{l}25.5 \\
23.2 \\
28.1 \\
27.9 \\
28.3\end{array}$ & $\begin{array}{l}31.5 \\
29.9 \\
26.6 \\
26.4 \\
28.1\end{array}$ & $\begin{array}{l}30.6 \\
30.2 \\
27.8 \\
31.2 \\
31.1\end{array}$ & $\begin{array}{l}30.9 \\
28.3 \\
30.7 \\
29.4 \\
27.7\end{array}$ & $\begin{array}{l}26.9 \\
29.9 \\
29.8 \\
29.7 \\
29.8\end{array}$ & $\begin{array}{l}25.4 \\
21.4 \\
24.5 \\
26.4 \\
27.4\end{array}$ & $\begin{array}{l}20.4 \\
17.3 \\
16.9 \\
12.2^{(2)} \\
14.2\end{array}$ & $\begin{array}{r}15.0 \\
11.4 \\
9.5 \\
14.8 \\
15.7\end{array}$ & $\begin{array}{l}23.4 \\
23.2 \\
22.7 \\
24.1 \\
23.9\end{array}$ \\
\hline $\begin{array}{l}1966 \\
1967 \\
1968 \\
1969 \\
1970\end{array}$ & $\begin{array}{l}14.3 \\
17.6 \\
17.2 \\
12.2 \\
12.0\end{array}$ & $\begin{array}{l}19.3 \\
24.0 \\
20.4 \\
14.1 \\
16.4\end{array}$ & $\begin{array}{l}24.4 \\
24.6 \\
23.5 \\
25.5 \\
23.8\end{array}$ & $\begin{array}{l}28.2 \\
24.4 \\
27.5 \\
24.5 \\
25.3\end{array}$ & $\begin{array}{l}30.7 \\
27.7 \\
27.5 \\
29.2 \\
29.2\end{array}$ & $\begin{array}{l}27.3 \\
28.7 \\
27.3 \\
27.7 \\
29.3\end{array}$ & $\begin{array}{l}28.7 \\
32.0 \\
31.2 \\
31.3 \\
31.7\end{array}$ & $\begin{array}{l}29.1 \\
34.5^{(2)} \\
26.2^{(2)} \\
33.3 \\
33.1\end{array}$ & $\begin{array}{l}28.4 \\
31.7 \\
28.2 \\
27.6 \\
27.2\end{array}$ & $\begin{array}{l}25.7 \\
25.5 \\
22.8 \\
25.0 \\
26.5\end{array}$ & $\begin{array}{l}18.1 \\
19.9 \\
14.2 \\
17.4 \\
17.8\end{array}$ & $\begin{array}{c}13.4 \\
15.7 \\
13.9 \\
9.2 \\
15.4\end{array}$ & $\begin{array}{l}24.0 \\
25.5^{(2)} \\
23.3 \\
23.1 \\
24.0\end{array}$ \\
\hline $\begin{array}{l}1971 \\
1972 \\
1973 \\
1974 \\
1975\end{array}$ & $\begin{array}{l}18.4 \\
17.3 \\
15.7 \\
17.8 \\
15.0\end{array}$ & $\begin{array}{l}21.1 \\
18.3 \\
16.6 \\
18.8 \\
17.0\end{array}$ & $\begin{array}{l}22.8 \\
25.2 \\
24.6 \\
23.2 \\
21.2\end{array}$ & $\begin{array}{l}26.8 \\
26.8 \\
29.6 \\
23.4 \\
24.8\end{array}$ & $\begin{array}{l}27.7 \\
27.2 \\
31.1 \\
27.3 \\
29.5\end{array}$ & $\begin{array}{l}26.9 \\
26.9 \\
29.7 \\
32.7^{(2)} \\
28.2\end{array}$ & $\begin{array}{l}32.0 \\
30.1 \\
32.1 \\
29.8 \\
30.3\end{array}$ & $\begin{array}{l}32.3 \\
30.6 \\
32.7 \\
31.9 \\
28.7\end{array}$ & $\begin{array}{l}27.8 \\
30.5 \\
27.0 \\
32.2 \\
32.2\end{array}$ & $\begin{array}{l}25.8 \\
27.5 \\
22.2 \\
28.3 \\
22.0\end{array}$ & $\begin{array}{l}17.8 \\
13.1 \\
12.6 \\
16.3 \\
20.9\end{array}$ & $\begin{array}{l}14.8 \\
17.2 \\
12.5 \\
16.5 \\
14.8\end{array}$ & $\begin{array}{l}24.5 \\
24.2 \\
23.9 \\
24.8 \\
23.7\end{array}$ \\
\hline $\begin{array}{l}1976 \\
1977 \\
1978 \\
1979 \\
1980\end{array}$ & $\begin{array}{l}15.2 \\
10.8 \\
11.4 \\
15.5 \\
13.2\end{array}$ & $\begin{array}{l}21.0 \\
20.7 \\
15.2 \\
18.7 \\
10.5^{(2)}\end{array}$ & $\begin{array}{l}25.3 \\
23.4 \\
23.0 \\
26.0 \\
22.1\end{array}$ & $\begin{array}{l}26.0 \\
30.6^{(2)} \\
23.8 \\
26.5 \\
27.1\end{array}$ & $\begin{array}{l}30.6 \\
26.0 \\
27.7 \\
29.4 \\
25.8\end{array}$ & $\begin{array}{l}28.8 \\
30.2 \\
31.3 \\
31.1 \\
25.8\end{array}$ & $\begin{array}{l}30.5 \\
30.5 \\
31.0 \\
32.9 \\
31.3\end{array}$ & $\begin{array}{l}28.0 \\
29.1 \\
29.0 \\
32.0 \\
29.9\end{array}$ & $\begin{array}{l}30.5 \\
23.8 \\
25.8 \\
31.1 \\
27.3\end{array}$ & $\begin{array}{l}27.5 \\
26.6 \\
30.3^{(2)} \\
25.6 \\
24.6\end{array}$ & $\begin{array}{l}20.3 \\
19.1 \\
18.2 \\
13.0 \\
15.3\end{array}$ & $\begin{array}{l}16.6 \\
15.1 \\
16.8 \\
12.9 \\
11.4\end{array}$ & $\begin{array}{l}25.0 \\
23.8 \\
23.6 \\
24.6 \\
22.0^{(2)}\end{array}$ \\
\hline $\begin{array}{l}1981 \\
1982 \\
1983 \\
1984 \\
1985\end{array}$ & $\begin{array}{r}9.9 \\
16.0 \\
15.5 \\
13.5 \\
6.8\end{array}$ & $\begin{array}{l}17.5 \\
21.4 \\
17.3 \\
15.2 \\
20.4\end{array}$ & $\begin{array}{l}25.9 \\
24.4 \\
20.7 \\
21.6 \\
25.4\end{array}$ & $\begin{array}{l}27.4 \\
28.2 \\
27.9 \\
23.9 \\
28.7\end{array}$ & $\begin{array}{l}27.1 \\
29.9 \\
28.4 \\
26.3 \\
29.2\end{array}$ & $\begin{array}{l}28.3 \\
28.0 \\
27.9 \\
26.1 \\
29.4\end{array}$ & $\begin{array}{l}31.7 \\
30.6 \\
26.3 \\
32.3 \\
32.0\end{array}$ & $\begin{array}{l}32.9 \\
29.5 \\
28.4 \\
32.0 \\
29.9\end{array}$ & $\begin{array}{l}30.8 \\
27.1 \\
27.5 \\
26.6 \\
24.3\end{array}$ & $\begin{array}{l}26.0 \\
24.9 \\
24.7 \\
25.5 \\
25.4\end{array}$ & $\begin{array}{l}20.0 \\
16.6 \\
15.5 \\
14.0 \\
15.8\end{array}$ & $\begin{array}{c}14.2 \\
13.0 \\
11.0 \\
15.7 \\
8.0^{(2)}\end{array}$ & $\begin{array}{l}24.3 \\
24.1 \\
22.6 \\
22.7 \\
22.9\end{array}$ \\
\hline $\begin{array}{l}1986 \\
1987 \\
1988 \\
1989 \\
1990\end{array}$ & $\begin{array}{l}12.5 \\
11.9 \\
13.4 \\
16.5 \\
15.7\end{array}$ & $\begin{array}{l}17.1 \\
19.0 \\
23.8 \\
17.3 \\
20.5\end{array}$ & $\begin{array}{l}22.1 \\
22.4 \\
25.1 \\
20.2 \\
26.5\end{array}$ & $\begin{array}{l}26.4 \\
28.2 \\
25.3 \\
26.2 \\
27.1\end{array}$ & $\begin{array}{l}26.6 \\
28.9 \\
27.4 \\
26.0 \\
24.4\end{array}$ & $\begin{array}{l}29.1 \\
31.2 \\
26.3 \\
28.5 \\
26.8\end{array}$ & $\begin{array}{l}28.6 \\
28.1 \\
30.0 \\
31.1 \\
28.8\end{array}$ & $\begin{array}{l}31.0 \\
30.1 \\
32.1 \\
27.3 \\
27.3\end{array}$ & $\begin{array}{l}23.5^{(2)} \\
33.0 \\
31.2 \\
31.1 \\
32.2\end{array}$ & $\begin{array}{l}26.8 \\
28.9 \\
26.2 \\
24.0 \\
23.6\end{array}$ & $\begin{array}{l}15.1 \\
19.8 \\
16.9 \\
16.0 \\
18.4\end{array}$ & $\begin{array}{r}8.4 \\
12.0 \\
11.4 \\
9.4 \\
16.0\end{array}$ & $\begin{array}{l}22.3 \\
24.5 \\
24.1 \\
22.8 \\
23.9\end{array}$ \\
\hline $\begin{array}{l}1991 \\
1992 \\
1993 \\
1994 \\
1995\end{array}$ & $\begin{array}{l}16.0 \\
12.5 \\
12.8 \\
15.7 \\
13.2\end{array}$ & $\begin{array}{l}21.4 \\
15.2 \\
15.1 \\
17.2 \\
20.1\end{array}$ & $\begin{array}{l}22.7 \\
25.9 \\
18.6 \\
28.4^{(2)} \\
23.7\end{array}$ & $\begin{array}{l}26.0 \\
24.6 \\
23.7 \\
26.0 \\
25.2\end{array}$ & $\begin{array}{l}25.1 \\
31.9^{(2)} \\
29.1 \\
26.5 \\
28.0\end{array}$ & $\begin{array}{l}25.6 \\
28.5 \\
27.1 \\
29.4 \\
26.3\end{array}$ & $\begin{array}{l}30.2 \\
28.0 \\
25.5^{(\mathfrak{)})} \\
33.0 \\
29.9\end{array}$ & $\begin{array}{l}29.6 \\
31.6 \\
29.5 \\
31.2 \\
30.6\end{array}$ & $\begin{array}{l}31.1 \\
28.1 \\
33.5^{(2)} \\
31.2 \\
30.2\end{array}$ & $\begin{array}{l}26.4 \\
24.4 \\
28.6 \\
25.4 \\
24.3\end{array}$ & $\begin{array}{l}13.1 \\
13.6 \\
23.9^{(2)} \\
17.2 \\
19.5\end{array}$ & $\begin{array}{l}13.5 \\
14.5 \\
10.5 \\
13.6 \\
11.7\end{array}$ & $\begin{array}{l}23.4 \\
23.2 \\
23.2 \\
24.6 \\
23.6\end{array}$ \\
\hline $\begin{array}{l}1996 \\
1997 \\
1998 \\
1999\end{array}$ & $\begin{array}{l}14.0 \\
15.6 \\
15.9 \\
16.4\end{array}$ & $\begin{array}{l}21.2 \\
18.8 \\
19.0 \\
18.0\end{array}$ & $\begin{array}{l}22.9 \\
22.1 \\
23.7 \\
22.2\end{array}$ & $\begin{array}{l}26.0 \\
25.7 \\
28.5 \\
28.9\end{array}$ & $\begin{array}{l}26.6 \\
28.1 \\
27.5 \\
28.0\end{array}$ & $\begin{array}{l}31.0 \\
27.3 \\
29.2 \\
28.0\end{array}$ & $\begin{array}{l}33.7 \\
31.2 \\
30.5 \\
30.2\end{array}$ & $\begin{array}{l}34.1 \\
31.2 \\
33.2 \\
29.0\end{array}$ & $\begin{array}{l}30.8 \\
26.7 \\
31.9 \\
32.9\end{array}$ & $\begin{array}{l}23.9 \\
24.3 \\
26.6 \\
25.7\end{array}$ & $\begin{array}{l}17.0 \\
17.6 \\
16.2 \\
17.7\end{array}$ & $\begin{array}{l}13.1 \\
14.2 \\
17.7 \\
13.5\end{array}$ & $\begin{array}{l}24.5 \\
23.6 \\
25.0 \\
24.2\end{array}$ \\
\hline $\begin{array}{l}\text { Average } \\
\text { Normal }\end{array}$ & $\begin{array}{l}14.4 \\
14.4\end{array}$ & $\begin{array}{l}18.3 \\
18.6\end{array}$ & $\begin{array}{l}23.1 \\
23.5\end{array}$ & $\begin{array}{l}26.3 \\
26.5\end{array}$ & $\begin{array}{l}27.7 \\
27.8\end{array}$ & $\begin{array}{l}28.1 \\
28.5\end{array}$ & $\begin{array}{l}30.6 \\
30.5\end{array}$ & $\begin{array}{l}30.3 \\
30.3\end{array}$ & $\begin{array}{l}29.0 \\
28.8\end{array}$ & $\begin{array}{l}25.1 \\
25.6\end{array}$ & $\begin{array}{l}17.0 \\
16.8\end{array}$ & $\begin{array}{l}13.4 \\
13.4\end{array}$ & $\begin{array}{l}23.6 \\
23.7\end{array}$ \\
\hline
\end{tabular}

(a) Greatest and least values. 
Table 3.12. Normal and Extreme Daily Maximum and Minimum Temperatures $\left({ }^{\circ} \mathrm{F}\right)$

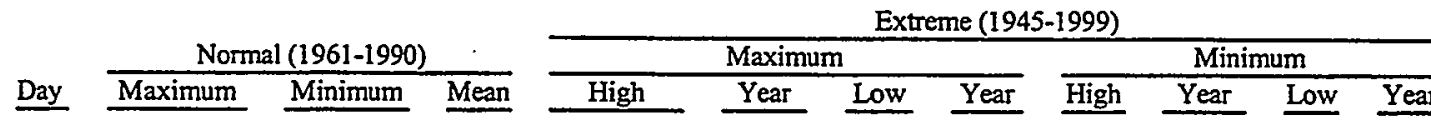

January

\begin{tabular}{rrrrrrrrrrrrr}
\hline 1 & 35 & 21 & 28 & 57 & $98^{(2)}$ & 8 & 69 & 38 & $81^{(2)}$ & -11 & 79 \\
2 & 35 & 21 & 28 & 57 & 97 & 15 & 69 & 42 & 63 & -9 & 78 \\
3 & 36 & 21 & 28 & 63 & 89 & 12 & 50 & 41 & 81 & 0 & 59 \\
4 & 36 & 21 & 29 & 60 & $94^{(2)}$ & 13 & 59 & 39 & 54 & -6 & 50 \\
5 & 36 & 22 & 29 & 59 & $90^{(a)}$ & 12 & 50 & 39 & 81 & -7 & 50 \\
6 & 36 & 22 & 29 & 59 & $90^{(2)}$ & 10 & 82 & 42 & 98 & -10 & 74 \\
7 & 36 & 22 & 29 & 63 & 62 & 9 & 79 & 45 & 90 & -8 & 74 \\
8 & 36 & 22 & 29 & 56 & $83^{(2)}$ & 5 & 74 & 44 & 53 & -9 & 74 \\
9 & 36 & 22 & 29 & 60 & $90^{(a)}$ & 5 & 74 & 41 & 90 & -13 & 74 \\
10 & 36 & 23 & 30 & 61 & 83 & 10 & 74 & 40 & 83 & -5 & 74 \\
11 & 37 & 23 & 30 & 60 & $83^{(2)}$ & 13 & 63 & 37 & 90 & -6 & 49 \\
12 & 38 & 24 & 31 & 59 & 53 & 15 & 63 & 47 & 53 & -4 & $63^{(a)}$ \\
13 & 38 & 24 & 31 & 61 & 94 & 10 & 50 & 42 & 66 & -4 & $93^{(2)}$ \\
14 & 39 & 25 & 32 & 62 & 99 & 7 & 50 & 48 & 61 & -11 & 50 \\
15 & 39 & 26 & 33 & 60 & $74^{(2)}$ & 5 & 50 & 50 & 74 & -5 & 50 \\
16 & 40 & 26 & 33 & 61 & 74 & 8 & 50 & 48 & 89 & -8 & 50 \\
17 & 40 & 26 & 33 & 57 & 98 & 5 & 50 & 40 & 89 & -11 & 50 \\
18 & 40 & 26 & 33 & 62 & 89 & 10 & 50 & 38 & 89 & -6 & 57 \\
19 & 40 & 26 & 33 & 63 & 68 & 13 & 50 & 47 & 68 & -2 & 57 \\
20 & 40 & 26 & 33 & 66 & 68 & 11 & 54 & 47 & 72 & -6 & 54 \\
21 & 40 & 26 & 33 & 65 & 68 & 14 & 54 & 42 & 72 & 0 & 54 \\
22 & 40 & 26 & 33 & 56 & 90 & 16 & 69 & 43 & 81 & -2 & 62 \\
23 & 40 & 26 & 32 & 63 & 53 & 10 & 69 & 43 & 81 & -8 & 69 \\
24 & 40 & 25 & 32 & 59 & $84^{(a)}$ & 13 & 57 & 43 & 58 & -6 & 49 \\
25 & 40 & 25 & 32 & 59 & $92^{(2)}$ & 6 & 50 & 41 & $74^{(a)}$ & -12 & 57 \\
26 & 40 & 25 & 32 & 61 & 71 & 2 & 57 & 46 & 62 & -22 & 57 \\
27 & 40 & 25 & 32 & 60 & $84^{(a)}$ & 0 & 57 & 36 & $95^{(2)}$ & -21 & 57 \\
28 & 40 & 25 & 32 & 61 & 67 & 6 & 57 & 42 & 99 & -14 & 57 \\
29 & 40 & 24 & 32 & 62 & 67 & 3 & 50 & 47 & 99 & -18 & 50 \\
30 & 40 & 24 & 32 & 67 & $89^{(a)}$ & 11 & 57 & 53 & 71 & -13 & 50 \\
31 & 40 & 24 & 32 & 72 & 71 & -2 & 50 & 45 & 53 & -21 & 50
\end{tabular}

February

\begin{tabular}{rrrrrrrrrrrrr}
\hline 1 & 40 & 24 & 32 & 63 & 71 & -3 & 50 & 42 & 92 & -23 & 50 \\
2 & 41 & 24 & 33 & 61 & $91^{(2)}$ & 0 & 50 & 42 & 68 & -21 & 50 \\
3 & 41 & 24 & 33 & 60 & $67^{(2)}$ & 1 & 50 & 46 & 91 & -23 & 50 \\
4 & 41 & 24 & 33 & 65 & 67 & 11 & 85 & 43 & 68 & -5 & 85 \\
5 & 41 & 25 & 33 & 61 & 65 & 23 & 85 & 39 & 61 & -5 & 89 \\
6 & 42 & 25 & 33 & 61 & 67 & 19 & 85 & 44 & 99 & -3 & 89 \\
7 & 42 & 25 & 34 & 65 & 45 & 20 & 48 & 41 & 55 & 2 & 89 \\
8 & 43 & 26 & 35 & 63 & 96 & 22 & 56 & 45 & 45 & 5 & $94^{(2)}$ \\
9 & 44 & 27 & 35 & 65 & 51 & 23 & 56 & 39 & $61^{(2)}$ & 4 & 85 \\
10 & 45 & 28 & 37 & 65 & 77 & 30 & $56^{(2)}$ & 50 & 51 & 7 & 85 \\
11 & 46 & 28 & 37 & 66 & 88 & 27 & 54 & 39 & $93^{(2)}$ & 9 & 48 \\
12 & 47 & 29 & 38 & 70 & 77 & 24 & 49 & 40 & 77 & 6 & 48 \\
13 & 48 & 30 & 39 & 66 & 71 & 25 & 49 & 42 & 47 & 3 & 49 \\
14 & 48 & 30 & 39 & 62 & $97^{(2)}$ & 22 & 80 & 54 & 82 & 8 & 95 \\
15 & 49 & 31 & 40 & 67 & 82 & 19 & 56 & 48 & 81 & 5 & 56 \\
16 & 49 & 31 & 40 & 69 & 77 & 20 & 56 & 48 & 81 & 4 & 56 \\
17 & 49 & 31 & 40 & 67 & $77^{(2)}$ & 22 & 56 & 48 & 48 & 9 & 56 \\
18 & 50 & 31 & 40 & 66 & 81 & 24 & 56 & 46 & 81 & 9 & 90 \\
19 & 50 & 31 & 41 & 68 & 95 & 28 & 56 & 56 & 95 & 14 & $90^{(2)}$
\end{tabular}


Table 3.12. (contd)

\begin{tabular}{|c|c|c|c|c|c|c|c|c|c|c|c|}
\hline \multirow[b]{3}{*}{ Day } & & & & \multicolumn{8}{|c|}{ Extreme (1945-1999) } \\
\hline & \multicolumn{3}{|c|}{ Normal (1961-1990) } & \multicolumn{4}{|c|}{ Maximum } & \multicolumn{4}{|c|}{ Minimum } \\
\hline & Maximum & Minimum & Mean & High & Year & Low & Year & High & Year & Low & Year \\
\hline 20 & 50 & 31 & 41 & 68 & 82 & 32 & $57^{(2)}$ & 51 & 61 & 15 & 86 \\
\hline 21 & 51 & 31 & 41 & 71 & 88 & 29 & 57 & 45 & 95 & 13 & 57 \\
\hline 22 & 51 & 31 & 41 & 62 & $95^{(2)}$ & 29 & 57 & 42 & $99^{(2)}$ & 11 & 93 \\
\hline 23 & 51 & 31 & 41 & 68 & 47 & 34 & $93^{(2)}$ & 43 & $83^{(2)}$ & 19 & 93 \\
\hline 24 & 52 & 31 & 41 & 72 & 86 & 32 & 62 & 60 & 86 & 11 & $93^{(2)}$ \\
\hline 25 & 52 & 31 & 42 & 72 & 86 & 28 & 93 & 49 & 86 & 4 & 93 \\
\hline 26 & 52 & 31 & 42 & 65 & $57^{(a)}$ & 28 & $93^{(2)}$ & 46 & 92 & 10 & 93 \\
\hline 27 & 52 & 31 & 42 & 68 & 72 & 26 & 93 & 44 & 92 & 7 & 62 \\
\hline 28 & 52 & 31 & 42 & 67 & 67 & 25 & 93 & 48 & 72 & 3 & 93 \\
\hline 29 & 52 & 31 & 42 & 63 & $88^{(z)}$ & 40 & 60 & 43 & 92 & 12 & 60 \\
\hline
\end{tabular}

March

\begin{tabular}{|c|c|c|c|c|c|c|c|c|c|c|c|}
\hline 1 & 52 & 31 & 42 & 67 & 94 & 26 & 93 & 44 & 94 & 15 & $71^{(a)}$ \\
\hline 2 & 52 & 31 & 42 & 66 & 68 & 26 & 60 & 46 & 87 & 14 & 60 \\
\hline 3 & 52 & 31 & 42 & 70 & 94 & 24 & 60 & 50 & 87 & 14 & 89 \\
\hline 4 & 52 & 31 & 42 & 63 & 53 & 26 & 55 & 46 & 87 & 7 & 55 \\
\hline 5 & 53 & 31 & 42 & 68 & 72 & 31 & 45 & 50 & 87 & 6 & 55 \\
\hline 6 & 54 & 32 & 43 & 65 & $67^{(2)}$ & 33 & 57 & 43 & 79 & 18 & 60 \\
\hline 7 & 55 & 32 & 44 & 66 & 53 & 41 & 45 & 42 & $86^{(2)}$ & 21 & $74^{(2)}$ \\
\hline 8 & 55 & 33 & 44 & 67 & 53 & 33 & 51 & 46 & 83 & 20 & 76 \\
\hline 9 & 56 & 33 & 44 & 69 & 53 & 40 & 51 & 47 & 83 & 22 & $94^{(2)}$ \\
\hline 10 & 56 & 33 & 45 & 69 & 72 & 40 & 48 & 45 & $87^{(2)}$ & 13 & 48 \\
\hline 11 & 56 & 33 & 45 & 67 & $95^{(2)}$ & 32 & 50 & 44 & 95 & 21 & 50 \\
\hline 12 & 57 & 34 & 45 & 68 & $98^{(2)}$ & 37 & 51 & 48 & 87 & 15 & 56 \\
\hline 13 & 57 & 34 & 45 & 72 & 98 & 38 & 51 & 44 & $98^{(2)}$ & 22 & $69^{(2)}$ \\
\hline 14 & 57 & 34 & 46 & 72 & 92 & 40 & 49 & 45 & 61 & 23 & 53 \\
\hline 15 & 58 & 34 & 46 & 73 & 94 & 37 & 49 & 50 & 92 & 23 & 76 \\
\hline 16 & 58 & 34 & 46 & 76 & 72 & 43 & 89 & 47 & 94 & 23 & 55 \\
\hline 17 & 59 & 35 & 47 & 76 & 72 & 38 & 65 & 48 & 69 & 17 & 65 \\
\hline 18 & 59 & 35 & 47 & 76 & 47 & 41 & 65 & 47 & 90 & 14 & 65 \\
\hline 19 & 59 & 35 & 47 & 76 & 47 & 48 & $65^{(2)}$ & 53 & 97 & 16 & 65 \\
\hline 20 & 59 & 35 & 47 & 76 & 47 & 49 & 50 & 49 & 88 & 22 & 74 \\
\hline 21 & 59 & 35 & 47 & 74 & 60 & 41 & 75 & 46 & $98^{(2)}$ & 26 & $82^{(a)}$ \\
\hline 22 & 59 & 35 & 47 & 74 & $78^{(\mathfrak{a})}$ & 47 & 71 & 47 & 78 & 19 & 94 \\
\hline 23 & 59 & 35 & 47 & 77 & 60 & 39 & 64 & 45 & $98^{(2)}$ & 20 & 48 \\
\hline 24 & 59 & 35 & 47 & 78 & 60 & 38 & 55 & 45 & $60^{(2)}$ & 15 & 64 \\
\hline 25 & 59 & 35 & 47 & 83 & 60 & 35 & 55 & 48 & 52 & 21 & 96 \\
\hline 26 & 60 & 35 & 47 & 76 & 46 & 38 & 65 & 49 & $92^{(2)}$ & 21 & 85 \\
\hline 27 & 60 & 35 & 48 & 77 & 94 & 47 & 79 & 46 & 89 & 24 & 75 \\
\hline 28 & 61 & 36 & 48 & 79 & 94 & 42 & 54 & 49 & 78 & 19 & 75 \\
\hline 29 & 61 & 36 & 49 & 78 & $94^{(2)}$ & 49 & 54 & 48 & $94^{(2)}$ & 18 & 54 \\
\hline 30 & 62 & 37 & 49 & 75 & 92 & 52 & 67 & 47 & $92^{(2)}$ & 20 & 54 \\
\hline 31 & 62 & 37 & 49 & 78 & 92 & 45 & 96 & 47 & 61 & 28 & 53 \\
\hline \multicolumn{12}{|c|}{ April } \\
\hline 1 & 63 & 37 & 50 & 80 & 90 & 45 & 76 & 50 & 59 & 24 & 82 \\
\hline 2 & 63 & 37 & 50 & 83 & 92 & 48 & 82 & 50 & 87 & 25 & 76 \\
\hline 3 & 64 & 37 & 50 & 76 & 77 & 50 & $63^{(2)}$ & 48 & 77 & 23 & 75 \\
\hline 4 & 64 & 38 & 51 & 82 & 60 & 45 & 75 & 56 & 91 & 27 & 50 \\
\hline 5 & 64 & 38 & 51 & 78 & $77^{(a)}$ & 51 & 75 & 54 & 60 & 21 & 75 \\
\hline 6 & 65 & 38 & 51 & 82 & 77 & 50 & 82 & 51 & 62 & 25 & 97 \\
\hline 7 & 65 & 38 & 52 & 85 & 77 & 41 & 45 & 53 & 60 & 26 & 54 \\
\hline 8 & 65 & 38 & 52 & 82 & 96 & 51 & 53 & 50 & 96 & 27 & $92^{(2)}$ \\
\hline 9 & 65 & 38 & 52 & 80 & 85 & 48 & 92 & 57 & 96 & 29 & $75^{(2)}$ \\
\hline 10 & 65 & 38 & 52 & 85 & 68 & 52 & 45 & 50 & 96 & 24 & 81 \\
\hline
\end{tabular}


Table 3.12. (contd)

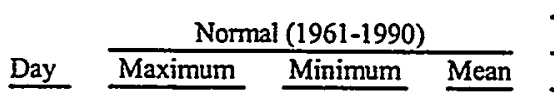

\begin{tabular}{|c|c|c|c|c|c|c|c|}
\hline \multicolumn{8}{|c|}{ Extreme (1945-1999) } \\
\hline \multicolumn{4}{|c|}{ Maximum } & \multicolumn{4}{|c|}{ Minimum } \\
\hline High & Year & Low & Year & High & Year & Low & Year \\
\hline 79 & 88 & 52 & 83 & 51 & $56^{(2)}$ & 27 & 83 \\
\hline 83 & 88 & 47 & 95 & 48 & $82^{(a)}$ & 26 & 97 \\
\hline 88 & 47 & 54 & 55 & 59 & 88 & 23 & 68 \\
\hline 85 & $62^{(2)}$ & 47 & 75 & 55 & 85 & 29 & 83 \\
\hline 82 & 88 & 55 & $75^{(2)}$ & 54 & 87 & 27 & 55 \\
\hline 83 & 54 & $53^{\circ}$ & 63 & 54 & 92 & 28 & 82 \\
\hline 88 & 94 & 48 & 63 & 52 & 90 & 26 & 55 \\
\hline 88 & 94 & 51 & 67 & 60 & 94 & 29 & 68 \\
\hline 78 & 56 & 50 & 51 & 56 & 94 & 27 & 66 \\
\hline 84 & 56 & 45 & 67 & 53 & $90^{(2)}$ & 28 & 82 \\
\hline 85 & 56 & 52 & 67 & 60 & 56 & 26 & $85^{(\mathrm{e})}$ \\
\hline 81 & $82^{(2)}$ & 53 & 88 & 55 & 98 & 28 & 72 \\
\hline 88 & $81^{(a)}$ & 56 & $79^{(2)}$ & 58 & 77 & 30 & 78 \\
\hline 94 & 77 & 53 & 75 & 56 & 52 & 28 & $86^{(2)}$ \\
\hline 91 & 46 & 56 & 58 & 59 & 52 & 31 & 55 \\
\hline 85 & 92 & 53 & 48 & 57 & 78 & 28 & 48 \\
\hline 90 & 87 & 50 & 90 & 57 & 92 & 27 & 70 \\
\hline 93 & 87 & 54 & 95 & 64 & 87 & 27 & 67 \\
\hline 90 & 68 & 47 & 67 & 60 & 87 & 29 & 52 \\
\hline 92 & 98 & 56 & $67^{(3)}$ & 60 & 98 & 29 & 86 \\
\hline
\end{tabular}

May

\begin{tabular}{|c|c|c|c|c|c|c|c|c|c|c|c|}
\hline 1 & 71 & 43 & 57 & 93 & 98 & 53 & 69 & 59 & $98^{(a)}$ & 28 & 54 \\
\hline 2 & 71 & 43 & 57 & 89 & $98^{(2)}$ & 56 & 88 & 60 & 71 & 30 & 97 \\
\hline 3 & 71 & 44 & 58 & 91 & 66 & 58 & 93 & 60 & 71 & 31 & 49 \\
\hline 4 & 72 & 44 & 58 & 94 & 66 & 56 & 63 & 57 & 46 & 31 & 62 \\
\hline 5 & 72 & 45 & 58 & 100 & 66 & 52 & 61 & 65 & 66 & 30 & 59 \\
\hline 6 & 73 & 45 & 59 & 98 & 92 & 56 & 86 & 62 & 87 & 34 & 77 \\
\hline 7 & 73 & 45 & 59 & 99 & 87 & 59 & 99 & 66 & 92 & 33 & 84 \\
\hline 8 & 73 & 46 & 59 & 102 & 87 & 56 & $99^{(2)}$ & 67 & $94^{(2)}$ & 29 & 96 \\
\hline 9 & 74 & 46 & 60 & 97 & 87 & 56 & 48 & 66 & 49 & 34 & $99^{(a)}$ \\
\hline 10 & 74 & 46 & 60 & 96 & 49 & 53 & 67 & 66 & 49 & 34 & 70 \\
\hline 11 & 74 & 46 & 60 & 98 & 49 & 51 & 67 & 68 & 49 & 30 & 70 \\
\hline 12 & 74 & 46 & 60 & 100 & 93 & 57 & 70 & 66 & 93 & 34 & 85 \\
\hline 13 & 74 & 46 & 60 & 94 & 97 & 57 & 55 & 66 & 97 & 34 & 85 \\
\hline 14 & 75 & 47 & 61 & 98 & 73 & 56 & 55 & 61 & $73^{(2)}$ & 31 & 55 \\
\hline 15 & 75 & 47 & 61 & 97 & 73 & 57 & 59 & 67 & 97 & 35 & 74 \\
\hline 16 & 75 & 47 & 61 & 95 & 73 & 54 & 55 & 60 & 73 & 32 & 74 \\
\hline 17 & 76 & 48 & 62 & 96 & 73 & 61 & 74 & 59 & 85 & 38 & $88^{(2)}$ \\
\hline 18 & 76 & 48 & 62 & 98 & 54 & 62 & 74 & 67 & 56 & 36 & 72 \\
\hline 19 & 77 & 48 & 62 & 92 & 93 & 56 & 62 & 70 & 56 & 33 & 75 \\
\hline 20 & 77 & 48 & 62 & 93 & 47 & 58 & 60 & 59 & 56 & 36 & 71 \\
\hline 21 & 77 & 49 & 63 & 94 & 58 & 62 & $60^{(a)}$ & 59 & 58 & 37 & $74^{(0)}$ \\
\hline 22 & 77 & 49 & 63 & 98 & 58 & 63 & 64 & 64 & 58 & 33 & 60 \\
\hline 23 & 77 & 49 & 63 & 95 & $85^{(2)}$ & 59 & 62 & 66 & 58 & 35 & 64 \\
\hline 24 & 77 & 49 & 63 & 97 & 99 & 54 & 62 & 63 & 81 & 35 & 75 \\
\hline 25 & 77 & 50 & 63 & 98 & $92^{(2)}$ & 61 & 98 & 65 & 83 & 38 & $91^{(2)}$ \\
\hline 26 & 77 & 50 & 64 & 101 & $58^{(2)}$ & 54 & 80 & 69 & 47 & 38 & 78 \\
\hline 27 & 78 & 50 & 64 & 93 & 83 & 62 & 89 & 69 & 58 & 37 & 73 \\
\hline 28 & 78 & 50 & 64 & 99 & 83 & 61 & 89 & 63 & $72^{(2)}$ & 38 & $79^{(a)}$ \\
\hline 29 & 78 & 51 & 64 & 103 & 83 & 68 & $98^{(2)}$ & 71 & 86 & 35 & 76 \\
\hline 30 & 79 & 51 & 65 & 104 & 86 & 62 & $76^{(2)}$ & 68 & 86 & 41 & $55^{(2)}$ \\
\hline 31 & 79 & 52 & 65 & 104 & 86 & 54 & 71 & 69 & 86 & 40 & $96^{(2)}$ \\
\hline \multicolumn{12}{|c|}{ June } \\
\hline 1 & 80 & 52 & 66 & 103 & 86 & 64 & 76 & 69 & 86 & 37 & 84 \\
\hline 2 & 80 & 52 & 66 & 99 & 70 & 65 & $99^{(a)}$ & 69 & $89^{(2)}$ & 37 & 76 \\
\hline
\end{tabular}


Table 3.12. (contd)

\begin{tabular}{|c|c|c|c|c|c|c|c|c|c|c|c|}
\hline \multirow[b]{3}{*}{ Day } & & & & \multicolumn{8}{|c|}{ Extreme (1945-1999) } \\
\hline & \multicolumn{3}{|c|}{ Normal (1961-1990) } & \multicolumn{4}{|c|}{ Maximum } & \multicolumn{4}{|c|}{ Minimum } \\
\hline & Maximum & Minimum & Mean & High & Year & Low & Year & High & Year & Low & Year \\
\hline 3 & 81 & 53 & 67 & 103 & 70 & 55 & 66 & 68 & $86^{(2)}$ & 37 & 62 \\
\hline 4 & 81 & 54 & 67 & 103 & 69 & 60 & 74 & 66 & $86^{(2)}$ & 40 & $80^{(2)}$ \\
\hline 5 & 81 & 54 & 68 & 101 & 78 & 60 & 88 & 73 & 69 & 43 & $76^{(2)}$ \\
\hline 6 & 81 & 54 & 68 & 102 & $70^{(2)}$ & 57 & 95 & 68 & 77 & 38 & 99 \\
\hline 7 & 82 & 54 & 68 & 100 & 77 & 56 & 50 & 69 & 77 & 42 & 99 \\
\hline 8 & 82 & 54 & 68 & 100 & 48 & 59 & 64 & 69 & 69 & 40 & 53 \\
\hline 9 & 82 & 54 & 68 & 98 & 55 & 68 & 59 & 68 & $69^{(2)}$ & 42 & 99 \\
\hline 10 & 82 & 54 & 68 & 98 & 55 & 69 & 72 & 68 & 79 & 41 & 59 \\
\hline 11 & 82 & 54 & 68 & 100 & 55 & 64 & 61 & 70 & 55 & 40 & 56 \\
\hline 12 & 83 & 55 & 69 & 98 & 74 & 68 & 54 & 67 & $87^{(2)}$ & 42 & 68 \\
\hline 13 & 83 & 55 & 69 & 99 & 74 & 59 & 80 & 70 & 99 & 42 & 52 \\
\hline 14 & 84 & 56 & 70 & 103 & 74 & 65 & 95 & 68 & 87 & 44 & $78^{(2)}$ \\
\hline 15 & 85 & 56 & 70 & 102 & $99^{(a)}$ & 70 & 65 & 72 & 63 & 44 & 54 \\
\hline 16 & 85 & 57 & 71 & 106 & 61 & 62 & 49 & 70 & 63 & 41 & 54 \\
\hline 17 & 86 & 57 & 72 & 108 & 61 & 70 & 73 & 75 & 61 & 40 & 81 \\
\hline 18 & 86 & 57 & 72 & 104 & 61 & 69 & 64 & 75 & 58 & 41 & 54 \\
\hline 19 & 86 & 57 & 72 & 102 & 85 & 63 & 95 & 73 & 58 & 43 & 86 \\
\hline 20 & 86 & 57 & 72 & 102 & 82 & 63 & 91 & 73 & 59 & 42 & 53 \\
\hline 21 & 86 & 57 & 72 & 104 & 70 & 62 & 84 & 73 & 58 & 45 & 56 \\
\hline 22 & 86 & 57 & 72 & 106 & $92^{(2)}$ & 71 & 93 & 74 & 92 & 46 & $97^{(2)}$ \\
\hline 23 & 86 & 57 & 72 & 111 & 92 & 68 & 72 & 75 & 58 & 44 & 52 \\
\hline 24 & 86 & 57 & 71 & 108 & 92 & 66 & 72 & 80 & 92 & 40 & 83 \\
\hline 25 & 86 & 56 & 71 & 107 & 92 & 70 & 46 & 79 & .92 & 42 & 76 \\
\hline 26 & 86 & 56 & 71 & 103 & 87 & 70 & 75 & 74 & 70 & 41 & $76^{(2)}$ \\
\hline 27 & 86 & 56 & 71 & 102 & 92 & 68 & 47 & 75 & 87 & 45 & $64^{(2)}$ \\
\hline 28 & 86 & 56 & 71 & 102 & $87^{(2)}$ & 64 & 46 & 68 & 87 & 38 & 75 \\
\hline 29 & 86 & 56 & 71 & 104 & 48 & 65 & 52 & 74 & 87 & 46 & $71^{(2)}$ \\
\hline 30 & 86 & 56 & 71 & 106 & 87 & 71 & 55 & 71 & 87 & 42 & 49 \\
\hline
\end{tabular}

July

\begin{tabular}{llllllllllll}
\hline 1 & 86 & 57 & 72 & 103 & 87 & 66 & 66 & 75 & 87 & 46 & $73^{(2)}$ \\
2 & 87 & 57 & 72 & 103 & 96 & 59 & 66 & 70 & 45 & 39 & 79 \\
3 & 87 & 58 & 72 & 105 & $91^{(2)}$ & 71 & 66 & 70 & 67 & 45 & 99 \\
4 & 88 & 58 & 73 & 108 & 68 & 71 & 86 & 75 & 70 & 48 & 66 \\
5 & 88 & 59 & 73 & 108 & 75 & 66 & 51 & 76 & 75 & 47 & $99^{(2)}$ \\
6 & 89 & 59 & 74 & 110 & 68 & 71 & 55 & 76 & 68 & 44 & 71 \\
7 & 89 & 59 & 74 & 105 & $68^{(2)}$ & 75 & 81 & 73 & 68 & 45 & 71 \\
8 & 89 & 59 & 74 & 108 & 68 & 71 & 72 & 74 & 85 & 45 & 81 \\
9 & 89 & 60 & 74 & 110 & 75 & 76 & 55 & 78 & 75 & 50 & $72^{(2)}$ \\
10 & 90 & 60 & 75 & 106 & 75 & 67 & 74 & 79 & 75 & 49 & $97^{(2)}$ \\
11 & 90 & 60 & 75 & 109 & 90 & 76 & 74 & 78 & 75 & 46 & 81 \\
12 & 90 & 60 & 75 & 110 & 90 & 75 & 88 & 75 & 90 & 50 & 74 \\
13 & 90 & 60 & 75 & 108 & 61 & 77 & $93^{(2)}$ & 73 & $90^{(2)}$ & 49 & 76 \\
14 & 90 & 61 & 76 & 107 & $87^{(2)}$ & 77 & 83 & 78 & 61 & 50 & 83 \\
15 & 91 & 61 & 76 & 108 & 96 & 71 & 82 & 76 & 55 & 45 & 82 \\
16 & 91 & 61 & 76 & 105 & 70 & 68 & 86 & 74 & 90 & 48 & 74 \\
17 & 92 & 61 & 76 & 110 & 60 & 73 & 93 & 77 & 58 & 48 & 86 \\
18 & 92 & 61 & 77 & 110 & 60 & 76 & 96 & 79 & 60 & 49 & $96^{(2)}$ \\
19 & 92 & 61 & 77 & 109 & 79 & 72 & 49 & 77 & 79 & 51 & 77 \\
20 & 93 & 62 & 77 & 110 & 79 & 75 & $65^{(2)}$ & 75 & 95 & 53 & $68^{(2)}$ \\
21 & 93 & 62 & 78 & 109 & 94 & 68 & 65 & 77 & 88 & 49 & 49 \\
22 & 94 & 62 & 78 & 111 & 94 & 74 & 92 & 75 & $94^{(2)}$ & 47 & 82 \\
23 & 94 & 63 & 78 & 109 & 94 & 69 & 92 & 82 & 94 & 49 & 63 \\
24 & 94 & 63 & 79 & 109 & 94 & 78 & 63 & 75 & 62 & 52 & $52^{(2)}$ \\
25 & 95 & 63 & 79 & 106 & 84 & 73 & 90 & 77 & 62 & 51 & $49^{(2)}$ \\
26 & 95 & 63 & 79 & 108 & $98^{(2)}$ & 66 & 55 & 76 & 88 & 53 & 99 \\
27 & 95 & 64 & 79 & 112 & 98 & 74 & 48 & 74 & $98^{(2)}$ & 52 & 86 \\
28 & 95 & 64 & 79 & 108 & $98^{(2)}$ & 77 & $50^{(2)}$ & 81 & 98 & 49 & 59
\end{tabular}


Table 3.12. (contd)

\begin{tabular}{|c|c|c|c|c|c|c|c|c|c|c|}
\hline \multirow[b]{3}{*}{ Day } & \multirow{2}{*}{\multicolumn{3}{|c|}{ Normal (1961-1990) }} & \multicolumn{7}{|c|}{ Extreme (1945-1999) } \\
\hline & & & & & Maxin & & & & Mini & \\
\hline & Maximum & Minimum & Mean & High & Year & Low & Year & High & Year & Low \\
\hline 29 & 95 & 63 & 79 & 107 & 82 & 80 & $93^{(2)}$ & 78 & 82 & 52 \\
\hline 30 & 95 & 63 & 79 & 107 & 71 & 78 & 75 & 74 & 90 & 49 \\
\hline 31 & 94 & 63 & 79 & 111 & 71 & 75 & 85 & 74 & $71^{(2)}$ & 52 \\
\hline
\end{tabular}

August

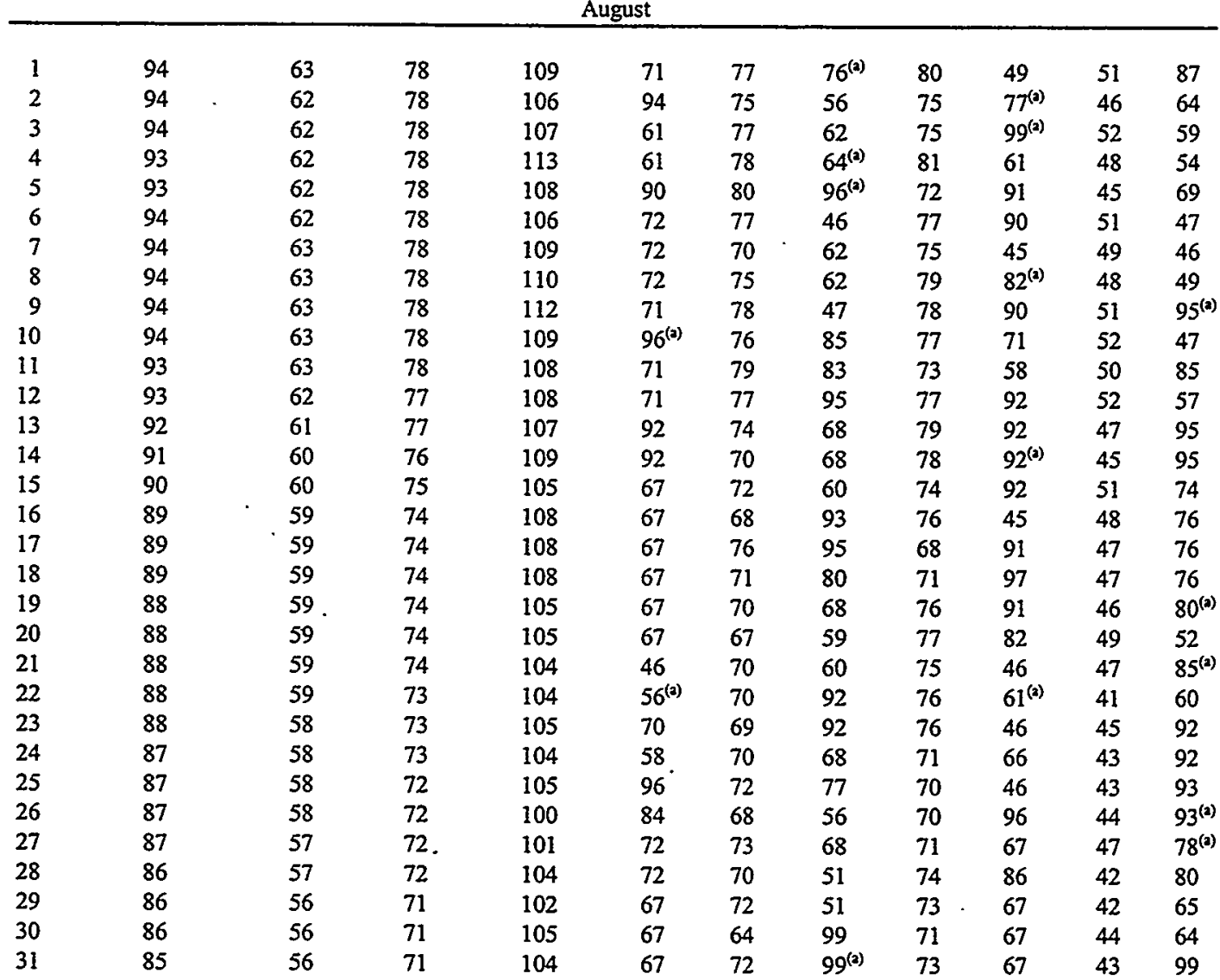

September

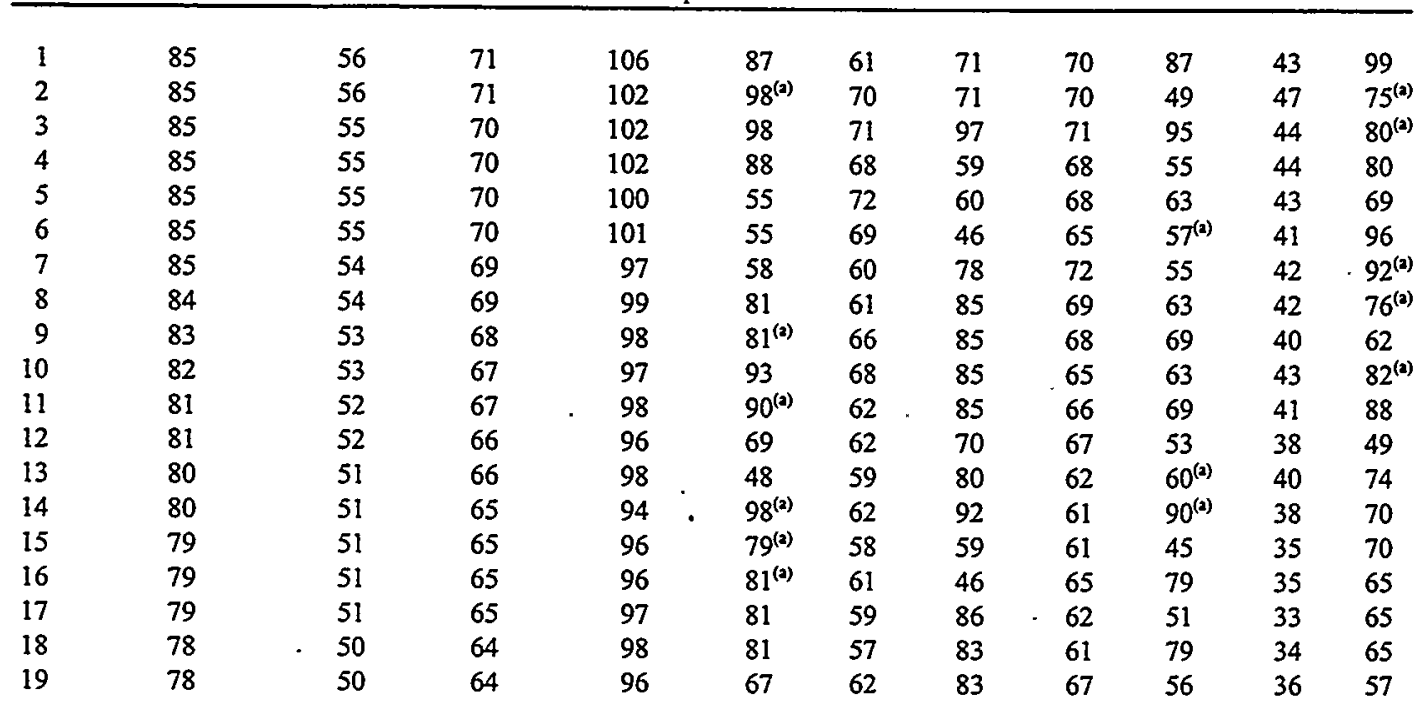


Table 3.12. (contd)

\begin{tabular}{|c|c|c|c|c|c|c|c|c|c|c|c|}
\hline \multirow[b]{3}{*}{ Day } & & & & \multicolumn{8}{|c|}{ Extreme (1945-1999) } \\
\hline & \multicolumn{3}{|c|}{ Normal (1961-1990) } & \multicolumn{4}{|c|}{ Maximum } & \multicolumn{4}{|c|}{ Minimum } \\
\hline & Maximum & Minimum & Mean & High & Year & Low & Year & High & Year & Low & Year \\
\hline 20 & 78 & 50 & 64 & 94 & 94 & 66 & $72^{(a)}$ & 67 & 94 & 37 & 83 \\
\hline 21 & 78 & 49 & 63 & 98 & 67 & 66 & 45 & 65 & 62 & 38 & $93^{(2)}$ \\
\hline 22 & 77 & 49 & 63 & 93 & 66 & 52 & 84 & 68 & 66 & 36 & $81^{(2)}$ \\
\hline 23 & 77 & 49 & 63 & 93 & 87 & 54 & 77 & 62 & 92 & 34 & 81 \\
\hline 24 & 77 & 49 & 63 & 94 & 52 & 60 & 72 & 60 & 50 & 34 & $72^{(2)}$ \\
\hline 25 & 77 & 48 & 63 & 97 & 52 & 56 & 77 & 66 & 49 & 30 & 72 \\
\hline 26 & 77 & 48 & 62 & 93 & 52 & 57 & 48 & 61 & 79 & 32 & 72 \\
\hline 27 & 76 & 48 & 62 & 92 & $63^{(2)}$ & 58 & 77 & 60 & 49 & 30 & 72 \\
\hline 28 & 76 & 48 & 62 & 92 & 67 & 58 & 77 & 62 & 76 & 33 & 85 \\
\hline 29 & 76 & 47 & 61 & 92 & $96^{(2)}$ & 57 & 77 & 61 & 89 & 34 & $85^{(2)}$ \\
\hline 30 & 75 & 46 & 61 & 88 & $93^{(2)}$ & 63 & $54^{(2)}$ & 64 & 93 & 35 & 85 \\
\hline \multicolumn{12}{|c|}{ October } \\
\hline
\end{tabular}

\begin{tabular}{|c|c|c|c|c|c|c|c|c|c|c|c|}
\hline 1 & 75 & 46 & 60 & 88 & $91^{(2)}$ & 61 & 59 & 59 & 92 & 30 & 54 \\
\hline 2 & 74 & 45 & 60 & 86 & $93^{(2)}$ & 56 & 67 & 60 & 88 & 32 & 54 \\
\hline 3 & 73 & 45 & 59 & 89 & 58 & 55 & 50 & 58 & $88^{(2)}$ & 33 & $99^{(2)}$ \\
\hline 4 & 73 & 44 & 58 & 89 & 80 & 55 & 50 & 57 & 88 & 32 & 73 \\
\hline 5 & 72 & 44 & 58 & 87 & 58 & 52 & 57 & 52 & $88^{(2)}$ & 34 & $82^{(2)}$ \\
\hline 6 & 72 & 44 & 58 & 85 & 80 & 53 & $57^{(2)}$ & 57 & 60 & 30 & 74 \\
\hline 7 & 71 & 44 & 57 & 86 & 80 & 48 & 57 & 57 & 88 & 29 & 74 \\
\hline 8 & 70 & 44 & 57 & 84 & 65 & 50 & 97 & 58 & 87 & 26 & 85 \\
\hline 9 & 70 & 43 & 56 & 84 & 45 & 52 & 58 & 56 & 96 & 26 & 85 \\
\hline 10 & 69 & 43 & 56 & 86 & 96 & 50 & 62 & 55 & 96 & 33 & 59 \\
\hline 11 & 69 & 43 & 56 & 84 & 52 & 52 & $68^{(2)}$ & 55 & 63 & 30 & 60 \\
\hline 12 & 68 & 43 & 55 & 84 & 45 & 54 & 66 & 56 & 52 & 34 & $85^{(2)}$ \\
\hline 13 & 68 & 42 & 55 & 81 & 99 & 57 & 69 & 55 & 88 & 31 & 69 \\
\hline 14 & 67 & 41 & 54 & 78 & 45 & 58 & $90^{(2)}$ & 59 & 88 & 24 & 69 \\
\hline 15 & 66 & 40 & 53 & 81 & 63 & 53 & 92 & 60 & 88 & 29 & 70 \\
\hline 16 & 66 & 39 & 53 & 79 & 63 & 54 & $92^{(2)}$ & 56 & 95 & 26 & 46 \\
\hline 17 & 65 & 39 & 52 & 77 & 97 & 45 & 96 & 49 & 55 & 26 & 96 \\
\hline 18 & 65 & 38 & 51 & 76 & 73 & 47 & 49 & 50 & 52 & 27 & 49 \\
\hline 19 & 64 & 38 & 51 & 78 & $92^{(2)}$ & 50 & 45 & 52 & 92 & 27 & $69^{(2)}$ \\
\hline 20 & 63 & 38 & 50 & 74 & 78 & 45 & 47 & 54 & 73 & 23 & 49 \\
\hline 21 & 63 & 38 & 50 & 73 & $91^{(2)}$ & 45 & 96 & 54 & 63 & 20 & 84 \\
\hline 22 & 63 & 38 & 50 & 74 & 59 & 46 & 50 & 51 & 52 & 20 & 84 \\
\hline 23 & 62 & 38 & 50 & 73 & $66^{(3)}$ & 39 & 84 & 51 & 60 & 25 & 84 \\
\hline 24 & 61 & 38 & 50 & 75 & $77^{(2)}$ & 49 & 57 & 49 & 46 & 26 & 75 \\
\hline 25 & 61 & 37 & 49 & 75 & $55^{(2)}$ & 49 & 57 & 60 & 45 & 26 & 78 \\
\hline 26 & 60 & 37 & 48 & 69 & 92 & 49 & 46 & 52 & 94 & 21 & 78 \\
\hline 27 & 59 & 36 & 48 & 74 & 85 & 43 & $99^{(0)}$ & 54 & 81 & 23 & 70 \\
\hline 28 & 58 & 36 & 47 & 68 & $65^{(2)}$ & 35 & 91 & 52 & 49 & 18 & 71 \\
\hline 29 & 58 & 36 & 47 & 70 & 53 & 42 & $9 \mathrm{I}^{(2)}$ & 50 & 97 & 13 & 71 \\
\hline 30 & 57 & 36 & 46 & 75 & 67 & 32 & 71 & 50 & 97 & 20 & 72 \\
\hline 31 & 56 & 36 & 46 & 75 & 67 & 34 & 84 & 54 & 67 & 12 & 84 \\
\hline \multicolumn{12}{|c|}{ November } \\
\hline 1 & 56 & 36 & 46 & 69 & 88 & 31 & 84 & 49 & 87 & 20 & 95 \\
\hline 2 & 56 & 36 & 46 & 70 & 45 & 38 & 91 & 51 & 85 & 17 & 95 \\
\hline 3 & 55 & 36 & 45 & 75 & 75 & 36 & 73 & 53 & 83 & 17 & 95 \\
\hline 4 & 55 & 36 & 45 & 71 & 75 & 32 & 73 & 46 & $89^{(2)}$ & 16 & 73 \\
\hline 5 & 54 & 35 & 44 & 63 & 89 & 31 & 73 & 48 & 88 & 20 & $73^{(2)}$ \\
\hline 6 & 53 & 35 & 44 & 64 & 58 & 32 & 73 & 49 & 89 & 19 & 73 \\
\hline 7 & 53 & 35 & 44 & 69 & 78 & 34 & 45 & 48 & $97^{(2)}$ & 19 & $93^{(2)}$ \\
\hline 8 & 52 & 34 & 43 & 69 & 95 & 34 & 45 & 48 & 89 & 16 & 45 \\
\hline 9 & 51 & 34 & 43 & 73 & 89 & 30 & 45 & 60 & 89 & 18 & 86 \\
\hline 10 & 51 & 34 & 42 & 73 & 89 & 32 & 45 & 56 & 89 & 16 & 86 \\
\hline 11 & 50 & 34 & 42 & 66 & 89 & 32 & 85 & 48 & 89 & 14 & 78 \\
\hline
\end{tabular}


Table 3.12. (contd)

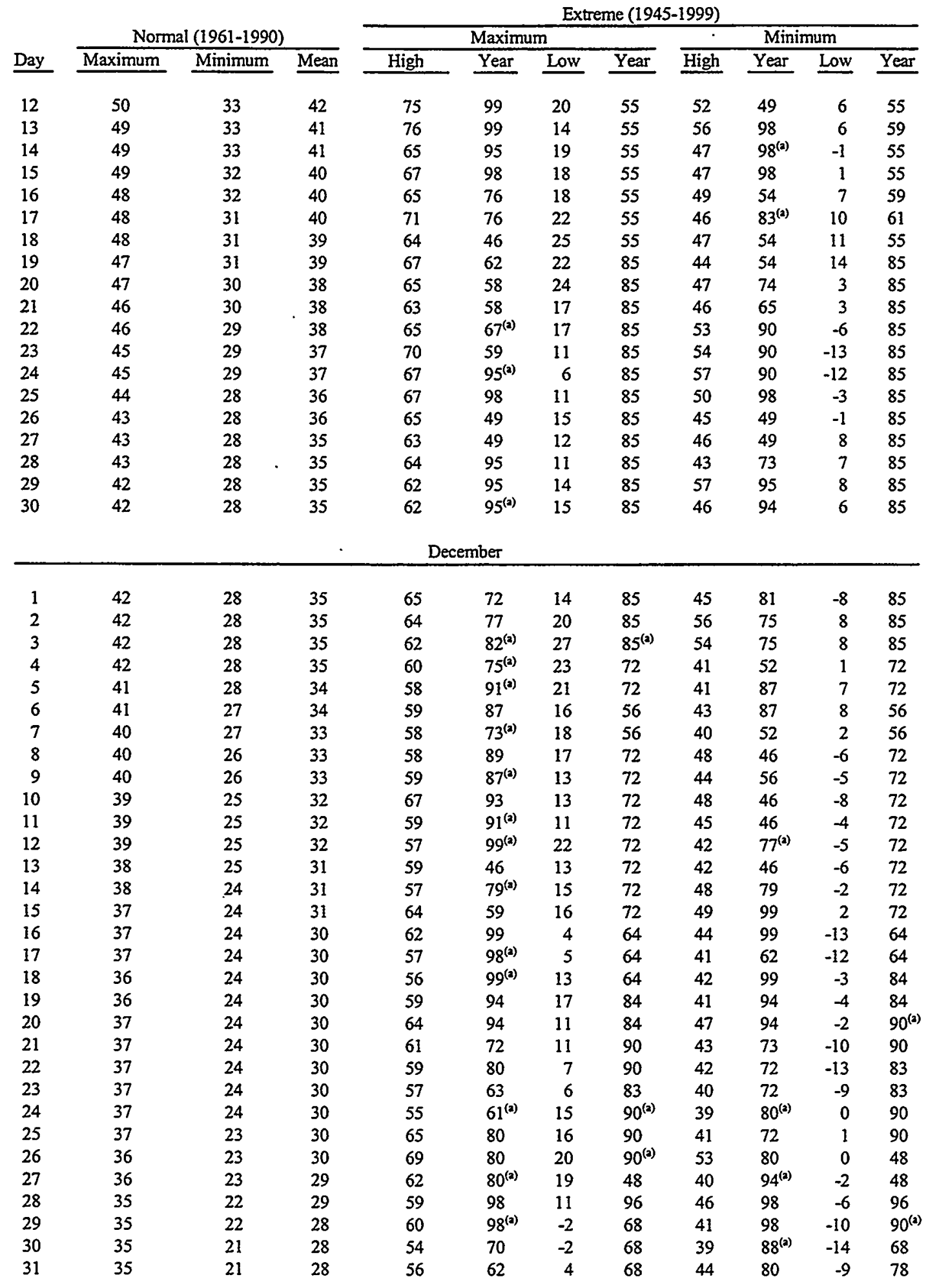

(a) Latest of several occurrences. 


\subsection{Subsurface Soil Temperatures}

Hourly subsurface soil temperature data at depths of $\sim 0.5$ inch, 15 inches, and 36 inches are available for the period from 1955 through 1999. The subsurface soil temperature sensors are installed in the natural soil of the area with the vegetation cover removed. The soil is sandy and mixed with large gravel.

Monthly averages and extremes of monthly averages are presented in Table 3.13. The absolute hourly extremes are also indicated in that table.

\subsection{Heating- and Cooling-Degree Days}

Data about heating- and cooling-degree days are generally used by the utility industry and those involved in building design to assess heating and cooling energy requirements. A temperature of $65^{\circ} \mathrm{F}$ is generally used as the basis for this calculation. To determine whether a day has either heating-degree days (HDDs) or cooling-degree days (CDDs), 65 is subtracted from the daily average temperature (computed by adding the daily maximum and minimum temperatures and dividing by two). If the difference is positive, the day has cooling-degree days. If the difference is negative, the day has heatingdegree days.

\begin{tabular}{llll}
\multicolumn{3}{c}{ Example Calculations } & \\
\cline { 2 - 2 } Daily high temperature & \multicolumn{1}{c}{ Summer Day } & & Winter Day \\
Daily low temperature & 60 & & 42 \\
Daily average temperature & $75(150 \div 2)$ & & $31(62 \div 2)$ \\
Threshold temperature & -65 & & -65 \\
Difference & $10(10 \mathrm{CDDs})$ & & $-34(34 \mathrm{HDDs})$
\end{tabular}

Tables 3.14 and 3.15 provide monthly HDD and CDD data, respectively, for the period 1945 through 1999. The HDDs are traditionally totaled for the 12-month period July through June of the following year. 


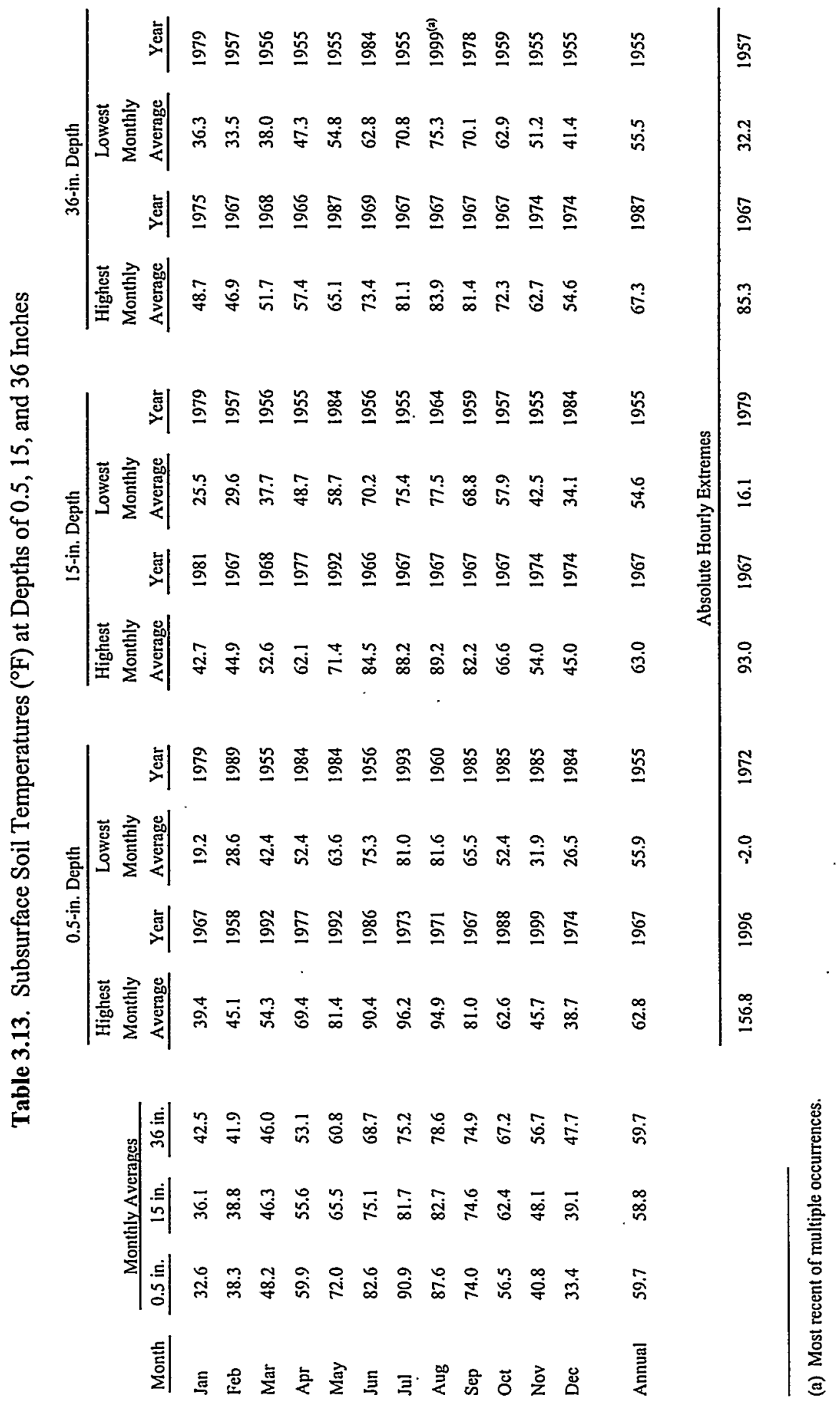


Table 3.14. Monthly and Seasonal Heating-Degree Days

\begin{tabular}{|c|c|c|c|c|c|c|c|c|c|c|c|c|c|}
\hline Year & Jul & Aug & Sep & Oct & Nov & Dec & $\operatorname{Jan}$ & $\mathrm{Feb}$ & Mar & Apr & May & Jun & Season \\
\hline $\begin{array}{l}1944-45 \\
1945-46 \\
1946-47 \\
1947-48 \\
1948-49 \\
1949-50\end{array}$ & $\begin{array}{l}- \\
0 \\
0 \\
0 \\
2 \\
1\end{array}$ & $\begin{array}{l}- \\
2 \\
1 \\
7 \\
0 \\
0\end{array}$ & $\begin{array}{r}- \\
97 \\
101 \\
70 \\
109 \\
52\end{array}$ & $\begin{array}{l}-\overline{277} \\
479 \\
351 \\
438 \\
456\end{array}$ & $\begin{array}{l}733 \\
875 \\
714 \\
725 \\
592\end{array}$ & $\begin{array}{r}- \\
1,000 \\
935 \\
989 \\
1,184 \\
927\end{array}$ & $\begin{array}{r}967 \\
949 \\
1,168 \\
1,024 \\
1,581 \\
1,640^{(2)}\end{array}$ & $\begin{array}{l}738 \\
710 \\
702 \\
963 \\
928 \\
959\end{array}$ & $\begin{array}{l}709 \\
603 \\
476 \\
709 \\
616 \\
704\end{array}$ & $\begin{array}{l}442 \\
331 \\
266 \\
471 \\
281 \\
452\end{array}$ & $\begin{array}{c}141 \\
79 \\
36^{(2)} \\
237 \\
85 \\
196\end{array}$ & $\begin{array}{r}38 \\
42 \\
25 \\
5 \\
36 \\
66\end{array}$ & $\begin{array}{c}- \\
4,823 \\
5,064 \\
5,540 \\
5,985 \\
6,045\end{array}$ \\
\hline $\begin{array}{l}1950-51 \\
1951-52 \\
1952-53 \\
1953-54 \\
1954-55\end{array}$ & $\begin{array}{r}0 \\
5 \\
0 \\
0 \\
10\end{array}$ & $\begin{array}{r}0 \\
19 \\
2 \\
3 \\
4\end{array}$ & $\begin{array}{l}64 \\
46 \\
34 \\
59 \\
79\end{array}$ & $\begin{array}{l}431 \\
421 \\
200^{(a)} \\
298 \\
423\end{array}$ & $\begin{array}{l}728 \\
763 \\
929 \\
649 \\
567\end{array}$ & $\begin{array}{r}895 \\
1,164 \\
934 \\
851 \\
957\end{array}$ & $\begin{array}{c}994 \\
1,235 \\
694^{(2)} \\
1,118 \\
1,090\end{array}$ & $\begin{array}{l}786 \\
823 \\
664 \\
720 \\
832\end{array}$ & $\begin{array}{l}773 \\
645 \\
585 \\
722 \\
794^{(2)}\end{array}$ & $\begin{array}{l}325 \\
311 \\
419 \\
408 \\
522^{(2)}\end{array}$ & $\begin{array}{l}146 \\
118 \\
228 \\
124 \\
253\end{array}$ & $\begin{array}{l}45 \\
45 \\
90^{(2)} \\
77 \\
23\end{array}$ & $\begin{array}{l}5,187 \\
5,595 \\
4,779 \\
5,029 \\
5,554\end{array}$ \\
\hline $\begin{array}{l}1955-56 \\
1956-57 \\
1957-58 \\
1958-59 \\
1959-60\end{array}$ & $\begin{array}{c}22^{(3)} \\
0 \\
0 \\
0 \\
4\end{array}$ & $\begin{array}{l}0 \\
6 \\
0 \\
0 \\
6\end{array}$ & $\begin{array}{r}108 \\
32 \\
37 \\
74 \\
118\end{array}$ & $\begin{array}{l}364 \\
399 \\
443 \\
339 \\
359\end{array}$ & $\begin{array}{r}1,008 \\
850 \\
739 \\
731 \\
855\end{array}$ & $\begin{array}{c}1,105 \\
940 \\
822^{(2)} \\
927 \\
987\end{array}$ & $\begin{array}{r}1,029 \\
1,499 \\
862 \\
1,025 \\
1,292\end{array}$ & $\begin{array}{l}1,147^{(2)} \\
862 \\
576 \\
827 \\
799\end{array}$ & $\begin{array}{l}655 \\
650 \\
666 \\
617 \\
616\end{array}$ & $\begin{array}{l}273 \\
308 \\
411 \\
325 \\
374\end{array}$ & $\begin{array}{r}110 \\
50 \\
72 \\
248 \\
227\end{array}$ & $\begin{array}{r}55 \\
11 \\
3 \\
29 \\
21\end{array}$ & $\begin{array}{l}5,876 \\
5,607 \\
4,631 \\
5,142 \\
5,658\end{array}$ \\
\hline $\begin{array}{l}1960-61 \\
1961-62 \\
1962-63 \\
1963-64 \\
1964-65\end{array}$ & $\begin{array}{r}0 \\
0 \\
12 \\
1 \\
0\end{array}$ & $\begin{array}{c}32^{(2)} \\
0 \\
1 \\
1 \\
21\end{array}$ & $\begin{array}{l}35 \\
91 \\
60 \\
25 \\
94\end{array}$ & $\begin{array}{l}330 \\
418 \\
385 \\
285 \\
360\end{array}$ & $\begin{array}{l}717 \\
893 \\
657 \\
668 \\
804\end{array}$ & $\begin{array}{r}1,114 \\
974 \\
874 \\
1,078 \\
1,224\end{array}$ & $\begin{array}{r}930 \\
1,090 \\
1,228 \\
913 \\
1,009\end{array}$ & $\begin{array}{l}598 \\
797 \\
747 \\
784 \\
686\end{array}$ & $\begin{array}{l}587 \\
698 \\
577 \\
656 \\
685\end{array}$ & $\begin{array}{l}380 \\
287 \\
456 \\
445 \\
307\end{array}$ & $\begin{array}{l}179 \\
255 \\
170 \\
195 \\
171\end{array}$ & $\begin{array}{l}16 \\
51 \\
25 \\
33 \\
16\end{array}$ & $\begin{array}{l}4,918 \\
5,554 \\
5,192 \\
5,084 \\
5,377\end{array}$ \\
\hline $\begin{array}{l}1965-66 \\
1966-67 \\
1967-68 \\
1968-69 \\
1969-70\end{array}$ & $\begin{array}{r}5 \\
17 \\
0 \\
0 \\
0\end{array}$ & $\begin{array}{r}14 \\
2 \\
0 \\
13 \\
5\end{array}$ & $\begin{array}{r}115 \\
26 \\
13 \\
50 \\
39\end{array}$ & $\begin{array}{l}247 \\
362 \\
305 \\
458 \\
431\end{array}$ & $\begin{array}{l}660 \\
638 \\
704 \\
702 \\
745\end{array}$ & $\begin{array}{c}995 \\
829 \\
993 \\
1,064 \\
941\end{array}$ & $\begin{array}{r}963 \\
782 \\
907 \\
1,399 \\
1,064\end{array}$ & $\begin{array}{l}702 \\
598 \\
670 \\
932 \\
683\end{array}$ & $\begin{array}{l}605 \\
639 \\
495 \\
591 \\
625\end{array}$ & $\begin{array}{l}311 \\
519 \\
416 \\
384 \\
480\end{array}$ & $\begin{array}{r}133 \\
175 \\
117 \\
88 \\
137\end{array}$ & $\begin{array}{r}58 \\
12 \\
23 \\
6 \\
23\end{array}$ & $\begin{array}{l}4,808 \\
4,599 \\
4,643 \\
5,687 \\
5,173\end{array}$ \\
\hline $\begin{array}{l}1970-71 \\
1971-72 \\
1972-73 \\
1973-74 \\
1974-75\end{array}$ & $\begin{array}{r}0 \\
13 \\
1 \\
2 \\
8\end{array}$ & $\begin{array}{l}0 \\
3 \\
3 \\
9 \\
0\end{array}$ & $\begin{array}{r}122 \\
133 \\
179 \\
73 \\
32\end{array}$ & $\begin{array}{l}439 \\
420 \\
397 \\
389 \\
388\end{array}$ & $\begin{array}{l}758 \\
728 \\
754 \\
798 \\
698\end{array}$ & $\begin{array}{c}1,063 \\
1,064 \\
1,168 \\
837 \\
892\end{array}$ & $\begin{array}{r}906 \\
1,065 \\
1,112 \\
1,104 \\
996\end{array}$ & $\begin{array}{l}726 \\
878 \\
742 \\
675 \\
880\end{array}$ & $\begin{array}{l}752 \\
560 \\
544 \\
611 \\
704\end{array}$ & $\begin{array}{l}392 \\
463 \\
338 \\
361 \\
504\end{array}$ & $\begin{array}{l}124 \\
112 \\
144 \\
236 \\
174\end{array}$ & $\begin{array}{l}50 \\
23 \\
38 \\
27 \\
31\end{array}$ & $\begin{array}{l}5,332 \\
5,462 \\
5,420 \\
5,122 \\
5,307\end{array}$ \\
\hline $\begin{array}{l}1975-76 \\
1976-77 \\
1977-78 \\
1978-79 \\
1979-80\end{array}$ & $\begin{array}{r}0 \\
5 \\
5 \\
1 \\
13\end{array}$ & $\begin{array}{r}13 \\
15 \\
7 \\
10 \\
0\end{array}$ & $\begin{array}{r}25 \\
23 \\
153 \\
76 \\
10\end{array}$ & $\begin{array}{l}388 \\
392 \\
401 \\
390 \\
266\end{array}$ & $\begin{array}{l}764 \\
736 \\
783 \\
981 \\
924\end{array}$ & $\begin{array}{r}949 \\
1,065 \\
967 \\
1,162 \\
887\end{array}$ & $\begin{array}{l}1,024 \\
1,232 \\
1,001 \\
1,582 \\
1,277\end{array}$ & $\begin{array}{l}796 \\
684 \\
761 \\
861 \\
888\end{array}$ & $\begin{array}{l}735 \\
608 \\
550 \\
571 \\
638\end{array}$ & $\begin{array}{l}422 \\
253 \\
393 \\
369 \\
302\end{array}$ & $\begin{array}{r}159 \\
258 \\
203 \\
94 \\
138\end{array}$ & $\begin{array}{l}74 \\
22 \\
22 \\
21 \\
68\end{array}$ & $\begin{array}{l}5,349 \\
5,293 \\
5,246 \\
6,118^{(2)} \\
5,411\end{array}$ \\
\hline $\begin{array}{l}1980-81 \\
1981-82 \\
1982-83 \\
1983-84 \\
1984-85\end{array}$ & $\begin{array}{r}5 \\
8 \\
10 \\
8 \\
0\end{array}$ & $\begin{array}{r}18 \\
0 \\
0 \\
0 \\
3\end{array}$ & $\begin{array}{r}53 \\
108 \\
75 \\
125 \\
145\end{array}$ & $\begin{array}{l}394 \\
402 \\
420 \\
387 \\
532^{(2)}\end{array}$ & $\begin{array}{l}723 \\
668 \\
844 \\
643 \\
768\end{array}$ & $\begin{array}{r}883 \\
998 \\
1,023 \\
1,357 \\
1,288\end{array}$ & $\begin{array}{r}838 \\
1,092 \\
855 \\
1,035 \\
1,245\end{array}$ & $\begin{array}{l}707 \\
754 \\
676 \\
763 \\
982\end{array}$ & $\begin{array}{l}503 \\
590 \\
511 \\
552 \\
651\end{array}$ & $\begin{array}{l}345 \\
469 \\
419 \\
432 \\
288\end{array}$ & $\begin{array}{l}165 \\
164 \\
151 \\
292^{(2)} \\
137\end{array}$ & $\begin{array}{l}51 \\
17 \\
50 \\
70 \\
21\end{array}$ & $\begin{array}{l}4,685 \\
5,270 \\
5,034 \\
5,664 \\
6,060\end{array}$ \\
\hline $\begin{array}{l}1985-86 \\
1986-87 \\
1987-88 \\
1988-89 \\
1989-90\end{array}$ & $\begin{array}{r}0 \\
18 \\
1 \\
3 \\
1\end{array}$ & $\begin{array}{r}10 \\
0 \\
0 \\
0 \\
0\end{array}$ & $\begin{array}{c}197^{(x)} \\
153 \\
32 \\
100 \\
12\end{array}$ & $\begin{array}{l}475 \\
319 \\
304 \\
208 \\
339\end{array}$ & $\begin{array}{c}1,206^{(2)} \\
680 \\
640 \\
.625 \\
621\end{array}$ & $\begin{array}{l}1,362^{(2)} \\
1,009 \\
1,040 \\
1,033 \\
985\end{array}$ & $\begin{array}{r}959 \\
1,066 \\
1,028 \\
859 \\
763\end{array}$ & $\begin{array}{c}724 \\
696 \\
695 \\
1,054 \\
767\end{array}$ & $\begin{array}{l}509 \\
522 \\
591 \\
658 \\
530\end{array}$ & $\begin{array}{l}426 \\
239 \\
301 \\
254 \\
217\end{array}$ & $\begin{array}{r}213 \\
85 \\
166 \\
141 \\
149\end{array}$ & $\begin{array}{r}10 \\
16 \\
65 \\
6 \\
28\end{array}$ & $\begin{array}{l}6,091 \\
4,803 \\
4,863 \\
4,941 \\
4,412\end{array}$ \\
\hline $\begin{array}{l}1990-91 \\
1991-92 \\
1992-93 \\
1993-94 \\
1994-95\end{array}$ & $\begin{array}{l}4 \\
0 \\
1 \\
1 \\
0\end{array}$ & $\begin{array}{c}0^{(2)} \\
0 \\
18 \\
13 \\
0\end{array}$ & $\begin{array}{l}0^{(2)} \\
12 \\
94 \\
89 \\
3\end{array}$ & $\begin{array}{l}401 \\
381 \\
298 \\
303 \\
332\end{array}$ & $\begin{array}{l}553^{(2)} \\
710 \\
716 \\
911 \\
759\end{array}$ & $\begin{array}{c}1,269 \\
842 \\
1,084 \\
914 \\
924\end{array}$ & $\begin{array}{r}1,124 \\
851 \\
1,247 \\
819 \\
954\end{array}$ & $\begin{array}{l}575^{(2)} \\
648 \\
958 \\
813 \\
614\end{array}$ & $\begin{array}{l}649 \\
418^{(2)} \\
674 \\
490 \\
581\end{array}$ & $\begin{array}{l}330 \\
278 \\
374 \\
217^{(2)} \\
372\end{array}$ & $\begin{array}{r}148 \\
77 \\
94 \\
97 \\
89\end{array}$ & $\begin{array}{l}55 \\
13 \\
23 \\
22 \\
46\end{array}$ & $\begin{array}{l}5,108 \\
4,230^{(2)} \\
5,581 \\
4,689 \\
4,674\end{array}$ \\
\hline $\begin{array}{l}1995-96 \\
1996-97 \\
1997-98\end{array}$ & $\begin{array}{l}0 \\
3 \\
2\end{array}$ & $\begin{array}{l}1 \\
0 \\
0\end{array}$ & $\begin{array}{l}24 \\
99 \\
38\end{array}$ & $\begin{array}{l}398 \\
401 \\
367\end{array}$ & $\begin{array}{l}623 \\
797 \\
658\end{array}$ & $\begin{array}{c}1,003 \\
1,090 \\
941\end{array}$ & $\begin{array}{r}1,124 \\
973 \\
892\end{array}$ & $\begin{array}{l}935 \\
692 \\
642\end{array}$ & $\begin{array}{l}623 \\
544 \\
521\end{array}$ & $\begin{array}{l}302 \\
395 \\
332\end{array}$ & $\begin{array}{r}225 \\
95 \\
131\end{array}$ & $\begin{array}{c}14 \\
2 \\
1^{(2)}\end{array}$ & $\begin{array}{l}5,272 \\
5,091 \\
4,523\end{array}$ \\
\hline $\begin{array}{l}1998-99 \\
1999-00\end{array}$ & $\begin{array}{l}0^{(2, b)} \\
8\end{array}$ & $\begin{array}{l}0^{(20)} \\
17\end{array}$ & $\begin{array}{l}15 \\
65\end{array}$ & $\begin{array}{l}393 \\
407\end{array}$ & $\begin{array}{l}582 \\
576\end{array}$ & $\begin{array}{r}1,000 \\
846\end{array}$ & $\begin{array}{r}829 \\
-\end{array}$ & $\begin{array}{r}651 \\
-\end{array}$ & 581 & $\begin{array}{r}424 \\
-\end{array}$ & $\begin{array}{r}265 \\
-\end{array}$ & $\underset{-}{-}$ & $\begin{array}{c}4,802 \\
-\end{array}$ \\
\hline $\begin{array}{l}\text { Average } \\
\text { Normal }\end{array}$ & $\begin{array}{r}4 \\
15\end{array}$ & $\begin{array}{l}5 \\
5\end{array}$ & $\begin{array}{l}71 \\
78\end{array}$ & $\begin{array}{l}374 \\
377\end{array}$ & $\begin{array}{l}743 \\
746\end{array}$ & $\begin{array}{l}1,012 \\
1,042\end{array}$ & $\begin{array}{l}1,059 \\
1,044\end{array}$ & $\begin{array}{l}772 \\
764\end{array}$ & $\begin{array}{l}612 \\
602\end{array}$ & $\begin{array}{l}366 \\
372\end{array}$ & $\begin{array}{l}154 \\
164\end{array}$ & $\begin{array}{l}33 \\
32\end{array}$ & $\begin{array}{l}5,206 \\
5,231\end{array}$ \\
\hline
\end{tabular}

(a) Greatest and least values.

(b) Most recent of numetous occurrences. 
Table 3.15. Monthly and Annual Cooling-Degree Days

\begin{tabular}{|c|c|c|c|c|c|c|c|c|c|c|c|c|c|}
\hline Year & Jan & Feb & Mar & Apr & May & Jun & Jul & Aug & Sep & Oct & Nov & Dec & Annual \\
\hline 1945 & 0 & 0 & 0 & 0 & 39 & 113 & 325 & 388 & 86 & 11 & 0 & 0 & 962 \\
\hline $\begin{array}{l}1946 \\
1947 \\
1948 \\
1949 \\
1950\end{array}$ & $\begin{array}{l}0 \\
0 \\
0 \\
0 \\
0\end{array}$ & $\begin{array}{l}0 \\
0 \\
0 \\
0 \\
0\end{array}$ & $\begin{array}{l}0 \\
0 \\
0 \\
0 \\
0\end{array}$ & $\begin{array}{l}9 \\
3 \\
0 \\
4 \\
0\end{array}$ & $\begin{array}{r}53 \\
153 \\
31 \\
147 \\
13\end{array}$ & $\begin{array}{l}100 \\
108 \\
230 \\
168 \\
112\end{array}$ & $\begin{array}{l}345 \\
321 \\
243 \\
311 \\
321\end{array}$ & $\begin{array}{l}360 \\
217 \\
213 \\
307 \\
353\end{array}$ & $\begin{array}{r}57 \\
86 \\
90 \\
146 \\
139\end{array}$ & $\begin{array}{r}0 \\
11 \\
0 \\
0 \\
0\end{array}$ & $\begin{array}{l}0 \\
0 \\
0 \\
0 \\
0\end{array}$ & $\begin{array}{l}0 \\
0 \\
0 \\
0 \\
0\end{array}$ & $\begin{array}{r}924 \\
899 \\
807 \\
1,083 \\
938\end{array}$ \\
\hline $\begin{array}{l}1951 \\
1952 \\
1953 \\
1954 \\
1955\end{array}$ & $\begin{array}{l}0 \\
0 \\
0 \\
0 \\
0\end{array}$ & $\begin{array}{l}0 \\
0 \\
0 \\
0 \\
0\end{array}$ & $\begin{array}{l}0 \\
0 \\
0 \\
0 \\
0\end{array}$ & $\begin{array}{r}1 \\
16 \\
0 \\
0 \\
0\end{array}$ & $\begin{array}{r}26 \\
46 \\
8 \\
55 \\
4\end{array}$ & $\begin{array}{c}173 \\
110 \\
26^{(2)} \\
90 \\
174\end{array}$ & $\begin{array}{l}370 \\
371 \\
336 \\
289 \\
270\end{array}$ & $\begin{array}{l}303 \\
281 \\
282 \\
204 \\
325\end{array}$ & $\begin{array}{c}123 \\
151 \\
143 \\
91 \\
13\end{array}$ & $\begin{array}{r}0 \\
16 \\
1 \\
0 \\
0\end{array}$ & $\begin{array}{l}0 \\
0 \\
0 \\
0 \\
0\end{array}$ & $\begin{array}{l}0 \\
0 \\
0 \\
0 \\
0\end{array}$ & $\begin{array}{l}996 \\
991 \\
796 \\
729 \\
786\end{array}$ \\
\hline $\begin{array}{l}1956 \\
1957 \\
1958 \\
1959 \\
1960\end{array}$ & $\begin{array}{l}0 \\
0 \\
0 \\
0 \\
0\end{array}$ & $\begin{array}{l}0 \\
0 \\
0 \\
0 \\
0\end{array}$ & $\begin{array}{l}0 \\
0 \\
0 \\
0 \\
0\end{array}$ & $\begin{array}{r}10 \\
12 \\
0 \\
0 \\
2\end{array}$ & $\begin{array}{c}122 \\
77 \\
167^{(2)} \\
15 \\
26\end{array}$ & $\begin{array}{r}78 \\
185 \\
282 \\
137 \\
174\end{array}$ & $\begin{array}{l}430 \\
289 \\
500 \\
397 \\
518\end{array}$ & $\begin{array}{l}322 \\
255 \\
447 \\
218 \\
233\end{array}$ & $\begin{array}{r}106 \\
160 \\
93 \\
45 \\
118\end{array}$ & $\begin{array}{r}0 \\
0 \\
14 \\
0 \\
3\end{array}$ & $\begin{array}{l}0 \\
0 \\
0 \\
0 \\
0\end{array}$ & $\begin{array}{l}0 \\
0 \\
0 \\
0 \\
0\end{array}$ & $\begin{array}{c}1,068 \\
978 \\
1,503^{(2)} \\
812 \\
1,074\end{array}$ \\
\hline $\begin{array}{l}1961 \\
1962 \\
1963 \\
1964 \\
1965\end{array}$ & $\begin{array}{l}0 \\
0 \\
0 \\
0 \\
0\end{array}$ & $\begin{array}{l}0 \\
0 \\
0 \\
0 \\
0\end{array}$ & $\begin{array}{l}0 \\
0 \\
0 \\
0 \\
0\end{array}$ & $\begin{array}{l}0 \\
5 \\
0 \\
0 \\
0\end{array}$ & $\begin{array}{l}23 \\
\mathbf{3}^{(2)} \\
67 \\
30 \\
31\end{array}$ & $\begin{array}{l}288 \\
148 \\
156 \\
115 \\
145\end{array}$ & $\begin{array}{l}447 \\
352 \\
232 \\
299 \\
362\end{array}$ & $\begin{array}{l}469 \\
215 \\
333 \\
171^{(2)} \\
314\end{array}$ & $\begin{array}{r}55 \\
125 \\
205 \\
34 \\
33\end{array}$ & $\begin{array}{l}0 \\
0 \\
5 \\
0 \\
2\end{array}$ & $\begin{array}{l}0 \\
0 \\
0 \\
0 \\
0\end{array}$ & $\begin{array}{l}0 \\
0 \\
0 \\
0 \\
0\end{array}$ & $\begin{array}{c}1,282 \\
848 \\
998 \\
649^{(3)} \\
887\end{array}$ \\
\hline $\begin{array}{l}1966 \\
1967 \\
1968 \\
1969 \\
1970\end{array}$ & $\begin{array}{l}0 \\
0 \\
0 \\
0 \\
0\end{array}$ & $\begin{array}{l}0 \\
0 \\
0 \\
0 \\
0\end{array}$ & $\begin{array}{l}0 \\
0 \\
0 \\
0 \\
0\end{array}$ & $\begin{array}{l}0 \\
0 \\
5 \\
0 \\
0\end{array}$ & $\begin{array}{l}80 \\
34 \\
35 \\
73 \\
29\end{array}$ & $\begin{array}{l}116 \\
237 \\
168 \\
310 \\
281\end{array}$ & $\begin{array}{l}274 \\
419 \\
451 \\
338 \\
421\end{array}$ & $\begin{array}{l}332 \\
508^{(2)} \\
213 \\
245 \\
351\end{array}$ & $\begin{array}{r}141 \\
216 \\
104 \\
110 \\
27\end{array}$ & $\begin{array}{l}1 \\
0 \\
0 \\
0 \\
1\end{array}$ & $\begin{array}{l}0 \\
0 \\
0 \\
0 \\
0\end{array}$ & $\begin{array}{l}0 \\
0 \\
0 \\
0 \\
0\end{array}$ & $\begin{array}{r}944 \\
1,414 \\
976 \\
1,076 \\
1,110\end{array}$ \\
\hline $\begin{array}{l}1971 \\
1972 \\
1973 \\
1974 \\
1975\end{array}$ & $\begin{array}{l}0 \\
0 \\
0 \\
0 \\
0\end{array}$ & $\begin{array}{l}0 \\
0 \\
0 \\
0 \\
0\end{array}$ & $\begin{array}{l}0 \\
0 \\
0 \\
0 \\
0\end{array}$ & $\begin{array}{l}0 \\
0 \\
0 \\
0 \\
0\end{array}$ & $\begin{array}{l}94 \\
87 \\
87 \\
12 \\
28\end{array}$ & $\begin{array}{r}59 \\
164 \\
149 \\
264 \\
102\end{array}$ & $\begin{array}{l}437 \\
339 \\
413 \\
303 \\
451\end{array}$ & $\begin{array}{l}481 \\
392 \\
285 \\
326 \\
202\end{array}$ & $\begin{array}{r}28 \\
67 \\
94 \\
125 \\
117\end{array}$ & $\begin{array}{r}10 \\
1 \\
0 \\
0 \\
0\end{array}$ & $\begin{array}{l}0 \\
0 \\
0 \\
0 \\
0\end{array}$ & $\begin{array}{l}0 \\
0 \\
0 \\
0 \\
0\end{array}$ & $\begin{array}{r}1,109 \\
1,050 \\
1,028 \\
1,030 \\
900\end{array}$ \\
\hline $\begin{array}{l}1976 \\
1977 \\
1978 \\
1979 \\
1980\end{array}$ & $\begin{array}{l}0 \\
0 \\
0 \\
0 \\
0\end{array}$ & $\begin{array}{l}0 \\
0 \\
0 \\
0 \\
0\end{array}$ & $\begin{array}{l}0 \\
0 \\
0 \\
0 \\
0\end{array}$ & $\begin{array}{r}0 \\
24 \\
0 \\
1 \\
7\end{array}$ & $\begin{array}{r}22 \\
5 \\
5 \\
65 \\
26\end{array}$ & $\begin{array}{r}91 \\
253 \\
182 \\
197 \\
57\end{array}$ & $\begin{array}{l}319 \\
276 \\
332 \\
394 \\
305\end{array}$ & $\begin{array}{l}195 \\
447 \\
248 \\
299 \\
207\end{array}$ & $\begin{array}{r}141 \\
46 \\
41 \\
138 \\
80\end{array}$ & $\begin{array}{l}3 \\
0 \\
0 \\
5 \\
9\end{array}$ & $\begin{array}{l}0 \\
0 \\
0 \\
0 \\
0\end{array}$ & $\begin{array}{l}0 \\
0 \\
0 \\
0 \\
0\end{array}$ & $\begin{array}{r}771 \\
1,051 \\
808 \\
1,099 \\
691\end{array}$ \\
\hline $\begin{array}{l}1981 \\
1982 \\
1983 \\
1984 \\
1985\end{array}$ & $\begin{array}{l}0 \\
0 \\
0 \\
0 \\
0\end{array}$ & $\begin{array}{l}0 \\
0 \\
0 \\
0 \\
0\end{array}$ & $\begin{array}{l}0 \\
0 \\
0 \\
0 \\
0\end{array}$ & $\begin{array}{r}16 \\
0 \\
0 \\
0 \\
3\end{array}$ & $\begin{array}{r}25 \\
20 \\
115 \\
11 \\
83\end{array}$ & $\begin{array}{r}82 \\
261 \\
61 \\
88 \\
175\end{array}$ & $\begin{array}{l}\mathbf{2 8 7} \\
315 \\
203 \\
340 \\
\mathbf{5 3 2}^{(\mathbf{2})}\end{array}$ & $\begin{array}{l}438 \\
333 \\
291 \\
280 \\
183\end{array}$ & $\begin{array}{l}144 \\
88 \\
26 \\
60 \\
11^{(0)}\end{array}$ & $\begin{array}{l}0 \\
0 \\
1 \\
0 \\
0\end{array}$ & $\begin{array}{l}0 \\
0 \\
0 \\
0 \\
0\end{array}$ & $\begin{array}{l}0 \\
0 \\
0 \\
0 \\
0\end{array}$ & $\begin{array}{r}992 \\
1,017 \\
697 \\
779 \\
987\end{array}$ \\
\hline $\begin{array}{l}1986 \\
1987 \\
1988 \\
1989 \\
1990\end{array}$ & $\begin{array}{l}0 \\
0 \\
0 \\
0 \\
0\end{array}$ & $\begin{array}{l}1^{(2)} \\
0 \\
0 \\
0 \\
0\end{array}$ & $\begin{array}{l}0 \\
0 \\
0 \\
0 \\
0\end{array}$ & $\begin{array}{c}3 \\
26^{(2)} \\
6 \\
1 \\
3\end{array}$ & $\begin{array}{r}125 \\
125 \\
45 \\
34 \\
16\end{array}$ & $\begin{array}{l}245 \\
265 \\
187 \\
215 \\
182\end{array}$ & $\begin{array}{l}192 \\
289 \\
385 \\
323 \\
491\end{array}$ & $\begin{array}{l}442 \\
359 \\
318 \\
260 \\
367\end{array}$ & $\begin{array}{c}68 \\
179 \\
113 \\
89 \\
222^{(2)}\end{array}$ & $\begin{array}{c}1 \\
11 \\
44^{(2)} \\
0 \\
3\end{array}$ & $\begin{array}{l}0 \\
0 \\
0 \\
1^{(2)} \\
0\end{array}$ & $\begin{array}{l}0 \\
0 \\
0 \\
0 \\
0\end{array}$ & $\begin{array}{r}1,077 \\
1,254 \\
1,098 \\
923 \\
1,284\end{array}$ \\
\hline $\begin{array}{l}1991 \\
1992 \\
1993 \\
1994 \\
1995\end{array}$ & $\begin{array}{l}0 \\
0 \\
0 \\
0 \\
0\end{array}$ & $\begin{array}{l}0 \\
0 \\
0 \\
0 \\
0\end{array}$ & $\begin{array}{l}0 \\
0 \\
0 \\
0 \\
0\end{array}$ & $\begin{array}{r}3 \\
11 \\
0 \\
15 \\
0\end{array}$ & $\begin{array}{r}6 \\
147 \\
139 \\
94 \\
73\end{array}$ & $\begin{array}{l}72 \\
365^{(2)} \\
127 \\
163 \\
142\end{array}$ & $\begin{array}{l}400 \\
362 \\
\mathbf{1 7 1}^{(2)} \\
501 \\
376\end{array}$ & $\begin{array}{l}427 \\
392 \\
265 \\
358 \\
216\end{array}$ & $\begin{array}{c}155 \\
81 \\
135 \\
167 \\
174\end{array}$ & $\begin{array}{r}7 \\
10 \\
6 \\
3 \\
0\end{array}$ & $\begin{array}{l}0 \\
0 \\
0 \\
0 \\
0\end{array}$ & $\begin{array}{l}0 \\
0 \\
0 \\
0 \\
0\end{array}$ & $\begin{array}{r}1,070 \\
1,368 \\
843 \\
1,301 \\
981\end{array}$ \\
\hline $\begin{array}{l}1996 \\
1997 \\
1998 \\
1999\end{array}$ & $\begin{array}{l}0 \\
0 \\
0 \\
0\end{array}$ & $\begin{array}{l}0 \\
0 \\
0^{(2,0)} \\
0^{(2,0)}\end{array}$ & $\begin{array}{l}0 \\
0 \\
0 \\
0\end{array}$ & $\begin{array}{c}4 \\
0 \\
16 \\
0^{\text {(2at }}\end{array}$ & $\begin{array}{l}14 \\
96 \\
55 \\
43\end{array}$ & $\begin{array}{l}134 \\
118 \\
183 \\
135\end{array}$ & $\begin{array}{l}450 \\
324 \\
527 \\
281 .\end{array}$ & $\begin{array}{l}324 \\
404 \\
398 \\
366\end{array}$ & $\begin{array}{r}79 \\
92 \\
195 \\
66\end{array}$ & $\begin{array}{l}9 \\
0 \\
3 \\
0^{(20)}\end{array}$ & $\begin{array}{l}0 \\
0 \\
0 \\
0^{(20)}\end{array}$ & $\begin{array}{l}0 \\
0 \\
0 \\
0\end{array}$ & $\begin{array}{r}1,014 \\
1,034 \\
1,377 \\
891\end{array}$ \\
\hline $\begin{array}{l}\text { Average } \\
\text { Normal }\end{array}$ & $\begin{array}{l}0 \\
0\end{array}$ & $\begin{array}{l}<1 \\
<1\end{array}$ & $\begin{array}{l}0 \\
0\end{array}$ & $\begin{array}{l}4 \\
3\end{array}$ & $\begin{array}{l}56 \\
48\end{array}$ & $\begin{array}{l}163 \\
175\end{array}$ & $\begin{array}{l}355 \\
351\end{array}$ & $\begin{array}{l}316 \\
317\end{array}$ & $\begin{array}{r}104 \\
98\end{array}$ & $\begin{array}{l}4 \\
3\end{array}$ & $\begin{array}{l}<1 \\
<1\end{array}$ & $\begin{array}{l}0 \\
0\end{array}$ & $\begin{array}{r}1,001 \\
994\end{array}$ \\
\hline
\end{tabular}

(a) Greatest and least values.

(b) Most recent of numerous occurrences. 


\subsection{Precipitation Climatology}

\subsection{Monthly and Annual Totals}

Table 4.1 shows monthly and annual precipitation totals for the period of record, 1946 through 1999. Normal monthly precipitation amounts for the period 1961 through 1990 and averages for the entire period of record are noted on the table, as are monthly and annual extremes. Normal annual precipitation at the Hanford Meteorology Station is 6.26 inches. The wettest year on record was 1995, with 12.31 inches; the driest was 1976, with 2.99 inches.

The months of November through February provide 3.35 inches (54\%) of the normal annual precipitation. December is the wettest month, receiving 1.03 inches; July is the driest, receiving only 0.18 inch. The wettest month on record was December 1996, with 3.69 inches. September 1999, September 1991, August 1988, and August 1955 received no precipitation.

\subsection{Total Monthly Precipitation Distributions}

A new method of presenting climatological data on daily temperature maxima and minima was described in Section 3.5. This method of presentation of climatological data is appropriate for monthly precipitation totals as well. Figure 4.1 shows climatological statistics for total precipitation for January at the Hanford Meteorology Station. Note that the median value is nearer the bottom of the expected range of values than it is to the top of the range. This is characteristic of precipitation distributions, and it the reason that it is not practical to show climatological statistics of daily precipitation totals using the new method. If the distribution of daily precipitation totals were shown, the median value, the bottom of the expected range, and record low value would all be zero because on most days there is no precipitation at all. The following information can be drawn from the figure:

- The median precipitation for January is about $0.8 \mathrm{inch}$ (the average precipitation is 0.93 inch).

- The record high precipitation for month is about 2.5 inches.

- The record low precipitation the month is about 0.1 inch.

- About 70 percent of the time ( 14 out of 20 years), the monthly total precipitation will be between 0.33 inch and 1.7 inches.

- About 15 percent of the time ( 3 out of 20 years), the monthly total precipitation will be more than 1.7 inches.

- About 35 percent of the time ( 7 out of 20 years), the monthly total precipitation will be between 0.8 inch and 1.7 inches. 


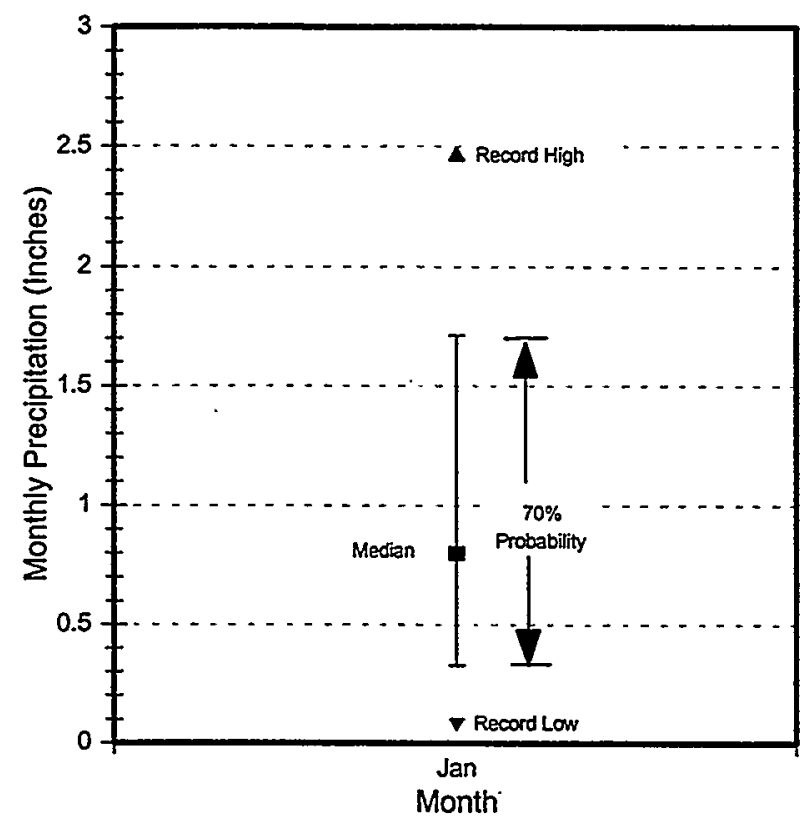

Figure 4.1. Graphical Representation of Total Monthly Precipitation

- About 35 percent of the time ( 7 out of 20 years), the monthly total precipitation will be between 0.33 inch and 0.8 inch.

- About 15 percent of the time ( 3 out of 20 years), the monthly total precipitation will be less than 0.33 inch.

Figure 4.2 shows the climatological statistics of total monthly precipitation. The statistics are based on the data in Table 4.1. The only months for which the median monthly total precipitation exceeds 0.5 inch are November, December, January, and February. Yet, 0.5 inch of rain is within the range of typical values for all months except July.

The climatological statistics of annual accumulation of precipitation are shown in Figure 4.3. The accumulation begins in July because July has the lowest expected monthly-total precipitation. In addition, the precipitation in December and the following January contributes to the water supply for the same growing season. July is therefore a natural starting point. This choice of starting points for precipitation accumulation does not affect the median accumulation (6.41 inches). However, it does increase the ranges of variability in accumulation during the 12 month period over the ranges determined starting accumulation in January. The range of variability is increased because there is a small but significant positive correlation between precipitation in December and precipitation in the following January. For example, if the precipitation in December is below average, the precipitation is more likely to be below average. Similarly, if the precipitation in December is above average, the precipitation in the following January is also more likely to be above average. This correlation is not found between the precipitation in January and the precipitation in the following December. 


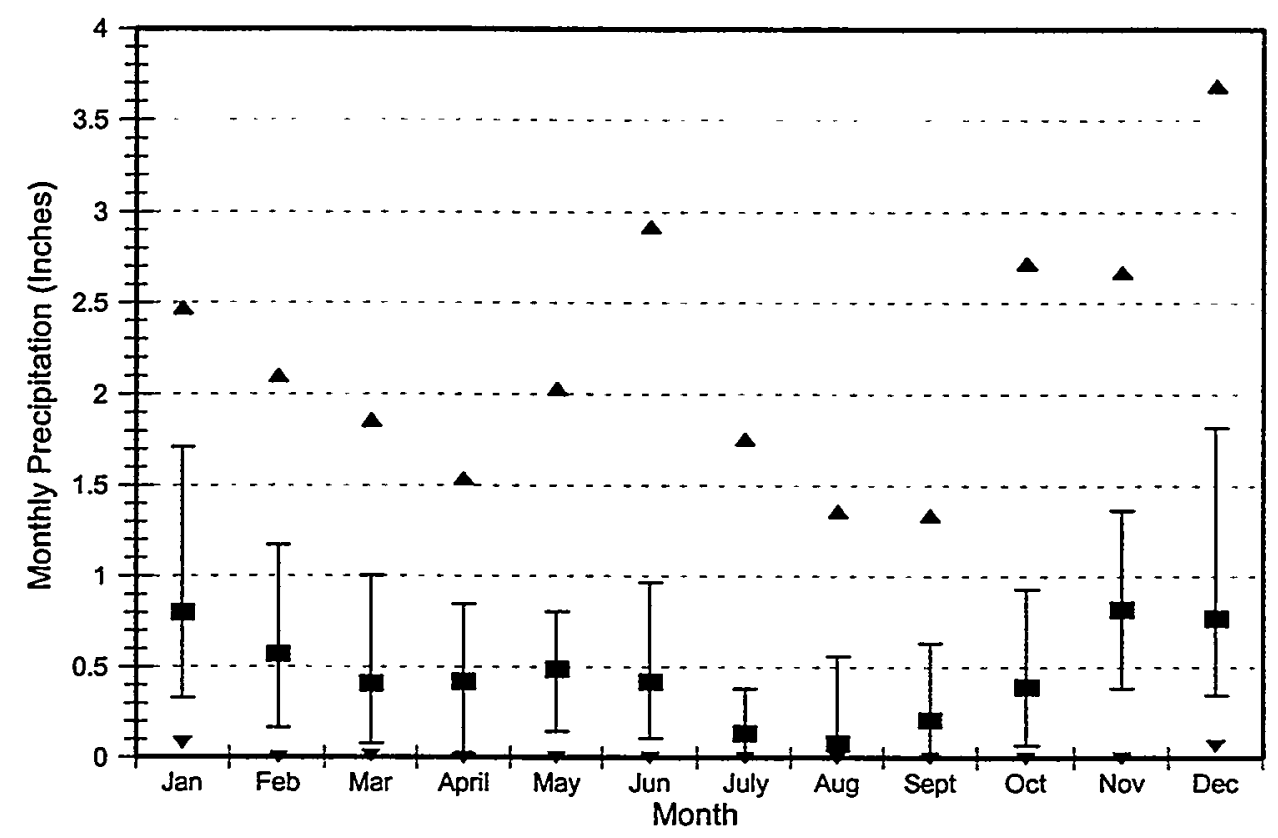

Figure 4.2. Climatological Statistics of Total Monthly Precipitation

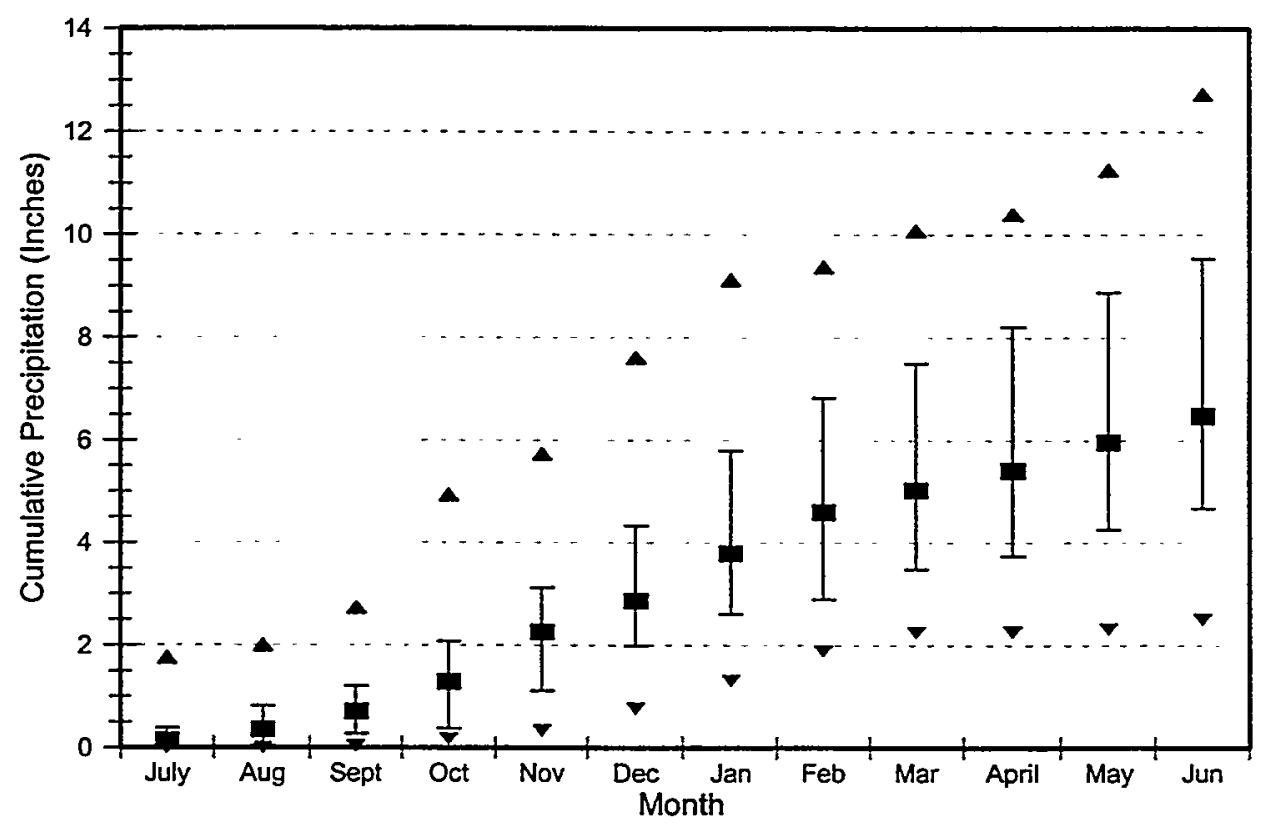

Figure 4.3. Climatological Statistics of Precipitation Accumulation 


\subsection{Seasonal Precipitation}

Table 4.2 provides seasonal precipitation information, with normal and average seasonal data noted. The extremes for each season are also noted. The wettest season was the winter of 1996-1997, with 5.45 inches; the driest received only 0.03 inch (summer 1973).

\subsection{Average Number of Days with Specified Amounts of Precipitation}

Table 4.3 presents information on the average number of days per year with precipitation events in six categories. A trace is less than 0.01 inch of precipitation. An average of 123 days per year have a trace or more of precipitation; however, only 23 days receive totals of 0.10 inch or more. During the 54-year period of record, only 4 days had an inch or more of precipitation.

\subsection{Total Time with Precipitation Observed}

The total time during which precipitation was observed at the Hanford Meteorology Station includes all types of precipitation. Observations of precipitation are recorded in hours and minutes, with the weather observer recording the starting and ending time of each precipitation event. These data are presented in Table 4.4. No record was kept for the hours 1600 through 2400 from July 1971 through June 1974; therefore, a 3-year gap exists in the record for those hours. Also, beginning in late April 1995, operations at the Hanford Meteorology Station were decreased to 8 hours $(0600$ to 1400$)$ on weekends and holidays. However, a combination of precipitation sensors and computer programs was initiated to help ascertain the beginning and ending times of precipitation events during periods when the Hanford Meteorology Station is not staffed. Table 4.5 lists total hours of precipitation by month for the period 1946 through 1999. As previously noted, complete precipitation duration data for the period July 1971 through June 1974 are not available, and incomplete data are not included.

The months of November through February, which contribute more than half of the annual precipitation, received precipitation $10.2 \%$ of the time, three times more than the other 8 months of the year (3.3\%). 
Table 4.1. Monthly and Annual Precipitation (inches)

\begin{tabular}{|c|c|c|c|c|c|c|c|c|c|c|c|c|c|}
\hline Year & Jan & Feb & Mar & Apr & May & Jun & Jul & Aug & Sep & Oct & Nov & Dec & Annual \\
\hline $\begin{array}{l}1946 \\
1947 \\
1948 \\
1949 \\
1950\end{array}$ & $\begin{array}{l}-\overline{32} \\
1.36 \\
0.13 \\
1.80\end{array}$ & $\begin{array}{l}-\overline{27} \\
0.69 \\
0.68 \\
1.06\end{array}$ & $\begin{array}{l}-\overline{42} \\
0.07 \\
1.12 \\
0.87\end{array}$ & $\begin{array}{l}-\overline{70} \\
0.95 \\
0.02 \\
0.47\end{array}$ & $\begin{array}{l}-\overline{0} \\
0.71 \\
1.71 \\
0.16 \\
0.27\end{array}$ & $\begin{array}{l}1 . \overline{07} \\
1.47 \\
0.01 \\
2.92^{(2)}\end{array}$ & $\begin{array}{l}0.15 \\
0.71 \\
0.40 \\
0.01 \\
0.07\end{array}$ & $\begin{array}{l}0.35 \\
0.68 \\
0.39 \\
0.03 \\
T\end{array}$ & $\begin{array}{l}0.52 \\
1.34^{(2)} \\
0.16 \\
0.23 \\
0.01\end{array}$ & $\begin{array}{l}0.65 \\
2.20 \\
0.45 \\
0.10 \\
2.46\end{array}$ & $\begin{array}{l}0.66 \\
0.81 \\
0.95 \\
1.47 \\
0.55\end{array}$ & $\begin{array}{l}0.11 \\
0.75 \\
1.11 \\
0.16 \\
0.97\end{array}$ & $\begin{array}{r}9 . \overline{29} \\
9.71 \\
4.12 \\
11.45\end{array}$ \\
\hline $\begin{array}{l}1951 \\
1952 \\
1953 \\
1954 \\
1955\end{array}$ & $\begin{array}{l}0.84 \\
0.65 \\
2.16 \\
1.48 \\
0.56\end{array}$ & $\begin{array}{l}0.51 \\
0.50 \\
0.25 \\
0.28 \\
0.22\end{array}$ & $\begin{array}{l}0.46 \\
0.06 \\
0.17 \\
0.59 \\
0.17\end{array}$ & $\begin{array}{l}0.53 \\
0.13 \\
0.77 \\
0.07 \\
0.40\end{array}$ & $\begin{array}{l}0.43 \\
0.58 \\
0.28 \\
0.41 \\
0.59\end{array}$ & $\begin{array}{l}1.38 \\
1.07 \\
0.55 \\
0.10 \\
0.28\end{array}$ & $\begin{array}{l}0.37 \\
T \\
T \\
0.22 \\
0.57\end{array}$ & $\begin{array}{l}0.15 \\
0.08 \\
0.96 \\
0.42 \\
0^{(26)}\end{array}$ & $\begin{array}{l}0.10 \\
0.08 \\
0.13 \\
0.51 \\
0.77\end{array}$ & $\begin{array}{l}0.71 \\
0.04 \\
0.20 \\
0.42 \\
0.40\end{array}$ & $\begin{array}{l}0.82 \\
0.20 \\
0.96 \\
0.86 \\
1.54\end{array}$ & $\begin{array}{l}0.70 \\
0.77 \\
0.49 \\
0.35 \\
2.03\end{array}$ & $\begin{array}{l}7.00 \\
4.16 \\
6.92 \\
5.71 \\
7.53\end{array}$ \\
\hline $\begin{array}{l}1956 \\
1957 \\
1958 \\
1959 \\
1960\end{array}$ & $\begin{array}{l}1.71 \\
0.48 \\
1.74 \\
2.05 \\
0.51\end{array}$ & $\begin{array}{l}0.56 \\
0.23 \\
1.48 \\
1.17 \\
0.58\end{array}$ & $\begin{array}{l}0.10 \\
1.86^{(2)} \\
0.46 \\
0.40 \\
0.67\end{array}$ & $\begin{array}{l}\mathrm{T} \\
0.38 \\
0.64 \\
0.20 \\
0.53\end{array}$ & $\begin{array}{l}0.22 \\
0.82 \\
0.74 \\
0.50 \\
0.71\end{array}$ & $\begin{array}{l}0.86 \\
0.47 \\
0.81 \\
0.23 \\
0.14\end{array}$ & $\begin{array}{l}T \\
0.05 \\
0.02 \\
T \\
T\end{array}$ & $\begin{array}{l}0.38 \\
0.02 \\
T \\
0.03 \\
0.26\end{array}$ & $\begin{array}{l}0.01 \\
0.34 \\
0.05 \\
1.26 \\
0.23\end{array}$ & $\begin{array}{l}1.03 \\
2.72^{(2)} \\
0.19 \\
0.56 \\
0.23\end{array}$ & $\begin{array}{l}0.15 \\
0.39 \\
0.77 \\
0.41 \\
0.92\end{array}$ & $\begin{array}{l}0.58 \\
0.53 \\
1.84 \\
0.26 \\
0.64\end{array}$ & $\begin{array}{l}5.60 \\
8.29 \\
8.74 \\
7.07 \\
5.42\end{array}$ \\
\hline $\begin{array}{l}1961 \\
1962 \\
1963 \\
1964 \\
1965\end{array}$ & $\begin{array}{l}0.33 \\
0.13 \\
0.95 \\
0.37 \\
0.93\end{array}$ & $\begin{array}{l}2.10^{(2)} \\
0.90 \\
0.69 \\
0.01 \\
0.14\end{array}$ & $\begin{array}{l}1.02 \\
0.14 \\
0.53 \\
0.03 \\
0.03\end{array}$ & $\begin{array}{l}0.48 \\
0.34 \\
1.17 \\
0.11 \\
0.09\end{array}$ & $\begin{array}{l}0.80 \\
1.35 \\
0.43 \\
0.04 \\
0.15\end{array}$ & $\begin{array}{l}0.42 \\
0.12 \\
0.28 \\
0.90 \\
0.49\end{array}$ & $\begin{array}{l}0.15 \\
T \\
0.31 \\
0.04 \\
0.11\end{array}$ & $\begin{array}{l}0.09 \\
0.50 \\
0.01 \\
0.24 \\
0.03\end{array}$ & $\begin{array}{l}T \\
0.38 \\
0.02 \\
0.09 \\
0.11\end{array}$ & $\begin{array}{l}0.07 \\
0.95 \\
0.04 \\
0.28 \\
0.01\end{array}$ & $\begin{array}{l}0.49 \\
0.65 \\
0.74 \\
0.94 \\
1.17\end{array}$ & $\begin{array}{l}0.89 \\
0.60 \\
1.14 \\
2.34 \\
0.39\end{array}$ & $\begin{array}{l}6.84 \\
6.06 \\
6.31 \\
5.39 \\
3.65\end{array}$ \\
\hline $\begin{array}{l}1966 \\
1967 \\
1968 \\
1969 \\
1970\end{array}$ & $\begin{array}{l}0.68 \\
0.32 \\
0.88 \\
1.24 \\
\mathbf{2 . 4 7 ^ { ( 2 ) }}\end{array}$ & $\begin{array}{l}0.03 \\
T \\
0.58 \\
0.54 \\
0.75\end{array}$ & $\begin{array}{l}0.39 \\
0.14 \\
0.02^{(2)} \\
0.10 \\
0.27\end{array}$ & $\begin{array}{l}0.03 \\
0.90 \\
0.01 \\
1.22 \\
0.45\end{array}$ & $\begin{array}{l}0.05 \\
0.56 \\
0.06 \\
0.51 \\
0.54\end{array}$ & $\begin{array}{l}0.43 \\
0.57 \\
0.19 \\
0.75 \\
0.25\end{array}$ & $\begin{array}{l}0.81 \\
T \\
0.04 \\
T \\
0.01\end{array}$ & $\begin{array}{l}\mathrm{T} \\
\mathrm{T} \\
0.51 \\
\mathrm{~T} \\
\mathrm{~T}\end{array}$ & $\begin{array}{l}0.27 \\
0.05 \\
0.25 \\
0.48 \\
0.03\end{array}$ & $\begin{array}{l}0.39 \\
0.13 \\
0.93 \\
0.10 \\
0.24\end{array}$ & $\begin{array}{l}2.25 \\
0.16 \\
1.23 \\
0.13 \\
0.71\end{array}$ & $\begin{array}{l}0.60 \\
0.43 \\
1.25 \\
1.29 \\
0.61\end{array}$ & $\begin{array}{l}5.93 \\
3.26 \\
5.95 \\
6.36 \\
6.33\end{array}$ \\
\hline $\begin{array}{l}1971 \\
1972 \\
1973 \\
1974 \\
1975\end{array}$ & $\begin{array}{l}0.78 \\
0.19 \\
0.90 \\
0.90 \\
1.43\end{array}$ & $\begin{array}{l}0.10 \\
0.27 \\
0.21 \\
0.41 \\
0.98\end{array}$ & $\begin{array}{l}1.02 \\
0.58 \\
0.08 \\
0.52 \\
0.33\end{array}$ & $\begin{array}{l}0.07 \\
0.10 \\
. T \\
0.46 \\
0.42\end{array}$ & $\begin{array}{l}0.56 \\
2.03^{(2)} \\
0.24 \\
0.28 \\
0.38\end{array}$ & $\begin{array}{l}0.71 \\
0.66 \\
0.01 \\
0.12 \\
0.24\end{array}$ & $\begin{array}{l}0.13 \\
0.16 \\
T \\
0.71 \\
0.32\end{array}$ & $\begin{array}{l}0.09 \\
0.56 \\
0.02 \\
T \\
1.16\end{array}$ & $\begin{array}{l}1.13 \\
0.02 \\
0.43 \\
0.01 \\
0.03\end{array}$ & $\begin{array}{l}0.18 \\
T \\
1.72 \\
0.21 \\
0.87\end{array}$ & $\begin{array}{l}0.46 \\
0.55 \\
2.64 \\
0.71 \\
0.60\end{array}$ & $\begin{array}{l}1.07 \\
1.27 \\
2.02 \\
0.97 \\
0.70\end{array}$ & $\begin{array}{l}6.30 \\
6.39 \\
8.27 \\
5.30 \\
7.46\end{array}$ \\
\hline $\begin{array}{l}1976 \\
1977 \\
1978 \\
1979 \\
1980\end{array}$ & $\begin{array}{l}0.56 \\
\mathbf{0 . 0 8} \\
1.72 \\
0.54 \\
1.32\end{array}$ & $\begin{array}{l}0.36 \\
0.57 \\
0.92 \\
0.17 \\
1.30\end{array}$ & $\begin{array}{l}0.23 \\
0.41 \\
0.30 \\
0.54 \\
0.30\end{array}$ & $\begin{array}{l}0.41 \\
T \\
0.46 \\
0.52 \\
0.86\end{array}$ & $\begin{array}{l}0.08 \\
0.65 \\
0.41 \\
0.10 \\
1.41\end{array}$ & $\begin{array}{l}0.11 \\
0.37 \\
0.09 \\
T \\
0.96\end{array}$ & $\begin{array}{l}0.13 \\
0.06 \\
0.52 \\
0.09 \\
T^{(0)}\end{array}$ & $\begin{array}{l}0.96 \\
1.36^{(2)} \\
0.57 \\
0.38 \\
0.02\end{array}$ & $\begin{array}{l}T \\
0.66 \\
0.11 \\
0.20 \\
0.85\end{array}$ & $\begin{array}{l}0.04 \\
0.15 \\
T \\
0.67 \\
0.33\end{array}$ & $\begin{array}{l}T \\
0.63 \\
1.21 \\
1.36 \\
0.44\end{array}$ & $\begin{array}{l}\mathbf{0 . 1 1} 1^{(2)} \\
1.47 \\
0.26 \\
0.99 \\
1.89\end{array}$ & $\begin{array}{l}2.99^{(2)} \\
6.41 \\
6.57 \\
5.56 \\
9.68\end{array}$ \\
\hline $\begin{array}{l}1981 \\
1982 \\
1983 \\
1984 \\
1985\end{array}$ & $\begin{array}{l}0.56 \\
0.33 \\
1.44 \\
0.23 \\
0.34\end{array}$ & $\begin{array}{l}0.60 \\
0.57 \\
1.36 \\
0.94 \\
0.82\end{array}$ & $\begin{array}{l}0.70 \\
0.30 \\
1.00 \\
1.01 \\
0.36\end{array}$ & $\begin{array}{l}0.02 \\
0.75 \\
0.42 \\
0.60 \\
0.01\end{array}$ & $\begin{array}{l}0.99 \\
0.28 \\
0.52 \\
0.55 \\
0.12\end{array}$ & $\begin{array}{l}0.43 \\
0.75 \\
0.68 \\
0.99 \\
0.15\end{array}$ & $\begin{array}{l}0.19 \\
0.22 \\
0.31 \\
0.06 \\
0.12\end{array}$ & $\begin{array}{l}0.03 \\
0.20 \\
0.12 \\
T \\
0.01\end{array}$ & $\begin{array}{l}0.60 \\
0.55 \\
0.46 \\
0.42 \\
0.63\end{array}$ & $\begin{array}{l}0.39 \\
1.33 \\
0.52 \\
0.07 \\
0.46\end{array}$ & $\begin{array}{l}1.08 \\
0.91 \\
2.12 \\
1.83 \\
1.24\end{array}$ & $\begin{array}{l}1.45 \\
1.79 \\
2.12 \\
0.57 \\
0.84\end{array}$ & $\begin{array}{r}7.04 \\
7.98 \\
11.07 \\
7.27 \\
5.10\end{array}$ \\
\hline $\begin{array}{l}1986 \\
1987 \\
1988 \\
1989 \\
1990\end{array}$ & $\begin{array}{l}1.76 \\
0.80 \\
0.48 \\
0.21 \\
0.77\end{array}$ & $\begin{array}{l}1.37 \\
0.19 \\
T^{(b)} \\
1.67 \\
0.09\end{array}$ & $\begin{array}{l}0.76 \\
1.05 \\
0.39 \\
1.56 \\
0.10\end{array}$ & $\begin{array}{l}\mathrm{T} \\
0.14 \\
1.12 \\
0.84 \\
0.40\end{array}$ & $\begin{array}{l}0.30 \\
0.17 \\
0.33 \\
0.59 \\
0.86\end{array}$ & $\begin{array}{l}\mathrm{T}^{(b)} \\
0.11 \\
0.11 \\
0.01 \\
0.36\end{array}$ & $\begin{array}{l}0.21 \\
0.50 \\
0.13 \\
0.01 \\
0.14\end{array}$ & $\begin{array}{l}0.02 \\
0.07 \\
0^{(b)} \\
0.26 \\
0.83\end{array}$ & $\begin{array}{l}0.96 \\
0.01 \\
0.39 \\
0.02 \\
T\end{array}$ & $\begin{array}{l}0.29 \\
T^{(b)} \\
0.01 \\
0.42 \\
0.78\end{array}$ & $\begin{array}{l}0.65 \\
0.40 \\
0.82 \\
1.04 \\
0.02\end{array}$ & $\begin{array}{l}0.77 \\
1.63 \\
0.40 \\
0.29 \\
0.72\end{array}$ & $\begin{array}{l}7.09 \\
5.07 \\
4.18 \\
6.92 \\
5.07\end{array}$ \\
\hline $\begin{array}{l}1991 \\
1992 \\
1993 \\
1994 \\
1995\end{array}$ & $\begin{array}{l}0.33 \\
0.44 \\
1.30 \\
0.44 \\
2.14\end{array}$ & $\begin{array}{l}0.19 \\
0.94 \\
1.17 \\
0.11 \\
0.69\end{array}$ & $\begin{array}{l}1.12 \\
0.09 \\
0.67 \\
0.03 \\
0.95\end{array}$ & $\begin{array}{l}0.45 \\
0.94 \\
0.71 \\
0.61 \\
1.54^{(2)}\end{array}$ & $\begin{array}{l}0.49 \\
T^{(2)} \\
0.60 \\
1.27 \\
0.79\end{array}$ & $\begin{array}{l}1.44 \\
1.14 \\
0.12 \\
0.38 \\
0.77\end{array}$ & $\begin{array}{l}0.29 \\
0.38 \\
1.76^{(2)} \\
0.15 \\
0.34\end{array}$ & $\begin{array}{l}0.07 \\
0.20 \\
0.24 \\
0.08 \\
0.07\end{array}$ & $\begin{array}{l}0 \\
0.27 \\
0.04 \\
0.08 \\
0.79\end{array}$ & $\begin{array}{l}0.53 \\
0.61 \\
0.09 \\
0.93 \\
0.87\end{array}$ & $\begin{array}{l}1.44 \\
1.07 \\
0.19 \\
0.68 \\
1.04\end{array}$ & $\begin{array}{l}0.40 \\
1.82 \\
0.94 \\
1.36 \\
2.32\end{array}$ & $\begin{array}{c}6.75 \\
7.90 \\
7.83 \\
6.12 \\
12.31^{(2)}\end{array}$ \\
\hline $\begin{array}{l}1996 \\
1997 \\
1998 \\
1999\end{array}$ & $\begin{array}{l}1.42 \\
1.51 \\
1.24 \\
0.89\end{array}$ & $\begin{array}{l}1.22 \\
0.25 \\
1.15 \\
0.70\end{array}$ & $\begin{array}{l}0.83 \\
0.70 \\
0.50 \\
0.06\end{array}$ & $\begin{array}{l}0.43 \\
0.33 \\
0.07 \\
T^{(\mathbf{b})}\end{array}$ & $\begin{array}{l}0.62 \\
0.33 \\
0.52 \\
0.34\end{array}$ & $\begin{array}{l}0.05 \\
0.46 \\
0.48 \\
0.31\end{array}$ & $\begin{array}{l}0.14 \\
0.19 \\
0.34 \\
0.07\end{array}$ & $\begin{array}{l}0.02 \\
0.06 \\
0.04 \\
0.57\end{array}$ & $\begin{array}{l}0.22 \\
0.32 \\
0.10 \\
0^{(2, b)}\end{array}$ & $\begin{array}{l}0.88 \\
0.92 \\
0.28 \\
0.48\end{array}$ & $\begin{array}{l}2.67^{(x)} \\
1.01 \\
1.29 \\
0.26\end{array}$ & $\begin{array}{l}3.69^{(2)} \\
0.31 \\
0.44 \\
0.07\end{array}$ & $\begin{array}{r}12.19 \\
6.39 \\
6.45 \\
3.75\end{array}$ \\
\hline $\begin{array}{l}\text { Average } \\
\text { Normal }\end{array}$ & $\begin{array}{l}0.93 \\
0.79\end{array}$ & $\begin{array}{l}0.63 \\
0.62\end{array}$ & $\begin{array}{l}0.50 \\
0.47\end{array}$ & $\begin{array}{l}0.44 \\
0.41\end{array}$ & $\begin{array}{l}0.52 \\
0.51\end{array}$ & $\begin{array}{l}0.52 \\
0.38\end{array}$ & $\begin{array}{l}0.22 \\
0.18\end{array}$ & $\begin{array}{l}0.24 \\
0.27\end{array}$ & $\begin{array}{l}0.31 \\
0.31\end{array}$ & $\begin{array}{l}0.55 \\
0.39\end{array}$ & $\begin{array}{l}0.89 \\
0.91\end{array}$ & $\begin{array}{l}0.99 \\
1.03\end{array}$ & $\begin{array}{l}6.76 \\
6.26\end{array}$ \\
\hline
\end{tabular}

(a) Greatest and least values.

(b) Most recent of multiple occurrences.

$\mathrm{T}=$ Trace. 
Table 4.2. Seasonal Precipitation (inches)

\begin{tabular}{|c|c|c|c|c|}
\hline Year & $\begin{array}{l}\text { Winter }{ }^{(a)} \\
\text { Dec-Feb }\end{array}$ & $\begin{array}{c}\text { Spring } \\
\text { Mar-May } \\
\end{array}$ & $\begin{array}{l}\text { Summer } \\
\text { Jun-Aug } \\
\end{array}$ & $\begin{array}{l}\text { Autumn } \\
\text { Sep-Nov } \\
\end{array}$ \\
\hline $\begin{array}{l}1946 \\
1947 \\
1948 \\
1949 \\
1950\end{array}$ & $\begin{array}{l}-\overline{0.70^{(b)}} \\
2.80 \\
1.92 \\
3.02\end{array}$ & $\begin{array}{l}-\overline{14} \\
2.73 \\
1.30 \\
1.61\end{array}$ & $\begin{array}{l}\overrightarrow{2.46} \\
2.26 \\
0.05 \\
2.99^{(b)}\end{array}$ & $\begin{array}{l}1.83 \\
4.35 \\
1.56 \\
1.80 \\
3.02\end{array}$ \\
\hline $\begin{array}{l}1951 \\
1952 \\
1953 \\
1954 \\
1955\end{array}$ & $\begin{array}{l}2.32 \\
1.85 \\
3.18 \\
2.25 \\
1.13\end{array}$ & $\begin{array}{l}1.42 \\
0.77 \\
1.22 \\
1.07 \\
1.16\end{array}$ & $\begin{array}{l}1.90 \\
1.15 \\
1.51 \\
0.74 \\
0.85\end{array}$ & $\begin{array}{l}1.63 \\
0.32 \\
1.29 \\
1.79 \\
2.71\end{array}$ \\
\hline $\begin{array}{l}1956 \\
1957 \\
1958 \\
1959 \\
1960\end{array}$ & $\begin{array}{l}4.30 \\
1.29 \\
3.75 \\
5.06 \\
1.35\end{array}$ & $\begin{array}{l}0.32 \\
3.06 \\
1.84 \\
1.10 \\
1.91\end{array}$ & $\begin{array}{l}1.24 \\
0.54 \\
0.83 \\
0.26 \\
0.40\end{array}$ & $\begin{array}{l}1.19 \\
3.45 \\
1.01 \\
2.23 \\
1.38\end{array}$ \\
\hline $\begin{array}{l}1961 \\
1962 \\
1963 \\
1964 \\
1965\end{array}$ & $\begin{array}{l}3.07 \\
1.92 \\
2.24 \\
1.52 \\
3.41\end{array}$ & $\begin{array}{l}2.30 \\
1.83 \\
2.13 \\
0.18 \\
0.27\end{array}$ & $\begin{array}{l}0.66 \\
0.62 \\
0.60 \\
1.18 \\
0.63\end{array}$ & $\begin{array}{l}0.56 \\
1.98 \\
0.80 \\
1.31 \\
1.29\end{array}$ \\
\hline $\begin{array}{l}1966 \\
1967 \\
1968 \\
1969 \\
1970\end{array}$ & $\begin{array}{l}1.10 \\
0.92 \\
1.89 \\
3.03 \\
4.51\end{array}$ & $\begin{array}{l}0.47 \\
1.60 \\
0.09^{(b)} \\
1.83 \\
1.26\end{array}$ & $\begin{array}{l}1.24 \\
0.57 \\
0.74 \\
0.75 \\
0.26\end{array}$ & $\begin{array}{l}2.91 \\
0.34 \\
2.41 \\
0.71 \\
0.98\end{array}$ \\
\hline $\begin{array}{l}1971 \\
1972 \\
1973 \\
1974 \\
1975\end{array}$ & $\begin{array}{l}1.49 \\
1.53 \\
2.38 \\
3.33 \\
3.65\end{array}$ & $\begin{array}{l}1.65 \\
2.71 \\
0.32 \\
1.26 \\
1.13\end{array}$ & $\begin{array}{l}0.93 \\
1.38 \\
\mathbf{0 . 0 3}^{(\mathfrak{b})} \\
0.83 \\
1.72\end{array}$ & $\begin{array}{l}1.77 \\
0.57 \\
4.79^{(0)} \\
0.93 \\
1.50\end{array}$ \\
\hline $\begin{array}{l}1976 \\
1977 \\
1978 \\
1979 \\
1980\end{array}$ & $\begin{array}{l}1.62 \\
0.76 \\
3.91 \\
0.97 \\
3.61\end{array}$ & $\begin{array}{l}0.72 \\
1.06 \\
1.17 \\
1.16 \\
2.57\end{array}$ & $\begin{array}{l}1.20 \\
1.79 \\
1.18 \\
0.47 \\
0.98\end{array}$ & $\begin{array}{l}0.04^{(0)} \\
1.44 \\
1.32 \\
2.23 \\
1.62\end{array}$ \\
\hline $\begin{array}{l}1981 \\
1982 \\
1983 \\
1984 \\
1985\end{array}$ & $\begin{array}{l}3.05 \\
2.35 \\
4.59 \\
3.29 \\
1.73\end{array}$ & $\begin{array}{l}1.71 \\
1.33 \\
1.94 \\
2.16 \\
0.49\end{array}$ & $\begin{array}{l}0.65 \\
1.17 \\
1.11 \\
1.05 \\
0.28\end{array}$ & $\begin{array}{l}2.07 \\
2.79 \\
3.10 \\
2.32 \\
2.33\end{array}$ \\
\hline $\begin{array}{l}1986 \\
1987 \\
1988 \\
1989 \\
1990\end{array}$ & $\begin{array}{l}3.97 \\
1.76 \\
2.11 \\
2.28 \\
1.15\end{array}$ & $\begin{array}{l}1.06 \\
1.36 \\
1.84 \\
2.99 \\
1.36\end{array}$ & $\begin{array}{l}0.23 \\
0.68 \\
0.24 \\
0.28 \\
1.33\end{array}$ & $\begin{array}{l}1.90 \\
0.41 \\
1.22 \\
1.48 \\
0.80\end{array}$ \\
\hline $\begin{array}{l}1991 \\
1992 \\
1993 \\
1994 \\
1995\end{array}$ & $\begin{array}{l}1.24 \\
1.78 \\
4.29 \\
1.49 \\
4.19\end{array}$ & $\begin{array}{l}2.06 \\
1.03 \\
1.98 \\
1.91 \\
3.28^{(b)}\end{array}$ & $\begin{array}{l}1.80 \\
1.72 \\
2.12 \\
0.61 \\
1.18\end{array}$ & $\begin{array}{l}1.97 \\
1.95 \\
0.32 \\
1.69 \\
2.70\end{array}$ \\
\hline $\begin{array}{l}1996 \\
1997 \\
1998 \\
1999\end{array}$ & $\begin{array}{l}4.96 \\
5.45^{(b)} \\
2.70 \\
2.03\end{array}$ & $\begin{array}{l}1.88 \\
1.36 \\
1.09 \\
0.40\end{array}$ & $\begin{array}{l}0.21 \\
0.71 \\
0.86 \\
0.95\end{array}$ & $\begin{array}{l}3.77 \\
2.25 \\
1.67 \\
0.74\end{array}$ \\
\hline $\begin{array}{l}\text { Average } \\
\text { Normal }\end{array}$ & $\begin{array}{l}2.57 \\
2.44\end{array}$ & $\begin{array}{l}1.46 \\
1.40\end{array}$ & $\begin{array}{l}0.98 \\
0.83\end{array}$ & $\begin{array}{l}1.75 \\
1.60\end{array}$ \\
\hline
\end{tabular}

(a) For the winter season, December is included in the previous year.

(b) Greatest and least values. 
Table 4.3. Average Number of Days with Precipitation of Specified Amount

\begin{tabular}{|c|c|c|c|c|c|c|}
\hline Month & $\begin{array}{c}\text { Trace or } \\
\text { more }\end{array}$ & $\begin{array}{l}0.01 \text { in. } \\
\text { or more }\end{array}$ & $\begin{array}{l}0.10 \mathrm{in} . \\
\text { or more }\end{array}$ & $\begin{array}{l}0.25 \mathrm{in} . \\
\text { or more }\end{array}$ & $\begin{array}{l}0.50 \mathrm{in} . \\
\text { or more }\end{array}$ & $\begin{array}{l}1.00 \mathrm{in} . \\
\text { or more }\end{array}$ \\
\hline Jan & 16 & 9 & 3 & 1 & (a) & 0 \\
\hline Feb & 12 & 7 & 2 & 1 & (a) & 0 \\
\hline Mar & 11 & 6 & 2 & (a) & (a) & 0 \\
\hline Apr & 10 & 5 & 2 & 1 & (a) & 0 \\
\hline May & 10 & 5 & 2 & 1 & (a) & 0 \\
\hline Jun & 9 & 5 & 2 & 1 & (a) & (a) \\
\hline Jul & 5 & 2 & 1 & (a) & (a) & (a) \\
\hline Aug & 5 & 3 & 1 & (a) & (a) & 0 \\
\hline Sep & 6 & 3 & 1 & (a) & (a) & 0 \\
\hline Oct & 9 & 5 & 2 & 1 & (a) & (a) \\
\hline Nov & 14 & 9 & 3 & 1 & (a) & (a) \\
\hline Dec & 16 & 10 & 3 & 1 & (a) & 0 \\
\hline Annual ${ }^{(b)}$ & 123 & 68 & 23 & 7 & $1^{(c)}$ & (a) \\
\hline
\end{tabular}

(a) Used to denote an average of less than $1 / 2$ day.

(b) Annual totals may differ from summation of monthly events because of rounding.

(c) Although the number of days with 0.50 inch or more averages less than 1/2 day for any one month, 72 such days were recorded during 52 years of record.

Table 4.4. Monthly and Annual Averages and Extremes in Total Time with Precipitation Observed: July 1946 Through June 1971, July 1974 Through December 1999

\begin{tabular}{|c|c|c|c|c|c|c|c|c|}
\hline \multirow[b]{2}{*}{ Month } & \multicolumn{2}{|c|}{ Averages } & \multicolumn{3}{|c|}{ Greatest } & \multicolumn{3}{|c|}{ Least } \\
\hline & $\begin{array}{l}\text { No. of } \\
\text { Hours }\end{array}$ & $\begin{array}{l}\% \text { of } \\
\text { Time }\end{array}$ & $\begin{array}{l}\text { No. of } \\
\text { Hours }\end{array}$ & $\begin{array}{l}\% \text { of } \\
\text { Time }\end{array}$ & Year & $\begin{array}{l}\text { No. of } \\
\text { Hours }\end{array}$ & $\begin{array}{l}\% \text { of } \\
\text { Time }\end{array}$ & Year \\
\hline Jan & 89.5 & 12.0 & 212.0 & 28.5 & 1969 & 29.2 & 3.9 & 1949 \\
\hline Feb & 56.2 & 8.3 & 151.6 & 22.6 & 1980 & 2.5 & 0.4 & 1988 \\
\hline Mar & 39.1 & 5.3 & 135.2 & 18.2 & 1957 & 6.4 & 0.9 & 1994 \\
\hline Apr & 29.0 & 4.0 & 69.2 & 9.6 & 1953 & 1.6 & 0.2 & 1985 \\
\hline May & 31.0 & 4.2 & 89.9 & 12.1 & 1948 & 1.2 & 0.2 & 1992 \\
\hline Jun & 26.8 & 3.7 & 80.8 & 11.2 & 1950 & 2.9 & 0.4 & 1986 \\
\hline Jul & 10.6 & 1.4 & 38.2 & 5.1 & 1966 & 0.5 & 0.1 & 1984 \\
\hline Aug & 12.0 & 1.6 & 61.7 & 8.3. & 1968 & 0.0 & 0.0 & $1988^{(a)}$ \\
\hline Sep & 15.5 & 2.2 & 66.4 & 9.2 & 1977 & 0.0 & 0.0 & $1999^{(a)}$ \\
\hline Oct & 31.7 & 4.3 & 119.9 & 16.1 & 1947 & 0.4 & 0.1 & 1978 \\
\hline Nov & 61.1 & 8.5 & 146.5 & 20.3 & 1985 & 4.8 & 0.7 & 1976 \\
\hline Dec & 88.2 & 11.9 & 230.5 & 31.0 & 1985 & 15.8 & 2.1 & 1976 \\
\hline Annual & 490.8 & 5.6 & 738.0 & 8.4 & 1950 & 286.7 & 3.3 & 1990 \\
\hline
\end{tabular}

(a) Most recent of several occurrences. 
Table 4.5. Total Duration (hours) of Precipitation by Month and Year

\begin{tabular}{|c|c|c|c|c|c|c|c|c|c|c|c|c|c|}
\hline Year & Jan & Feb & Mar & Apr & May & Jun & Jul & Aug & Sep & Oct & Nov & Dec & Annual \\
\hline $\begin{array}{l}1946 \\
1947 \\
1948 \\
1949 \\
1950\end{array}$ & $\begin{array}{c}- \\
34.6 \\
70.7 \\
29.2^{(2)} \\
147.4\end{array}$ & $\begin{array}{c}- \\
29.8 \\
70.4 \\
69.9 \\
78.0\end{array}$ & $\begin{array}{c}- \\
30.2 \\
16.6 \\
63.5 \\
72.0\end{array}$ & $\begin{array}{r}- \\
32.7 \\
43.8 \\
5.6 \\
23.3\end{array}$ & $\begin{array}{c}\overline{-} \\
4.8 \\
89.9^{(0)} \\
13.2 \\
13.9\end{array}$ & $\begin{array}{c}- \\
38.4 \\
36.4 \\
3.2 \\
\mathbf{8 0 . 8}\end{array}$ & $\begin{array}{r}6.0 \\
17.4 \\
15.2 \\
2.2 \\
5.0\end{array}$ & $\begin{array}{r}16.3 \\
12.6 \\
16.6 \\
1.6 \\
2.3\end{array}$ & $\begin{array}{r}12.2 \\
36.4 \\
15.8 \\
21.2 \\
1.3\end{array}$ & $\begin{array}{c}38.2 \\
119.9^{(2)} \\
19.7 \\
15.0 \\
112.3\end{array}$ & $\begin{array}{l}66.0 \\
48.1 \\
44.4 \\
98.7 \\
92.9\end{array}$ & $\begin{array}{r}31.5 \\
71.1 \\
81.9 \\
32.1 \\
108.8\end{array}$ & $\begin{array}{l}-\overline{476.0} \\
521.4 \\
355.4 \\
738.0^{(2)}\end{array}$ \\
\hline $\begin{array}{l}1951 \\
1952 \\
1953 \\
1954 \\
1955\end{array}$ & $\begin{array}{r}66.3 \\
151.0 \\
89.3 \\
92.6 \\
116.4\end{array}$ & $\begin{array}{l}55.9 \\
57.8 \\
29.7 \\
90.0 \\
33.4\end{array}$ & $\begin{array}{l}34.8 \\
19.3 \\
32.3 \\
35.8 \\
20.0\end{array}$ & $\begin{array}{l}21.5 \\
22.1 \\
69.2^{(2)} \\
18.4 \\
57.2\end{array}$ & $\begin{array}{l}23.7 \\
25.4 \\
20.6 \\
33.7 \\
47.8\end{array}$ & $\begin{array}{l}60.8 \\
77.7 \\
37.1 \\
32.4 \\
10.2\end{array}$ & $\begin{array}{r}12.8 \\
4.3 \\
2.0 \\
17.8 \\
36.9\end{array}$ & $\begin{array}{r}17.7 \\
5.4 \\
25.2 \\
22.2 \\
0.0\end{array}$ & $\begin{array}{r}26.6 \\
7.1 \\
7.1 \\
15.1 \\
40.1\end{array}$ & $\begin{array}{r}66.5 \\
3.6 \\
23.6 \\
17.9 \\
42.3\end{array}$ & $\begin{array}{r}66.0 \\
71.2 \\
59.4 \\
74.3 \\
132.2\end{array}$ & $\begin{array}{r}86.7 \\
137.0 \\
32.2 \\
39.9 \\
141.0\end{array}$ & $\begin{array}{l}539.3 \\
581.9 \\
427.7 \\
490.1 \\
677.5\end{array}$ \\
\hline $\begin{array}{l}1956 \\
1957 \\
1958 \\
1959 \\
1960\end{array}$ & $\begin{array}{r}126.9 \\
140.6 \\
82.8 \\
129.5 \\
86.8\end{array}$ & $\begin{array}{r}74.4 \\
46.4 \\
106.8 \\
98.2 \\
48.0\end{array}$ & $\begin{array}{c}15.9 \\
135.2^{(2)} \\
37.5 \\
32.6 \\
49.9\end{array}$ & $\begin{array}{r}1.8 \\
19.5 \\
54.5 \\
17.5 \\
32.8\end{array}$ & $\begin{array}{l}35.8 \\
43.4 \\
24.2 \\
33.0 \\
47.2\end{array}$ & $\begin{array}{r}30.8 \\
20.8 \\
24.2 \\
29.8 \\
6.3\end{array}$ & $\begin{array}{l}4.2 \\
2.6 \\
1.2 \\
4.2 \\
3.5\end{array}$ & $\begin{array}{r}17.4 \\
5.7 \\
2.0 \\
15.9 \\
27.3\end{array}$ & $\begin{array}{r}6.6 \\
23.1 \\
13.4 \\
52.2 \\
15.8\end{array}$ & $\begin{array}{l}65.7 \\
72.0 \\
13.6 \\
27.2 \\
34.8\end{array}$ & $\begin{array}{l}71.0 \\
21.4 \\
58.5 \\
44.8 \\
64.1\end{array}$ & $\begin{array}{r}98.8 \\
49.3 \\
107.7 \\
51.8 \\
120.8\end{array}$ & $\begin{array}{l}549.3 \\
580.0 \\
526.4 \\
536.7 \\
537.3\end{array}$ \\
\hline $\begin{array}{l}1961 \\
1962 \\
1963 \\
1964 \\
1965\end{array}$ & $\begin{array}{r}91.8 \\
43.9 \\
56.3 \\
49.1 \\
153.3\end{array}$ & $\begin{array}{r}94.4 \\
58.8 \\
88.4 \\
5.2 \\
18.8\end{array}$ & $\begin{array}{r}60.7 \\
55.1 \\
31.2 \\
8.3 \\
14.2\end{array}$ & $\begin{array}{l}39.2 \\
24.8 \\
66.5 \\
15.7 \\
30.9\end{array}$ & $\begin{array}{r}48.7 \\
80.2 \\
51.3 \\
6.1 \\
15.2\end{array}$ & $\begin{array}{l}23.9 \\
13.9 \\
37.1 \\
46.8 \\
28.6\end{array}$ & $\begin{array}{r}4.2 \\
4.3 \\
20.9 \\
14.5 \\
6.8\end{array}$ & $\begin{array}{r}17.2 \\
24.9 \\
4.4 \\
14.1 \\
18.4\end{array}$ & $\begin{array}{r}2.0 \\
21.6 \\
11.2 \\
7.2 \\
11.2\end{array}$ & $\begin{array}{l}15.9 \\
71.5 \\
26.4 \\
19.2 \\
11.2\end{array}$ & $\begin{array}{r}57.2 \\
44.4 \\
61.0 \\
109.0 \\
89.1\end{array}$ & $\begin{array}{r}99.0 \\
139.6 \\
179.6 \\
149.0 \\
57.8\end{array}$ & $\begin{array}{l}554.2 \\
583.0 \\
634.3 \\
444.2 \\
455.5\end{array}$ \\
\hline $\begin{array}{l}1966 \\
1967 \\
1968 \\
1969 \\
1970\end{array}$ & $\begin{array}{c}51.7 \\
34.1 \\
99.1 \\
212.0^{(2)} \\
157.2\end{array}$ & $\begin{array}{r}12.4 \\
4.7 \\
42.0 \\
75.4 \\
72.9\end{array}$ & $\begin{array}{r}42.9 \\
30.6 \\
7.3 \\
9.7 \\
34.0\end{array}$ & $\begin{array}{r}9.1 \\
60.9 \\
18.6 \\
52.2 \\
19.2\end{array}$ & $\begin{array}{r}7.2 \\
52.9 \\
29.9 \\
51.9 \\
27.2\end{array}$ & $\begin{array}{l}30.4 \\
23.3 \\
38.3 \\
38.7 \\
31.1\end{array}$ & $\begin{array}{c}38.2^{(2)} \\
2.2 \\
5.6 \\
1.3 \\
6.9\end{array}$ & $\begin{array}{c}3.7 \\
1.7 \\
61.7^{(2)} \\
0.3 \\
2.3\end{array}$ & $\begin{array}{r}15.9 \\
12.1 \\
17.2 \\
26.8 \\
5.3\end{array}$ & $\begin{array}{l}26.3 \\
29.4 \\
45.3 \\
20.4 \\
32.2\end{array}$ & $\begin{array}{r}103.5 \\
27.0 \\
68.9 \\
44.1 \\
85.8\end{array}$ & $\begin{array}{r}75.6 \\
88.2 \\
134.2 \\
148.3 \\
83.9\end{array}$ & $\begin{array}{l}416.9 \\
367.1 \\
568.1 \\
681.1 \\
558.0\end{array}$ \\
\hline $\begin{array}{l}1971 \\
1972 \\
1973 \\
1974 \\
1975\end{array}$ & $\begin{array}{c}49.5 \\
\text { (b) } \\
\text { (b) } \\
\text { (b) } \\
105.7\end{array}$ & $\begin{array}{r}14.8 \\
\text { (b) } \\
\text { (b) } \\
\text { (b) } \\
103.8\end{array}$ & $\begin{array}{r}68.0 \\
\text { (b) } \\
\text { (b) } \\
\text { (b) } \\
42.3\end{array}$ & $\begin{array}{c}25.0 \\
\text { (b) } \\
\text { (b) } \\
\text { (b) } \\
29.5\end{array}$ & $\begin{array}{c}43.7 \\
\text { (b) } \\
\text { (b) } \\
\text { (b) } \\
29.2\end{array}$ & $\begin{array}{r}52.7 \\
\text { (b) } \\
\text { (b) } \\
\text { (b) } \\
20.8\end{array}$ & $\begin{array}{r}\text { (b) } \\
\text { (b) } \\
\text { (b) } \\
21.8 \\
13.5\end{array}$ & $\begin{array}{c}\text { (b) } \\
\text { (b) } \\
\text { (b) } \\
0.8 \\
26.8\end{array}$ & $\begin{array}{l}\text { (b) } \\
\text { (b) } \\
\text { (b) } \\
1.3 \\
1.0\end{array}$ & $\begin{array}{c}\text { (b) } \\
\text { (b) } \\
\text { (b) } \\
26.2 \\
65.6\end{array}$ & $\begin{array}{r}\text { (b) } \\
(\mathrm{b}) \\
(\mathrm{b}) \\
58.9 \\
56.1\end{array}$ & $\begin{array}{r}\text { (b) } \\
(b) \\
(b) \\
64.9 \\
76.6\end{array}$ & $\begin{array}{r}(b) \\
(b) \\
(b) \\
(b) \\
570.9\end{array}$ \\
\hline $\begin{array}{l}1976 \\
1977 \\
1978 \\
1979 \\
1980\end{array}$ & $\begin{array}{r}79.8 \\
138.7 \\
147.9 \\
111.5 \\
118.5\end{array}$ & $\begin{array}{c}28.6 \\
37.1 \\
98.6 \\
73.7 \\
151.6^{(\mathfrak{)})}\end{array}$ & $\begin{array}{l}35.2 \\
37.8 \\
46.2 \\
45.0 \\
35.4\end{array}$ & $\begin{array}{r}36.8 \\
4.6 \\
53.4 \\
29.6 \\
33.8\end{array}$ & $\begin{array}{l}16.7 \\
45.9 \\
45.2 \\
14.0 \\
60.2\end{array}$ & $\begin{array}{r}10.1 \\
24.8 \\
8.1 \\
4.5 \\
45.0\end{array}$ & $\begin{array}{r}15.1 \\
14.2 \\
27.6 \\
8.6 \\
1.5\end{array}$ & $\begin{array}{r}38.8 \\
28.9 \\
37.7 \\
27.4 \\
8.8\end{array}$ & $\begin{array}{c}2.2 \\
66.4^{(2)} \\
27.9 \\
10.0 \\
24.5\end{array}$ & $\begin{array}{c}10.7 \\
15.8 \\
0.4^{(2)} \\
47.3 \\
22.0\end{array}$ & $\begin{array}{l}4.8^{(2)} \\
77.1 \\
71.6 \\
86.9 \\
44.2\end{array}$ & $\begin{array}{c}15.8^{(2)} \\
98.1 \\
43.7 \\
104.2 \\
121.2\end{array}$ & $\begin{array}{l}294.6 \\
589.4 \\
608.3 \\
562.7 \\
666.7\end{array}$ \\
\hline $\begin{array}{l}1981 \\
1982 \\
1983 \\
1984 \\
1985\end{array}$ & $\begin{array}{r}72.0 \\
47.5 \\
72.5 \\
32.6 \\
151.2\end{array}$ & $\begin{array}{l}31.7 \\
29.2 \\
76.1 \\
58.1 \\
54.9\end{array}$ & $\begin{array}{l}32.0 \\
27.8 \\
59.4 \\
40.8 \\
26.0\end{array}$ & $\begin{array}{c}3.4 \\
33.6 \\
15.2 \\
35.2 \\
\mathbf{1 . 6}^{(2)}\end{array}$ & $\begin{array}{r}28.4 \\
9.4 \\
13.2 \\
34.2 \\
7.0\end{array}$ & $\begin{array}{l}21.1 \\
21.8 \\
22.4 \\
37.9 \\
17.4\end{array}$ & $\begin{array}{c}7.5 \\
5.8 \\
17.9 \\
0.5^{(2)} \\
3.2\end{array}$ & $\begin{array}{r}1.0 \\
5.3 \\
13.7 \\
0.5 \\
1.2\end{array}$ & $\begin{array}{l}17.4 \\
23.2 \\
12.5 \\
20.5 \\
30.8\end{array}$ & $\begin{array}{r}24.8 \\
40.1 \\
19.8 \\
7.1 \\
17.2\end{array}$ & $\begin{array}{c}34.1 \\
42.8 \\
79.3 \\
97.6 \\
146.5^{(2)}\end{array}$ & $\begin{array}{c}91.8 \\
81.7 \\
133.2 \\
75.5 \\
230.5^{(2)}\end{array}$ & $\begin{array}{l}365.2 \\
368.2 \\
535.2 \\
440.5 \\
687.5\end{array}$ \\
\hline $\begin{array}{l}1986 \\
1987 \\
1988 \\
1989 \\
1990\end{array}$ & $\begin{array}{r}107.6 \\
64.6 \\
92.4 \\
35.2 \\
34.6\end{array}$ & $\begin{array}{c}68.9 \\
20.8 \\
2.5^{(\mathrm{e})} \\
114.4 \\
20.5\end{array}$ & $\begin{array}{r}47.4 \\
74.0 \\
24.8 \\
102.2 \\
18.2\end{array}$ & $\begin{array}{l}14.4 \\
10.8 \\
36.2 \\
36.8 \\
29.9\end{array}$ & $\begin{array}{l}22.3 \\
14.6 \\
18.3 \\
25.8 \\
31.0\end{array}$ & $\begin{array}{c}2.9^{(2)} \\
11.8 \\
17.4 \\
5.4 \\
8.6\end{array}$ & $\begin{array}{r}13.3 \\
16.1 \\
6.2 \\
3.8 \\
7.3\end{array}$ & $\begin{array}{l}1.1 \\
4.5 \\
0.0^{(2, c)} \\
14.4 \\
15.4\end{array}$ & $\begin{array}{r}28.2 \\
0.5 \\
13.8 \\
0.7 \\
0.1\end{array}$ & $\begin{array}{r}10.3 \\
1.8 \\
2.2 \\
23.1 \\
49.1\end{array}$ & $\begin{array}{r}31.3 \\
21.1 \\
55.8 \\
33.2 \\
7.1\end{array}$ & $\begin{array}{r}143.8 \\
125.6 \\
62.7 \\
57.9 \\
64.9\end{array}$ & $\begin{array}{l}491.5 \\
366.2 \\
332.3 \\
452.9 \\
286.7^{(2)}\end{array}$ \\
\hline $\begin{array}{l}1991 \\
1992 \\
1993 \\
1994 \\
1995\end{array}$ & $\begin{array}{r}57.5 \\
36.2 \\
171.0 \\
40.5 \\
113.8\end{array}$ & $\begin{array}{l}28.0 \\
56.2 \\
64.4 \\
55.8 \\
39.2\end{array}$ & $\begin{array}{c}43.8 \\
7.9 \\
65.2 \\
6.4^{(\mathrm{a})} \\
47.3\end{array}$ & $\begin{array}{l}15.8 \\
42.0 \\
57.0 \\
43.2 \\
56.2\end{array}$ & $\begin{array}{c}39.0 \\
1.2^{(a)} \\
38.7 \\
40.7 \\
27.3\end{array}$ & $\begin{array}{l}41.8 \\
35.9 \\
13.0 \\
21.2 \\
52.6\end{array}$ & $\begin{array}{r}9.8 \\
22.5 \\
35.2 \\
4.7 \\
8.3\end{array}$ & $\begin{array}{r}4.2 \\
6.6 \\
12.1 \\
3.8 \\
7.9\end{array}$ & $\begin{array}{r}0.0 \\
23.6 \\
3.0 \\
8.9 \\
14.5\end{array}$ & $\begin{array}{r}42.9 \\
36.6 \\
6.8 \\
37.6 \\
33.0\end{array}$ & $\begin{array}{l}70.2 \\
53.0 \\
30.3 \\
52.9 \\
47.1\end{array}$ & $\begin{array}{l}48.2 \\
92.6 \\
58.2 \\
72.2 \\
62.2\end{array}$ & $\begin{array}{l}401.2 \\
414.3 \\
554.9 \\
387.9 \\
509.4\end{array}$ \\
\hline $\begin{array}{l}1996 \\
1997 \\
1998 \\
1999\end{array}$ & $\begin{array}{r}102.1 \\
69.5 \\
60.0 \\
52.9\end{array}$ & $\begin{array}{l}73.0 \\
17.7 \\
72.3 \\
56.6\end{array}$ & $\begin{array}{r}55.4 \\
36.1 \\
34.2 \\
4.8\end{array}$ & $\begin{array}{r}23.2 \\
13.7 \\
8.6 \\
3.1\end{array}$ & $\begin{array}{l}30.8 \\
19.2 \\
45.7 \\
15.2\end{array}$ & $\begin{array}{r}3.6 \\
14.7 \\
13.7 \\
9.4\end{array}$ & $\begin{array}{r}8.2 \\
12.7 \\
12.7 \\
2.1\end{array}$ & $\begin{array}{l}3.3 \\
4.1 \\
1.8 \\
9.4\end{array}$ & $\begin{array}{c}13.7 \\
19.7 \\
6.8 \\
\mathbf{0 . 0} 0^{(\mathbf{a})}\end{array}$ & $\begin{array}{l}46.8 \\
29.4 \\
14.8 \\
20.9\end{array}$ & $\begin{array}{l}81.5 \\
43.6 \\
45.1 \\
44.8\end{array}$ & $\begin{array}{r}124.4 \\
15.5 \\
54.1 \\
35.2\end{array}$ & $\begin{array}{l}566.0 \\
295.9 \\
369.8 \\
254.4\end{array}$ \\
\hline $\begin{array}{l}\text { Average } \\
\text { Normal }\end{array}$ & $\begin{array}{l}89.5 \\
89.3\end{array}$ & $\begin{array}{l}56.2 \\
54.0\end{array}$ & $\begin{array}{l}39.1 \\
39.9\end{array}$ & $\begin{array}{l}29.0 \\
28.4\end{array}$ & $\begin{array}{l}31.0 \\
30.7\end{array}$ & $\begin{array}{l}26.8 \\
23.9\end{array}$ & $\begin{array}{l}10.6 \\
10.7\end{array}$ & $\begin{array}{l}12.0 \\
13.9\end{array}$ & $\begin{array}{l}15.5 \\
15.2\end{array}$ & $\begin{array}{l}31.7 \\
25.2\end{array}$ & $\begin{array}{l}61.1 \\
60.7\end{array}$ & $\begin{array}{r}88.2 \\
101.8\end{array}$ & $\begin{array}{l}490.8 \\
495.4\end{array}$ \\
\hline
\end{tabular}

(a) Greatest and least values.

(b) Incomplete data not included. See Section 4.5.

(c) Most recent of numerous occurrences. 


\subsection{Notable Wet Periods}

Nine periods are listed when precipitation was particularly high:

\begin{tabular}{|c|c|c|c|c|c|}
\hline \multirow[b]{2}{*}{ Period } & \multicolumn{2}{|c|}{$\begin{array}{l}\text { Number of Days with } \\
\text { Trace or More }\end{array}$} & \multicolumn{3}{|c|}{ Total Amount, inches } \\
\hline & Altogether & $\begin{array}{c}\text { Greatest } \\
\text { Consecutive }\end{array}$ & $\begin{array}{l}\text { Measurable } \\
\text { Precipitation }\end{array}$ & $\begin{array}{c}\text { Water } \\
\text { Equivalent }\end{array}$ & $\begin{array}{c}\text { Snow- } \\
\text { fall }\end{array}$ \\
\hline Oct 7 - Nov 4, 1947 & 23 out of 29 & 10 & 17 & 2.21 & 0.0 \\
\hline $\operatorname{Jan} 3-28,1950$ & 21 out of 26 & 10 & 15 & 1.80 & 23.4 \\
\hline Nov 11 - Dec 19, 1950 & 33 out of 39 & 12 & 20 & 1.37 & 3.7 \\
\hline Nov 16 - Dec 22, 1955 & 31 out of 37 & 15 & 24 & 3.19 & 22.7 \\
\hline Oct $31-\operatorname{Dec} 7,1973$ & 32 out of 38 & 14 & 20 & 3.45 & 8.1 \\
\hline Nov $15-\operatorname{Dec} 7,1985$ & 17 out of 23 & 8 & 14 & 1.96 & 25.2 \\
\hline $\operatorname{Dec} 27,1992-\operatorname{Jan} 23,1993$ & 26 out of 29 & 12 & 19 & 2.02 & 26.8 \\
\hline Nov $13-27,1996$ & 12 out of 15 & 7 & 10 & 2.66 & 11.9 \\
\hline Dec $20-31,1996$ & 11 out of 12 & 9 & 9 & 3.00 & 20.1 \\
\hline
\end{tabular}

From a precipitation standpoint, 1973 was an unusual year. Total precipitation for 1973 was 8.27 inches, $132 \%$ of normal (6.26 inches). The period March 30 through September 18, 1973, was extremely dry, receiving only 0.29 inch of precipitation during that 173-day period; however, the period October 31 through December 7, 1973 was a notable wet period. During the months of October, November, and December 1973, 6.38 inches of precipitation were recorded, $289 \%$ of normal ( 2.21 inches) for those months. November and December 1996 received 6.36 inches of precipitation, $328 \%$ of normal (1.94 inches) for those months, which is greater than the normal precipitation amount for an entire year (6.26 inches).

\subsection{Notable Dry Periods}

The Hanford Meteorology Station is in a semiarid region; thus, it experiences many dry periods. January, March, and December are the only months that have always received measurable precipitation (1946 through 1999). A total of 39 months during the period of record have been without measurable precipitation, with the months of July and August accounting for 20 of those months. The record number of consecutive days with no precipitation (not even a trace) occurred in 1988, when the period July 14 through September 17 (66 days) was totally dry. The following list indicates some long periods with small amounts of precipitation. 


\begin{tabular}{|c|c|c|c|c|}
\hline \multicolumn{5}{|c|}{ Notable Dry Periods } \\
\hline Year & From & To & $\begin{array}{l}\text { Number } \\
\text { of Days }\end{array}$ & $\begin{array}{c}\text { Total Precipitation, } \\
\text { inch }\end{array}$ \\
\hline 1952 & Jun 30 & Nov 10 & 134 & 0.20 \\
\hline 1967 & Jun 22 & Nov 7 & 139 & 0.18 \\
\hline 1968 & Feb 24 & Aug 13 & 172 & 0.32 \\
\hline 1973 & $\operatorname{Mar} 30$ & Sep 18 & 173 & 0.29 \\
\hline 1976 & Aug 26 & $\operatorname{Dec} 31$ & 128 & 0.15 \\
\hline 1985 & $\operatorname{Mar} 31$ & $\operatorname{Sep} 7$ & 161 & 0.43 \\
\hline 1986 & May 6 & Sep 12 & 129 & 0.30 \\
\hline 1987 & Jul 19 & Oct 31 & 105 & 0.08 \\
\hline 1988 & Jun 6 & Sep 17 & 105 & 0.13 \\
\hline
\end{tabular}

The driest year on record was 1976, which had 2.99 inches recorded (less than $50 \%$ of normal). During the period September through December 1976, total precipitation was 0.15 inch, which was $6 \%$ of normal (2.52 inches) for those months.

\subsection{Snowfall}

Snowfall, which includes all frozen precipitation, varied from a seasonal total of 0.3 to 56.1 inches in 1957-1958 and 1992-1993, respectively. Table 4.6 provides information on monthly and seasonal snowfall amounts, as well as the dates and amounts of earliest and latest snowfall each season. The earliest measurable snowfall ( 0.3 inch) was recorded on October 26, 1957; the latest measurable snowfall (1.0 inch) was recorded on April 6, 1982. The average date of the first measurable snow is November 30; the average last measurable snow date is February 11. Normal snowfall for the period 1961 through 1990 and averages for the entire period of record are noted on the table, as are monthly and seasonal extremes.

Table 4.7 lists the greatest single storm snowfall amounts by month for the period 1946 through 1999. The greatest single snowstorm, on February 18-20, 1993, produced 12.4 inches of snow. During the winter of 1957-58 (actually the only snowfall was recorded in October - not yet winter), the greatest single snowstorm produced only 0.3 inch.

Table 4.8 lists some miscellaneous snowfall statistics for the Hanford Meteorology Station for the period 1946 through 1999. Included in this table are average number of days per month with snow depth above certain threshold values, greatest number of days per month with snow depth above certain threshold values, record number of consecutive days with snow depth above certain threshold values, record monthly snow depth, and 24-hour snowfall amounts. The record snow depth at the Hanford Meteorology Station is 15.6 inches, recorded in December 1985. The record number of days with snow depth $\geq 6$ inches was 43 days in the winter of 1992-1993. 


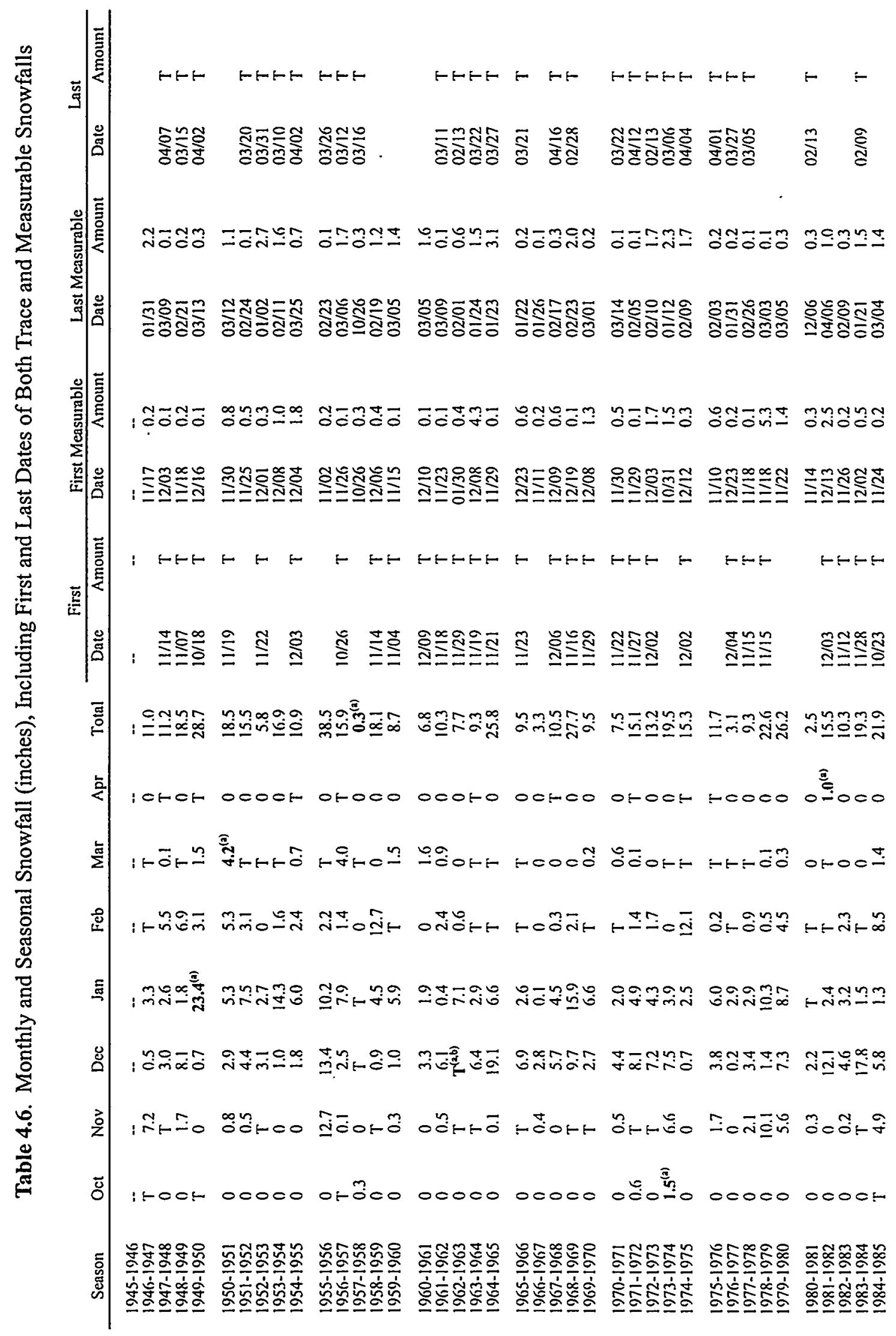




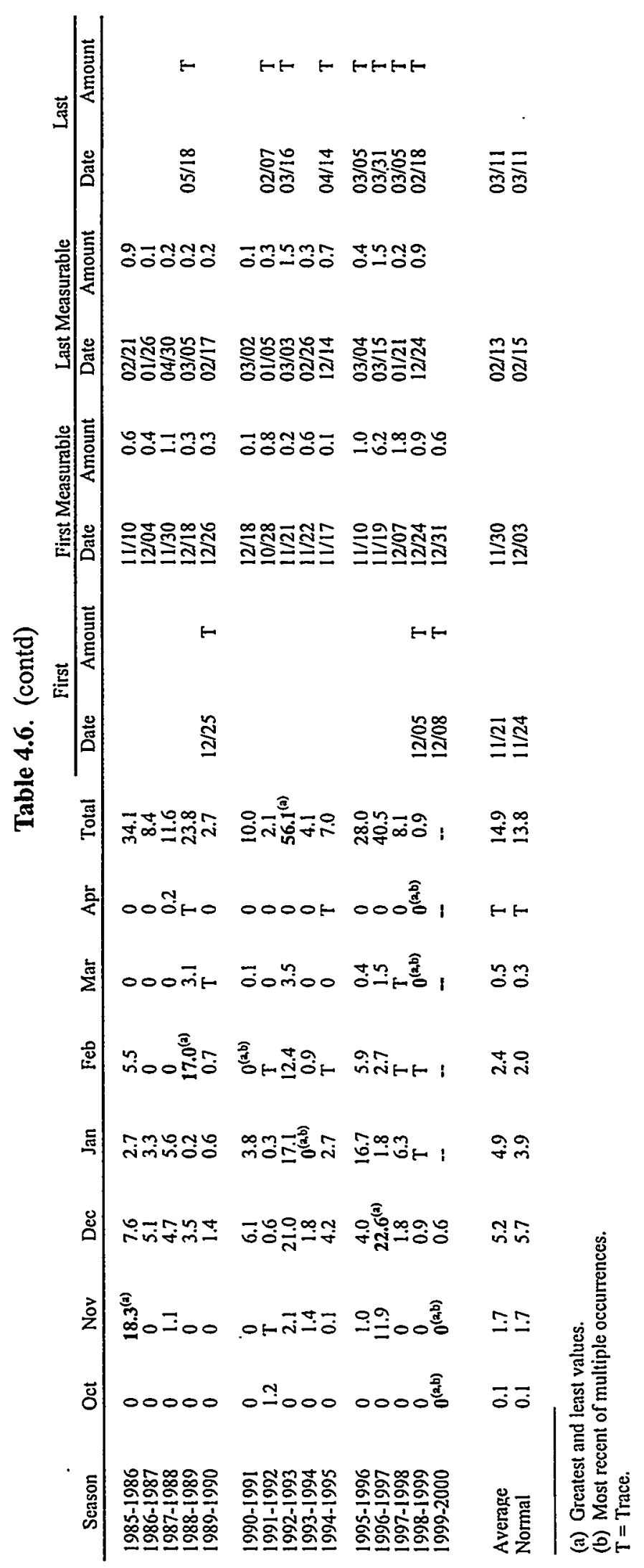


Table 4.7. Snowfall (inches) - Greatest Amount from a Single Storm

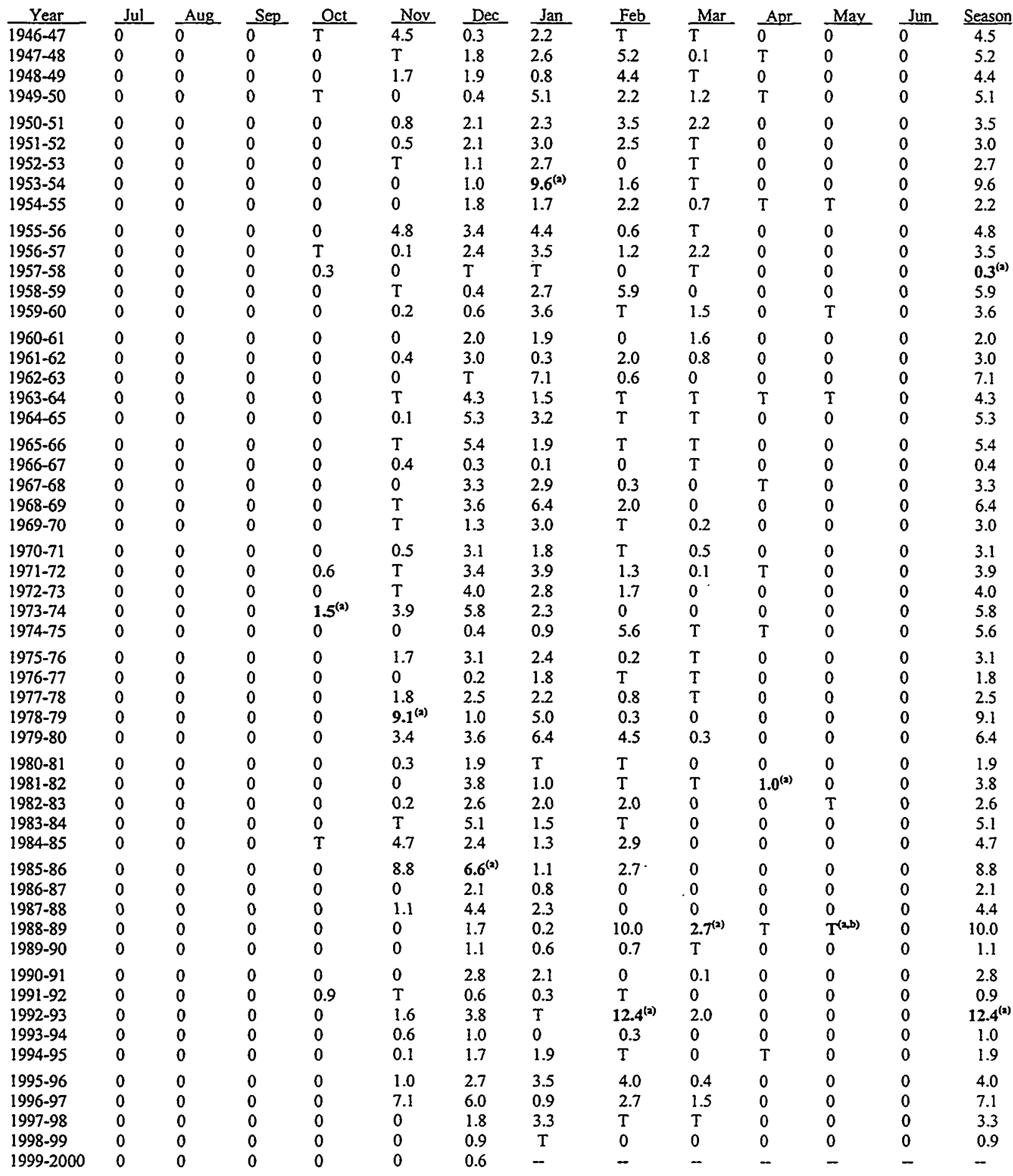

(a) Greatest value.

(b) Most recent of multiple occurrences.

$\mathrm{T}=$ Trace 
Table 4.8. Miscellaneous Snowfall Statistics, 1946 Through 1999

\begin{tabular}{|c|c|c|c|c|c|c|c|}
\hline & Oct & Nov & Dec & Jan & Feb & Mar & Season \\
\hline \multicolumn{8}{|c|}{$\begin{array}{l}\text { Average Numbet } \\
\text { of Days of Given } \\
\text { Depth at } 0400 \text { PST }\end{array}$} \\
\hline 21 inch & (a) & 1 & 7 & 9 & 4 & (a) & 22 \\
\hline$\geq 3$ inches & 0 & 1 & 3 & 6 & 3 & (a) & 12 \\
\hline$\geq 6$ inches & 0 & (a) & 1 & 3 & 1 & (a) & 5 \\
\hline$\geq 12$ inches & 0 & 0 & (a) & (a) & 0 & 0 & (a) \\
\hline
\end{tabular}

Record Greatest

Number of Days of Given Depth at 0400 PST

$\geq 1$ inch

$\geq 3$ inches

$\geq 6$ inches

$0 \quad 12(1996)$

31

31 (1985) $\quad 31$ (1969)

20 (1989)

7 (1993)

72 (1992-93)

$0 \quad .12(1996)$

31 (1985) 27 (1993)

$16(1950)$

6 (1993)

$58(1985-86)$

$\geq 12$ inches

0 . $9(1985)$

23 (1985) 25 (1993) 9 (1993)

5 (1993)

43 (1992-93)

212 inches

0

$10(1993) \quad 0$

$10(1992-93)$

\begin{tabular}{llllllll}
$\begin{array}{l}\text { Record Greatest } \\
\text { Depth }\end{array}$ & $1.5(1973)$ & $10.0(1985)$ & $15.6(1985)$ & $15.0(1993)$ & $10.0(1969)$ & $9.1(1993)$ & $15.6($ Dec 1985) \\
\hline $\begin{array}{l}\text { Geatest in } \\
24 \text { hours }\end{array}$ & $1.5(1973)$ & $8.8(1985)$ & $6.6(1985)$ & $7.1(1954)$ & $10.2(1993)$ & $2.7(1989)$ & 10.2 (Feb 1993) \\
\hline
\end{tabular}

\section{Record Consecutive}

Number of Days of

Given Depth at

0400 PST

$\geq 1$ inch

$\frac{\begin{array}{l}\text { Number } \\ \text { of Days }\end{array}}{60}$

$\geq 3$ inches

$\geq 6$ inches

$\geq 12$ inches
57

32

6

From
November 20, 1985
November 22, 1985
December 20, 1964
January 15,1993

January 15, 1993

\begin{tabular}{l} 
To \\
\hline January 18,1986 \\
January 17,1986 \\
January 20,1965 \\
January 20,1993
\end{tabular}

(a) Denotes less than $1 / 2$ day.

(b) Year of occurrence in parentheses.

PST $=$ Pacific Standard Time. 


\subsection{Normal and Maximum Daily Precipitation}

Table 4.9 contains annual maximum precipitation statistics for the time periods $1,2,3,6,12$, and 24 hours, including the dates of occurrence for each time period, 1947 through 1999. Table 4.10 contains normal and maximum values of precipitation (minimum values are not needed because every day of the year has a minimum value of 0). The normal precipitation values are based on the period 1961 through 1990; the daily maximum values are for the entire period of record (1945 through 1999). The maximum daily value for each month is noted on the table.

Climatologically speaking, the wettest period of the year is from December 14 through 24 , with each day having a normal precipitation value of 0.04 inch. Although previously stated in Section 4.2 , most days do not receive any precipitation, those that do typically receive considerably more than 0.04 inch. The driest period is from July 23 through August 11, with each day having a normal value of only a trace. October 1, 1957, recorded the greatest precipitation in one day, 1.60 inches. There have been only 4 days during the period of record that have never received measurable precipitation. However, all have received a trace. 
Table 4.9. Maximum Precipitation (inches)

\begin{tabular}{|c|c|c|c|c|c|c|c|c|c|c|c|c|}
\hline Year & ith & Date & $2 \mathrm{~h}$ & Date & $3 \mathrm{~h}$ & Date & $6 \mathrm{~h}$ & Date & $12 \mathrm{~b}$ & Date & $24 \mathrm{~h}$ & Date \\
\hline 1947 & 0.48 & $08-29$ & 0.51 & $06-07$ & 0.54 & $06-07$ & 0.67 & $09-15$ & 0.75 & $09 / 16-17$ & 0.88 & $10 / 19-20$ \\
\hline 1948 & 0.24 & $05-19$ & 0.30 & $06-11$ & 0.31 & $06 / 10-11$ & 0.50 & $01 / 6-7$ & 0.65 & $01-06$ & 1.08 & $01 / 06-07$ \\
\hline 1949 & 0.18 & $11-23$ & 0.28 & $11-23$ & 0.41 & $11-23$ & 0.60 & $11-23$ & 0.63 & $11-23$ & 0.65 & $11 / 23-24$ \\
\hline 1950 & 0.30 & $06-17$ & 0.52 & $06-17$ & 0.58 & $06-17$ & 0.87 & $06-17$ & 1.05 & $06-17$ & 1.24 & $06 / 16-17$ \\
\hline 1951 & 0.28 & $04-28$ & 0.41 & $04-28$ & 0.44 & $04-28$ & 0.45 & $06-06$ & 0.47 & $06-06$ & 0.70 & $06 / 05-06$ \\
\hline 1952 & 0.27 & $05-10$ & 0.27 & $05-10$ & 0.27 & $05-10$ & 0.29 & $06-29$ & 0.39 & $06-29$ & 0.48 & $06-29$ \\
\hline 1953 & 0.35 & $08-26$ & 0.35 & $08-26$ & 0.35 & $08-26$ & 0.44 & $01 / 08-09$ & 0.77 & $01 / 08-09$ & 0.83 & $01 / 08-09$ \\
\hline 1954 & 0.16 & 03-19 & 0.19 & $05-26$ & 0.27 & $01-16$ & 0.52 & $01-16$ & 0.72 & $01 / 15-16$ & 0.77 & $01 / 15-16$ \\
\hline 1955 & 0.13 & $12-31$ & 0.21 & $12-21$ & 0.31 & $12-21$ & 0.49 & $12-21$ & 0.61 & $12-21$ & 0.64 & $11 / 26-27$ \\
\hline 1956 & 0.16 & $06-14$ & 0.22 & $06-14$ & 0.27 & $06-14$ & $0.28^{(a)}$ & $01-15$ & 0.44 & $01 / 14-15$ & 0.73 & $01 / 14-15$ \\
\hline 1957 & 0.47 & $10-01$ & $0.88^{(\Delta)}$ & $10-01$ & $1.08^{(0)}$ & $10-01$ & $1.68^{(\mathrm{z})}$ & 10/01-02 & $1.88^{(2)}$ & $10 / 01-02$ & $1.91^{(a)}$ & $10 / 01-02$ \\
\hline 1958 & 0.43 & $06-12$ & 0.43 & $06-12$ & 0.43 & $06-12$ & 0.65 & $12 / 10-11$ & 0.88 & $12 / 10-11$ & 1.00 & $12 / 10-11$ \\
\hline 1959 & 0.18 & $05-17$ & 0.18 & $05-17^{(0)}$ & 0.23 & $09-14^{(B)}$ & 0.40 & $01-11$ & 0.54 & $01 / 11-12$ & 0.82 & $01 / 11-12$ \\
\hline 1960 & 0.22 & $03-27$ & 0.23 & $03-27$ & 0.33 & $05 / 06-07$ & 0.43 & $05 / 06-07$ & 0.44 & $05 / 06-07$ & 0.44 & $05 / 06-07$ \\
\hline 1961 & 0.21 & $02-01$ & 0.39 & 02-01 & 0.42 & 02-01 & 0.46 & $05 / 09-10$ & 0.72 & 02-01 & 0.72 & $02-01$ \\
\hline 1962 & 0.19 & $11-30$ & 0.27 & $11-30$ & 0.34 & $02-09$ & 0.40 & $10-12$ & 0.52 & $10-12$ & 0.52 & $10-12$ \\
\hline 1963 & 0.22 & $01-31$ & 0.37 & $01-31$ & 0.44 & $01-31$ & 0.54 & $01-31$ & 0.94 & $01-31 / 02-01$ & 0.98 & $01-31 / 02-01$ \\
\hline 1964 & 0.16 & $12-22$ & 0.20 & $06-08$ & 0.32 & $12-21$ & 0.42 & $12-21$ & 0.54 & $12-21$ & 0.60 & $12 / 21-22$ \\
\hline 1965 & $0.10^{(a)}$ & $05-19$ & $0.14^{(1)}$ & $11-24^{(B)}$ & $0.18^{(\text {(n) }}$ & $06-17$ & 0.29 & $06-17$ & 0.39 & $06-17$ & 0.48 & $06-17$ \\
\hline 1966 & 0.14 & $07-02$ & 0.17 & $07-02$ & 0.22 & $11-19$ & 0.37 & $11-19$ & 0.74 & $11 / 19-20$ & 0.78 & $11 / 19-20$ \\
\hline 1967 & 0.15 & $04-18$ & 0.26 & $06-21$ & 0.31 & $06-21$ & 0.31 & $06-21$ & $0.32^{(0)}$ & $06-21$ & $0.37^{(2)}$ & $04 / 17-18$ \\
\hline 1968 & 0.12 & $12-24$ & 0.21 & $12-24$ & 0.28 & $12-24$ & 0.36 & $12-24$ & 0.43 & 10-11 & 0.54 & $10 / 07-08$ \\
\hline 1969 & $0.55^{(2)}$ & $06-12$ & 0.59 & $06-12$ & 0.59 & $06-12$ & 0.60 & $06-12$ & 0.60 & $06 / 12-13$ & 0.60 & $06-12$ \\
\hline 1970 & 0.15 & $05-12$ & 0.29 & $05-12$ & 0.37 & $05-12$ & 0.47 & $05-12$ & 0.50 & $05-12$ & 0.61 & $01 / 22-23$ \\
\hline 1971 & 0.15 & $03-15$ & 0.26 & $03-25$ & 0.35 & $01-16$ & 0.48 & $03 / 25-26$ & 0.53 & $01-16$ & 0.53 & $01-16$ \\
\hline 1972 & 0.18 & $05-20$ & 0.32 & $05-30$ & 0.45 & $05-20$ & 0.80 & $05 / 20-21$ & 1.24 & $5 / 20-21$ & 1.39 & $05 / 20-21$ \\
\hline 1973 & 0.15 & $10-31$ & 0.21 & $10-31^{(0)}$ & 0.30 & $11-12$ & 0.53 & $10-31$ & 0.64 & $10-31$ & 0.64 & $11 / 11-12$ \\
\hline 1974 & 0.45 & $07-19$ & 0.45 & $07-19$ & 0.45 & $07-19$ & 0.45 & $07-19$ & 0.45 & $07-19$ & 0.45 & $07-19$ \\
\hline 1975 & 0.30 & 08-18 & 0.47 & $08-18$ & 0.55 & $08-18$ & 0.69 & $08-18$. & 0.69 & $08-18$ & 0.69 & $08-18$ \\
\hline 1976 & 0.32 & $08-07$ & 0.33 & $08-07$ & 0.33 & $08-07$ & 0.33 & $08-07$ & 0.33 & $08-07$ & 0.40 & $08 / 24-25$ \\
\hline 1977 & 0.16 & $12-13$ & 0.28 & $12-13$ & 0.36 & $12-13$ & 0.61 & $12-13$ & 0.75 & $12-13$ & 0.89 & $08 / 29-30$ \\
\hline 1978 & 0.15 & $04-27$ & 0.22 & $04-27$ & 0.23 & $04-27$ & 0.31 & $11-18^{(B)}$ & 0.58 & $11 / 18-19$ & 0.67 & $11 / 18-19$ \\
\hline 1979 & 0.11 & $04-17$ & 0.18 & $03-27$ & 0.22 & $03-27$ & 0.29 & $03-27$ & 0.40 & $03-27$ & 0.42 & $11-16+$ \\
\hline 1980 & 0.14 & $12-25$ & 0.24 & $04-20$ & 0.29 & $04-20$ & 0.47 & $05 / 25-26$ & 0.74 & $09-13$ & 0.90 & $05 / 25-26$ \\
\hline 1981 & 0.22 & $05-25$ & 0.34 & $05-25$ & 0.38 & $05-25$ & 0.73 & $05-25$ & 0.74 & $05-25$ & 0.74 & $05-25$ \\
\hline 1982 & 0.22 & $07-07$ & 0.33 & $11-18$ & 0.40 & $11-18$ & 0.64 & $10-28$ & 0.95 & $10 / 28-29$ & 0.97 & $10 / 28-29$ \\
\hline 1983 & 0.24 & $09-01$ & 0.31 & $11-10$ & 0.39 & $11-10$ & 0.45 & $11 / 23-24$ & 0.60 & $11 / 23-24$ & 0.66 & $11-10$ \\
\hline 1984 & 0.20 & $06-28$ & 0.38 & $03-20$ & 0.39 & $03-20$ & 0.48 & $03 / 20-21$ & 0.51 & $03 / 20-21$ & 0.53 & $03 / 20-21$ \\
\hline 1985 & 0.14 & $12-07$ & 0.22 & $11-21$ & 0.29 & $11-21$ & 0.46 & $11-21$ & 0.52 & $11 / 21-22$ & 0.52 & $11 / 21-22$ \\
\hline 1986 & 0.24 & $09-15$ & 0.43 & $09-15$ & 0.45 & $09-15$ & 0.47 & $09-15$ & 0.47 & $09-15$ & 0.54 & $09-15$ \\
\hline 1987 & 0.21 & 07-09 & 0.24 & 07.09 & 0.27 & 07-09 & 0.31 & $12-09$ & 0.34 & $12-09$ & 0.55 & $12-09$ \\
\hline 1988 & 0.31 & $04-28$ & 0.42 & $04-28$ & 0.42 & 04.28 & 0.42 & $04-28$ & 0.48 & $04-28$ & 0.49 & $04 / 27-28$ \\
\hline 1989 & 0.16 & $04-25$ & 0.25 & $04-25$ & 0.26 & $04-25$ & .0 .31 & $05-23$ & 0.38 & $02 / 16-17$ & 0.56 & $02 / 16-17$ \\
\hline 1990 & 0.25 & $06-06$ & 0.33 & $08-21^{(b)}$ & 0.43 & $08-21$ & 0.66 & $08-21$ & 0.77 & $08 / 20-21$ & 0.77 & $08 / 20-21$ \\
\hline 1991 & 0.49 & $06-29$ & 0.50 & $06-29$ & 0.51 & $06-29$ & 0.51 & $06-29$ & 0.53 & $06-29$ & 0.59 & $06 / 05-06$ \\
\hline 1992 & 0.17 & $06-12$ & 0.25 & $06-12$ & 0.31 & $06-12$ & 0.44 & $06-12$ & 0.70 & $06-12$ & 0.79 & $06-12$ \\
\hline 1993 & 0.32 & $07-17$ & 0.45 & $07-17$ & 0.55 & $07-17$ & 0.82 & $07-17$ & 1.01 & $07 / 16-17$ & 1.39 & $07 / 16-17$ \\
\hline 1994 & 0.27 & $05-15$ & 0.32 & $10-14$ & 0.37 & $05-15$ & 0.49 & $05-15$ & 0.58 & $05-15$ & 0.59 & $05 / 14-15$ \\
\hline 1995 & 0.48 & $05-09$ & 0.53 & $05-09$ & 0.53 & $05-09$ & 0.55 & $12-12$ & 0.65 & $12-12$ & 1.04 & $12 / 11-12$ \\
\hline 1996 & 0.16 & $12-31^{(0)}$ & 0.29 & $12-29$ & 0.40 & $12-29$ & 0.65 & $12-29$ & 0.90 & $11-19$ & 1.70 & $11 / 18-19$ \\
\hline 1997 & 0.27 & $10-08$ & 0.36 & $10-08$ & 0.40 & $10-08$ & 0.48 & $01-31$ & 0.57 & $11-07$ & 0.70 & $01-17$ \\
\hline 1998 & 0.19 & $11-05$ & 0.29 & $11-05$ & 0.36 & $11-05$ & 0.49 & $11-05$ & 0.62 & $11-05$ & 0.62 & $11-05$ \\
\hline 1999 & 0.40 & $08-05$ & 0.40 & $08-05$ & 0.47 & $08-05$ & 0.48 & 08.05 & 0.48 & $08-05$ & 0.51 & $01 / 22-23$ \\
\hline
\end{tabular}

(a) Greatest and least values.

(b) Last of multiple occurrences. 
Table 4.10. Normal and Maximum Daily Precipitation (inches)

\begin{tabular}{|c|c|c|c|c|c|c|c|}
\hline \multirow[b]{3}{*}{ Day } & \multicolumn{5}{|c|}{ Normal Period (1961-1990) } & \multicolumn{2}{|c|}{$\begin{array}{c}\text { Historical Period } \\
(1945-1999)\end{array}$} \\
\hline & \multirow[b]{2}{*}{$\underline{\text { Normal }}$} & \multicolumn{2}{|c|}{ Number of Years } & \multirow[b]{2}{*}{ Maximum } & \multirow[b]{2}{*}{ Year } & \multirow{3}{*}{ Maximum } & \multirow{3}{*}{ Year } \\
\hline & & W/Meas. & W/Trace & & & & \\
\hline \multicolumn{6}{|c|}{ January } & & \\
\hline 1 & 0.02 & 5 & 10 & 0.20 & 1987 & 0.20 & 1987 \\
\hline 2 & 0.02 & 9 & 9 & 0.17 & 1983 & 0.27 & 1953 \\
\hline 3 & 0.02 & 8 & 7 & 0.28 & 1966 & 0.28 & 1966 \\
\hline 4 & 0.02 & 7 & 10 & 0.17 & 1976 & 0.25 & 1956 \\
\hline 5 & 0.02 & 9 & 8 & 0.23 & 1966 & 0.23 & 1966 \\
\hline 6 & 0.02 & 4 & 8 & 0.50 & 1983 & 0.87 & 1948 \\
\hline 7 & 0.02 & 8 & 5 & 0.31 & 1990 & 0.31 & 1990 \\
\hline 8 & 0.02 & 7 & 9 & 0.25 & 1978 & 0.59 & 1953 \\
\hline 9 & 0.02 & 8 & 9 & 0.20 & $1980^{(a)}$ & 0.41 & $1995^{(a)}$ \\
\hline 10 & 0.03 & 10 & 5 & 0.20 & $1979^{(2)}$ & 0.22 & 1995 \\
\hline 11 & 0.03 & 4 & 10 & 0.17 & 1980 & 0.48 & 1959 \\
\hline 12 & 0.03 & 9 & 10 & 0.32 & 1973 & 0.58 & 1958 \\
\hline 13 & 0.03 & 10 & 8 & 0.33 & 1980 & 0.37 & 1950 \\
\hline 14 & 0.03 & 10 & 3 & 0.43 & 1978 & 0.43 & 1978 \\
\hline 15 & 0.03 & 7 & 5 & 0.18 & 1968 & 0.34 & 1956 \\
\hline 16 & 0.03 & 10 & 6 & 0.53 & 1971 & 0.70 & 1954 \\
\hline 17 & 0.02 & 5 & 7 & 0.10 & 1986 & 0.31 & 1997 \\
\hline 18 & 0.02 & 7 & 8 & 0.25 & 1974 & 0.28 & 1996 \\
\hline 19 & 0.02 & 8 & 1 & 0.32 & 1970 & 0.39 & 1950 \\
\hline 20 & 0.02 & 5 & 6 & 0.26 & 1985 & 0.32 & 1953 \\
\hline 21 & 0.02 & 6 & 5 & 0.15 & 1984 & 0.16 & $1997^{(2)}$ \\
\hline 22 & 0.02 & 6 & 7 & 0.54 & 1970 & 0.54 & 1970 \\
\hline 23 & 0.03 & 8 & 9 & 0.27 & 1965 & 0.27 & 1965 \\
\hline 24 & 0.03 & 8 & 6 & 0.22 & 1970 & 0.26 & 1996 \\
\hline 25 & 0.03 & 6 & 6 & 0.72 & 1975 & 0.72 & 1975 \\
\hline 26 & 0.03 & 8 & 4 & 0.36 & 1970 & 0.36 & 1970 \\
\hline 27 & 0.03 & 6 & 6 & 0.20 & 1981 & 0.32 & 1954 \\
\hline 28 & 0.03 & 6 & 7 & 0.19 & 1986 & 0.19 & $1995^{(2)}$ \\
\hline 29 & 0.03 & 9 & 4 & 0.31 & 1965 & 0.33 & 1958 \\
\hline 30 & 0.03 & 8 & 8 & 0.12 & 1986 & 0.24 & 1995 \\
\hline 31 & 0.03 & 9 & 10 & 0.94 & 1963 & $0.94^{(b)}$ & $1963^{(b)}$ \\
\hline \multicolumn{8}{|c|}{ February } \\
\hline 1 & 0.03 & 8 & 4 & 0.72 & 1961 & 0.72 & 1961 \\
\hline 2 & 0.02 & 5 & 8 & 0.26 & 1963 & 0.26 & 1963 \\
\hline 3 & 0.02 & 5 & 6 & 0.06 & 1990 & 0.31 & 1998 \\
\hline 4 & 0.02 & 5 & 5 & 0.28 & 1975 & 0.28 & 1975 \\
\hline 5 & 0.02 & 7 & 7 & 0.07 & 1978 & 0.15 & 1953 \\
\hline 6 & 0.02 & 10 & 2 & 0.18 & 1961 & 0.18 & 1961 \\
\hline 7 & 0.02 & 5 & 4 & 0.27 & 1985 & 0.27 & 1985 \\
\hline 8 & 0.02 & 5 & 8 & 0.12 & 1985 & 0.12 & 1985 \\
\hline 9 & 0.02 & 9 & 5 & 0.37 & 1962 & 0.43 & 1959 \\
\hline 10 & 0.02 & 5 & 5 & 0.64 & 1961 & 0.64 & 1961 \\
\hline 11 & 0.02 & 10 & 3 & 0.30 & 1969 & 0.30 & 1969 \\
\hline 12 & 0.03 & 9 & 6 & 0.20 & 1986 & 0.42 & 1958 \\
\hline 13 & 0.02 & 10 & 6 & 0.21 & 1981 & 0.21 & 1981 \\
\hline 14 & 0.03 & 5 & 11 & 0.39 & 1986 & 0.39 & 1986 \\
\hline 15 & 0.03 & 12 & 7 & 0.30 & 1970 & 0.30 & 1970 \\
\hline 16 & 0.03 & 7 & 7 & 0.42 & 1989 & 0.42 & 1989 \\
\hline 17 & 0.03 & 10 & 7 & 0.42 & 1989 & 0.42 & 1989 \\
\hline 18 & 0.03 & 9 & 2 & 0.34 & 1983 & 0.34 & $1983^{(0)}$ \\
\hline 19 & 0.02 & 9 & 5 & 0.14 & 1989 & $0.78^{(\mathrm{b})}$ & $1993^{(b)}$ \\
\hline
\end{tabular}


Table 4.10. (contd)

\begin{tabular}{|c|c|c|c|c|c|c|c|}
\hline \multirow[b]{3}{*}{ Day } & \multicolumn{5}{|c|}{ Normal Period (1961-1990) } & \multicolumn{2}{|c|}{$\begin{array}{c}\text { Historical Period } \\
(1945-1999)\end{array}$} \\
\hline & \multirow[b]{2}{*}{ Normal } & \multicolumn{2}{|c|}{ Number of Years } & \multirow[b]{2}{*}{ Maximum } & \multirow[b]{2}{*}{ Year } & \multirow[b]{2}{*}{ Maximum } & \multirow[b]{2}{*}{ Year } \\
\hline & & W/Meas. & W/Trace & & & & \\
\hline 20 & 0.02 & 5 & 3 & 0.18 & 1984 & 0.18 & 1984 \\
\hline 21 & 0.02 & 8 & 3 & 0.20 & 1986 & 0.36 & 1956 \\
\hline 22 & 0.02 & 4 & 5 & 0.15 & 1989 & 0.21 & 1949 \\
\hline 23 & 0.02 & 5 & 4 & 0.22 & 1968 & 0.22 & 1968 \\
\hline 24 & 0.02 & 5 & 5 & 0.10 & 1981 & 0.33 & 1950 \\
\hline 25 & 0.02 & 9 & 3 & 0.22 & 1983 & 0.25 & 1948 \\
\hline 26 & 0.02 & 6 & 4 & 0.30 & 1976 & 0.30 & 1976 \\
\hline 27 & 0.02 & 9 & 3 & 0.24 & 1980 & 0.32 & 1999 \\
\hline 28 & 0.02 & 7 & 4 & 0.33 & 1977 & 0.33 & 1977 \\
\hline 29 & 0.02 & 1 & 1 & 0.04 & 1984 & 0.04 & 1984 \\
\hline \multicolumn{8}{|c|}{ March } \\
\hline 1 & 0.02 & 8 & 4 & 0.15 & 1972 & 0.15 & 1972 \\
\hline 2 & 0.01 & 4 & 8 & 0.07 & 1989 & 0.20 & $1991^{(2)}$ \\
\hline 3 & 0.01 & 3 & 7 & 0.15 & 1977 & 0.15 & $1991^{(2)}$ \\
\hline 4 & 0.01 & 9 & 6 & 0.16 & 1985 & 0.44 & 1957 \\
\hline 5 & 0.01 & 6 & 7 & 0.23 & 1989 & 0.23 & 1989 \\
\hline 6 & 0.02 & 7 & 3 & 0.07 & $1971^{(2)}$ & 0.24 & 1957 \\
\hline 7 & 0.02 & 6 & 4 & 0.21 & 1986 & 0.21 & 1986 \\
\hline 8 & 0.02 & 8 & 2 & 0.19 & 1988 & 0.23 & 1951 \\
\hline 9 & 0.02 & 6 & 6 & 0.31 & 1989 & 0.42 & 1995 \\
\hline 10 & 0.02 & 11 & 4 & 0.06 & $1980^{(2)}$ & 0.21 & 1995 \\
\hline 11 & 0.02 & 8 & 6 & 0.24 & 1989 & 0.24 & 1989 \\
\hline 12 & 0.02 & 9 & 6 & 0.42 & 1987 & 0.42 & 1987 \\
\hline 13 & 0.02 & 7 & 8 & 0.35 & 1983 & 0.35 & 1983 \\
\hline 14 & 0.02 & 9 & 3 & 0.14 & 1970 & 0.16 & 1995 \\
\hline 15 & 0.02 & 7 & 4 & 0.18 & 1987 & 0.25 & 1949 \\
\hline 16 & 0.01 & 7 & 5 & 0.22 & 1989 & 0.34 & $1997^{(2)}$ \\
\hline 17 & 0.01 & 3 & 7 & 0.05 & 1967 & .0 .16 & 1949 \\
\hline 18 & 0.01 & 7 & 5 & 0.04 & 1989 & 0.25 & 1949 \\
\hline 19 & 0.01 & 3 & 4 & 0.12 & 1987 & 0.12 & 1987 \\
\hline 20 & 0.01 & 5 & 4 & 0.43 & 1984 & 0.43 & 1984 \\
\hline 21 & 0.01 & 5 & 2 & 0.10 & $1984^{(2)}$ & 0.18 & 1958 \\
\hline 22 & 0.02 & 4 & 8 & 0.22 & 1961 & 0.22 & 1961 \\
\hline 23 & 0.02 & 7 & 4 & 0.26 & 1986 & 0.26 & 1986 \\
\hline 24 & 0.02 & 4 & 2 & 0.12 & 1961 & $0.52^{(b)}$ & $1991^{(b)}$ \\
\hline 25 & 0.02 & 9 & 3 & 0.43 & 1971 & 0.43 & 1971 \\
\hline 26 & 0.02 & 5 & 6 & 0.50 & 1981 & 0.50 & 1981 \\
\hline 27 & 0.02 & 5 & 4 & 0.42 & 1979 & 0.42 & 1979 \\
\hline 28 & 0.02 & 5 & 3 & 0.13 & 1982 & 0.13 & 1982 \\
\hline 29 & 0.01 & 8 & 1 & 0.15 & 1983 & 0.15 & 1983 \\
\hline 30 & 0.01 & 4 & 7 & 0.23 & 1974 & 0.23 & 1974 \\
\hline 31 & 0.01 & 4 & 6 & 0.14 & 1976 & 0.26 & 1996 \\
\hline \multicolumn{8}{|c|}{ April } \\
\hline 1 & 0.01 & 3 & 12 & 0.18 & 1983 & 0.22 & 1958 \\
\hline 2 & 0.01 & 6 & 3 & 0.10 & 1979 & 0.18 & 1948 \\
\hline 3 & 0.01 & 2 & 3 & 0.03 & 1963 & 0.18 & 1947 \\
\hline 4 & 0.01 & 5 & 9 & 0.13 & 1984 & 0.18 & 1948 \\
\hline 5 & 0.01 & 6 & 6 & 0.44 & 1969 & 0.44 & 1969 \\
\hline 6 & 0.01 & 6 & 3 & 0.36 & 1982 & 0.36 & 1982 \\
\hline 7 & 0.01 & 3 & 1 & 0.22 & 1984 & 0.30 & 1953 \\
\hline 8 & 0.01 & 5 & 2 & 0.17 & 1976 & 0.18 & 1991 \\
\hline 9 & 0.01 & 3 & 10 & 0.12 & 1980 & 0.32 & 1992 \\
\hline
\end{tabular}


Table 4.10. (contd)

\begin{tabular}{|c|c|c|c|c|c|c|c|}
\hline \multirow[b]{3}{*}{ Day } & \multicolumn{5}{|c|}{ Normal Period (1961-1990) } & \multicolumn{2}{|c|}{$\begin{array}{c}\text { Historical Period } \\
(1945-1999)\end{array}$} \\
\hline & \multirow[b]{2}{*}{ Normal } & \multicolumn{2}{|c|}{ Number of Years } & \multirow[b]{2}{*}{ Maximum } & \multirow[b]{2}{*}{ Year } & \multirow[b]{2}{*}{ Maximum } & \multirow[b]{2}{*}{ Year } \\
\hline & & W/Meas. & W/Trace & & & & \\
\hline 10 & 0.01 & 2 & 6 & 0.03 & 1969 & 0.10 & 1958 \\
\hline 11 & 0.01 & 4 & 9 & 0.23 & 1982 & 0.23 & 1982 \\
\hline 12 & 0.01 & 4 & 5 & 0.08 & 1961 & 0.36 & 1995 \\
\hline 13 & 0.01 & 1 & 3 & 0.01 & 1990 & 0.39 & 1992 \\
\hline 14 & 0.01 & 4 & 4 & 0.17 & $1975^{(2)}$ & 0.17 & $1975^{(2)}$ \\
\hline 15 & 0.01 & 3 & 8 & 0.09 & 1962 & 0.17 & 1991 \\
\hline 16 & 0.02 & 4 & 7 & 0.08 & $1979^{(2)}$ & 0.11 & 1948 \\
\hline 17 & 0.02 & 6 & 6 & 0.36 & 1988 & 0.36 & 1988 \\
\hline 18 & 0.02 & 4 & 6 & 0.31 & 1967 & 0.31 & 1967 \\
\hline 19 & 0.02 & 7 & 9 & 0.41 & 1970 & 0.41 & 1970 \\
\hline 20 & 0.02 & 6 & 6 & 0.56 & 1980 & $0.56^{(b)}$ & $1980^{(0)}$ \\
\hline 21 & 0.02 & 2 & 7 & 0.07 & 1989 & 0.07 & 1989 \\
\hline 22 & 0.02 & 7 & 6 & 0.12 & 1974 & 0.28 & 1996 \\
\hline 23 & 0.01 & 6 & 5 & 0.22 & 1974 & 0.22 & 1974 \\
\hline 24 & 0.01 & 4 & 8 & 0.22 & 1975 & 0.22 & 1975 \\
\hline 25 & 0.02 & 3 & 6 & 0.35 & 1989 & 0.35 & 1989 \\
\hline 26 & 0.02 & 2 & 7 & 0.04 & 1989 & 0.25 & 1955 \\
\hline 27 & 0.02 & 7 & 5 & 0.28 & $1989^{(2)}$ & 0.34 & 1995 \\
\hline 28 & 0.02 & 6 & 5 & 0.48 & 1988 & 0.51 & 1951 \\
\hline 29 & 0.02 & 2 & 3 & 0.30 & 1961 & 0.30 & 1961 \\
\hline \multirow[t]{2}{*}{30} & 0.02 & 6 & 4 & 0.12 & 1984 & 0.12 & 1984 \\
\hline & & \multicolumn{4}{|c|}{ May } & & \\
\hline 1 & 0.02 & 5 & 6 & 0.19 & 1984 & 0.19 & 1984 \\
\hline 2 & 0.01 & 5 & 1 & 0.17 & 1975 & 0.17 & 1975 \\
\hline 3 & 0.01 & 4 & 3 & 0.29 & 1977 & 0.29 & 1977 \\
\hline 4 & 0.01 & 6 & 5 & 0.10 & 1967 & 0.10 & 1967 \\
\hline 5 & 0.02 & 8 & 3 & 0.28 & 1963 & 0.28 & 1963 \\
\hline 6 & 0.02 & 4 & 6 & 0.20 & 1986 & 0.20 & 1986 \\
\hline 7 & 0.02 & 3 & 1 & 0.39 & 1983 & 0.39 & 1983 \\
\hline 8 & 0.02 & 5 & 7 & 0.55 & 1972 & 0.55 & 1972 \\
\hline 9 & 0.02 & 5 & 5 & 0.25 & 1961 & 0.53 & 1995 \\
\hline 10 & 0.02 & 6 & 7 & 0.39 & 1961 & 0.39 & 1961 \\
\hline 11 & 0.02 & 4 & 4 & 0.19 & 1967 & 0.39 & 1951 \\
\hline 12 & 0.02 & 4 & 7 & 0.50 & 1970 & 0.50 & 1970 \\
\hline 13 & 0.01 & 4 & 2 & 0.11 & 1985 & 0.15 & 1952 \\
\hline 14 & 0.01 & 4 & 6 & 0.25 & 1978 & 0.25 & 1978 \\
\hline 15 & 0.01 & 3 & 6 & 0.06 & 1975 & 0.58 & 1994 \\
\hline 16 & 0.01 & 0 & 8 & $\mathrm{~T}$ & $1988^{(2)}$ & 0.14 & 1991 \\
\hline 17 & 0.01 & 4 & 3 & 0.13 & 1982 & 0.25 & 1959 \\
\hline 18 & 0.01 & 5 & 2 & 0.13 & 1981 & 0.13 & 1981 \\
\hline 19 & 0.01 & 7 & 3 & 0.15 & 1965 & 0.55 & 1948 \\
\hline 20 & 0.02 & 4 & 1 & 0.70 & 1972 & 0.70 & 1972 \\
\hline 21 & 0.02 & 3 & 4 & 0.69 & 1972 & 0.69 & 1972 \\
\hline 22 & 0.02 & 6 & 5 & 0.12 & 1984 & 0.12 & 1984 \\
\hline 23 & 0.03 & 5 & 6 & 0.33 & 1990 & 0.33 & 1990 \\
\hline 24 & 0.03 & 4 & 5 & 0.51 & 1962 & 0.51 & 1962 \\
\hline 25 & 0.02 & 6 & 2 & 0.74 & 1981 & 0.74 & 1981 \\
\hline 26 & 0.02 & 4 & 6 & 0.79 & 1980 & $0.79^{(0)}$ & $1980^{(b)}$ \\
\hline 27 & 0.02 & 6 & 2 & 0.11 & 1990 & 0.11 & 1990 \\
\hline 28 & 0.02 & 5 & 7 & 0.28 & 1988 & 0.28 & 1988 \\
\hline 29 & 0.02 & 5 & 3 & 0.11 & 1961 & 0.11 & 1961 \\
\hline 30 & 0.01 & 6 & 1 & 0.14 & 1987 & 0.14 & 1987 \\
\hline 31 & 0.02 & 2 & 5 & 0.35 & 1971 & 0.35 & 1971 \\
\hline
\end{tabular}


Table 4.10. (contd)

\begin{tabular}{|c|c|c|c|c|c|c|c|}
\hline \multirow[b]{3}{*}{ Day } & \multicolumn{5}{|c|}{ Normal Period (1961-1990) } & \multicolumn{2}{|c|}{$\begin{array}{c}\text { Historical Period } \\
(1945-1999)\end{array}$} \\
\hline & \multirow[b]{2}{*}{ Normal } & \multicolumn{2}{|c|}{ Number of Years } & \multirow[b]{2}{*}{ Maximum } & \multirow{3}{*}{ Year } & \multirow{3}{*}{ Maximum } & \multirow{3}{*}{ Year } \\
\hline & & W/Meas. & W/Trace & & & & \\
\hline \multicolumn{5}{|c|}{ June } & & & \\
\hline 1 & 0.02 & 3 & 5 & 0.29 & 1977 & 0.29 & 1977 \\
\hline 2 & 0.02 & 5 & 6 & 0.12 & 1966 & 0.12 & 1966 \\
\hline 3 & 0.02 & 6 & 9 & 0.30 & 1971 & 0.30 & 1971 \\
\hline 4 & 0.02 & 6 & 3 & 0.25 & 1984 & 0.45 & 1951 \\
\hline 5 & 0.02 & 4 & 4 & 0.14 & 1981 & 0.49 & 1991 \\
\hline 6 & 0.02 & 6 & 3 & 0.36 & 1990 & 0.54 & 1951 \\
\hline 7 & 0.01 & 5 & 5 & 0.15 & 1972 & 0.71 & 1947 \\
\hline 8 & 0.01 & 6 & 6 & 0.49 & 1964 & 0.49 & 1964 \\
\hline 9 & 0.02 & 3 & 4 & 0.07 & 1963 & 0.22 & 1948 \\
\hline 10 & 0.02 & 2 & 7 & 0.08 & 1983 & 0.14 & 1956 \\
\hline 11 & 0.01 & 3 & 5 & 0.13 & 1961 & 0.39 & 1950 \\
\hline 12 & 0.01 & 4 & 8 & 0.60 & 1969 & 0.79 & 1992 \\
\hline 13 & 0.01 & 6 & 4 & 0.35 & 1980 & 0.49 & 1948 \\
\hline 14 & 0.01 & 4 & 2 & 0.04 & 1983 & 0.37 & 1956 \\
\hline 15 & 0.01 & 4 & 1 & 0.15 & 1964 & 0.15 & 1964 \\
\hline 16 & 0.01 & 2 & 5 & 0.14 & 1980 & 0.18 & 1948 \\
\hline 17 & 0.01 & 2 & 5 & 0.48 & 1965 & $1.09^{(b)}$ & $1950^{(b)}$ \\
\hline 18 & 0.01 & 2 & 5 & 0.07 & 1983 & 0.09 & 1994 \\
\hline 19 & 0.01 & 1 & 3 & 0.01 & 1983 & 0.29 & 1998 \\
\hline 20 & 0.01 & 5 & 2 & 0.24 & 1984 & 0.24 & 1984 \\
\hline 21 & 0.01 & 5 & 2 & 0.32 & 1967 & 0.32 & 1967 \\
\hline 22 & 0.01 & 4 & 7 & 0.14 & 1971 & 0.14 & 1971 \\
\hline 23 & 0.01 & 7 & 2 & 0.17 & 1963 & 0.17 & 1963 \\
\hline 24 & 0.01 & 5 & 3 & 0.21 & 1972 & 0.21 & 1972 \\
\hline 25 & 0.01 & 3 & 4 & 0.02 & 1980 & 0.03 & 1954 \\
\hline 26 & 0.01 & 2 & 6 & 0.27 & 1982 & 0.27 & 1982 \\
\hline 27 & 0.01 & 4 & 3 & 0.37 & 1983 & 0.37 & 1983 \\
\hline 28 & 0.01 & 2 & 8 & 0.23 & 1984 & 0.24 & 1992 \\
\hline 29 & 0.01 & 4 & 2 & 0.16 & 1984 & 0.53 & 1991 \\
\hline 30 & 0.01 & 1 & 1 & 0.06 & 1976 & 0.06 & 1976 \\
\hline \multicolumn{8}{|c|}{ July } \\
\hline 1 & 0.01 & 5 & 3 & 0.31 & 1966 & 0.31 & 1966 \\
\hline 2 & 0.01 & 3 & 6 & 0.34 & 1966 & 0.34 & 1966 \\
\hline 3 & 0.01 & 3 & 3 & 0.31 & 1978 & 0.31 & 1978 \\
\hline 4 & 0.01 & 3 & 4 & 0.10 & 1986 & 0.10 & 1986 \\
\hline 5 & 0.01 & 1 & 3 & 0.19 & 1981 & 0.36 & 1951 \\
\hline 6 & 0.01 & $i$ & 3 & 0.02 & 1979 & 0.04 & 1993 \\
\hline 7 & 0.01 & 3 & 2 & 0.30 & 1963 & 0.30 & 1963 \\
\hline 8 & 0.01 & 5 & 3 & 0.14 & 1974 & 0.20 & 1995 \\
\hline 9 & 0.01 & 3 & 2 & 0.27 & 1987 & 0.27 & 1987 \\
\hline 10 & 0.01 & 2 & 5 & 0.05 & 1972 & 0.16 & 1954 \\
\hline 11 & 0.01 & 2 & 4 & 0.04 & 1979 & 0.04 & 1979 \\
\hline 12 & $\mathrm{~T}$ & 0 & 4 & $T$ & $1982^{(2)}$ & $\mathrm{T}$ & $1982^{(0)}$ \\
\hline 13 & $\mathrm{~T}$ & 2 & 5 & 0.28 & 1975 & 0.28 & 1975 \\
\hline 14 & $T$ & 1 & 2 & 0.03 & 1966 & 0.05 & 1957 \\
\hline 15 & $T$ & 2 & 2 & 0.04 & 1975 & 0.08 & 1991 \\
\hline 16 & $T$ & 4 & 3 & 0.07 & 1966 & 0.50 & 1993 \\
\hline 17 & $T$ & 3 & 1 & 0.05 & $1987^{(2)}$ & $0.89^{(b)}$ & $1993^{(b)}$ \\
\hline 18 & $T$ & 2 & 2 & 0.12 & 1987 & 0.12 & 1987 \\
\hline 19 & $T$ & 2 & 2 & 0.45 & 1974 & 0.45 & 1974 \\
\hline 20 & $T$ & 2 & 2 & 0.09 & 1965 & 0.09 & 1965 \\
\hline 21 & $T$ & 1 & 2 & 0.02 & 1965 & 0.02 & 1965 \\
\hline
\end{tabular}


Table 4.10. (contd)

$\begin{array}{ll}\text { Day } & \text { Normal } \\ 22 & \text { T } \\ 23 & \text { T } \\ 24 & \text { T } \\ 25 & \text { T } \\ 26 & \text { T } \\ 27 & \text { T } \\ 28 & \text { T } \\ 29 & \text { T } \\ 30 & \text { T } \\ 31 & \text { T }\end{array}$

Normal Period (1961-1990)

Number of Years

$\begin{array}{lll}0 & & \text { W/Teas. } \\ 2 & \\ 2 & \\ 3 & \\ 0 & \\ 1 & \\ 1 & \\ 1 & \\ 0 & \\ 1 & \end{array}$

W/Trace
2
0
2
2
1
1
3
1
3
2

\begin{tabular}{lll}
\cline { 1 - 1 } Maximum & Year \\
\cline { 1 - 1 }$T$ & & $1987^{(\text {(a) }}$ \\
0.15 & 1961 \\
0.06 & 1990 \\
0.23 & 1983 \\
$\mathrm{~T}$ & 1978 \\
0.02 & 1983 \\
0.06 & 1984 \\
0.01 & 1964 \\
$\mathrm{~T}$ & $1987^{(2)}$ \\
0.12 & 1985
\end{tabular}

\begin{tabular}{|c|c|}
\hline Maximum & Year \\
\hline $\mathrm{T}$ & $1993^{(2)}$ \\
\hline 0.28 & 1992 \\
\hline 0.07 & 1955 \\
\hline 0.23 & 1983 \\
\hline 0.22 & 1955 \\
\hline 0.31 & 1947 \\
\hline 0.28 & 1947 \\
\hline 0.05 & 1997 \\
\hline$T$ & $1997^{(2)}$ \\
\hline 0.16 & 1998 \\
\hline
\end{tabular}

\begin{tabular}{|c|c|c|c|c|c|c|c|}
\hline \multicolumn{8}{|c|}{ August } \\
\hline 1 & $T$ & 2 & 1 & 0.08 & $1976^{(2)}$ & 0.08 & $1976^{(a)}$ \\
\hline 2 & I & 1 & 3 & 0.01 & 1976 & 0.01 & $1996^{(a)}$ \\
\hline 3 & $T$ & 1 & 2 & 0.29 & 1962 & 0.29 & 1962 \\
\hline 4 & $T$ & 1 & 2 & 0.01 & 1985 & 0.04 & 1948 \\
\hline 5 & $T$ & 0 & 3 & $\mathrm{~T}$ & $1984^{(2)}$ & 0.48 & 1999 \\
\hline 6 & $T$ & 1 & 2 & 0.11 & 1976 & 0.11 & 1976 \\
\hline 7 & $T$ & 2 & 1 & 0.33 & 1976 & 0.33 & 1976 \\
\hline 8 & $T$ & 0 & 4 & $\mathrm{~T}$ & $1989^{(2)}$ & 0.08 & 1952 \\
\hline 9 & $T$ & 2 & 2 & 0.10 & 1982 & 0.10 & 1982 \\
\hline 10 & $\mathrm{~T}$ & 0 & 3 & $T$ & $1984^{(2)}$ & 0.01 & 1947 \\
\hline 11 & $T$ & 1 & 4 & 0.01 & 1983 & 0.11 & 1947 \\
\hline 12 & 0.01 & 3 & 5 & 0.18 & 1962 & 0.18 & 1962 \\
\hline 13 & 0.01 & 4 & 2 & 0.04 & $1987^{(2)}$ & 0.06 & 1995 \\
\hline 14 & 0.01 & 5 & 3 & 0.09 & 1979 & 0.09 & 1979 \\
\hline 15 & 0.01 & 3 & 1 & 0.42 & 1972 & 0.42 & 1972 \\
\hline 16 & 0.01 & 1 & 1 & 0.01 & 1968 & 0.24 & 1993 \\
\hline 17 & 0.01 & 0 & 3 & $T$ & $1980^{(2)}$ & $\mathrm{T}$ & $1995^{(2)}$ \\
\hline 18 & 0.01 & 4 & 3 & 0.69 & 1975 & 0.69 & 1975 \\
\hline 19 & 0.01 & 3 & 5 & 0.05 & 1979 & 0.18 & 1954 \\
\hline 20 & 0.01 & 4 & 6 & 0.03 & 1978 & 0.22 & 1953 \\
\hline 21 & 0.02 & 3 & 4 & 0.76 & 1990 & $0.76^{(b)}$ & $1990^{(b)}$ \\
\hline 22 & 0.01 & 6 & 1 & 0.18 & 1978 & 0.18 & 1978 \\
\hline 23 & 0.02 & 4 & 1 & 0.14 & 1975 & 0.14 & 1975 \\
\hline 24 & 0.02 & 6 & 1 & 0.38 & 1977 & 0.38 & 1977 \\
\hline 25 & 0.01 & 2 & 6 & 0.29 & 1976 & 0.29 & 1976 \\
\hline 26 & 0.01 & 3 & 4 & 0.19 & 1968 & 0.38 & 1953 \\
\hline 27 & 0.01 & 5 & 3 & 0.14 & 1989 & 0.14 & 1989 \\
\hline 28 & 0.01 & 2 & 4 & 0.13 & 1975 & 0.13 & 1975 \\
\hline 29 & 0.01 & $6^{\circ}$ & 1 & 0.28 & 1977 & 0.51 & 1947 \\
\hline 30 & 0.01 & 2 & 4 & 0.61 & 1977 & 0.61 & 1977 \\
\hline 31 & 0.01 & 2 & 3 & 0.02 & 1961 & 0.02 & $1961^{(2)}$ \\
\hline \multicolumn{8}{|c|}{ September } \\
\hline 1 & 0.01 & 7 & 2 & 0.43 & 1971 & 0.43 & 1971 \\
\hline 2 & 0.01 & 2 & 4 & 0.17 & 1971 & 0.17 & 1971 \\
\hline 3 & 0.01 & 3 & 1 & 0.13 & 1979 & 0.15 & 1997 \\
\hline 4 & 0.01 & 1 & 1 & 0.02 & 1977 & 0.19 & 1960 \\
\hline 5 & $\mathrm{~T}$ & 3 & 2 & 0.19 & 1971 & 0.19 & 1971 \\
\hline 6 & $\mathrm{~T}$ & 3 & 0 & 0.29 & 1971 & 0.48 & 1995 \\
\hline 7 & $\mathrm{~T}$ & 2 & 0 & 0.04 & 1978 & 0.23 & 1947 \\
\hline 8 & $\mathrm{~T}$ & 2 & 3 & 0.10 & 1985 & 0.10 & 1985 \\
\hline 9 & $T$ & 2 & 4 & 0.07 & 1985 & 0.07 & 1985 \\
\hline 10 & 0.01 & 3 & 3 & 0.06 & 1985 & 0.06 & 1985 \\
\hline
\end{tabular}


Table 4.10. (contd)

\begin{tabular}{|c|c|c|c|c|c|c|c|}
\hline \multirow[b]{3}{*}{ Day } & \multicolumn{5}{|c|}{ Normal Period (1961-1990) } & \multicolumn{2}{|c|}{$\begin{array}{l}\text { Historical Period } \\
(1945-1999)\end{array}$} \\
\hline & \multirow[b]{2}{*}{ Normal } & \multicolumn{2}{|c|}{ Number of Years } & \multirow[b]{2}{*}{ Maximum } & \multirow[b]{2}{*}{ Year } & \multirow[b]{2}{*}{ Maximum } & \multirow[b]{2}{*}{ Year } \\
\hline & & W/Meas. & W/Trace & & & & \\
\hline 11 & 0.01 & 4 & 1 & 0.10 & 1966 & 0.10 & 1966 \\
\hline 12 & 0.01 & 0 & 3 & $T$ & $1980^{(2)}$ & 0.03 & 1958 \\
\hline 13 & 0.01 & 5 & 2 & 0.79 & 1980 & $0.79^{(\mathrm{b})}$ & $1980^{(b)}$ \\
\hline 14 & 0.01 & 3 & 4 & 0.04 & 1985 & 0.41 & 1959 \\
\hline 15 & 0.01 & 2 & 5 & 0.54 & 1986 & 0.54 & 1986 \\
\hline 16 & 0.02 & 2 & 7 & 0.03 & 1985 & 0.66 & 1947 \\
\hline 17 & 0.01 & 4 & 5 & 0.26 & 1969 & 0.26 & 1969 \\
\hline 18 & 0.01 & 4 & 6 & 0.22 & 1983 & 0.41 & 1959 \\
\hline 19 & 0.01 & 6 & 4 & 0.26 & 1973 & 0.26 & 1973 \\
\hline 20 & 0.01 & 4 & 8 & 0.13 & 1988 & 0.13 & 1988 \\
\hline 21 & 0.01 & 2 & 4 & 0.03 & 1971 & 0.03 & 1971 \\
\hline 22 & 0.01 & 4 & 4 & 0.20 & 1984 & 0.20 & 1984 \\
\hline 23 & 0.01 & 4 & 2 & 0.21 & 1986 & 0.21 & 1986 \\
\hline 24 & 0.01 & 4 & 0 & 0.10 & 1977 & 0.10 & 1977 \\
\hline 25 & 0.01 & 3 & 3 & 0.25 & 1982 & 0.25 & 1982 \\
\hline 26 & 0.01 & 4 & 1 & 0.22 & 1981 & 0.22 & 1981 \\
\hline 27 & 0.01 & 4 & 2 & 0.38 & 1981 & 0.43 & 1955 \\
\hline 28 & 0.01 & 5 & 3 & 0.34 & 1962 & 0.34 & 1962 \\
\hline 29 & 0.01 & 3 & 1 & 0.07 & 1986 & 0.07 & 1986 \\
\hline 30 & 0.01 & 2 & 3 & 0.02 & 1969 & 0.03 & $1953^{(a)}$ \\
\hline \multicolumn{8}{|c|}{ October } \\
\hline 1 & $T$ & 2 & 5 & 0.01 & $1969^{(a)}$ & $1.60^{(b)}$ & $1957^{(b)}$ \\
\hline 2 & $\mathrm{~T}$ & 5 & 5 & 0.06 & 1967 & 0.31 & 1957 \\
\hline 3 & $\mathrm{~T}$ & 2 & 1 & 0.04 & 1975 & 0.38 & 1995 \\
\hline 4 & $\mathrm{~T}$ & 1 & 4 & 0.01 & 1970 & 0.15 & 1950 \\
\hline 5 & $T$ & 0 & 9 & $\mathrm{~T}$ & $1981^{(2)}$ & 0.25 & 1950 \\
\hline 6 & $I$ & 5 & 4 & 0.22 & 1973 & 0.22 & 1973 \\
\hline 7 & 0.01 & 3 & 3 & 0.25 & 1985 & 0.25 & 1985 \\
\hline 8 & 0.01 & 1 & 5 & 0.06 & 1964 & 0.49 & 1950 \\
\hline 9 & 0.01 & 5 & 2 & 0.09 & 1968 & 0.32 & 1947 \\
\hline 10 & 0.01 & 5 & 4 & 0.09 & 1985 & 0.32 & 1959 \\
\hline 11 & 0.01 & 3 & 7 & 0.43 & 1968 & 0.43 & 1968 \\
\hline 12 & 0.01 & 6 & 3 & 0.52 & 1962 & 0.52 & 1962 \\
\hline 13 & 0.01 & 2 & 3 & 0.04 & 1980 & 0.16 & 1994 \\
\hline 14 & 0.01 & 4 & 3 & 0.11 & 1962 & 0.43 & 1950 \\
\hline 15 & 0.01 & 1 & 1 & 0.01 & 1980 & 0.15 & 1947 \\
\hline 16 & 0.01 & 0 & 1 & $T$ & 1975 & 0.24 & 1947 \\
\hline 17 & 0.01 & 2 & 3 & 0.16 & 1968 & 0.23 & 1950 \\
\hline 18 & 0.01 & 4 & 3 & 0.28 & 1979 & 0.28 & 1979 \\
\hline 19 & 0.02 & 5 & 4 & 0.12 & 1979 & 0.64 & 1947 \\
\hline 20 & 0.02 & 5 & 5 & 0.15 & 1968 & 0.37 & 1947 \\
\hline 21 & 0.02 & 8 & 3 & 0.45 & 1975 & 0.45 & 1975 \\
\hline 22 & 0.02 & 5 & 9 & 0.20 & 1983 & 0.23 & 1957 \\
\hline 23 & 0.02 & 4 & 2 & 0.39 & 1973 & 0.39 & 1973 \\
\hline 24 & 0.02 & 4 & 5 & 0.11 & 1979 & 0.12 & 1991 \\
\hline 25 & 0.02 & 5 & 3 & 0.22 & 1975 & 0.22 & 1975 \\
\hline 26 & 0.02 & 6 & 3 & 0.12 & 1989 & 0.18 & 1956 \\
\hline 27 & 0.02 & 7 & 4 & 0.09 & 1969 & 0.36 & 1999 \\
\hline 28 & 0.02 & 4 & 6 & 0.93 & 1982 & 0.93 & 1982 \\
\hline 29 & 0.02 & 7 & 8 & 0.18 & 1986 & 0.38 & 1950 \\
\hline 30 & 0.02 & 3 & 7 & 0.52 & 1990 & 0.52 & 1990 \\
\hline 31 & 0.02 & 3 & 6 & 0.64 & 1973 & 0.64 & 1973 \\
\hline
\end{tabular}


Table 4.10. (contd)

\begin{tabular}{|c|c|c|c|c|c|c|c|}
\hline \multirow[b]{3}{*}{ Day } & \multicolumn{5}{|c|}{ Normal Period (1961-1990) } & \multicolumn{2}{|c|}{$\begin{array}{c}\text { Historical Period } \\
(1945-1999)\end{array}$} \\
\hline & \multirow[b]{2}{*}{ Normal } & \multicolumn{2}{|c|}{ Number of Years } & \multirow[b]{2}{*}{ Maximum } & \multirow[b]{2}{*}{ Year } & \multirow{3}{*}{ Maximum } & \multirow{3}{*}{ Year } \\
\hline & & W/Meas. & W/Trace & & & & \\
\hline \multicolumn{6}{|c|}{ November } & & \\
\hline 1 & 0.02 & 7 & 3 & 0.18 & 1987 & 0.26 & 1948 \\
\hline 2 & 0.02 & 6 & 2 & 0.25 & 1984 & 0.25 & 1984 \\
\hline 3 & 0.02 & 8 & 3 & 0.28 & 1965 & 0.28 & 1965 \\
\hline 4 & 0.02 & 9 & 5 & 0.19 & 1973 & 0.24 & 1991 \\
\hline 5 & 0.02 & 9 & 2 & 0.19 & 1963 & 0.62 & 1998 \\
\hline 6 & 0.02 & 8 & 4 & 0.30 & 1980 & 0.30 & $1980^{(2)}$ \\
\hline 7 & 0.03 & 5 & 7 & 0.23 & 1963 & 0.57 & 1967 \\
\hline 8 & 0.03 & 9 & 6 & 0.50 & 1968 & 0.50 & 1968 \\
\hline 9 & 0.04 & 8 & 7 & 0.16 & 1973 & 0.27 & 1949 \\
\hline 10 & 0.04 & 11 & 4 & 0.66 & 1983 & 0.66 & 1983 \\
\hline 11 & 0.04 & 12 & 3 & 0.26 & 1970 & 0.26 & 1970 \\
\hline 12 & 0.04 & 10 & 4 & 0.57 & 1973 & 0.57 & 1973 \\
\hline 13 & 0.04 & 7 & 4 & 0.47 & 1981 & 0.47 & 1981 \\
\hline 14 & 0.04 & 7 & 6 & 0.35 & 1966 & 0.35 & 1966 \\
\hline 15 & 0.04 & 12 & 5 & 0.17 & 1985 & 0.19 & 1994 \\
\hline 16 & 0.03 & 9 & 5 & 0.42 & 1979 & 0.42 & 1979 \\
\hline 17 & 0.03 & 9 & 7 & 0.12 & 1974 & 0.18 & 1955 \\
\hline 18 & 0.03 & 7 & 4 & 0.46 & 1982 & 0.51 & 1996 \\
\hline 19 & 0.03 & 7 & 5 & 0.37 & 1966 & $1.39^{(8)}$ & $1996^{(0)}$ \\
\hline 20 & 0.03 & 4 & 7 & 0.42 & 1966 & 0.42 & 1966 \\
\hline 21 & 0.03 & 6 & 8 & 0.50 & 1985 & 0.50 & 1985 \\
\hline 22 & 0.03 & 6 & 10 & 0.30 & 1979 & 0.30 & 1979 \\
\hline 23 & 0.03 & 12 & 5 & 0.42 & 1983 & 0.63 & 1949 \\
\hline 24 & 0.03 & 10 & 7 & 0.37 & 1965 & 0.37 & 1965 \\
\hline 25 & 0.03 & 5 & 10 & 0.25 & 1977 & 0.25 & 1977 \\
\hline 26 & 0.03 & 8 & 6 & 0.11 & $1986^{(a)}$ & 0.54 & 1955 \\
\hline 27 & 0.03 & 9 & 6 & 0.49 & 1984 & 0.49 & 1984 \\
\hline 28 & 0.02 & 9 & 7 & 0.19 & 1986 & 0.19 & 1986 \\
\hline 29 & 0.03 & 8 & 7 & 0.18 & 1978 & 0.18 & 1978 \\
\hline 30 & 0.03 & 9 & 5 & 0.30 & 1962 & 0.34 & 1998 \\
\hline \multicolumn{8}{|c|}{ December } \\
\hline 1 & 0.03 & 8 & 4 & 0.19 & 1966 & 0.29 & 1955 \\
\hline 2 & 0.04 & 8 & 8 & 0.34 & $1985^{(2)}$ & 0.34 & $1985^{(2)}$ \\
\hline 3 & 0.04 & 7 & 9 & 0.56 & 1980 & 0.56 & 1980 \\
\hline 4 & 0.03 & 13 & 3 & 0.28 & 1974 & 0.28 & 1974 \\
\hline 5 & 0.04 & 11 & 7 & 0.43 & 1963 & 0.43 & 1963 \\
\hline 6 & 0.04 & 12 & 8 & 0.18 & 1985 & 0.18 & 1985 \\
\hline 7 & 0.03 & 8 & 5 & 0.32 & 1983 & 0.36 & 1948 \\
\hline 8 & 0.03 & 5 & 7 & 0.36 & 1963 & 0.36 & 1963 \\
\hline 9 & 0.03 & 9 & 8 & 0.55 & 1987 & 0.55 & 1987 \\
\hline 10 & 0.03 & 9 & 4 & 0.25 & 1990 & 0.54 & 1958 \\
\hline 11 & 0.04 & 6 & 8 & 0.33 & 1969 & 0.53 & 1958 \\
\hline 12 & 0.04 & 10 & 5 & 0.29 & 1982 & 0.65 & 1995 \\
\hline 13 & 0.03 & 8 & 5 & 0.76 & 1977 & 0.76 & 1977 \\
\hline 14 & 0.04 & 12 & 5 & 0.25 & 1964 & 0.25 & 1964 \\
\hline 15 & 0.04 & 15 & 2 & 0.22 & 1981 & 0.22 & 1981 \\
\hline 16 & 0.04 & 9 & 9 & 0.30 & 1961 & 0.37 & 1994 \\
\hline 17 & 0.04 & 10 & 5 & 0.22 & 1973 & 0.22 & 1973 \\
\hline 18 & 0.04 & 11 & 8 & 0.22 & 1981 & 0.27 & 1960 \\
\hline 19 & 0.04 & 15 & 7 & 0.19 & 1964 & 0.20 & 1953 \\
\hline 20 & 0.04 & 13 & 7 & 0.33 & 1982 & 0.33 & 1982 \\
\hline 21 & 0.04 & 10 & 10 & 0.54 & 1964 & 0.61 & 1955 \\
\hline
\end{tabular}


Table 4.10. (contd)

\begin{tabular}{|c|c|c|c|c|c|c|c|}
\hline \multirow[b]{3}{*}{ Day } & \multicolumn{5}{|c|}{ Normal Period (1961-1990) } & \multicolumn{2}{|c|}{$\begin{array}{l}\text { Historical Period } \\
(1945-1999)\end{array}$} \\
\hline & \multirow[b]{2}{*}{ Normal } & \multicolumn{2}{|c|}{ Number of Years } & \multirow[b]{2}{*}{ Maximum } & \multirow[b]{2}{*}{ Year } & & \\
\hline & & W/Meas. & W/Trace & & & Maximum & Year \\
\hline 22 & 0.04 & 9 & 5 & 0.59 & 1964 & 0.59 & 1964 \\
\hline 23 & 0.04 & 7 & 7 & 0.31 & 1975 & 0.31 & 1975 \\
\hline 24 & 0.04 & 11 & 8 & 0.36 & 1968 & 0.36 & 1968 \\
\hline 25 & 0.03 & 12 & 6 & 0.26 & 1980 & 0.32 & 1996 \\
\hline 26 & 0.03 & 9 & 6 & 0.19 & 1973 & 0.58 & 1996 \\
\hline 27 & 0.03 & 11 & 7 & 0.36 & 1973 & 0.36 & 1973 \\
\hline 28 & 0.02 & 10 & 11 & 0.09 & $1990^{(\mathrm{a})}$ & 0.10 & 1951 \\
\hline 29 & 0.02 & 9 & 7 & 0.55 & 1983 & $0.80^{(\mathrm{b})}$ & $1996^{(b)}$ \\
\hline 30 & 0.02 & 5 & 9 & 0.15 & 1979 & 0.28 & 1995 \\
\hline 31 & 0.02 & 4 & 9 & 0.29 & 1968 & 0.72 & 1996 \\
\hline
\end{tabular}

(a) Most recent of several occurrences.

(b) Greatest and year of occurrence.

$T=$ Trace. 


\subsection{Wind Climatology}

\subsection{Monthly and Annual Prevailing Wind Directions, Average Speeds, and Peak Gusts}

At the Hanford Meteorology Station, the prevailing wind direction for every month of the year is either WNW or NW (Table 5.1), and the peak gusts for every month are from the SSW, SW, or WSW. The highest monthly average wind speeds occur in June, the lowest in December. The variability in monthly average wind speeds is much greater in the winter months than during the remainder of the year. The highest January average $(10.3 \mathrm{mph})$ is less than 3.5 times greater than the lowest $(2.9 \mathrm{mph})$; however, in June, the highest average (10.7 $\mathrm{mph})$ is only 1.4 times greater than the lowest $(7.7 \mathrm{mph})$.

Table 5.1. Monthly and Annual Prevailing Wind Directions, Average Speeds, and Peak Gusts at 50-Foot Level, 1945 Through 1999

\begin{tabular}{|c|c|c|c|c|c|c|c|c|c|}
\hline \multirow[b]{2}{*}{ Month } & \multirow[b]{2}{*}{$\begin{array}{l}\text { Prevailing } \\
\text { Direction } \\
\end{array}$} & \multirow{2}{*}{$\begin{array}{c}\text { Average } \\
\text { Speed, } \\
\text { mph }\end{array}$} & \multirow{2}{*}{$\begin{array}{c}\text { Highest } \\
\text { Average, } \\
\text { mph } \\
\end{array}$} & \multirow{2}{*}{\multicolumn{2}{|c|}{$\begin{array}{c}\text { Lowest } \\
\text { Average, } \\
\text { mph }\end{array}$}} & \multirow[b]{2}{*}{ Year } & \multicolumn{3}{|c|}{ Peak Gusts } \\
\hline & & & & & & & $\begin{array}{c}\text { Speed, } \\
\text { mph }\end{array}$ & Direction & Year \\
\hline Jan & NW & 6.3 & 10.3 & 1972 & 2.9 & 1985 & 80 & SW & 1972 \\
\hline Feb & NW & 7.1 & 11.1 & 1999 & 4.6 & 1963 & 65 & Sw & 1971 \\
\hline Mar & WNW & 8.2 & 10.7 & $1977^{(2)}$ & 5.9 & 1958 & 70 & sw & 1956 \\
\hline Apr & WNW & 8.9 & 11.1 & $1972^{(2)}$ & 7.4 & $1989^{(2)}$ & 73 & SSW & 1972 \\
\hline May & WNW & 8.8 & 10.7 & 1983 & $5.8^{\circ}$ & 1957 & 71 & SSW & 1948 \\
\hline Jun & NW & 9.1 & 10.7 & $1983^{(2)}$ & 7.7 & $1950^{(2)}$ & 72 & SW & 1957 \\
\hline Jul & NW & 8.6 & 10.7 & 1983 & 6.8 & 1955 & 69 & wsw & 1979 \\
\hline Aug & WNW & 8.0 & 9.5 & 1996 & 6.0 & 1956 & 66 & SW & 1961 \\
\hline Sep & WNW & 7.5 & 9.2 & 1961 & 5.4 & 1957 & 65 & SSW & 1953 \\
\hline Oct & NW & 6.6 & 9.1 & 1946 & 4.4 & 1952 & 72 & Sw & 1997 \\
\hline Nov & NW & 6.3 & 10.0 & 1990 & 2.9 & 1956 & 67 & WSW & 1993 \\
\hline Dec & NW & 6.0 & 8.3 & 1968 & 3.3 & 1985 & 71 & SW & 1955 \\
\hline Annual & NW & 7.6 & 8.8 & 1999 & 6.2 & 1989 & 80 & SW & $\operatorname{Jan} 1972$ \\
\hline
\end{tabular}

(a) Also in earlier years.

\subsection{Days with Peak Gusts Above or Below Specific Thresholds}

Table 5.2 lists the number of days by month and year with peak wind gusts (at 50 foot) above or below specific threshold wind speeds. June and July have the highest average number of days with gusts $\geq 25 \mathrm{mph}$ (nearly 20 each); however, January, March, and April have the highest average number of days with gusts $\geq 40 \mathrm{mph}$ (nearly 3 days) and January has the highest average number of days with gusts $\geq 50 \mathrm{mph}$ ( 0.9 day). January also has the record highest number of gusts $\geq 40$ and $\geq 50 \mathrm{mph}$ at 11 and 


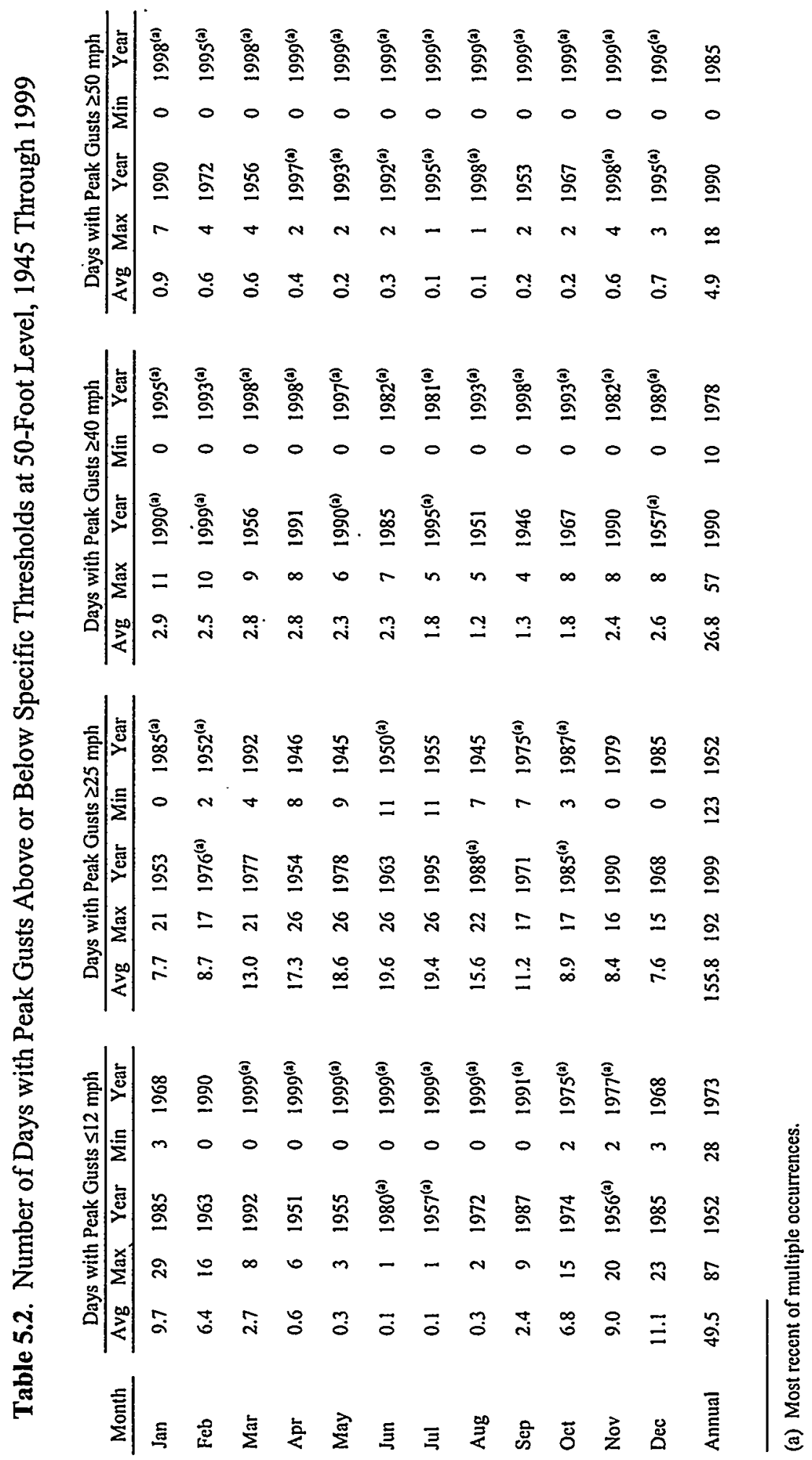


7 days, respectively, in 1990 . Calendar year 1990 recorded the most days with gusts $\geq 40$ and $\geq 50 \mathrm{mph}$ at 57 and 18 days, respectively. Of particular interest is that previous.records for these categories were 41 days $\geq 40 \mathrm{mph}$ in 1961 and 10 days $\geq 50 \mathrm{mph}$ in 1972 .

\subsection{Frequency of Monthly and Annual Wind Direction and Speed at 50-Foot Level}

Table 5.3 presents Hanford Meteorology Station data on the percent frequency of monthly and annual wind direction and wind speed at 50 foot. This table shows that for every month of the year the prevailing wind direction is either from the WNW or NW. Winds are relatively evenly distributed from the NNE through the SSW at between $2 \%$ and $4 \%$ on an annual average for each direction.

Table 5.3. Frequency (\%) of Monthly and Annual Wind Direction and Speed at 50-Foot Level, 1955 Through 1999

\begin{tabular}{|c|c|c|c|c|c|c|c|c|c|c|c|c|c|}
\hline Direction & Jan & $\mathrm{Feb}$ & Mar & Apr & May & Jun & Jul & Aug & Sep & Oct & Nov & Dec & Total \\
\hline $\begin{array}{l}N \\
N N E \\
N E \\
\text { ENE } \\
\text { E } \\
\text { ESE } \\
\text { SE } \\
\text { SSE } \\
\text { S }\end{array}$ & $\begin{array}{l}4.3 \\
3.7 \\
3.0 \\
2.3 \\
2.7 \\
2.8 \\
4.1 \\
3.4 \\
3.4\end{array}$ & $\begin{array}{l}4.7 \\
4.6 \\
3.7 \\
2.1 \\
2.2 \\
2.6 \\
3.6 \\
3.4 \\
3.3\end{array}$ & $\begin{array}{l}4.7 \\
4.5 \\
3.5 \\
2.1 \\
2.2 \\
2.7 \\
3.7 \\
3.4 \\
3.5\end{array}$ & $\begin{array}{l}4.1 \\
3.7 \\
3.5 \\
2.4 \\
2.5 \\
2.5 \\
3.0 \\
3.0 \\
3.1\end{array}$ & $\begin{array}{l}3.8 \\
3.5 \\
3.4 \\
2.5 \\
2.4 \\
2.6 \\
3.1 \\
3.0 \\
2.7\end{array}$ & $\begin{array}{l}3.6 \\
3.2 \\
3.2 \\
2.2 \\
2.5 \\
2.7 \\
2.9 \\
2.8 \\
2.7\end{array}$ & $\begin{array}{l}4.6 \\
4.3 \\
3.7 \\
2.6 \\
2.9 \\
2.8 \\
2.9 \\
2.5 \\
2.4\end{array}$ & $\begin{array}{l}4.7 \\
4.0 \\
3.5 \\
2.5 \\
3.1 \\
3.3 \\
3.5 \\
2.8 \\
2.6\end{array}$ & $\begin{array}{l}5.6 \\
5.5 \\
4.2 \\
2.4 \\
3.1 \\
3.2 \\
3.8 \\
3.3 \\
2.6\end{array}$ & $\begin{array}{l}5.0 \\
4.1 \\
3.5 \\
2.6 \\
2.9 \\
3.7 \\
5.0 \\
4.0 \\
3.6\end{array}$ & $\begin{array}{l}4.3 \\
3.5 \\
3.0 \\
2.5 \\
2.7 \\
3.2 \\
4.4 \\
3.9 \\
4.1\end{array}$ & $\begin{array}{l}4.3 \\
3.3 \\
2.9 \\
2.5 \\
2.7 \\
3.2 \\
4.5 \\
3.7 \\
3.6\end{array}$ & $\begin{array}{l}4.5 \\
4.0 \\
3.4 \\
2.4 \\
2.7 \\
3.0 \\
3.7 \\
3.3 \\
3.1\end{array}$ \\
\hline $\begin{array}{l}\text { SSW } \\
\text { SW } \\
\text { WSW } \\
\text { W } \\
\text { WNW } \\
\text { NW } \\
\text { NNW } \\
\text { Calm }\end{array}$ & $\begin{array}{r}4.9 \\
6.5 \\
6.5 \\
6.6 \\
15.2 \\
19.3 \\
7.4 \\
4.1\end{array}$ & $\begin{array}{r}4.5 \\
7.9 \\
7.5 \\
8.2 \\
14.6 \\
17.8 \\
6.8 \\
2.4\end{array}$ & $\begin{array}{r}5.0 \\
9.2 \\
10.4 \\
9.5 \\
14.6 \\
14.5 \\
5.8 \\
0.8\end{array}$ & $\begin{array}{r}4.3 \\
8.7 \\
11.8 \\
11.6 \\
16.4 \\
14.4 \\
4.6 \\
0.5\end{array}$ & $\begin{array}{r}3.6 \\
6.8 \\
10.4 \\
11.5 \\
18.4 \\
17.5 \\
4.3 \\
0.5\end{array}$ & $\begin{array}{r}3.4 \\
6.5 \\
9.7 \\
10.9 \\
19.1 \\
19.5 \\
4.8 \\
0.4\end{array}$ & $\begin{array}{r}2.7 \\
5.6 \\
8.3 \\
9.6 \\
19.4 \\
20.1 \\
5.3 \\
0.3\end{array}$ & $\begin{array}{r}3.0 \\
5.9 \\
8.8 \\
10.7 \\
17.9 \\
17.8 \\
5.4 \\
0.5\end{array}$ & $\begin{array}{r}3.3 \\
5.8 \\
9.3 \\
11.2 \\
15.1 \\
14.7 \\
5.9 \\
0.9\end{array}$ & $\begin{array}{r}4.2 \\
6.9 \\
8.6 \\
10.3 \\
13.3 \\
13.6 \\
6.5 \\
2.2\end{array}$ & $\begin{array}{r}4.1 \\
5.2 \\
7.7 \\
7.9 \\
8.2 \\
12.7 \\
15.8 \\
7.1 \\
3.7\end{array}$ & $\begin{array}{r}3.0 \\
4.7 \\
6.9 \\
7.3 \\
7.2 \\
13.6 \\
17.9 \\
7.0 \\
4.7\end{array}$ & $\begin{array}{r}5.1 \\
4.1 \\
7.0 \\
8.9 \\
9.6 \\
15.8 \\
16.9 \\
5.9 \\
1.7\end{array}$ \\
\hline Total & 100.0 & 100.0 & 100.0 & 100.0 & 100.0 & 100.0 & 100.0 & 100.0 & 100.0 & 100.0 & 100.0 & 100.0 & 100.0 \\
\hline Speed, mph & Jan & $\mathrm{Feb}$ & Mar & Apr & May & Jun & Jul & Aug & Sep & Oct & Nov & Dec & Total \\
\hline $\begin{array}{l}\text { Calm } \\
1-3 \\
4-7 \\
8-12 \\
13-18 \\
19-24 \\
25-31 \\
32-38 \\
39-46 \\
>46\end{array}$ & $\begin{array}{r}4.1 \\
30.7 \\
34.5 \\
20.1 \\
6.8 \\
2.5 \\
1.0 \\
0.3 \\
0.0 \\
0.0\end{array}$ & $\begin{array}{r}2.4 \\
24.6 \\
35.4 \\
23.9 \\
8.8 \\
3.3 \\
1.3 \\
0.3 \\
0.0 \\
0.0\end{array}$ & $\begin{array}{r}0.8 \\
16.8 \\
36.5 \\
27.6 \\
12.1 \\
4.5 \\
1.5 \\
0.2 \\
0.0 \\
0.0\end{array}$ & $\begin{array}{r}0.5 \\
13.1 \\
34.7 \\
28.7 \\
15.7 \\
5.7 \\
1.5 \\
0.1 \\
0.0 \\
0.0\end{array}$ & $\begin{array}{r}0.5 \\
11.7 \\
35.1 \\
30.6 \\
15.7 \\
5.5 \\
0.9 \\
0.0 \\
0.0 \\
0.0\end{array}$ & $\begin{array}{r}0.4 \\
9.6 \\
35.9 \\
30.3 \\
16.5 \\
6.2 \\
1.1 \\
0.0 \\
0.0 \\
0.0\end{array}$ & $\begin{array}{r}0.3 \\
10.7 \\
39.2 \\
29.3 \\
14.3 \\
5.3 \\
0.9 \\
0.0 \\
0.0 \\
0.0\end{array}$ & $\begin{array}{r}0.5 \\
13.4 \\
42.2 \\
27.7 \\
11.8 \\
3.9 \\
0.6 \\
0.0 \\
0.0 \\
0.0\end{array}$ & $\begin{array}{r}0.9 \\
18.1 \\
41.5 \\
25.9 \\
9.8 \\
3.2 \\
0.6 \\
0.0 \\
0.0 \\
0.0\end{array}$ & $\begin{array}{r}2.2 \\
26.3 \\
39.0 \\
21.7 \\
7.7 \\
2.6 \\
0.6 \\
0.0 \\
0.0 \\
0.0\end{array}$ & $\begin{array}{r}3.7 \\
29.4 \\
35.6 \\
20.2 \\
7.1 \\
2.8 \\
1.0 \\
0.2 \\
0.0 \\
0.0\end{array}$ & $\begin{array}{r}4.7 \\
33.4 \\
33.5 \\
18.2 \\
6.4 \\
2.6 \\
0.9 \\
0.2 \\
0.0 \\
0.0\end{array}$ & $\begin{array}{r}1.7 \\
19.8 \\
36.9 \\
25.3 \\
11.0 \\
4.0 \\
1.0 \\
0.1 \\
0.0 \\
0.0\end{array}$ \\
\hline Total & 100.0 & 100.0 & 100.0 & 100.0 & 100.0 & 100.0 & 100.0 & 100.0 & 100.0 & 100.0 & 100.0 & 100.0 & 100.0 \\
\hline
\end{tabular}

The wind speed class with the highest frequency of occurrence is 4 to $7 \mathrm{mph}$, with winds in that category $37 \%$ of the time. The speed class with the second highest frequency is 8 to $12 \mathrm{mph}$, at $25 \%$. Winds averaging greater than $25 \mathrm{mph}$ occur only $1 \%$ of the time on an annual basis, with the highest frequency occurring in March (1.7\%). 


\subsection{Composite Wind Roses and Joint Frequency Distributions for the Hanford Meteorological Monitoring Network}

Figure 5.1 and Table 5.4 contain composite wind roses and joint frequency distributions at the 10-meter level for the entire Hanford Meteorological Monitoring Network (see Table 1.1 and Figure 1.1) for the period 1982 through 1999.

Figure 5.2 and Table 5.5 contain composite wind roses and joint frequency distributions at the 60-meter level for stations 9, 11, 13, and 21 for the period 1986 through 1999. 


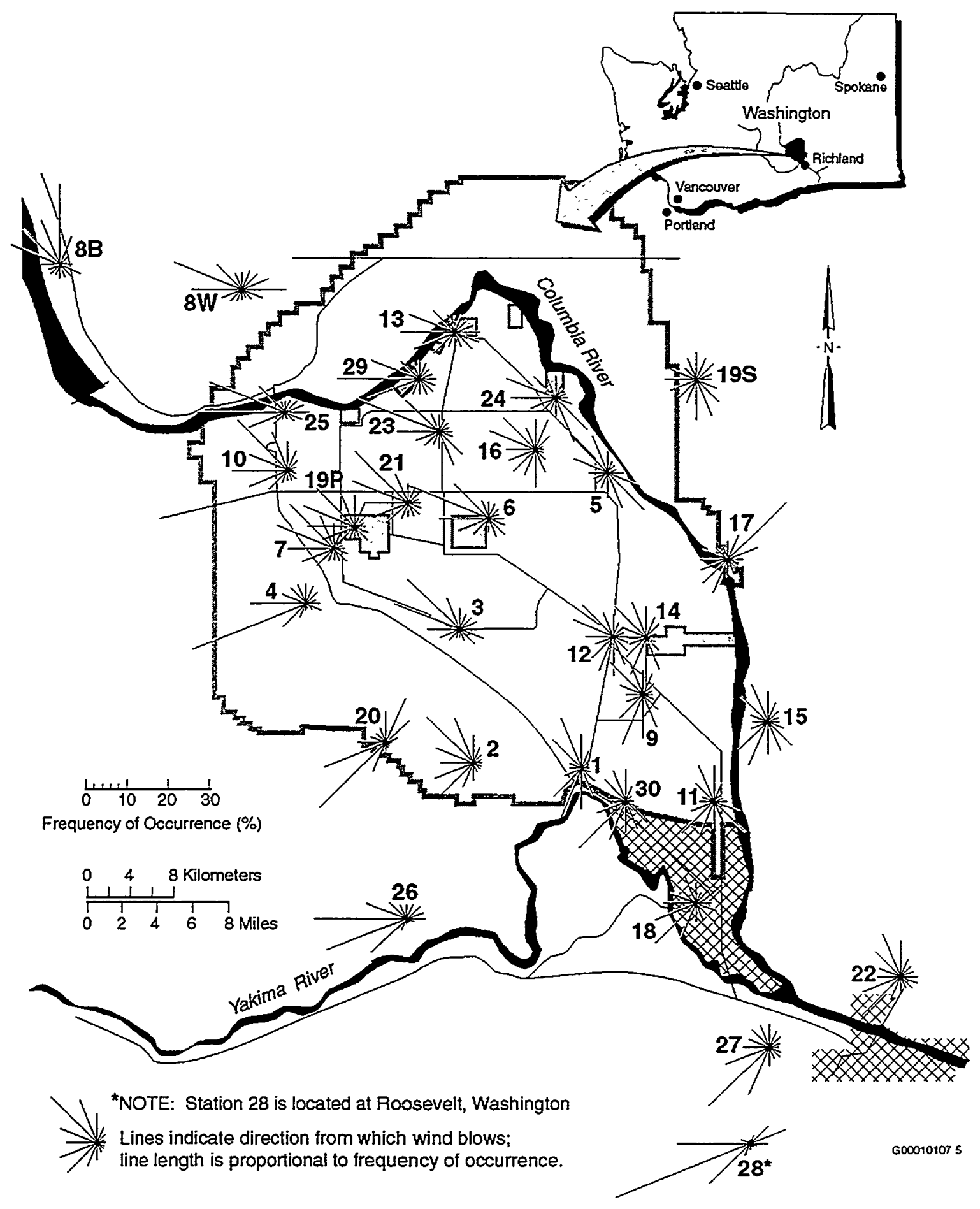

Figure 5.1. Hanford Meteorological Monitoring Network Wind Roses at 10-Meter Level, 1982 Through 1999 
Table 5.4. Joint Frequency Distributions (\%) for Hanford Meteorological Monitoring Network Wind Stations at 10-Meter Level, 1982 Through 1999

Station: (1) PROS

Begin: $1 / 82$

End: $12 / 99$

Total Hours: 153237

DIRECTION

\begin{tabular}{|c|c|c|c|c|c|c|c|c|c|c|c|c|c|c|c|c|c|c|}
\hline & $\mathbf{N}$ & NNE & NE & ENE & E & ESE & SE & SSE & $S$ & SSW & SW & WSW & W & WNW & NW & NNW & CALM & TOTAI \\
\hline CALM & 0.0 & 0.0 & 0.0 & 0.0 & 0.0 & 0.0 & 0.0 & 0.0 & 0.0 & 0.0 & 0.0 & 0.0 & 0.0 & 0.0 & 0.0 & 0.0 & 1.2 & 1.2 \\
\hline $1-3$ & 2.2 & 1.5 & 1.3 & 0.9 & 1.0 & 1.2 & 1.7 & 2.2 & 2.4 & 2.2 & 1.7 & 1.2 & 1.1 & & 2.1 & 2.6 & 0.0 & 26.8 \\
\hline $4-7$ & 3.3 & 1.9 & 1.1 & 0.7 & 0.8 & 1.0 & 2.1 & 4.0 & 4.6 & 3.6 & 2.0 & 0.8 & 0.7 & 1.0 & 3.0 & 5.0 & 0.0 & 35.5 \\
\hline $8-12$ & 1.9 & 0.9 & 0.3 & 0.1 & 0.1 & 0.2 & 0.5 & 1.0 & 2.4 & 4.4 & 2.5 & 0.8 & 0.5 & 0.5 & 2.6 & 4.0 & 0.0 & 22.8 \\
\hline $13-18$ & 0.6 & 0.2 & 0.1 & 0.0 & 0.0 & 0.0 & 0.0 & 0.0 & 0.4 & 2.5 & 2.0 & 0.9 & 0.4 & 0.2 & 1.5 & 1.3 & 0.0 & 10.3 \\
\hline $19-24$ & 0.1 & 0.1 & 0.0 & 0.0 & 0.0 & 0.0 & 0.0 & 0.0 & 0.0 & 0.4 & 0.7 & 0.4 & 0.2 & 0.0 & 0.4 & 0.2 & 0.0 & 2.6 \\
\hline $25-31$ & 0.0 & 0.0 & 0.0 & 0.0 & 0.0 & 0.0 & 0.0 & 0.0 & 0.0 & 0.1 & 0.3 & 0.1 & 0.0 & 0.0 & 0.1 & 0.0 & 0.0 & 0.7 \\
\hline $32-38$ & 0.0 & 0.0 & 0.0 & 0.0 & 0.0 & 0.0 & 0.0 & 0.0 & 0.0 & 0.0 & 0.1 & 0.0 & 0.0 & 0.0 & 0.0 & 0.0 & 0.0 & 0.1 \\
\hline $39-4$ & 0.0 & 0.0 & 0.0 & 0.0 & 0.0 & 0.0 & 0.0 & 0.0 & 0.0 & 0.0 & 0.0 & 0.0 & 0.0 & 0.0 & 0.0 & 0.0 & 0.0 & 0.0 \\
\hline$>$ & 0.0 & 0.0 & 0.0 & 0.0 & 0.0 & 0. & 0.0 & 0. & 0.0 & 0.0 & 0.0 & 0. & 0.0 & 0. & 0.0 & 0.0 & 0.0 & 0.0 \\
\hline DTAL & 8.1 & 4.6 & 2.8 & 1.8 & 1.9 & 2.5 & 4.3 & 7.2 & 9.8 & 13.2 & 9.4 & 4.3 & 3.0 & 3.2 & 9.7 & 13.1 & 1.2 & 100.0 \\
\hline
\end{tabular}

Station: (2) EOC

Begin: $1 / 82$

End: $12 / 99$

Total Hours: 154098

DIRECTION

$N$ NNE NE ENE E ESE SE SSE S SSW SW WSW W WNW NW NNW CALM TOTAL

\begin{tabular}{|c|c|c|c|c|c|c|c|c|c|c|c|c|c|c|c|c|c|c|}
\hline CALM & 0.0 & 0.0 & 0.0 & 0.0 & 0.0 & 0.0 & 0.0 & 0.0 & 0.0 & 0.0 & 0.0 & 0.0 & 0.0 & 0.0 & 0.0 & 0.0 & 1.0 & 1.0 \\
\hline $1-3$ & 1.3 & 1.2 & 1.2 & 1.0 & 0.9 & 0.9 & 0.8 & 0.9 & 1.2 & 1.2 & 1.2 & 1.2 & 1.6 & 1.8 & 1.8 & 1.4 & 0.0 & 19.6 \\
\hline $4-7$ & 2.8 & 1.9 & 1.2 & 0.9 & 1.0 & 1.1 & 1.1 & 1.2 & 1.9 & 2.0 & 1.5 & 1.2 & 2.0 & 3.6 & 4.1 & 3.3 & 0.0 & 30.8 \\
\hline $8-12$ & 1.8 & 0.6 & 0.2 & 0.1 & 0.0 & 0.1 & 0.2 & 0.4 & 1.0 & 2.2 & 2.7 & 1.9 & 1.5 & 3.2 & 5.9 & 4.0 & 0.0 & 25.6 \\
\hline $13-18$ & 0.6 & 0.2 & 0.1 & 0.0 & 0.0 & 0.0 & 0.0 & 0.0 & 0.1 & 0.6 & 2.2 & 2.3 & 1.4 & 0.7 & 3.1 & 2.4 & 0.0 & 13.7 \\
\hline $19-24$ & 0.1 & 0.0 & 0.0 & 0.0 & 0.0 & 0.0 & 0.0 & 0.0 & 0.0 & 0.2 & 1.6 & 2.0 & 0.8 & 0.2 & 0.5 & 0.4 & 0.0 & 5.9 \\
\hline $25-31$ & 0.0 & 0.0 & 0.0 & 0.0 & 0.0 & 0.0 & 0.0 & 0.0 & 0.0 & 0.0 & 1.1 & 1.0 & 0.3 & 0.1 & 0.1 & 0.0 & 0.0 & 2.6 \\
\hline $32-38$ & 0.0 & 0.0 & 0.0 & 0.0 & 0.0 & 0.0 & 0.0 & 0.0 & 0.0 & 0.0 & 0.3 & 0.2 & 0.1 & 0.0 & 0.0 & 0.0 & 0.0 & 0.6 \\
\hline $39-46$ & 0.0 & 0.0 & 0.0 & 0.0 & 0.0 & 0.0 & 0.0 & 0.0 & 0.0 & 0.0 & 0.1 & 0.0 & 0.0 & 0.0 & 0.0 & 0.0 & 0.0 & \\
\hline$>46$ & 0.0 & 0.0 & 0.0 & 0.0 & 0.0 & 0.0 & 0.0 & 0.0 & 0.0 & 0.0 & 0.0 & 0.0 & 0.0 & 0.0 & 0.0 & 0.0 & 0.0 & 0.0 \\
\hline OTAL & 6.6 & 3.9 & 2.7 & 2.0 & 2.0 & 2.0 & 2.2 & 2.5 & 4.3 & 6.3 & 10.5 & 9.8 & 7.7 & 9.5 & 15.5 & 11.5 & 1.0 & 100.0 \\
\hline
\end{tabular}

Station: (3) ARMY

Begin: $1 / 82$

End: $12 / 99$

Total Hours: 153884

DIRECTION

\begin{tabular}{|c|c|c|c|c|c|c|c|c|c|c|c|c|c|c|c|c|c|c|}
\hline & $\mathrm{N}$ & NNE & $\mathrm{NE}$ & ENE & $E$ & ESE & SE & SSE & S & SSW & SW & WSW & W & WNW & NW & NNW & CALM & TOTAI \\
\hline CALM & 0.0 & 0.0 & 0.0 & 0.0 & 0.0 & 0.0 & 0.0 & 0.0 & 0.0 & 0.0 & 0.0 & 0.0 & 0.0 & 0.0 & 0.0 & 0.0 & 1.0 & 1.0 \\
\hline $1-3$ & 1.9 & 1.7 & 2.0 & 2.3 & 2.6 & 2.4 & 1.8 & 1.2 & 0.9 & 0.8 & 0.9 & 1.2 & 2.2 & 3.2 & 3.2 & 2.4 & 0.0 & \\
\hline $4-7$ & 2.1 & 1.4 & 1.5 & 1.9 & 2.5 & 2.8 & 2.1 & 0.9 & 0.6 & 0.5 & 0.6 & 0.9 & 2.4 & 7.2 & 7.6 & 3.4 & 0.0 & \\
\hline $8-12$ & 0.9 & 0.5 & 0.3 & 0.2 & 0.4 & 0.7 & 0.9 & 0.6 & 0.4 & 0.4 & 0.6 & 1.1 & 1.9 & 4.6 & 4.0 & 1.5 & 0.0 & \\
\hline $13-18$ & 0.2 & 0.2 & 0.1 & 0.0 & 0.0 & 0.1 & 0.2 & 0.2 & 0.2 & 0.3 & 0.6 & 1.1 & 1.0 & 1.1 & 1.5 & 0.4 & 0.0 & \\
\hline $19-24$ & 0.0 & 0.1 & 0.0 & 0.0 & 0.0 & 0.0 & 0.0 & 0.0 & 0.0 & 0.2 & 0.5 & 0.6 & 0.3 & 0.2 & 0.6 & 0.1 & 0.0 & \\
\hline $25-31$ & 0.0 & 0.0 & 0.0 & 0.0 & 0.0 & 0.0 & 0.0 & 0.0 & 0.0 & 0.1 & 0.3 & 0.2 & 0.1 & 0.0 & 0.1 & 0.0 & 0.0 & \\
\hline $32-38$ & 0.0 & 0.0 & 0.0 & 0.0 & 0.0 & 0.0 & 0.0 & 0.0 & 0.0 & 0.0 & 0.1 & 0.0 & 0.0 & 0.0 & 0.0 & 0.0 & 0.0 & \\
\hline $39-46$ & 0.0 & 0.0 & 0.0 & 0.0 & 0.0 & 0.0 & 0.0 & 0.0 & 0.0 & 0.0 & 0.0 & 0.0 & 0.0 & 0. & 0.0 & 0.0 & 0.0 & \\
\hline$>46$ & 0.0 & 0.0 & 0.0 & 0.0 & 0.0 & 0.0 & 0.0 & 0.0 & 0.0 & 0.0 & 0.0 & 0.0 & 0.0 & 0.0 & 0.0 & 0.0 & 0.0 & \\
\hline TOTAL & 5.1 & 3.8 & 3.9 & 4.4 & 5.5 & 6.0 & 5.1 & 2.9 & 2.1 & 2.2 & 3.7 & 5.1 & 7.8 & 16.2 & 17.1 & 7.9 & 1.0 & \\
\hline
\end{tabular}

Station: (4) RSPG

Begin: $1 / 82$

End: $12 / 99$

Total Hours: 153037

DIRECTION

\begin{tabular}{|c|c|c|c|c|c|c|c|c|c|c|c|c|c|c|c|c|c|c|}
\hline & $\mathrm{N}$ & NNE & NE & ENE & E & ESE & SE & SSE & $\underline{S}$ & SSW & SW & WSW & W & WNW & NW & NNW & CALM & TOTAI \\
\hline CALM & 0.0 & 0.0 & 0.0 & 0.0 & 0.0 & 0.0 & 0.0 & 0.0 & 0.0 & 0.0 & 0.0 & 0.0 & 0.0 & 0.0 & 0.0 & 0.0 & 1.1 & 1.1 \\
\hline $1-3$ & 1.7 & 1.9 & 2.0 & 1.7 & 1.8 & 1.7 & 1.2 & 0.8 & 0.8 & 1.0 & 1.9 & 2.8 & 1.9 & 1.2 & 1.1 & 1.3 & 0.0 & \\
\hline $4-7$ & 2.6 & 2.2 & 1.5 & 1.0 & 1.6 & 2.1 & 0.9 & 0.4 & 0.5 & 0.7 & 2.4 & 10.0 & 4.5 & 1.8 & 1.8 & 2.2 & 0.0 & \\
\hline $8-12$ & 0.5 & 0.4 & 0.2 & 0.1 & 0.1 & 0.3 & 0.1 & 0.1 & 0.2 & 0.7 & 1.7 & 13.9 & 5.1 & & 1.5 & 1.1 & 0.0 & \\
\hline $13-18$ & 0.0 & 0.1 & 0.1 & 0.0 & 0.0 & 0.0 & 0.0 & 0.0 & 0.1 & 0.5 & 1.0 & 2.0 & 1. & 0. & 0. & 0.4 & 0.0 & \\
\hline $19-24$ & 0.0 & 0.0 & 0.0 & 0.0 & 0.0 & 0.0 & 0.0 & 0.0 & 0.0 & 0.3 & 0.5 & 0.4 & 0.2 & 0.1 & 0.1 & 0.1 & 0.0 & \\
\hline $25-31$ & 0.0 & 0.0 & 0.0 & 0.0 & 0.0 & 0.0 & 0.0 & 0.0 & 0.0 & 0.1 & 0.2 & 0.1 & 0.0 & 0.0 & 0.0 & 0.0 & 0.0 & \\
\hline $32-38$ & 0.0 & 0.0 & 0.0 & 0.0 & 0.0 & 0.0 & 0.0 & 0.0 & 0.0 & 0.0 & 0.0 & 0.0 & 0.0 & 0.0 & 0.0 & 0.0 & 0.0 & \\
\hline $39-46$ & 0.0 & 0.0 & 0.0 & 0.0 & 0.0 & 0.0 & 0.0 & 0.0 & 0.0 & 0.0 & 0.0 & 0.0 & 0.0 & 0.0 & 0.0 & 0.0 & 0.0 & \\
\hline$>46$ & 0.0 & 0.0 & 0.0 & 0.0 & 0.0 & 0.0 & 0.0 & 0.0 & 0.0 & 0.0 & 0.0 & 0.0 & 0.0 & 0.0 & 0.0 & 0.0 & 0.0 & \\
\hline DTA & 4.8 & 4.6 & 3.8 & 2.7 & 3.6 & 4.1 & 2.1 & 1.2 & 1.6 & 3.4 & 7.7 & 29.2 & 13.5 & 6.2 & 5.2 & 5.1 & 1.1 & \\
\hline
\end{tabular}


Table 5.4. (contd)

Station: (5) EDNA

DIRECTION

\begin{tabular}{|c|c|c|c|c|c|c|c|c|c|c|c|c|c|c|c|c|c|c|}
\hline & $\mathbf{N}$ & NNE & NE & ENE & $\mathrm{E}$ & ESE & SE & SSE & S & SSW & SW & WSW & W & WNW & NW & NNW & CALM & TOTAL \\
\hline CALM & 0.0 & 0.0 & 0.0 & 0.0 & 0.0 & 0.0 & 0.0 & 0.0 & 0.0 & 0.0 & 0.0 & 0.0 & 0.0 & 0.0 & 0.0 & 0.0 & 1.3 & 1.3 \\
\hline $1-3$ & 1.3 & 0.8 & 0.7 & 0.8 & 1.2 & 2.1 & 3.6 & 3.9 & 3.1 & 1.9 & 1.4 & 1.2 & 1.7 & 2.8 & 3.3 & 2.3 & 0.0 & 32.2 \\
\hline $4-7$ & 2.3 & 1.1 & 0.9 & 1.0 & 2.0 & 4.5 & 7.1 & 3.7 & 1.6 & 0.8 & 0.7 & 0.7 & 1.0 & 2.4 & 5.9 & 4.8 & 0.0 & 40.7 \\
\hline $8-12$ & 1.2 & 0.6 & 0.4 & 0.2 & 0.7 & 1.7 & 1.4 & 1.1 & 0.9 & 0.6 & 0.6 & 0. & 1.1 & 1.7 & 2.2 & 1.9 & 0.0 & 17.0 \\
\hline $13-18$ & 0.2 & & 0.2 & 0.1 & 0.0 & 0.1 & 0.2 & 0. & 0.4 & 0.4 & 0.5 & 0. & 0 & & & 0.2 & 0.0 & 6.4 \\
\hline $19-24$ & 0.0 & 0.1 & 0.1 & 0.0 & 0.0 & 0.0 & 0.0 & 0. & 0.1 & 0.2 & 0.2 & 0. & 0.2 & 0.5 & 0. & 0.0 & 0.0 & 1.9 \\
\hline $25-31$ & 0.0 & 0.0 & 0.0 & 0.0 & 0.0 & 0.0 & 0.0 & 0.0 & 0.0 & 0.0 & 0.0 & 0.1 & 0.0 & 0.1 & 0.0 & 0.0 & 0.0 & 0.4 \\
\hline $32-$ & 0.0 & 0.0 & 0.0 & 0.0 & 0.0 & 0.0 & 0.0 & 0.0 & 0.0 & 0.0 & 0.0 & 0. & 0.0 & 0.0 & 0.0 & 0.0 & 0.0 & 0.0 \\
\hline $39-46$ & 0.0 & 0.0 & 0.0 & 0.0 & 0.0 & 0.0 & 0.0 & 0 & 0.0 & 0.0 & 0.0 & 0. & 0.0 & 0.0 & 0.0 & 0.0 & 0.0 & 0.0 \\
\hline & 0.0 & 0.0 & 0.0 & 0.0 & 0.0 & 0.0 & 0.0 & 0. & 0.0 & 0.0 & 0.0 & 0. & 0.0 & 0.0 & 0.0 & 0.0 & 0.0 & 0.0 \\
\hline COTAL & 5.0 & 2.8 & 2.3 & 2.2 & 3.9 & 8.4 & 12.3 & 9.0 & 6.2 & 3.9 & 3.5 & 3.8 & 4.6 & 8.8 & 12.7 & 9.2 & 1.3 & 100.0 \\
\hline
\end{tabular}

Station: (6) $200 \mathrm{E}$

DIRECTION

\begin{tabular}{|c|c|c|c|c|c|c|c|c|c|c|c|c|c|c|c|c|c|c|}
\hline & $\mathbf{N}$ & NNE & $\mathrm{NE}$ & ENE & E & ESE & SE & SSE & S & SSW & SW & WSW & W & WNW & NW & NNW & CALM & TOTAL \\
\hline CALM & 0.0 & 0.0 & 0.0 & 0.0 & 0.0 & 0.0 & 0.0 & 0.0 & 0.0 & 0.0 & 0.0 & 0.0 & 0.0 & 0.0 & 0.0 & 0.0 & 0.9 & 0.9 \\
\hline $1-3$ & 1.5 & 1.4 & 1.5 & 1.5 & 1.7 & 1.7 & 1.7 & 1. & 1.1 & 1 & 1.0 & & 1.4 & & 1.9 & 1.7 & 0.0 & 23.4 \\
\hline 4-7 & 1.6 & 1.4 & 1.0 & 1.0 & 1.5 & 2.1 & 2.9 & 2.4 & 1.5 & 1.1 & 1.5 & 2.3 & 4.3 & 6.2 & 4.4 & 2.2 & 0.0 & 37.4 \\
\hline $8-12$ & 0.7 & 0.7 & 0.3 & 0.1 & 0.2 & 0.4 & 0.8 & 1. & 0.7 & 0.5 & 1.0 & 2.1 & 4.3 & 7.6 & 2.8 & 0.7 & 0.0 & 24.1 \\
\hline $13-18$ & 0.2 & 0 & 0.1 & 0.0 & 0.0 & 0.0 & 0.1 & 0.2 & 0.2 & 0.3 & 0.8 & 1.2 & 1.3 & 3.5 & 1.4 & 0.1 & 0.0 & 9.9 \\
\hline & 0.0 & & 0.0 & 0.0 & 0.0 & 0.0 & 0.0 & 0 & 0.0 & 0 & 0. & 0 & 0 & & 0 & & .0 & 3.4 \\
\hline $25-31$ & 0.0 & 0.0 & 0.0 & 0.0 & 0.0 & 0.0 & 0.0 & 0 & 0.0 & 0. & 0.2 & 0. & 0.1 & 0 & 0 & & .0 & 0.8 \\
\hline $32-38$ & 0.0 & 0.0 & 0.0 & 0.0 & 0.0 & 0.0 & 0.0 & 0.0 & 0.0 & 0.0 & 0.0 & 0. & 0.0 & 0.0 & 0.0 & 0.0 & 0.0 & 0.1 \\
\hline $39-4$ & 0.0 & 0.0 & 0.0 & 0.0 & 0.0 & 0.0 & 0.0 & 0.0 & 0.0 & 0.0 & 0.0 & 0. & 0.0 & 0.0 & 0.0 & 0.0 & 0.0 & 0.0 \\
\hline$>4$ & 0.0 & 0.0 & 0.0 & 0.0 & 0.0 & 0.0 & 0.0 & 0. & 0.0 & 0.0 & 0.0 & 0.0 & 0.0 & 0.0 & 0.0 & 0.0 & 0.0 & 0.0 \\
\hline TOTAL & 4.0 & 3.8 & 3.1 & 2.7 & 3.5 & 4.2 & 5.5 & 5.0 & 3.6 & 3.1 & 5.1 & 7.7 & 11.7 & 20.1 & 11.5 & 4.6 & 0.9 & 100.0 \\
\hline
\end{tabular}

Station: (7) $200 \mathrm{~W}$

Begin: 1/82

End: $12 / 99$

Total Hours: 140096

DIRECTION

\begin{tabular}{|c|c|c|c|c|c|c|c|c|c|c|c|c|c|c|c|c|c|c|}
\hline & $\mathbf{N}$ & NNE & $\mathrm{NE}$ & ENE & E & ESE & SE & SSE & $S$ & SSW & SW & WSW & W & WNW & $\mathrm{NW}$ & NNW & CALM & TOTAL \\
\hline CALM & 0.0 & 0.0 & 0.0 & 0.0 & 0.0 & 0.0 & 0.0 & 0.0 & 0.0 & 0.0 & 0.0 & 0.0 & 0.0 & 0.0 & 0.0 & 0.0 & 1.7 & 1.7 \\
\hline $1-3$ & 2.2 & 1.8 & 1.5 & 1.4 & 1.6 & 1.7 & 2.2 & 2.0 & 1.8 & 1.7 & 2.0 & 2.6 & 3.6 & 4.4 & 3.7 & 2.6 & 0.0 & 36.8 \\
\hline 4. & 3.0 & 1.6 & 1.0 & 0.8 & 0.9 & 1.5 & 1.7 & 1.0 & 0.7 & 0.8 & 1.2 & 1.5 & 4.0 & 6.8 & 5. & & 0.0 & 35.4 \\
\hline $8-12$ & 0.7 & 0.5 & 0.2 & 0.1 & 0.1 & 0.3 & 0.4 & 0.2 & 0.2 & 0.6 & 1.1 & 1. & 2.5 & 2. & 3.2 & 1.8 & 0.0 & 16.4 \\
\hline $13-1$ & 0.1 & 0.2 & 0.1 & 0.0 & 0.0 & 0.0 & 0.0 & 0.0 & 0.1 & 0.3 & 1.0 & & 0.7 & 0.7 & 2.0 & 0.4 & 0.0 & 7.1 \\
\hline 19. & 0.0 & 0.0 & 0.0 & 0.0 & 0.0 & 0.0 & 0.0 & 0 & 0.0 & 0. & 0.5 & 0 & 0.2 & & 0.8 & & 0.0 & 2.2 \\
\hline 25 & 0.0 & 0.0 & 0.0 & 0.0 & 0.0 & 0.0 & 0.0 & 0. & 0.0 & 0.0 & 0.1 & 0. & 0.0 & 0. & 0. & 0.0 & 0.0 & 0.4 \\
\hline 32 & 0.0 & 0.0 & 0.0 & 0.0 & 0.0 & 0.0 & 0.0 & 0.0 & 0.0 & 0.0 & 0.0 & 0.0 & 0.0 & 0.0 & 0.0 & 0.0 & 0.0 & 0.0 \\
\hline & 0.0 & 0.0 & 0.0 & 0.0 & 0.0 & 0.0 & 0.0 & 0.0 & 0.0 & 0.0 & 0.0 & 0.0 & 0.0 & 0.0 & 0.0 & 0.0 & 0.0 & 0.0 \\
\hline$>4$ & 0.0 & 0.0 & 0.0 & 0.0 & 0.0 & 0.0 & 0.0 & 0.0 & 0.0 & 0.0 & 0.0 & 0.0 & 0.0 & 0.0 & 0.0 & 0.0 & 0.0 & 0.0 \\
\hline TOTAL & 6.0 & 4.I & 2.9 & 2.2 & 2.6 & 3.5 & 4.2 & 3.2 & 2.9 & 3.5 & 5.9 & 8.2 & 11.0 & 14.9 & 14.9 & 8.3 & 1.7 & 100.0 \\
\hline
\end{tabular}

Station: (8) BVLY

Begin: $8 / 91$

End: $12 / 99$

Total Hours: 73038

DIRECTION

\begin{tabular}{|c|c|c|c|c|c|c|c|c|c|c|c|c|c|c|c|c|c|c|}
\hline & $\mathrm{N}$ & NNE & NE & ENE & E & ESE & SE & SSE & $\underline{S}$ & SSW & SW & WSW & W & WNW & NW & NNW & CALM & TOTAL \\
\hline CALM & 0.0 & 0.0 & 0.0 & 0.0 & 0.0 & 0.0 & 0.0 & 0.0 & 0.0 & 0.0 & 0.0 & 0.0 & 0.0 & 0.0 & 0.0 & 0.0 & 1.0 & 1.0 \\
\hline 1-3 & 2.7 & 2.1 & 1.6 & 1.3 & 1.6 & 1.8 & 1.9 & 1.7 & 1.7 & 1.3 & 1.1 & 1.1 & 1.2 & 1.5 & 2.0 & 2.4 & 0.0 & 27.0 \\
\hline 4-7 & 8.4 & 3.0 & 0.6 & 0.4 & 1.2 & 2.9 & 1.8 & 1.2 & 1.1 & 0.8 & 0.7 & 0.8 & 1.4 & 2 & 4.5 & 6.7 & 0.0 & 38.0 \\
\hline $8-12$ & 7.3 & 1.9 & 0.2 & 0.0 & 0.3 & 0.8 & 0.3 & 0.3 & 0.3 & 0.3 & 0.4 & 0.4 & 1.2 & 3.3 & 2.9 & 2.2 & 0.0 & 22.1 \\
\hline $13-18$ & 0.5 & 0.3 & 0.1 & 0.0 & 0.0 & 0.0 & 0.0 & 0.1 & 0.1 & 0.2 & 0.5 & 0.3 & 0.5 & 3.4 & 2.1 & 0.1 & 0.0 & 8.3 \\
\hline $19-24$ & 0.0 & 0.0 & 0.0 & 0.0 & 0.0 & 0.0 & 0.0 & 0.0 & 0.0 & 0.1 & 0.2 & 0. & 0.1 & 1. & 0. & 0.0 & 0.0 & 2.8 \\
\hline $25-3$ & 0.0 & 0.0 & 0.0 & 0.0 & 0.0 & 0.0 & 0.0 & 0.0 & 0.0 & 0.0 & 0.1 & 0.0 & 0.0 & 0.4 & 0.2 & 0.0 & 0.0 & 0.7 \\
\hline $32-38$ & 0.0 & 0.0 & 0.0 & 0.0 & 0.0 & 0.0 & 0.0 & 0.0 & 0.0 & 0.0 & 0.0 & 0.0 & 0.0 & 0.0 & 0.0 & 0.0 & 0.0 & 0.1 \\
\hline $39-46$ & 0.0 & 0.0 & 0.0 & 0.0 & 0.0 & 0.0 & 0.0 & 0.0 & 0.0 & 0.0 & 0.0 & 0.0 & 0.0 & 0.0 & 0.0 & 0.0 & 0.0 & 0.0 \\
\hline & 0.0 & 0. & 0.0 & 0.0 & 0.0 & 0.0 & 0.0 & 0.0 & 0.0 & 0.0 & 0.0 & 0. & 0.0 & 0.0 & 0.0 & 0.0 & 0.0 & 0.0 \\
\hline COTAL & 19.0 & 7.3 & 2.5 & 1.8 & 3.1 & 5.6 & 4.0 & 3.3 & 3.2 & 2.6 & 3.1 & 2.7 & 4.4 & 12.5 & 12.5 & 11.4 & 1.0 & 100.0 \\
\hline
\end{tabular}


Table 5.4. (contd)

Station: (9) FFTF

Begin: 1/82

End: $12 / 99$

Total Hours: 153413

DIRECTION

\begin{tabular}{|c|c|c|c|c|c|c|c|c|c|c|c|c|c|c|c|c|c|c|}
\hline & $N$ & NNE & $\mathrm{NE}$ & ENE & $\mathrm{E}$ & ESE & SE & SSE & $\mathrm{s}$ & SSW & SW & WSW & W & WNW & NW & NNW & CALM & TOTAL \\
\hline CALM & 0.0 & 0.0 & 0.0 & 0.0 & 0.0 & 0.0 & 0.0 & 0.0 & 0.0 & 0.0 & 0.0 & 0.0 & 0.0 & 0.0 & 0.0 & 0.0 & 0.5 & 0.5 \\
\hline $1-3$ & 1.2 & 1.1 & 0.9 & 0.8 & 0.9 & 1.0 & 1.1 & 1.0 & 1.2 & 1.2 & 1.2 & 1.0 & 1.0 & 1.1 & 1.3 & 1.3 & 0.0 & 17.2 \\
\hline $4-7$ & 2.8 & 2.6 & 1.9 & 1.1 & 1.1 & 1.4 & 2.6 & 3.7 & 4.1 & 3.6 & 2.0 & 1.3 & 1.4 & 2.1 & 3.6 & 3.3 & 0.0 & 38. \\
\hline $8-12$ & 1.4 & 1.3 & 0.7 & 0.2 & 0.2 & 0.2 & 1.1 & 3.2 & 3.9 & 4.4 & 1.8 & 0.8 & 0.9 & 1.8 & 3.9 & 2.6 & 0.0 & \\
\hline $13-18$ & 0.3 & 0.3 & 0.2 & 0.0 & 0.0 & 0.0 & 0.1 & 0.3 & 0.9 & 3.0 & 1.8 & 0.7 & 0.5 & 0.8 & 1.6 & 0.5 & 0.0 & 11 \\
\hline $19-24$ & 0.0 & 0.1 & 0.1 & 0.0 & 0.0 & 0.0 & 0.0 & 0.0 & 0.1 & 0.6 & 0.9 & 0.4 & 0.2 & 0.2 & 0.5 & 0.1 & 0.0 & \\
\hline $25-31$ & 0.0 & 0.0 & 0.0 & 0.0 & 0.0 & 0.0 & 0.0 & 0.0 & 0.0 & 0.1 & 0.3 & 0.1 & 0.0 & 0.0 & 0.1 & 0.0 & 0.0 & \\
\hline $32-38$ & 0.0 & 0.0 & 0.0 & 0.0 & 0.0 & 0.0 & 0.0 & 0.0 & 0.0 & 0.0 & 0.1 & 0.0 & 0.0 & 0.0 & 0.0 & 0.0 & 0.0 & \\
\hline $39-46$ & 0.0 & 0.0 & 0.0 & 0.0 & 0.0 & 0.0 & 0.0 & 0.0 & 0.0 & 0.0 & 0.0 & 0.0 & 0.0 & 0.0 & 0.0 & 0.0 & 0.0 & \\
\hline$>46$ & 0.0 & 0.0 & 0.0 & 0.0 & 0.0 & 0.0 & 0.0 & 0.0 & 0.0 & 0.0 & 0.0 & 0.0 & 0.0 & 0.0 & 0.0 & 0.0 & 0.0 & \\
\hline TOTAL & 5.7 & 5.5 & 3.8 & 2.2 & 2.2 & 2.6 & 4.9 & 8.3 & 10.2 & 13.0 & 8.1 & 43 & 4.0 & 6.1 & 109 & 77 & 0.6 & 100. \\
\hline
\end{tabular}

Station: (10) YAKB

Begin: 1/82

End: $12 / 99$

Total Hours: 154089

DIRECTION

\begin{tabular}{|c|c|c|c|c|c|c|c|c|c|c|c|c|c|c|c|c|c|c|}
\hline & & $\sqrt{E}$ & $\mathrm{VE}$ & ENE & $\mathbf{E}$ & ESE & SE & SSE & $\mathbf{S}$ & SW & SW & SW & W & WNWW & $N W$ & NW & & \\
\hline CALM & 0.0 & 0.0 & 0.0 & 0.0 & 0.0 & 0.0 & 0.0 & 0.0 & 0.0 & 0.0 & 0.0 & 0.0 & 0.0 & 0.0 & 0.0 & 0.0 & 0.6 & 0.6 \\
\hline $1-3$ & 1.7 & 1.6 & 1.5 & 1.1 & 0.9 & 1.0 & 1.1 & 1.3 & 1.2 & 1.2 & 1.5 & 2.0 & 2.5 & 2.0 & 1.7 & 1.6 & 0.0 & 24.0 \\
\hline $4-7$ & 3.7 & 2.8 & 1.6 & 0.9 & 0.8 & 1.0 & 1.4 & 1.2 & 0.9 & 1.0 & 1.6 & 3.6 & 7.0 & 4.4 & 3.8 & 3.6 & 0.0 & 9.8 \\
\hline $8-12$ & 1.3 & 0.5 & 0.2 & 0.1 & 0.1 & 0.1 & 0.3 & 0.2 & 0.3 & 0.6 & 1.5 & 2.6 & 3.1 & 2.4 & 5.5 & 3.2 & 0.0 & \\
\hline $13-18$ & 0.2 & 0.2 & 0.1 & 0.0 & 0.0 & 0.0 & 0.0 & 0.0 & 0.1 & 0.3 & 1.2 & 1.4 & 0.6 & 0.9 & 3.9 & 1.0 & 0.0 & \\
\hline $19-24$ & 0.0 & 0.0 & 0.0 & 0.0 & 0.0 & 0.0 & 0.0 & 0.0 & 0.0 & 0.2 & 0.6 & 0.4 & 0.1 & 0.2 & 1.8 & 0.1 & 0.0 & \\
\hline $25-31$ & 0.0 & 0.0 & 0.0 & 0.0 & 0.0 & 0.0 & 0.0 & 0.0 & 0.0 & 0.1 & 0.2 & 0.1 & 0.0 & 0.0 & 0.3 & 0.0 & 0.0 & \\
\hline $32-38$ & 0.0 & 0.0 & 0.0 & 0.0 & 0.0 & 0.0 & 0.0 & 0.0 & 0.0 & 0.0 & 0.0 & 0.0 & 0.0 & 0.0 & 0.0 & 0.0 & 0.0 & \\
\hline $39-46$ & 0.0 & 0.0 & 0.0 & 0.0 & 0.0 & 0.0 & 0.0 & 0.0 & 0.0 & 0.0 & 0.0 & 0.0 & 0.0 & 0.0 & 0.0 & 0.0 & 0.0 & \\
\hline$>46$ & 0.0 & 0.0 & 0.0 & 0.0 & 0.0 & 0.0 & 0.0 & 0.0 & 0.0 & 0.0 & 0.0 & 0.0 & 0.0 & 0.0 & 0.0 & 0.0 & 0.0 & \\
\hline TOTAL & 6.9 & 5.2 & 3.4 & 2.1 & 1.8 & 2.1 & 2.8 & 2.7 & 2.6 & 3.3 & 6.5 & 10.0 & 13.4 & 10.0 & 17.0 & 9.5 & 0.6 & in \\
\hline
\end{tabular}

Station: (11) $300 \mathrm{~A}$

Begin: 1/82

End: $12 / 99$

Total Hours: 140865

DIRECTION

\begin{tabular}{|c|c|c|c|c|c|c|c|c|c|c|c|c|c|c|c|c|c|c|}
\hline & $\mathrm{N}$ & NNE & $\mathrm{NE}$ & ENE & E & ESE & SE & SSE & $\underline{S}$ & SSW & SW & WSW & W & WNW & NW & NNW & CALM & TOTAL \\
\hline CALM & 0.0 & 0.0 & 0.0 & 0.0 & 0.0 & 0.0 & 0.0 & 0.0 & 0.0 & 0.0 & 0.0 & 0.0 & 0.0 & 0.0 & 0.0 & 0.0 & 0.6 & 0.6 \\
\hline $1-3$ & 1.2 & 0.7 & 0.6 & 0.6 & 0.8 & 1.2 & 1.7 & 1.8 & 1.7 & 1.4 & 1.4 & 1.2 & 1.3 & 1.4 & 1.8 & 1.7 & 0.0 & \\
\hline $4-7$ & 3.5 & 1.6 & 1.1 & 1.1 & 1.9 & 4.2 & 6.8 & 3.7 & 3.1 & 2.5 & 1.9 & 1.2 & 0.9 & 1.0 & 2.1 & 4.0 & 0.0 & \\
\hline $8-12$ & 3.5 & 1.9 & 0.8 & 0.3 & 0.4 & 1.2 & 1.6 & 0.8 & 1.6 & 3.1 & 3.3 & 1.7 & 0.6 & 0.4 & 1.1 & 2.6 & 0.0 & \\
\hline $13-18$ & 0.6 & 0.5 & 0.2 & 0.1 & 0.0 & 0.0 & 0.1 & 0.1 & 0.4 & 1.5 & 2.5 & 1.3 & 0.4 & 0.2 & 0.8 & 0.9 & 0.0 & 9 \\
\hline $19-24$ & 0.1 & 0.1 & 0.0 & 0.0 & 0.0 & 0.0 & 0.0 & 0.0 & 0.1 & 0.4 & 0.9 & 0.4 & 0.2 & 0.0 & 0.3 & 0.2 & 0.0 & \\
\hline $25-31$ & 0.0 & 0.0 & 0.0 & 0.0 & 0.0 & 0.0 & 0.0 & 0.0 & 0.0 & 0.1 & 0.4 & 0.1 & 0.1 & 0.0 & 0.1 & 0.0 & 0.0 & \\
\hline $32-38$ & 0.0 & 0.0 & 0.0 & 0.0 & 0.0 & 0.0 & 0.0 & 0.0 & 0.0 & 0.0 & 0.1 & 0.0 & 0.0 & 0.0 & 0.0 & 0.0 & 0.0 & \\
\hline & 0.0 & 0.0 & 0.0 & 0.0 & 0.0 & 0.0 & 0.0 & 0.0 & 0.0 & 0.0 & 0.0 & 0.0 & 0.0 & 0.0 & 0.0 & 0.0 & 0.0 & \\
\hline$>46$ & 0.0 & 0.0 & 0.0 & 0.0 & 0.0 & 0.0 & 0.0 & 0.0 & 0.0 & 0.0 & 0.0 & 0.0 & 0.0 & 0.0 & 0.0 & 0.0 & 0.0 & \\
\hline TOTAL & 8.8 & 4.8 & 2.7 & 2.1 & 3.0 & 6.7 & 10.3 & 6.5 & 6.8 & 9.2 & 10.6 & 6.0 & 3.4 & 3.0 & 6.2 & 9.4 & 0.6 & 100 \\
\hline
\end{tabular}

Station: (12) WYEB

Begin: 1/82

End: $12 / 99$

Total Hours: 153413

DIRECTION

\begin{tabular}{|c|c|c|c|c|c|c|c|c|c|c|c|c|c|c|c|c|c|c|}
\hline & $\mathbf{N}$ & NNE & NE & ENE & $\mathrm{E}$ & ESE & SE & SSE & $\mathbf{S}$ & SSW & SW & WSW & W & WNW & NW & NNW & CALM & TOTAL \\
\hline CALM & 0.0 & 0.0 & 0.0 & 0.0 & 0.0 & 0.0 & 0.0 & 0.0 & 0.0 & 0.0 & 0.0 & 0.0 & 0.0 & 0.0 & 0.0 & 0.0 & 0.4 & 0.4 \\
\hline $1-3$ & 1.3 & 1.1 & 1.2 & 1.2 & 1.4 & 1.5 & 1.4 & 1.5 & 1.5 & 1.3 & 1.3 & 1.2 & 1.2 & 1.2 & 1.4 & 1.3 & 0.0 & 20.8 \\
\hline $4-7$ & 2.5 & 1.6 & 1.3 & 1.3 & 2.0 & 2.0 & 2.7 & 3.4 & 3.9 & 2.9 & 2.4 & 2.2 & 2.5 & 3.1 & 3.8 & 3.0 & 0.0 & 40. \\
\hline $8-12$ & 1.2 & 0.6 & 0.4 & 0.2 & 0.3 & 0.3 & 0.8 & 1.8 & 2.9 & 2.5 & 1.6 & 1.3 & 2.0 & 4.1 & 3.2 & 1.5 & 0.0 & 24. \\
\hline $13-18$ & 0.3 & 0.2 & 0.1 & 0.0 & 0.0 & 0.0 & 0.1 & 0.3 & 1.0 & 1.5 & 1.1 & 0.7 & 0.8 & 1.5 & 1.4 & 0.3 & 0.0 & \\
\hline $19-24$ & 0.0 & 0.1 & 0.0 & 0.0 & 0.0 & 0.0 & 0.0 & 0.0 & 0.1 & 0.5 & 0.6 & 0.3 & 0.2 & 0.3 & 0.6 & 0.1 & 0.0 & \\
\hline $25-31$ & 0.0 & 0.0 & 0.0 & 0.0 & 0.0 & 0.0 & 0.0 & 0.0 & 0.0 & 0.1 & 0.2 & 0.1 & 0.1 & 0.1 & 0.2 & 0.0 & 0.0 & \\
\hline $32-38$ & 0.0 & 0.0 & 0.0 & 0.0 & 0.0 & 0.0 & 0.0 & 0.0 & 0.0 & 0.0 & 0.1 & 0.0 & 0.0 & 0.0 & 0.0 & 0.0 & 0.0 & \\
\hline $39-46$ & 0.0 & 0.0 & 0.0 & 0.0 & 0.0 & 0.0 & 0.0 & 0.0 & 0.0 & 0.0 & 0.0 & 0.0 & 0.0 & 0.0 & 0.0 & 0.0 & 0.0 & \\
\hline$>46$ & 0.0 & 0.0 & 0.0 & 0.0 & 0.0 & 0.0 & 0.0 & 0.0 & 0.0 & 0.0 & 0.0 & 0.0 & 0.0 & 0.0 & 0.0 & 0.0 & 0.0 & 0 \\
\hline TOTAL & 5.3 & 3.7 & 3.0 & 2.7 & 3.7 & 3.8 & 5.0 & 7.0 & 9.3 & 8.9 & 7.3 & 5.9 & 6.8 & 10.4 & 10.6 & 6.3 & 0.4 & 100 \\
\hline
\end{tabular}


Table 5.4. (contd)

Station: (13) $100 \mathrm{~N}$

Begin: 1/82

End: $12 / 99$

Total Hours: 153625

DIRECTION

\begin{tabular}{|c|c|c|c|c|c|c|c|c|c|c|c|c|c|c|c|c|c|c|}
\hline & $N$ & NNE & $\mathrm{NE}$ & ENE & $E$ & ESE & SE & SSE & $S$ & SSW & SW & WSW & W & WNW & NW & NNW & CALM & TOTAL \\
\hline CALM & 0.0 & 0.0 & 0.0 & 0.0 & 0.0 & 0.0 & 0.0 & 0.0 & 0.0 & 0.0 & 0.0 & 0.0 & 0.0 & 0.0 & 0.0 & 0.0 & 0.9 & 0.9 \\
\hline $1-3$ & 2.1 & 1.8 & 2.0 & 2.3 & 3.1 & 3.1 & 2.6 & 1.9 & 1.7 & 1.7 & 2.2 & 2.7 & 3.2 & 3.3 & 3.1 & 2.5 & 0.0 & 39.3 \\
\hline $4-7$ & 1.4 & 1.6 & 1.6 & 1.9 & 2.7 & 2.6 & 2.3 & 1.3 & 0.9 & 1.0 & 2.3 & 4.4 & 4.8 & & 2.1 & 1.5 & 0.0 & 35.6 \\
\hline 8-12 & 0.4 & 0.8 & 0.6 & 0.2 & 0.3 & 0.4 & 0. & 0.5 & 0.3 & 0.5 & 1.2 & 2. & 3.2 & 2 & 0.7 & 0 & 0.0 & 14.5 \\
\hline $13-18$ & 0.2 & 0.4 & 0.3 & 0.1 & 0.0 & 0.0 & 0.1 & 0.1 & 0.1 & 0.3 & 0.8 & 0.6 & 1.2 & 1. & 0.6 & 0 & 0.0 & 6.9 \\
\hline $19-24$ & 0.0 & 0.1 & 0.1 & 0.0 & 0.0 & 0.0 & 0.0 & 0.0 & 0.0 & 0.2 & 0.3 & 0.2 & 0.2 & 0.7 & 0.4 & 0.0 & 0.0 & 2.3 \\
\hline $25-31$ & 0.0 & 0.0 & 0.0 & 0.0 & 0.0 & 0.0 & 0.0 & 0.0 & 0.0 & 0.1 & 0.1 & 0.0 & 0.0 & 0.2 & 0.1 & 0.0 & 0.0 & 0.5 \\
\hline 32 & 0.0 & 0.0 & 0.0 & 0.0 & 0.0 & 0.0 & 0.0 & 0.0 & 0.0 & 0.0 & 0.0 & 0.0 & 0.0 & 0.0 & 0.0 & 0.0 & 0.0 & 0.0 \\
\hline & 0.0 & 0.0 & 0.0 & 0.0 & 0.0 & 0.0 & 0. & 0.0 & 0. & 0.0 & 0.0 & 0.0 & 0.0 & 0. & 0 & 0.0 & 0.0 & 0.0 \\
\hline & 0.0 & 0.0 & 0.0 & 0.0 & 0.0 & 0.0 & 0.0 & 0.0 & 0.0 & 0.0 & 0.0 & 0.0 & 0.0 & 0.0 & 0.0 & 0.0 & 0.0 & 0.0 \\
\hline OTAL & 4.1 & 4.7 & 4.6 & 4.6 & 6.0 & 6.2 & 5.8 & 3.7 & 3.1 & 3.7 & 6.9 & 10.1 & 12.7 & 11.4 & 7.0 & 4.5 & 0.9 & 100.0 \\
\hline
\end{tabular}

Station: (14) WPPS

Begin: 1/82

End: $12 / 99$

Total Hours: 154121

DIRECTION

\begin{tabular}{|c|c|c|c|c|c|c|c|c|c|c|c|c|c|c|c|c|c|c|}
\hline & $\mathrm{N}$ & NNE & $\mathrm{NE}$ & ENE & E & ESE & $\mathrm{SE}$ & SSE & $S$ & SSW & SW & WSW & $\mathrm{W}$ & WNW & NW & NNW & CALM & TOTAL \\
\hline CALM & 0.0 & 0.0 & 0.0 & 0.0 & 0.0 & 0.0 & 0.0 & 0.0 & 0.0 & 0.0 & 0.0 & 0.0 & 0.0 & 0.0 & 0.0 & 0.0 & 0.9 & 0.9 \\
\hline 1 & 2.5 & 2.1 & 1.9 & 1.3 & 1.1 & 1.1 & 1.4 & 1.9 & 2.2 & 2.0 & 1.7 & 1.4 & 1.6 & 1.8 & 2.7 & 2.8 & 0.0 & 29.7 \\
\hline 4-7 & 3.4 & 2.3 & 2.1 & 1.2 & 0.7 & 0.9 & 1.9 & 4.2 & 5.2 & 3.0 & 1.7 & 1.2 & 1.3 & 1. & 3.8 & 4.4 & 0.0 & 39.1 \\
\hline $8-12$ & 1.2 & 0.7 & 0.5 & 0.2 & 0.1 & 0.2 & 0.7 & 1.9 & 3. & 2.6 & 1.4 & 0.8 & 0.9 & I. & 2.3 & 1.4 & 0.0 & 19.9 \\
\hline $13-1$ & 0.3 & 0.2 & 0.1 & 0.0 & 0.0 & 0.0 & 0.1 & 0.2 & 0.7 & 1.6 & 1.2 & 0.5 & 0.4 & 0.7 & 1.3 & 0.3 & 0.0 & 7.7 \\
\hline & 0.0 & 0.1 & 0.0 & 0.0 & 0.0 & 0.0 & 0.0 & 0.0 & 0.1 & 0.4 & 0.6 & 0.2 & 0.1 & 0.1 & 0.5 & 0.0 & 0.0 & 2.2 \\
\hline & 0.0 & 0.0 & 0.0 & 0.0 & 0.0 & 0.0 & 0.0 & 0.0 & 0. & 0. & 0.2 & 0 & 0 & & 0 & & & 0.5 \\
\hline $32-3$ & 0.0 & 0.0 & 0.0 & 0.0 & 0.0 & 0.0 & 0.0 & 0.0 & 0.0 & 0.0 & 0.0 & 0.0 & 0. & 0. & 0. & 0 & 0 & 0.1 \\
\hline $39-4$ & 0.0 & 0.0 & 0.0 & 0.0 & 0.0 & 0.0 & 0.0 & 0.0 & 0.0 & 0.0 & 0.0 & 0.0 & 0.0 & 0.0 & 0.0 & 0.0 & 0.0 & 0.0 \\
\hline & 0.0 & 0.0 & 0.0 & 0.0 & 0.0 & 0.0 & 0.0 & 0.0 & 0.0 & 0.0 & 0.0 & 0.0 & 0.0 & 0.0 & 0.0 & 0.0 & 0.0 & 0.0 \\
\hline TOTAL & 7.4 & 5.4 & 4.6 & 2.7 & 1.9 & 2.2 & 4.1 & 8.2 & 11.4 & 9.8 & 6.8 & 4.2 & 4.4 & 6.3 & 10.6 & 9.0 & 0.9 & 100.0 \\
\hline
\end{tabular}

Station: (15) FRNK

Begin: $1 / 82$

End: $12 / 99$

Total Hours: 153691

DIRECTION

\begin{tabular}{|c|c|c|c|c|c|c|c|c|c|c|c|c|c|c|c|c|c|c|}
\hline & $\mathrm{N}$ & NNE & $\mathrm{NE}$ & ENE & $E$ & ESE & SE & SSE & $\mathrm{s}$ & SSW & SW & WSW & W & WNW & NW & NNW & CALM & TOTAL \\
\hline CALM & 0.0 & 0.0 & 0.0 & 0.0 & 0.0 & 0.0 & 0.0 & 0.0 & 0.0 & 0.0 & 0.0 & 0.0 & 0.0 & 0.0 & 0.0 & 0.0 & 0.8 & 0.8 \\
\hline $1-3$ & 1.2 & 1.0 & 1.0 & 0.8 & 0.9 & 1.1 & 1.5 & 1.5 & 1.3 & 1. & 1.2 & & 1.3 & & 1.6 & 1.4 & 0.0 & 19.3 \\
\hline $4-7$ & 4.1 & 2.7 & 1.7 & 1.1 & 1.5 & 2.3 & 4.8 & 4.2 & 3.5 & 3. & 2. & 1. & 1.4 & & & & 0.0 & 46.0 \\
\hline $8-1$ & 1.6 & 0.9 & 0.6 & 0.3 & 0.3 & 0.6 & 1. & 1. & 2. & 4. & 3 & & 5 & & & & .0 & 24.9 \\
\hline & 0.1 & 0.2 & 0.2 & 0.1 & 0.0 & 0.0 & 0.1 & 0.2 & 0.4 & 2. & 2. & 0.6 & 0.2 & & 0.5 & & 0.0 & 7.1 \\
\hline & 0.0 & 0.1 & 0.0 & 0.0 & 0.0 & 0.0 & 0.0 & 0.0 & 0.0 & 0. & 0.5 & 0.2 & 0.0 & 0. & 0.1 & 0.0 & 0.0 & 1.4 \\
\hline & 0.0 & & 0.0 & 0.0 & 0.0 & 0.0 & 0.0 & 0.0 & 0.0 & 0. & 0.2 & 0 & 0.0 & 0. & & & 0.0 & 0.4 \\
\hline & 0.0 & 0. & 0.0 & 0.0 & 0.0 & 0. & 0. & 0. & 0.0 & 0. & 0. & & & & & & & 0.1 \\
\hline & 0.0 & 0.0 & 0.0 & 0.0 & 0.0 & 0.0 & 0.0 & 0.0 & 0.0 & 0. & 0. & 0. & 0.0 & 0 & 0. & & 0.0 & 0.0 \\
\hline$>46$ & 0.0 & 0.0 & 0.0 & 0.0 & 0.0 & 0.0 & 0.0 & 0.0 & 0.0 & 0.0 & 0.0 & 0.0 & 0.0 & 0.0 & 0.0 & 0.0 & 0.0 & 0.0 \\
\hline TOTAL & 7.1 & 4.9 & 3.5 & 2.4 & 2.7 & 4.1 & 8.0 & 7.4 & 7.7 & 11.3 & 9.8 & 4.3 & 3.5 & 4.2 & 8.8 & 9.5 & 0.8 & 100.0 \\
\hline
\end{tabular}

Station: (16) GABL

Begin: 1/82

End: $12 / 99$

Total Hours: 153183

DIRECTION

\begin{tabular}{|c|c|c|c|c|c|c|c|c|c|c|c|c|c|c|c|c|c|c|}
\hline & $\mathrm{N}$ & NNE & $\mathrm{NE}$ & ENE & E & ESE & SE & SSE & $S$ & SSW & SW & WSW & W & WNW & NW & NNW & CALM & TOTAL \\
\hline CALM & 0.0 & 0.0 & 0.0 & 0.0 & 0.0 & 0.0 & 0.0 & 0.0 & 0.0 & 0.0 & 0.0 & 0.0 & 0.0 & 0.0 & 0.0 & 0.0 & 0.7 & 0.7 \\
\hline $1-3$ & 1.0 & 0.9 & 0.9 & 0.7 & 0.6 & 0.6 & 0.7 & 0.8 & 1.0 & 1.0 & 0.9 & 0. & 0.8 & 0.7 & 0.8 & 0.9 & 0.0 & 13.1 \\
\hline $4-7$ & 2.3 & 2.3 & 1.6 & 0.9 & 0.9 & 0.9 & 1.3 & 2. & 3.2 & 2.4 & 1.8 & 1.5 & $1 . J$ & 1.7 & 2.2 & 2.1 & 0.0 & 28.8 \\
\hline $8-12$ & 2.0 & 2.2 & 1.0 & 0.3 & 0.4 & 0.5 & 0.9 & 1.7 & 2.4 & 1.5 & 1.5 & 1.6 & 1.7 & 2.2 & 2.9 & 1.8 & 0.0 & 24.6 \\
\hline $13-1$ & 1.3 & 1.4 & 0.5 & 0.1 & 0.1 & 0.1 & 0.3 & 1.0 & 1.4 & 0. & 1.3 & 1. & 1.8 & 2. & 2.5 & 0.7 & 0.0 & 17.6 \\
\hline & 0.4 & 0.5 & 0.3 & 0.0 & 0.0 & 0. & 0.0 & 0. & 0.6 & & 0. & 0 & 0.5 & 2 & & & & \\
\hline $25-$ & 0.1 & 0.1 & 0.2 & 0.0 & 0.0 & 0. & 0.0 & 0. & 0.2 & 0.3 & 0.6 & 0. & 0.2 & 1. & 0.5 & & 0.0 & \\
\hline $32-3$ & 0.0 & 0.0 & 0.1 & 0.0 & 0.0 & 0.0 & 0.0 & 0.0 & 0.1 & 0.2 & 0.3 & 0. & 0.0 & 0.2 & 0.0 & 0.0 & 0.0 & 8 \\
\hline & 0.0 & 0.0 & 0.0 & 0.0 & 0.0 & 0.0 & 0.0 & 0.0 & 0.0 & 0.1 & 0.1 & 0.0 & 0.0 & 0.0 & 0.0 & 0.0 & 0.0 & 0.3 \\
\hline & 0.0 & 0.0 & 0.0 & 0.0 & 0.0 & 0.0 & 0.0 & 0.0 & 0.0 & 0.0 & 0.0 & & 0.0 & 0.0 & 0.0 & 0.0 & 0.0 & \\
\hline TOTAL & 7.1 & 7.5 & 4.6 & 2.0 & 2.0 & 2.1 & 3.2 & 6.0 & 8.9 & 6.8 & 7.4 & 6.8 & 7.0 & 11.6 & 10.6 & 5.8 & 0.8 & 100 \\
\hline
\end{tabular}


Table 5.4. (contd)

Station: (17) RING DIRECTION

\begin{tabular}{|c|c|c|c|c|c|c|c|c|c|c|c|c|c|c|c|c|c|c|}
\hline & & & & & & & & & & & & & & NW & Now & NNW & $L M$ & IOLIAL \\
\hline CALM & 0.0 & 0.0 & 0.0 & 0.0 & 0.0 & 0.0 & 0.0 & 0.0 & 0.0 & 0.0 & 0.0 & 0.0 & 0.0 & 0.0 & 0.0 & 0.0 & 1.1 & 1.1 \\
\hline $1-3$ & 2.2 & 3.6 & 7.5 & 3.7 & 2.3 & 1.8 & 1.5 & 1.4 & 1.6 & 1.7 & 2.1 & 2.5 & 2.2 & 1.5 & 1.5 & 1.8 & 0.0 & 38. \\
\hline $4-7$ & 1.6 & 2.1 & 10.5 & 3.2 & 1.1 & 0.9 & 1.1 & 1.4 & 1.8 & 2.9 & 2.5 & 2.7 & 2.3 & 1.3 & 1.2 & 1.2 & 0.0 & \\
\hline $8-12$ & 0.6 & 0.6 & 0.8 & 0.4 & 0.1 & 0.1 & 0.2 & 0.5 & 1.1 & 3.3 & 2.1 & 1.1 & 1.5 & 1.9 & 0.9 & 0.2 & 0.0 & \\
\hline $13-18$ & 0.1 & 0.2 & 0.2 & 0.0 & 0.0 & 0.0 & 0.0 & 0.1 & 0.1 & 1.0 & 1.0 & 0.5 & 0.5 & 1.4 & 0.4 & 0.0 & 0.0 & \\
\hline $19-24$ & 0.0 & 0.1 & 0.1 & 0.0 & 0.0 & 0.0 & 0.0 & 0.0 & 0.0 & 0.1 & 0.3 & 0.2 & 0.1 & 0.4 & 0.1 & 0.0 & 0.0 & \\
\hline $25-31$ & 0.0 & 0.0 & 0.0 & 0.0 & 0.0 & 0.0 & 0.0 & 0.0 & 0.0 & 0.0 & 0.1 & 0.0 & 0.0 & 0.0 & 0.0 & 0.0 & 0.0 & \\
\hline $32-38$ & 0.0 & 0.0 & 0.0 & 0.0 & 0.0 & 0.0 & 0.0 & 0.0 & 0.0 & 0.0 & 0.0 & 0.0 & 0.0 & 0.0 & 0.0 & 0.0 & 0.0 & \\
\hline $39-46$ & 0.0 & 0.0 & 0.0 & 0.0 & 0.0 & 0.0 & 0.0 & 0.0 & 0.0 & 0.0 & 0.0 & 0.0 & 0.0 & 0.0 & 0.0 & 0.0 & 0.0 & \\
\hline$>46$ & 0.0 & 0.0 & 0.0 & 0.0 & 0.0 & 0.0 & 0.0 & 0.0 & 0.0 & 0.0 & 0.0 & 0.0 & 0.0 & 0.0 & 0.0 & 0.0 & 0.0 & \\
\hline TOTAL & 4.5 & 6.5 & 19.2 & 7.3 & 3.5 & 2.7 & 2.9 & 3.3 & 4.7 & 8.9 & 8.1 & 6.9 & 6.6 & 6.5 & 4.1 & 3.2 & 1.2 & 100 \\
\hline
\end{tabular}

Station: (18) RICH

\begin{tabular}{|c|c|c|c|c|c|c|c|c|c|c|c|c|c|c|c|c|c|c|}
\hline \multicolumn{2}{|c|}{ N NNE } & \multicolumn{2}{|c|}{ NE ENE } & \multicolumn{2}{|c|}{ E ESE } & \multicolumn{2}{|c|}{ SE SSE } & \multicolumn{2}{|c|}{ S SSW } & \multicolumn{2}{|c|}{ SW WSW } & \multicolumn{2}{|c|}{ W WNW } & \multirow{2}{*}{$\frac{\text { NW }}{0.0}$} & \multicolumn{3}{|c|}{ NNW CALM TOTAL } & \\
\hline CALM & 0.0 & 0.0 & 0.0 & 0.0 & 0.0 & 0.0 & 0.0 & 0.0 & 0.0 & 0.0 & 0.0 & 0.0 & 0.0 & & 0.0 & 0.0 & 1.1 & 1.1 \\
\hline $1-3$ & 1.3 & 0.9 & 0.9 & 1.0 & 1.6 & 2.6 & 3.0 & 2.5 & 2.1 & 2.1 & 2.4 & 2.5 & & 2.7 & & 1.9 & 0.0 & 32.5 \\
\hline $4-7$ & 1.9 & 0.9 & 0.9 & 0.9 & 1.8 & 2.8 & 3.1 & 1.7 & 1.6 & 2.9 & 4.2 & 3.2 & 2.5 & 2.8 & 3.0 & 2.6 & 0.0 & 36.8 \\
\hline $8-12$ & 1.3 & 0.7 & 0.4 & 0.3 & 0.2 & 0.2 & 0.3 & 0.2 & 0.6 & 2.6 & 4.0 & 2.8 & 1.5 & 0.9 & 1.3 & 1.5 & 0.0 & 18.6 \\
\hline $13-18$ & 0.4 & 0.2 & 0.1 & 0.0 & 0.0 & 0.0 & 0.0 & 0.0 & 0.1 & 1.2 & 2.4 & 1.3 & 0.9 & 0.3 & 0 & 0 & 0.0 & .5 \\
\hline $19-24$ & 0.1 & 0.0 & 0.0 & 0.0 & 0.0 & 0.0 & 0.0 & 0.0 & 0.0 & 0.2 & 0.7 & 0.3 & 0. & 0.1 & 0.2 & 0.2 & 0.0 & 2.1 \\
\hline $25-31$ & 0.0 & 0.0 & 0.0 & 0.0 & 0.0 & 0.0 & 0.0 & 0.0 & 0.0 & 0.0 & 0.2 & 0.1 & 0.0 & 0.0 & 0.1 & 0.0 & 0.0 & 0.4 \\
\hline $32-38$ & 0.0 & 0.0 & 0.0 & 0.0 & 0.0 & 0.0 & 0.0 & 0.0 & 0.0 & 0.0 & 0.0 & 0.0 & 0.0 & 0.0 & 0.0 & 0.0 & 0.0 & 0.0 \\
\hline $39-46$ & 0.0 & 0.0 & 0.0 & 0.0 & 0.0 & 0.0 & 0.0 & 0.0 & 0.0 & 0.0 & 0.0 & 0.0 & 0.0 & 0.0 & 0.0 & 0.0 & 0.0 & 0.0 \\
\hline$>46$ & 0.0 & 0.0 & 0.0 & 0.0 & 0.0 & 0.0 & 0.0 & 0.0 & 0.0 & 0.0 & 0.0 & 0.0 & 0.0 & 0.0 & 0.0 & & 0.0 & 0.0 \\
\hline IOTAL & 4.9 & 2.7 & 2.3 & 2.3 & 3.6 & 5.5 & 6.4 & 4.4 & 4.5 & 9.0 & 13.8 & 10.3 & 7.7 & 6.8 & 7.9 & 6.8 & 1.1 & 100.0 \\
\hline
\end{tabular}

Station: (19) PFP

\begin{tabular}{|c|c|c|c|c|c|c|c|c|c|c|c|c|c|c|c|c|c|c|}
\hline & $\mathbf{N}$ & NNE & $N E$ & ENE & $\mathbf{E}$ & ESE & SE & SSE & S & SSW & SW & WSW & W & WNW & NW & NNW & CALM & TOTAL \\
\hline CALM & 0.0 & 0.0 & 0.0 & 0.0 & 0.0 & 0.0 & 0.0 & 0.0 & 0.0 & 0.0 & 0.0 & 0.0 & 0.0 & 0.0 & 0.0 & 0.0 & 2.2 & 2.2 \\
\hline $1-3$ & 3.5 & 3.2 & 2.1 & 1.4 & 1.5 & 1.7 & 2.2 & 1.9 & 1.9 & 1.8 & 2.4 & 3.4 & 5.3 & 5.2 & 3. & 3.1 & 0.0 & 44.4 \\
\hline $4-7$ & 3.7 & 2.3 & 1.1 & 0.7 & 0.8 & 1.4 & 1.7 & 0.7 & 0.6 & 0.7 & 1.3 & 2.0 & 4.1 & 6.3 & 4.9 & & 0.0 & \\
\hline $8-12$ & 0.4 & 0.5 & 0.3 & 0.1 & 0.1 & 0.2 & 0.3 & 0.2 & 0.3 & 0. & 1.4 & 1.9 & 1.4 & 1 & 3. & & 0.0 & \\
\hline $13-18$ & 0.0 & 0.0 & 0.1 & 0.0 & 0.0 & 0.0 & 0.0 & 0.0 & 0.1 & 0. & 0.8 & 0.9 & 0. & 0.1 & & 0 & .0 & \\
\hline $19-24$ & 0.0 & 0.0 & 0.0 & 0.0 & 0.0 & 0.0 & 0.0 & 0.0 & 0.0 & 0.1 & 0.2 & 0.1 & 0. & 0.0 & 0.0 & 0.0 & 0.0 & .5 \\
\hline $25-31$ & 0.0 & 0.0 & 0.0 & 0.0 & 0.0 & 0.0 & 0.0 & 0.0 & 0.0 & 0.0 & 0.0 & 0.0 & 0.0 & 0.0 & 0.0 & 0.0 & 0.0 & 0.1 \\
\hline $32-38$ & 0.0 & 0.0 & 0.0 & 0.0 & 0.0 & 0.0 & 0.0 & 0.0 & 0.0 & 0.0 & 0.0 & 0.0 & 0.0 & 0.0 & 0.0 & 0. & 0.0 & 1.0 \\
\hline & 0.0 & 0.0 & 0.0 & 0.0 & 0.0 & 0. & 0.0 & 0.0 & 0.0 & 0. & 0. & 0.0 & 0. & 0 & 0. & & & \\
\hline$>46$ & 0.0 & 0.0 & 0.0 & 0.0 & 0.0 & 0. & 0.0 & 0.0 & 0.0 & 0. & 0. & 0. & 0.0 & 0.0 & 0. & & 0.0 & \\
\hline TAL & 7.5 & 6.1 & 3.6 & 2.1 & 2.4 & 3.3 & 4.2 & 2.9 & 2.9 & 3.5 & 6.1 & 8.3 & 11.2 & 13.1 & 12.8 & 8.1 & 2.2 & 100 \\
\hline
\end{tabular}

Station: (20) RMTN

\begin{tabular}{|c|c|c|c|c|c|c|c|c|c|c|c|c|c|c|c|c|c|c|}
\hline & $\mathbf{N}$ & NNE & $\mathrm{NE}$ & ENE & E & ESE & SE & SSE & S & SSW & SW & WSW & W & WNW & NW & NNW & CALM & TOTA \\
\hline CALM & 0.0 & 0.0 & 0.0 & 0.0 & 0.0 & 0.0 & 0.0 & 0.0 & 0.0 & 0.0 & 0.0 & 0.0 & 0.0 & 0.0 & 0.0 & 0.0 & 0.7 & \\
\hline $1-3$ & 0.5 & 0.5 & 0.5 & 0.5 & 0.4 & 0.4 & 0.4 & 0.4 & 0.5 & 0.6 & 0.7 & 0.5 & 0.4 & 0.4 & 0.4 & 0.4 & 0.0 & \\
\hline $4-7$ & 1.4 & 1.5 & 1.4 & 1.0 & 0.7 & 0.5 . & 0.4 & 0.5 & 0.8 & 1.4 & 2.1 & 1.4 & 1.0 & 0.7 & 0.7 & 0.9 & 0.0 & \\
\hline $8-12$ & 2.2 & 2.5 & 1.8 & 0.8 & 0.4 & 0.2 & 0.2 & 0.3 & 0.7 & 1.7 & 3.5 & 2 & 1.4 & 0. & 0.8 & & 0.0 & \\
\hline $13-18$ & 1.8 & 2.7 & 1.3 & 0.3 & 0.1 & 0.0 & 0.1 & 0.1 & 0.4 & 1.5 & 4.5 & 3.0 & 1.6 & 0. & 0. & 0.9 & 0.0 & \\
\hline $19-24$ & 0.9 & 2.0 & 0.9 & 0.1 & 0.0 & 0.0 & 0.0 & 0.0 & 0.2 & 0.8 & 3.4 & 2.8 & 1.2 & 0.5 & 0.3 & 0.2 & 0.0 & \\
\hline $25-31$ & 0.4 & 1.3 & 0.7 & 0.1 & 0.0 & 0.0 & 0.0 & 0.0 & 0.1 & 0.6 & 3.1 & 2.6 & 0.7 & 0.2 & 0.1 & 0.0 & 0.0 & \\
\hline $32-38$ & 0.1 & 0.6 & 0.4 & 0.1 & 0.0 & 0.0 & 0.0 & 0.0 & 0.1 & 0.4 & 2.4 & 1.8 & 0.3 & 0.0 & 0.0 & 0.0 & 0.0 & \\
\hline & 0.0 & 0.3 & 0.2 & 0.0 & 0.0 & 0.0 & 0.0 & 0. & 0.0 & 0.2 & 1.6 & 1.0 & 0.1 & 0. & 0.0 & 0.0 & 0.0 & \\
\hline$>46$ & 0.0 & 0.1 & 0.2 & 0.0 & 0.0 & 0.0 & 0.0 & 0. & 0.0 & 0.1 & 1.0 & 0.5 & 0.0 & & 0.0 & & 0.0 & \\
\hline TOTAL & 7.3 & 11.4 & 7.4 & 2.8 & 1.5 & 1.1 & 1.1 & 1.3 & 2.7 & 7.4 & 22.3 & 15.9 & 6.8 & 3.6 & 3.0 & 3.6 & 0.7 & \\
\hline
\end{tabular}


Table 5.4. (contd)

Station: (21) HMS

Begin: $1 / 82$

End: $12 / 99$

Total Hours: 156491

DIRECTION

\begin{tabular}{|c|c|c|c|c|c|c|c|c|c|c|c|c|c|c|c|c|c|c|}
\hline & $\mathrm{N}$ & NNE & NE & ENE & $\underline{E}$ & ESE & SE & SSE & S & SSW & SW & WSW & W & WNW & NW & NNW & CALM & TOTAI \\
\hline CALM & 0.0 & 0.0 & 0.0 & 0.0 & 0.0 & 0.0 & 0.0 & 0.0 & 0.0 & 0.0 & 0.0 & 0.0 & 0.0 & 0.0 & 0.0 & 0.0 & 1.2 & 1.2 \\
\hline $1-3$ & 2.1 & 1.8 & 1.8 & 1.4 & 1.5 & 1.6 & 1.7 & 1.3 & 1.2 & 1.2 & 1.4 & 1.4 & 1.7 & 1.9 & 2.2 & 2.2 & 0.0 & 26.3 \\
\hline 4-7 & 2.2 & 1.4 & 1.1 & 1.0 & 1.2 & 1.3 & 1.7 & 1.5 & 1.3 & 1.4 & 2.3 & 3.6 & 5.0 & 6.3 & 6.7 & 3.9 & 0.0 & 41.9 \\
\hline $8-12$ & 0.5 & 0.5 & 0.3 & 0.1 & 0.1 & 0.1 & 0.2 & 0.4 & 0.4 & 0.6 & 1.3 & & 2.5 & & 5.2 & 1.1 & 0.0 & 20.1 \\
\hline $13-18$ & 0.1 & 0.2 & 0.1 & 0.0 & 0.0 & 0.0 & 0.0 & 0.1 & 0.2 & 0. & 1.1 & 1 & 0.6 & 1. & 2.4 & 0.3 & 0.0 & 8.1 \\
\hline $19-24$ & 0.0 & 0.0 & 0.0 & 0.0 & 0.0 & 0.0 & 0.0 & 0.0 & 0.0 & 0.2 & 0.5 & 0. & 0.1 & 0.2 & 0.7 & 0.0 & 0.0 & 2.1 \\
\hline 25 & 0.0 & 0.0 & 0.0 & 0.0 & 0.0 & 0.0 & 0.0 & 0.0 & 0.0 & 0.1 & 0.1 & 0.1 & 0.0 & 0.0 & 0.1 & 0.0 & 0.0 & 0.3 \\
\hline & 0.0 & 0.0 & 0.0 & 0.0 & 0.0 & 0.0 & 0.0 & 0.0 & 0.0 & 0.0 & 0.0 & 0.0 & 0.0 & 0.0 & 0.0 & 0.0 & 0.0 & 0.0 \\
\hline & 0.0 & 0.0 & 0.0 & 0.0 & 0.0 & 0.0 & 0.0 & 0.0 & 0.0 & 0.0 & 0.0 & 0.0 & 0.0 & 0.0 & 0.0 & 0.0 & 0.0 & 0.0 \\
\hline & 0.0 & 0.0 & 0.0 & 0.0 & 0.0 & 0.0 & 0.0 & 0.0 & 0.0 & 0.0 & 0.0 & 0. & 0.0 & 0.0 & 0.0 & 0.0 & 0.0 & 0.0 \\
\hline TOTAL & 4.8 & 4.0 & 3.3 & 2.6 & 2.8 & 3.0 & 3.6 & 3.3 & 3.2 & 4.0 & 6.6 & 8.9 & 9.9 & 14.1 & 17.3 & 7.5 & 1.2 & 100.0 \\
\hline
\end{tabular}

Station: (22) PASC

Begin: 10/87

End: $12 / 99$

Total Hours: 102207

DIRECTION

\begin{tabular}{|c|c|c|c|c|c|c|c|c|c|c|c|c|c|c|c|c|c|c|}
\hline & $\mathrm{N}$ & NNE & NE & ENE & $E$ & ESE & SE & SSE & S & SSW & SW & WSW & W & WNW & NW & NNW & CALM & TOTAL \\
\hline CALM & 0.0 & 0.0 & 0.0 & 0.0 & 0.0 & 0.0 & 0.0 & 0.0 & 0.0 & 0.0 & 0.0 & 0.0 & 0.0 & 0.0 & 0.0 & 0.0 & 1.1 & 1.1 \\
\hline $1-3$ & 4.3 & .7 & 2.4 & 2.4 & 2.5 & 2.2 & 1.8 & 1.3 & 1.3 & 1.2 & 1.1 & 1.2 & 1.5 & & 3.2 & 4.6 & 0.0 & 35.7 \\
\hline 4. & 2.9 & 1.3 & 0.7 & 0.9 & 1.4 & 2.0 & 1.9 & 1. & 1.6 & 2.4 & 2.9 & 2.0 & 2.1 & 2.3 & 3.5 & 4.3 & 0.0 & 33.8 \\
\hline $8-12$ & 1.1 & 0.4 & 0.2 & 0.1 & 0.1 & 0.2 & 0.3 & 0.3 & 0.5 & 2.0 & 4.5 & 2.3 & 1.0 & 0.7 & 1.2 & 1.6 & 0.0 & 16.6 \\
\hline $13-$ & 0.2 & 0.2 & 0.1 & 0.0 & 0.0 & 0.0 & 0.0 & 0.0 & 0.1 & 0.6 & 3.1 & 2.1 & 0.6 & 0.3 & 0.4 & 0.4 & 0.0 & 8.2 \\
\hline & 0.0 & 0.0 & 0.0 & 0.0 & 0.0 & 0.0 & 0.0 & 0.0 & 0.0 & 0.1 & 1.1 & 1.2 & 0.3 & & 0.1 & 0.0 & 0.0 & 3.0 \\
\hline $25-3$ & 0.0 & 0.0 & 0.0 & 0.0 & 0.0 & 0.0 & 0.0 & 0.0 & 0.0 & 0.0 & 0.4 & 0.6 & 0.1 & & 0 & & & 1.2 \\
\hline & 0.0 & 0.0 & 0.0 & 0.0 & 0.0 & 0.0 & 0.0 & 0.0 & 0.0 & 0.0 & 0.1 & 0.2 & 0.0 & 0.0 & 0.0 & 0.0 & 0.0 & 0.3 \\
\hline & 0.0 & 0.0 & 0.0 & 0.0 & 0.0 & 0.0 & 0.0 & 0.0 & 0.0 & 0.0 & 0.0 & 0.1 & 0.0 & 0.0 & 0.0 & 0.0 & 0.0 & 0.1 \\
\hline & 0.0 & 0.0 & 0.0 & 0.0 & 0.0 & 0.0 & 0.0 & 0.0 & 0.0 & 0.0 & 0.0 & 0.0 & 0.0 & 0.0 & 0.0 & 0.0 & 0.0 & 0.0 \\
\hline OTAl & 8.5 & 4.7 & 3.4 & 3.4 & 4.0 & 4.4 & 4.1 & 3.1 & 3.5 & 6.2 & 13.3 & 9.7 & 5.6 & 5.4 & 8.5 & 11.0 & 1.1 & 100.0 \\
\hline
\end{tabular}

Station: (23) GABW

Begin: $3 / 86$

End: $12 / 99$

Total Hours: 118409

DIRECTION

\begin{tabular}{|c|c|c|c|c|c|c|c|c|c|c|c|c|c|c|c|c|c|c|}
\hline & $\mathbf{N}$ & NNE & $\mathrm{NE}$ & ENE & $\mathrm{E}$ & ESE & SE & SSE & $\mathrm{S}$ & SSW & SW & WSW & W & WNW & NW & NNW & CALM & TOTAL \\
\hline CALM & 0.0 & 0.0 & 0.0 & 0.0 & 0.0 & 0.0 & 0.0 & 0.0 & 0.0 & 0.0 & 0.0 & 0.0 & 0.0 & 0.0 & 0.0 & 0.0 & 1.2 & 1.2 \\
\hline $1-3$ & 1.6 & 1.3 & 1.2 & 1.3 & 1.5 & 1.7 & 2.3 & 2.5 & 2.1 & 1.6 & 1.6 & 1.7 & 2.6 & 3.6 & 3.2 & 2.0 & 0.0 & 31.9 \\
\hline 4 & 1.6 & 1.1 & 1.0 & 1.0 & 1.4 & 1.3 & 4.0 & 4.9 & 1.6 & 0.8 & 1.0 & 1.5 & 3.0 & 7.2 & 4.0 & 2.0 & 0.0 & 37.3 \\
\hline $8-12$ & 0.6 & 0.5 & 0.3 & 0.2 & 0.2 & 0.3 & 1.2 & 1.0 & 0.3 & 0.5 & 1.0 & 1.5 & 2.7 & 5.2 & 1.8 & 0.6 & 0.0 & 17.9 \\
\hline $13-1$ & 0.2 & 0.2 & 0.1 & 0.0 & 0.0 & 0.0 & 0.1 & 0.1 & 0.2 & 0.3 & 0.8 & 0.9 & 1.0 & 3.3 & 1.1 & 0.2 & 0.0 & 8.5 \\
\hline $19-2$ & 0.0 & 0.1 & 0.0 & 0.0 & 0.0 & 0.0 & 0.0 & 0.0 & 0.0 & 0.2 & 0.4 & 0.3 & 0.2 & 1.0 & 0.4 & 0.0 & 0.0 & 2.6 \\
\hline $25=$ & 0.0 & 0.0 & 0.0 & 0.0 & 0.0 & 0.0 & 0.0 & 0.0 & 0.0 & 0.0 & 0.1 & 0.1 & 0.0 & 0.2 & 0.1 & 0.0 & 0.0 & 0.5 \\
\hline 3 & 0.0 & 0.0 & 0.0 & 0.0 & 0.0 & 0.0 & 0.0 & 0.0 & 0.0 & 0.0 & 0.0 & 0.0 & 0.0 & 0.0 & 0.0 & 0.0 & 0.0 & 0.0 \\
\hline & 0.0 & 0.0 & 0.0 & 0.0 & 0.0 & 0.0 & 0.0 & 0.0 & 0.0 & 0.0 & 0.0 & 0.0 & 0.0 & 0.0 & 0.0 & 0.0 & 0.0 & 0.0 \\
\hline & 0.0 & 0.0 & 0.0 & 0. & 0.0 & 0. & 0. & 0. & 0.0 & 0. & 0.0 & 0 & 0.0 & 0.0 & 0.0 & 0.0 & 0.0 & 0.0 \\
\hline TOTAL & 3.9 & 3.2 & 2.7 & 2.5 & 3.1 & 3.4 & 7.7 & 8.6 & 4.2 & 3.5 & 4.9 & 5.9 & 9.5 & 20.4 & 10.5 & 4.8 & 1.2 & 100.0 \\
\hline
\end{tabular}

Station: (24) 100F

Begin: 3/86

End: $12 / 99$

Total Hours: 118503

DIRECTION

\begin{tabular}{|c|c|c|c|c|c|c|c|c|c|c|c|c|c|c|c|c|c|c|}
\hline & $\mathbf{N}$ & NNE & $\mathrm{NE}$ & ENE & $E$ & ESE & $\mathrm{SE}$ & SSE & $\mathrm{S}$ & SSW & SW & WSW & W & WNW & NW & NNW & CALM & TOTAL \\
\hline CALM & 0.0 & 0.0 & 0.0 & 0.0 & 0.0 & 0.0 & 0.0 & 0.0 & 0.0 & 0.0 & 0.0 & 0.0 & 0.0 & 0.0 & 0.0 & 0.0 & 1.2 & 1.2 \\
\hline $1-3$ & 1.8 & 1.4 & 1.2 & 1.1 & 1.4 & 1.9 & 2.8 & 2.8 & 2.2 & & 1.8 & & 3. & 4 & 3.5 & & 0.0 & 36.3 \\
\hline & 2.0 & 1.4 & 1.1 & 1.2 & 1.3 & 1.7 & 5. & 5. & 1.2 & 0 & & & 3.4 & & & & 0.0 & 36.1 \\
\hline $8-12$ & 1.0 & 0.7 & 0.3 & 0.3 & 0.2 & 0.6 & 2.5 & 2. & 0.6 & 0.5 & 0.8 & 1. & 2.5 & 2.5 & 0.6 & 0.5 & 0.0 & 17.3 \\
\hline & 0.2 & 0.2 & 0.1 & 0.1 & 0.0 & 0.0 & 0.3 & 0.3 & 0.3 & 0.3 & 0.6 & 0. & 1.0 & 1. & 0.4 & 0.1 & 0.0 & 6.6 \\
\hline 19 & 0.0 & 0.1 & 0.1 & 0.0 & 0.0 & 0.0 & 0.0 & 0. & 0.1 & 0 & 0.3 & & 0. & 0 & 0.2 & & 0.0 & 2.0 \\
\hline & 0.0 & 0.0 & 0.0 & 0.0 & 0.0 & 0.0 & 0. & 0. & 0.0 & 0 & 0. & 0 & 0 & 0 & 0 & & 0 & 0.4 \\
\hline 32 & 0.0 & 0.0 & 0.0 & 0.0 & 0.0 & 0.0 & 0.0 & 0.0 & 0.0 & 0.0 & 0.0 & 0. & 0.0 & 0. & 0.0 & 0. & 0.0 & 0.0 \\
\hline & 0.0 & 0.0 & 0.0 & 0.0 & 0.0 & 0.0 & 0.0 & 0.0 & 0.0 & 0.0 & 0.0 & 0. & 0.0 & 0.0 & 0.0 & 0.0 & 0.0 & 0.0 \\
\hline & 0.0 & 0.0 & 0.0 & 0.0 & 0.0 & 0.0 & 0.0 & 0.0 & 0.0 & 0.0 & 0.0 & 0.0 & 0.0 & 0.0 & 0.0 & 0.0 & 0.0 & 0.0 \\
\hline rOTAI & 5.0 & 3.7 & 2.8 & 2.7 & 3.0 & 4.2 & 10.8 & 11.4 & 4.8 & 3.7 & 4.6 & 6.4 & 10.7 & 12.8 & 7.3 & 5.0 & 1.2 & 100.0 \\
\hline
\end{tabular}


Table 5.4. (contd)

Station: (25) VERN

Begin: 2/88

End: $12 / 99$

Total Hours: 102617

DIRECTION

\begin{tabular}{|c|c|c|c|c|c|c|c|c|c|c|c|c|c|c|c|c|c|c|}
\hline & $N$ & NNE & NE & ENE & $\mathrm{E}$ & ESE & SE & SSE & $\mathbf{S}$ & SSW & SW & WSW & W & WNW & NW & NNW & CALM & TOTAL \\
\hline CALM & 0.0 & 0.0 & 0.0 & 0.0 & 0.0 & 0.0 & 0.0 & 0.0 & 0.0 & 0.0 & 0.0 & 0.0 & 0.0 & 0.0 & 0.0 & 0.0 & 1.0 & 1.0 \\
\hline 1-3 & 1.1 & 1.2 & 1.3 & 1.6 & 2.1 & 2.0 & 1.8 & 1.2 & 0.9 & 0.9 & 1.2 & 2.3 & 2.7 & 1.7 & 1.2 & 1.0 & 0.0 & 24.2 \\
\hline $4-7$ & 0.8 & 1.3 & 2.1 & 2.7 & 3.5 & 2.1 & 1.0 & 0.5 & 0.4 & 0.4 & 0.6 & 4.1 & 7.6 & 4.0 & 1.9 & 1.0 & 0.0 & 33.9 \\
\hline 8-12 & 0.5 & 0.4 & 0.4 & 0.5 & 0.4 & 0.2 & 0.1 & 0.1 & 0.2 & 0.4 & 0.7 & 2.0 & 7.8 & 7.2 & 2.6 & 0.6 & 0.0 & 24.2 \\
\hline $13-18$ & 0.2 & 0.2 & 0.1 & 0.0 & 0.0 & 0.0 & 0.0 & 0.0 & 0.1 & 0.3 & 0.7 & 0.7 & 2.4 & 5.2 & 2.2 & 0.3 & 0.0 & 12.5 \\
\hline $19-24$ & 0.1 & 0.0 & 0.0 & 0.0 & 0.0 & 0.0 & 0.0 & 0.0 & 0.0 & 0.2 & 0.4 & 0.2 & 0.4 & 1.4 & 0.7 & 0.1 & 0.0 & 3.5 \\
\hline $25-31$ & 0.0 & 0.0 & 0.0 & 0.0 & 0.0 & 0.0 & 0.0 & 0.0 & 0.0 & 0.1 & 0.2 & 0.0 & 0.0 & 0.2 & 0.1 & 0.0 & 0.0 & 0.6 \\
\hline $32-38$ & 0.0 & 0.0 & 0.0 & 0.0 & 0.0 & 0.0 & 0.0 & 0.0 & 0.0 & 0.0 & 0.0 & 0.0 & 0.0 & 0.0 & 0.0 & 0.0 & 0.0 & 0.1 \\
\hline & 0.0 & 0.0 & 0.0 & 0.0 & 0.0 & 0.0 & 0.0 & 0.0 & 0.0 & 0.0 & 0.0 & 0.0 & 0.0 & 0.0 & 0.0 & 0.0 & 0.0 & 0.0 \\
\hline$>46$ & 0.0 & 0.0 & 0.0 & 0.0 & 0.0 & 0.0 & 0.0 & 0.0 & 0.0 & 0.0 & 0.0 & 0.0 & 0.0 & 0.0 & 0.0 & 0.0 & 0.0 & 0.0 \\
\hline TOTAL & 2.7 & 3.1 & 4.0 & 4.9 & 6.0 & 4.3 & 2.9 & 1.8 & 1.6 & 2.1 & 3.8 & 9.4 & 21.1 & 19.6 & 8.7 & 2.9 & 1.0 & 100. \\
\hline
\end{tabular}

Station: (26) BENT

Begin: 2/95

End: $12 / 99$

Total Hours: 42850

DIRECTION

\begin{tabular}{|c|c|c|c|c|c|c|c|c|c|c|c|c|c|c|c|c|c|c|}
\hline & $\mathrm{N}$ & NNE & NE & ENE & $E$ & ESE & SE & SSE & $\underline{\mathbf{S}}$ & SSW & SW & WSW & $\mathrm{W}$ & WNW & $\mathrm{NW}$ & NNW & CALM & TOTAL \\
\hline CALM & 0.0 & 0.0 & 0.0 & 0.0 & 0.0 & 0.0 & 0.0 & 0.0 & 0.0 & 0.0 & 0.0 & 0.0 & 0.0 & 0.0 & 0.0 & 0.0 & 0.2 & 0.2 \\
\hline $1-3$ & 1.1 & 1.1 & 0.9 & 0.8 & 0.8 & 0.6 & 0.5 & 0.4 & 0. & 0.6 & 1.1 & 1.7 & 2.4 & 1.9 & 1.5 & 1.1 & 0.0 & 16.9 \\
\hline $4-7$ & 1.4 & 1.2 & 1.7 & 2.2 & 2.4 & 1.1 & 0.3 & 0.2 & 0.5 & 1.2 & 4.8 & 11.5 & 13.9 & 5.6 & 2.7 & 1.9 & 0.0 & 52.6 \\
\hline $8-12$ & 0.5 & 0.7 & 1.2 & 1.1 & 1.1 & 0.3 & 0.0 & 0.0 & 0.2 & 0.8 & 3.1 & 6.0 & 5.0 & & 1.2 & 0.4 & 0.0 & 23.5 \\
\hline $13-18$ & 0.3 & 0.7 & 0.4 & 0.0 & 0.0 & 0.0 & 0.0 & 0.0 & 0.1 & 0.4 & 1.6 & 1.0 & 0.4 & 0. & 0.1 & 0.1 & 0.0 & 5.4 \\
\hline $19-2$ & 0.1 & 0.2 & 0.1 & 0.0 & 0.0 & 0.0 & 0.0 & 0.0 & 0.0 & 0.1 & 0.5 & 0.1 & 0.1 & 0. & 0.0 & 0.0 & 0.0 & 1.2 \\
\hline & 0.0 & 0.0 & 0.0 & 0.0 & 0.0 & 0.0 & 0.0 & 0.0 & 0.0 & 0.0 & 0.1 & 0.0 & 0.0 & 0.0 & 0.0 & 0.0 & 0.0 & 07 \\
\hline & 0.0 & 0.0 & 0.0 & 0.0 & 0.0 & 0.0 & 0.0 & 0.0 & 0.0 & 0.0 & 0.0 & 0.0 & 0.0 & 0.0 & 0.0 & 0.0 & 0.0 & 0.0 \\
\hline & 0.0 & 0. & 0.0 & 0.0 & 0.0 & 0.0 & 0.0 & 0.0 & 0.0 & 0.0 & 0.0 & 0.0 & 0.0 & 0.0 & 0.0 & 0.0 & 0.0 & 0.0 \\
\hline$>46$ & 0.0 & 0.0 & 0.0 & 0.0 & 0.0 & 0. & 0.0 & 0. & 0. & 0. & 0.0 & 0.0 & 0.0 & 0. & 0.0 & 0.0 & 0.0 & 0.0 \\
\hline TOTAL & 3.4 & 3.8 & 4.2 & 4.0 & 4.3 & 2.0 & 0.9 & 0.7 & 1.3 & 3.1 & 11.2 & 20.3 & 21.8 & 9.8 & 5.5 & 3.6 & 0.2 & 100.0 \\
\hline
\end{tabular}

Station: (27) VSTA

Begin: 2/91

End: $12 / 99$

Total Hours: 76598

DIRECTION

\begin{tabular}{|c|c|c|c|c|c|c|c|c|c|c|c|c|c|c|c|c|c|c|}
\hline & $\mathbf{N}$ & NNE & NE & ENE & $E$ & ESE & SE & SSE & $\mathbf{S}$ & SSW & SW & WSW & W & WNW & NW & NNW & CALM & TOTAL \\
\hline CALM & 0.0 & 0.0 & 0.0 & 0.0 & 0.0 & 0.0 & 0.0 & 0.0 & 0.0 & 0.0 & 0.0 & 0.0 & 0.0 & 0.0 & 0.0 & 0.0 & 0.8 & 0.8 \\
\hline $1-3$ & 2.2 & 2.2 & 2.0 & 1.6 & 1.6 & 1.8 & 2.0 & 1.7 & 1.9 & 2.4 & 2.7 & 2.5 & 2.3 & 1.8 & 1.8 & 1.6 & 0.0 & 32.1 \\
\hline $4-7$ & 3.0 & 2.0 & 1.5 & 1.2 & 0.8 & 1.1 & 1.2 & 1.2 & 1.9 & 4.1 & 5.9 & 4.2 & 2.6 & 2.6 & 3.1 & 2.9 & 0.0 & 39.2 \\
\hline $8-12$ & 0.6 & 0.2 & 0.1 & 0.0 & 0.0 & 0.1 & 0.1 & 0.2 & 0.6 & 3.8 & 6.1 & 2.7 & 0.8 & 0.4 & 0.9 & 1.2 & 0.0 & 18.0 \\
\hline $13-18$ & 0.1 & 0.1 & 0.0 & 0.0 & 0.0 & 0.0 & 0.0 & 0.0 & 0.2 & 1.7 & 4.0 & 1.1 & 0.4 & 0.2 & 0.1 & 0.2 & 0.0 & 8.0 \\
\hline $19-24$ & 0.0 & 0.0 & 0.0 & 0.0 & 0.0 & 0.0 & 0.0 & 0.0 & 0.0 & 0.2 & 1.1 & 0.3 & 0.1 & 0.0 & 0.0 & 0.0 & 0.0 & 1.7 \\
\hline $25-31$ & 0.0 & 0.0 & 0.0 & 0.0 & 0.0 & 0.0 & 0.0 & 0.0 & 0.0 & 0.0 & 0.1 & 0.0 & 0.0 & 0.0 & 0.0 & 0.0 & 0.0 & 0.2 \\
\hline $32-3$ & 0.0 & 0.0 & 0.0 & 0.0 & 0.0 & 0.0 & 0.0 & 0.0 & 0.0 & 0.0 & 0.0 & 0.0 & 0.0 & 0.0 & 0.0 & 0.0 & 0.0 & 0.0 \\
\hline $39-46$ & 0.0 & 0.0 & 0.0 & 0.0 & 0.0 & 0.0 & 0.0 & 0.0 & 0.0 & 0.0 & 0.0 & 0.0 & 0.0 & 0.0 & 0.0 & 0.0 & 0.0 & 0.0 \\
\hline$>46$ & 0.0 & 0.0 & 0.0 & 0.0 & 0.0 & 0.0 & 0.0 & 0.0 & 0.0 & 0.0 & 0.0 & 0.0 & 0.0 & 0.0 & 0.0 & 0.0 & 0.0 & 0.0 \\
\hline TOTAL & 5.8 & 4.5 & 3.7 & 2.8 & 2.4 & 3.0 & 3.3 & 3.1 & 4.6 & 12.2 & 19.9 & 10.9 & 6.1 & 5.0 & 5.9 & 5.9 & 0.8 & 100.0 \\
\hline
\end{tabular}

Station: (28) SURF

Begin: $9 / 94$

End: $12 / 99$

Total Hours: 46370

DIRECTION

\begin{tabular}{|c|c|c|c|c|c|c|c|c|c|c|c|c|c|c|c|c|c|c|}
\hline & $\mathbf{N}$ & NNE & NE & ENE & E & ESE & $S E$ & SSE & $S$ & SSW & SW & WSW & W & WNW & NW & NNW & CALM & TOTAL \\
\hline CALM & 0.0 & 0.0 & 0.0 & 0.0 & 0.0 & 0.0 & 0.0 & 0.0 & 0.0 & 0.0 & 0.0 & 0.0 & 0.0 & 0.0 & 0.0 & 0.0 & 0.8 & 0.8 \\
\hline $1-3$ & 0 & & 1.2 & 1.3 & 1.2 & 0.8 & 0.7 & 0.7 & 1.1 & 2.2 & 3.6 & 3.8 & 1.8 & 0.7 & & 0.3 & & 20.5 \\
\hline $4-7$ & 0 & 0.4 & 2.4 & 3.8 & 2.0 & 0.7 & 0.3 & 0.2 & 0.4 & 1.0 & 3.9 & 6.2 & 1.7 & 0. & 0. & 0.1 & 0.0 & 23.4 \\
\hline $8-12$ & 0.2 & 0 & 2.0 & 3.1 & 0.7 & 0.0 & 0.0 & 0. & 0.0 & & 4.1 & 10. & 3.7 & & 0. & 0.0 & 0.0 & 25.3 \\
\hline $13-1$ & 0.2 & 0.3 & 0.2 & 0.5 & 0.2 & 0.0 & 0.0 & 0. & 0.0 & 0.0 & 1.9 & 8. & 6.0 & 0.6 & 0.0 & 0.0 & 0.0 & 19.0 \\
\hline $19-2$ & 0.1 & 0.1 & 0.1 & 0.0 & 0.0 & 0.0 & 0.0 & 0. & 0.0 & 0.0 & 0.4 & 4.3 & 3.1 & 0.3 & 0.0 & 0.0 & 0.0 & 8.3 \\
\hline $25-3$ & 0.0 & 0.0 & 0.0 & 0.0 & 0.0 & 0.0 & 0.0 & 0. & 0.0 & 0.0 & 0.1 & 1. & 0.9 & & 0.1 & 0.0 & & \\
\hline $32-38$ & 0.0 & 0.0 & 0.0 & 0.0 & 0.0 & 0.0 & 0.0 & 0.0 & 0.0 & 0.0 & 0.0 & 0.1 & 0.1 & & & & & 0.2 \\
\hline & 0.0 & 0.0 & 0.0 & 0.0 & 0.0 & 0.0 & 0.0 & 0. & 0.0 & 0.0 & 0.0 & 0.0 & 0.0 & & 0.0 & 0.0 & 0.0 & 0.0 \\
\hline & 0.0 & 0. & 0.0 & 0.0 & 0.0 & 0.0 & 0.0 & 0. & 0.0 & 0.0 & 0.0 & 0.0 & 0.0 & 0.0 & 0.0 & 0.0 & 0.0 & 0.0 \\
\hline OTAL & 1.0 & 2.1 & 5.9 & 8.7 & 4.1 & 1.5 & 1.0 & 0.9 & 1.5 & 3.2 & 13.9 & 34.8 & 17.3 & 2.3 & 0.6 & 0.5 & 0.8 & 100.0 \\
\hline
\end{tabular}


Table 5.4. (contd)

Station: (29) $100 \mathrm{~K}$

Begin: 3/96

End: $12 / 99$

Total Hours: 33143

DIRECTION

\begin{tabular}{|c|c|c|c|c|c|c|c|c|c|c|c|c|c|c|c|c|c|c|}
\hline & $\mathrm{N}$ & NNE & $\mathrm{NE}$ & ENE & E & ESE & SE & SSE & $\mathbf{S}$ & SSW & SW & WSW & W & WNW & NW & NNW & CALM & TOTAL \\
\hline CALM & 0.0 & 0.0 & 0.0 & 0.0 & 0.0 & 0.0 & 0.0 & 0.0 & 0.0 & 0.0 & 0.0 & 0.0 & 0.0 & 0.0 & 0.0 & 0.0 & 1.4 & 1.4 \\
\hline $1-3$ & 2.1 & 1.7 & 1.6 & 1.9 & 2.2 & 2.1 & 2.1 & 1.8 & 1.7 & 1.7 & 2.2 & 3.4 & 4.4 & 3. & 2.5 & 2.0 & 0.0 & 36.4 \\
\hline $4-7$ & 1.7 & 1.3 & 0.9 & 1.0 & 1.5 & 1.6 & 1.7 & 1.5 & 1.1 & 0.9 & 1. & 4.9 & 6.8 & & 2.0 & & 0.0 & 33.6 \\
\hline $8-12$ & 0.4 & 0.6 & 0.4 & 0.1 & 0.2 & 0.4 & 0.7 & 0.7 & 0.5 & 0.6 & 1.1 & 3.2 & 5.7 & 2.4 & 0.6 & 0.3 & 0.0 & 18.0 \\
\hline $13-1$ & 0.1 & .2 & 0.1 & 0.0 & 0.0 & 0.0 & 0.1 & 0.1 & 0.2 & 0.4 & 1.0 & 0.9 & 1.9 & 2.1 & 0.6 & 0.1 & 0.0 & 7.9 \\
\hline $19-24$ & 0.0 & 0.0 & 0.0 & 0.0 & 0.0 & 0.0 & 0.0 & 0.0 & 0.0 & 0.2 & 0.4 & 0.2 & 0.3 & 0.8 & 0.2 & 0.0 & 0.0 & 2.2 \\
\hline 25 & 0.0 & 0.0 & 0.0 & 0.0 & 0.0 & 0.0 & 0.0 & 0.0 & 0.0 & 0.0 & 0.0 & 0.0 & 0.0 & 0.2 & 0.0 & 0.0 & 0.0 & 0.3 \\
\hline & 0.0 & & 0.0 & 0.0 & 0.0 & 0. & 0 & 0 & 0.0 & 0.0 & 0.0 & 0. & 0.0 & 0. & 0.0 & 0.0 & 0.0 & 0.0 \\
\hline & 0.0 & 0.0 & 0.0 & 0.0 & 0.0 & 0.0 & 0.0 & 0.0 & 0.0 & 0.0 & 0.0 & 0.0 & 0.0 & 0.0 & 0.0 & & 0.0 & 0.0 \\
\hline$>46$ & 0.0 & 0.0 & 0.0 & 0.0 & 0.0 & 0.0 & 0.0 & 0.0 & 0.0 & 0.0 & 0.0 & 0.0 & 0.0 & 0.0 & 0.0 & 0.0 & 0.0 & 0.0 \\
\hline OTAL & 4.4 & 3.9 & 3.0 & 3.0 & 4.0 & 4.0 & 4.6 & 4.2 & 3.6 & 3.9 & 6.3 & 12.7 & 19.2 & 11.8 & 6.0 & 4.1 & 1.4 & 100.0 \\
\hline
\end{tabular}

Station: (30) HAMR

Begin: 1/98

End: $12 / 99$

Total Hours: 17459

DIRECTION

\begin{tabular}{|c|c|c|c|c|c|c|c|c|c|c|c|c|c|c|c|c|c|c|}
\hline & $\mathrm{N}$ & NNE & $\mathrm{NE}$ & ENE & $E$ & ESE & SE & SSE & $\underline{s}$ & SSW & SW & WSW & W & WNW & NW & NNW & CALM & TOTAL \\
\hline CALM & 0.0 & 0.0 & 0.0 & 0.0 & 0.0 & 0.0 & 0.0 & 0.0 & 0.0 & 0.0 & 0.0 & 0.0 & 0.0 & 0.0 & 0.0 & 0.0 & 0.9 & 0.9 \\
\hline $1-3$ & 1.2 & 0.8 & 0.8 & 0.5 & 0.7 & 1.0 & 1.8 & 2.0 & 2.0 & 1.5 & 1.3 & 1.1 & 1.2 & 1.2 & 1.3 & 1.2 & 0.0 & 19.6 \\
\hline $4-7$ & 3.6 & 1.7 & 0.8 & 0.8 & 1.0 & 2.0 & 4.9 & 4.4 & 3.9 & 3.6 & 4.1 & 2.1 & 1.6 & 2.0 & 3.0 & 3.8 & 0.0 & 43.3 \\
\hline $8-12$ & 2.3 & 1.1 & 0.3 & 0.1 & 0.2 & 0.3 & 0.5 & 0.5 & 1.2 & 3.1 & 6.8 & 2.7 & 0.8 & 0.6 & 1.5 & 2.2 & 0.0 & 24.2 \\
\hline $13-18$ & 0.6 & 0.3 & 0.1 & 0.0 & 0.0 & 0.0 & 0.0 & 0.1 & 0.4 & 1.4 & 2.7 & 1.2 & 0.4 & 0.2 & 1.0 & 0.8 & 0.0 & 9.2 \\
\hline $19-24$ & 0.0 & 0.0 & 0.0 & 0.0 & 0.0 & 0.0 & 0.0 & 0.0 & 0.1 & 0.3 & 0.8 & 0.3 & 0.1 & 0.1 & 0.3 & 0.1 & 0.0 & 2.2 \\
\hline $25-31$ & 0.0 & 0.0 & 0.0 & 0.0 & 0.0 & 0.0 & 0.0 & 0.0 & 0.0 & 0.1 & 0.3 & 0.1 & 0.0 & 0.0 & 0.0 & 0.0 & 0.0 & 0.5 \\
\hline $32-38$ & 0.0 & 0.0 & 0.0 & 0.0 & 0.0 & 0.0 & 0.0 & 0.0 & 0.0 & 0.0 & 0.0 & 0.0 & 0.0 & 0.0 & 0.0 & 0.0 & 0.0 & 0.1 \\
\hline $39-46$ & 0.0 & 0.0 & 0.0 & 0.0 & 0.0 & 0.0 & 0.0 & 0.0 & 0.0 & 0.0 & 0.0 & 0.0 & 0.0 & 0.0 & 0.0 & 0.0 & 0.0 & 0.0 \\
\hline$>46$ & 0.0 & 0.0 & 0.0 & 0.0 & 0.0 & 0.0 & 0.0 & 0.0 & 0.0 & 0.0 & 0.0 & 0.0 & 0.0 & 0.0 & 0.0 & 0.0 & 0.0 & 0.0 \\
\hline TOTAL & 7.7 & 3.9 & 2.0 & 1.4 & 2.0 & 3.3 & 7.2 & 7.0 & 7.6 & 10.0 & 16.1 & 7.5 & 4.0 & 4.1 & 7.2 & 8.0 & 0.9 & 100.0 \\
\hline
\end{tabular}

Station: (8W) WAHL

Begin: 1/82

End: $7 / 91$

Total Hours: 79579

DIRECTION

\begin{tabular}{|c|c|c|c|c|c|c|c|c|c|c|c|c|c|c|c|c|c|c|}
\hline & $\mathrm{N}$ & NNE & $\mathrm{NE}$ & ENE & $\mathrm{E}$ & ESE & SE & SSE & $S$ & SSW & SW & WSW & W & WNW & NW & NNW & CALM & TOTAL \\
\hline CALM & 0.0 & 0.0 & 0.0 & 0.0 & 0.0 & 0.0 & 0.0 & 0.0 & 0.0 & 0.0 & 0.0 & 0.0 & 0.0 & 0.0 & 0.0 & 0.0 & 1.3 & 1.3 \\
\hline $1-3$ & 1.9 & 2.0 & 2.9 & 3.4 & 4.6 & 3.1 & 2.2 & 1.6 & 1.7 & 1.7 & 1.8 & 2.1 & 2.8 & 2.7 & 2.4 & 2.1 & 0.0 & 39.2 \\
\hline $4-7$ & 1.3 & 1.7 & 1.8 & 2.8 & 5.2 & 1.9 & 0.8 & 0.5 & 0.6 & 0.8 & 0.9 & 1.8 & 4.7 & 5.3 & 2.7 & 1.2 & 0.0 & 33.8 \\
\hline $8-12$ & 0.6 & 0.9 & 0.5 & 0.5 & 0.8 & 0.1 & 0.1 & 0.1 & 0.2 & 0.3 & 0.5 & 0.7 & 2.9 & 4.4 & 1.5 & 0.5 & 0.0 & 14.5 \\
\hline $13-18$ & 0.3 & 0.3 & 0.2 & 0.1 & 0.1 & 0.0 & 0.0 & 0.0 & 0.1 & 0.2 & 0.5 & 0.4 & 1.2 & 3.3 & 1.2 & 0.2 & 0.0 & 7.8 \\
\hline $19-24$ & 0.1 & 0.1 & 0.0 & 0.0 & 0.0 & 0.0 & 0.0 & 0.0 & 0.0 & 0.1 & 0.2 & 0.1 & 0.2 & 1.2 & 0.5 & 0.1 & 0.0 & \\
\hline $25-31$ & 0.0 & 0.0 & 0.0 & 0.0 & 0.0 & 0.0 & 0.0 & 0.0 & 0.0 & 0.0 & 0.1 & 0.0 & 0.0 & 0.3 & 0.1 & 0.0 & 0.0 & \\
\hline $32-38$ & 0.0 & 0.0 & 0.0 & 0.0 & 0.0 & 0.0 & 0.0 & 0.0 & 0.0 & 0.0 & 0.0 & 0.0 & 0.0 & 0.0 & 0.0 & 0.0 & 0.0 & \\
\hline $39-46$ & 0.0 & 0.0 & 0.0 & 0.0 & 0.0 & 0.0 & 0.0 & 0.0 & 0.0 & 0.0 & 0.0 & 0.0 & 0.0 & 0.0 & 0.0 & 0.0 & 0.0 & \\
\hline$>46$ & 0.0 & 0.0 & 0.0 & 0.0 & 0.0 & 0.0 & 0.0 & 0.0 & 0.0 & 0.0 & 0.0 & 0.0 & 0.0 & 0.0 & 0.0 & 0.0 & 0.0 & \\
\hline TOTAL & 4.0 & 5.0 & 5.3 & 6.7 & 10.7 & 5.2 & 3.1 & 2.2 & 2.5 & 3.0 & 4.0 & 5.2 & 11.9 & 17.2 & 8.4 & 4.1 & 1.3 & 100.0 \\
\hline
\end{tabular}

Station: (19S) SAGE

Begin: 3/82

End: $12 / 92$

Total Hours: 91756

DIRECTION

\begin{tabular}{|c|c|c|c|c|c|c|c|c|c|c|c|c|c|c|c|c|c|c|}
\hline & $\mathrm{N}$ & NNE & $\mathrm{NE}$ & ENE & E & ESE & SE & SSE & $\underline{S}$ & SSW & SW & WSW & W & WNW & NW & NNW & CALM & TOTAL \\
\hline CALM & 0.0 & 0.0 & 0.0 & 0.0 & 0.0 & 0.0 & 0.0 & 0.0 & 0.0 & 0.0 & 0.0 & 0.0 & 0.0 & 0.0 & 0.0 & 0.0 & 0.7 & 0.7 \\
\hline 1-3 & 1.3 & 1.1 & 1.2 & 1.0 & 1.1 & 1.4 & 1.7 & 1.9 & 1.8 & 1.6 & 1.7 & 1.5 & 1.5 & 1.3 & 1.6 & 1.6 & 0.0 & 23.3 \\
\hline $4-7$ & 3.6 & 2.9 & 3.3 & 2.5 & 2.1 & 3.2 & 4.2 & 4.9 & 4.3 & 2.4 & 2.2 & 3.4 & 4.4 & 2.5 & 1.9 & 3.4 & 0.0 & 51.2 \\
\hline $8-12$ & 1.4 & 1.3 & 0.9 & 0.4 & 0.4 & 0.5 & 1.3 & 2.3 & 2.7 & 1.3 & 1.1 & 1.3 & 1.5 & 1.8 & 0.4 & 0.6 & 0.0 & 19.1 \\
\hline $13-18$ & 0.2 & 0.3 & 0.1 & 0.0 & 0.0 & 0.0 & 0.1 & 0.3 & 0.7 & 0.6 & 0.5 & 0. & 0.2 & 0.8 & 0.2 & 0.1 & 0.0 & 4.5 \\
\hline $19-24$ & 0.0 & 0.0 & 0.1 & 0.0 & 0.0 & 0.0 & 0.0 & 0.0 & 0.1 & 0.2 & 0.2 & 0.1 & 0.0 & 0.1 & 0.0 & 0.0 & 0.0 & 0.9 \\
\hline $25-31$ & 0.0 & 0.0 & 0.0 & 0.0 & 0.0 & 0.0 & 0.0 & 0.0 & 0.0 & 0.1 & 0.1 & 0.0 & 0.0 & 0.0 & 0.0 & 0.0 & 0.0 & 0.2 \\
\hline $32-38$ & 0.0 & 0.0 & 0.0 & 0.0 & 0.0 & 0.0 & 0.0 & 0.0 & 0.0 & 0.0 & 0.0 & 0.0 & 0.0 & 0.0 & 0.0 & 0.0 & 0.0 & 0.0 \\
\hline & 0.0 & 0.0 & 0.0 & 0.0 & 0.0 & 0.0 & 0.0 & 0. & 0.0 & 0.0 & 0.0 & 0. & 0.0 & 0.0 & 0.0 & 0.0 & 0.0 & 0.0 \\
\hline$>46$ & 0.0 & 0.0 & 0.0 & 0.0 & 0.0 & 0.0 & 0.0 & 0. & 0.0 & 0.0 & 0.0 & 0 & 0.0 & 0.0 & 0.0 & 0.0 & 0.0 & 0.0 \\
\hline OTAL & 6.6 & 5.7 & 5.6 & 3.9 & 3.6 & 5.2 & 7.2 & 9.3 & 9.6 & 6.1 & 5.9 & 6.8 & 7.5 & 6.5 & 4.1 & 5.7 & 0.7 & 100.0 \\
\hline
\end{tabular}




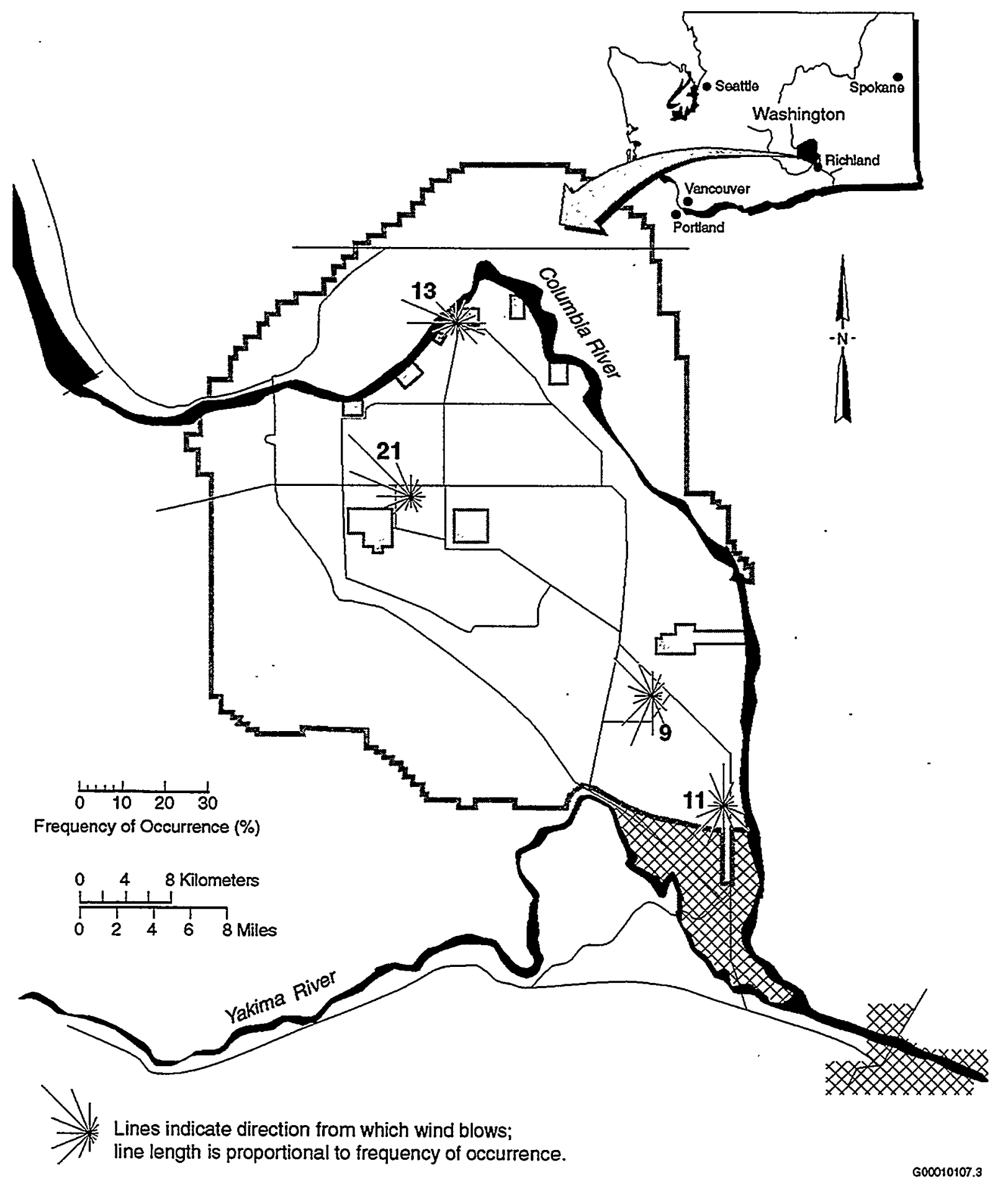

Figure 5.2. Hanford Meteorological Monitoring Network Wind Roses at 60-Meter Level, 1986 Through 1999 
Table 5.5. Joint Frequency Distributions (\%) for Hanford Meteorological Monitoring Network Wind Stations at 60-Meter Level, 1986 Through 1999

Tower: 100 Area

Begin: $1 / 86$

End: $12 / 99$

Total Hours: 117014

DIRECTION

\begin{tabular}{|c|c|c|c|c|c|c|c|c|c|c|c|c|c|c|c|c|c|c|}
\hline & $\mathbf{N}$ & NE & NE & ENE & $\mathrm{E}$ & ESE & SE & SSE & $\underline{S}$ & SSW & SW & WSW & W & WNW & NW & NNW & CALM & TOTAL \\
\hline CALM & 0.0 & 0.0 & 0.0 & 0.0 & 0.0 & 0.0 & 0.0 & 0.0 & 0.0 & 0.0 & 0.0 & 0.0 & 0.0 & 0.0 & 0.0 & 0.0 & 0.7 & 0.7 \\
\hline 1-3 & 1.7 & 1.6 & 1.8 & 2.2 & 3.1 & 2.9 & 2.3 & 1.6 & 1.3 & 1.2 & 1.3 & 1.5 & 1.8 & 1.9 & 1.9 & 1.8 & 0.0 & 29.9 \\
\hline $4-7$ & 1.8 & 1.9 & 1.6 & 1.7 & 3.0 & 3.1 & 2.6 & 1.5 & 0.9 & 0.7 & 1.2 & 1.9 & 3.0 & 3.2 & 2.2 & 1. & 0.0 & 31.8 \\
\hline $8-12$ & 0.7 & 1.1 & 0.8 & 0.5 & 0.5 & 0.7 & 1.2 & 0.6 & 0.4 & 0.4 & 0.9 & 1.3 & 2.7 & 2.6 & 1.0 & 0.6 & 0.0 & 16.0 \\
\hline $13-18$ & 0.4 & 0.6 & 0.4 & 0.2 & 0.2 & 0.2 & 0.5 & 0.4 & 0.2 & 0.3 & 0.8 & 0.8 & 2.6 & 3.2 & 0.7 & 0.2 & 0.0 & 11.7 \\
\hline $19-24$ & 0.2 & 0.3 & 0.2 & 0.1 & 0.1 & 0.1 & 0.1 & 0.1 & 0.1 & 0.2 & 0.5 & 0.4 & 1.0 & 2.1 & 0.6 & 0.1 & 0.0 & 6.4 \\
\hline $25-31$ & 0.1 & 0.2 & 0.1 & 0.0 & 0.0 & 0.0 & 0.0 & 0.1 & 0.0 & 0.2 & 0.3 & 0.2 & 0.3 & 0.8 & 0.4 & 0.0 & 0.0 & 2.7 \\
\hline $32-38$ & 0.0 & 0.0 & 0.0 & 0.0 & 0.0 & 0. & 0.0 & 0.0 & 0.0 & 0.1 & 0.1 & 0.1 & 0.1 & 0.2 & 0.1 & 0.0 & 0.0 & 0.6 \\
\hline & 0.0 & 0.0 & 0.0 & 0.0 & 0.0 & 0.0 & 0.0 & 0.0 & 0.0 & 0.0 & 0.0 & 0.0 & 0.0 & 0.0 & 0.0 & 0.0 & 0.0 & 0.1 \\
\hline$>46$ & 0.0 & 0.0 & 0.0 & 0.0 & 0.0 & 0.0 & 0.0 & 0.0 & 0.0 & 0.0 & 0.0 & 0.0 & 0.0 & 0.0 & 0.0 & 0.0 & 0.0 & 0.0 \\
\hline TOTAL & 5.0 & 5.8 & 4.9 & 4.8 & 6.9 & 7.0 & 6.8 & 4.3 & 3.1 & 3.2 & 5.1 & 6.1 & 11.5 & .14 .0 & 6.7 & 4.1 & 0.7 & 100.0 \\
\hline
\end{tabular}

Tower: 200 Area

Begin: $1 / 86$

End: $12 / 99$

Total Hours: 122608

DIRECTION

$N$ NNE NE ENE E ESE SE SSE S SSW SW WSW W WNW NW NNW CALM TOTAL

\begin{tabular}{|c|c|c|c|c|c|c|c|c|c|c|c|c|c|c|c|c|c|c|}
\hline CALM & 0.0 & 0.0 & 0.0 & 0.0 & 0.0 & 0.0 & 0.0 & 0.0 & 0.0 & 0.0 & 0.0 & 0.0 & 0.0 & 0.0 & 0.0 & 0.0 & 0.4 & 0.4 \\
\hline $1-3$ & 1.3 & 1.1 & 1.1 & 1.0 & 1.1 & 1.0 & 1.2 & 0.9 & 0.6 & 0.6 & 0.6 & 0.6 & 0.7 & 0.9 & 1.1 & 1.3 & 0.0 & 15.1 \\
\hline 4-7 & 2.6 & 2.0 & 1.5 & 1.4 & 1.7 & 1.3 & 1.7 & 1.6 & 1.0 & 1.0 & 1.2 & 1.3 & 1.9 & 2.9 & 4.2 & 3.8 & 0.0 & 31.0 \\
\hline $8-12$ & 1.0 & 0.7 & 0.5 & 0.3 & 0.4 & 0.3 & 0.4 & 0.7 & 0.5 & 0.6 & 1.2 & 1.9 & 2.7 & 4.8 & 6.2 & 2.3 & 0.0 & 24.6 \\
\hline $13-18$ & 0.3 & 0.3 & 0.2 & 0.1 & 0.1 & 0.0 & 0.1 & 0.3 & 0.3 & 0.5 & 1.1 & 1.9 & 2.0 & 4.5 & 5.5 & 0.6 & 0.0 & 17.7 \\
\hline $19-24$ & 0.0 & 0.1 & 0.1 & 0.0 & 0.0 & 0.0 & 0.0 & 0.1 & 0.1 & 0.3 & 0.9 & 0.9 & 0.5 & 1.7 & 2.3 & 0.1 & 0.0 & 7.2 \\
\hline $25-31$ & 0.0 & 0.0 & 0.0 & 0.0 & 0.0 & 0.0 & 0.0 & 0.0 & 0.1 & 0.3 & 0.6 & 0.4 & 0.1 & 0.5 & 1.0 & 0.0 & 0.0 & 3.1 \\
\hline $32-38$ & 0.0 & 0.0 & 0.0 & 0.0 & 0.0 & 0.0 & 0.0 & 0.0 & 0.0 & 0.1 & 0.2 & 0.1 & 0.0 & 0.1 & 0.1 & 0.0 & 0.0 & 0.6 \\
\hline & 0.0 & 0.0 & 0.0 & 0.0 & 0.0 & 0.0 & 0.0 & 0.0 & 0.0 & 0.0 & 0.1 & 0.0 & 0.0 & 0.0 & 0.0 & 0.0 & 0.0 & 0.1 \\
\hline$>46$ & 0.0 & 0.0 & 0.0 & 0.0 & 0.0 & 0.0 & 0.0 & 0.0 & 0.0 & 0.0 & 0.0 & 0.0 & 0.0 & 0.0 & 0.0 & 0.0 & 0.0 & 0.0 \\
\hline OTAL & 5.2 & 4.3 & 3.5 & 2.7 & 3.2 & 2.5 & 3.4 & 3.7 & 2.6 & 3.5 & 5.9 & 7.1 & 7.9 & 15.3 & 20.6 & 8.3 & 0.4 & 100 \\
\hline
\end{tabular}

Tower: 300 Area

DIRECTION

\begin{tabular}{|c|c|c|c|c|c|c|c|c|c|c|c|c|c|c|c|c|c|c|}
\hline & $\mathrm{N}$ & NNE & NE & ENE & $E$ & ESE & $S E$ & SSE & $\underline{S}$ & SSW & SW & WSW & $\mathrm{W}$ & WNW & NW & NNW & CALM & TOTAL \\
\hline CALM & 0.0 & 0.0 & 0.0 & 0.0 & 0.0 & 0.0 & 0.0 & 0.0 & 0.0 & 0.0 & 0.0 & 0.0 & 0.0 & 0.0 & 0.0 & 0.0 & 0.5 & 0.5 \\
\hline $1-3$ & 1.0 & 0.8 & 0.7 & 0.6 & 0.8 & 1.0 & 1.2 & 1.2 & 1.1 & 0.9 & 0.8 & 0.6 & 0.7 & 0.7 & 0.9 & 1.0 & 0.0 & 14.3 \\
\hline $4-7$ & 3.0 & 1.8 & 1.2 & 1.2 & 1.8 & 2.8 & .4 .1 & 3.1 & 2.7 & 2.4 & 1.9 & 1.2 & 0.9 & 0.8 & 1.2 & 2.4 & 0.0 & 32.8 \\
\hline $8-12$ & 3.5 & 2.1 & 0.9 & 0.4 & 0.4 & 1.3 & 2.6 & 1.4 & 1.8 & 3.3 & 3.2 & 1.7 & 0.8 & 0.5 & 1.0 & 2.5 & 0.0 & 27.8 \\
\hline $13-18$ & 2.0 & 0.9 & 0.2 & 0.1 & 0.0 & 0.2 & 0.4 & 0.3 & 0.5 & 2.0 & 3.2 & 2.0 & 0.6 & 0.4 & 1.0 & 1.8 & 0.0 & 15.8 \\
\hline $19-24$ & 0.3 & 0.2 & 0.1 & 0.0 & 0.0 & 0.0 & 0.0 & 0.1 & 0.1 & 0.7 & 1.5 & 0.9 & 0.4 & 0.1 & 0.7 & 0.5 & 0.0 & 5.6 \\
\hline $25-3$ & 0.0 & 0.1 & 0.0 & 0.0 & 0.0 & 0.0 & 0.0 & 0.0 & 0.0 & 0.3 & 0.8 & 0.3 & 0.2 & 0.0 & 0.2 & 0.1 & 0.0 & 2.2 \\
\hline $32-3$ & 0.0 & 0.0 & 0.0 & 0.0 & 0.0 & 0.0 & 0.0 & 0.0 & 0.0 & 0.1 & 0.3 & 0.1 & 0.0 & 0.0 & 0.0 & 0.0 & 0.0 & 0.6 \\
\hline & 0.0 & 0. & 0.0 & 0.0 & 0.0 & 0.0 & 0.0 & 0.0 & 0.0 & 0.0 & 0.2 & 0.0 & 0.0 & 0.0 & 0.0 & 0.0 & 0.0 & 0.2 \\
\hline & 0.0 & 0. & 0.0 & 0.0 & 0.0 & 0.0 & 0.0 & 0.0 & 0.0 & 0.0 & 0.0 & 0.0 & 0.0 & 0.0 & 0.0 & 0.0 & 0.0 & 0.0 \\
\hline TOTAL & 9.8 & 5.9 & 3.1 & 2.4 & 3.0 & 5.3 & 8.4 & 6.1 & 6.4 & 9.5 & 12.0 & 6.9 & 3.5 & 2.6 & 5.0 & 8.2 & 0.5 & 100.0 \\
\hline
\end{tabular}

Tower: 400 Area

DIRECTION

\begin{tabular}{|c|c|c|c|c|c|c|c|c|c|c|c|c|c|c|c|c|c|c|}
\hline & $\mathbf{N}$ & NNE & NE & ENE & $E$ & ESE & SE & SSE & S & SSW & SW & WSW & W & WNW & NW & NNW & CALM & TOTAL \\
\hline CALM & 0.0 & 0.0 & 0.0 & 0.0 & 0.0 & 0.0 & 0.0 & 0.0 & 0.0 & 0.0 & 0.0 & 0.0 & 0.0 & 0.0 & 0.0 & 0.0 & 0.3 & 0.3 \\
\hline $1-3$ & 0.8 & 0.8 & 0.7 & 0.6 & 0.8 & 0.8 & 0.9 & 0.9 & 0.9 & 0.8 & 0.8 & 0.6 & 0.7 & 0.6 & 0.7 & 0.8 & 0.0 & 12.2 \\
\hline 4-7 & 2.1 & 2.0 & 1.7 & 1.1 & 1.1 & 1.3 & 1.9 & 2.7 & 2.9 & 2.3 & 1.6 & 1.2 & 1.2 & 1.4 & 2.1 & 2.2 & 0.0 & 28.8 \\
\hline $8-12$ & 1.9 & 1.8 & 1.1 & 0.4 & 0.3 & 0.3 & 1.3 & 2.3 & 3.4 & 3.6 & 2.1 & 0.9 & 0.9 & 1.4 & 3.0 & 2.6 & 0.0 & 27.1 \\
\hline $13-18$ & 0.7 & 0.6 & 0.3 & 0.1 & 0.0 & 0.0 & 0.5 & 0.8 & 1.6 & 3.8 & 2.4 & 0.8 & 0.6 & 1.5 & 3.6 & 1.8 & 0.0 & 19.1 \\
\hline $19-2$ & 0.1 & 0.2 & 0.2 & 0.0 & 0.0 & 0.0 & 0.1 & 0.1 & 0.3 & 1.4 & 1.5 & 0.6 & 0.3 & 0.9 & 2.2 & 0.4 & 0.0 & 8.4 \\
\hline 25 & 0.0 & 0.1 & 0.1 & 0.0 & 0.0 & 0.0 & 0.0 & 0.0 & 0.1 & 0.4 & 0.8 & 0.3 & 0.1 & 0.2 & 0.6 & 0.1 & 0.0 & 2.9 \\
\hline $32-3$ & 0.0 & 0.0 & 0.0 & 0.0 & 0.0 & 0. & 0.0 & 0.0 & 0.0 & 0.2 & 0.3 & 0. & 0.0 & 0.0 & 0.1 & 0.0 & 0.0 & 0.8 \\
\hline & 0.0 & 0.0 & 0.0 & 0.0 & 0.0 & 0.0 & 0.0 & 0.0 & 0.0 & 0.1 & 0.1 & 0.0 & 0.0 & 0.0 & 0.0 & 0.0 & 0.0 & 0.2 \\
\hline$>46$ & 0.0 & 0.0 & 0.0 & 0.0 & 0.0 & 0.0 & 0.0 & 0.0 & 0.0 & 0.0 & 0.1 & 0.0 & 0.0 & 0.0 & 0.0 & 0.0 & 0.0 & 0. \\
\hline TOTAL & 5.7 & 5.4 & 4.0 & 2.3 & 2.2 & 2.5 & 4.8 & 6.9 & 9.1 & 12.5 & 9.7 & 4.6 & 3.9 & 6.0 & 12.3 & 7.8 & 0.3 & 100.0 \\
\hline
\end{tabular}




\subsection{Miscellaneous Climatological Statistics}

\subsection{Sky Cover}

The term sky cover is used to express the portion of the celestial dome that is 1) covered, but not necessarily hidden, by clouds or obscuring phenomena aloft; 2) hidden by an obscuring phenomenon on the ground (such as fog or smoke); or 3) a combination of both 1 and 2. The sky cover is determined hourly by scanning the sky and estimating the number of tenths that are covered ( 0 denotes clear and 10 denotes overcast). Average monthly sunrise-to-sunset sky covers for the period 1946 through 1999 are shown in Table 6.1. Also shown in Table 6.1 are the number of clear, partly cloudy, and cloudy days for the period 1954 through 1999. The number of clear, partly cloudy, and cloudy days is the result of assigning each day to one of the following categories based on its average sky cover for that day:

\begin{tabular}{lll}
\multicolumn{1}{c}{ Category } & \multicolumn{1}{c}{$\begin{array}{c}\text { Average } \\
\text { Sky Cover }\end{array}$} \\
Clear & & $0-3$ tenths \\
Partly cloudy & & $4-7$ tenths \\
Cloudy & & $8-10$ tenths
\end{tabular}

During the period of record (1954 through 1999), an average of 200 sunny days (the sum of the clear and partly cloudy days) was recorded per year at the Hanford Meteorology Station.

\subsection{Fog and Dense Fog}

Table 6.2 shows the average monthly and annual number of days with fog and dense fog. Fog is reported any time horizontal visibility is reduced to 6 miles or less because of the suspension of water droplets in the surface layer of the atmosphere. Dense fog is reported when horizontal visibility is reduced to 0.25 mile or less. Most fog at the Hanford Meteorology Station is radiation fog, a common type of fog that forms on nights characterized by light wind, clear sky, and moist air in the lower levels of the atmosphere. Nearly $90 \%$ of both fog and dense fog at the Hanford Meteorology Station occurs during the late autumn and winter months, though fog is observed every month of the year.

\subsection{Psychrometric Data}

Psychrometric data include observations of dry bulb, wet bulb, dew point temperatures, and relative humidity. The dry bulb temperature is the temperature of the ambient air; the wet bulb temperature is the lowest temperature to which a parcel of air, under constant pressure, can be cooled by evaporating water into it. The dew point temperature is the temperature to which a given parcel of air, under constant watervapor content, must be cooled to attain saturation. Relative humidity is the ratio of the actual water-vapor content of the air to the one where saturation would occur if the pressure and temperature remained unchanged. 


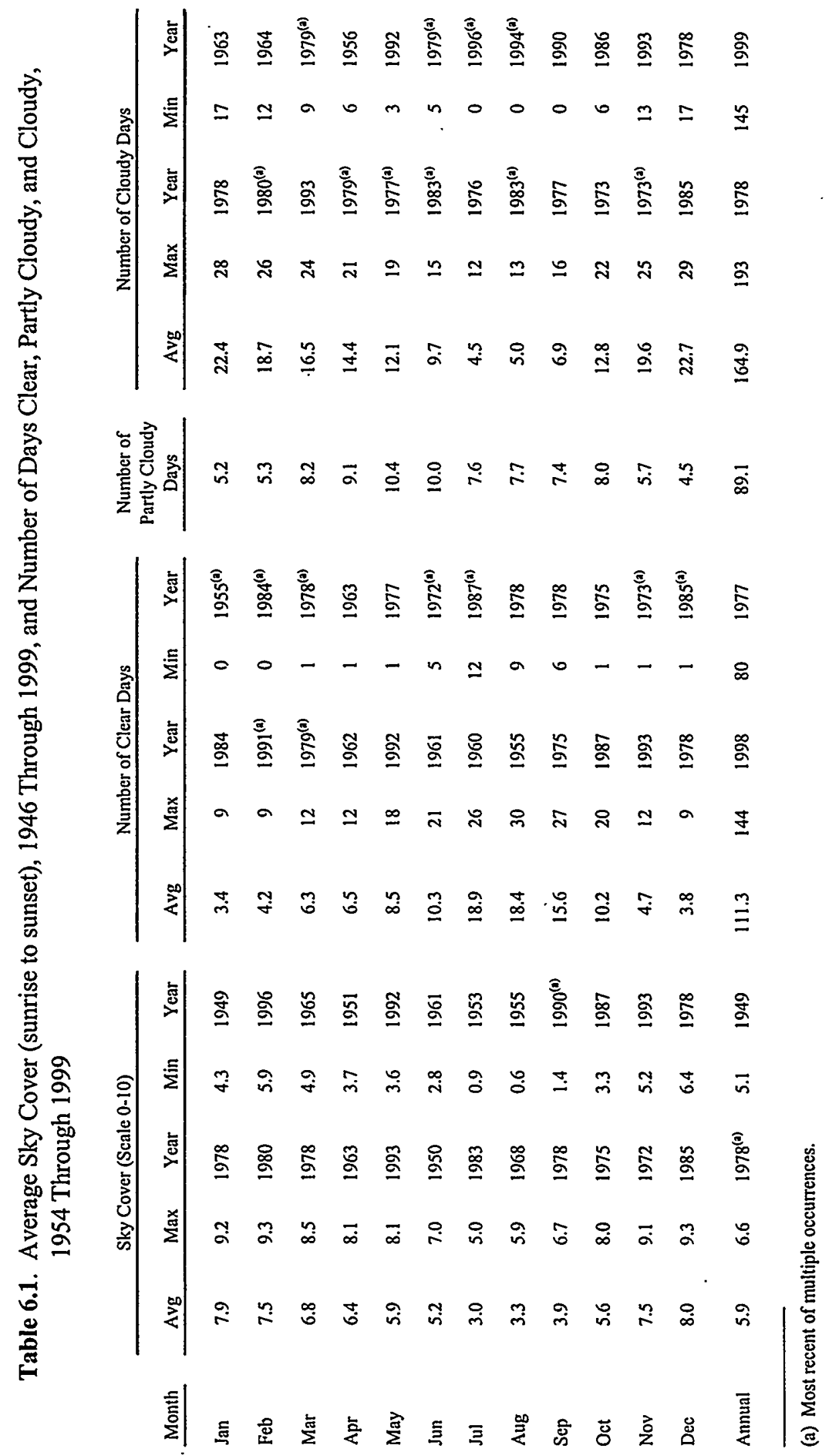


Table 6.2. Monthly and Annual Number of Days with Fog and Dense Fog, 1945 Through 1999

\begin{tabular}{|c|c|c|c|c|c|c|c|c|c|c|}
\hline \multirow[b]{2}{*}{ Month } & \multicolumn{5}{|c|}{ Days with Fog (Visibility $\leq 6$ miles) } & \multicolumn{5}{|c|}{ Days with Dense Fog (Visibility $\leq 0.25$ mile) } \\
\hline & Avg & $\operatorname{Max}$ & Year & Min & Year & Avg & $\operatorname{Max}$ & Year & Min & Year \\
\hline Jan & 11.5 & 25 & 1979 & 0 & 1949 & 6.2 & 15 & $1994^{(2)}$ & 0 & 1949 \\
\hline Feb & 6.5 & 20 & 1963 & 0 & $1988^{(2)}$ & 3.2 & 11 & 1963 & 0 & $1999^{(2)}$ \\
\hline Mar & 2.1 & 10 & 1993 & 0 & $1999^{(2)}$ & 0.8 & 5 & $1993^{(2)}$ & 0 & $1999^{(2)}$ \\
\hline Apr & 0.5 & 3 & 1992 & 0 & $1999^{(a)}$ & 0.1 & 1 & $1993^{(2)}$ & 0 & $1999^{(a)}$ \\
\hline May & 0.2 & 3 & 1948 & 0 & $1999^{(2)}$ & $<0.1$ & 1 & 1958 & 0 & $1999^{(2)}$ \\
\hline Jun & 0.1 & 2 & 1971 & 0 & $1999^{(a)}$ & $<0.1$ & 1 & 1971 & 0 & $1999^{(2)}$ \\
\hline Jul & $<0.1$ & 1 & 1966 & 0 & $1999^{(a)}$ & 0 & 0 & 0 & & \\
\hline Aug & 0.1 & 1 & $1985^{(2)}$ & 0 & $1999^{(2)}$ & $<0.1$ & 1 & $1985^{(2)}$ & 0 & $1999^{(2)}$ \\
\hline Sep & 0.3 & 2 & $1985^{(2)}$ & 0 & $1999^{(a)}$ & 0.1 & 1 & $1995^{(2)}$ & 0 & $1999^{(2)}$ \\
\hline Oct & 2.0 & 9 & 1962 & 0 & $1989^{(a)}$ & 1.0 & 7 & 1980 & 0 & $1998^{(a)}$ \\
\hline Nov & 9.7 & 19 & $1985^{(2)}$ & 0 & 1990 & 5.5 & 13 & 1965 & 0 & $1990^{(2)}$ \\
\hline Dec & 14.0 & 25 & $1989^{(2)}$ & 2 & 1968 & 7.5 & 17 & 1950 & 2 & $1996^{(2)}$ \\
\hline Annual & 47.0 & 84 & $1985-86$ & 22 & $1948-49$ & 24.5 & 42 & $1950-51$ & 9 & $1948-49$ \\
\hline
\end{tabular}

(a) Most recent of multiple occurrences.

Longest duration of fog: 113.7 hours, December 16-20, 1985.

Longest duration of dense fog: 47.0 hours, December 1957.

Table 6.3 presents monthly averages and extremes of dry bulb, wet bulb, dew point temperatures, and relative humidity from the Hanford Meteorology Station for the period 1950 through 1999 . These parameters are collected hourly and are averaged on a monthly (as opposed to a daily) basis. Prior to 1975, wet bulb temperatures $\geq 75^{\circ} \mathrm{F}$ had never been observed at the Hanford Meteorology Station. On July 8, 9, and $10,1975,7$ hourly observations were made of wet bulb temperatures $\geq 75^{\circ} \mathrm{F}$.

\subsection{Solar Radiation}

Table 6.4 presents average and extreme daily solar radiation values by month for the period 1953 through 1999. These data are reported in langleys (a langley is a unit defined as 1 gram calorie per square centimeter), and are integrated over an hour period and totaled for a daily value.

The highest daily values occur with a clear sky and clean air; the lowest commonly occur on days overcast with low stratus clouds. The lowest midday values of hourly solar radiation occurred on May 18, 1980, as the dense ash cloud from the morning eruption of Mount St. Helens passed over eastern Washington. Hourly solar radiation values dropped to 0 at 1100 hours and remained at 0 for the rest of that day. 
Table 6.3. Monthly Averages and Extremes of Psychrometric Data, 1950 Through 1999

\begin{tabular}{|c|c|c|c|c|c|c|c|c|c|c|c|c|c|}
\hline \multirow[b]{2}{*}{ Category ${ }^{(2)}$} & \multicolumn{13}{|c|}{ Monthly Averages } \\
\hline & $\operatorname{Jan}$ & Feb & Mar & Apr & May & Jun & $\mathrm{Jul}$ & Aug & Sep & Oct & Nov & Dec & Annual \\
\hline Dry bulb & 31.1 & 37.6 & 45.2 & 53.2 & 62.2 & 69.9 & 77.3 & 75.7 & 66.5 & 53.0 & 40.2 & 32.5 & 53.7 \\
\hline Wet bulb & 29 & 34 & 38 & 44 & 50 & 55 & 58 & 58 & 53 & 45 & 37 & 30 & 44 \\
\hline Rel. hum. & 77.2 & 70.4 & 56.5 & 47.3 & 43.0 & 39.6 & 33.4 & 35.6 & 42.1 & 56.3 & 73.5 & 80.0 & 54.5 \\
\hline \multirow[t]{2}{*}{ Dew point } & 24.4 & 27.8 & 28.9 & 31.6 & 37.0 & 41.6 & 43.7 & 44.0 . & 40.4 & 36.0 & 31.5 & 26.5 & 34.4 \\
\hline & \multicolumn{12}{|c|}{ Extremes of Monthly Averages } & \\
\hline Dry Bulb & & & & & & & & & & & & & \\
\hline Highest & 43.0 & 44.6 & 51.6 & 58.6 & 68.7 & 77.3 & 83.3 & 82.5 & 72.7 & 59.5 & 46.4 & 38.8 & 56.6 \\
\hline Year & 1953 & 1991 & 1992 & 1987 & 1958 & 1992 & 1985 & 1967 & 1990 & 1988 & 1999 & 1953 & 1992 \\
\hline Lowest & 12.9 & 25.8 & 39.6 & 48.3 & 57.0 & 64.2 & 71.3 & 70.6 & 58.9 & 48.1 & 25.7 & 21.9 & 50.2 \\
\hline Year & 1950 & 1956 & 1955 & 1955 & 1984 & 1953 & 1986 & 1964 & 1985 & 1984 & 1985 & 1985 & 1985 \\
\hline \multicolumn{14}{|l|}{ Wet Bulb } \\
\hline Highest & 39 & 41 & 44 & 47 & 55 & 59 & 63 & 61 & 56 & 50 & 42 & 36 & 47 \\
\hline Year & 1953 & 1956 & 1992 & 1992 & 1958 & $1992^{(b)}$ & 1998 & $1999^{(b)}$ & $1995^{(b)}$ & 1988 & $1999^{(b)}$ & $1991^{(b)}$ & 1992 \\
\hline Lowest & 12 & 23 & 33 & 39 & 45 & 51 & 56 & 55 & 48 & 40 & 24 & 21 & 41 \\
\hline Year & 1950 & 1956 & 1955 & 1955 & 1959 & $1983^{(b)}$ & $1986^{\text {(b) }}$ & $1980^{(b)}$ & 1970 & 1984 & 1985 & 1985 & 1985 \\
\hline \multicolumn{14}{|c|}{ Relative Humidity } \\
\hline Highest & 88.8 & 86.9 & 69.1 & 64.5 & 61.9 & 53.5 & 45.6 & 47.8 & 55.5 & 74.2 & 88.7 & 90.5 & 58.9 \\
\hline Year & 1960 & 1963 & 1993 & 1963 & 1948 & 1950 & 1993 & 1976 & 1977 & 1962 & 1979 & 1950 & 1978 \\
\hline Lowest & 60.0 & 54.0 & 44.0 & 36.9 & 31.2 & 30.0 & 21.9 & 24.5 & 33.2 & 42.5 & 62.8 & 69.0 & 49.4 \\
\hline Year & 1963 & 1967 & 1965 & 1966 & 1966 & 1949 & 1959 & 1967 & 1974 & 1952 & 1976 & 1968 & 1967 \\
\hline \multicolumn{14}{|l|}{ Dew Point } \\
\hline Highest & 34.4 & 36.7 & 37.2 & 37.1 & 43.9 & 47.5 & 50.1 & 48.4 & 45.4 & 43.5 & 38.3 & 34.3 & 37.7 \\
\hline Year & 1953 & $1992^{(b)}$ & 1986 & $1992^{(b)}$ & 1998 & 1958 & 1975 & 1976 & 1963 & 1962 & 1954 & 1950 & 1958 \\
\hline Lowest & 6.5 & 17.3 & 20.8 & 26.0 & 30.4 & 37.5 & 35.4 & 38.4 & 33.8 & 30.2 & 19.4 & 15.1 & 31.5 \\
\hline Year & 1950 & 1956 & $1965^{(b)}$ & 1982 & 1964 & 1954 & 1959 & 1955 & 1970 & 1984 & 1985 & 1983 & 1955 \\
\hline
\end{tabular}

(a) Dry bulb, wet bulb, and dew point temperatures in ${ }^{\circ} \mathrm{F}$, relative humidity in $\%$.

(b) Most recent of multiple occurrences.

Table 6.4. Average and Extreme Solar Radiation Daily Values (langley), 1953 Through 1999

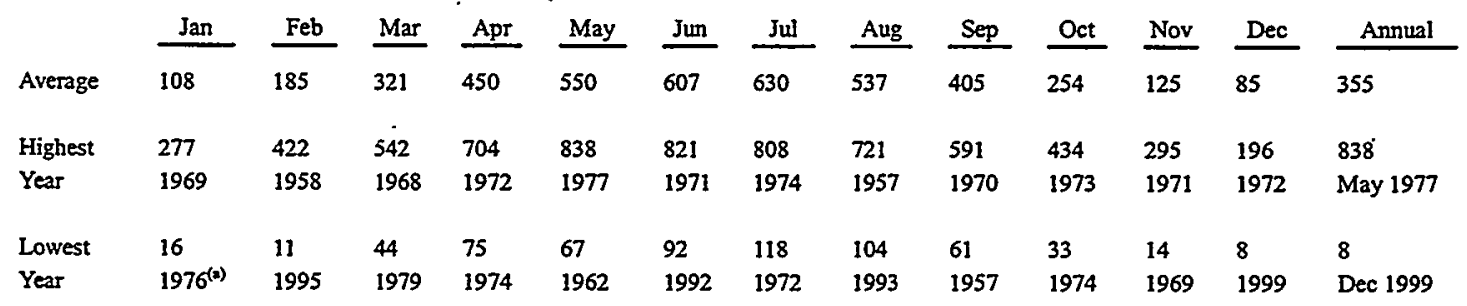

(a) Most recent of multiple occurrences. 


\subsection{Thunderstorms, Dust and Glaze}

A thunderstorm day is one in which thunder is heard at the observing station one or more times during a calendar day. If a thunderstorm were to begin before midnight and continue until after midnight, it is possible to have two thunderstorm days from a single storm.

Table 6.5 shows that thunderstorms occurred in every month of the year, except January and November. The thunderstorm season is essentially from April through September. The average number of thunderstorm days per year is 10; however, the total varies from a low of 3 in 1949 to a high of 23 in 1948. The largest number of thunderstorms in any single month was 8 in July 1998, July 1983, June 1972, and August 1953.

The criterion for both dust and blowing dust is that horizontal visibility be reduced to 6 miles or less. Dust is carried into the area from a distant source and may occur without strong winds. Blowing dust occurs when dust is being picked up locally and occurs with stronger winds. Both dust and blowing dust occurred at the Hanford Meteorology Station; however, in most cases, it is blowing dust. Table 6.5 presents the average number of days per month and year of dust and blowing dust during the period 1945 through 1999. The average number of days per year with dust or blowing dust is 5 . The greatest number of such days in any year was 20 in 1980; the fewest was 0 in 1987 and earlier years. The greatest number of days with dust or blowing dust in any month was 9 in May 1980. This peak in the number of days with dust or blowing dust resulted from the eruption of Mount St. Helens on May 18, 1980, and subsequent dates.

Glaze is a coating of ice formed when rain or drizzle freezes on contact with any surface having a temperature that is below freezing. Table 6.5 provides data on the number of days per month and year with glaze for the period 1945 through 1999. The average number of days with freezing rain or freezing drizzle is 6 . The highest number of days with glaze in any winter season was 18 during the winter of 1969-1970; the least, 1 day during the winter of 1987-1988 and earlier winters. The greatest number of such days in any single month was 9 in January 1970.

Table 6.5. Average Number of Days of Various Meteorological Phenomena, 1945 Through 1999

\begin{tabular}{|c|c|c|c|c|c|c|c|c|c|c|c|c|c|}
\hline Phenomenon & Jan & Feb & Mar & Apr & May & Jun & Jul & Aug & Sep & Oct & Nov & Dec & Annual \\
\hline Thunderstorm & 0 & $\leq 0.1$ & 0.1 & 0.8 & 1.5 & 2.3 & 2.2 & 2.0 & 0.8 & 0.2 & 0 & $\leq 0.1$ & 10.0 \\
\hline Dust or blowing dust & 0.4 & 0.4 & 0.5 & 0.6 & 0.7 & 0.4 & 0.4 & 0.3 & 0.5 & 0.3 & 0.2 & 0.2 & 4.6 \\
\hline Glaze & 2.2 & 0.7 & $\leq 0.1$ & 0 & 0 & 0 & 0 & 0 & 0 & 0 & 0.8 & 2.4 & 6.1 \\
\hline
\end{tabular}

\subsection{Atmospheric Pressure}

Table 6.6 contains atmospheric pressure data for the period 1955 through 1999 . This table lists both station and sea-level pressure, including extremes and years of occurrence. Atmospheric pressure may be indicated in several different units, including inches of mercury, millimeters of mercury, millibars, or 
Pascals; however, in this table, pressure is stated in inches of mercury. Station pressure is the barometric pressure measured at the Hanford Meteorology Station (at an elevation of 733 feet); sea-level pressure is the station pressure adjusted to sea level. Most are familiar with barometric pressure adjusted to sea level, which allows atmospheric pressures for all locations to be compared, regardless of the elevation of the station where the data are measured.

The highest sea-level pressure ever recorded at the Hanford Meteorology Station was 31.12 inches in January 1979; the lowest was 28.94 inches in December 1995 and on previous occasions.

Some rapid pressure changes occurred on November 3, 1958, falling 0.492 inch over a 6-hours period ( 0.082 inch per hour), including a 1 -hour fall of 0.160 inch. On the same day, the pressure rose 0.554 inch during a 6-hour period ( 0.090 inch per hour), including a 1-hour rise of 0.145 inch. The greatest sea-level pressure change during a 1-day period was 1.02 inches (December 8, 1971). 
Table 6.6. Average and Extreme Station and Sea-Level Pressure Data, 1955 Through 1999 (inches of mercury)

\begin{tabular}{|c|c|c|c|c|c|c|c|c|c|c|}
\hline \multirow[b]{2}{*}{ Month } & \multicolumn{6}{|c|}{ Station Pressure } & \multicolumn{4}{|c|}{ Sea-Level Pressure } \\
\hline & Average & High & Year & Low & Year & $\begin{array}{c}\text { Greatest Daily } \\
\text { Range } \\
\end{array}$ & High & Year & Low & Year \\
\hline Jan & 29.31 & 30.23 & $1979^{(2)}$ & 28.18 & 1980 & 0.77 & 31.12 & 1979 & 28.94 & 1964 \\
\hline Feb & 29.27 & 30.08 & 1956 & 28.23 & $1958^{(2)}$ & 0.79 & 30.97 & $1956^{(2)}$ & 28.98 & $1958^{(n)}$ \\
\hline Mar & 29.20 & 29.92 & 1955 & 28.34 & 1995 & 0.85 & 30.79 & 1955 & 29.11 & 1995 \\
\hline Apr & 29.19 & 29.91 & 1999 & 28.49 & $1962^{(a)}$ & 0.81 & 30.73 & 1999 & 29.26 & 1962 \\
\hline May & 29.16 & 29.68 & $1970^{(2)}$ & 28.61 & 1999 & 0.46 & 30.48 & $1970^{(a)}$ & 29.38 & $1957^{(4)}$ \\
\hline Jun & 29.13 & 29.60 & $1987^{(2)}$ & 28.67 & $1992^{(a)}$ & 0.54 & 30.40 & 1987 & 29.42 & 1992 \\
\hline Jul & 29.14 & 29.56 & $1993^{(a)}$ & 28.80 & $1979^{(2)}$ & 0.48 & 30.34 & $1993^{(2)}$ & 29.55 & $1979^{(2)}$ \\
\hline Aug & 29.14 & 29.55 & 1968 & 28.75 & 1980 & 0.39 & 30.32 & 1968 & 29.52 & 1980 \\
\hline Sep & 29.18 & 29.79 & $1983^{(2)}$ & 28.48 & $1986^{(2)}$ & 0.56 & 30.60 & 1983 & 29.25 & 1986 \\
\hline Nov & 29.28 & 30.06 & $1979^{(a)}$ & 28.36 & $1982^{(a)}$ & 0.78 & 30.90 & $1979^{(a)}$ & 29.13 & 1982 \\
\hline Dec & 29.32 & 30.20 & 1978 & 28.16 & 1995 & 1.02 & 31.07 & 1978 & 28.94 & $1995^{(a)}$ \\
\hline Annual & 29.22 & 30.23 & $\operatorname{Jan} 1979^{(2)}$ & 28.16 & Dec 1995 & 1.02 & 31.12 & Jan 1979 & 28.94 & Dec $1995^{(a)}$ \\
\hline
\end{tabular}

(a) Most recent of several occurrences. 


\subsection{Extreme Values}

Extreme values are generally described in terms of probability of occurrence or in terms of return period. For low probability events, the return period is simply the reciprocal of the probability when the probability is expressed as the likelihood of the event occurring in a given year. As with all estimate extreme values, the uncertainty in the estimates increases as the return period increases.

\subsection{Annual Temperature Extremes}

Annual maximum and minimum temperatures with return periods from 2 to 1,000 years are listed in Table 7.1. The probabilities of exceeding various maximum and minimum temperatures are shown in Figures 7.1 and 7.2 along with the maximum and minimum temperatures observed at the Hanford Meteorology Station from 1945 through 1998. The curves were estimated by assuming that the annual extreme temperatures may be fit using a normal distribution and calculating distribution parameters from the observed data.

\subsection{Precipitation Rates}

Maximum precipitation rates for return periods of 2 to 1,000 years are listed in Table 7.2. The corresponding precipitation amounts are listed in Table 7.3. The precipitation rate estimates are based on precipitation measurements made at the Hanford Meteorology Station from 1947 through 1998. The precipitation rates were estimated for each return period assuming a lognormal distribution and distribution parameters calculated from the data. Figure 7.3 shows the predicted rates for $1,3,6$, and 12 hours duration along with the observed data.

Table 7.1. Return Periods for Annual Maximum and Minimum Temperatures

\begin{tabular}{|c|c|c|}
\hline $\begin{array}{l}\text { Return Period } \\
\text { (years) }\end{array}$ & $\begin{array}{c}\text { Maximum Temperature } \\
\left({ }^{\circ} \mathrm{F}\right)\end{array}$ & $\begin{array}{c}\text { Minimum Temperature } \\
\left({ }^{\circ} \mathrm{F}\right)\end{array}$ \\
\hline 2 & 106.1 & -0.9 \\
\hline 5 & 107.3 & -9.2 \\
\hline 10 & 108.7 & -13.5 \\
\hline 20 & 110.1 & -17.1 \\
\hline 50 & 111.2 & -21.2 \\
\hline 100 & 113.3 & -23.9 \\
\hline 200 & 114.0 & -26.3 \\
\hline 500 & 115.0 & -29.3 \\
\hline 1000 & 115.6 & -31.4 \\
\hline
\end{tabular}




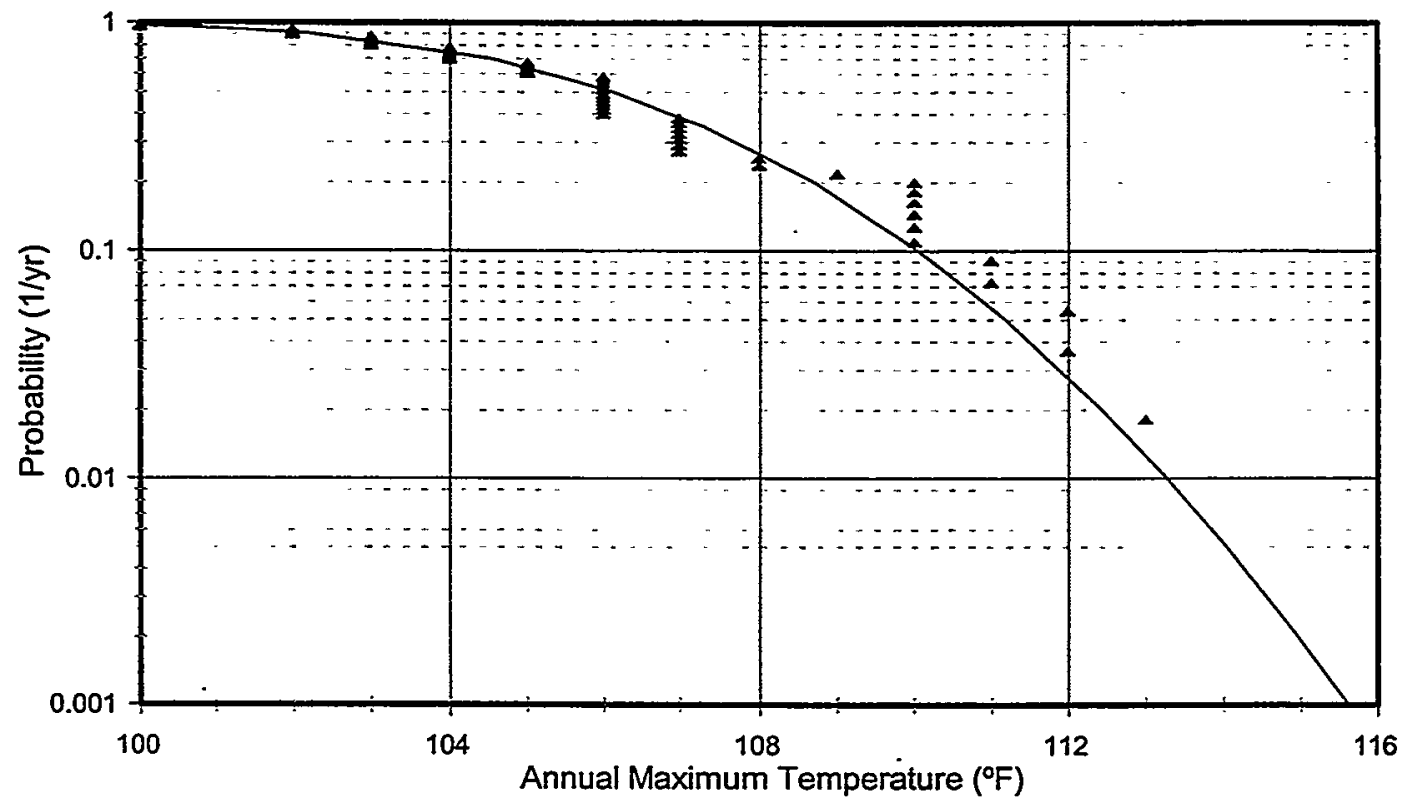

Figure 7.1. Probability of an Annual Maximum Temperature Exceeding a Given Value

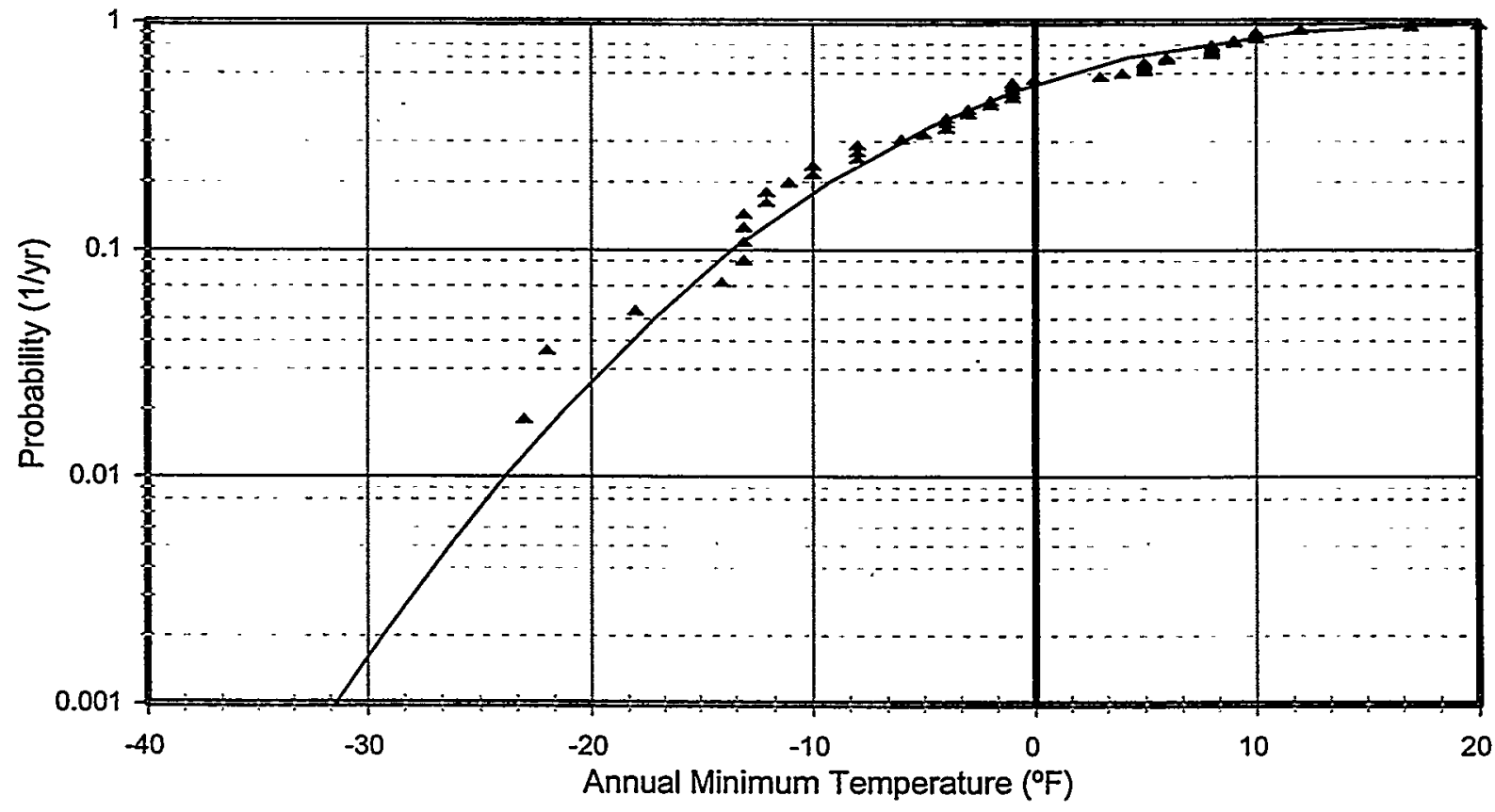

Figure 7.2. Probability of an Annual Minimum Temperature Being Less Than a Given Value 
Table 7.2. Precipitation Rates (inches per hour) for 1 to 24 Hours Duration and Return Periods from 2 to 1,000 Years

\begin{tabular}{|c|c|c|c|c|c|c|}
\hline \multirow{2}{*}{$\begin{array}{l}\text { Return Period } \\
\text { (years) }\end{array}$} & \multicolumn{6}{|c|}{ Duration } \\
\hline & 1 hour & 2 hours & 3 hours & 6 hours & 12 hours & 24 hours \\
\hline 2 & 0.22 & 0.15 & 0.12 & 0.081 & 0.051 & 0.029 \\
\hline 5 & 0.31 & 0.21 & 0.16 & 0.11 & 0.067 & 0.039 \\
\hline 10 & 0.38 & 0.24 & 0.18 & 0.12 & 0.078 & 0.046 \\
\hline 20 & 0.44 & 0.28 & 0.20 & 0.14 & 0.089 & 0.052 \\
\hline 50 & 0.52 & 0.32 & 0.23 & 0.16 & 0.10 & 0.061 \\
\hline 100 & 0.58 & 0.36 & 0.25 & 0.18 & 0.11 & 0.0067 \\
\hline 200 & 0.65 & 0.39 & 0.27 & 0.19 & 0.12 & 0.073 \\
\hline 500 & 0.73 & 0.43 & 0.30 & 0.21 & 0.14 & 0.081 \\
\hline 1,000 & 0.80 & 0.47 & 0.32 & 0.22 & 0.15 & 0.088 \\
\hline
\end{tabular}

Table 7.3. Precipitation Amounts (inches) for 1 to 24 Hours in Periods and Return Periods from 2 to 1,000 Years

\begin{tabular}{|c|c|c|c|c|c|c|}
\hline \multirow{2}{*}{$\begin{array}{l}\text { Return Period } \\
\text { (years) }\end{array}$} & \multicolumn{6}{|c|}{ Duration } \\
\hline & 1 hour & 2 hours & 3 hours & 6 hours & 12 hours & 24 hours \\
\hline 2 & 0.22 & 0.31 & 0.36 & 0.48 & 0.61 & 0.70 \\
\hline 5 & 0.31 & 0.42 & 0.47 & 0.64 & 0.81 & 0.95 \\
\hline 10 & 0.38 & 0.49 & 0.54 & 0.74 & 0.94 & 1.11 \\
\hline 20 & 0.44 & 0.56 & 0.61 & 0.84 & 1.07 & 1.26 \\
\hline 50 & 0.52 & 0.65 & 0.69 & 0.96 & 1.23 & 1.46 \\
\hline 100 & 0.58 & 0.71 & 0.75 & 1.05 & 1.35 & 1.61 \\
\hline 200 & 0.65 & 0.78 & 0.81 & 1.14 & 1.47 & 1.75 \\
\hline 500 & 0.73 & 0.87 & 0.89 & 1.26 & 1.63 & 1.95 \\
\hline 1,000 & 0.80 & 0.94 & 0.95 & 1.35 & 1.75 & 2.11 \\
\hline
\end{tabular}

\subsection{Snow}

Snow extremes for return periods from 2 to 1,000 years are listed in Table 7.4. The estimates are based on data from the Hanford Meteorology Station for the 1946-1947 through 1997-1998 snow seasons. The values in the tables were estimated assuming a Type 1 (Gumbel) extreme value distribution (Johnson et al. 1995) using maximum-likelihood estimates (Kinnison 1985) of the distribution parameter values calculated from the Hanford Meteorology Station data. Figures 7.4, 7.5, and 7.6 show the probabilities of seasonal maximum snowfall, maximum single storm snowfall, and maximum snow depth, respectively with the corresponding Hanford Meteorology Station data. 


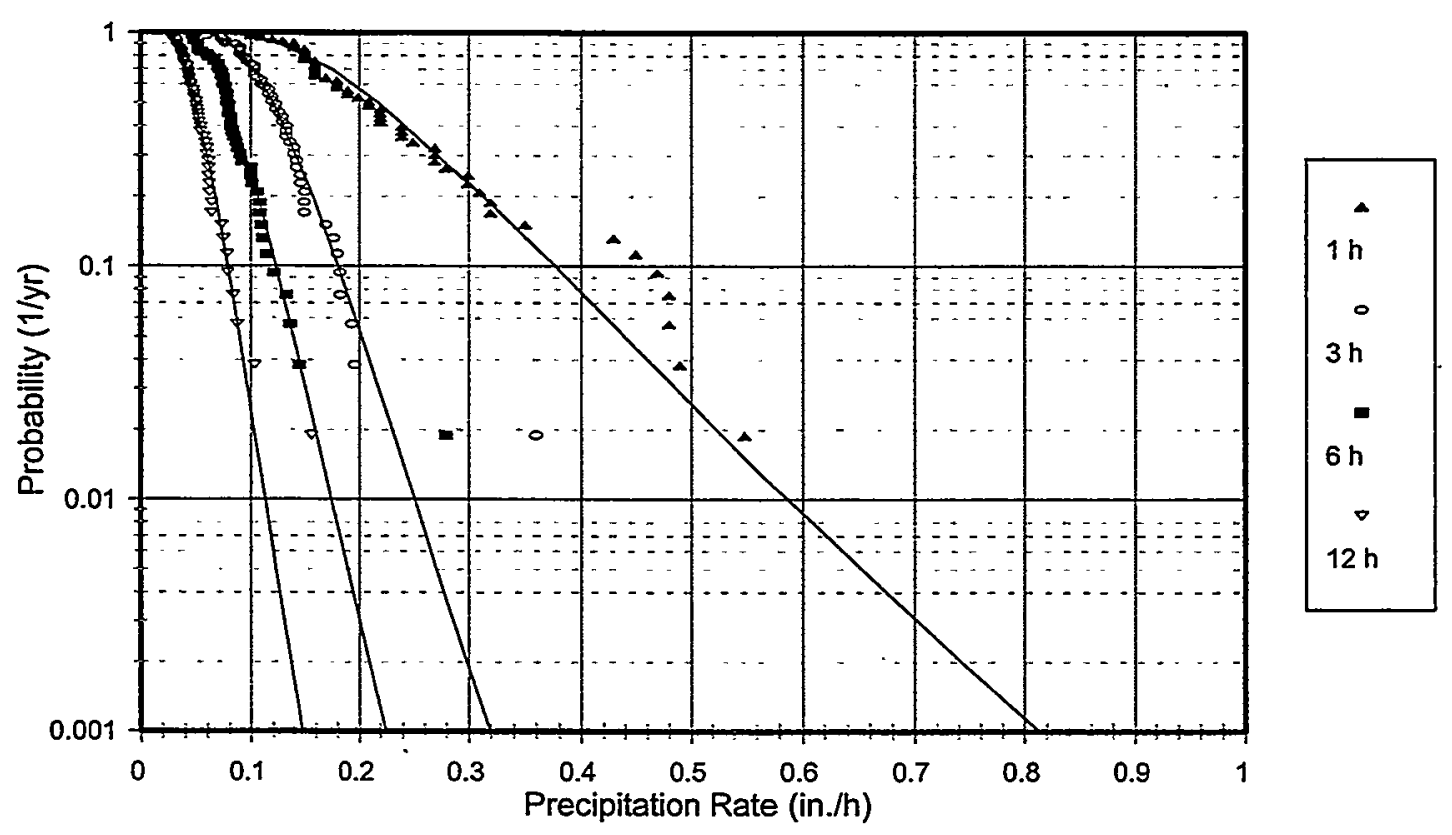

Figure 7.3. Probability of Precipitation Rate Exceeding Given Values by Duration

Table 7.4. Snowfall Extremes for Return Periods from 2 to 1,000 Years

\begin{tabular}{|c|c|c|c|}
\hline $\begin{array}{l}\text { Return Period } \\
\text { (years) }\end{array}$ & $\begin{array}{l}\text { Seasonal Total } \\
\text { (inches) }\end{array}$ & $\begin{array}{l}\text { Single Storm } \\
\text { (inches) }\end{array}$ & $\begin{array}{l}\text { Maximum on } \\
\text { Ground (inches) }\end{array}$ \\
\hline 2 & 13.6 & 3.8 & 5.1 \\
\hline 5 & 23.3 & 6.0 & 8.2 \\
\hline 10 & 29.6 & 7.5 & 10.3 \\
\hline 20 & 35.7 & 8.9 & 12.3 \\
\hline 50 & 43.7 & 10.7 & 14.9 \\
\hline 100 & 49.6 & 12.1 & 16.9 \\
\hline 200 & 55.5 & 13.4 & 18.8 \\
\hline 500 & 63.3 & 15.2 & 21.4 \\
\hline 1,000 & 69.2 & 16.5 & 23.3 \\
\hline
\end{tabular}




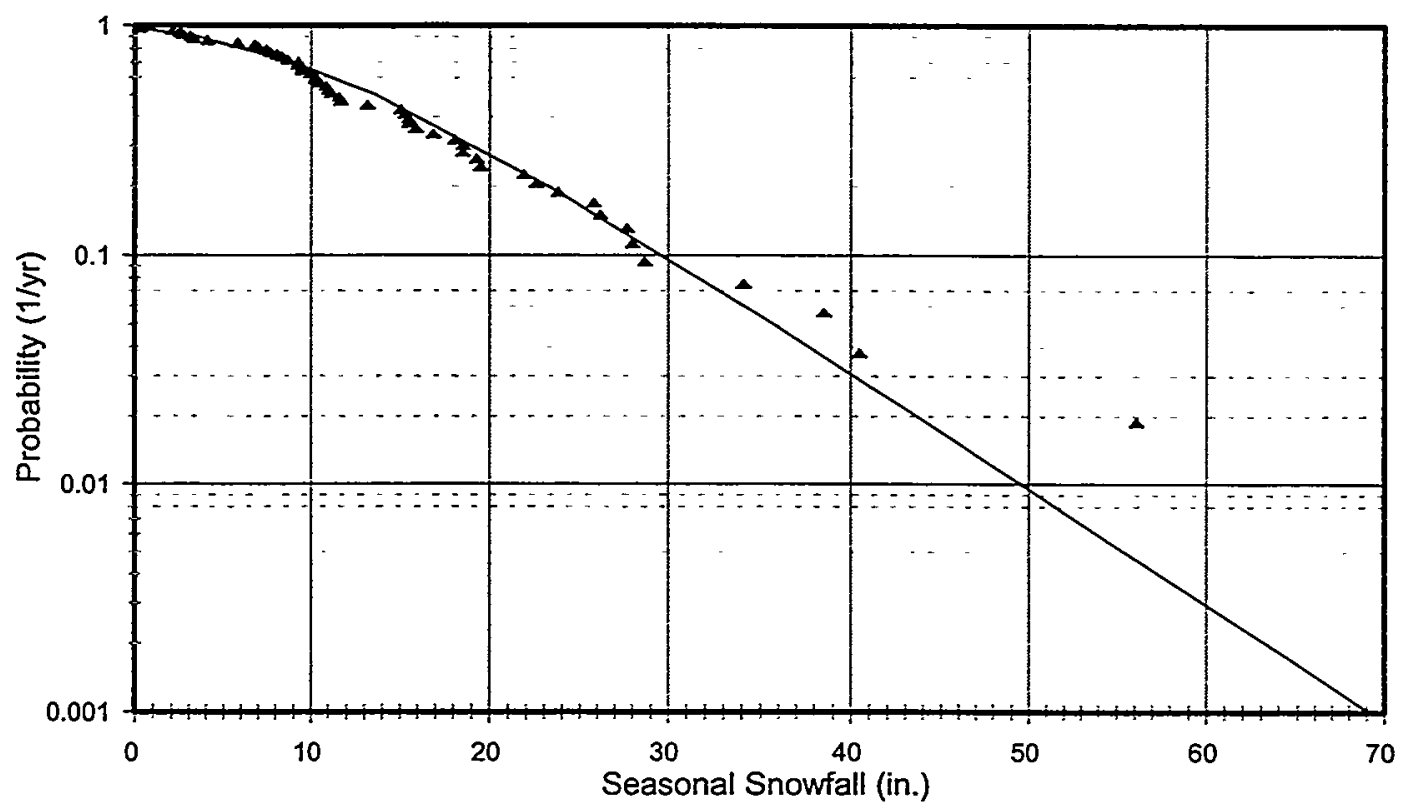

Figure 7.4. Probability of Exceeding a Given Seasonal Snowfall

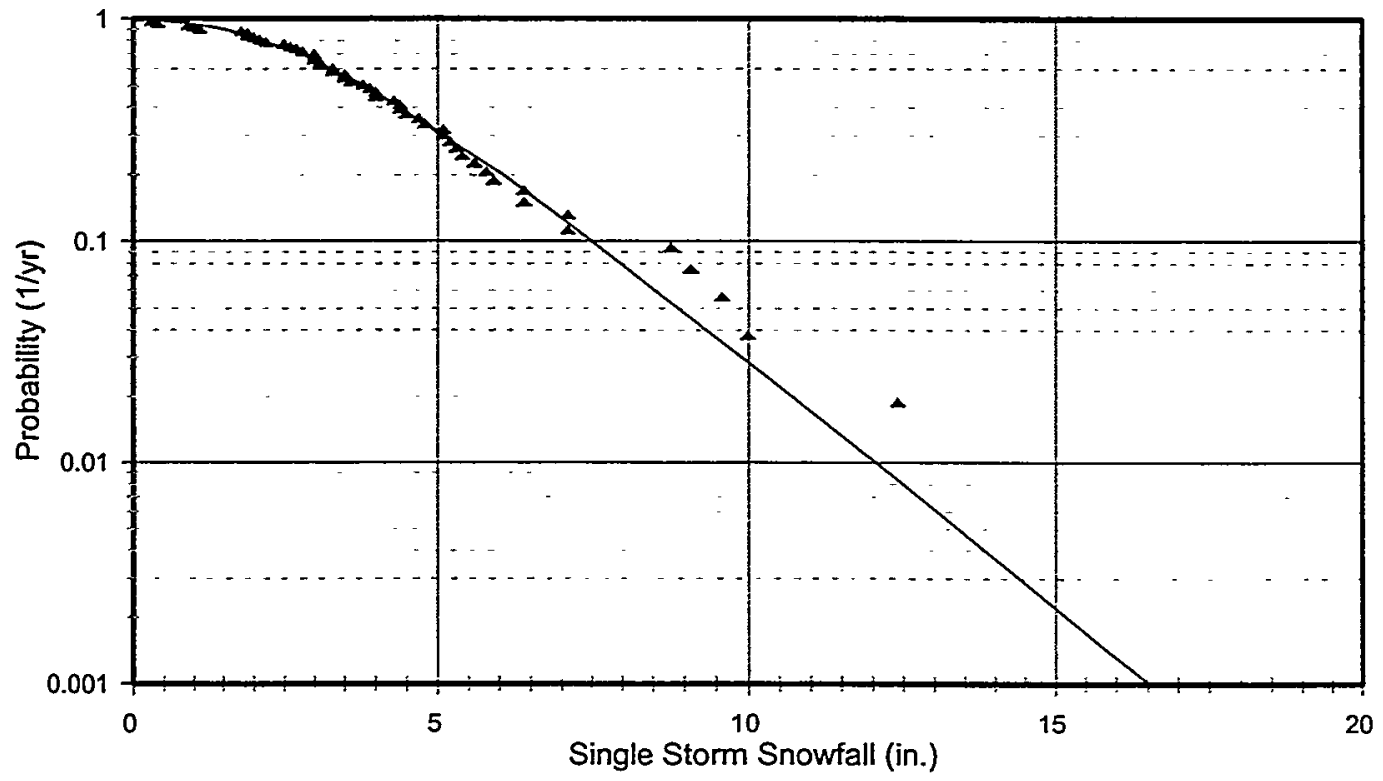

Figure 7.5. Probability of Exceeding a Given Snowfall in a Single Storm

\subsection{Peak Wind Gusts}

Peak wind gusts for return periods of 2 to 10,000 years are listed in Table 7.5 for heights of $30.5,50$, 200 , and 400 feet above ground. The peak wind gust estimates are based on wind measurements made at the 50, 200, 400-feet levels of the tower at the Hanford Meteorology Station. The peak wind gusts for 


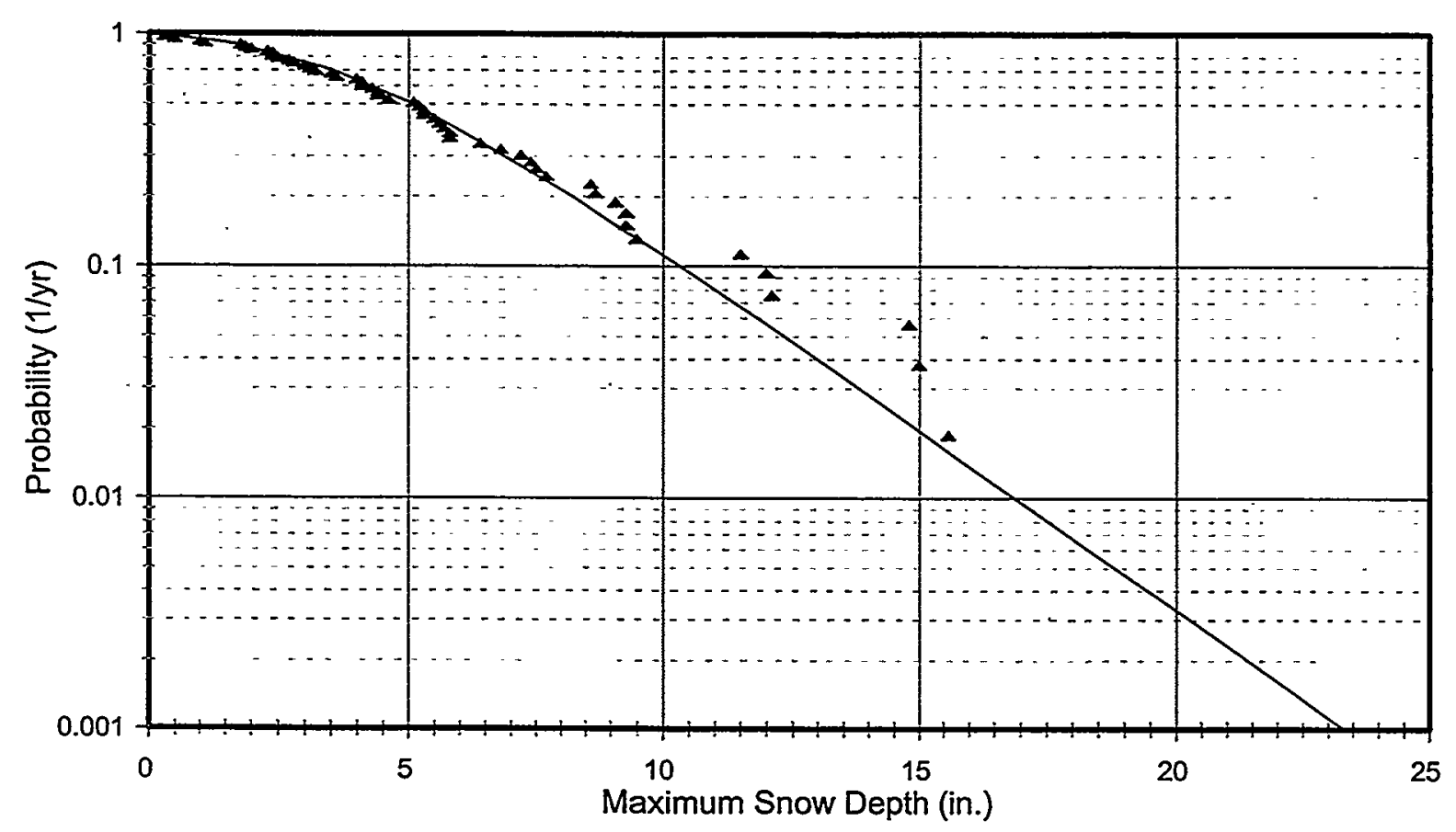

Figure 7.6. Probability of Exceeding a Given Snow Depth

Table 7.5. Peak Wind Gusts (mph) for Return Periods from 2 to 10,000 Years

\begin{tabular}{|c|c|c|c|c|}
\hline \multirow{2}{*}{$\begin{array}{l}\text { Return Period } \\
\text { (years) }\end{array}$} & \multicolumn{4}{|c|}{ Height Above Ground } \\
\hline & 10 meters & 50 feet & 200 feet & 400 feet \\
\hline 2 & 57.4 & 60.2 & 67.5 & 71.6 \\
\hline 5 & 63.6 & 66.7 & 75.3 & 80.5 \\
\hline 10 & 67.8 & 71.0 & 80.5 & 86.4 \\
\hline 20 & 71.7 & 75.2 & 85.4 & 92.1 \\
\hline 50 & 76.9 & 80.5 & 91.8 & 99.4 \\
\hline 100 & 80.7 & 84.6 & 96.6 & 104.8 \\
\hline 200 & 84.5 & 88.6 & 101.4 & 110.3 \\
\hline 500 & 89.6 & 93.9 & 107.7 & 117.5 \\
\hline 1,000 & 93.4 & 97.9 & 112.4 & 122.9 \\
\hline 2,000 & 97.2 & 101.9 & 117.2 & 128.4 \\
\hline 5,000 & 102.2 & 107.1 & 123.5 & 135.6 \\
\hline 10,000 & 106.1 & 111.1 & 128.2 & 141.0 \\
\hline
\end{tabular}

each return period for these levels were estimated assuming a Type 1 extreme value distribution and maximum likelihood distribution parameters calculated from the Hanford Meteorology Station data. The peak wind gusts for the 30.5-feet (10-meters) level were made by first adjusting the peak gusts observed 
at 50 feet to 30.5 feet using the technique described by Peterka and Shahid (1998) and then calculating the distribution parameters using maximum likelihood techniques. Figure 7.7 shows the probabilities of peak wind gusts at all four levels along with the Hanford Meteorology Station peak wind gust data for 50, 200, and 400 feet.

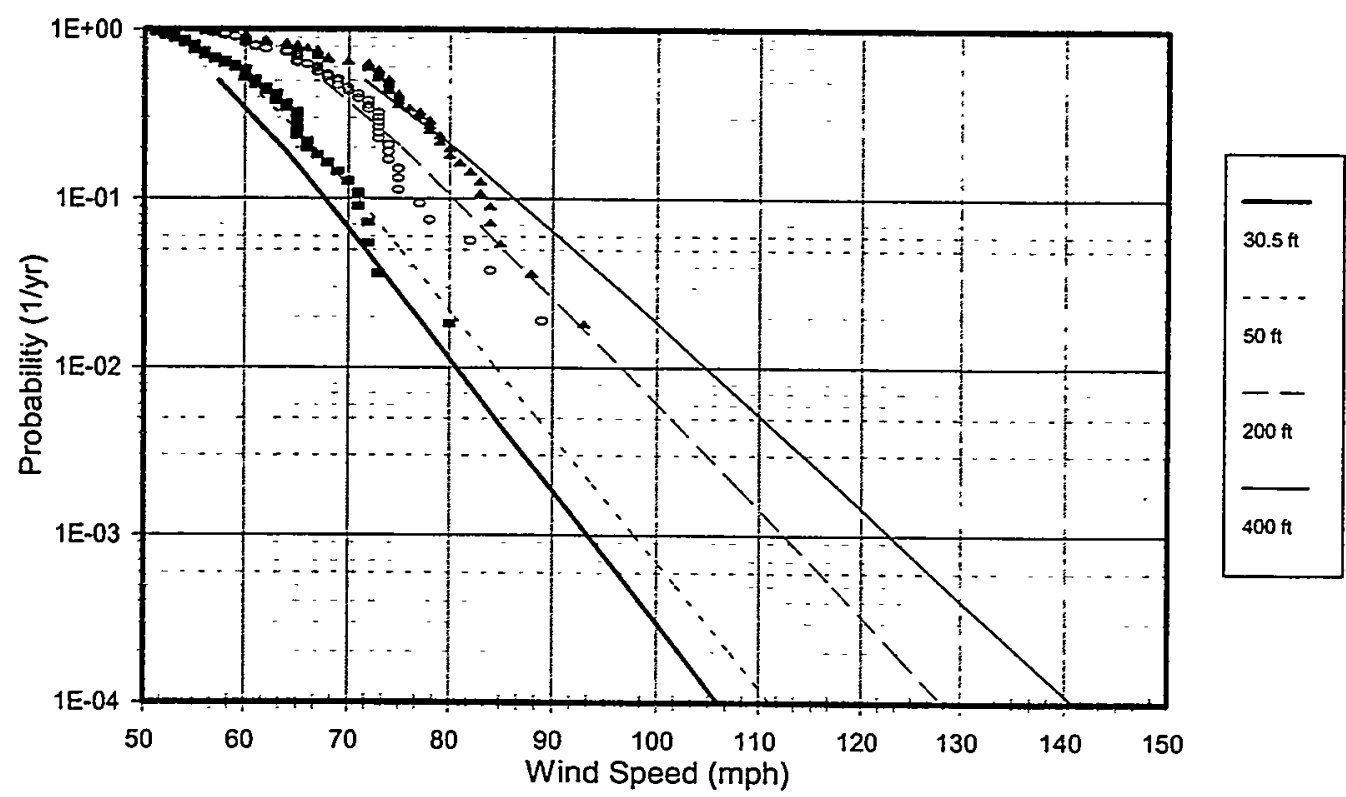

Figure 7.7. Probabilities of Peak Wind Gusts Exceeding Given Values 


\subsection{References}

Glantz, C. S., and M. M. Islam. 1988. The Data Collection Component of the Hanford Meteorology Monitoring Program. PNL-6684, Pacific Northwest Laboratory, Richland, Washington.

Hoitink, D. J., and K. W. Burk. 1994. Climatological Data Summary 1993, with Historical Data. PNL-9809, Pacific Northwest Laboratory, Richland, Washington.

Hoitink, D. J., and K. W. Burk. 1995. Climatological Data Summary 1994, with Historical Data. PNL-10553, Pacific Northwest Laboratory, Richland, Washington.

Hoitink, D. J., and K. W. Burk. 1996. Climatological Data Summary 1995, with Historical Data. PNNL-11107, Pacific Northwest National Laboratory, Richland, Washington.

Hoitink, D. J., and K. W. Burk. 1997. Climatological Data Summary 1996, with Historical Data. PNNL-11471, Pacific Northwest National Laboratory, Richland, Washington.

Hoitink, D. J., and K. W. Burk. 1998. Climatological Data Summary 1997, with Historical Data. PNNL-11794, Pacific Northwest National Laboratory, Richland, Washington.

Hoitink, D. J., K. W. Burk, and J. V. Ramsdell, Jr. 1999. Climatological Data Summary 1998, with Historical Data. PNNL-12087. Pacific Northwest National Laboratory, Richland, Washington.

Jenne, D. E., and R. E. Kems. 1959. A Climatological Study of the Hanford Area. HW-57722, General Electric, Hanford Atomic Products Operation, Richland, Washington.

Johnson, N. L., S. Kotz, and N. Balakrishnan. 1995. Continuous Univariate Distributions, Vol. 2, John Wiley \& Sons, New York.

Kinnison, R. R. 1985. Applied Extreme Value Statistics. Macmillan, New York.

Personnel from Pacific Northwest National Laboratory, Fluor Daniel Hanford, Inc., Waste Management Federal Services of Hanford, Inc., and Bechtel Hanford, Inc. for U.S. Department of Energy. 1997. Environmental Monitoring Plan, United States Department of Energy, Richland Operation Office. DOE/RL-91-50, Rev. 2. United States Department of Energy, Richland, Washington.

Peterka, J. A., and S. Shahid. 1998. "Design Gust Wind Speeds in the United States." Journal of Structural Engineering, 124:207-214.

Stone, W. A., D. E. Jenne, and J. M. Thorp. 1972. Climatography of the Hanford Area. BNWL-1605, Pacific Northwest Laboratory, Richland, Washington.

Stone, W. A., J. M. Thorp, O. P. Gifford, and D. J. Hoitink. 1983. Climatological Summary for the Hanford Area. PNL-4622, Pacific Northwest Laboratory, Richland, Washington. 
Appendix

1999 Wind Climatology 


\section{Appendix}

\section{Wind Climatology}

This appendix provides the 1999 station-specific wind roses (Figures A.1[a] and A.2[a]) from the Hanford Meteorological Monitoring Network. Each petal of the wind rose represents the proportional amount of time that the wind blew from that direction. The width of the petal corresponds to each wind speed category. Starting from the center of the rose, the narrowest petal represents winds in the 1- to 3-mph class, the next widest represents the 4- to 7-mph class, and so forth. The length of each of these petals is proportional to the frequency of occurrence for each speed class.

The wind speed histograms (Figures A.1[b] and A.2[b]) represent the proportional amount of time in each speed class.

Table A.1 lists joint frequency distributions (at 10 meters) of wind direction versus wind speed class for the individual stations (see Figure 2.1 in text for locations). Table A.2 lists joint frequency distributions (at 60 meters) for stations $9,11,13$, and 21 (see Figure 2.2 in text for locations). 


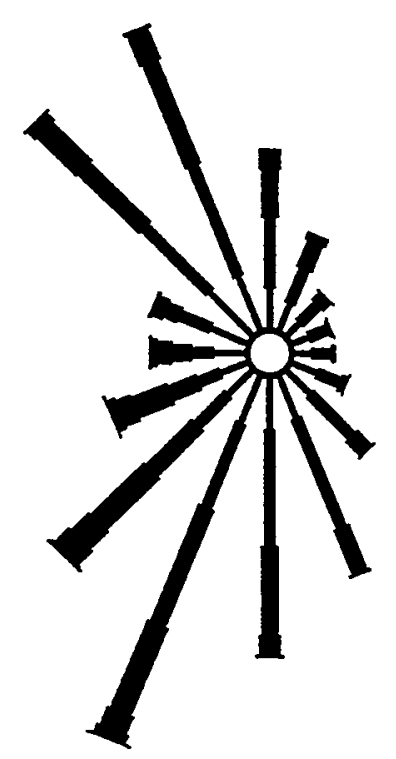

(a) Wind Rose

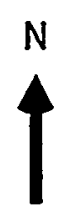

Station \# 1 - PROS

Period: $1 / 99-12 / 99$

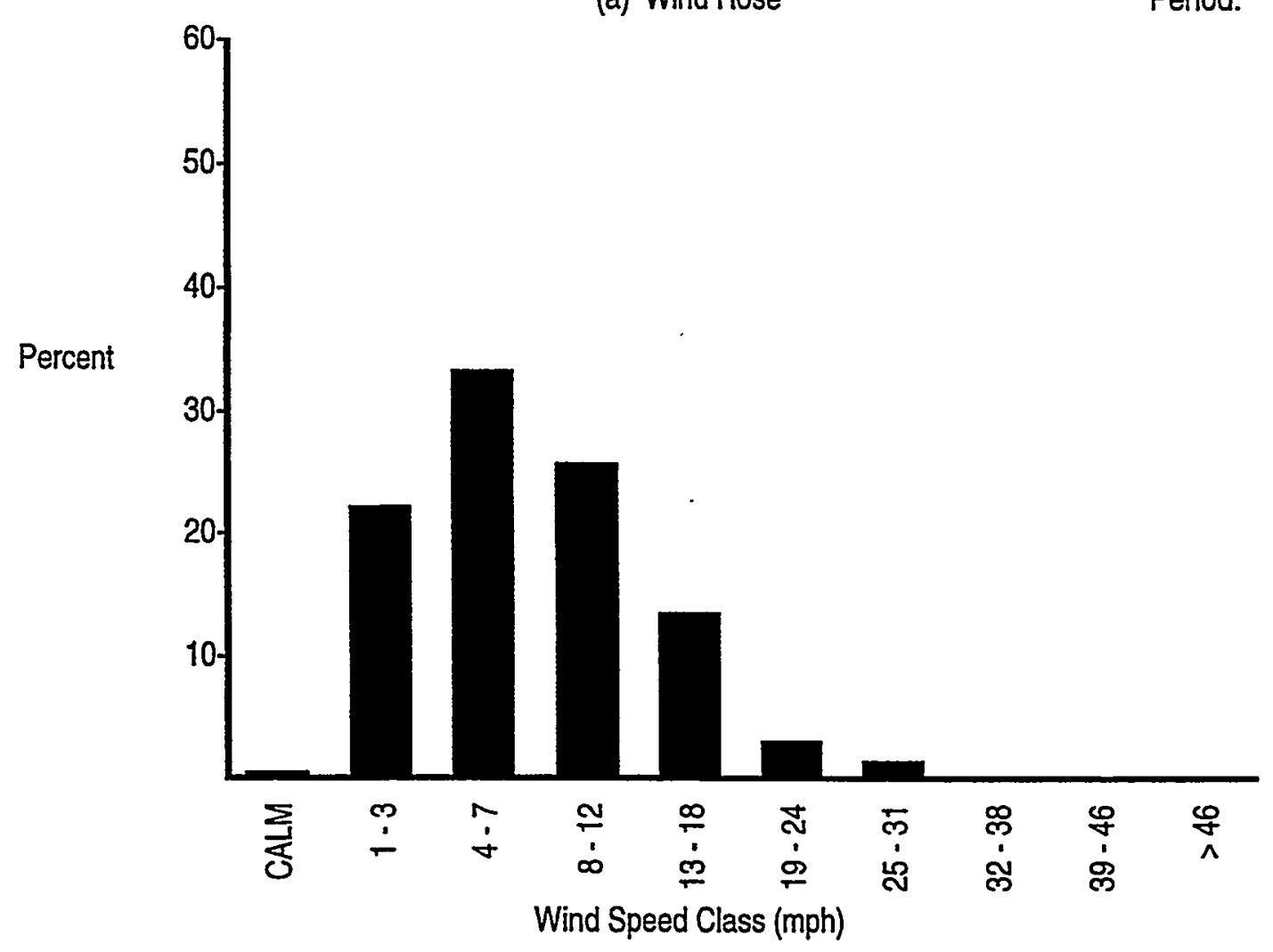

(b) Wind Speed Histogram

Figure A.1. Wind Rose and Wind Speed Histogram, 10 Meters 

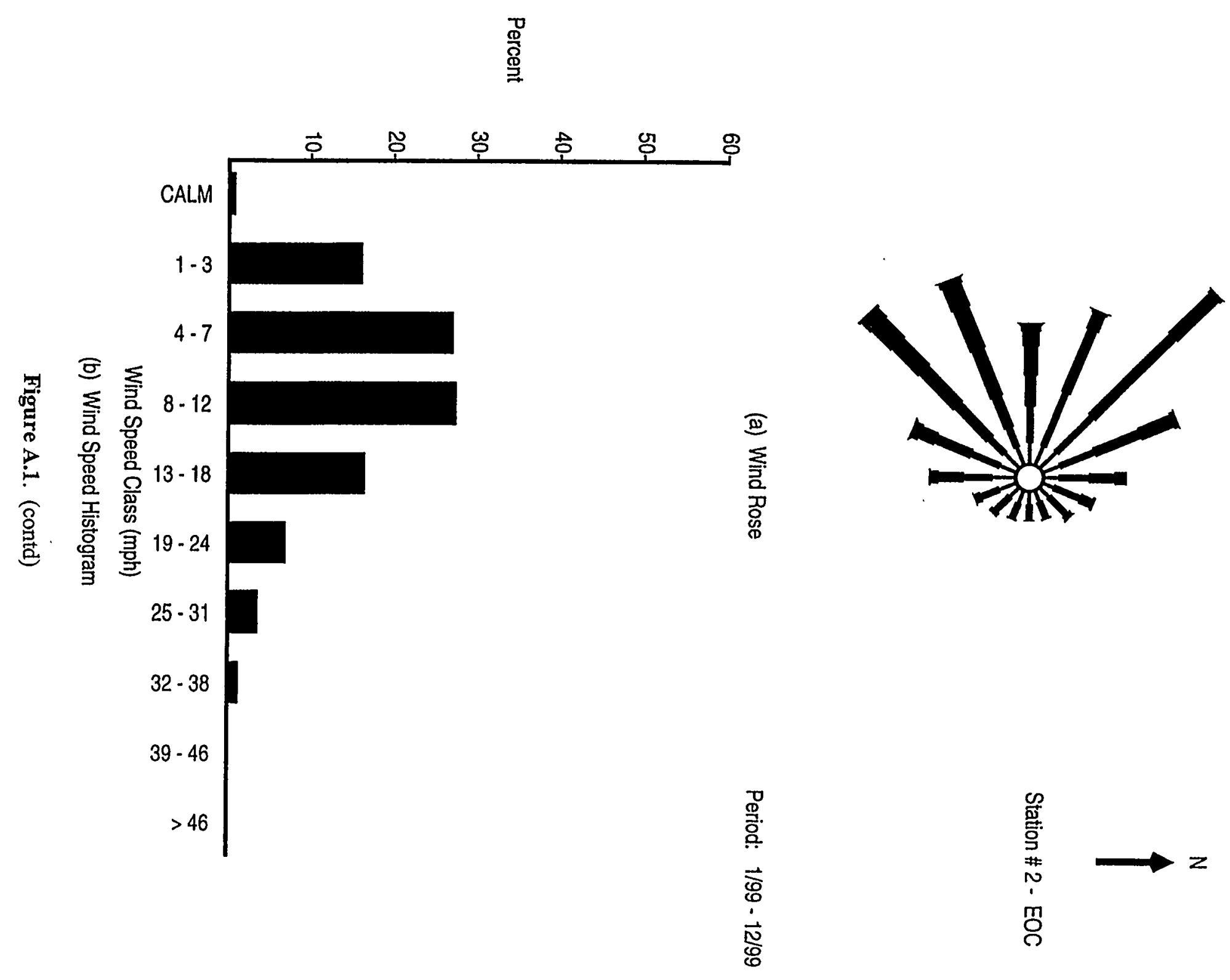

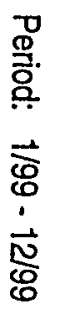

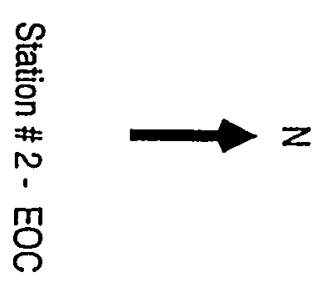




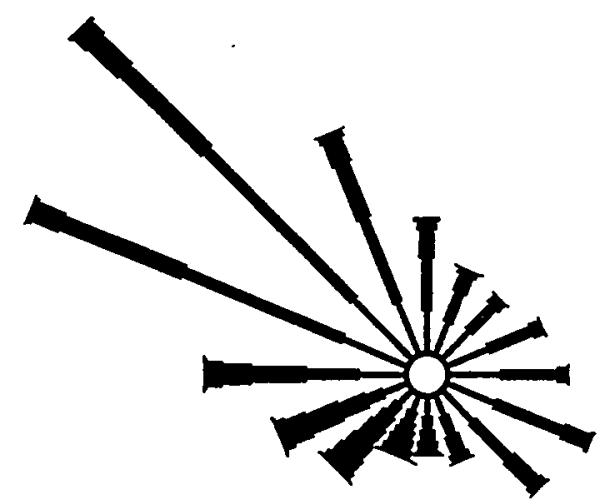

(a) Wind Rose

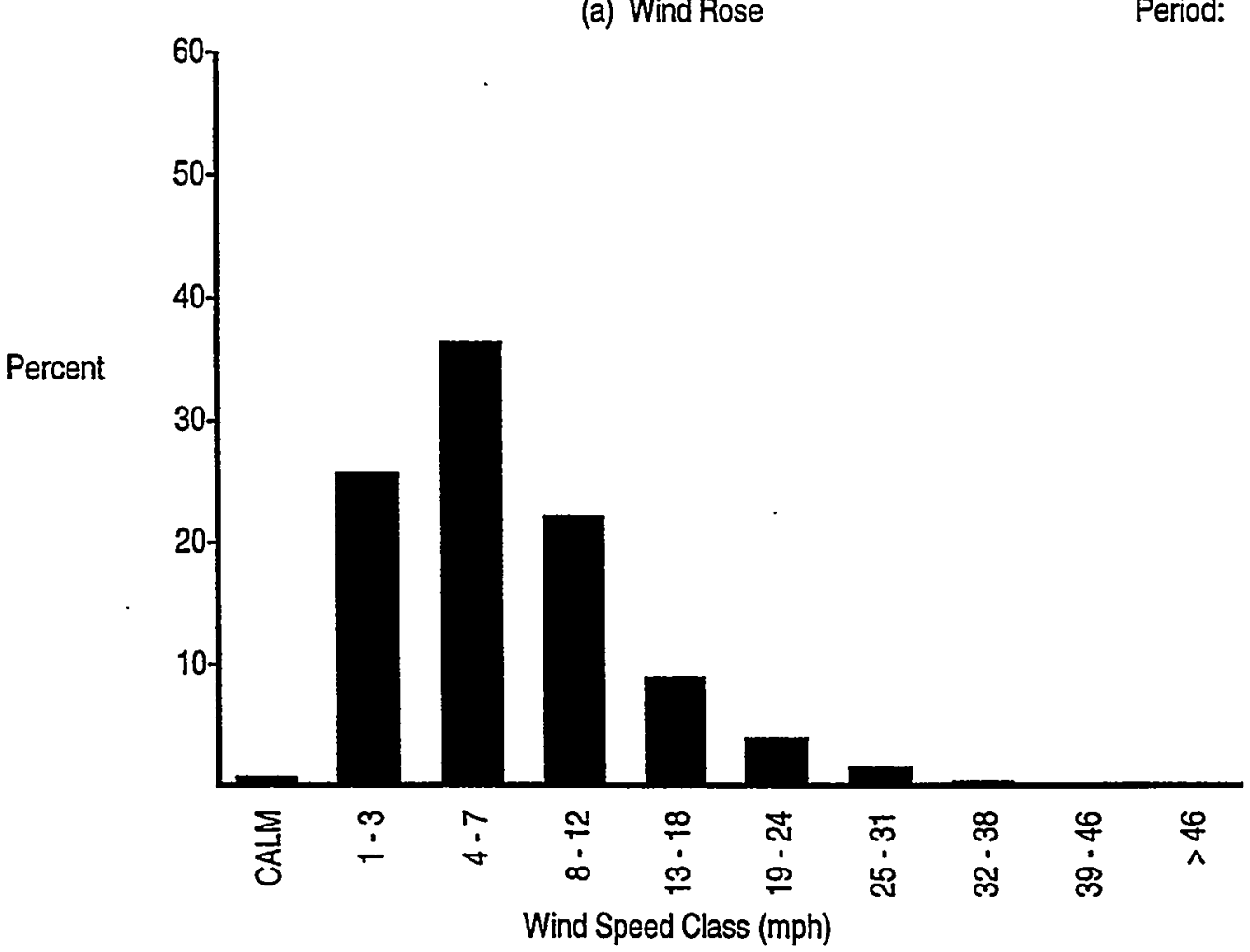

(b) Wind Speed Histogram

Figure A.1. (contd)

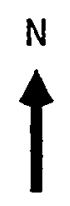

Station \# 3 - ARMY

Period: $1 / 99-12 / 99$ 

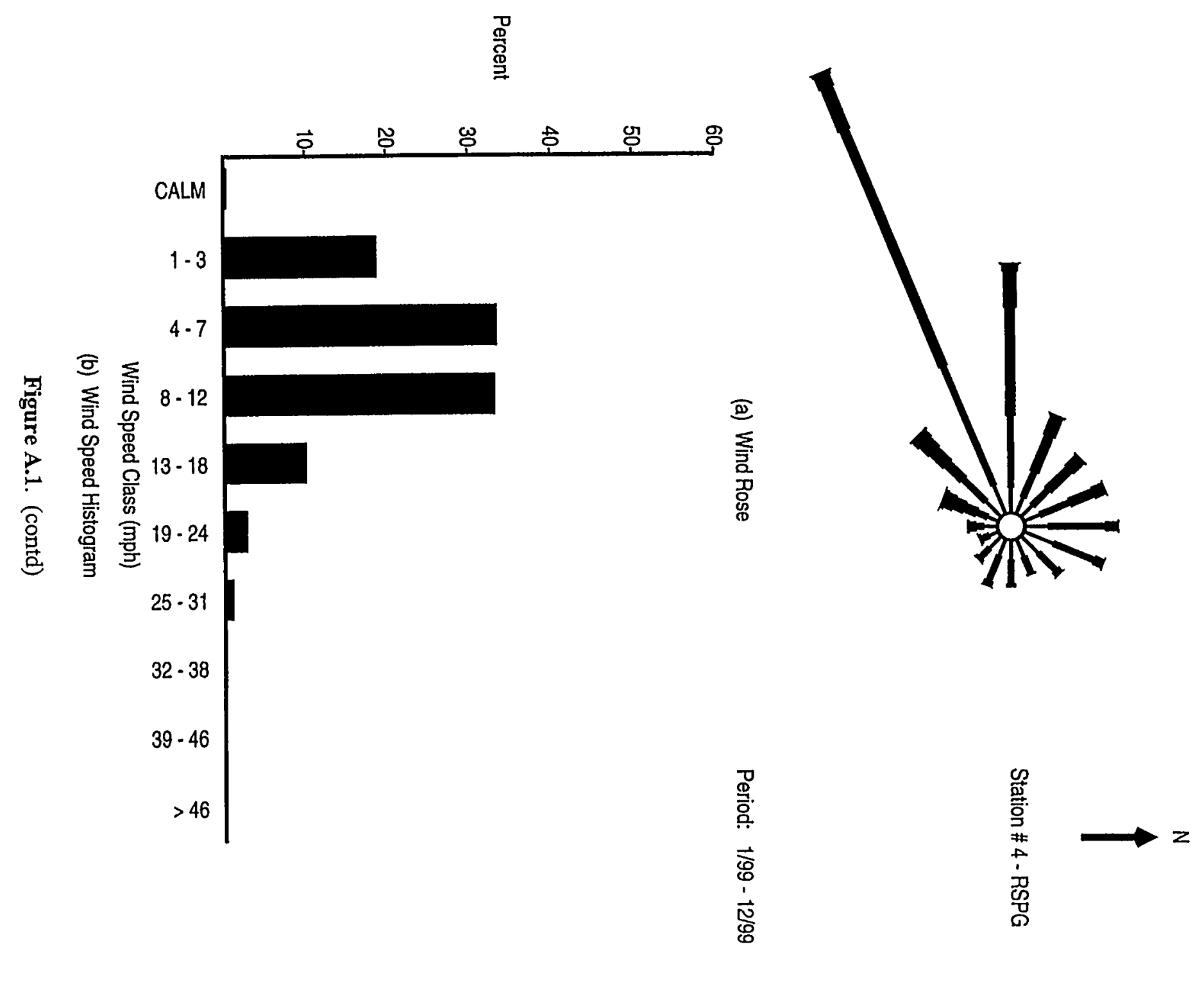


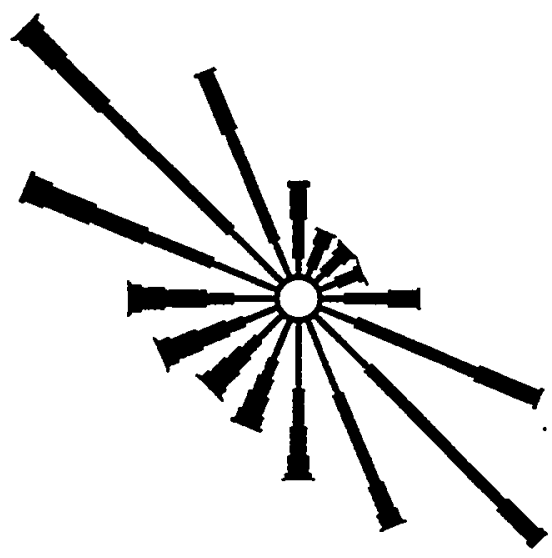

(a) Wind Rose

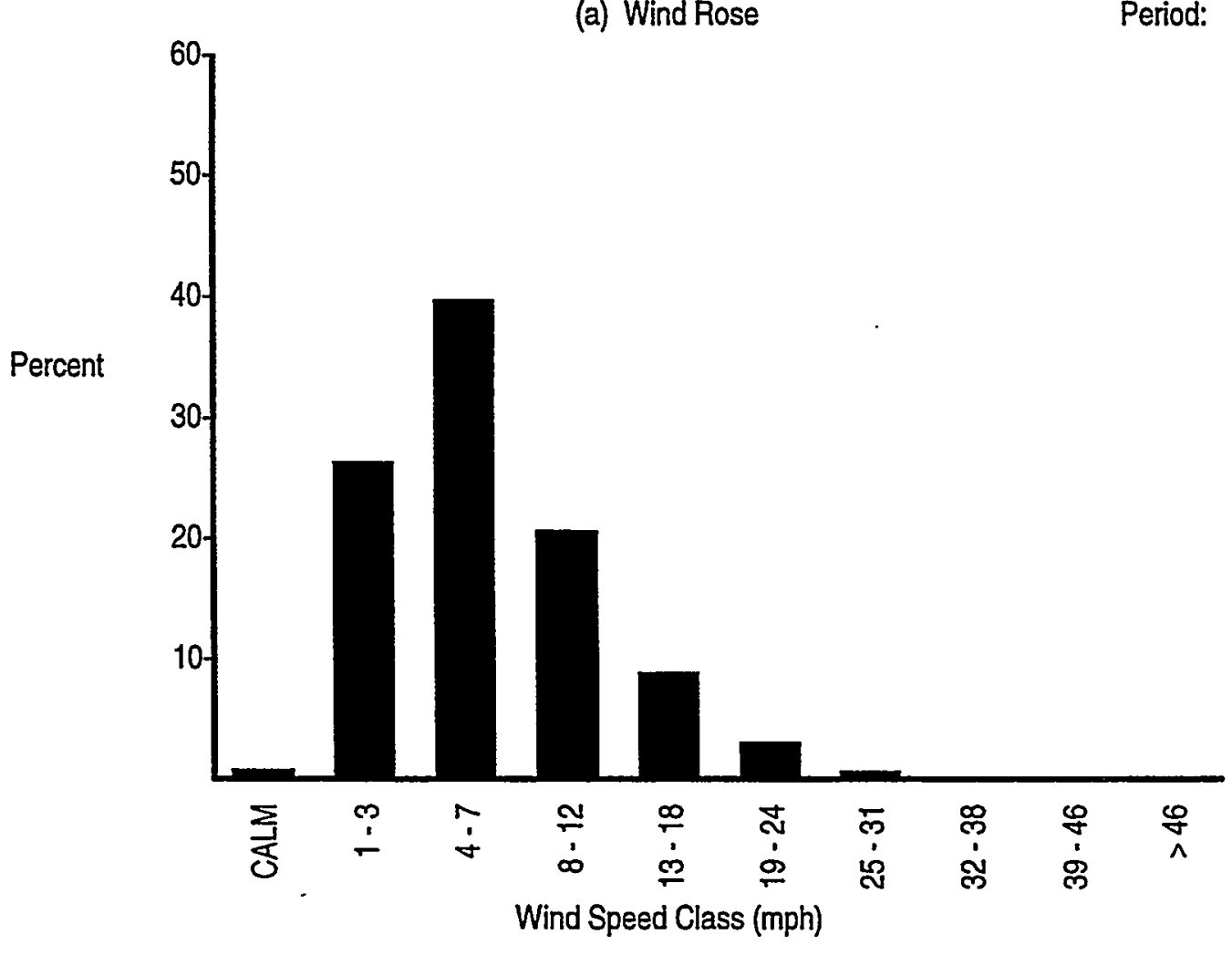

(b) Wind Speed Histogram

- Figure A.1. (contd)

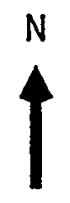

Station \# 5 - EDNA

Period: 1/99-12/99 

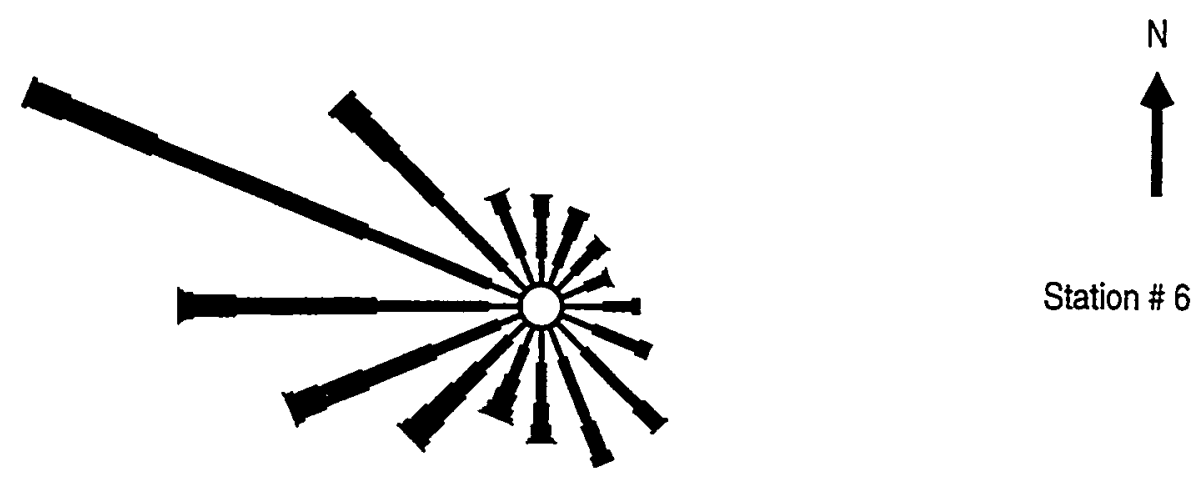

Station \# 6 - 200E

(a) Wind Rose

Period: $1 / 99-12 / 99$

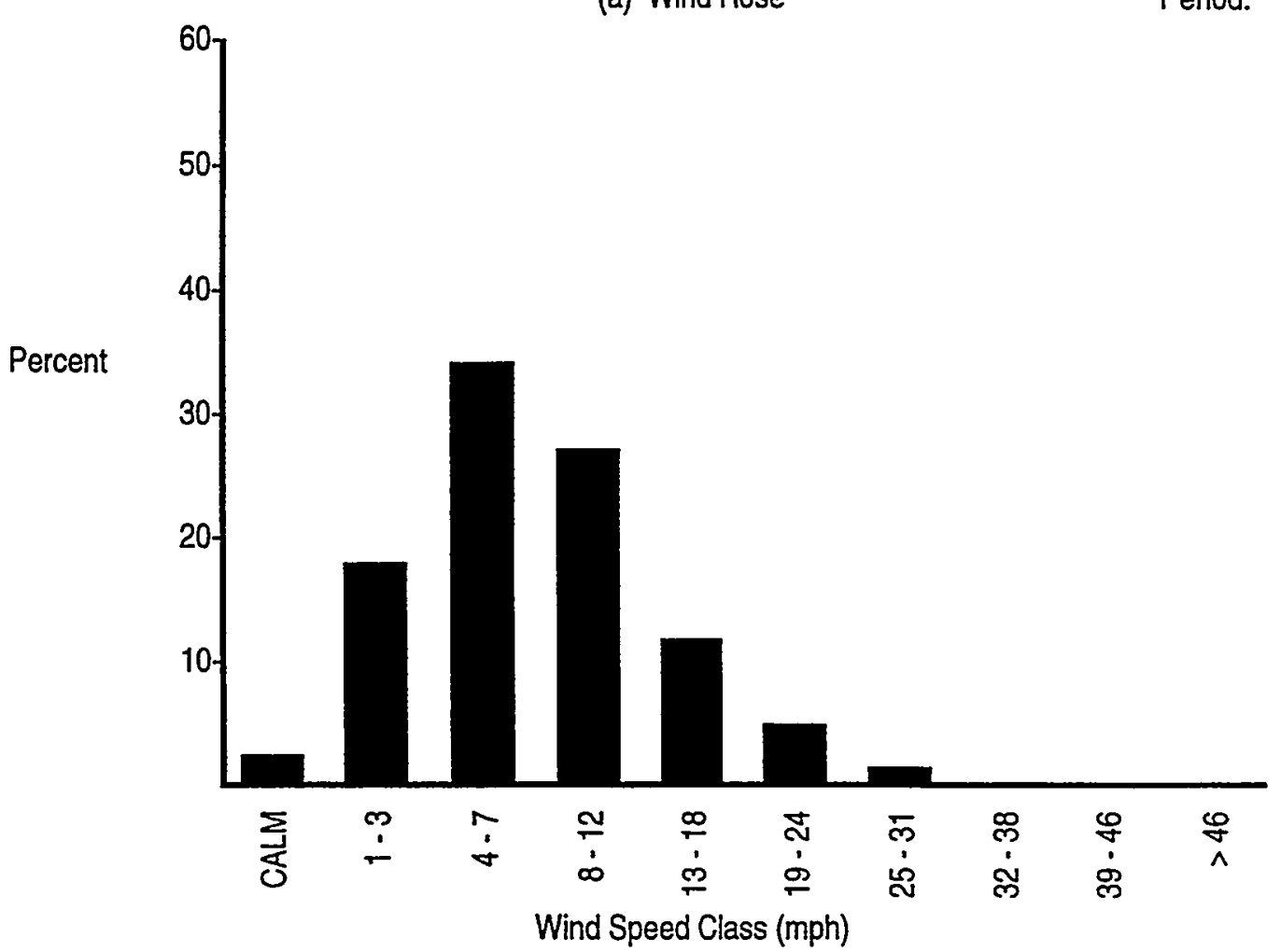

(b) Wind Speed Histogram

Figure A.1. (contd) 

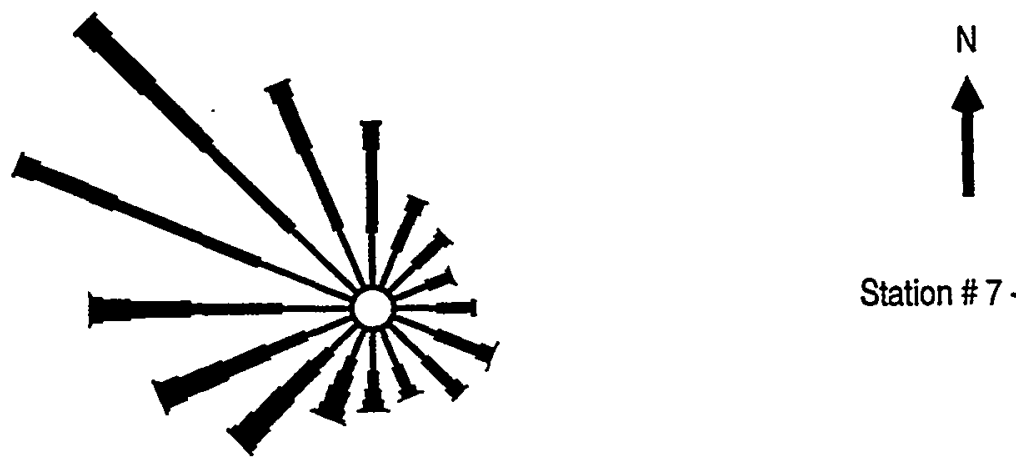

Station \# 7-200W

(a) Wind Rose

Period: $1 / 99-12 / 99$

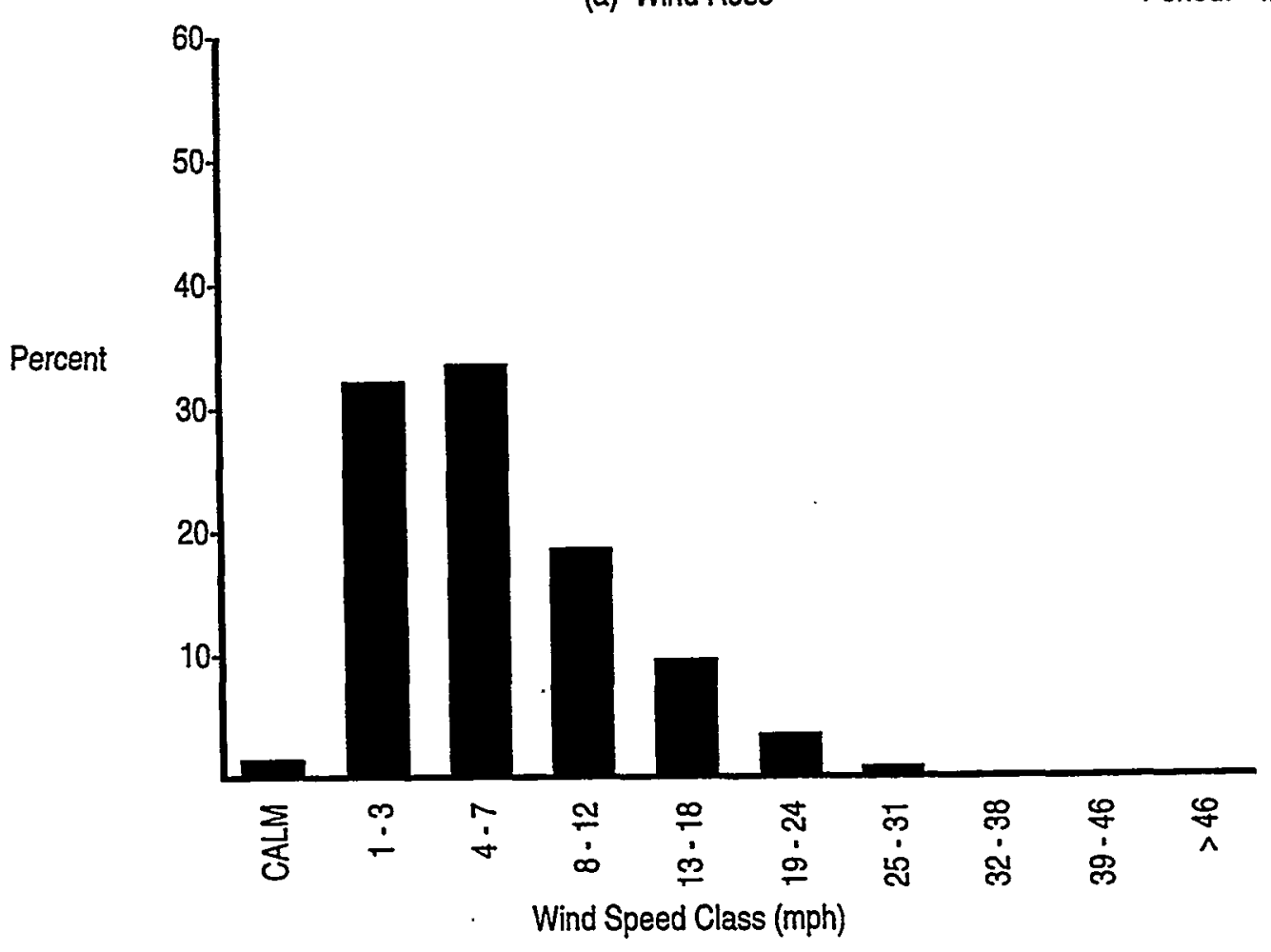

(b) Wind Speed Histogram

Figure A.1. (contd) 

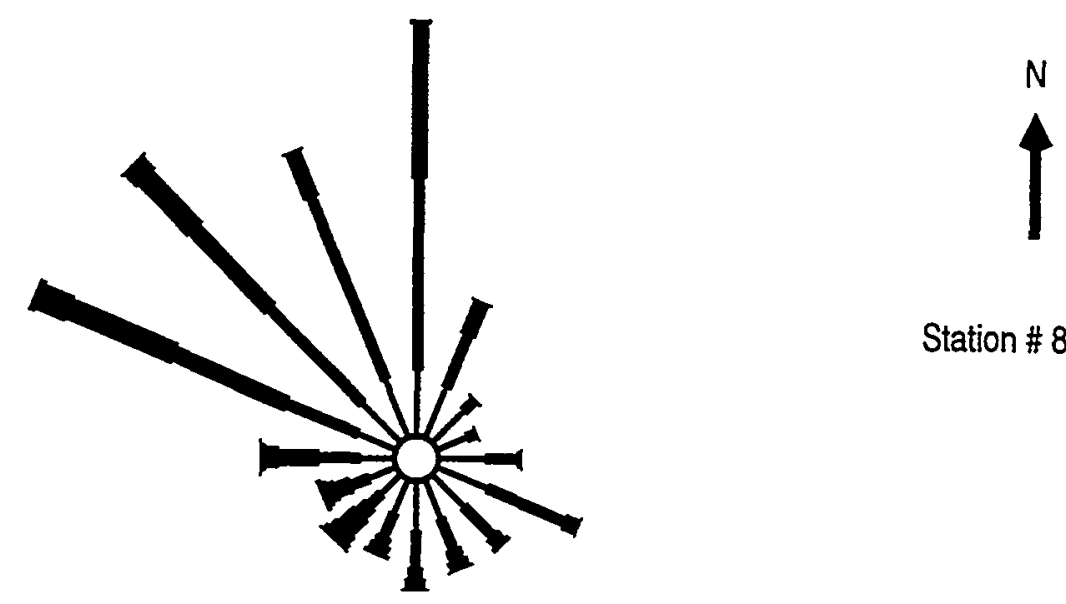

Station \# 8 - BVLY

(a) Wind Rose

Period: $1 / 99-12 / 99$

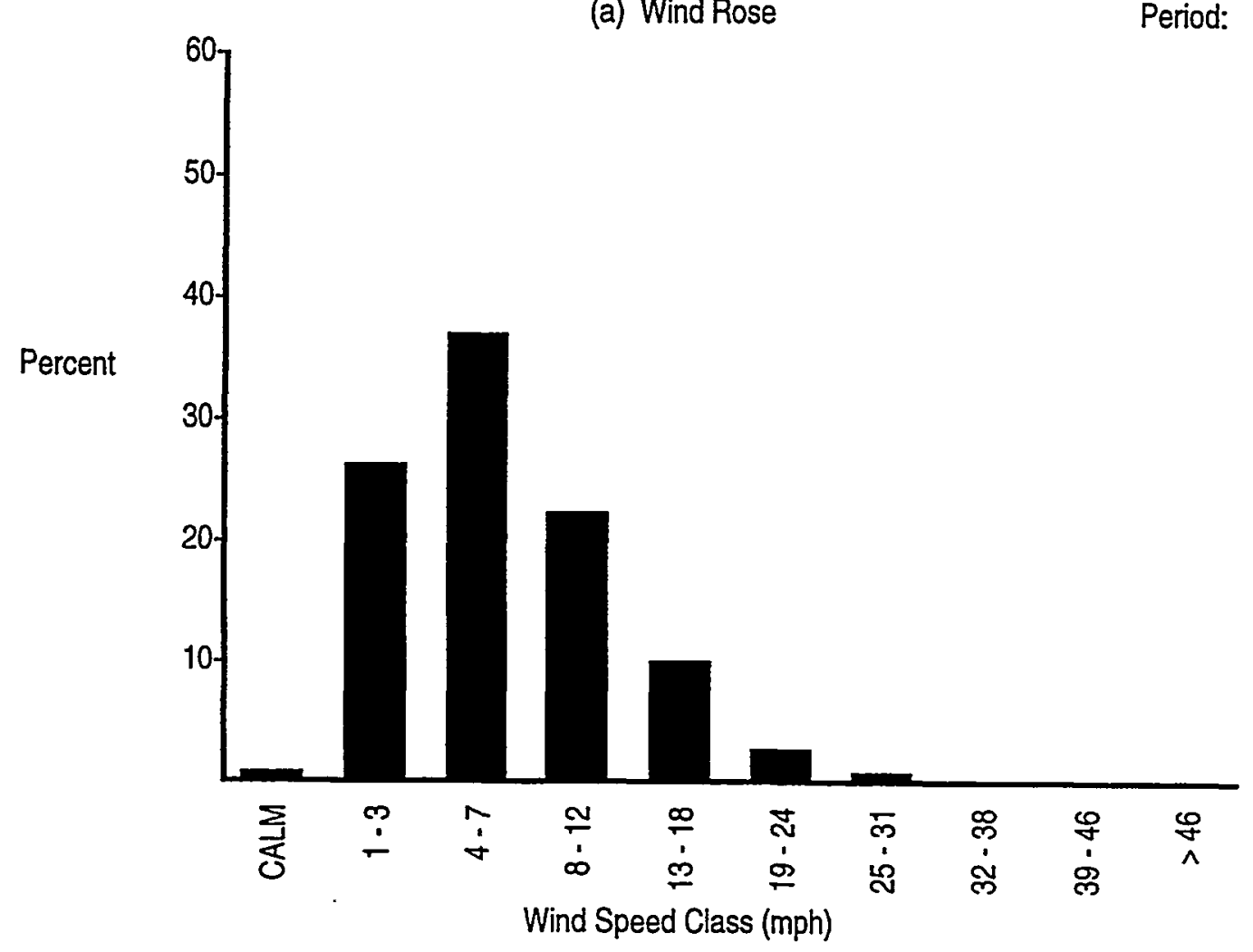

(b) Wind Speed Histogram

Figure A.1. (contd) 


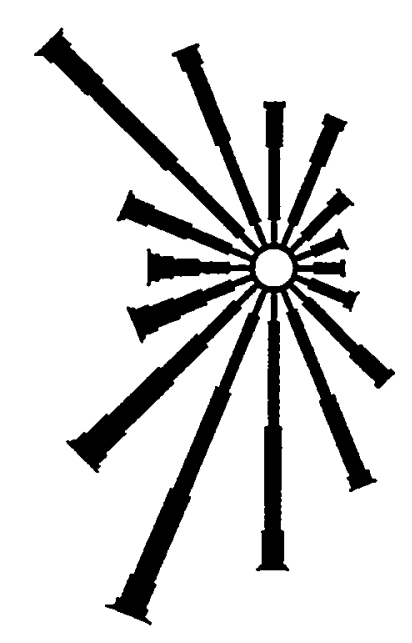

(a) Wind Rose

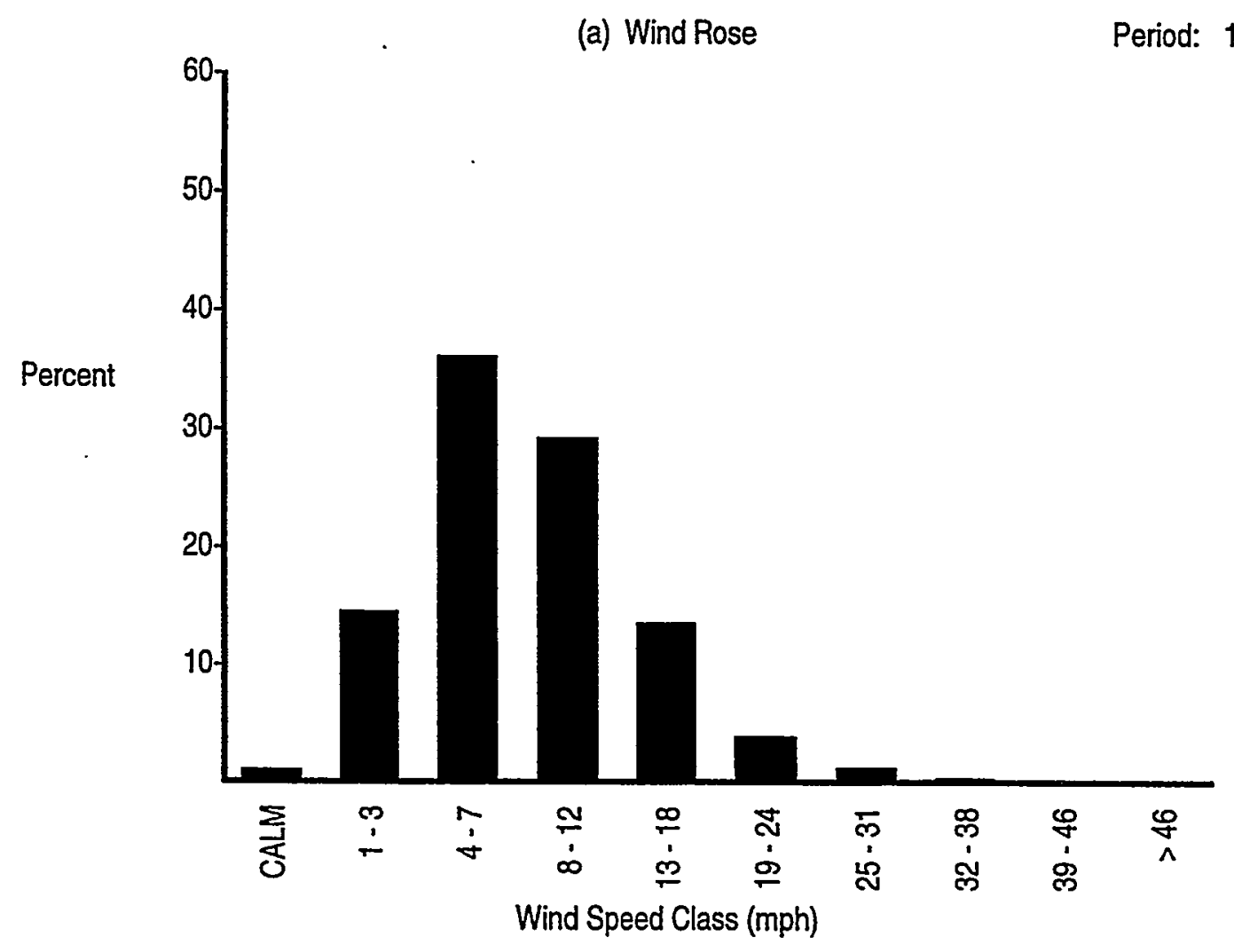

(b) Wind Speed Histogram

Figure A.1. (contd) 

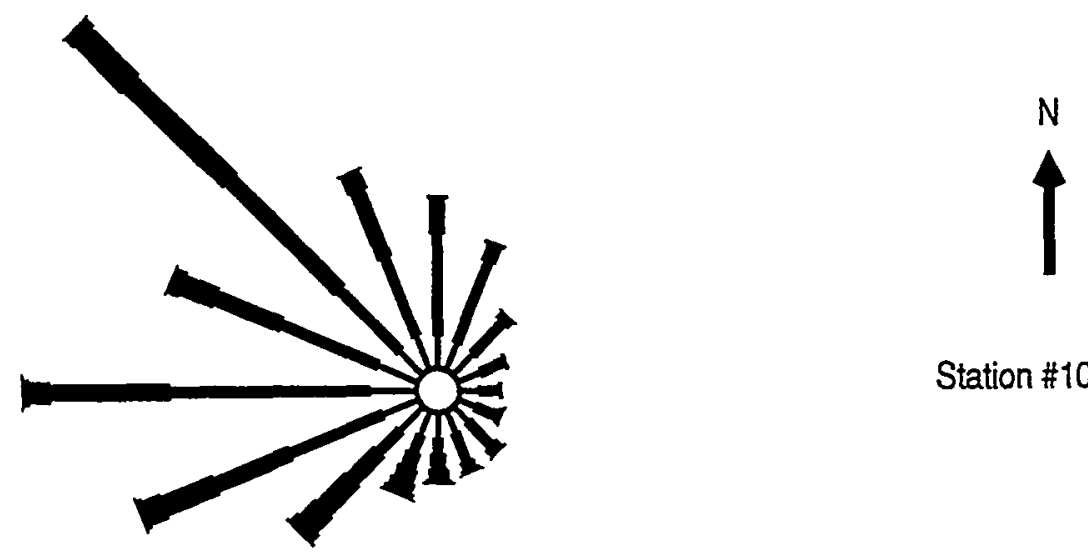

Station \#10 - YAKB

(a) Wind Rose

Period: $1 / 99-12 / 99$

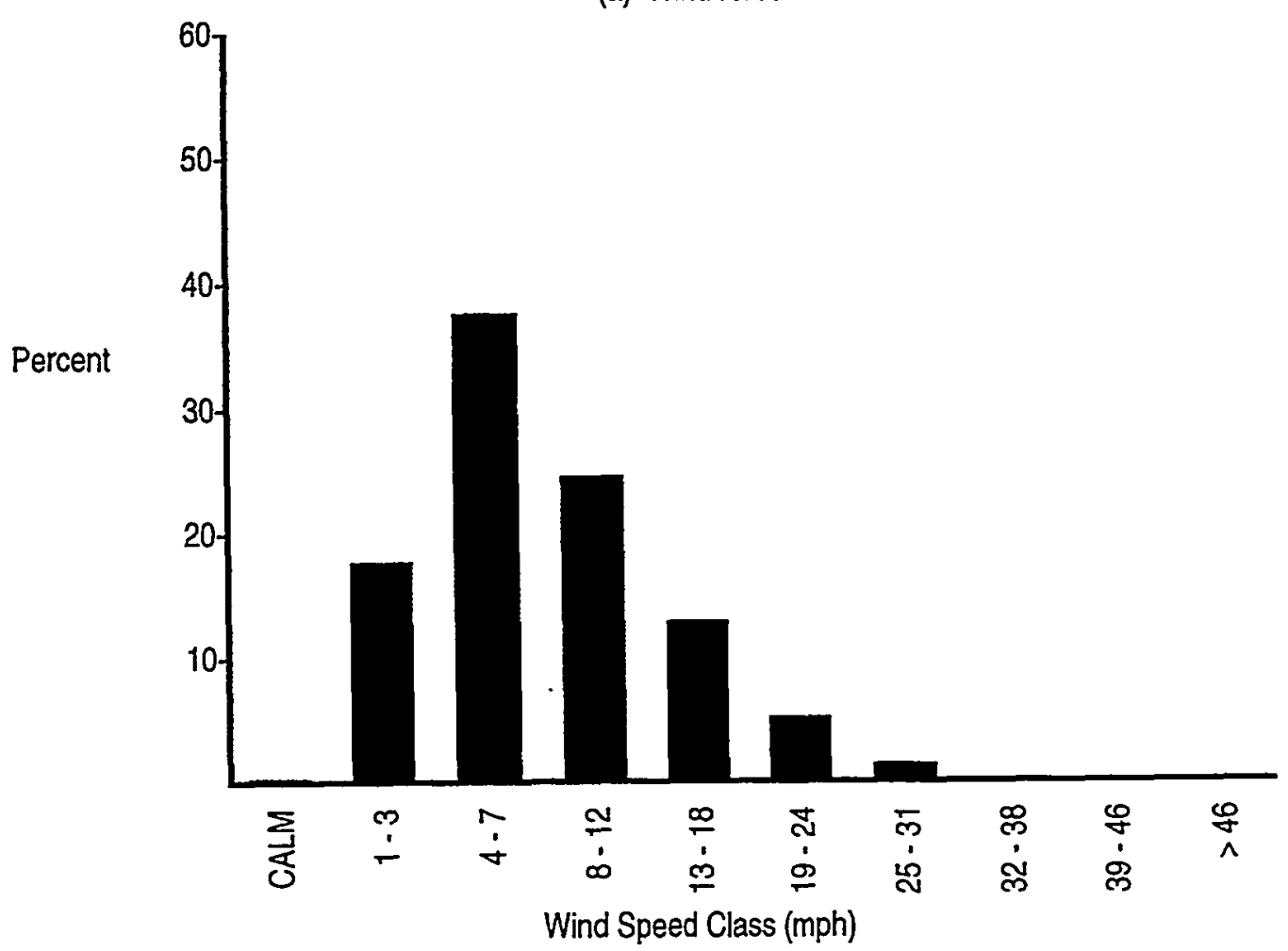

(b) Wind Speed Histogram

Figure A.1. (contd) 


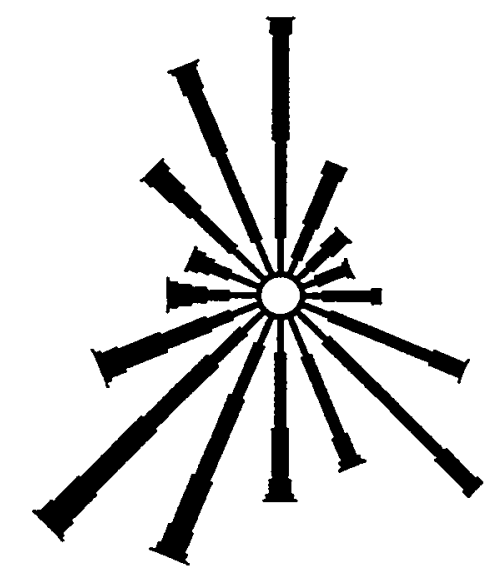

(a) Wind Rose

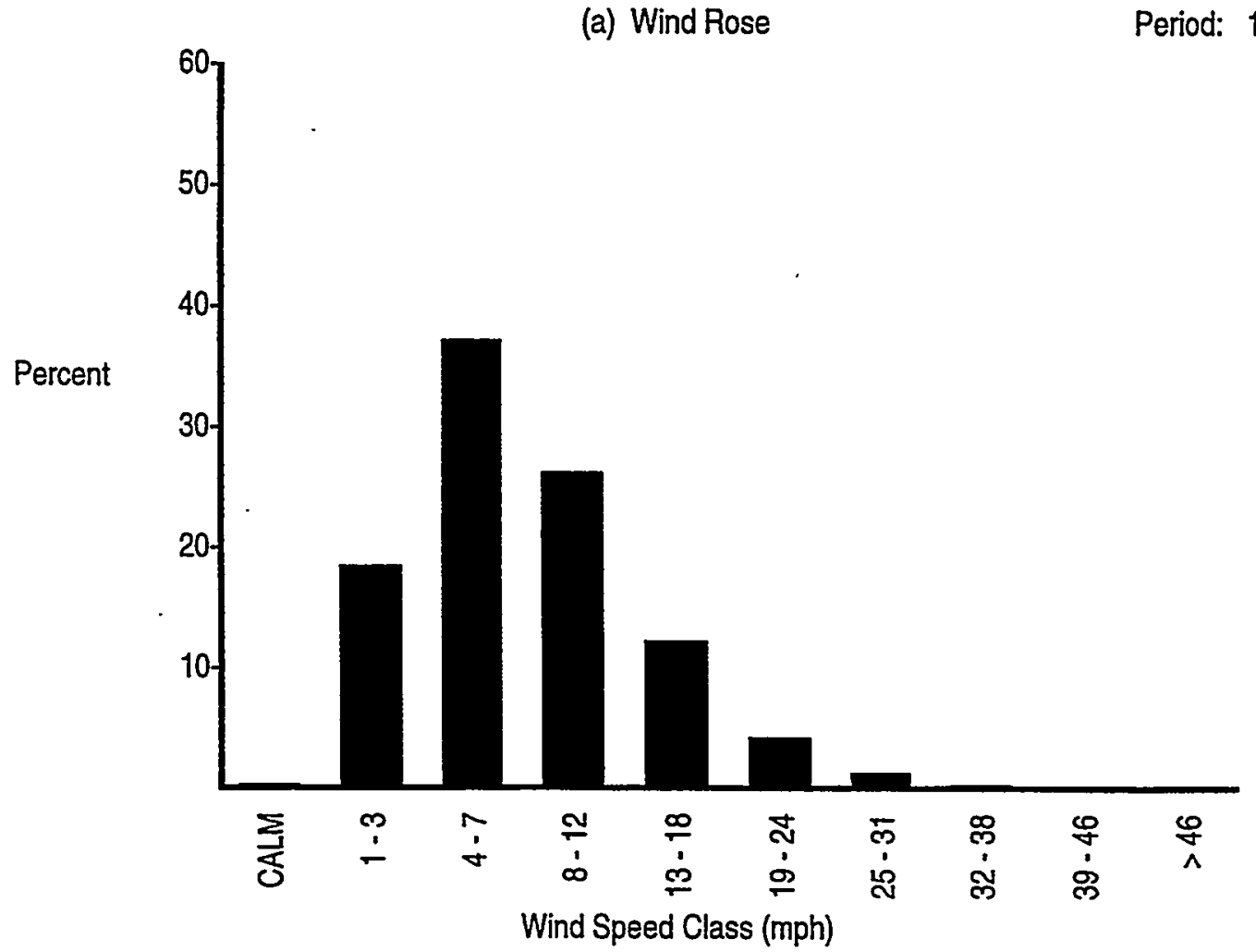

(b) Wind Speed Histogram

Figure A.1. (contd)
Station \#11 - 300A

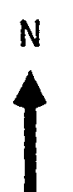

Period: 1/99-12/99 


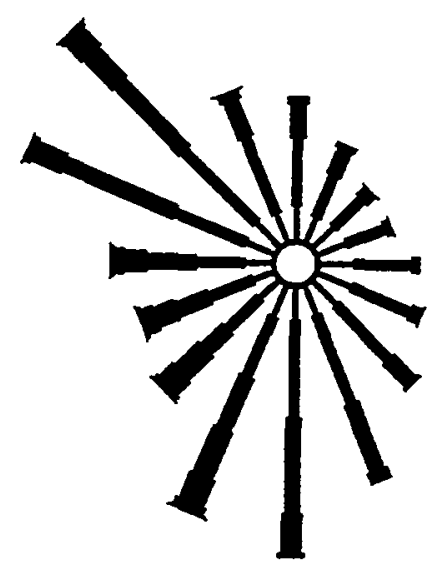

(a) Wind Rose

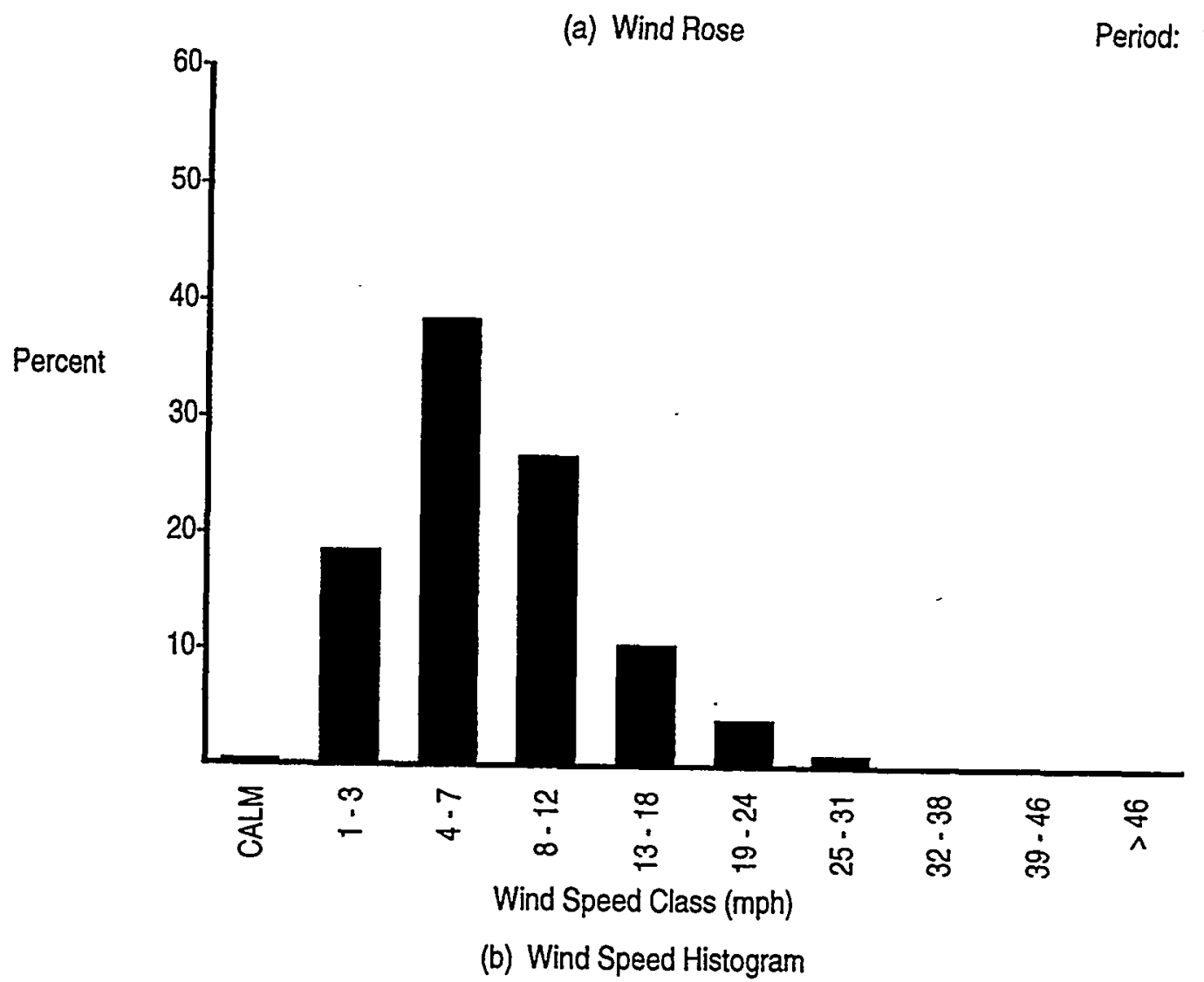

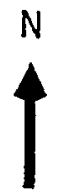

Station \#12 - WYEB

Figure A.1. (contd) 

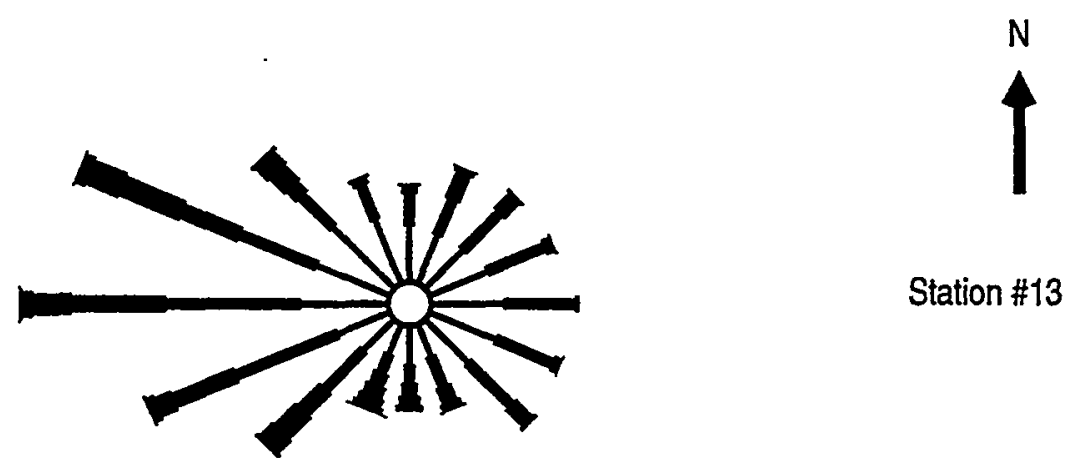

Station \#13 - 100N

(a) Wind Rose

Period: $1 / 99-12 / 99$

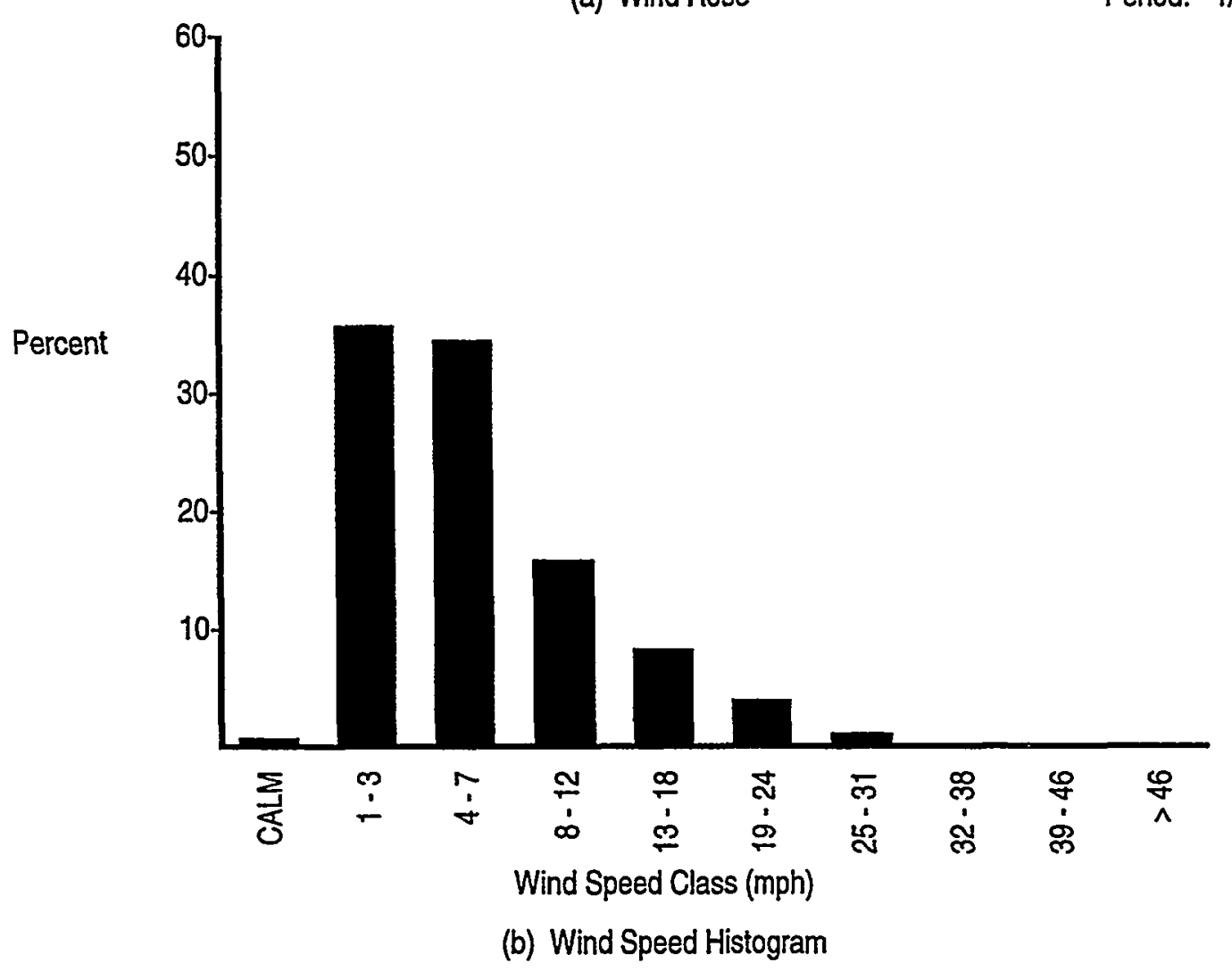

Figure A.1. (contd) 


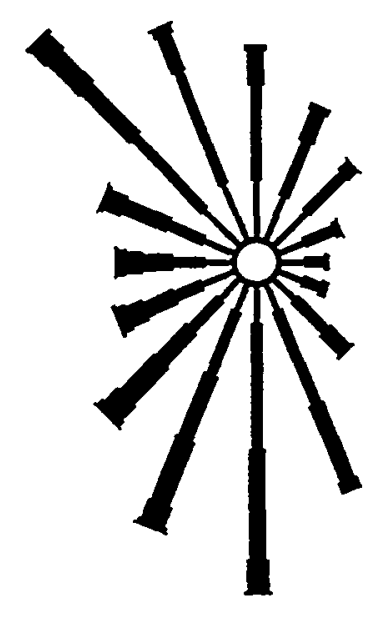

(a) Wind Rose

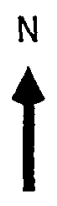

Station \#14 - WPPS

Period: $1 / 99 \cdot 12 / 99$

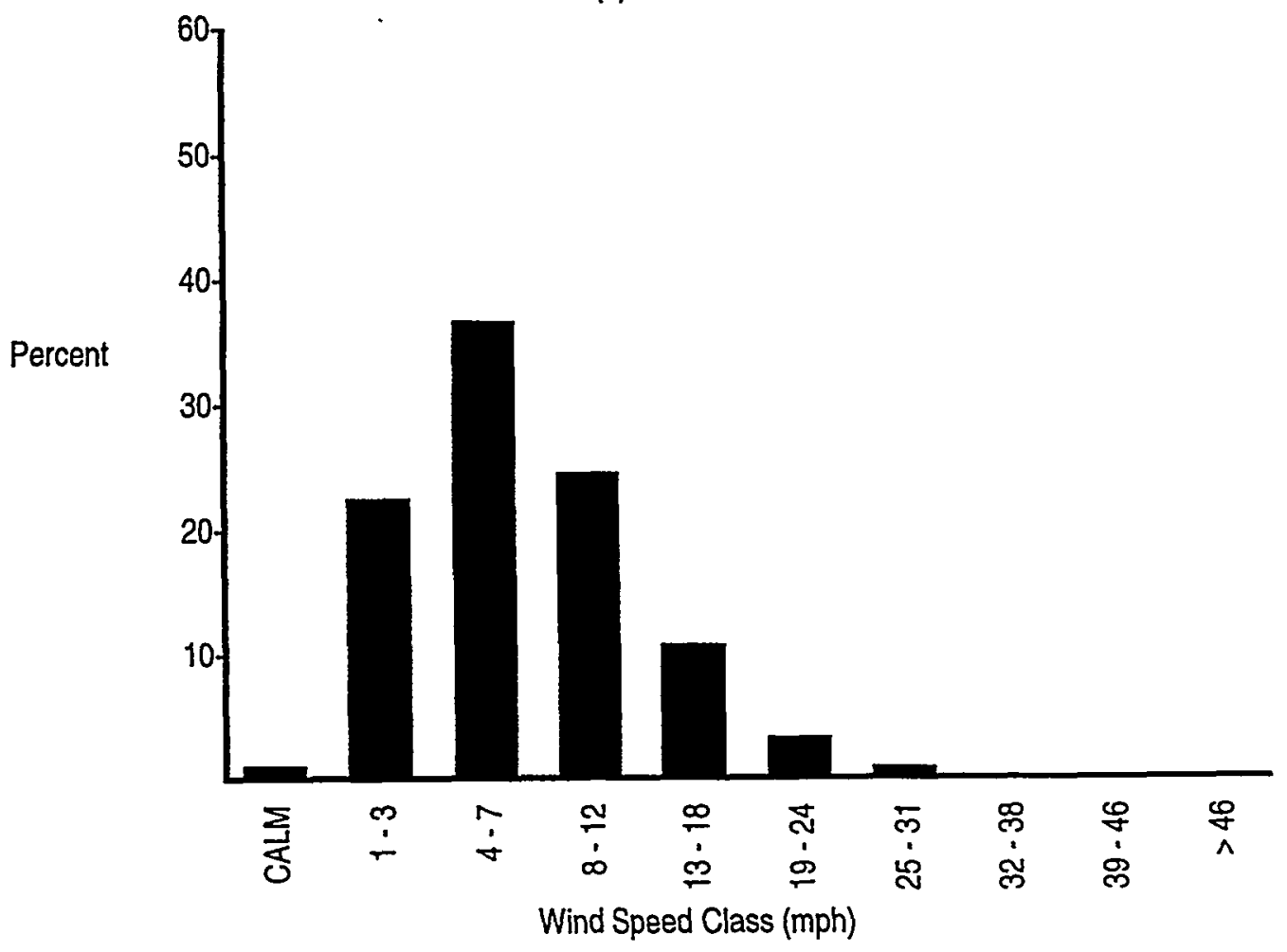

(b) Wind Speed Histogram

Figure A.1. (contd) 


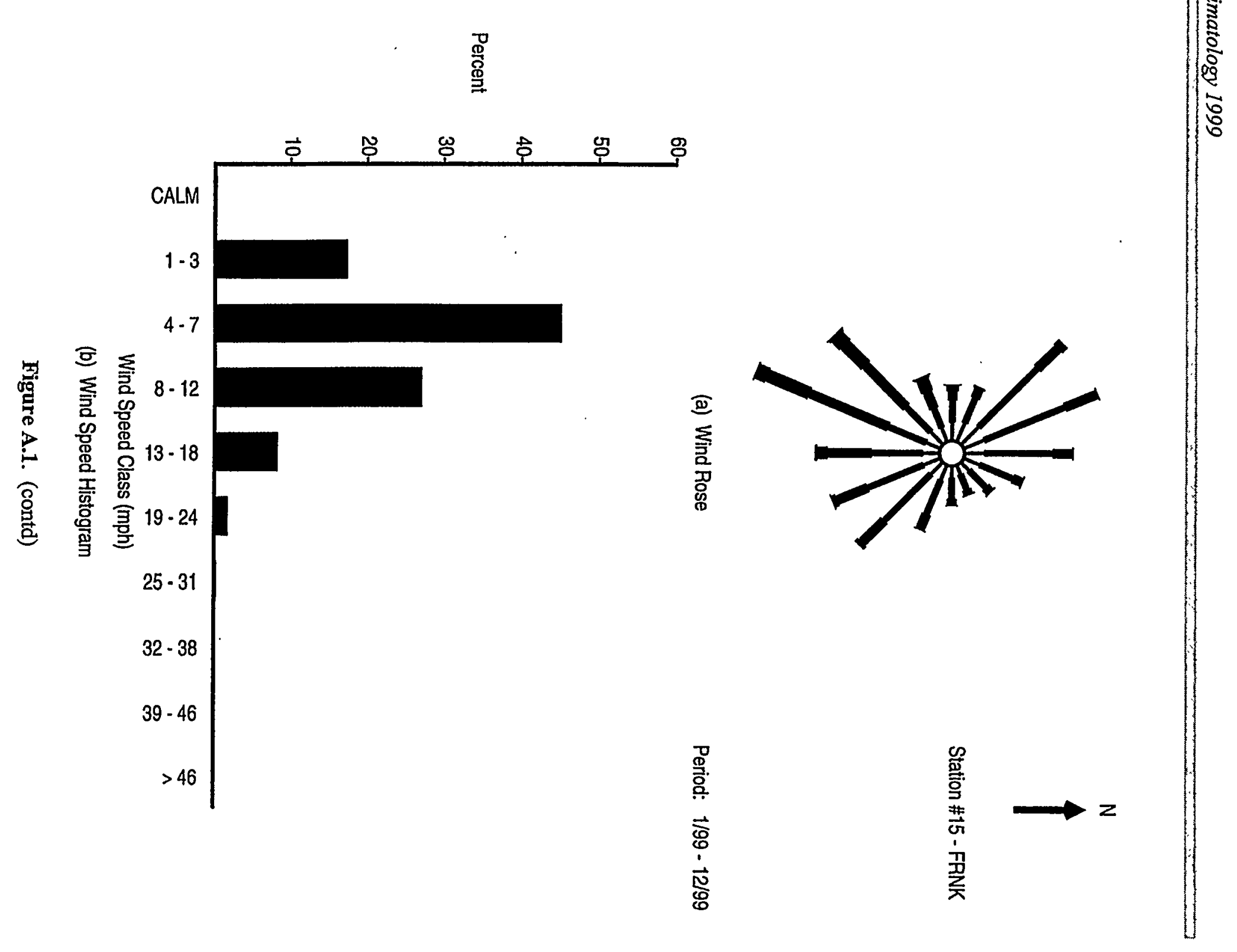



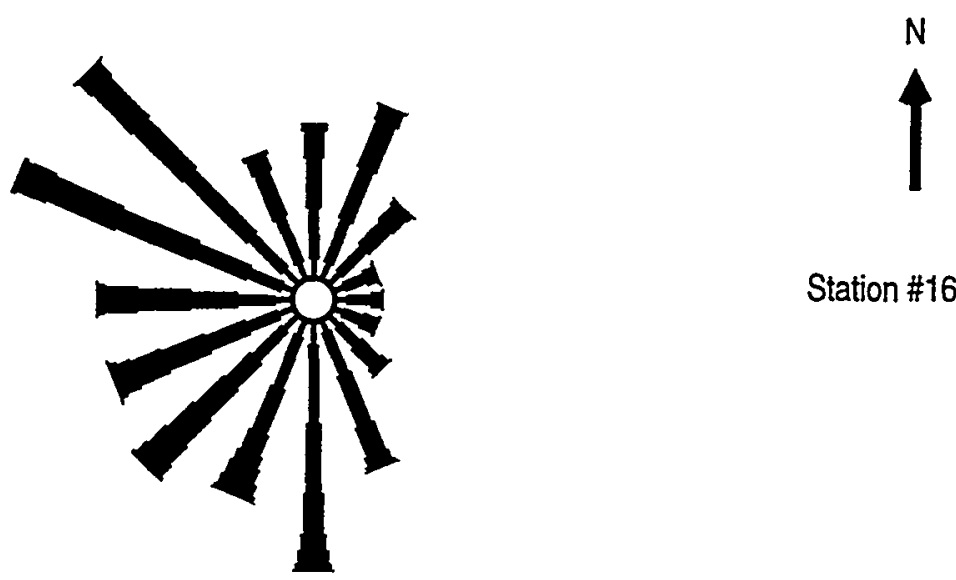

Station \#16 - GABL

(a) Wind Rose

Period: $1 / 99-12 / 99$

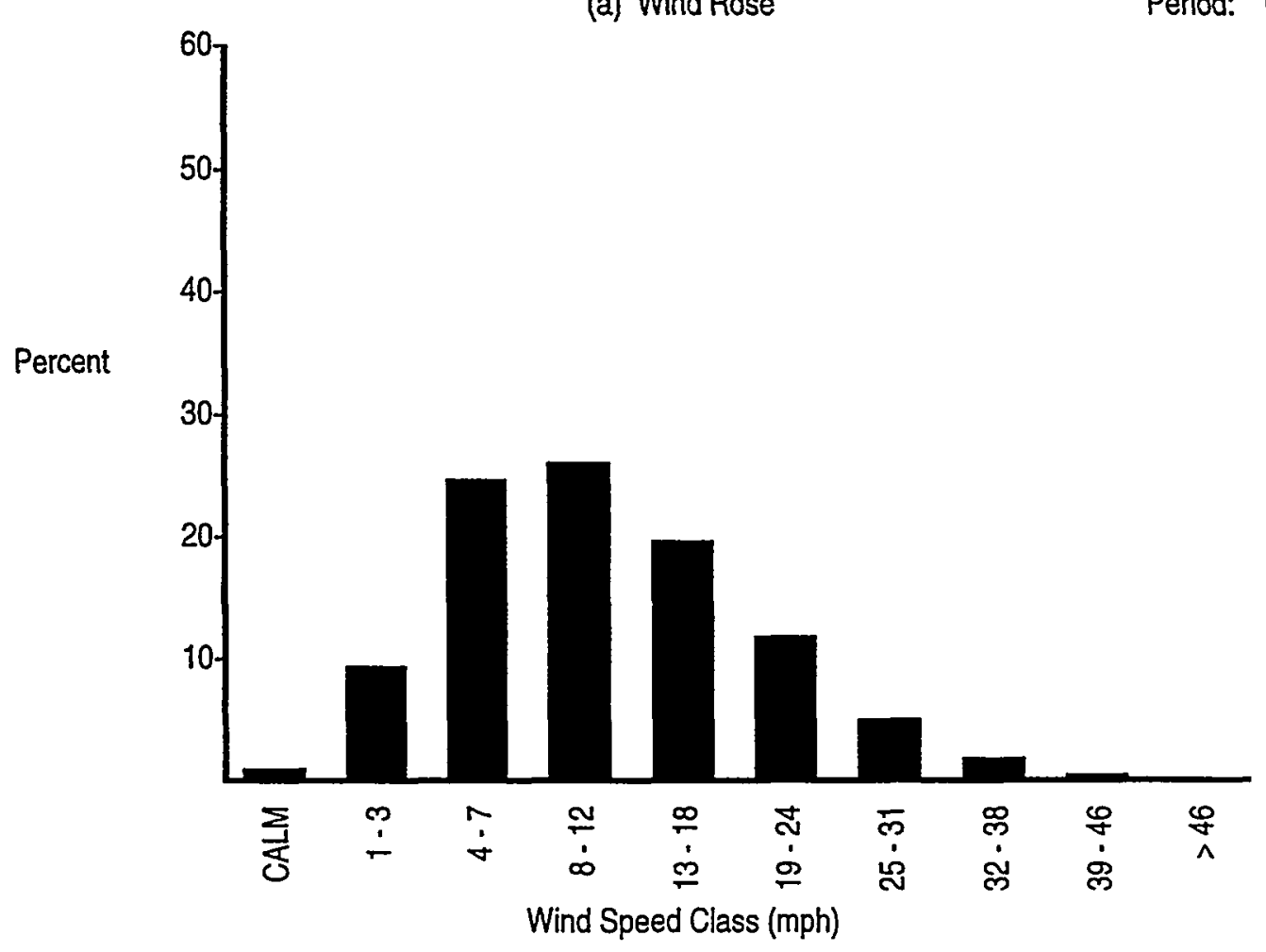

(b) Wind Speed Histogram

Figure A.1. (contd) 

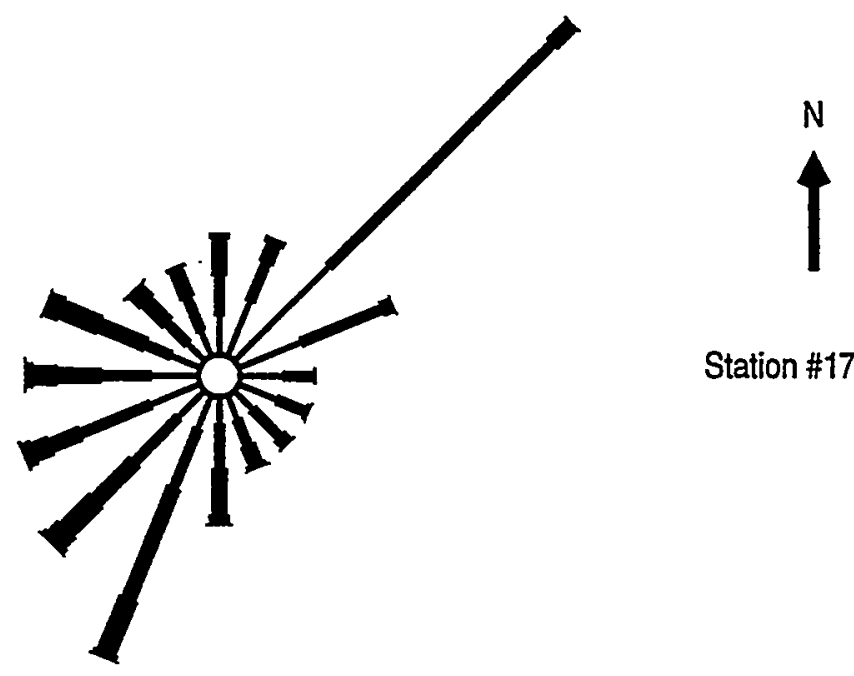

Station \#17 - RING

(a) Wind Rose

Period: $1 / 99-12 / 99$

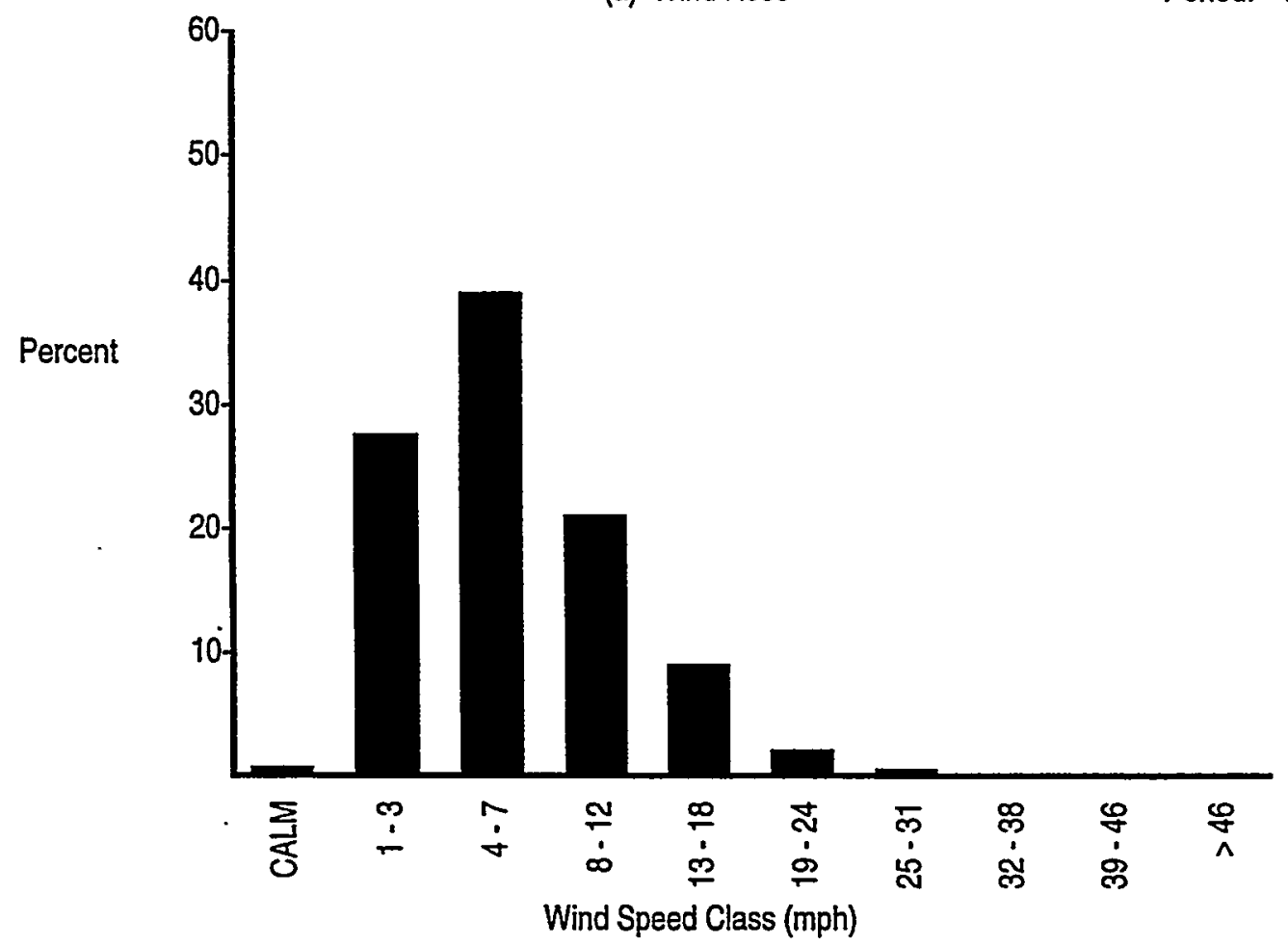

(b) Wind Speed Histogram

Figure A.1. (contd) 


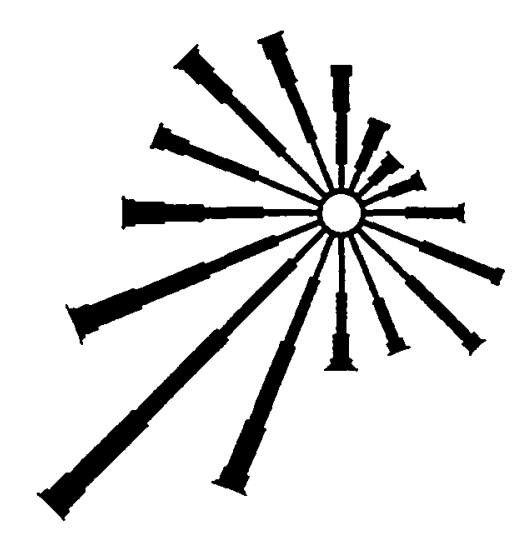

(a) Wind Rose

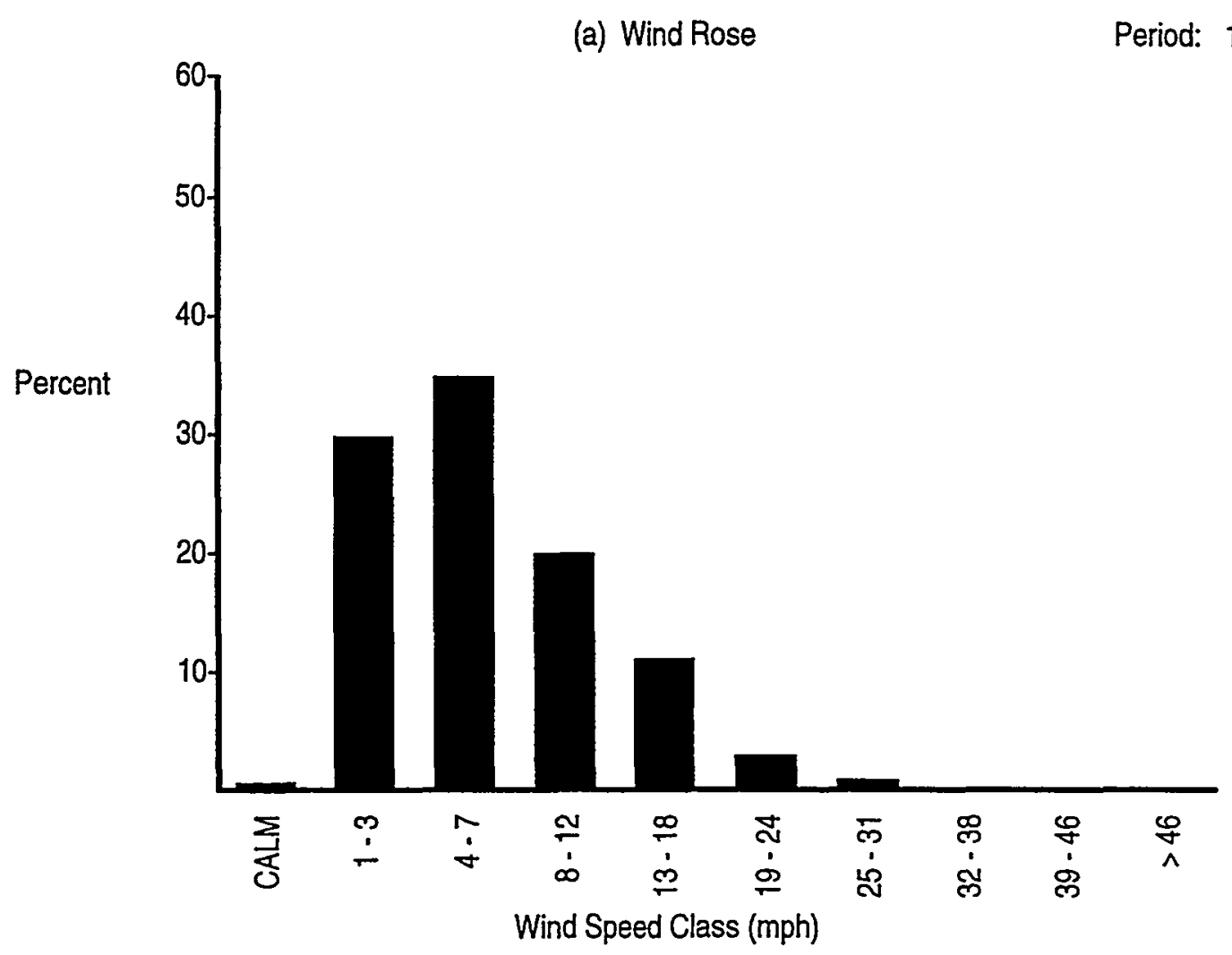

(b) Wind Speed Histogram

Figure A.1. (contd)

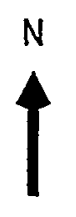

Station \#18 - RICH 

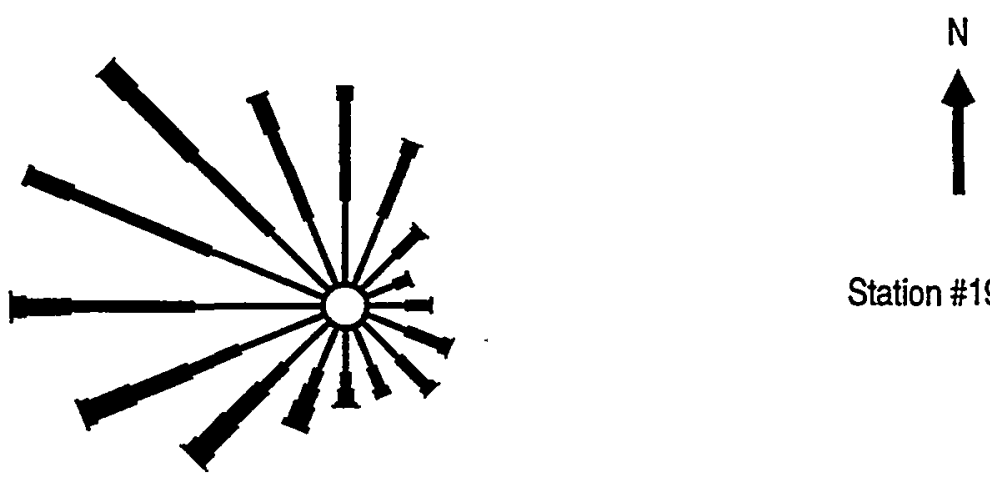

Station \#19 - PFP

(a) Wind Rose

Period: $1 / 99-12 / 99$

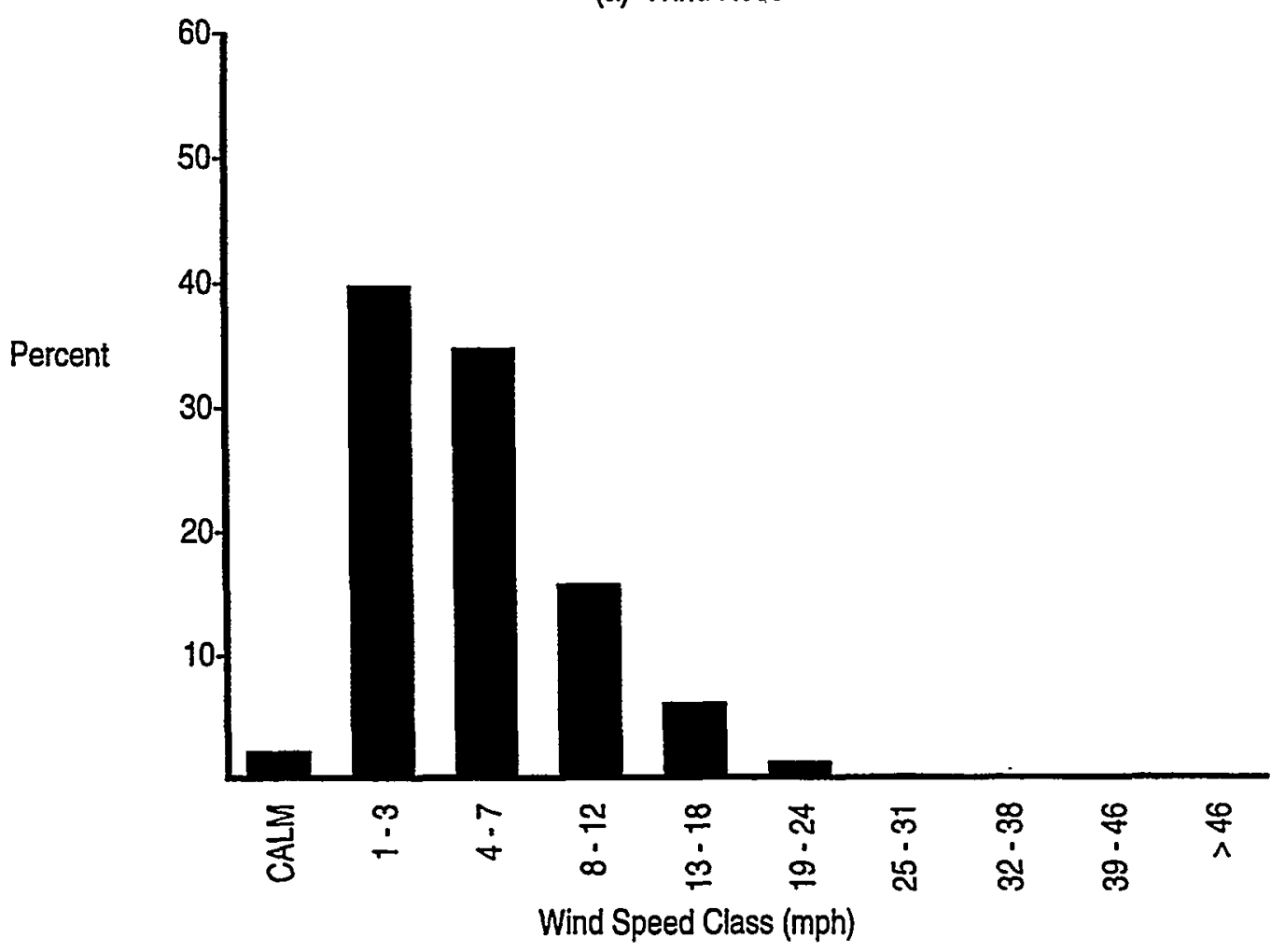

(b) Wind Speed Histogram

Figure A.1. (contd) 


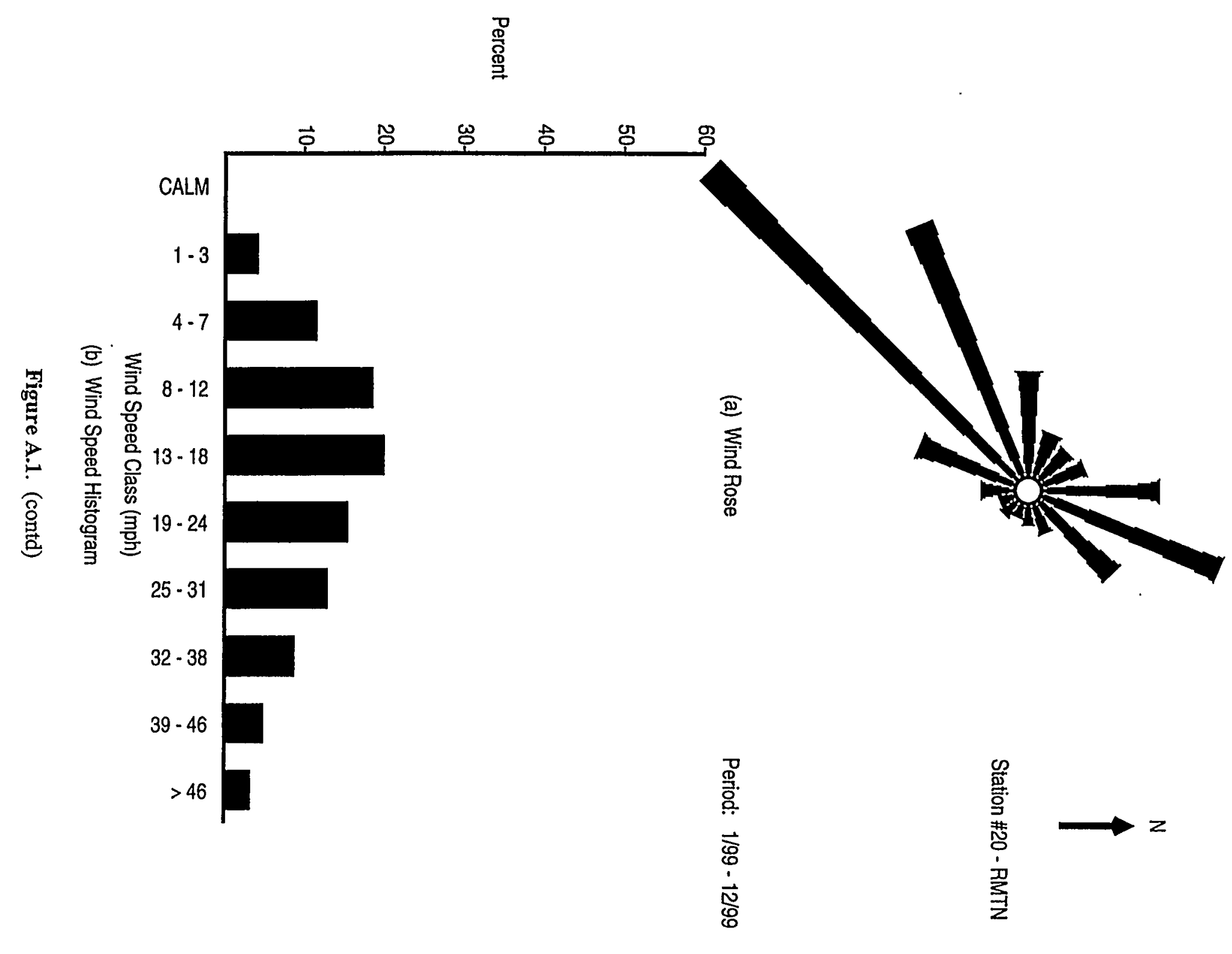




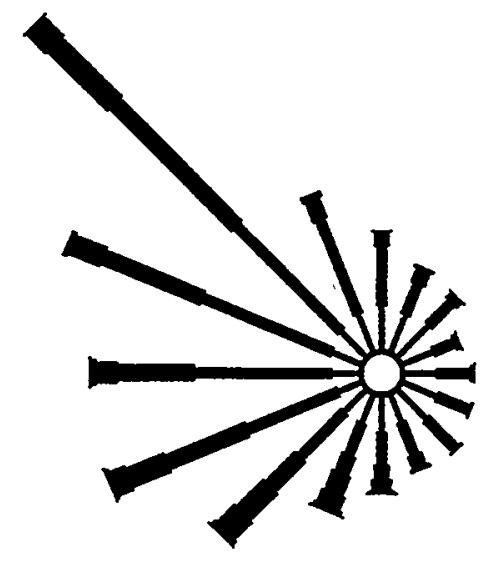

(a) Wind Rose

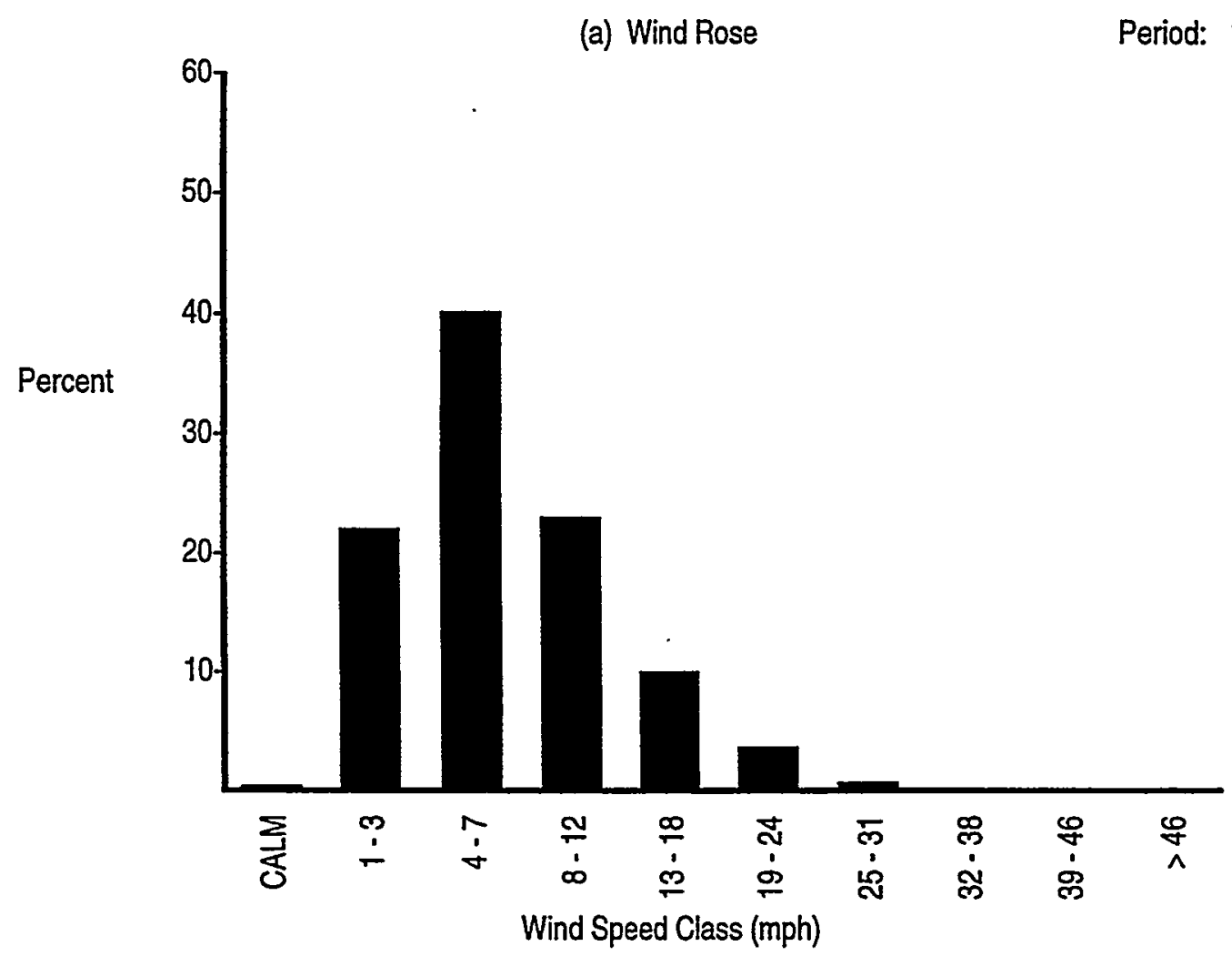

(b) Wind Speed Histogram

Figure A.1. (contd)

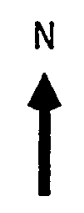

Station \#21 - HMS

Period: 1/99-12/99 


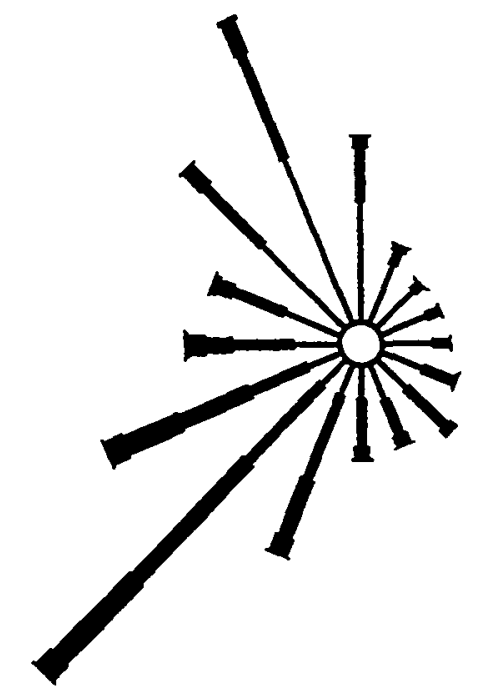

(a) Wind Rose

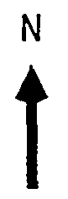

Station \#22 - PASC

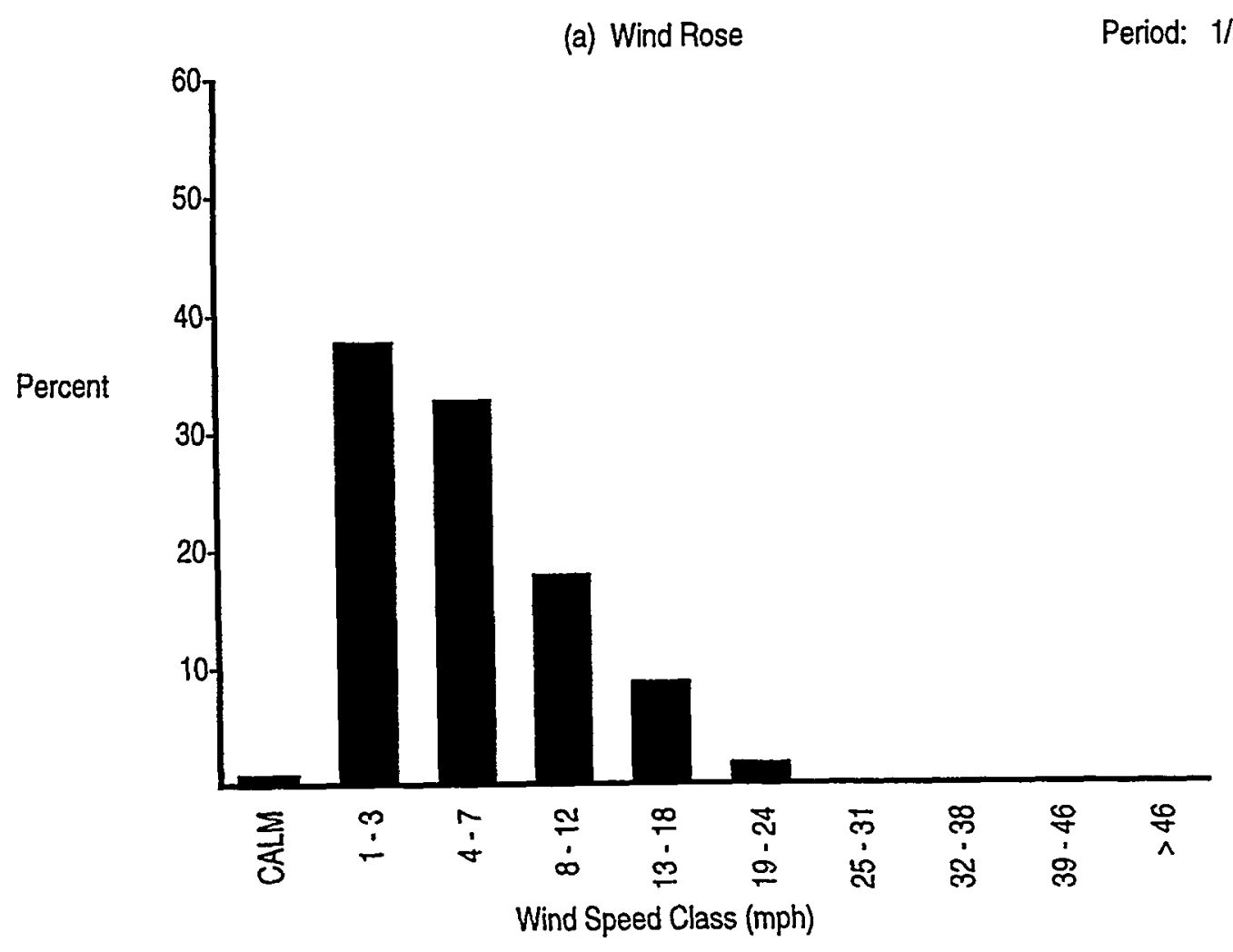

(b) Wind Speed Histogram

Figure A.1. (contd) 

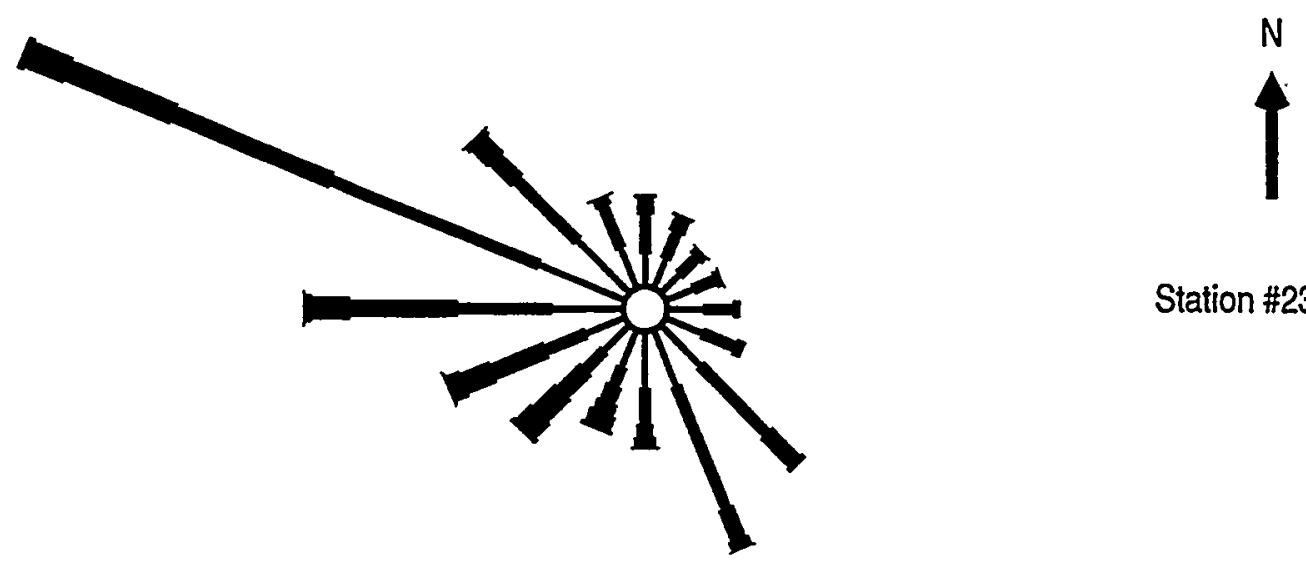

Station \#23 - GABW

(a) Wind Rose

Period: $1 / 99-12 / 99$

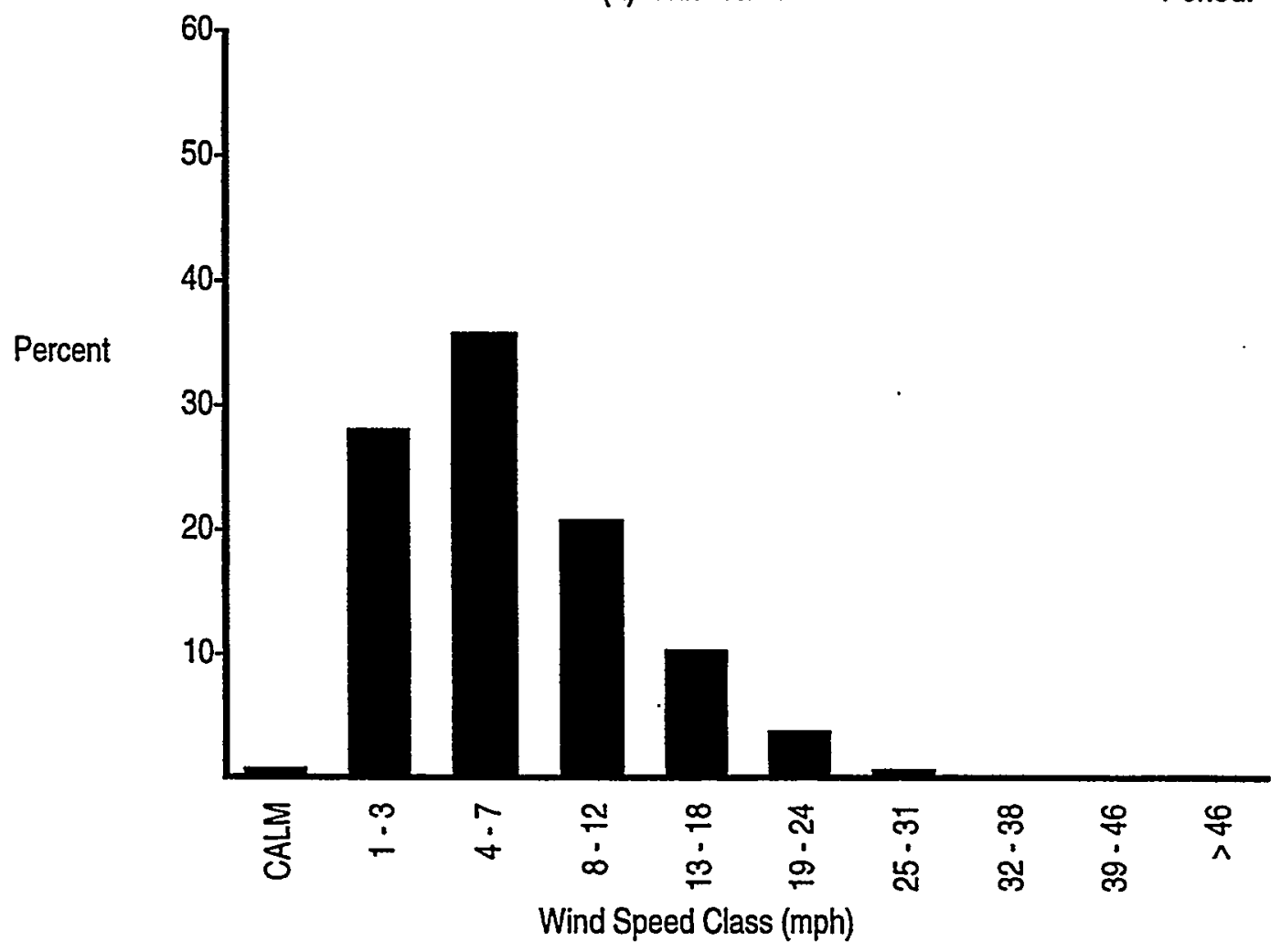

(b) Wind Speed Histogram

Figure A.1. (contd) 

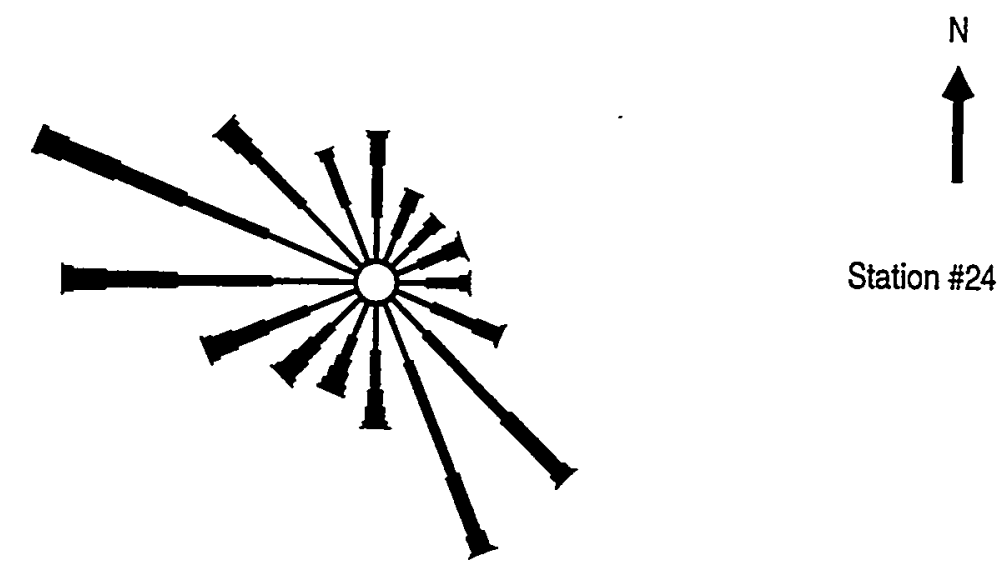

Station \#24 - 100F

(a) Wind Rose

Period: 1/99-12/99

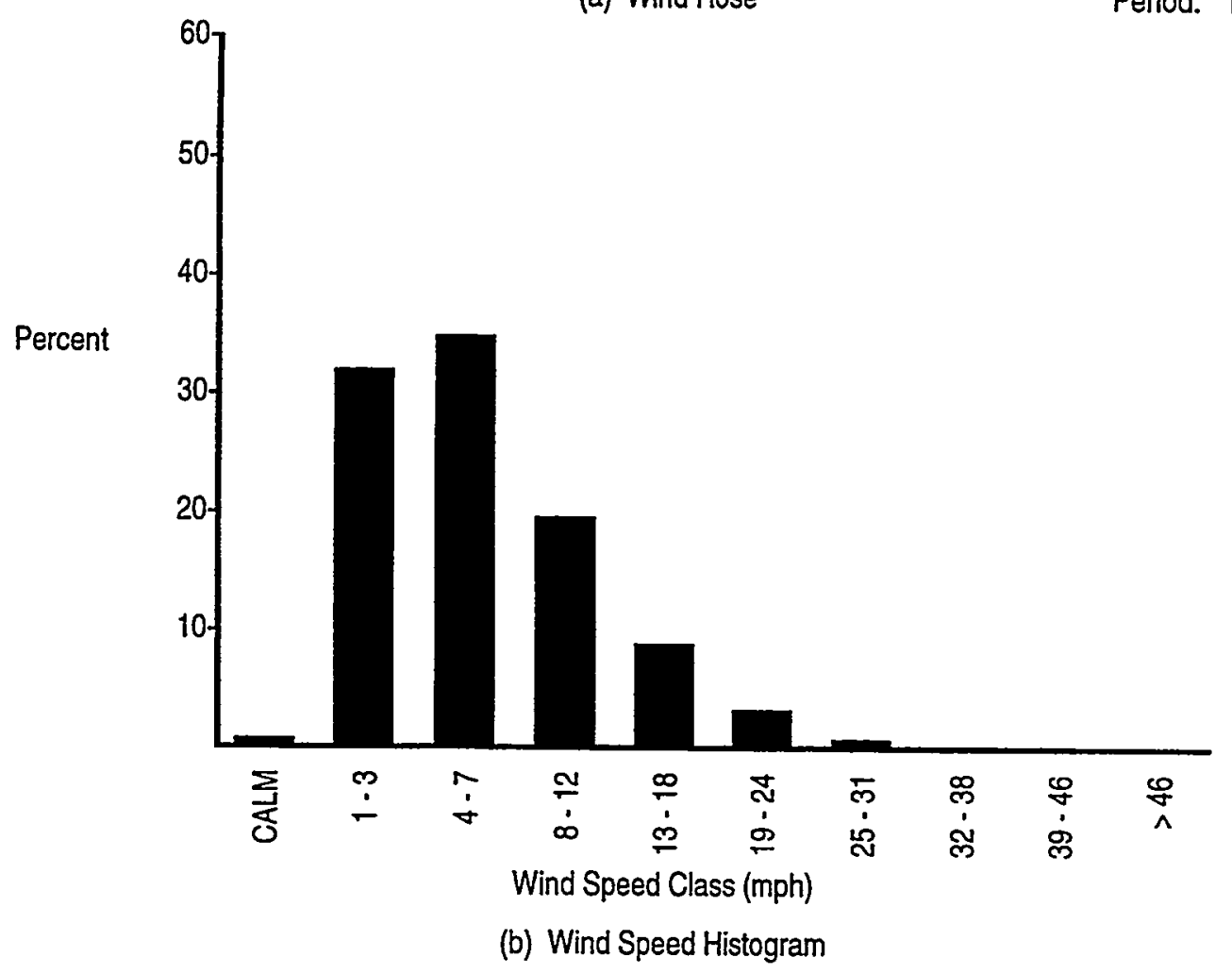

Figure A.1. (contd) 

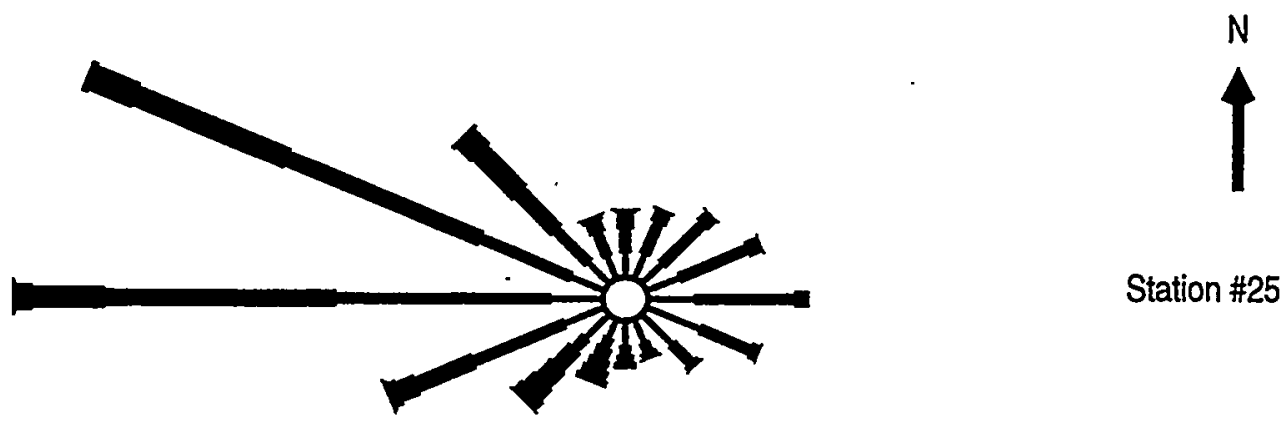

Station \#25 - VERN

(a) Wind Rose

Period: 1/99-12/99

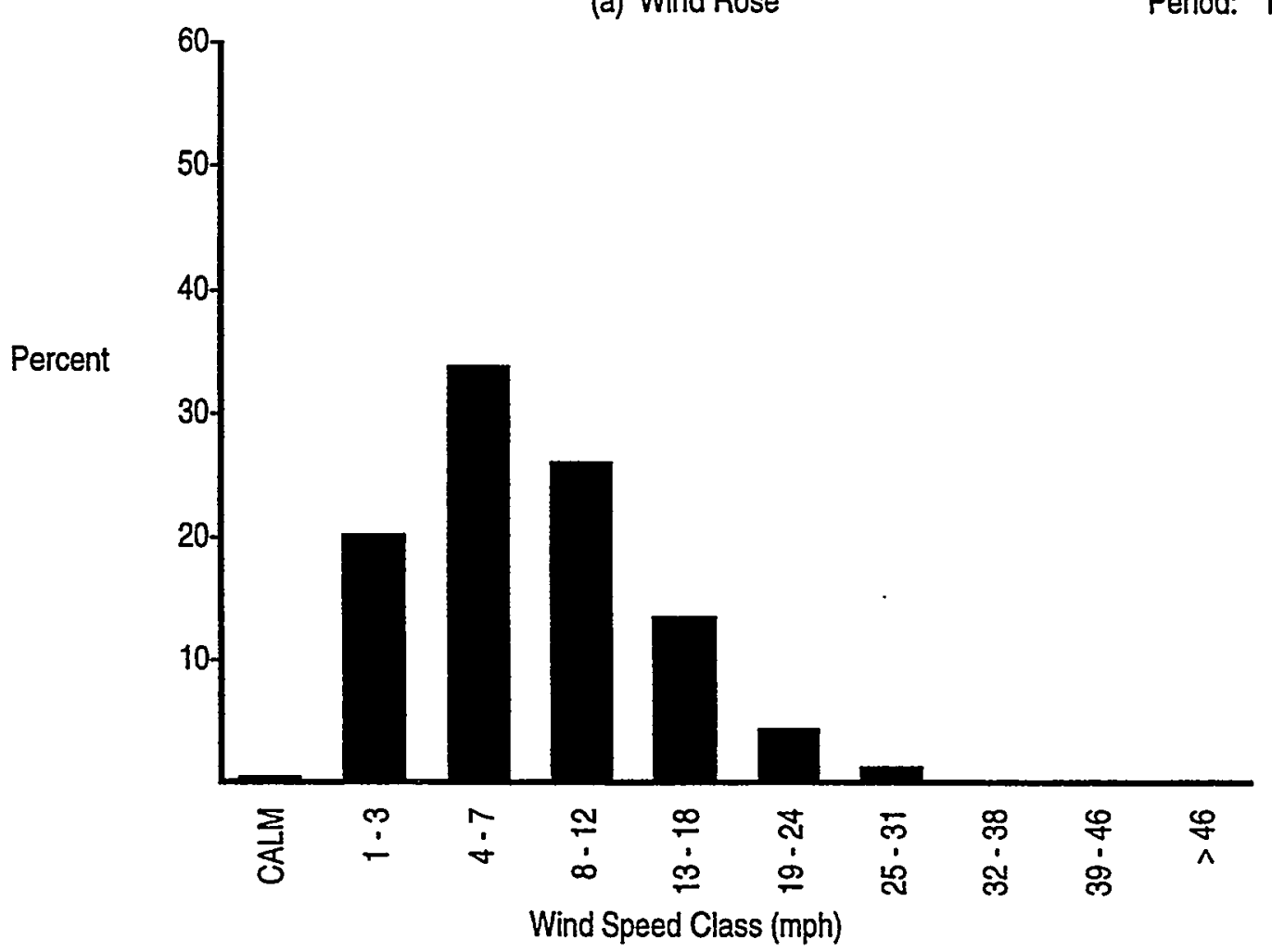

(b) Wind Speed Histogram

Figure A.1. (contd) 

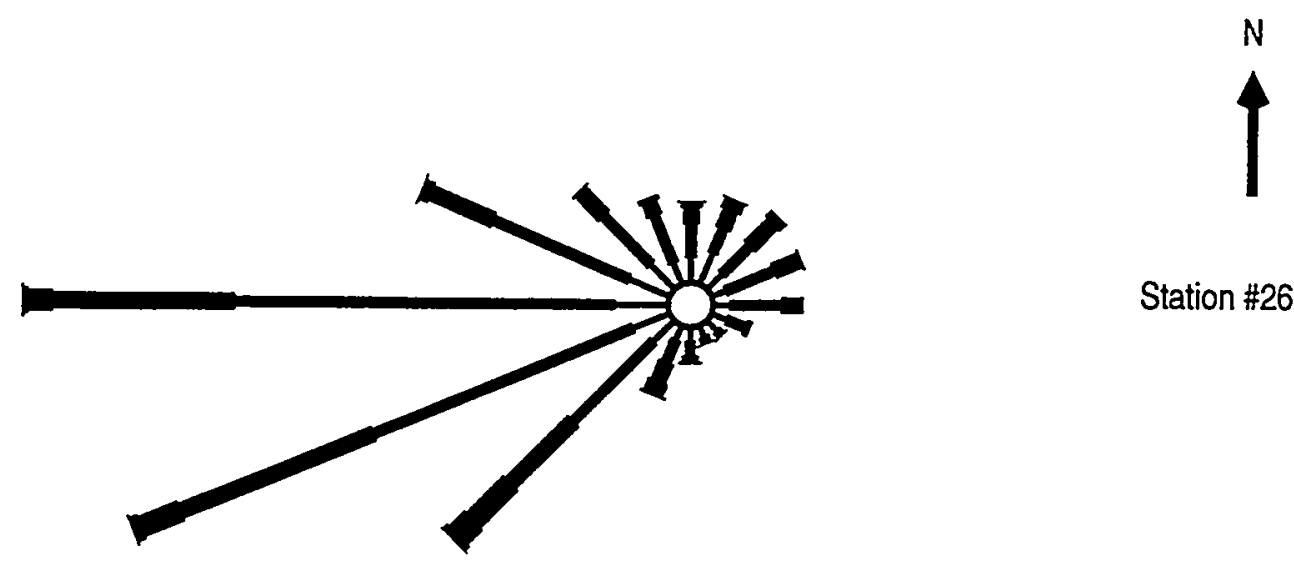

Station \#26 - BENT

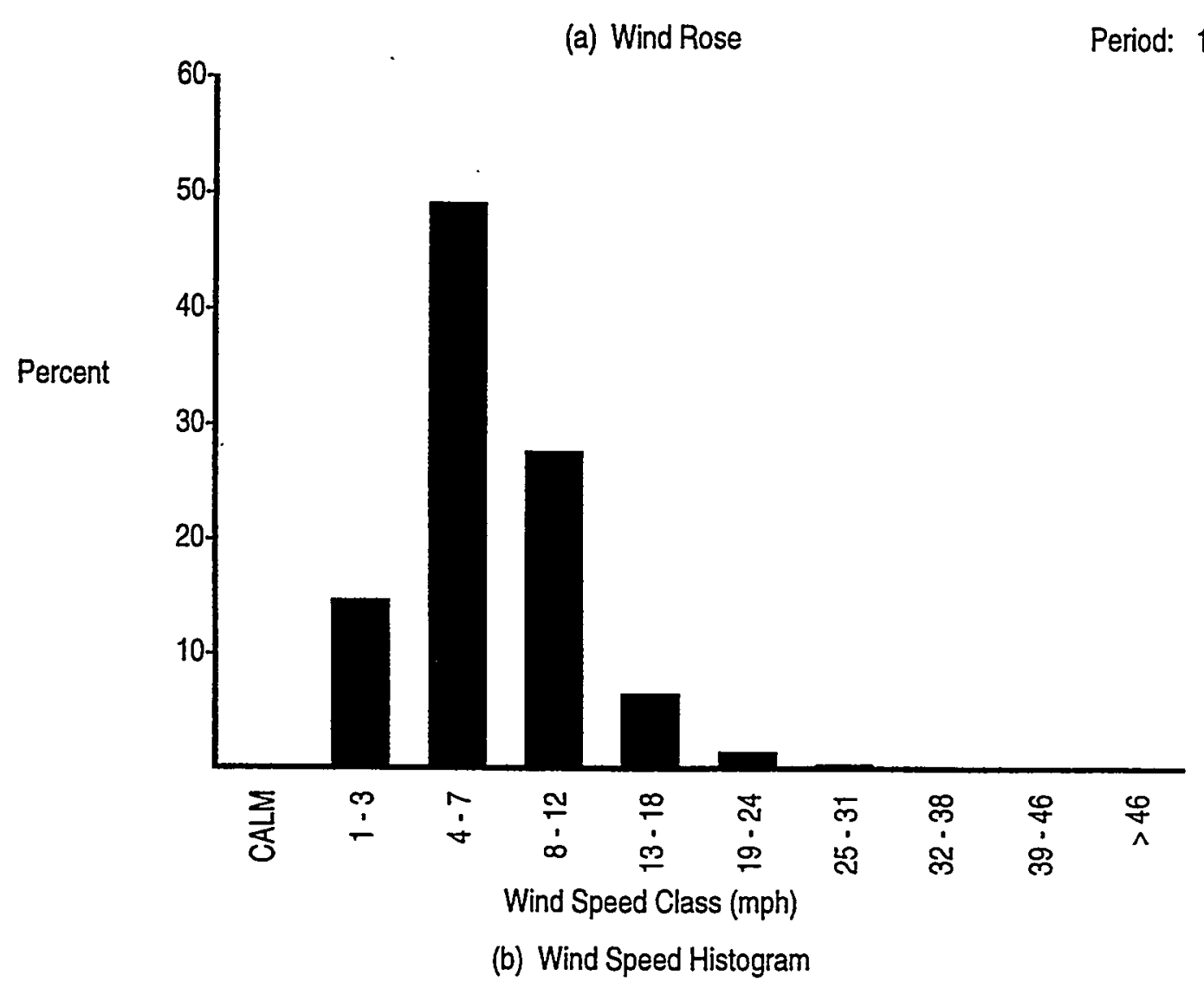

Figure A.1. (contd) 

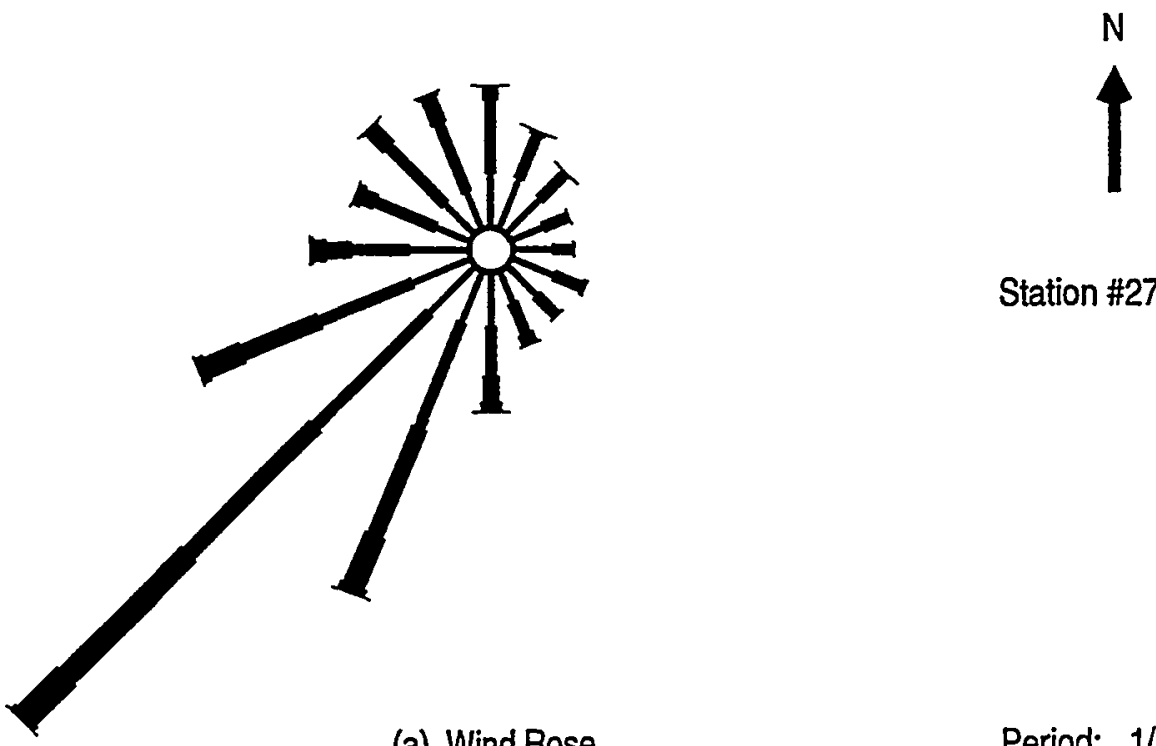

(a) Wind Rose

Period: $1 / 99-12 / 99$

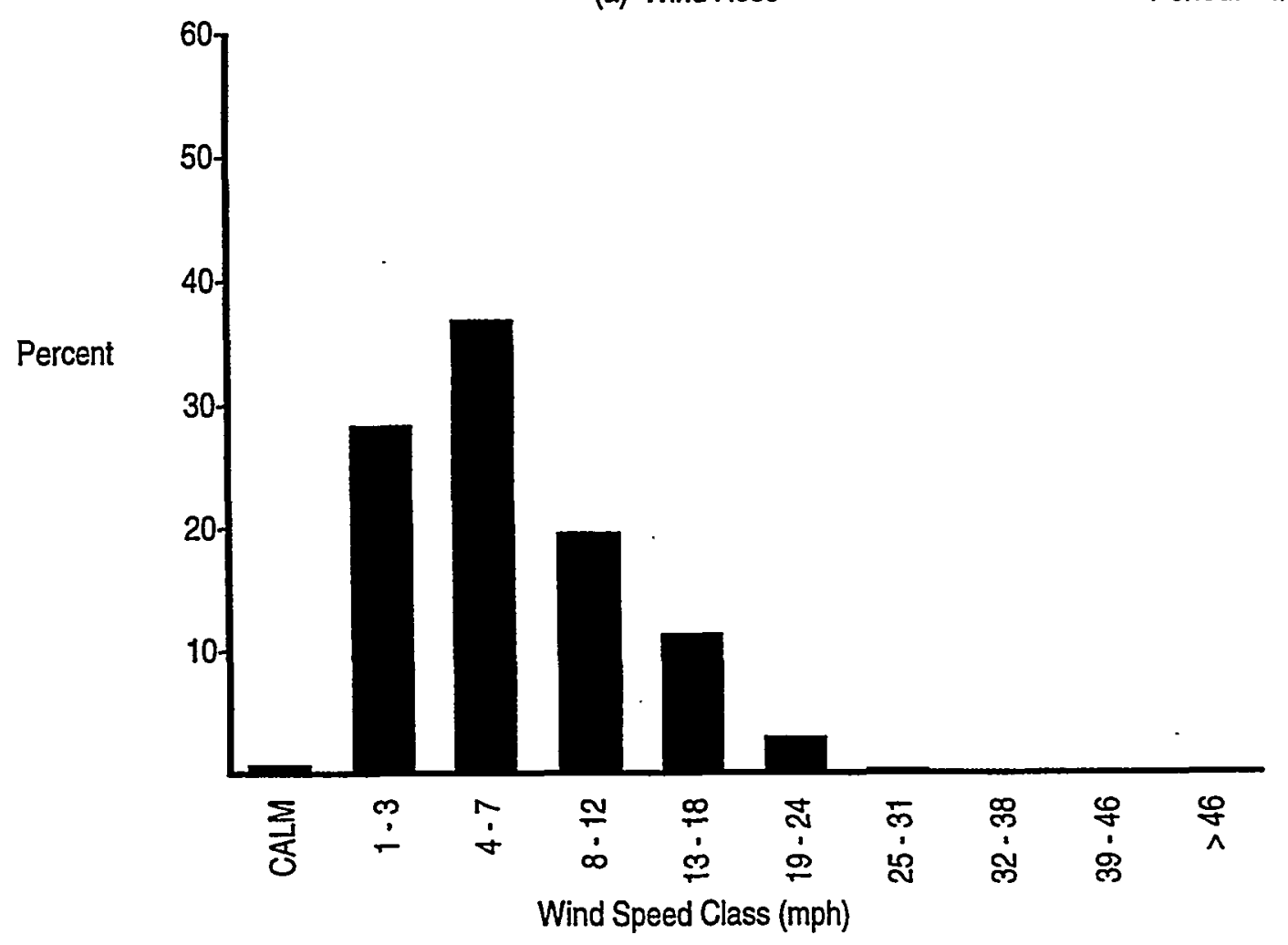

(b) Wind Speed Histogram

Figure A.1. (contd) 

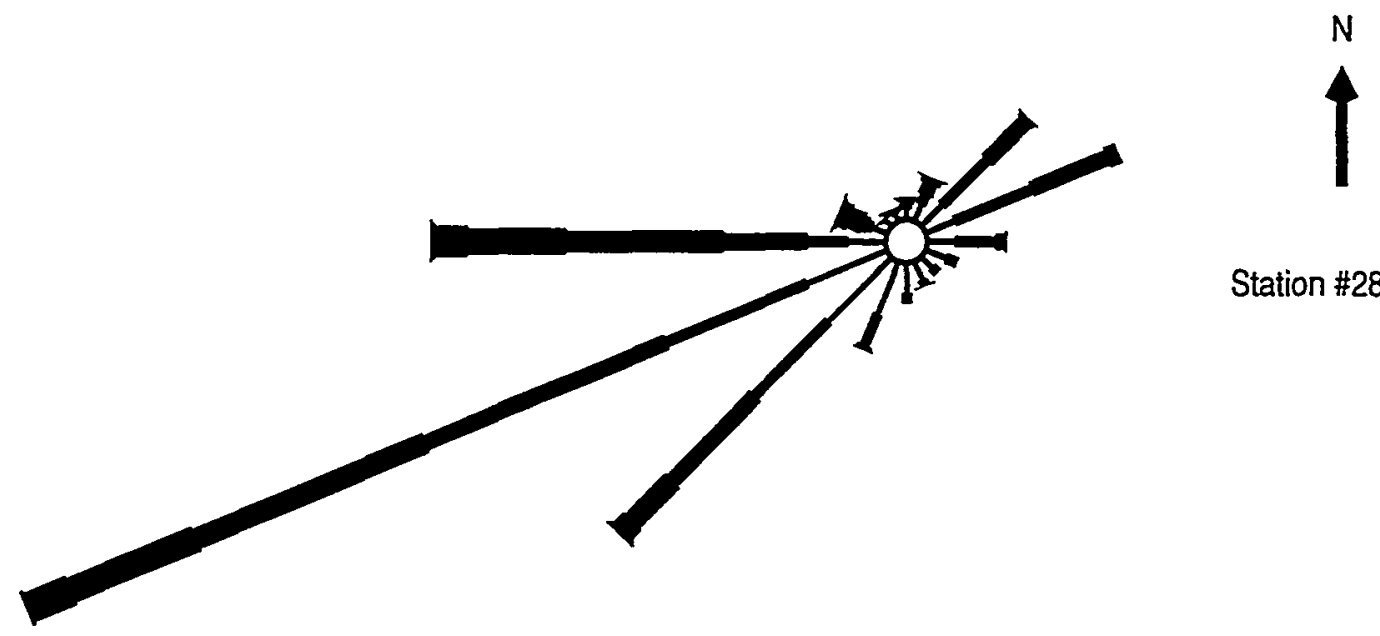

Station \#28 - SURF

(a) Wind Rose

Period: 1/99-12/99

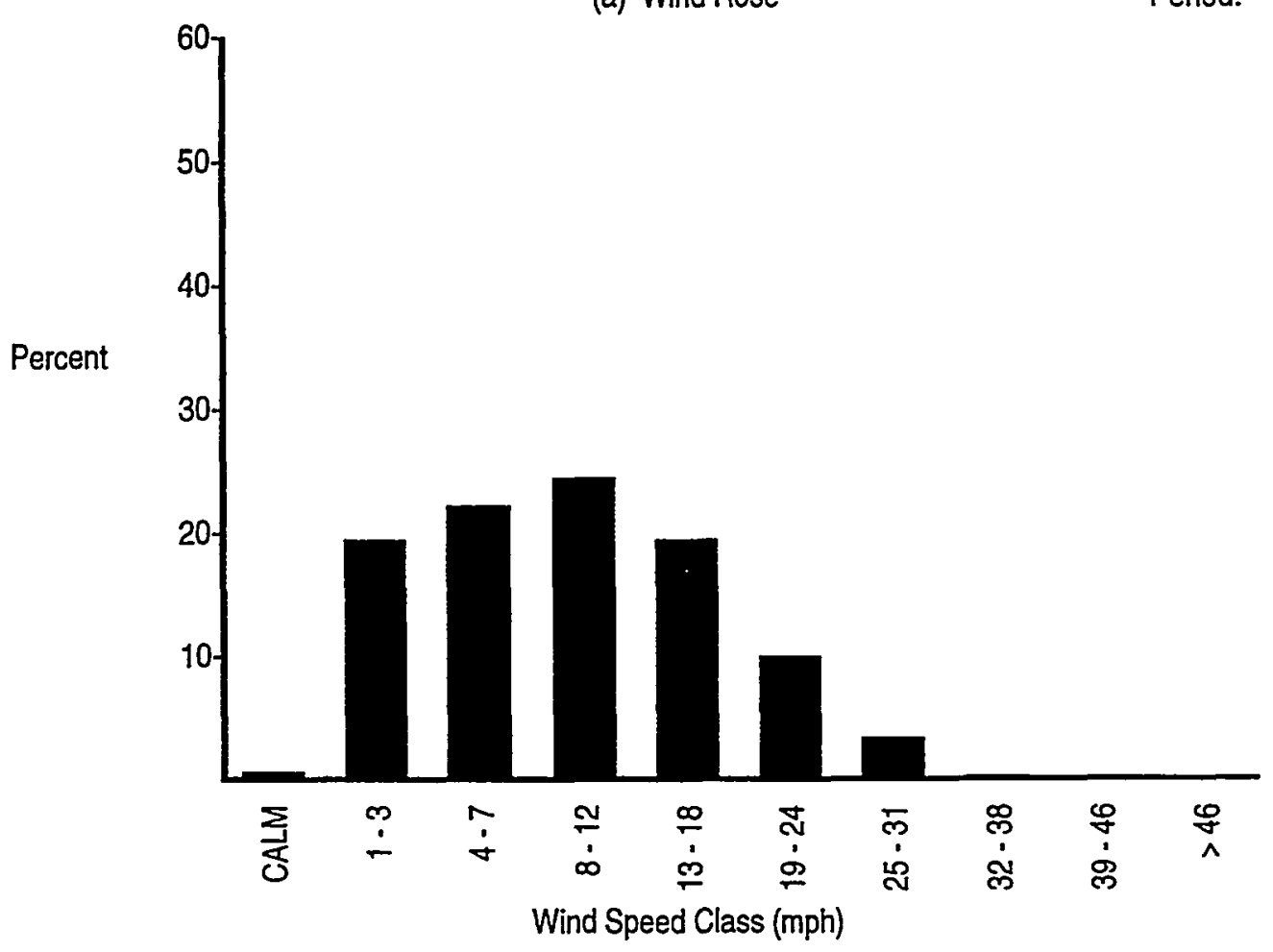

(b) Wind Speed Histogram

Figure A.1. (contd) 

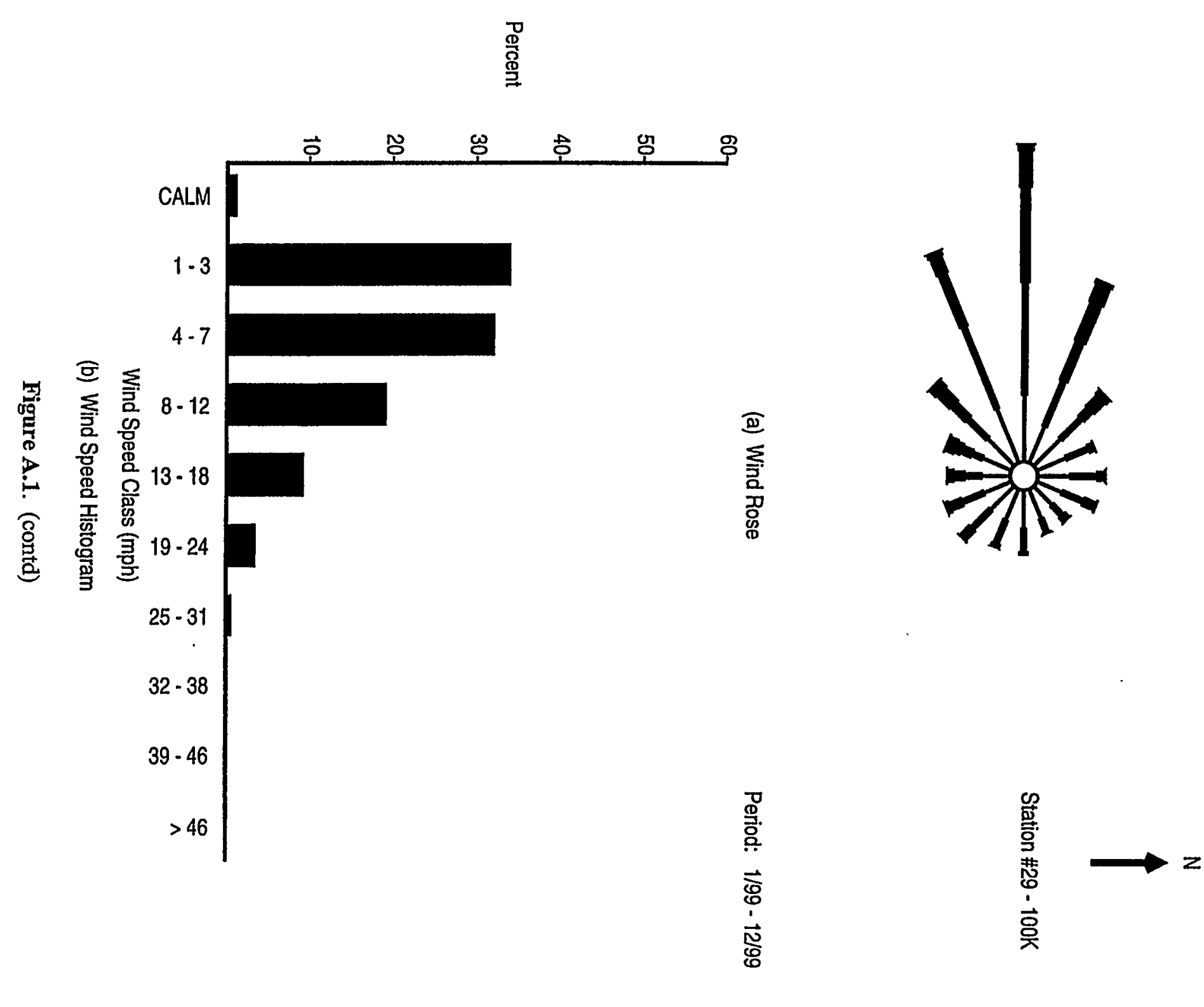

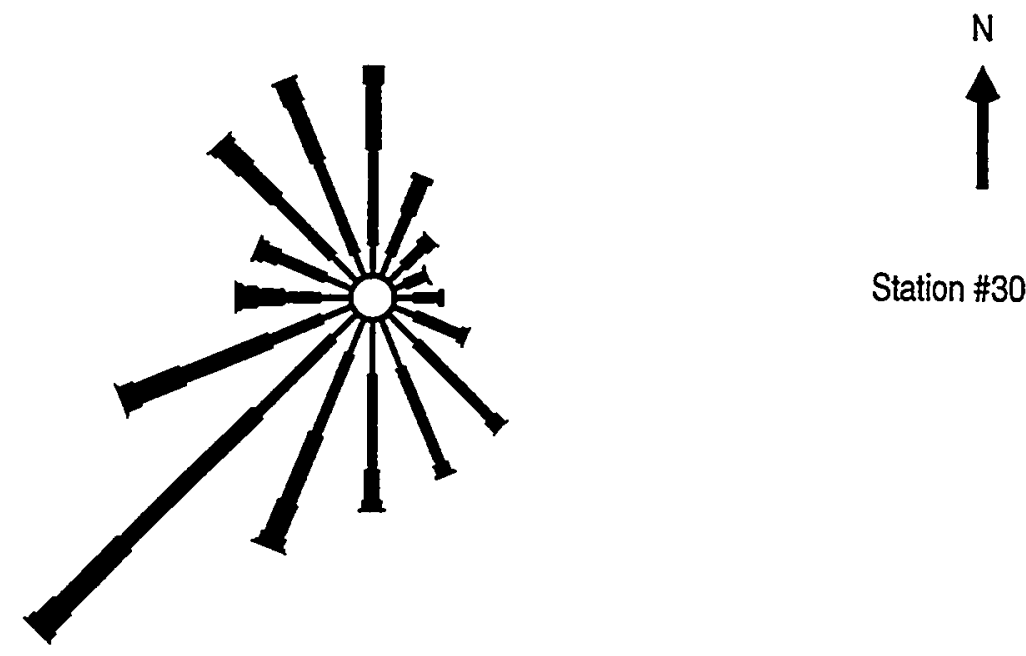

Station \#30 - HAMR

(a) Wind Rose

Period: $1 / 99-12 / 99$

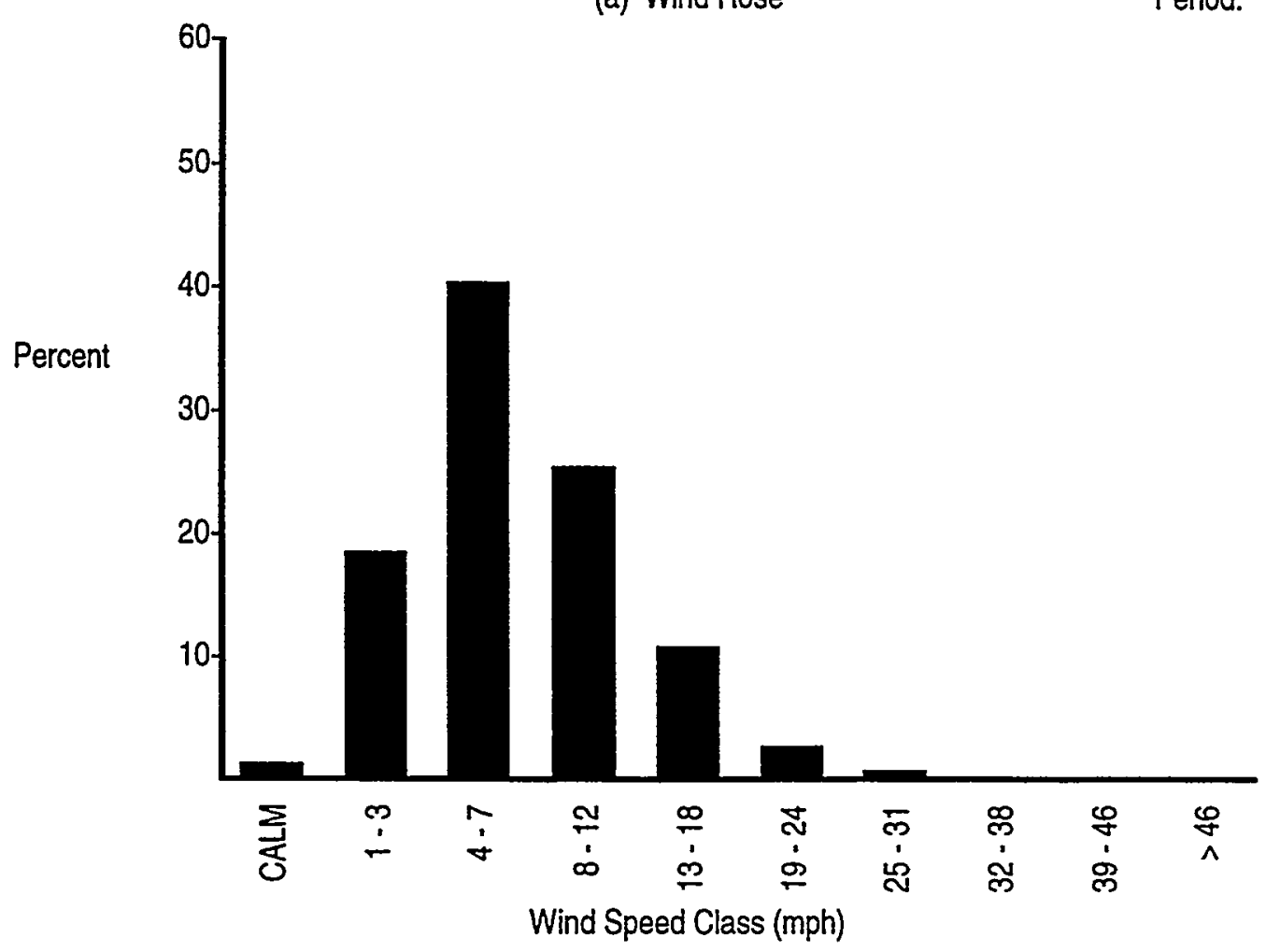

(b) Wind Speed Histogram

Figure A.1. (contd) 


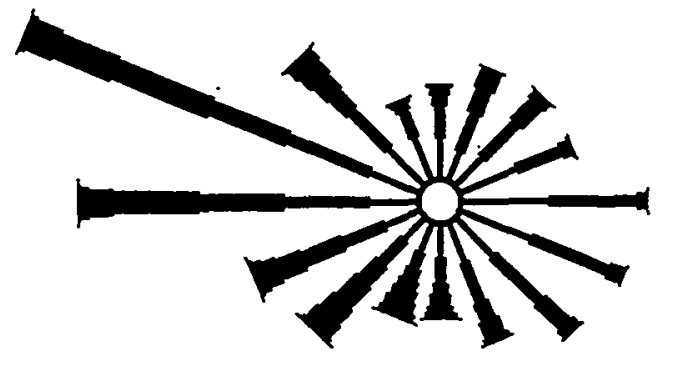

(a) Wind Rose

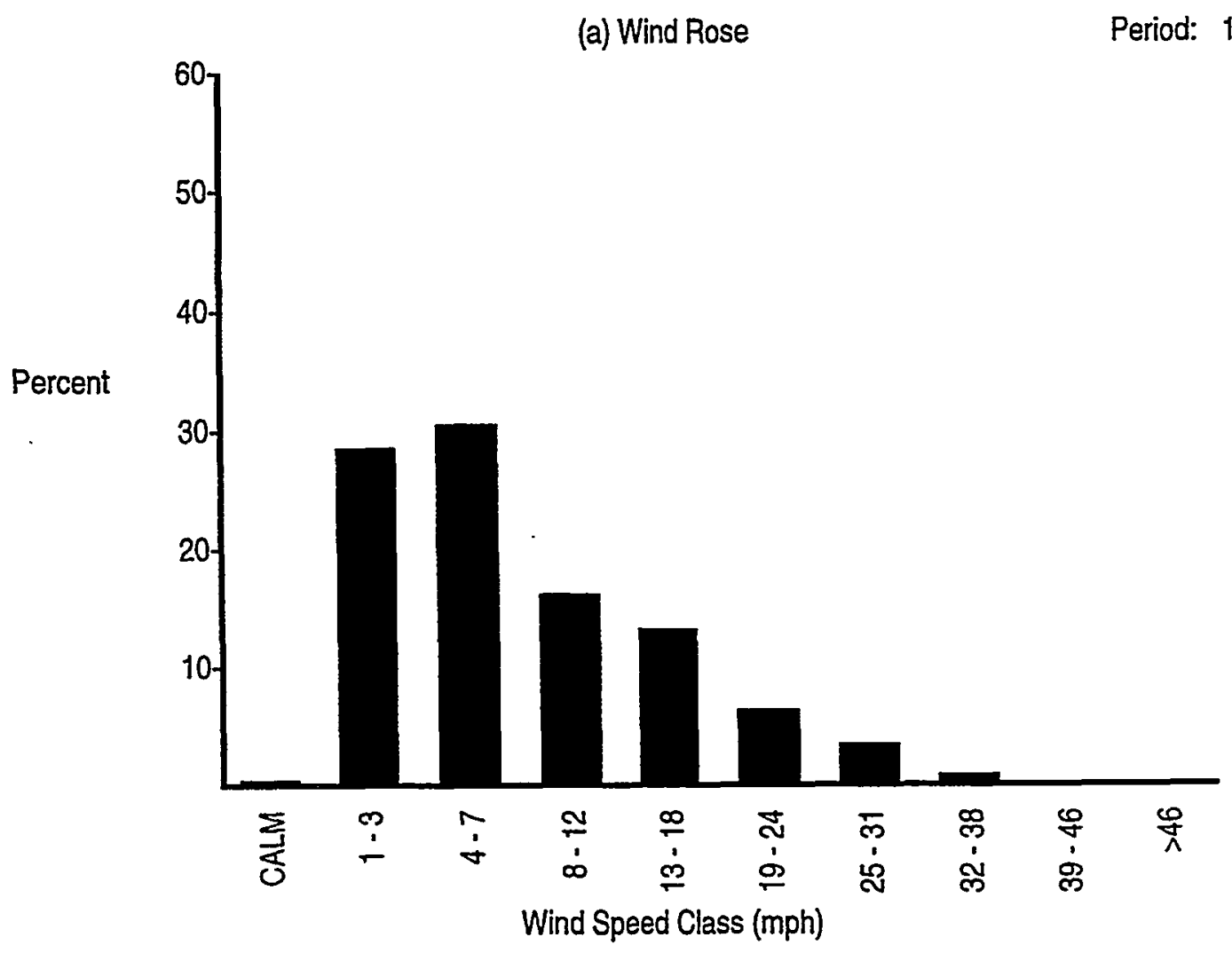

(b) Wind Speed Histogram

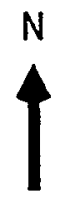

100 Area Tower

Period: $1 / 99-12 / 99$ 


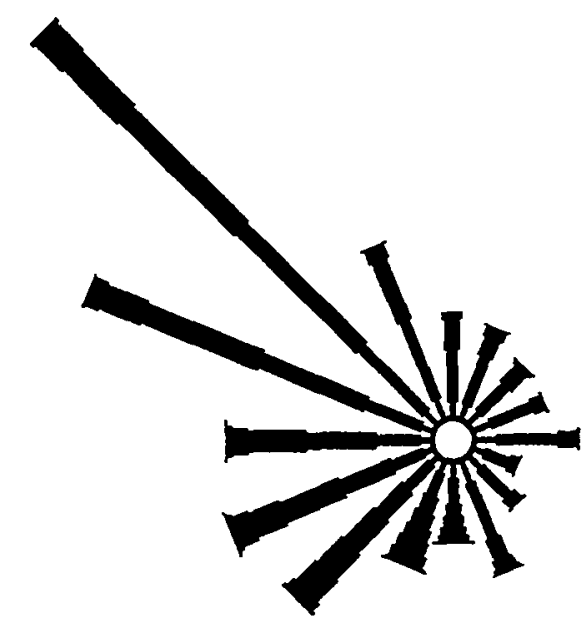

(a) Wind Rose

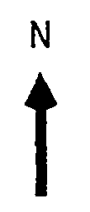

200 Area Tower

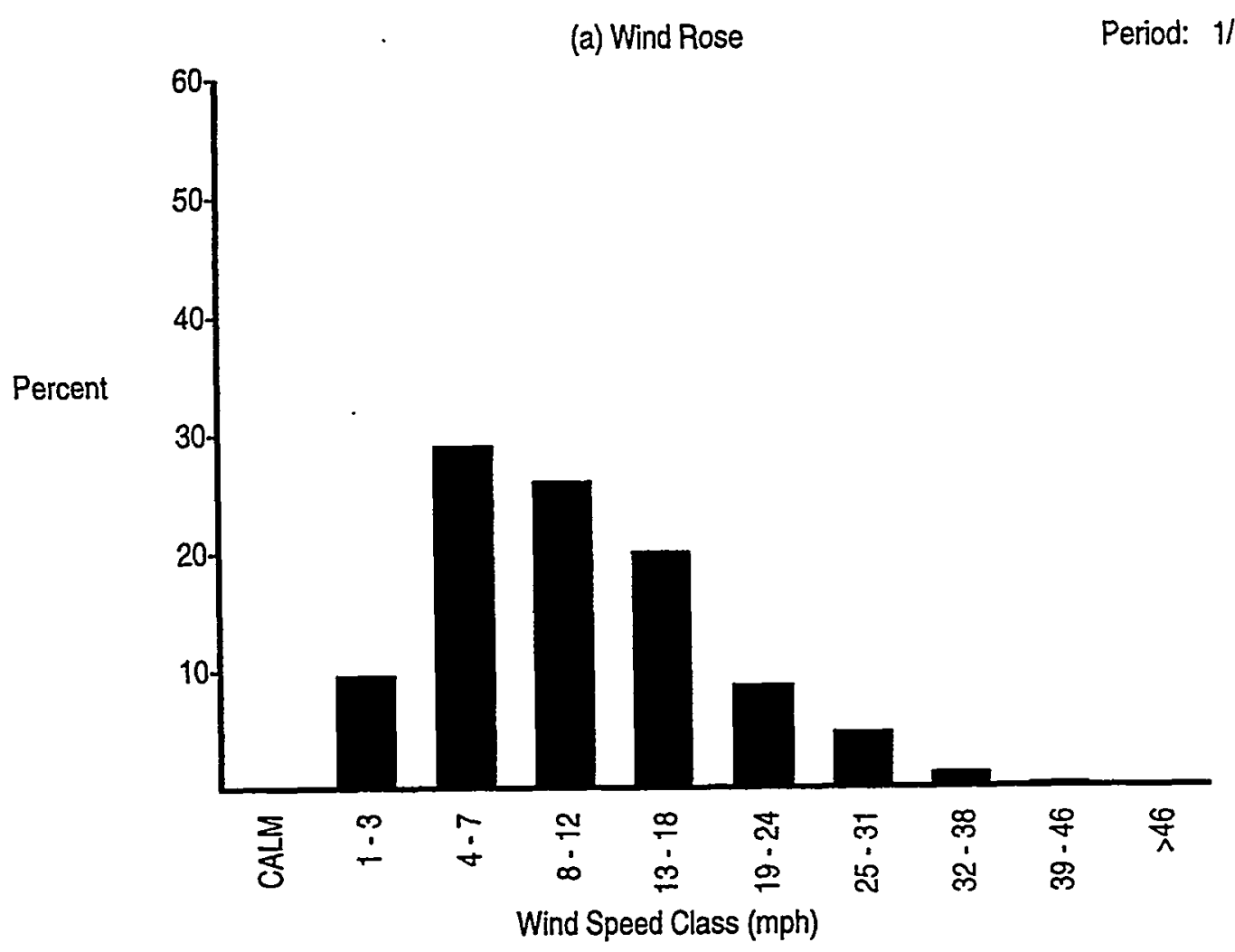

(b) Wind Speed Histogram

Figure A.2. (contd) 


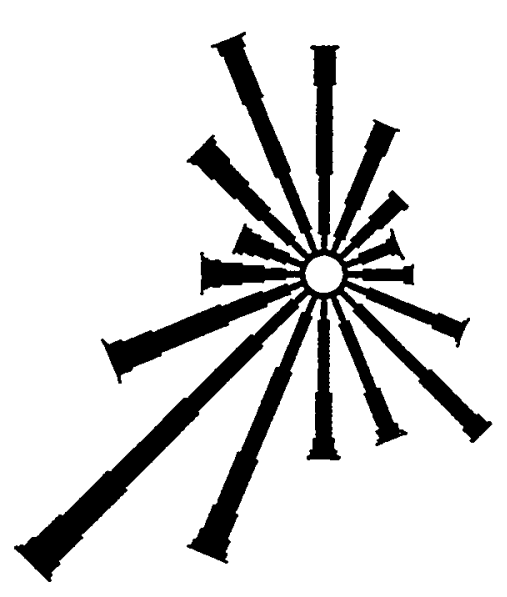

(a) Wind Rose

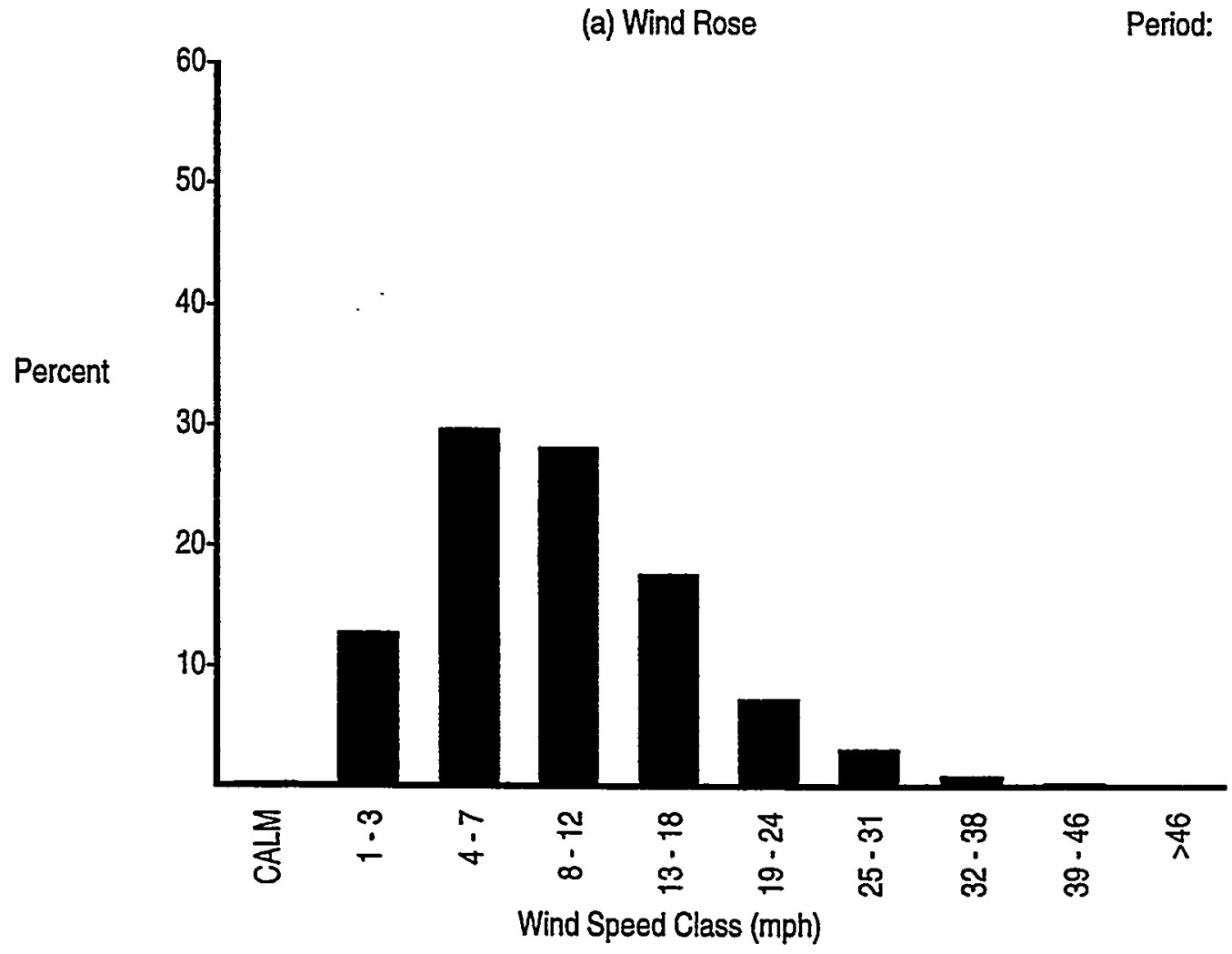

(b) Wind Speed Histogram

Figure A.2. (contd)

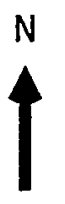

300 Area Tower

Period: 1/99-12/99 


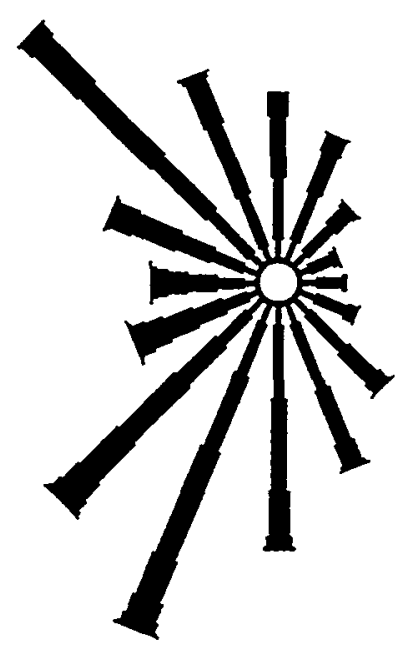

(a) Wind Rose

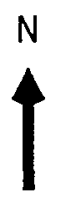

400 Area Tower

Period: $1 / 99-12 / 99$

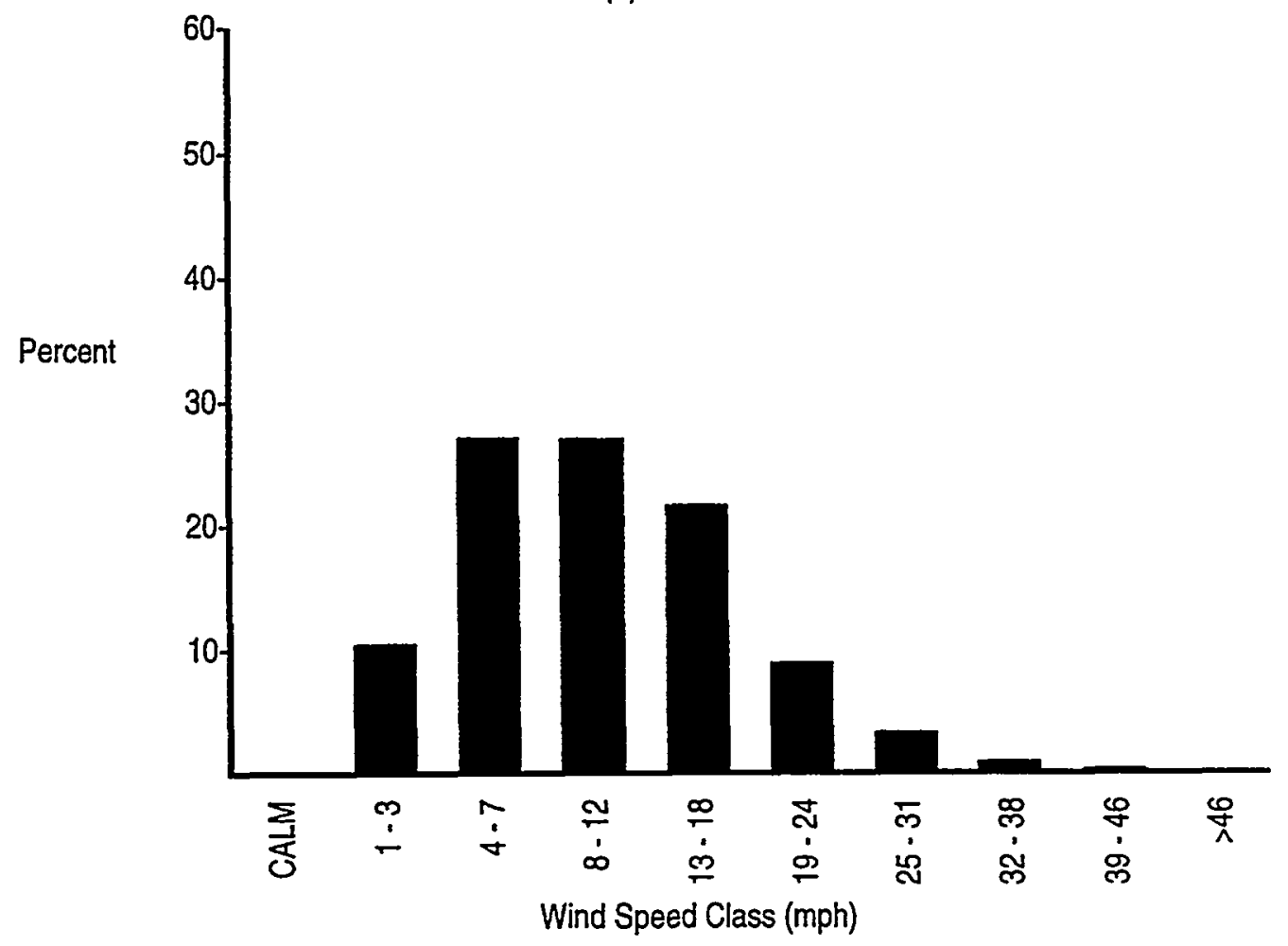

(b) Wind Speed Histogram

Figure A.2. (contd) 
Table A.1. Joint Frequency Distributions (\%) for Hanford Meteorological Monitoring Network Wind Stations at 10-Meter Level, 1999

Station: (1) PROS

Begin: 1/99

End: $12 / 99$

Total Hours: 8742

DIRECTION

\begin{tabular}{|c|c|c|c|c|c|c|c|c|c|c|c|c|c|c|c|c|c|c|}
\hline & $\mathbf{N}$ & NNE & NE & ENE & $E$ & ESE & SE & SSE & $S$ & SSW & SW & WSW & W & WNW & NW & NNW & CALM & TOTAL \\
\hline CALM & 0.0 & 0.0 & 0.0 & 0.0 & 0.0 & 0.0 & 0.0 & 0.0 & 0.0 & 0.0 & 0.0 & 0.0 & 0.0 & 0.0 & 0.0 & 0.0 & 0.5 & 0.5 \\
\hline $1-3$ & 1.5 & .1 & 0.7 & 0.7 & 0.7 & 1.0 & 1.3 & 1.7 & 1.9 & 1.9 & 1.3 & 1.1 & 1.2 & 1.5 & 2.3 & 2.2 & 0.0 & 22.2 \\
\hline $4-7$ & 2.6 & 1.4 & 0.9 & 0.7 & 0.7 & 0.5 & 2.1 & 4.3 & 4.3 & 3. & 1. & 0. & 0.8 & 1. & 3. & 4.3 & 0.0 & 33.3 \\
\hline $8-12$ & 1.7 & 1.0 & 0.4 & 0.1 & 0.2 & 0.2 & 0.8 & 1.7 & 3.2 & 4. & 2. & 1 & 0.7 & 0.5 & & & & 25.7 \\
\hline $13-1$ & 0.7 & 0.3 & 0.0 & 0.0 & 0.0 & 0.0 & 0.0 & 0.1 & 0.7 & 3. & 2.6 & 1. & 0.5 & 0. & 2.0 & 1.2 & 0.0 & 13.5 \\
\hline $19-2$ & 0.0 & 0.0 & 0.0 & 0.0 & 0.0 & 0.0 & 0.0 & 0.0 & 0.1 & 0. & 0.9 & 0. & 0.2 & 0.1 & 0.4 & 0.1 & 0.0 & 3.1 \\
\hline $25-3$ & 0.0 & 0.0 & 0.0 & 0.0 & 0.0 & 0.0 & 0.0 & 0.0 & 0.0 & 0 & 0. & 0 & 0.1 & 0.0 & 0.1 & 0.0 & 0.0 & 1.5 \\
\hline $32-3$ & 0.0 & 0.0 & 0.0 & 0.0 & 0.0 & 0.0 & 0.0 & 0.0 & 0.0 & 0. & 0.1 & 0. & 0.0 & 0. & 0 & 0.0 & & 0.2 \\
\hline & 0.0 & 0.0 & 0.0 & 0.0 & 0.0 & 0.0 & 0.0 & 0.0 & 0.0 & 0.0 & 0.1 & 0. & 0.0 & 0. & 0 & 0.0 & 0.0 & 0.1 \\
\hline & 0.0 & 0.0 & 0.0 & 0.0 & 0.0 & 0.0 & 0.0 & 0.0 & 0.0 & 0.0 & 0.0 & 0.0 & 0.0 & 0.0 & 0.0 & 0.0 & 0.0 & 0.0 \\
\hline OTAL & 6.6 & 3.8 & 2.1 & 1.6 & 1.6 & 2.2 & 4.2 & 7.8 & 10.2 & 14.4 & 9.6 & 5.3 & 3.5 & 3.7 & 11.0 & 11.9 & 0.5 & 100.0 \\
\hline
\end{tabular}

Station: (2) EOC

Begin: $1 / 99$

End: $12 / 99$

Total Hours: 8742

DIRECTION

\begin{tabular}{|c|c|c|c|c|c|c|c|c|c|c|c|c|c|c|c|c|c|c|}
\hline & $\mathrm{N}$ & NNE & NE & ENE & $E$ & ESE & $\mathrm{SE}$ & SSE & $S$ & SSW & SW & WSW & W & WNW & NW & NNW & CALM & TOTAL \\
\hline CALM & 0.0 & 0.0 & 0.0 & 0.0 & 0.0 & 0.0 & 0.0 & 0.0 & 0.0 & 0.0 & 0.0 & 0.0 & 0.0 & 0.0 & 0.0 & 0.0 & 0.8 & 0.8 \\
\hline $1-3$ & 0.9 & 0.7 & 0.8 & 0.7 & 0.8 & 0.8 & 0.8 & 1.1 & 1.4 & & 1.2 & & 1.3 & & & & 0.0 & 16.1 \\
\hline 4-7 & 1.8 & 1.7 & 1.4 & 1.0 & 1.0 & 1. & 1.2 & 1. & 1.7 & 1 & 1.3 & 1 & 2. & & & & 0.0 & 27.0 \\
\hline $8-12$ & 1.5 & 0.7 & 0.3 & 0.1 & 0.1 & 0.1 & 0.3 & 0. & 1.5 & & 2. & 1 & 1. & 4. & 6 & & 0.0 & 27.4 \\
\hline $13-$ & 0.7 & 0.2 & 0.0 & 0.0 & 0.0 & 0.0 & 0.0 & 0.0 & 0.5 & 1. & 2.9 & 3. & 1.7 & 1 & 3 & & 0.0 & 16.5 \\
\hline $19=$ & 0.0 & 0 & 0.0 & 0.0 & 0.0 & 0.0 & 0.0 & 0.0 & 0.1 & 0. & 2.2 & 2.7 & 0.9 & 0.2 & 0 & 0.3 & 0.0 & 7.0 \\
\hline $25-3$ & 0.0 & 0.0 & 0.0 & 0.0 & 0.0 & 0. & 0.0 & 0.0 & 0.0 & 0. & 1.6 & 1.5 & 0.3 & 0.0 & 0 & 0.0 & 0.0 & 3.6 \\
\hline $32-3$ & 0.0 & 0.0 & 0.0 & 0.0 & 0.0 & 0. & 0.0 & 0.0 & 0.0 & 0 & 0.8 & 0 & 0.1 & & & & 0.0 & 1.3 \\
\hline & 0.0 & 0.0 & 0.0 & 0.0 & 0.0 & 0.0 & 0.0 & 0.0 & 0.0 & 0 & 0.2 & 0 & 0. & 0 & & & 0.0 & 0.2 \\
\hline & 0.0 & 0.0 & 0.0 & 0.0 & 0.0 & 0.0 & 0.0 & 0.0 & 0.0 & 0 & 0.0 & 0.0 & 0.0 & 0.0 & 0.0 & 0.0 & 0.0 & 0.0 \\
\hline OTAL & 4.9 & 3.3 & 2.5 & 1.9 & 1.8 & 1.9 & 2.2 & 2.6 & 5.1 & 6.7 & 12.8 & 11.9 & 8.4 & 9.9 & 14.7 & 8.6 & 0.8 & 100.0 \\
\hline
\end{tabular}

Station: (3) ARMY

Begin: 1/99

End: $12 / 99$

Total Hours: 8624

DIRECTION

\begin{tabular}{|c|c|c|c|c|c|c|c|c|c|c|c|c|c|c|c|c|c|c|}
\hline & $\mathrm{N}$ & NNE & NE & ENE & $E$ & ESE & SE & SSE & $\underline{S}$ & SSW & SW & WSW & $\mathrm{W}$ & WNW & NW & NNW & CALM & TOTAL \\
\hline CALM & 0.0 & 0.0 & 0.0 & 0.0 & 0.0 & 0.0 & 0.0 & 0.0 & 0.0 & 0.0 & 0.0 & 0.0 & 0.0 & 0.0 & 0.0 & 0.0 & 0.8 & 0.8 \\
\hline $1-3$ & 1.6 & 1.3 & 1.5 & 1.7 & 2.0 & 2.0 & 1.7 & 0.8 & 0.7 & 0.7 & 0.7 & 1.0 & 1.8 & 2.7 & 3.2 & 2.1 & 0.0 & 25.7 \\
\hline $4-7$ & 2.0 & 1.2 & 1.3 & 1.8 & 2.1 & 2.8 & 2.0 & 0.8 & 0.5 & 0.4 & 0.5 & 0.7 & 2.1 & 6.6 & 8.0 & 3.4 & 0.0 & 36.4 \\
\hline $8-12$ & 1.1 & 0.7 & 0.4 & 0.4 & 0.4 & 1.0 & 1.3 & 0.7 & 0.4 & 0.3 & 0.6 & 1.1 & 2.1 & 4.9 & 4.3 & 2.3 & 0.0 & 22.1 \\
\hline $13-18$ & 0.3 & 0.2 & 0.0 & 0.0 & 0.1 & 0.1 & 0.3 & 0.3 & 0.4 & 0.3 & 0.8 & 1.3 & 1.1 & 1.1 & 1.6 & 1.0 & 0.0 & 9.0 \\
\hline $19-24$ & 0.1 & 0.1 & 0.0 & 0.0 & 0.0 & 0.0 & 0.0 & 0.0 & 0.1 & 0.4 & 0.6 & 0.8 & 0.6 & 0.2 & 0.7 & 0.3 & 0.0 & 3.9 \\
\hline $25-3$ & 0.0 & 0.0 & 0.0 & 0.0 & 0.0 & 0.0 & 0.0 & 0.0 & 0.0 & 0.3 & 0.6 & 0.3 & 0.1 & 0.0 & 0.1 & 0.0 & 0.0 & 1.6 \\
\hline $32-38$ & 0.0 & 0.0 & 0.0 & 0.0 & 0.0 & 0.0 & 0.0 & 0.0 & 0.0 & 0.1 & 0.3 & 0.1 & 0.1 & 0.0 & 0.0 & 0.0 & 0.0 & 0.5 \\
\hline $39-46$ & 0.0 & 0.0 & 0.0 & 0.0 & 0.0 & 0.0 & 0.0 & 0.0 & 0.0 & 0.0 & 0.1 & 0. & 0.0 & 0.0 & 0.0 & 0.0 & 0.0 & 0.1 \\
\hline$>46$ & 0.0 & 0.0 & 0.0 & 0.0 & 0.0 & 0.0 & 0.0 & 0.0 & 0.0 & 0.0 & 0.0 & 0.0 & 0.0 & 0.0 & 0.0 & 0.0 & 0.0 & 0.0 \\
\hline TOTAL & 5.2 & 3.5 & 3.3 & 4.0 & 4.6 & 5.9 & 5.3 & 2.7 & 2.2 & 2.5 & 4.3 & 5.4 & 7.8 & 15.6 & 17.9 & 9.1 & 0.8 & 100.0 \\
\hline
\end{tabular}

Station: (4) RSPG

Begin: 1/99

End: $12 / 99$

Total Hours: 8749

DIRECTION

\begin{tabular}{|c|c|c|c|c|c|c|c|c|c|c|c|c|c|c|c|c|c|c|}
\hline & $\mathrm{N}$ & NNE & NE & ENE & E & ESE & SE & SSE & $S$ & SSW & SW & WSW & W & WNW & NW & NNW & CALM & TOTAL \\
\hline CALM & 0.0 & 0.0 & 0.0 & 0.0 & 0.0 & 0.0 & 0.0 & 0.0 & 0.0 & 0.0 & 0.0 & 0.0 & 0.0 & 0.0 & 0.0 & 0.0 & 0.4 & 0.4 \\
\hline $1-3$ & 1.4 & 1.9 & 1.4 & 1.1 & 1.2 & 1.0 & 1.0 & 0.6 & 0.8 & 0.7 & 1.3 & 1.9 & 1.5 & 1.1 & 0.9 & 1.0 & 0.0 & 18.8 \\
\hline 4-7 & 3.5 & 2.7 & 1.4 & 1.1 & 1.5 & 1.6 & 0.8 & 0.5 & 0.5 & 0.7 & 1.9 & 7.9 & 4.4 & 1.7 & 1.3 & 1.9 & 0.0 & 33.4 \\
\hline $8-12$ & 0.8 & 0.6 & 0.3 & 0.1 & 0.2 & 0.4 & 0.2 & 0.2 & 0.4 & 0.8 & 1.4 & 15.6 & 6.5 & 2.4 & 1.8 & 1.5 & 0.0 & 33.2 \\
\hline $13-18$ & 0.0 & 0.1 & 0.1 & 0.0 & 0.0 & 0.0 & 0.0 & 0.0 & 0.1 & 0.7 & 1.1 & 2.8 & 2.2 & 1.2 & 0.9 & 0.8 & 0.0 & 10.1 \\
\hline $19-24$ & 0.0 & 0.0 & 0.0 & 0.0 & 0.0 & 0.0 & 0.0 & 0.0 & 0.0 & 0.5 & 0.8 & 0.8 & 0.4 & 0.1 & 0.1 & 0.1 & 0.0 & 2.8 \\
\hline $25-31$ & 0.0 & 0.0 & 0.0 & 0.0 & 0.0 & 0.0 & 0.0 & 0.0 & 0.0 & 0.2 & 0.5 & 0.2 & 0.1 & 0.0 & 0.0 & 0.0 & 0.0 & 1.0 \\
\hline $32-38$ & 0.0 & 0.0 & 0.0 & 0.0 & 0.0 & 0.0 & 0.0 & 0.0 & 0.0 & 0.0 & 0.1 & 0.1 & 0.0 & 0.0 & 0.0 & 0.0 & 0.0 & 0.2 \\
\hline $39-46$ & 0.0 & 0.0 & 0.0 & 0.0 & 0.0 & 0.0 & 0.0 & 0.0 & 0.0 & 0.0 & 0.0 & 0.0 & 0.0 & 0.0 & 0.0 & 0.0 & 0.0 & 0.0 \\
\hline$>46$ & 0.0 & 0.0 & 0.0 & 0.0 & 0.0 & 0.0 & 0.0 & 0.0 & 0.0 & 0.0 & 0.0 & 0.0 & 0.0 & 0.0 & 0.0 & 0.0 & 0.0 & 0.0 \\
\hline TOTAL & 5.6 & 5.2 & 3.3 & 2.3 & 2.9 & 3.1 & 2.0 & 1.3 & 1.8 & 3.6 & 7.1 & 29.1 & 15.2 & 6.6 & .5 .1 & 5.3 & 0.4 & 100.0 \\
\hline
\end{tabular}


Table A.1. (contd)

Station: (5) EDNA

DIRECTION

\begin{tabular}{|c|c|c|c|c|c|c|c|c|c|c|c|c|c|c|c|c|c|c|}
\hline & $\mathbf{N}$ & NNE & $\mathrm{NE}$ & ENE & $\mathrm{E}$ & ESE & SE & SSE & $S$ & SSW & SW & WSW & $\mathrm{W}$ & WNW & NW & NNW & CALM & TOTAL \\
\hline CALM & 0.0 & 0.0 & 0.0 & 0.0 & 0.0 & 0.0 & 0.0 & 0.0 & 0.0 & 0.0 & 0.0 & 0.0 & 0.0 & 0.0 & 0.0 & 0.0 & 0.8 & 0.8 \\
\hline 1-3 & 0.7 & 0.4 & 0.5 & 0.7 & 0.9 & 1.5 & 2.8 & 3.1 & 2.6 & 1.6 & 1.3 & 1.4 & 1.6 & 2.7 & 2.8 & 1.5 & 0.0 & 26.3 \\
\hline $4-7$ & 1.5 & 0.9 & 0.8 & 0.8 & 1.6 & 4.9 & 7.2 & 3.7 & 1.4 & 0.7 & 0.7 & 0. & 1.0 & 2 & & 4.5 & 0.0 & 39.7 \\
\hline $8-12$ & 1.2 & 0 & 0.4 & 0.2 & 1.1 & 2. & 1.7 & 1. & 1.1 & 0. & 0.7 & 1 & 1.5 & 2 & 2 & 2 & 0.0 & 20.6 \\
\hline $13-1$ & 0.2 & 0. & 0.1 & 0.0 & 0.1 & 0.1 & 0.3 & 0.6 & 0.7 & 0. & 0.8 & 1. & 0.9 & 1 & 1. & & 0.0 & 8. \\
\hline $19-24$ & 0.0 & 0.0 & 0.0 & 0.0 & 0.0 & 0.0 & 0.0 & 0.0 & 0.2 & 0.4 & 0.4 & 0.4 & 0.4 & 0. & 0. & & 0.0 & 3.1 .1 \\
\hline 25 . & 0.0 & 0.0 & 0.0 & 0.0 & 0.0 & 0.0 & 0.0 & 0.0 & 0.0 & 0.1 & 0.1 & 0.1 & 0.1 & 0.2 & 0.1 & 0.0 & 0.0 & 0.7 \\
\hline & 0.0 & 0 & 0.0 & 0.0 & 0.0 & 0.0 & 0.0 & 0.0 & 0.0 & 0.0 & 0.0 & 0.0 & 0.0 & 0.0 & 0.0 & 0 & 0.0 & \\
\hline & 0.0 & 0 & 0.0 & 0.0 & 0.0 & 0.0 & 0.0 & 0.0 & 0.0 & 0.0 & 0.0 & 0.0 & 0.0 & 0.0 & 0.0 & .0 & 0.0 & 0.0 \\
\hline & 0.0 & 0.0 & 0.0 & 0.0 & 0.0 & 0.0 & 0.0 & 0.0 & 0.0 & 0.0 & 0.0 & 0.0 & 0.0 & 0.0 & 0.0 & & 0.0 & 0.0 \\
\hline TOTAL & 3.6 & 1.9 & 1.8 & 1.8 & 3.7 & 9.0 & 12.1 & 8.4 & 6.0 & 4.4 & 3.9 & 4.8 & 5.5 & 10.2 & 13.6 & 8.5 & 0.8 & 100.0 \\
\hline
\end{tabular}

Station: (6) $200 \mathrm{E}$

DIRECTION

\begin{tabular}{|c|c|c|c|c|c|c|c|c|c|c|c|c|c|c|c|c|c|c|}
\hline & $\mathbf{N}$ & NNE & NE & ENE & E & ESE & $\mathrm{SE}$ & SSE & S & SSW & SW & WSW & W & WNW & NW & NNW & CALM & TOTAL \\
\hline CALM & 0.0 & 0.0 & 0.0 & 0.0 & 0.0 & 0.0 & 0.0 & 0.0 & 0.0 & 0.0 & 0.0 & 0.0 & 0.0 & 0.0 & 0.0 & 0.0 & 2.5 & 2.5 \\
\hline $1-3$ & 1.0 & 0.8 & 0.9 & 1.0 & 1.5 & 1.3 & 1.5 & 1.3 & 1.3 & 0.8 & 0.9 & 0.9 & 1.2 & 1.3 & 1.2 & 1.1 & 0.0 & 17.9 \\
\hline 4-7 & 1.5 & 1.2 & 1.0 & 0.8 & 1.1 & 1.8 & 2.9 & 2.6 & 1.5 & 1. & 1.5 & 2. & 4.2 & & 3 & & 0.0 & 34.2 \\
\hline $8-1$ & 0.7 & 0.8 & 0.5 & 0.2 & 0.3 & 0. & 1.0 & 1.2 & 0.8 & 0. & 1.2 & 2. & 5. & & & & 0.0 & 27.1 \\
\hline 13. & 0.1 & 0.2 & 0.0 & 0.0 & 0.0 & 0. & 0.1 & 0.4 & 0.5 & 0.5 & 1.3 & 1.7 & 1.6 & 3. & 1.6 & 0.1 & 0.0 & 11.8 \\
\hline & 0.0 & 0.0 & 0.0 & 0.0 & 0.0 & 0.0 & 0.0 & 0.0 & 0.1 & 0.3 & 0.9 & 0.9 & 0.4 & & 0.9 & 0.0 & 0.0 & 4. \\
\hline & 0.0 & 0.0 & 0.0 & 0.0 & 0.0 & 0.0 & 0.0 & 0.0 & 0.0 & 0.2 & 0.4 & 0. & 0.2 & 0. & 0.2 & 0 & 0.0 & \\
\hline & 0 & 0.0 & 0.0 & 0. & 0.0 & 0 & 0.0 & 0.0 & 0.0 & 0. & 0. & 0 & 0.0 & 0 & & & 0.0 & \\
\hline & 0.0 & 0.0 & 0.0 & 0. & 0.0 & 0. & 0.0 & 0.0 & 0.0 & 0. & 0 & 0 & 0.0 & 0 & & & 1.0 & \\
\hline & 0.0 & 0.0 & 0.0 & 0.0 & 0.0 & 0.0 & 0.0 & 0.0 & 0.0 & 0.0 & 0.0 & 0.0 & 0.0 & 0.0 & 0. & 0.0 & 0.0 & \\
\hline TOTAL & 3.3 & 3.0 & 2.5 & 2.0 & 2.9 & 3.6 & 5.5 & 5.5 & 4.3 & 3.7 & 6.1 & 9.3 & 12.6 & 19.8 & 9.8 & 3.6 & 2.5 & $100 . C$ \\
\hline
\end{tabular}

Station: (7) $200 \mathrm{~W}$

DIRECTION

\begin{tabular}{|c|c|c|c|c|c|c|c|c|c|c|c|c|c|c|c|c|c|c|}
\hline & $\mathrm{N}$ & NNE & $\mathrm{NE}$ & ENE & $E$ & ESE & SE & SSE & $S$ & SSW & SW & WSW & W & WNW & NW & NNW & CALM & TOTAL \\
\hline CALM & 0.0 & 0.0 & 0.0 & 0.0 & 0.0 & 0.0 & 0.0 & 0.0 & 0.0 & 0.0 & 0.0 & 0.0 & 0.0 & 0.0 & 0.0 & 0.0 & 1.5 & 1.5 \\
\hline 1-3 & 2.1 & 1.6 & 1.5 & 1.4 & 1.6 & 1.8 & 2.0 & 1.6 & 1.6 & 1. & 1. & 1.5 & 2.6 & 3. & 3.5 & 2.5 & 0.0 & 32.2 \\
\hline 4-7 & 3.2 & 1.6 & 1.3 & 0.9 & 1.3 & 1.8 & 1.4 & 0.9 & 0.7 & 0. & 0.8 & 1.6 & 3.6 & 6.1 & 4. & & 0.0 & 3.6 \\
\hline $8-12$ & 0.9 & 0.5 & 0.3 & 0.1 & 0.2 & 0.5 & 0.4 & 0.3 & 0.4 & 0.6 & 1.5 & 1.8 & 2.3 & 3.2 & 3.2 & & 0.0 & 18.6 \\
\hline 13. & 0.1 & 0.1 & 0.0 & 0.0 & 0.0 & 0.0 & 0.1 & 0.0 & 0.3 & 0.7 & 1.5 & 1. & 1.1 & 0.7 & 2. & 0.6 & 0.0 & 9.0 \\
\hline & 0.0 & 0.0 & 0.0 & 0.0 & 0.0 & 0 & 0.0 & 0. & 0. & 0 & 0. & 0. & 0.4 & 0.1 & 0. & & 0.0 & \\
\hline $25-3$ & 0.0 & 0.0 & 0.0 & 0.0 & 0.0 & 0. & 0.0 & 0. & 0.0 & 0. & 0. & 0. & 0 . & 0 & 0 & & .0 & \\
\hline & 0.0 & 0.0 & 0.0 & 0.0 & 0.0 & 0. & 0.0 & 0.0 & 0.0 & 0.0 & 0.1 & 0.1 & 0.0 & 0. & 0. & 0 & 0.0 & \\
\hline & 0.0 & 0.0 & 0.0 & 0.0 & 0.0 & 0.0 & 0.0 & 0.0 & 0.0 & 0.0 & 0.0 & 0.0 & 0.0 & 0.0 & 0.0 & 0.0 & 0.0 & \\
\hline$>46$ & 0.0 & 0.0 & 0.0 & 0.0 & 0.0 & 0.0 & 0.0 & 0.0 & 0.0 & 0.0 & 0.0 & 0.0 & 0.0 & 0.0 & 0.0 & 0.0 & 0.0 & \\
\hline TOTAL & 6.4 & 3.7 & 3.1 & 2.4 & 3.1 & 4.1 & 3.8 & 2.9 & 3.1 & 3.7 & 6.5 & 8.1 & 10.1 & 13.9 & 14.8 & 8.6 & 1.5 & 100. \\
\hline
\end{tabular}

Station: (8) BVLY

\begin{tabular}{|c|c|c|c|c|c|c|c|c|c|c|c|c|c|c|c|c|c|c|}
\hline & $\mathrm{N}$ & NNE & $\mathrm{NE}$ & ENE & E & ESE & $\mathrm{SE}$ & SSE & S & SSW & SW & WSW & $\mathrm{W}$ & WNW & NW & NNW & CALM & TOTAL \\
\hline CALM & 0.0 & 0.0 & 0.0 & 0.0 & 0.0 & 0.0 & 0.0 & 0.0 & 0.0 & 0.0 & 0.0 & 0.0 & 0.0 & 0.0 & 0.0 & 0.0 & 0.8 & 0.8 \\
\hline $1-3$ & 2.4 & 2.0 & 1.6 & 1.2 & 1.7 & & 1.7 & 1.5 & 1.8 & 1.3 & 1.0 & 1.1 & 1.2 & 1.5 & 2 & 2.3 & 0.0 & 26.3 \\
\hline $4-7$ & 7.1 & 2.1 & 0.6 & 0.4 & 1.2 & 3.1 & 1.5 & 1.2 & 1.4 & 0.9 & 0.6 & 0. & 1.5 & & 4.8 & 7.2 & .0 & 37.0 \\
\hline $8-12$ & 5.7 & 1.3 & 0.1 & 0.0 & 0.2 & 0.6 & 0.4 & 0.4 & 0.4 & 0.3 & 0.5 & 0. & 1.5 & 4.7 & 4.1 & 1.8 & 0.0 & 22.4 \\
\hline $13-18$ & 0.1 & 0.1 & 0.0 & 0.0 & 0.0 & 0.0 & 0.2 & 0.3 & 0.4 & 0.3 & 0.8 & 0.3 & 0.5 & 4.2 & 2.6 & 0.1 & 0.0 & 10.0 \\
\hline & 0.0 & 0.0 & 0.0 & 0.0 & 0.0 & 0. & 0.0 & 0.1 & 0.0 & 0.1 & 0.3 & 0. & 0.2 & 1 & 0 & 0.0 & 0.0 & 2.7 \\
\hline $25-3$ & 0.0 & 0.0 & 0.0 & 0.0 & 0.0 & 0.0 & 0.0 & 0.0 & 0.0 & 0.0 & 0.2 & 0.2 & 0.0 & 0. & 0. & 0.0 & 0.0 & \\
\hline $32-=$ & 0.0 & 0.0 & 0.0 & 0.0 & 0.0 & 0.0 & 0.0 & 0.0 & 0.0 & 0.0 & 0.0 & 0.0 & 0.0 & 0.0 & 0.0 & 0.0 & 0.0 & \\
\hline & 0.0 & 0.0 & 0.0 & 0.0 & 0.0 & 0.0 & 0.0 & 0.0 & 0.0 & 0.0 & 0.0 & 0.0 & 0.0 & 0.0 & 0.0 & 0.0 & 0.0 & \\
\hline$>46$ & 0.0 & 0.0 & 0.0 & 0.0 & 0.0 & 0. & 0.0 & 0.0 & 0.0 & 0.0 & 0.0 & 0.0 & 0.0 & 0.0 & 0.0 & 0.0 & 0.0 & \\
\hline TOTAL & 15.3 & 5.4 & 2.3 & 1.6 & 3.1 & 5.7 & 3.7 & 3.6 & 4.1 & 3.0 & 3.5 & 2.9 & 4.9 & 14.6 & 14.3 & 11.3 & 0.8 & 100. \\
\hline
\end{tabular}


Table A.1. (contd)

Station: (9) FFTF

Begin: 1/99

End: 12/99

Total Hours: 8740

DIRECTION

\begin{tabular}{|c|c|c|c|c|c|c|c|c|c|c|c|c|c|c|c|c|c|c|}
\hline & $\mathrm{N}$ & NNE & NE & ENE & $E$ & ESE & SE & SSE & S & SSW & SW & VSW & W & WNW & NW & NNW & CALM & TOTAI \\
\hline CALM & 0.0 & 0.0 & 0.0 & 0.0 & 0.0 & 0.0 & 0.0 & 0.0 & 0.0 & 0.0 & 0.0 & 0.0 & 0.0 & 0.0 & 0.0 & 0.0 & 1.1 & 1.1 \\
\hline $1-3$ & 1.0 & 1.0 & 0.8 & 0.8 & 0.7 & 0.6 & 0.9 & 0.9 & 1.2 & 1.1 & 1. & 0 & 0.9 & 1. & 0.9 & 1.0 & 0.0 & 14.5 \\
\hline $4-7$ & 2.7 & 2.4 & 1.5 & 1.0 & 1.0 & 1.5 & 2.6 & 3.5 & 3.9 & 3.1 & 1.9 & 1.2 & 1.1 & 1.7 & 3.7 & 3.3 & 0.0 & 66 \\
\hline $8-12$ & 1.5 & 1.5 & 0.6 & 0.2 & 0.1 & 0.3 & 1.4 & 3.1 & 3.9 & 4.2 & 1.9 & 1.1 & 0.9 & 1.5 & 4.0 & 3.0 & & \\
\hline $13-1$ & 0.2 & 0.4 & 0.1 & 0.0 & 0.0 & 0.0 & 0.2 & 0.4 & 1.3 & 3.5 & 2.6 & 0. & 0.5 & 0. & 1.8 & 0.6 & 0 & \\
\hline $19-2$ & 0.0 & 0.0 & 0.0 & 0.0 & 0.0 & 0.0 & 0.0 & 0.1 & 0.2 & 1.0 & 1.1 & 0 & 0.3 & 0.2 & 0.5 & 0.1 & 0.0 & \\
\hline $25-31$ & 0.0 & 0.0 & 0.0 & 0.0 & 0.0 & 0.0 & 0.0 & 0.0 & 0.0 & 0.3 & 0.5 & 0.2 & 0.1 & 0.0 & 0.1 & 0.0 & 0.0 & \\
\hline $32-38$ & 0.0 & 0.0 & 0.0 & 0.0 & 0.0 & 0.0 & 0.0 & 0.0 & 0.0 & 0.1 & 0.2 & 0.1 & 0.0 & 0.0 & 0.0 & 0.0 & 0.0 & \\
\hline & 0.0 & 0.0 & 0.0 & 0.0 & 0.0 & 0.0 & 0.0 & 0.0 & 0.0 & 0.0 & 0.0 & 0.0 & 0.0 & 0.0 & 0.0 & 0.0 & 0.0 & \\
\hline & 0.0 & 0.0 & 0.0 & 0.0 & 0.0 & 0.0 & 0.0 & 0.0 & 0.0 & 0.0 & 0.0 & 0. & 0.0 & 0.0 & 0.0 & 0.0 & 0.0 & \\
\hline OTAL & 5.4 & 5.3 & 3.0 & 2.0 & 1.8 & 2.4 & 5.1 & 8.0 & 10.5 & 13.3 & 9.2 & 4.8 & 3.9 & 5.2 & 11.1 & 8.0 & 1.1 & \\
\hline
\end{tabular}

Station: (10) YAKB

Begin: $1 / 99$

End: 12/99

Total Hours: 8742

DIRECTION

\begin{tabular}{|c|c|c|c|c|c|c|c|c|c|c|c|c|c|c|c|c|c|c|}
\hline & $\mathbf{N}$ & NNE & $\mathrm{NE}$ & ENE & $\mathrm{E}$ & ESE & SE & SSE & S & SSW & SW & WSW & W & WNW & NW & NNW & CALM & TOTAL \\
\hline CALM & 0.0 & 0.0 & 0.0 & 0.0 & 0.0 & 0.0 & 0.0 & 0.0 & 0.0 & 0.0 & 0.0 & 0.0 & 0.0 & 0.0 & 0.0 & 0.0 & 0.3 & 0.3 \\
\hline $1-3$ & 1.2 & 1.0 & 1.1 & 0.9 & 0.7 & 0.7 & 0.8 & 0.9 & 0.9 & 0.9 & 1.2 & 1.5 & 1.8 & 1.6 & 1.2 & 1.3 & 0.0 & 17.7 \\
\hline $4-7$ & 3.8 & 3.3 & 1.6 & 1.0 & 0.8 & 0.9 & 1.3 & 1.1 & 0.6 & 0.7 & 1.1 & 3.8 & 7.4 & $4.0^{\circ}$ & 3.2 & 3.1 & 0.0 & 37.6 \\
\hline $8-12$ & 1.3 & 0.7 & 0.3 & 0.1 & 0.1 & 0.2 & 0.4 & 0.3 & 0.6 & 0.6 & 1.5 & 3.0 & 4.5 & 2.6 & 5.8 & 2.6 & 0.0 & 24.6 \\
\hline $13-18$ & 0.1 & 0.1 & 0.0 & 0.0 & 0.0 & 0.0 & 0.1 & 0.2 & 0.4 & 0.5 & 1.5 & 2.0 & 0.8 & 1.3 & 5.1 & 0.7 & 0.0 & 12.9 \\
\hline $19-24$ & 0.0 & 0.0 & 0.0 & 0.0 & 0.0 & 0.0 & 0.0 & 0.0 & 0.1 & 0.4 & 0.8 & 0.7 & 0.2 & 0.4 & 2.5 & 0.1 & 0.0 & \\
\hline $25-31$ & 0.0 & 0.0 & 0.0 & 0.0 & 0.0 & 0.0 & 0.0 & 0.0 & 0.0 & 0.2 & 0.4 & 0.2 & 0.1 & 0.0 & 0.5 & 0.0 & 0.0 & \\
\hline $32-38$ & 0.0 & 0.0 & 0.0 & 0.0 & 0.0 & 0.0 & 0.0 & 0.0 & 0.0 & 0.0 & 0.1 & 0.1 & 0.0 & 0.0 & 0.0 & 0.0 & 0.0 & \\
\hline $39-46$ & 0.0 & 0.0 & 0.0 & 0.0 & 0.0 & 0.0 & 0.0 & 0.0 & 0.0 & 0.0 & 0.0 & 0.0 & 0.0 & 0.0 & 0.0 & 0.0 & 0.0 & \\
\hline$>46$ & 0.0 & 0.0 & 0.0 & 0.0 & 0.0 & 0.0 & 0.0 & 0.0 & 0.0 & 0.0 & 0.0 & 0.0 & 0.0 & 0.0 & 0.0 & 0.0 & 0.0 & \\
\hline TOTAL & 6.4 & 5.1 & 3.0 & 2.0 & 1.6 & 1.8 & 2.4 & 2.5 & 2.7 & 3.4 & 6.7 & 11.2 & 14.8 & 10.0 & 18.3 & 7.9 & 0.3 & \\
\hline
\end{tabular}

Station: (11) 300A

Begin: 1/99

End: 12/99

Total Hours: 8723

DIRECTION

\begin{tabular}{|c|c|c|c|c|c|c|c|c|c|c|c|c|c|c|c|c|c|c|}
\hline & $\mathbf{N}$ & NNE & $\mathrm{NE}$ & ENE & E & ESE & SE & SSE & S & SSW & SW & WSW & W & WNW & NW & NNW & CALM & TOTAL \\
\hline CALM & 0.0 & 0.0 & 0.0 & 0.0 & 0.0 & 0.0 & 0.0 & 0.0 & 0.0 & 0.0 & 0.0 & 0.0 & 0.0 & 0.0 & 0.0 & 0.0 & 0.3 & 0.3 \\
\hline $1-3$ & 1.4 & 0.7 & 0.7 & 0.6 & 0.7 & 1.1 & 1.5 & 1.6 & 1.3 & 1.2 & 1.1 & 1.1 & 1.1 & 1.2 & 1.6 & 1.4 & 0.0 & 18.4 \\
\hline 4-7 & 3.7 & 1.4 & 0.9 & 1.1 & 1.8 & 4.1 & 6.1 & 3.3 & 2.9 & 2.2 & 1.6 & 0.9 & 0.8 & 0.7 & 2.0 & 3.6 & 0.0 & 37. \\
\hline $8-12$ & 4.0 & 2.0 & 0.8 & 0.2 & 0.4 & 1.3 & 1.6 & 0.9 & 1.9 & 3.3 & 3.4 & 1.8 & 0.6 & 0.4 & 0.9 & 2.5 & 0.0 & \\
\hline $13-18$ & 0.6 & 0.5 & 0.1 & 0.0 & 0.0 & 0.0 & 0.3 & 0.3 & 0.6 & 2.1 & 3.3 & 1.7 & 0.5 & 0.4 & 0.9 & 0.9 & 0.0 & \\
\hline $19-24$ & 0.0 & 0.0 & 0.0 & 0.0 & 0.0 & 0.0 & 0.0 & 0.1 & 0.2 & 0.8 & 1.4 & 0.7 & 0.3 & 0.1 & 0.4 & 0.1 & 0.0 & \\
\hline $25-31$ & 0.0 & 0.0 & 0.0 & 0.0 & 0.0 & 0.0 & 0.0 & 0.0 & 0.0 & 0.3 & 0.6 & 0.2 & 0.1 & 0.0 & 0.0 & 0.0 & 0.0 & \\
\hline $32-38$ & 0.0 & 0.0 & 0.0 & 0.0 & 0.0 & 0.0 & 0.0 & 0.0 & 0.0 & 0.1 & 0.2 & 0.0 & 0.0 & 0.0 & 0.0 & 0.0 & 0.0 & \\
\hline $39-46$ & 0.0 & 0.0 & 0.0 & 0.0 & 0.0 & 0.0 & 0.0 & 0.0 & 0.0 & 0.0 & 0.0 & 0.0 & 0.0 & 0.0 & 0.0 & 0.0 & 0.0 & \\
\hline$>46$ & 0.0 & 0.0 & 0.0 & 0.0 & 0.0 & 0.0 & 0.0 & 0.0 & 0.0 & 0.0 & 0.0 & 0.0 & 0.0 & 0.0 & 0.0 & 0.0 & 0.0 & \\
\hline TOTAL & 9.7 & 4.6 & 2.5 & 2.0 & 2.9 & 6.6 & 9.5 & 6.2 & 7.0 & 9.9 & 11.6 & 6.5 & 3.4 & 2.8 & 5.9 & 8.6 & 0.3 & \\
\hline
\end{tabular}

Station: (12) WYEB

Begin: 1/99

End: $12 / 99$

Total Hours: 8732

DIRECTION

\begin{tabular}{|c|c|c|c|c|c|c|c|c|c|c|c|c|c|c|c|c|c|c|}
\hline & $\mathbf{N}$ & NNE & NE & ENE & $E$ & ESE & SE & SSE & S & SSW & SW & WSW & W & WNW & NW & NNW & CALM & TOTAL \\
\hline CALM & 0.0 & 0.0 & 0.0 & 0.0 & 0.0 & 0.0 & 0.0 & 0.0 & 0.0 & 0.0 & 0.0 & 0.0 & 0.0 & 0.0 & 0.0 & 0.0 & 0.5 & 0.5 \\
\hline 1-3 & 1.3 & 1.0 & 1.1 & 1.1 & 1.3 & 1.4 & 1.3 & 1.2 & 1.2 & 1. & 1.0 & & 1.0 & 1.1 & 1.3 & 1.1 & 0.0 & 18. \\
\hline $4-7$ & 2.5 & 1.7 & 1.5 & 1.5 & 2.1 & 2.3 & 2.8 & 3.5 & 3.6 & 2.4 & 1.6 & 1.7 & 1.8 & 2.8 & 3.7 & 3.0 & 0.0 & \\
\hline $8-12$ & 1.2 & 1.0 & 0.4 & 0.2 & 0.3 & 0.4 & 1.2 & 2.6 & 3.5 & 2.1 & 1.1 & 1.3 & 1.8 & 4.5 & 3.5 & 1.5 & 0.0 & \\
\hline $13-1$ & 0.3 & 0.2 & 0.1 & 0.0 & 0.0 & 0.0 & 0.1 & 0.4 & 1.4 & 1.9 & 1.3 & 0. & 0.9 & & 1.5 & 0.3 & 0.0 & \\
\hline $19-24$ & 0.0 & 0.0 & 0.0 & 0.0 & 0.0 & 0.0 & 0.0 & 0.0 & 0.2 & 1. & 0.7 & 0.5 & 0.4 & 0.3 & 0.9 & 0.1 & .0 & \\
\hline $25-31$ & 0.0 & 0.0 & 0.0 & 0.0 & 0.0 & 0.0 & 0.0 & 0.0 & 0.0 & 0.3 & 0.2 & 0.2 & 0.1 & 0.0 & 0.2 & 0.0 & 0.0 & \\
\hline $32-38$ & 0.0 & 0.0 & 0.0 & 0.0 & 0.0 & 0.0 & 0.0 & 0.0 & 0.0 & 0.1 & 0.1 & 0.0 & 0.0 & 0.0 & 0.0 & 0.0 & 0.0 & \\
\hline & 0.0 & 0.0 & 0.0 & 0.0 & 0.0 & 0.0 & 0.0 & 0.0 & 0.0 & 0.0 & 0.0 & 0.0 & 0.0 & 0.0 & 0.0 & 0.0 & 0.0 & \\
\hline & 0.0 & 0.0 & 0.0 & 0.0 & 0.0 & 0.0 & 0.0 & 0.0 & 0.0 & 0.0 & 0.0 & 0.0 & 0.0 & 0.0 & 0.0 & 0.0 & 0.0 & \\
\hline OTAL & 5.3 & 3.9 & 3.0 & 2.9 & 3.7 & 4.2 & 5.3 & 7.8 & 10.0 & 9.1 & 5.9 & 5.3 & 6.0 & 9.9 & 11.1 & 6.0 & 0.5 & 100 \\
\hline
\end{tabular}


Table A.1. (contd)

Station: (13) $100 \mathrm{~N}$

Begin: $1 / 99$

End: $12 / 99$

Total Hours: 8648

DIRECTION

$N$ NNE NE ENE E ESE SE SSE S SSW SW WSW W WNW NW NNW CALM TOTAL

\begin{tabular}{rrrrrrrrrrrrrrrrrrr}
\hline CALM & 0.0 & 0.0 & 0.0 & 0.0 & 0.0 & 0.0 & 0.0 & 0.0 & 0.0 & 0.0 & 0.0 & 0.0 & 0.0 & 0.0 & 0.0 & 0.0 & 0.7 & 0.7 \\
$1-3$ & 2.1 & 1.9 & 2.0 & 2.3 & 2.7 & 2.7 & 2.3 & 1.3 & 1.4 & 1.2 & 1.6 & 2.1 & 3.3 & 2.9 & 3.2 & 2.6 & 0.0 & 35.7 \\
$4-7$ & 1.2 & 1.5 & 2.0 & 2.4 & 2.7 & 2.4 & 2.3 & 1.2 & 0.7 & 0.8 & 2.0 & 4.1 & 5.0 & 3.1 & 1.7 & 1.4 & 0.0 & 34.4 \\
$8-12$ & 0.3 & 1.1 & 0.8 & 0.3 & 0.1 & 0.3 & 0.9 & 0.7 & 0.5 & 0.5 & 1.2 & 2.5 & 3.6 & 2.3 & 0.5 & 0.2 & 0.0 & 15.8 \\
$13-18$ & 0.1 & 0.2 & 0.1 & 0.1 & 0.0 & 0.0 & 0.2 & 0.3 & 0.3 & 0.4 & 1.1 & 0.7 & 1.4 & 2.4 & 0.8 & 0.1 & 0.0 & 8.3 \\
$19-24$ & 0.0 & 0.0 & 0.0 & 0.0 & 0.0 & 0.0 & 0.0 & 0.0 & 0.2 & 0.3 & 0.7 & 0.3 & 0.4 & 1.3 & 0.6 & 0.0 & 0.0 & 3.9 \\
$25-31$ & 0.0 & 0.0 & 0.0 & 0.0 & 0.0 & 0.0 & 0.0 & 0.0 & 0.0 & 0.2 & 0.1 & 0.1 & 0.1 & 0.4 & 0.1 & 0.0 & 0.0 & 1.1 \\
$32-38$ & 0.0 & 0.0 & 0.0 & 0.0 & 0.0 & 0.0 & 0.0 & 0.0 & 0.0 & 0.0 & 0.0 & 0.0 & 0.0 & 0.0 & 0.0 & 0.0 & 0.0 & 0.1 \\
$39-46$ & 0.0 & 0.0 & 0.0 & 0.0 & 0.0 & 0.0 & 0.0 & 0.0 & 0.0 & 0.0 & 0.0 & 0.0 & 0.0 & 0.0 & 0.0 & 0.0 & 0.0 & 0.0 \\
$>46$ & 0.0 & 0.0 & 0.0 & 0.0 & 0.0 & 0.0 & 0.0 & 0.0 & 0.0 & 0.0 & 0.0 & 0.0 & 0.0 & 0.0 & 0.0 & 0.0 & 0.0 & 0.0 \\
TOTAL & 3.7 & 4.7 & 4.9 & 5.1 & 5.6 & 5.3 & 5.6 & 3.5 & 3.2 & 3.5 & 6.8 & 9.7 & 13.9 & 12.6 & 7.0 & 4.3 & 0.7 & 100.0
\end{tabular}

Station: (14) WPPS

Begin: 1/99

End: 12/99

Total Hours: 8742

DIRECTION

$N$ NNE NE ENE E ESE SE SSE S SSW SW WSW W WNW NW NNW CALM TOTAL

\begin{tabular}{rrrrrrrrrrrrrrrrrrr}
\hline CALM & 0.0 & 0.0 & 0.0 & 0.0 & 0.0 & 0.0 & 0.0 & 0.0 & 0.0 & 0.0 & 0.0 & 0.0 & 0.0 & 0.0 & 0.0 & 0.0 & 1.0 & 1.0 \\
$1-3$ & 2.2 & 1.8 & 1.8 & 1.0 & 0.9 & 1.0 & 1.1 & 1.1 & 1.4 & 1.1 & 1.1 & 1.3 & 1.1 & 1.3 & 1.9 & 2.3 & 0.0 & 22.6 \\
$4-7$ & 3.5 & 2.2 & 2.0 & 1.2 & 0.7 & 0.7 & 1.6 & 3.8 & 4.7 & 2.5 & 1.6 & 1.2 & 1.3 & 1.5 & 3.8 & 4.5 & 0.0 & 36.7 \\
$8-12$ & 1.3 & 1.1 & 0.5 & 0.2 & 0.2 & 0.3 & 1.0 & 3.1 & 4.2 & 2.6 & 1.7 & 1.3 & 1.2 & 1.9 & 2.6 & 1.5 & 0.0 & 24.6 \\
$13-18$ & 0.2 & 0.3 & 0.0 & 0.0 & 0.0 & 0.0 & 0.1 & 0.4 & 1.2 & 2.6 & 1.9 & 0.7 & 0.6 & 0.7 & 1.7 & 0.3 & 0.0 & 10.8 \\
$19-24$ & 0.0 & 0.0 & 0.0 & 0.0 & 0.0 & 0.0 & 0.0 & 0.0 & 0.1 & 0.8 & 0.7 & 0.4 & 0.4 & 0.2 & 0.8 & 0.1 & 0.0 & 3.3 \\
$25-31$ & 0.0 & 0.0 & 0.0 & 0.0 & 0.0 & 0.0 & 0.0 & 0.0 & 0.0 & 0.2 & 0.3 & 0.1 & 0.1 & 0.0 & 0.2 & 0.0 & 0.0 & 0.9 \\
$32-38$ & 0.0 & 0.0 & 0.0 & 0.0 & 0.0 & 0.0 & 0.0 & 0.0 & 0.0 & 0.0 & 0.0 & 0.0 & 0.0 & 0.0 & 0.0 & 0.0 & 0.0 & 0.1 \\
$39-46$ & 0.0 & 0.0 & 0.0 & 0.0 & 0.0 & 0.0 & 0.0 & 0.0 & 0.0 & 0.0 & 0.0 & 0.0 & 0.0 & 0.0 & 0.0 & 0.0 & 0.0 & 0.0 \\
$>46$ & 0.0 & 0.0 & 0.0 & 0.0 & 0.0 & 0.0 & 0.0 & 0.0 & 0.0 & 0.0 & 0.0 & 0.0 & 0.0 & 0.0 & 0.0 & 0.0 & 0.0 & 0.0 \\
TOTAL & 7.3 & 5.4 & 4.3 & 2.5 & 1.8 & 2.0 & 3.9 & 8.4 & 11.6 & 9.9 & 7.3 & 4.9 & 4.6 & 5.5 & 10.9 & 8.7 & 1.0 & 100.0
\end{tabular}

Station: (15) FRNK

Begin: 1/99

DIRECTION

End: 12/99

Total Hours: 8739

\begin{tabular}{|c|c|c|c|c|c|c|c|c|c|c|c|c|c|c|c|c|c|c|}
\hline & $\mathrm{N}$ & NNE & $\mathrm{NE}$ & ENE & $E$ & ESE & SE & SSE & $S$ & SSW & SW & WSW & $\mathrm{W}$ & WNW & NW & NNW & CALM & TOTAL \\
\hline CALM & 0.0 & 0.0 & 0.0 & 0.0 & 0.0 & 0.0 & 0.0 & 0.0 & 0.0 & 0.0 & 0.0 & 0.0 & 0.0 & 0.0 & 0.0 & 0.0 & 0.3 & 0.3 \\
\hline $1-3$ & 1.3 & 1.1 & 0.7 & 0.6 & 0.8 & 1.0 & 1.1 & 1.2 & 1.0 & 1.0 & 1.1 & 1.0 & 1.2 & & 1.6 & 1.4 & 0.0 & 17.3 \\
\hline $4-7$ & 4.4 & 2.3 & 1.5 & 1.1 & 1.4 & 2.4 & 4.3 & 3.5 & 3.3 & 2.7 & 2.2 & 1. & 1.4 & 1. & 5.3 & 5.6 & 0.0 & 45.1 \\
\hline $8-1$ & 1.3 & 0.6 & 0.4 & 0.3 & 0.4 & 1.1 & 2.0 & 2.2 & 2.9 & 5.6 . & 3.8 & 1. & 0.6 & 0. & 1.9 & 2.2 & 0.0 & 27.0 \\
\hline $13-$ & 0.1 & 0.1 & 0.1 & 0.1 & 0.0 & 0.0 & 0.3 & 0.3 & 0.7 & 3.0 & 1.9 & 0.8 & 0.4 & 0. & 0.5 & 0.1 & 0.0 & 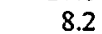 \\
\hline & 0.0 & 0.0 & 0.0 & 0.0 & 0.0 & 0.0 & 0.0 & 0.0 & 0.1 & 0.7 & 0.7 & 0.1 & 0.0 & 0.0 & 0.0 & 0.0 & 0.0 & 1.8 \\
\hline & 0.0 & 0 & 0.0 & 0 & 0.0 & 0 & 0.0 & 0. & 0.0 & 0.1 & 0.2 & 0. & 0.0 & 0.0 & 0.0 & 0.0 & 0.0 & 0.3 \\
\hline $32-3$ & 0.0 & 0.0 & 0.0 & 0. & 0.0 & 0. & 0.0 & 0. & 0.0 & 0.0 & 0.1 & 0 & 0. & 0. & 0.0 & 0.0 & 0.0 & \\
\hline & 0.0 & 0.0 & 0.0 & 0.0 & 0.0 & 0.0 & 0.0 & 0.0 & 0.0 & 0.0 & 0.0 & 0.0 & 0.0 & 0. & 0. & & 0.0 & \\
\hline$>46$ & 0.0 & 0.0 & 0.0 & 0.0 & 0.0 & 0.0 & 0.0 & 0.0 & 0.0 & 0.0 & 0.0 & 0.0 & 0.0 & 0.0 & 0.0 & 0.0 & 0.0 & \\
\hline TOTAL & 7.0 & 4.1 & 2.8 & 2.1 & 2.6 & 4.5 & 7.6 & 7.5 & 8.1 & 13.0 & 10.0 & 4.5 & 3.6 & 3.8 & 9.2 & 9.3 & 0.3 & 100.0 \\
\hline
\end{tabular}

Station: (16) GABL

Begin: $1 / 99$

End: $12 / 99$

Total Hours: 8742

DIRECTION

$N$ NNE NE ENE E ESE SE SSE S SSW SW WSW W WNW NW NNW CALM TOTAL

\begin{tabular}{rrrrrrrrrrrrrrrrrrrr}
\hline CALM & 0.0 & 0.0 & 0.0 & 0.0 & 0.0 & 0.0 & 0.0 & 0.0 & 0.0 & 0.0 & 0.0 & 0.0 & 0.0 & 0.0 & 0.0 & 0.0 & 1.0 & 1.0 \\
$1-3$ & 0.7 & 0.6 & 0.5 & 0.4 & 0.4 & 0.4 & 0.5 & 0.5 & 0.9 & 0.6 & 0.7 & 0.6 & 0.6 & 0.5 & 0.7 & 0.6 & 0.0 & 9.4 \\
$4-7$ & 1.9 & 1.8 & 1.3 & 0.9 & 1.0 & 0.8 & 1.0 & 1.7 & 3.0 & 2.1 & 1.5 & 1.3 & 1.4 & 1.4 & 1.8 & 1.9 & 0.0 & 24.7 \\
$8-12$ & 1.9 & 2.1 & 1.2 & 0.4 & 0.4 & 0.6 & 1.0 & 2.1 & 2.7 & 1.8 & 1.4 & 1.6 & 1.7 & 2.1 & 3.3 & 1.8 & 0.0 & 26.1 \\
$13-18$ & 1.0 & 1.9 & 0.7 & 0.1 & 0.1 & 0.1 & 0.3 & 1.2 & 1.5 & 0.9 & 1.5 & 1.8 & 2.0 & 3.2 & 2.7 & 0.6 & 0.0 & 19.6 \\
$19-24$ & 0.3 & 0.6 & 0.2 & 0.0 & 0.0 & 0.0 & 0.0 & 0.4 & 0.6 & 0.5 & 1.4 & 1.3 & 1.3 & 2.9 & 2.1 & 0.2 & 0.0 & 11.8 \\
$25-31$ & 0.0 & 0.0 & 0.0 & 0.0 & 0.0 & 0.0 & 0.0 & 0.2 & 0.5 & 0.6 & 0.9 & 0.6 & 0.3 & 1.2 & 0.7 & 0.0 & 0.0 & 5.0 \\
$32-38$ & 0.0 & 0.0 & 0.0 & 0.0 & 0.0 & 0.0 & 0.0 & 0.0 & 0.3 & 0.4 & 0.6 & 0.2 & 0.1 & 0.2 & 0.1 & 0.0 & 0.0 & 1.9 \\
$39-46$ & 0.0 & 0.0 & 0.0 & 0.0 & 0.0 & 0.0 & 0.0 & 0.0 & 0.0 & 0.2 & 0.2 & 0.1 & 0.0 & 0.0 & 0.0 & 0.0 & 0.0 & 0.5 \\
$>46$ & 0.0 & 0.0 & 0.0 & 0.0 & 0.0 & 0.0 & 0.0 & 0.0 & 0.0 & 0.0 & 0.0 & 0.0 & 0.0 & 0.0 & 0.0 & 0.0 & 0.0 & 0.1 \\
TOTAL & 5.8 & 7.0 & 4.1 & 1.8 & 1.9 & 1.8 & 2.9 & 6.0 & 9.5 & 7.2 & 8.1 & 7.4 & 7.5 & 11.5 & 11.4 & 5.1 & 1.0 & 100.0
\end{tabular}


Table A.1. (contd)

Station: (17) RING

Begin: 1/99

End: $12 / 99$

Total Hours: 8684

DIRECTION

\begin{tabular}{|c|c|c|c|c|c|c|c|c|c|c|c|c|c|c|c|c|c|c|}
\hline & $\mathrm{N}$ & NNE & $\mathrm{NE}$ & ENE & E & ESE & SE & SSE & S & SSW & SW & WSW & $\mathrm{W}$ & WNW & NW & NNW & CALM & IOTAL \\
\hline CALM & 0.0 & 0.0 & 0.0 & 0.0 & 0.0 & 0.0 & 0.0 & 0.0 & 0.0 & 0.0 & 0.0 & 0.0 & 0.0 & 0.0 & 0.0 & 0.0 & 0.7 & 0.7 \\
\hline $1-3$ & 1.5 & 2.3 & 5.2 & 2.6 & 1.7 & 1.6 & 1.0 & 1.0 & 1.0 & 1.4 & 1.4 & 2.1 & 1.8 & 1.2 & 0.9 & 1.0 & 0.0 & 27.6 \\
\hline $4-7$ & 1.6 & 1.6 & 12.3 & 3.4 & 1.2 & 1.2 & 1.5 & 1.1 & 1.7 & 2.5 & 2.4 & 2.9 & 2.0 & 1.1 & 1.1 & 1.4 & 0.0 & 39.0 \\
\hline $8-12$ & 1.5 & 0.8 & 1.1 & 0.4 & 0.1 & 0.1 & 0.4 & 0.8 & 1.8 & 4.7 & 2.1 & 1. & 1.7 & 2.0 & 1.2 & 1.2 & 0.0 & 21.0 \\
\hline 13-18 & 0.2 & 0.3 & 0.1 & 0.0 & 0.0 & 0.0 & 0.1 & 0.2 & 0.4 & 2.3 & 1.6 & 0.7 & 0.9 & 1.6 & 0.6 & 0.1 & 0.0 & 9.0 \\
\hline $19-24$ & 0.0 & 0.0 & 0.0 & 0.0 & 0.0 & 0.0 & 0.0 & 0.0 & 0.0 & 0.2 & 0.5 & 0.3 & 0.3 & 0 & 0.1 & 0.0 & 0.0 & 2.1 \\
\hline $25-3$ & 0.0 & 0.0 & 0.0 & 0.0 & 0.0 & 0.0 & 0.0 & 0.0 & 0.0 & 0.0 & 0.3 & 0.1 & 0. & 0.0 & 0.0 & 0.0 & 0.0 & 0.5 \\
\hline $32-38$ & 0.0 & 0.0 & 0.0 & 0.0 & 0.0 & 0.0 & 0.0 & 0.0 & 0.0 & 0.0 & 0.0 & 0.0 & 0.0 & 0.0 & 0.0 & 0.0 & 0.0 & 0.0 \\
\hline $39-46$ & 0.0 & 0.0 & 0.0 & 0.0 & 0.0 & 0.0 & 0.0 & 0.0 & 0.0 & 0.0 & 0.0 & 0.0 & 0.0 & 0.0 & 0.0 & 0.0 & 0.0 & 0.0 \\
\hline & 0.0 & 0.0 & 0.0 & 0.0 & 0.0 & 0.0 & 0.0 & 0.0 & 0.0 & 0.0 & 0.0 & 0.0 & 0.0 & 0.0 & 0.0 & 0.0 & 0.0 & 0.0 \\
\hline OTAL & 4.7 & 4.9 & 18.6 & 6.4 & 2.9 & 2.9 & 2.9 & 3.0 & 5.0 & 11.1 & 8.4 & 7.4 & 6.8 & 6.5 & 3.9 & 3.8 & 0.7 & 100.0 \\
\hline
\end{tabular}

Station: (18) RICH

Begin: 1/99

End: $12 / 99$

Total Hours: 8743

DIRECTION

\begin{tabular}{|c|c|c|c|c|c|c|c|c|c|c|c|c|c|c|c|c|c|c|}
\hline & & & JE & NE & $\mathbf{E}$ & ESE & $S E$ & $\mathbf{S E}$ & $\mathbf{S}$ & SSW & SW & isw & $w$ & WNW & NW & NNW & CAI & TOT \\
\hline CALM & 0.0 & 0.0 & 0.0 & 0.0 & 0.0 & 0.0 & 0.0 & 0.0 & 0.0 & 0.0 & 0.0 & 0.0 & 0.0 & 0.0 & 0.0 & 0.0 & 0.6 & 0.6 \\
\hline $1-3$ & 1.0 & 0.9 & 0.8 & 1.1 & 1.5 & 2.4 & 2.6 & 2.7 & 2.1 & 1.8 & 1.8 & 1.8 & 2.1 & 2.6 & 2.4 & 2.1 & 0.0 & 29.8 \\
\hline 4-7 & 1.9 & 0.9 & 0.7 & 0.9 & 1.9 & 2.8 & 3.1 & 1.6 & 1.5 & 2.6 & 3.7 & 2.8 & 2.2 & 2.9 & 2.9 & 2.2 & 0.0 & 34.8 \\
\hline $8-12$ & 1.3 & 0.8 & 0.4 & 0.3 & 0.2 & 0.3 & 0.5 & 0.4 & 0.9 & 3.2 & 4.3 & 2.8 & 1.5 & 0.8 & 1.1 & 12 & 0.0 & \\
\hline $13-18$ & 0.4 & 0.2 & 0.0 & 0.0 & 0.0 & 0.0 & 0.0 & 0.0 & 0.3 & 1.9 & 3.3 & 1.8 & 1.1 & 0.4 & 0.9 & 0.7 & 0.0 & 11.1 \\
\hline $19-24$ & 0.0 & 0.0 & 0.0 & 0.0 & 0.0 & 0.0 & 0.0 & 0.0 & 0.1 & 0.6 & 0.9 & 0.5 & 0.4 & 0.1 & 0.2 & 0.1 & 0.0 & 2.9 \\
\hline $25-31$ & 0.0 & 0.0 & 0.0 & 0.0 & 0.0 & 0.0 & 0.0 & 0.0 & 0.0 & 0.1 & 0.4 & 0.2 & 0.1 & 0.0 & 0.0 & 0.0 & 0.0 & \\
\hline $32-38$ & 0.0 & 0.0 & 0.0 & 0.0 & 0.0 & 0.0 & 0.0 & 0.0 & 0.0 & 0.0 & 0.0 & 0.0 & 0.0 & 0.0 & 0.0 & 0.0 & 0.0 & \\
\hline $39-46$ & 0.0 & 0.0 & 0.0 & 0.0 & 0.0 & 0.0 & 0.0 & 0.0 & 0.0 & 0.0 & 0.0 & 0.0 & 0.0 & 0.0 & 0.0 & 0.0 & 0.0 & \\
\hline$>46$ & 0.0 & 0.0 & 0.0 & 0.0 & 0.0 & 0.0 & 0.0 & 0.0 & 0.0 & 0.0 & 0.0 & 0.0 & 0.0 & 0.0 & 0.0 & 0.0 & 0.0 & \\
\hline TOTAL & 4.7 & 2.9 & 2.0 & 2.3 & 3.6 & 5.5 & 6.2 & 4.7 & 5.0 & 10.2 & 14.5 & 10.0 & 7.4 & 6.7 & 7.5 & 6.3 & 0.6 & 100 \\
\hline
\end{tabular}

Station: (19) PFP

Begin: 1/99

End: $12 / 99$

Total Hours: 8743

DIRECTION

\begin{tabular}{|c|c|c|c|c|c|c|c|c|c|c|c|c|c|c|c|c|c|c|}
\hline & $\mathrm{N}$ & NNE & $\mathrm{NE}$ & ENE & $E$ & ESE & SE & SSE & $s$ & SSW & SW & WSW & $\mathrm{W}$ & WNW & NW & NNW & CALM & TOTA \\
\hline CALM & 0.0 & 0.0 & 0.0 & 0.0 & 0.0 & 0.0 & 0.0 & 0.0 & 0.0 & 0.0 & 0.0 & 0.0 & 0.0 & 0.0 & 0.0 & 0.0 & 2.2 & 2.2 \\
\hline $1-3$ & 3.1 & 2.8 & 1.8 & 1.2 & 1.4 & 1.6 & 2.0 & 1.7 & 1.7 & 1.8 & 2.3 & 3.4 & 4.7 & 4.6 & 3.0 & 2.7 & 0.0 & 39.7 \\
\hline $4-7$ & 3.7 & 2.5 & 1.1 & 0.5 & 0.9 & 1.4 & 1.4 & 0.8 & 0.6 & 0.7 & 1.2 & 2.0 & 4.7 & 5.7 & 4.1 & 3.5 & 0.0 & \\
\hline $8-12$ & 0.5 & 0.5 & 0.2 & 0.1 & 0.1 & 0.4 & 0.3 & 0.3 & 0.5 & 0.7 & 1.7 & 2.3 & 1.7 & 1.7 & 3.3 & 1.4 & 0.0 & \\
\hline $13-18$ & 0.0 & 0.0 & 0.0 & 0.0 & 0.0 & 0.0 & 0.0 & 0.0 & 0.1 & 0.7 & 1.3 & 1.6 & 0.5 & 0.2 & 1.3 & 0.1 & 0.0 & \\
\hline $19-24$ & 0.0 & 0.0 & 0.0 & 0.0 & 0.0 & 0.0 & 0.0 & 0.0 & 0.1 & 0.2 & 0.5 & 0.4 & 0.1 & 0. & 0.0 & 0.0 & 0.0 & \\
\hline $25-3$ & 0.0 & 0.0 & 0.0 & 0.0 & 0.0 & 0.0 & 0.0 & 0.0 & 0.0 & 0.0 & 0.1 & 0.1 & 0.0 & 0.0 & 0.0 & 0.0 & 0.0 & \\
\hline 32 & 0.0 & 0.0 & 0.0 & 0.0 & 0.0 & 0.0 & 0.0 & 0.0 & 0.0 & 0.0 & 0.0 & 0.0 & 0.0 & 0.0 & 0.0 & 0.0 & 0.0 & \\
\hline & 0.0 & 0.0 & 0.0 & 0.0 & 0.0 & 0.0 & 0.0 & 0.0 & 0.0 & 0.0 & 0.0 & 0.0 & 0.0 & 0.0 & 0.0 & 0.0 & 0.0 & \\
\hline & 0.0 & 0.0 & 0.0 & 0. & 0.0 & 0. & 0. & 0. & 0.0 & 0.0 & 0.0 & 0.0 & 0.0 & 0.0 & 0.0 & 0.0 & 0.0 & \\
\hline DTAL & 7.4 & 5.8 & 3.2 & 1.8 & 2.4 & 3.4 & 3.7 & 2.8 & 3.0 & 4.1 & 7.2 & 9.8 & 11.7 & 12.1 & 11.8 & 7.7 & 2.2 & \\
\hline
\end{tabular}

Station: (20) RMTN

Begin: 1/99

End: $12 / 99$

Total Hours: 8749

DIRECTION

\begin{tabular}{|c|c|c|c|c|c|c|c|c|c|c|c|c|c|c|c|c|c|c|}
\hline & $\mathrm{N}$ & NNE & NE & ENE & E & ESE & SE & SSE & $\mathrm{S}$ & SSW & SW & WSW & W & WNW & NW & NNW & CALM & TOTA \\
\hline CALM & 0.0 & 0.0 & 0.0 & 0.0 & 0.0 & 0.0 & 0.0 & 0.0 & 0.0 & 0.0 & 0.0 & 0.0 & 0.0 & 0.0 & 0.0 & 0.0 & 0.2 & 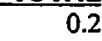 \\
\hline $1-3$ & 0.3 & 0.2 & 0.2 & 0.2 & 0.2 & 0.2 & 0.3 & 0.2 & 0.4 & 0.2 & 0.4 & 0.3 & 0.2 & 0.3 & 0.2 & 0.2 & 0.0 & \\
\hline 4-7 & 1.2 & 0.8 & 0.7 & 0.7 & 0.6 & 0.4 & 0. & 0. & 0.5 & 1.0 & 1.4 & 1. & 0.6 & 0.6 & 0.6 & 0.7 & 0.0 & \\
\hline $8-12$ & 2.4 & 2.1 & 1.8 & 0.8 & 0.4 & 0.3 & 0.4 & 0.3 & 0.5 & 1.3 & 2.6 & 2.0 & 1.3 & 0.7 & 0.7 & 1.0 & 0.0 & \\
\hline $13-1$ & 2.0 & 3.0 & 1.3 & 0.2 & 0.1 & 0.1 & 0.1 & 0.2 & 0.5 & 1.6 & 4.5 & 2.8 & 1.7 & 0.6 & 0.7 & 0.8 & 0.0 & \\
\hline $19-2$ & 0.8 & 2.3 & 0.9 & 0.0 & 0.0 & 0.0 & 0.0 & 0. & 0.2 & 1.1 & 4.6 & 2.9 & 1.5 & 0 . & 0.3 & 0.2 & 0.0 & \\
\hline $25-3$ & 0.4 & 2.2 & 1.0 & 0.0 & 0.0 & 0.0 & 0.0 & 0.1 & 0.1 & 0.7 & 4.4 & 3.0 & 0.9 & 0 & 0. & 0.0 & 0.0 & \\
\hline $32-38$ & 0.1 & 1.0 & 0.4 & 0.0 & 0.0 & 0.0 & 0.0 & 0.0 & 0.0 & 0.2 & 4.0 & 2. & 0.2 & 0. & 0.0 & 0.0 & 0.0 & \\
\hline & 0.0 & 0.4 & 0.1 & 0.0 & 0.0 & 0.0 & 0.0 & 0.0 & 0.0 & 0.2 & 2.9 & 1.3 & 0.0 & 0.0 & 0.0 & 0.0 & 0.0 & \\
\hline & 0.0 & 0.0 & 0.0 & 0.0 & 0.0 & 0.0 & 0.0 & 0.0 & 0.0 & 0.0 & 2.2 & 1.0 & 0.0 & 0.0 & 0.0 & 0.0 & 0.0 & \\
\hline OTAL & 7.3 & 11.9 & 6.5 & 2.0 & 1.3 & 0.9 & 1.2 & 1.0 & 2.1 & 6.4 & 27.1 & 17.0 & 6.6 & 3.0 & 2.6 & 2.9 & 0.2 & \\
\hline
\end{tabular}


Table A.1. (contd)

Station: (21) HMS

Begin: 1/99

End: $12 / 99$

Total Hours: 8750

DIRECTION

\begin{tabular}{|c|c|c|c|c|c|c|c|c|c|c|c|c|c|c|c|c|c|c|}
\hline & $\mathrm{N}$ & NNE & NE & ENE & E & ESE & SE & SSE & $\mathbf{S}$ & SSW & SW & WSW & $\mathrm{W}$ & WNW & NW & NNW & CAIM & TOTAL \\
\hline CALM & 0.0 & 0.0 & 0.0 & 0.0 & 0.0 & 0.0 & 0.0 & 0.0 & 0.0 & 0.0 & 0.0 & 0.0 & 0.0 & 0.0 & 0.0 & 0.0 & 0.4 & 0.4 \\
\hline 1-3 & 1.7 & 1.6 & 1.7 & 1.3 & 1.3 & & 1. & 1.2 & 1.4 & & 1.2 & 1.0 & 1.1 & 1.3 & 1.4 & 1.6 & 0.0 & 22.0 \\
\hline $4-7$ & 2.3 & 1.3 & 1.2 & 0.9 & 1.3 & 1.3 & 1. & 1.3 & 1.2 & 1.3 & 2.4 & 3.8 & 5.2 & 5. & 5.7 & 3.9 & 0.0 & 40.1 \\
\hline $8-12$ & 0.6 & 0.6 & 0.3 & 0.2 & 0.2 & 0.1 & 0. & 0.5 & 0.6 & 0.8 & 1.6 & 3.3 & 2.9 & 4.0 & 6.5 & 0.8 & 0.0 & 23.0 \\
\hline $13-1$ & 0.0 & 0.1 & 0.0 & 0.0 & 0.0 & 0.0 & 0.0 & 0.1 & 0.4 & 0.8 & 1.4 & 1. & 0.8 & 1.3 & 3.1 & 0.2 & 0.0 & 10.0 \\
\hline $19-2$ & 0.0 & 0.0 & 0.0 & 0.0 & 0.0 & 0.0 & 0.0 & 0.0 & 0.1 & 0.5 & 0.9 & 0. & 0.2 & 0.2 & 1.1 & 0.0 & 0.0 & 3.7 \\
\hline $25-$ & 0.0 & 0.0 & 0.0 & $0 . C$ & 0.0 & $0 . c$ & 0.0 & 0. & 0.0 & 0.2 & 0.2 & 0.1 & 0.0 & 0. & 0.0 & 0.0 & 0.0 & 0.7 \\
\hline 32 & 0.0 & 0.0 & 0.0 & 0. & 0.0 & $0 . C$ & 0.0 & 0. & 0.0 & 0.0 & 0.0 & 0.0 & 0.0 & 0.0 & 0.0 & 0.0 & 0.0 & 0.1 \\
\hline & 0.0 & 0.0 & 0.0 & 0.0 & 0.0 & 0. & 0.0 & 0.0 & 0.0 & 0.0 & 0.0 & 0.0 & 0.0 & 0.0 & 0.0 & 0.0 & 0.0 & 0.0 \\
\hline & 0.0 & 0.0 & 0.0 & 0.0 & 0.0 & 0. & 0.0 & 0.0 & 0.0 & 0.0 & 0.0 & 0.0 & 0.0 & 0.0 & 0.0 & 0.0 & 0.0 & 0.0 \\
\hline IOTAL & 4.6 & 3.5 & 3.3 & 2.4 & 2.8 & 2.9 & 3.3 & 3.1 & 3.8 & 4.8 & 7.9 & 10.4 & 10.3 & 12.1 & 17.8 & 6.5 & 0.4 & 100.0 \\
\hline
\end{tabular}

Station: (22) PASC

Begin: 1/99

End: $12 / 99$

Total Hours: 8743

DIRECTION

\begin{tabular}{|c|c|c|c|c|c|c|c|c|c|c|c|c|c|c|c|c|c|c|}
\hline & $\mathrm{N}$ & NNE & $\mathrm{NE}$ & ENE & E & ESE & SE & SSE & $S$ & SSW & SW & WSW & W & WNW & $\mathrm{NW}$ & NNW & CALM & TOTAL \\
\hline CALM & 0.0 & 0.0 & 0.0 & 0.0 & 0.0 & 0.0 & 0.0 & 0.0 & 0.0 & 0.0 & 0.0 & 0.0 & 0.0 & 0.0 & 0.0 & 0.0 & 0.9 & 0.9 \\
\hline $1-3$ & 4.6 & 2.4 & 2.0 & 1.8 & 1.9 & 1.7 & 1. & 1.5 & 1.2 & 1.2 & 1.3 & 1. & 1.7 & 2.3 & 4.5 & 6.7 & 0.0 & 37.7 \\
\hline 4-7 & 2.0 & 0.5 & 0.4 & 0.5 & 0.7 & 1.3 & 2.0 & 1.5 & 1.8 & 3.5 & 4.1 & 2.4 & 2.4 & 2.3 & 3.1 & 4.3 & 0.0 & 32.8 \\
\hline $8-12$ & 0.5 & 0.2 & 0.1 & 0.1 & 0.1 & 0.1 & 0.4 & 0.5 & 0.5 & 2.3 & 6.2 & 3.0 & 1.1 & 0.6 & 1.0 & 1.3 & 0.0 & 17.9 \\
\hline $13-18$ & 0.1 & 0.1 & 0.0 & 0.0 & 0.0 & 0.0 & 0.0 & 0.0 & 0.1 & 0. & 4.2 & 2.3 & 0.7 & 0.2 & 0.1 & 0.2 & 0.0 & 8.7 \\
\hline $19-2$ & 0.0 & 0.0 & 0.0 & 0.0 & 0.0 & 0.0 & 0.0 & 0.0 & 0.0 & 0. & 0.8 & 0.7 & 0.1 & 0.0 & 0.0 & 0.0 & 0.0 & 1.8 \\
\hline $25-31$ & 0.0 & 0.0 & 0.0 & 0.0 & 0.0 & 0.0 & 0.0 & 0.0 & 0.0 & 0.0 & 0.1 & 0. & 0.0 & 0.0 & 0.0 & 0.0 & 0.0 & 0.2 \\
\hline $32-3$ & 0.0 & 0.0 & 0.0 & 0.0 & 0.0 & 0.0 & 0.0 & 0.0 & 0.0 & 0.0 & 0.0 & 0.0 & 0.0 & 0.0 & 0.0 & 0.0 & 0.0 & 0.0 \\
\hline & 0.0 & 0.0 & 0.0 & 0.0 & 0.0 & 0.0 & 0.0 & 0.0 & 0.0 & 0.0 & 0.0 & 0.0 & 0.0 & 0.0 & 0.0 & 0.0 & 0.0 & 0.0 \\
\hline & 0.0 & 0.0 & 0.0 & 0.0 & 0.0 & 0.0 & 0.0 & 0.0 & 0.0 & 0.0 & 0.0 & 0.0 & 0.0 & 0.0 & 0.0 & 0.0 & 0.0 & 0.0 \\
\hline COTAL & 7.1 & 3.2 & 2.4 & 2.5 & 2.6 & 3.1 & 4.0 & 3.4 & 3.7 & 7.8 & 16.7 & 9.9 & 6.0 & 5.4 & 8.7 & 12.6 & 0.9 & 100.0 \\
\hline
\end{tabular}

Station: (23) GABW

Begin: 1/99

End: $12 / 99$

Total Hours: 8740

DIRECTION

\begin{tabular}{|c|c|c|c|c|c|c|c|c|c|c|c|c|c|c|c|c|c|c|}
\hline & $\mathbf{N}$ & NNE & NE & ENE & $E$ & ESE & SE & SSE & $S$ & SSW & SW & WSW & $\mathrm{W}$ & WNW & NW & NNW & CALM & TOTAL \\
\hline CALM & 0.0 & 0.0 & 0.0 & 0.0 & 0.0 & 0.0 & 0.0 & 0.0 & 0.0 & 0.0 & 0.0 & 0.0 & 0.0 & 0.0 & 0.0 & 0.0 & 0.7 & 0.7 \\
\hline $1-3$ & 1.2 & 1.1 & 0.9 & 1.1 & 1.3 & 1.5 & 2.1 & 2.2 & 2.1 & 1.4 & 1.3 & 1.5 & 2.6 & 3.4 & 2.7 & 1.6 & 0.0 & 28.0 \\
\hline $4-7$ & 1.4 & 1.1 & 0.9 & 0.9 & 1.1 & 1.3 & 3.5 & 4.8 & 1.3 & 0.7 & 1.0 & 1.6 & 3.4 & 8.0 & 3.1 & 1.7 & 0.0 & 35.8 \\
\hline $8-12$ & 0.6 & 0.6 & 0.3 & 0.2 & 0.3 & 0.3 & 1.4 & 1.2 & 0.5 & 0.6 & 1.2 & 2.1 & 3.8 & 6.2 & 1.1 & 0.3 & 0.0 & 20.7 \\
\hline $13-18$ & 0.1 & 0.1 & 0.0 & 0.0 & 0.0 & 0.0 & 0.2 & 0.4 & 0.3 & 0.4 & 1.1 & 1.1 & 1.4 & 4.1 & 0.8 & 0.0 & 0.0 & 10.3 \\
\hline $19-24$ & 0.0 & 0.0 & 0.0 & 0.0 & 0.0 & 0.0 & 0.0 & 0.0 & 0.1 & 0.5 & 0.7 & 0.5 & 0.2 & 1.5 & 0.2 & 0.0 & 0.0 & 3.8 \\
\hline $25-$ & 0.0 & 0.0 & 0.0 & 0.0 & 0.0 & 0.0 & 0.0 & 0.0 & 0.0 & 0.1 & 0.2 & 0.1 & 0.0 & 0.2 & 0.0 & 0.0 & 0.0 & 0.7 \\
\hline 32 & 0.0 & 0.0 & 0.0 & 0.0 & 0.0 & 0.0 & 0.0 & 0.0 & 0.0 & 0.0 & 0.0 & 0.0 & 0.0 & 0.0 & 0.0 & 0.0 & 0.0 & 0.0 \\
\hline & 0.0 & 0.0 & 0.0 & 0.0 & 0.0 & 0.0 & 0.0 & 0.0 & 0.0 & 0.0 & 0.0 & 0.0 & 0.0 & 0.0 & 0.0 & 0.0 & 0.0 & 0.0 \\
\hline & 0.0 & 0.0 & 0.0 & 0.0 & 0.0 & 0.0 & 0. & 0.0 & 0.0 & 0 & 0.0 & 0.0 & 0.0 & 0.0 & 0.0 & 0.0 & 0.0 & 0.0 \\
\hline OTAL & 3.4 & 2.9 & 2.1 & 2.2 & 2.7 & 3.1 & 7.2 & 8.6 & 4.3 & 3.9 & 5.5 & 6.9 & 11.5 & 23.5 & 8.0 & 3.6 & 0.7 & 100.0 \\
\hline
\end{tabular}

Station: (24) $100 \mathrm{~F}$

Begin: 1/99

End: $12 / 99$

Total Hours: 8743

DIRECTION

\begin{tabular}{|c|c|c|c|c|c|c|c|c|c|c|c|c|c|c|c|c|c|c|}
\hline & $N$ & NNE & NE & ENE & E & ESE & SE & SSE & $\underline{S}$ & SSW & SW & WSW & W & WNW & NW & NNW & CALM & TOTAL \\
\hline CALM & 0.0 & 0.0 & 0.0 & 0.0 & 0.0 & 0.0 & 0.0 & 0.0 & 0.0 & 0.0 & 0.0 & 0.0 & 0.0 & 0.0 & 0.0 & 0.0 & 0.8 & 0.8 \\
\hline $1-3$ & 1.7 & 1.1 & 1.1 & 1.0 & 1.2 & & 2.0 & 2.5 & 1.8 & 1.5 & 1.6 & 2.1 & 3.3 & 3.8 & 3.3 & 2.4 & 0.0 & 32.0 \\
\hline 4. & 2.1 & 1.1 & 1.0 & 1.1 & 1.3 & 1.9 & 4.6 & 4.8 & 1.6 & 0.9 & 0.9 & 1.8 & 3.7 & 3.5 & 2.6 & 2.0 & 0.0 & 34.8 \\
\hline $8-12$ & 1.1 & 0.7 & 0.5 & 0.6 & 0.4 & 1.0 & 2.9 & 2.5 & 0.7 & 0.5 & 0.8 & 1.3 & 2.7 & 2.9 & 0.7 & 0.4 & 0.0 & 19.5 \\
\hline $13-1$ & 0.3 & 0.1 & 0.1 & 0.2 & 0.1 & 0.1 & 0.4 & 0.7 & 0.6 & 0.5 & 0.8 & & 1.3 & 2.2 & 0.8 & 0.0 & 0.0 & 8.8 \\
\hline $19-2$ & 0.0 & 0.0 & 0.0 & $0 . C$ & 0.0 & 0.1 & 0.1 & 0.1 & 0.3 & 0 & 0. & 0 & 0.4 & 0.9 & 0.3 & 0.0 & 0.0 & 3.2 \\
\hline $25-31$ & 0.0 & 0.0 & 0.0 & 0.0 & 0.0 & 0.0 & 0.0 & 0.0 & 0.0 & 0. & 0.1 & 0.1 & 0.1 & 0. & 0.0 & 0.0 & 0.0 & 0.8 \\
\hline $32-3$ & 0.0 & 0.0 & 0.0 & 0.0 & 0.0 & 0.0 & 0.0 & 0.0 & 0.0 & 0.0 & 0.0 & 0.0 & 0.0 & 0.0 & 0.0 & 0.0 & 0.0 & 0.0 \\
\hline $39-4$ & 0.0 & 0.0 & 0.0 & 0.0 & 0.0 & 0.0 & 0.0 & 0.0 & 0.0 & 0.0 & 0.0 & 0.0 & 0.0 & 0.0 & 0.0 & 0.0 & 0.0 & 0.0 \\
\hline$>46$ & 0.0 & 0.0 & 0.0 & 0.0 & 0.0 & 0.0 & 0.0 & 0.0 & 0.0 & 0. & 0.0 & 0.0 & 0.0 & 0.0 & 0.0 & 0.0 & 0.0 & 0.0 \\
\hline OTAL & 5.1 & 3.0 & 2.6 & 2.9 & 3.0 & 4.6 & 10.0 & 10.6 & 4.9 & 3.9 & 4.5 & 6.4 & 11.5 & 13.6 & 7.8 & 4.7 & 0.8 & 100.0 \\
\hline
\end{tabular}


Table A.1. (contd)

Station: (25) VERN

Begin: 1/99

End: $12 / 99$

Total Hours: 8743

DIRECTION

\begin{tabular}{|c|c|c|c|c|c|c|c|c|c|c|c|c|c|c|c|c|c|c|}
\hline & $\mathbf{N}$ & NNE & NE & ENE & $E$ & ESE & $\mathrm{SE}$ & SSE & $\mathbf{S}$ & SSW & SW & VSW & $\mathrm{W}$ & WNW & NW & NNW & CALM & TOTAL \\
\hline CALM & 0.0 & 0.0 & 0.0 & 0.0 & 0.0 & 0.0 & 0.0 & 0.0 & 0.0 & 0.0 & 0.0 & 0.0 & 0.0 & 0.0 & 0.0 & 0.0 & 0.5 & 0.5 \\
\hline $1-3$ & 0.9 & 1.0 & 1.0 & 1.3 & 1.7 & 2.1 & 1.6 & 0.9 & 0.9 & 0.7 & 1.2 & 1.6 & 1.9 & & & 0.9 & 0.0 & 20.2 \\
\hline $4-7$ & 0.9 & 1.3 & 2.1 & 2.8 & 3.8 & 2.1 & 1.0 & 0.4 & 0.4 & 0.4 & 0.6 & & 8.1 & 3.7 & & 1.0 & 0.0 & 33.9 \\
\hline $8-12$ & 0.6 & 0.5 & 0.5 & 0.5 & 0.5 & 0.3 & 0.2 & 0.2 & 0.3 & 0.4 & 0.9 & 2.3 & 8.6 & 7.8 & 2.0 & 0.4 & 0.0 & 20.1 \\
\hline $13-18$ & 0.1 & 0.1 & 0.0 & 0.0 & 0.0 & 0.0 & 0.0 & 0.0 & 0.2 & 0.4 & 0.8 & 0.7 & 2.7 & 6.1 & 2.1 & 0.2 & 0.0 & 13.5 \\
\hline $19-24$ & 0.0 & 0.0 & 0.0 & 0.0 & 0.0 & $\cdot 0.0$ & 0.0 & 0.0 & 0.0 & 0.4 & 0.6 & 0. & 0.6 & 1 & 0 & .0 & & 4.4 \\
\hline $25-31$ & 0.0 & 0.0 & 0.0 & 0.0 & 0.0 & 0.0 & 0.0 & 0.0 & 0.0 & 0.2 & 0.5 & 0. & 0.1 & 0.3 & 0. & 0.0 & 0.0 & 1.3 \\
\hline $32-38$ & 0.0 & 0.0 & 0.0 & 0.0 & 0.0 & 0.0 & 0.0 & 0.0 & 0.0 & 0.0 & 0.0 & 0.0 & 0.0 & 0.0 & 0.0 & 0.0 & 0.0 & 0 \\
\hline $39-46$ & 0.0 & 0.0 & 0.0 & 0.0 & 0.0 & 0.0 & 0.0 & 0.0 & 0.0 & 0.0 & 0.0 & 0.0 & 0.0 & 0.0 & 0.0 & 0.0 & 0.0 & 0.0 \\
\hline & 0.0 & 0.0 & 0.0 & 0.0 & 0.0 & 0.0 & 0.0 & 0. & 0.0 & 0. & 0.0 & 0.0 & 0.0 & 0.0 & 0.0 & 0.0 & 0.0 & 0.0 \\
\hline TOTAL & 2.6 & 2.8 & 3.6 & 4.6 & 6.0 & 4.5 & 2.7 & 1.6 & 1.8 & 2.5 & 4.6 & 8.8 & 22.0 & 20.9 & 7.8 & 2.5 & 0.5 & 100 \\
\hline
\end{tabular}

Station: (26) BENT

Begin: 1/99

End: $12 / 99$

Total Hours: 8707

DIRECTION

\begin{tabular}{|c|c|c|c|c|c|c|c|c|c|c|c|c|c|c|c|c|c|c|}
\hline & $\mathrm{N}$ & NNE & NE & ENE & $E$ & ESE & SE & SSE & $\mathrm{s}$ & SSW & SW & WSW & W & WNW & NW & NNW & CALM & TOTAL \\
\hline CALM & 0.0 & 0.0 & 0.0 & 0.0 & 0.0 & 0.0 & 0.0 & 0.0 & 0.0 & 0.0 & 0.0 & 0.0 & 0.0 & 0.0 & 0.0 & 0.0 & 0.2 & 0.2 \\
\hline $1-3$ & 0.9 & 1.2 & 0.8 & 0.5 & 0.6 & 0.6 & 0.5 & 0.4 & 0.5 & 0 & 1.7 & 1. & 2.0 & & & & 0.0 & 14.7 \\
\hline 4 & 1.2 & 1.0 & 1.4 & 2.0 & 1.9 & 0.7 & 0.2 & 0.3 & 0.5 & 0 & 4.3 & 10.5 & 14.2 & 5.5 & 2.5 & .9 & 0.0 & 49.1 \\
\hline $8-1$ & 0.6 & 0.7 & 1.2 & 1.0 & 0.8 & 0. & 0.0 & 0. & 0.2 & & 3.3 & 7. & 6.7 & 2.5 & 1.3 & & 0.0 & 27.6 \\
\hline $13-1$ & 0.2 & 0.4 & 0.3 & 0.1 & 0.0 & 0.0 & 0.0 & 0.0 & 0.1 & 0. & 2.0 & 1. & 0.9 & 0. & 0 & & 0.0 & 6.5 \\
\hline & 0.0 & 0.0 & 0.0 & 0.0 & 0.0 & 0. & 0.0 & 0.0 & 0.0 & 0 & 0.8 & 0. & 0.1 & 0.0 & 0.0 & & &. \\
\hline & 0.0 & 0.0 & 0.0 & 0.0 & 0.0 & 0.0 & 0.0 & 0.0 & 0.0 & 0 & 0.3 & 0.0 & 0.1 & 0.0 & 0.0 & 0.0 & 0.0 & 0.4 \\
\hline & 0.0 & 0. & 0.0 & 0.0 & 0.0 & 0. & 0.0 & 0. & 0. & 0 & 0.0 & 0.0 & 0.0 & 0.0 & 0.0 & & 0.0 & 0.0 \\
\hline & 0 & & 0.0 & .0 .0 & 0. & 0 & 0.0 & 0. & 0.0 & 0 & 0.0 & 0. & 0 & & & & 0 & \\
\hline & 0.0 & 0.0 & 0.0 & 0.0 & 0.0 & 0.0 & 0.0 & 0.0 & 0.0 & 0. & 0.0 & 0.0 & 0.0 & 0.0 & & & 0.0 & \\
\hline TOTAL & 3.0 & 3.4 & 3.7 & 3.6 & 3.3 & 1.5 & $0.8^{\circ}$ & 0.7 & 1.3 & 2.8 & 11.6 & 21.5 & 23.9 & 10.1 & 5.1 & 3.4 & 0.2 & 100.0 \\
\hline
\end{tabular}

Station: (27) VSTA

Begin: 1/99

End: $12 / 99$

Total Hours: 8735

DIRECTION

\begin{tabular}{rrrrrrrrrrrrrrrrrrr} 
& N & NNE & NE & ENE & E & ESE & SE & SSE & S & SSW & SW & WSW & W & WNW & NW & NNW & CALM & TOTAL \\
\hline CALM & 0.0 & 0.0 & 0.0 & 0.0 & 0.0 & 0.0 & 0.0 & 0.0 & 0.0 & 0.0 & 0.0 & 0.0 & 0.0 & 0.0 & 0.0 & 0.0 & 0.7 & 0.7 \\
$1-3$ & 2.0 & 2.0 & 1.8 & 1.3 & 1.4 & 1.7 & 1.6 & 1.3 & 2.0 & 2.0 & 2.4 & 2.3 & 2.2 & 1.3 & 1.6 & 1.5 & 0.0 & 28.3 \\
$4-7$ & 2.7 & 1.7 & 1.3 & 1.0 & 0.8 & 1.1 & 1.0 & 1.2 & 1.8 & 4.2 & 5.9 & 3.6 & 2.1 & 2.4 & 3.0 & 2.7 & 0.0 & 36.8 \\
$8-12$ & 0.5 & 0.1 & 0.0 & 0.0 & 0.1 & 0.2 & 0.1 & 0.4 & 1.0 & 4.3 & 6.7 & 3.0 & 0.9 & 0.5 & 0.9 & 1.0 & 0.0 & 19.6 \\
$13-18$ & 0.0 & 0.0 & 0.0 & 0.0 & 0.0 & 0.0 & 0.0 & 0.1 & 0.3 & 2.0 & 6.4 & 1.4 & 0.5 & 0.3 & 0.1 & 0.1 & 0.0 & 11.3 \\
$19-24$ & 0.0 & 0.0 & 0.0 & 0.0 & 0.0 & 0.0 & 0.0 & 0.0 & 0.0 & 0.3 & 2.1 & 0.3 & 0.1 & 0.0 & 0.0 & 0.0 & 0.0 & 2.9 \\
$25-31$ & 0.0 & 0.0 & 0.0 & 0.0 & 0.0 & 0.0 & 0.0 & 0.0 & 0.0 & 0.0 & 0.2 & 0.0 & 0.0 & 0.0 & 0.0 & 0.0 & 0.0 & 0.3 \\
$32-38$ & 0.0 & 0.0 & 0.0 & 0.0 & 0.0 & 0.0 & 0.0 & 0.0 & 0.0 & 0.0 & 0.0 & 0.0 & 0.0 & 0.0 & 0.0 & 0.0 & 0.0 & 0.1 \\
$39-46$ & 0.0 & 0.0 & 0.0 & 0.0 & 0.0 & 0.0 & 0.0 & 0.0 & 0.0 & 0.0 & 0.0 & 0.0 & 0.0 & 0.0 & 0.0 & 0.0 & 0.0 & 0.0 \\
$>46$ & 0.0 & 0.0 & 0.0 & 0.0 & 0.0 & 0.0 & 0.0 & 0.0 & 0.0 & 0.0 & 0.0 & 0.0 & 0.0 & 0.0 & 0.0 & 0.0 & 0.0 & 0.0 \\
TOTAL & 5.2 & 3.9 & 3.2 & 2.3 & 2.3 & 2.9 & 2.6 & 3.0 & 5.2 & 12.9 & 23.7 & 10.8 & 5.8 & 4.6 & 5.5 & 5.4 & 0.7 & 100.0
\end{tabular}

Station: (28) SURF

Begin: 1/99

End: $12 / 99$

Total Hours: 8631

DIRECTION

\begin{tabular}{|c|c|c|c|c|c|c|c|c|c|c|c|c|c|c|c|c|c|c|}
\hline & $\mathrm{N}$ & NNE & NE & ENE & $\mathrm{E}$ & ESE & SE & SSE & $\mathrm{S}$ & SSW & SW & WSW & $\mathrm{W}$ & WNW & NW & NNW & CALM & TOTAL \\
\hline CALM & 0.0 & 0.0 & 0.0 & 0.0 & 0.0 & 0.0 & 0.0 & 0.0 & 0.0 & 0.0 & 0.0 & 0.0 & 0.0 & 0.0 & 0.0 & 0.0 & 0.6 & 0.6 \\
\hline $1-3$ & 0.4 & 0.7 & 1.1 & 1.2 & 1.0 & 0. & 0.6 & 0. & 1.2 & 2. & 3.5 & 3 & 1.5 & 0.6 & 0.3 & 0.3 & 0.0 & 19.5 \\
\hline 4 & 0.2 & 0.4 & 2.4 & 3.2 & 1.6 & 0.5 & 0.3 & 0.2 & 0.4 & 1. & 4.0 & 5 & $i$ & 0 & 0 & 0 & 0.0 & 2.2 \\
\hline $8-12$ & 0.1 & 0.5 & 2.1 & 3.1 & 0.4 & 0.0 & 0.0 & 0. & 0.0 & 0 & 4.6 & 10. & 3 & & & 0.0 & 0.0 & 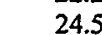 \\
\hline $13-1$ & 0.1 & 0.2 & 0.2 & 0.5 & 0.0 & 0.0 & 0.0 & 0.0 & 0.0 & 0.0 & 2.2 & 9 & 6.1 & 0.6 & 0.0 & 0.0 & 0.0 & 19.2 \\
\hline & 0.0 & 0 & 0.0 & 0.0 & 0.0 & 0.0 & 0.0 & 0.0 & 0.0 & 0.0 & 0.6 & 5.2 & 3.7 & 0.3 & 0.0 & 0.0 & 0.0 & 10.0 \\
\hline 25 & 0.0 & 0.0 & 0.0 & 0.0 & 0.0 & 0.0 & 0.0 & 0. & 0.0 & 0.0 & 0. & 1 & & & & & 0.0 & \\
\hline $32-38$ & 0.0 & 0.0 & 0.0 & 0.0 & 0.0 & 0.0 & 0.0 & 0. & 0.0 & 0. & 0. & 0 & 0 & & & & 0.0 & \\
\hline $39-46$ & 0.0 & 0.0 & 0.0 & 0.0 & 0.0 & 0.0 & 0.0 & 0.0 & 0.0 & 0.0 & 0.0 & 0.0 & 0.1 & 0.0 & 0.0 & 0.0 & 0.0 & \\
\hline$>46$ & 0.0 & 0.0 & 0.0 & 0.0 & 0.0 & 0.0 & 0.0 & 0.0 & 0.0 & 0.0 & 0.0 & 0.0 & 0.0 & 0.0 & 0.0 & 0.0 & 0.0 & \\
\hline TOTAL & 0.8 & 1.8 & 5.8 & 8.0 & 3.0 & 1.3 & 0.8 & 1.0 & 1.5 & 3.7 & 15.0 & 35.9 & 17.6 & 2.1 & 0.5 & 0.5 & 0.6 & 100 \\
\hline
\end{tabular}


Table A.1. (contd)

Station: (29) $100 \mathrm{~K}$

Begin: 1/99

End: $12 / 99$

Total Hours: 8743

DIRECTION

\begin{tabular}{|c|c|c|c|c|c|c|c|c|c|c|c|c|c|c|c|c|c|c|}
\hline & $\mathrm{N}$ & NNE & NE & ENE & $E$ & ESE & SE & SSE & $S$ & SSW & SW & WSW & $\mathrm{W}$ & WNW & NW & NNW & CALM & TOTAL \\
\hline CALM & 0.0 & 0.0 & 0.0 & 0.0 & 0.0 & 0.0 & 0.0 & 0.0 & 0.0 & 0.0 & 0.0 & 0.0 & 0.0 & 0.0 & 0.0 & 0.0 & 1.1 & 1.1 \\
\hline 1-3 & 1.9 & 1.6 & 1.5 & 1.8 & 2.2 & 1.9 & 1.9 & & 1.7 & & & & 3.9 & & 2.2 & 1.8 & 0.0 & 34.1 \\
\hline $4-$ & 1.6 & 1.4 & 0.9 & 0.9 & 1.4 & 1.5 & 1.6 & 1.4 & 1.0 & 0 & 1. & 5 & 6 & & 1.7 & 1.5 & 0.0 & 32.1 \\
\hline $8-1$ & 0.5 & 0.8 & 0.4 & 0.1 & 0.2 & 0.3 & 0.8 & 0.8 & 0.7 & 0 & 1.2 & & 5.7 & & 0.7 & 0.4 & 0.0 & 19.2 \\
\hline 13. & 0.1 & 0.1 & 0.0 & $0 . c$ & 0.0 & 0.0 & 0.1 & 0.3 & 0.4 & 0. & 1.1 & 1.1 & 2.2 & 2. & 0.9 & 0.1 & 0.0 & 9.3 \\
\hline & 0.0 & 0.0 & 0.0 & 0.0 & 0.0 & 0. & 0.0 & 0. & 0.1 & 0.5 & 0.6 & 0. & 0.4 & 1. & 0.4 & 0.0 & & 3.5 \\
\hline & 0.0 & 0.0 & 0.0 & 0 & 0.0 & 0. & 0.0 & 0.0 & 0.0 & 0. & 0.1 & 0. & 0.1 & 0. & 0.1 & 0.0 & 0.0 & 0.7 \\
\hline & 0.0 & 0.0 & 0.0 & 0.0 & 0.0 & & 0.0 & 0.0 & 0.0 & 0. & 0.0 & 0. & 0 & 0 & 0.0 & 0.0 & 0.0 & 0.0 \\
\hline & 0.0 & 0. & 0.0 & 0. & 0.0 & & 0.0 & 0.0 & 0.0 & 0.0 & 0.0 & 0.0 & 0.0 & 0.0 & 0.0 & 0.0 & 0.0 & 0.0 \\
\hline & 0.0 & 0.0 & 0.0 & 0.0 & 0.0 & 0 & 0.0 & 0.0 & 0.0 & 0. & 0.0 & 0.0 & 0.0 & 0.0 & 0.0 & 0.0 & 0.0 & 0.0 \\
\hline TOTAL & 4.1 & 3.9 & 2.8 & 2.9 & 3.8 & 3.7 & 4.4 & 4.2 & 3.8 & 4.2 & 6.8 & 13.6 & 19.0 & 11.7 & 6.0 & 3.7 & 1.1 & 100.0 \\
\hline
\end{tabular}

Station: (30) HAMR

Begin: 1/99

End: $12 / 99$

Total Hours: 8748

DIRECTION

\begin{tabular}{|c|c|c|c|c|c|c|c|c|c|c|c|c|c|c|c|c|c|c|}
\hline & $\mathrm{N}$ & NNE & $\mathrm{NE}$ & ENE & $E$ & ESE & SE & SSE & $s$ & SSW & SW & WSW & W & WNW & NW & NNW & CALM & TOTAL \\
\hline CALM & 0.0 & 0.0 & 0.0 & 0.0 & 0.0 & 0.0 & 0.0 & 0.0 & 0.0 & 0.0 & 0.0 & 0.0 & 0.0 & 0.0 & 0.0 & 0.0 & 1.3 & 1.3 \\
\hline $1-3$ & 1.1 & 0.9 & 0.8 & 0.5 & 0.7 & 0.9 & 1.5 & 1.9 & 2.0 & & 1.3 & & & & & 0.9 & 0.0 & 18.5 \\
\hline $4-7$ & 3.6 & 1.7 & 0.9 & 0.8 & 0.9 & 1.8 & 3.9 & 3.9 & 3.6 & 3.3 & 4.0 & 2.1 & 1.4 & & 3.0 & 3.7 & 0.0 & 40.4 \\
\hline $8-1$ & 2.4 & 1.3 & 0.5 & 0.1 & 0.2 & 0.3 & 0.5 & 0.4 & 1.1 & 2.7 & 6.9 & 3.5 & 1.0 & 0.5 & 1.6 & 2.4 & 0.0 & 25.5 \\
\hline 13 & 0.7 & 0.2 & 0.0 & 0.0 & 0.0 & 0.0 & 0.0 & 0.1 & 0.3 & 1.3 & 3.4 & 1.8 & 0. & 0. & & .9 & 0.0 & 10.8 \\
\hline & 0.0 & 0.0 & 0.0 & 0.0 & 0.0 & 0.0 & 0.0 & 0.0 & 0.1 & 0.4 & 0.9 & 0.6 & 0.2 & 0 & 0. & 0.1 & 0.0 & 2.7 \\
\hline & 0.0 & & 0.0 & 0.0 & 0.0 & 0.0 & 0.0 & 0.0 & 0.0 & 0.1 & 0.4 & 0.1 & 0.0 & 0. & 0.0 & 0.0 & 0.0 & 0.7 \\
\hline & 0.0 & & 0.0 & 0.0 & 0.0 & 0.0 & 0 & 0. & 0.0 & 0.6 & 0. & 0.0 & 0.0 & 0.0 & 0.0 & 0.0 & 0.0 & 0.1 \\
\hline & 0.0 & 0.0 & 0.0 & 0.0 & 0.0 & 0.0 & 0. & 0. & 0. & 0. & 0. & 0 & 0. & & & & 0.0 & 0.0 \\
\hline$>46$ & 0.0 & 0.0 & 0.0 & 0.0 & 0.0 & 0.0 & 0.0 & 0.0 & 0.0 & 0.0 & 0.0 & 0. & 0 & & & & 0.0 & 0.0 \\
\hline TOTAL & 7.9 & 4.1 & 2.2 & 1.4 & 1.8 & 3.0 & 6.0 & 6.4 & 7.1 & 9.3 & 16.9 & 9.3 & 4.3 & 3.9 & 7.3 & 8.0 & 1.3 & 100.0 \\
\hline
\end{tabular}


Table A.2. Joint Frequency Distributions (\%) for Hanford Meteorological Monitoring Network Wind Stations at 60-Meter Level, 1999

Tower: 100 Area

Begin: 1/99

End: $12 / 99$

Total Hours: 8724

DIRECTION

\begin{tabular}{rrrrrrrrrrrrrrrrrrr} 
& N & NNE & NE & ENE & E & ESE & SE & SSE & S & SSW & SW & WSW & W & WNW & NW & NNW & CALM & TOTAL \\
\hline CALM & 0.0 & 0.0 & 0.0 & 0.0 & 0.0 & 0.0 & 0.0 & 0.0 & 0.0 & 0.0 & 0.0 & 0.0 & 0.0 & 0.0 & 0.0 & 0.0 & 0.5 & 0.5 \\
$1-3$ & 1.5 & 1.2 & 1.5 & 2.3 & 3.2 & 2.8 & 2.0 & 1.6 & 1.2 & 1.2 & 1.1 & 1.4 & 1.8 & 2.0 & 1.9 & 1.7 & 0.0 & 28.6 \\
$4-7$ & 1.2 & 1.5 & 1.8 & 1.7 & 3.2 & 3.0 & 2.4 & 1.6 & 1.0 & 0.6 & 1.2 & 1.7 & 3.2 & 3.3 & 1.9 & 1.1 & 0.0 & 30.5 \\
$8-12$ & 0.4 & 1.0 & 1.0 & 0.3 & 0.3 & 0.6 & 1.2 & 0.7 & 0.4 & 0.5 & 0.9 & 1.6 & 3.1 & 3.0 & 0.9 & 0.2 & 0.0 & 16.2 \\
$13-18$ & 0.3 & 0.8 & 0.4 & 0.1 & 0.1 & 0.0 & 0.3 & 0.6 & 0.5 & 0.4 & 1.1 & 1.0 & 3.1 & 3.8 & 0.7 & 0.1 & 0.0 & 13.2 \\
$19-24$ & 0.1 & 0.0 & 0.0 & 0.0 & 0.0 & 0.0 & 0.1 & 0.2 & 0.2 & 0.4 & 0.7 & 0.5 & 0.9 & 2.3 & 0.6 & 0.1 & 0.0 & 6.3 \\
$25-31$ & 0.0 & 0.0 & 0.0 & 0.0 & 0.0 & 0.0 & 0.0 & 0.0 & 0.2 & 0.4 & 0.6 & 0.3 & 0.3 & 1.1 & 0.5 & 0.0 & 0.0 & 3.5 \\
$32-38$ & 0.0 & 0.0 & 0.0 & 0.0 & 0.0 & 0.0 & 0.0 & 0.0 & 0.0 & 0.2 & 0.1 & 0.1 & 0.1 & 0.2 & 0.1 & 0.0 & 0.0 & 0.9 \\
$39-46$ & 0.0 & 0.0 & 0.0 & 0.0 & 0.0 & 0.0 & 0.0 & 0.0 & 0.0 & 0.0 & 0.0 & 0.0 & 0.0 & 0.0 & 0.0 & 0.0 & 0.0 & 0.1 \\
$>46$ & 0.0 & 0.0 & 0.0 & 0.0 & 0.0 & 0.0 & 0.0 & 0.0 & 0.0 & 0.0 & 0.0 & 0.0 & 0.0 & 0.0 & 0.0 & 0.0 & 0.0 & 0.1 \\
TOTAL & 3.5 & 4.5 & 4.7 & 4.5 & 6.9 & 6.5 & 6.0 & 4.8 & 3.5 & 3.8 & 6.0 & 6.7 & 12.5 & 15.8 & 6.7 & 3.3 & 0.5 & 100.0
\end{tabular}

Tower: 200 Area

Begin: 1/9

End: $12 / 99$

Total Hours: 8736

DIRECTION

\begin{tabular}{|c|c|c|c|c|c|c|c|c|c|c|c|c|c|c|c|c|c|c|}
\hline & $\mathrm{N}$ & NNE & $\mathrm{NE}$ & ENE & $E$ & ESE & SE & SSE & $\underline{\mathbf{S}}$ & SSW & SW & WSW & W & WNW & NW & NNW & CALM & TOTAL \\
\hline CALM & 0.0 & 0.0 & 0.0 & 0.0 & 0.0 & 0.0 & 0.0 & 0.0 & 0.0 & 0.0 & 0.0 & 0.0 & 0.0 & 0.0 & 0.0 & 0.0 & 0.0 & 0.0 \\
\hline $1-3$ & 0.6 & 0.6 & 0.6 & 0.7 & 0.8 & 0.4 & 0.6 & 0.9 & 0.6 & 0.4 & 0.4 & 0.4 & 0.4 & 0.5 & 0.6 & 0.9 & 0.0 & 9.6 \\
\hline $4-7$ & 2.0 & 2.0 & 1.4 & 1.6 & 2.2 & 1.0 & 1.5 & 2.2 & 1.0 & 1.0 & 1.3 & 1.3 & 1.7 & 2.3 & 3.4 & 3.2 & 0.0 & 29.1 \\
\hline $8-12$ & 1.2 & 0.8 & 0.8 & 0.4 & 0.7 & 0.3 & 0.4 & 0.8 & 0.5 & 0.7 & 1.4 & 2.1 & 2.7 & 4.3 & 6.4 & 2.5 & 0.0 & 26.1 \\
\hline $13-18$ & 0.3 & 0.4 & 0.2 & 0.1 & 0.1 & 0.0 & 0.0 & 0.4 & 0.4 & 0.6 & 1.6 & 2. & 2.2 & 4.7 & 6.1 & 0.5 & 0.0 & 20.1 \\
\hline $19-24$ & 0.0 & 0.0 & 0.0 & 0.0 & 0.0 & 0.0 & 0.0 & 0.2 & 0.3 & 0.5 & 1.3 & 1.2 & 0.6 & 1.7 & 2.9 & 0.1 & 0.0 & 8.8 \\
\hline $25-31$ & 0.0 & 0.0 & 0.0 & 0.0 & 0.0 & 0.0 & 0.0 & 0.0 & 0.2 & 0.5 & 1.1 & 0.6 & 0.2 & 0.6 & 1.4 & 0.0 & 0.0 & 4.7 \\
\hline $32-38$ & 0.0 & 0.0 & 0.0 & 0.0 & 0.0 & 0.0 & 0.0 & 0.0 & 0.0 & 0.5 & 0.4 & 0.2 & 0.0 & 0.0 & 0.2 & 0.0 & 0.0 & 1.3 \\
\hline $39-46$ & 0.0 & 0.0 & 0.0 & 0.0 & 0.0 & 0.0 & 0.0 & 0.0 & 0.0 & 0.1 & 0.1 & 0.1 & 0.0 & 0.0 & 0.0 & 0.0 & 0.0 & 0.4 \\
\hline$>46$ & 0.0 & 0.0 & 0.0 & 0.0 & 0.0 & 0.0 & 0.0 & 0.0 & 0.0 & 0.0 & 0.0 & 0.0 & 0.0 & 0.0 & 0.0 & 0.0 & 0.0 & 0.1 \\
\hline TOTAL & 4.0 & 3.7 & 3.0 & 2.9 & 3.9 & 1.8 & 2.6 & 4.5 & 3.0 & 4.3 & 7.7 & 8.4 & 7.9 & 14.1 & 21.1 & 7.1 & 0.1 & 100.0 \\
\hline
\end{tabular}

Tower: 300 Area

Begin: 1/99

End: $12 / 99$

Total Hours: 8711

DIRECTION

\begin{tabular}{|c|c|c|c|c|c|c|c|c|c|c|c|c|c|c|c|c|c|c|}
\hline & $\mathbf{N}$ & NNE & NE & ENE & E & ESE & SE & SSE & $S$ & SSW & SW & WSW & $\mathrm{W}$ & WNW & NW & NNW & CALM & TOTAL \\
\hline CALM & 0.0 & 0.0 & 0.0 & 0.0 & 0.0 & 0.0 & 0.0 & 0.0 & 0.0 & 0.0 & 0.0 & 0.0 & 0.0 & 0.0 & 0.0 & 0.0 & 0.3 & 0.3 \\
\hline $1-3$ & 0.7 & 0.6 & 0.8 & 0.7 & 0.6 & 1.0 & 0.9 & 1.2 & 0.9 & 0.8 & 0.7 & 0.6 & 0.6 & 0.7 & 0.9 & 0.9 & 0.0 & \\
\hline 4-7 & 2.3 & 1.3 & 1.1 & 1.2 & 1.6 & 2.7 & 3.6 & 2.8 & 2.8 & 2.3 & 2.0 & & 0.8 & 0.7 & 1.4 & 2.3 & 0.0 & \\
\hline $8-12$ & 3.3 & 2.2 & 1.1 & 0.2 & 0.3 & 1.0 & 2.5 & 1.5 & 1.7 & 3.5 & 3.5 & & 0.8 & 0.6 & 1.2 & 3.0 & 0.0 & \\
\hline $13-18$ & 1.4 & 1.1 & 0.2 & 0.1 & 0.0 & 0.1 & 0.5 & 0.3 & 0.4 & 2.2 & 4.0 & 2.8 & 0.8 & 0.4 & 1.1 & 2.0 & 0.0 & 17. \\
\hline $19-24$ & 0.1 & 0.0 & 0.0 & 0.0 & 0.0 & 0.0 & 0.1 & 0.1 & 0.2 & 1.0 & 2.4 & 1.2 & 0.5 & 0.3 & 0.9 & 0.4 & 0.0 & \\
\hline $25-31$ & 0.0 & 0.0 & 0.0 & 0.0 & 0.0 & 0.0 & 0.0 & 0.0 & 0.1 & 0.5 & 1.3 & 0.5 & 0.2 & 0.1 & 0.3 & 0.1 & 0.0 & \\
\hline $32-38$ & 0.0 & 0.0 & 0.0 & 0.0 & 0.0 & 0.0 & 0.0 & 0.0 & 0.0 & 0.3 & 0.5 & 0.1 & 0.0 & 0.0 & 0.0 & 0.0 & 0.0 & \\
\hline $39-46$ & 0.0 & 0.0 & 0.0 & 0.0 & 0.0 & 0.0 & 0.0 & 0.0 & 0.0 & 0.1 & 0.2 & 0.0 & 0.0 & 0.0 & 0.0 & 0.0 & 0.0 & \\
\hline$>46$ & 0.0 & 0.0 & 0.0 & 0.0 & 0.0 & 0.0 & 0.0 & 0.0 & 0.0 & 0.0 & 0.1 & 0.0 & 0.0 & 0.0 & 0.0 & 0.0 & 0.0 & \\
\hline TOTAL & 7.7 & 5.3 & 3.2 & 2.2 & 2.5 & 4.8 & 7.6 & 5.9 & 6.1 & 10.7 & 14.8 & 7.9 & 3.8 & 2.6 & 5.8 & 8.7 & 0.3 & 100 \\
\hline
\end{tabular}

Tower: 400 Area

Begin: 1/99

End: $12 / 99$

Total Hours: 8728

DIRECTION

\begin{tabular}{|c|c|c|c|c|c|c|c|c|c|c|c|c|c|c|c|c|c|c|}
\hline & $\mathrm{N}$ & NNE & $\mathrm{NE}$ & ENE & $\mathrm{E}$ & ESE & SE & SSE & $S$ & SSW & SW & NSW & $\mathrm{w}$ & WNW & NW & NNW & CALM & TOTAL \\
\hline CALM & 0.0 & 0.0 & 0.0 & 0.0 & 0.0 & 0.0 & 0.0 & 0.0 & 0.0 & 0.0 & 0.0 & 0.0 & 0.0 & 0.0 & 0.0 & 0.0 & 0.1 & 0.1 \\
\hline 1-3 & 0.8 & .8 & 0.6 & 0.4 & 0.6 & 0.7 & 0.8 & 0.8 & 0.8 & 0.8 & 0.7 & 0.4 & 0.5 & 0. & 0.6 & 0.6 & 0.0 & 10.5 \\
\hline 4. & 2.3 & 1.9 & 1.4 & 0.9 & 1.0 & 1.2 & 1.9 & 2.6 & 2.8 & 2. & 1.5 & 1.0 & 1.0 & 1.3 & 2.2 & 2.1 & 0.0 & 27.1 \\
\hline $8-12$ & 2.3 & 1.6 & 0.8 & 0.2 & 0.2 & 0.4 & 1.5 & 2.2 & 3.5 & 3. & 2.0 & 1. & 1.1 & 1. & 2.7 & 2.8 & 0.0 & 27.0 \\
\hline $13-$ & 0.9 & 0.7 & 0.2 & 0.1 & 0.0 & 0.0 & 0.5 & 0.9 & 1.7 & 4.4 & 3.0 & 1.7 & 0.7 & 1. & 3.8 & 1.8 & 0.0 & 21.7 \\
\hline $19-2$ & 0.0 & 0.0 & 0.0 & 0.0 & 0.0 & 0.0 & 0.1 & 0.2 & 0.4 & 1.7 & 2.0 & 0.7 & 0.4 & 0. & 2.4 & 0.2 & 0.0 & 8.9 \\
\hline 25 & 0.0 & 0.0 & 0.0 & 0.0 & 0.0 & 0.0 & 0.0 & 0.0 & 0.1 & 0.8 & 0.9 & 0. & 0.2 & 0. & 0.6 & 0.0 & 0.0 & \\
\hline $32-$ & 0.0 & 0.0 & 0.0 & 0.0 & 0.0 & 0.0 & 0.0 & 0.0 & 0.0 & 0.4 & 0.4 & 0. & 0.0 & 0. & 0.1 & 0.0 & 0.0 & \\
\hline 39 & 0.0 & 0.0 & 0.0 & 0.0 & 0.0 & 0.0 & 0.0 & 0.0 & 0.0 & 0.1 & 0.2 & 0. & 0.0 & 0. & 0.0 & 0.0 & 0.0 & \\
\hline & 0.0 & 0.0 & 0.0 & 0.0 & 0.0 & 0.0 & 0.0 & 0.0 & 0.0 & 0.1 & 0.0 & 0.0 & 0.0 & 0.0 & 0.0 & 0.0 & 0.0 & 0. \\
\hline TOTAL & 6.3 & 5.1 & 3.1 & 1.7 & 1.8 & 2.4 & 4.7 & 6.7 & 9.3 & 13.3 & 10.8 & 5.0 & 4.0 & 6.0 & 12.4 & 7.6 & 0.1 & 100. \\
\hline
\end{tabular}




\section{Distribution}

No. of

Copies

\section{OFFSITE}

All Seasons Heating \& Air

Conditioning, Inc.

ATTN: Terry Poe

P.O. Box 9006

Yakima, WA 98909-0006

B. Allen

611 Vaughan Road

Yakima, WA 98908

T. Allen

Power Resource Managers

2100 112th Ave. NE, Suite 100

Bellevue, WA 98004

D. Batchelor

732 W. Park

Pasco, WA 99301

Benton County Public Works 1709 S. Ely

Kennewick, WA 99336

2 Benton County PUD

P.O. Box 6270

524 S. Auburn

Kennewick, WA 99332

ATTN: Ken Mey

M. Sheeran

R. C. Blewett

4409 Arlington Drive

West Richland, WA 99352

T. Brazil

Cascade Natural Gas

P.O. Box 6899

Kennewick, WA 99336
No. of

Copies

CENEX Land O'Lakes

5115 W. Brinkley Rd.

Kennewick, WA 99338

E. Charvet

MeGregor Company

5251 Eltopia West Road

Eltopia, WA 99330

City of Benton City

ATTN: City Clerk

P.O. Box 70

Benton City, WA 99320

4 City of Richland

P.O. Box 190

Richland, WA 99352

ATTN: L. D. Dunbar, C.E.M.

L. Herbert

M. E. Lippold

R. Overman

W. T. Cobb

Cobb Consulting Services

815 S. Kellogg

Kennewick, WA 99336

Columbia Basin College Library

ATTN: Sharon Bailey

2600 N. 20th Ave.

Pasco, WA 99301

Columbia National Wildlife Refuge

U.S. Fish \& Wildlife Service

U.S. Department of the Interior

P.O. Box F

Othello, WA 99344

Distr.1 
No. of

Copies

Farmers Insurance Claims Office

P.O. Box 6998

Kennewick, WA 99336

D. Fiskum

Kadlec Medical Center

888 Swift Boulevard

Richland, WA 99352

Franklin County

ASCS Office

1620 Road 44

Pasco, WA 99301

Franklin County Planning

ATTN: James Cherry

1016 N. 4th

Pasco, WA 99301

E. Frimodt III

Franklin County Road Department

3416 Stearman Ave.

Pasco, WA 99301-7104

K. Gregg

North Franklin School District

P.O. Box 829

Connell, WA 99326

B. Hachey

Mid-Columbia Library - Pasco

$1320 \mathrm{~W}$. Hopkins

Pasco, WA 99301

P. M. Hamilton, Jr.

Assistant Director

Physical Plant

Central Washington University

Ellensburg, WA 98926

J. Hancock

U.S. Ecology

P.O. Box 638

Richland, WA 99352
No. of

Copies

L. Harms

Harms \& Associates

$1632 \mathrm{~W}$. Slyvester

Pasco, WA 99301

D. Harper

Wilber-Ellis Company

P.O. Box 764

Pasco, WA 99301

A. Haymaker

1721 Cottonwood Drive

Pasco, WA 99301

D. Kennell

Central Pre-mix Concrete Co.

11919 Harris Road

Pasco, WA 99301

Jacob and Rhodes

P.O. Box 6881

Kennewick, WA 99336

B. Johnson

556 Columbia Center

Kennewick, WA 99336

B. Kelley

614 S. Kellogg Street

Kennewick, WA 99336

J. Kleckner

D. Hittle Associates

757 Columbia Drive, SE

Richland, WA 99352

R. A. Kyle

Richland School District

615 Snow Ave.

Richland, WA 99352

Distr.2 
No. of

\section{Copies}

R. LaFramboise

Central Wash. Farm Crops Assoc.

P.O. Box 202

Yakima, WA 98907

K. Lettau

George Grant Construction Co.

P.O. Box 789

Richland, WA 99352

J. Mannix

Kennewick School Dist.

622 N. Kellogg Street

Kennewick, WA 99336

R. Mazurkiewicz

Bonneville Power Admin.

P.O. Box 968, MD-399

Richland, WA 99352

D. McBaugh

State of Washington Department of Health

Division of Radiation Protection

P.O. Box 47827

Olympia, WA 98504

W. A. McCullough

2402 Lariat Lane

Richland, WA 99352

J. McDonald

Washington Public Power

Supply System, MS 1025

P.O. Box 968

Richland, WA 99352

G. McMurphy

Valley Electric Co.

1010 E. Bruneau Ave.

Kennewick, WA 99336-3775

National Weather Service

2001 NW 56th Drive

Pendleton, OR 97801
No. of

Copies

R. Okarma

Kennewick High School

500 S. Dayton

Kennewick, WA 99336

M. Orgill, CCM

342 S. 200 West

Cedar City, UT 84720

R. Parker

Pavement Surface Control

307 N. Dayton, Bldg 1

Kennewick, WA 99336

Pasco School District

ATTN: Kathy, Bldg 210

3412 N. Stearman Ave.

Tri-Cities Airport

Pasco, WA 99301

Pioneer Hi-Bred

1040 Settler Road

Connell, WA 99326

Potato Growers of Washington

P.O. Box 563

Othello, WA 99344-0563

D. C. Powell

2110 Hudson

Richland, WA 99352

Professional Ag Services

P.O. Box 3053

Pasco, WA 99302

J. Pryce

Franklin County PUD

P.O. Box 2407

Pasco, WA 99302

Distr.3

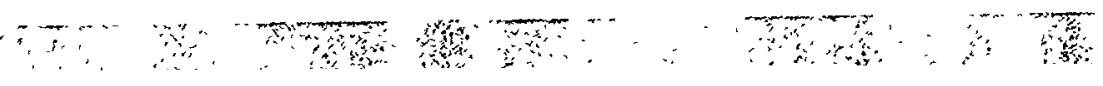


No. of

Copies

Raekes, Rettig, Osborne

ATTN: B. Iller

6725 W. Clearwater

Kennewick, WA 99336

J. T. Raese

Tree Fruit Research Lab

1104 N. Western Avenue

Wenatchee, WA 98801

K. Redmond

Western Regional Climate Center

Desert Research Institute

2215 Raggio Parkway

Reno, NV 89512-1095

R. R. Reid

1884 Rustic Road

Walla Walla, WA 99362

Richland Public Library

ATTN: Reference Librarian

955 Northgate

Richland, WA 99352

P. Roberts

6309 James St.

West Richland, WA 99353

W. Sahli

P.O. Box 912

Mattawa, WA 99344

W. Sealock

622 N. Kellogg

Kennewick, WA 99336

J. St. Clair

Benton-Franklin County Clean

Air Authority

650 George Washington Way

Richland, WA 99352
No. of

Copies

D. Smith

Lamb-Weston, Inc.

2005 Saint Street

Richland, WA 99352

Smith Frozen Foods

ATTN: Cliff Burt

205204 East Bowles Road

Kennewick, WA 99337

Soil Conservation Service

1620 Road 44, North

Pasco, WA 99301-2667

L. Stewart

Benton Rural Electric Assoc.

P.O. Box 1150

Prosser, WA 99350

K. Tanaka

Siemens Power Corporation

P.O. Box 130

2101 Horn Rapids Rd.

Richland, WA 99352

G. Taylor, Climatologist

Oregon Climate Service

Strand 326

Oregon State University

Corvallis, OR 97331-2209

J. Thorp

2110 Dover

Richland, WA 99352

Tri-City Sign \& Barricade, Inc.

307 N. Dayton

Kennewick, WA 99336

M. Troutman

1207 Sunnyvale Road

Kennewick, WA 99337

Distr.4 
No. of

Copies

K. Tucker

TRIDEC

901 N. Colorado

Kennewick, WA 99336

2 UNOCAL

P.O. Box 5797

Kennewick, WA 99336

ATTN: J. Akers

G. Stroud

USACE-Hydrology Branch

201 S. 3rd St.

Walla Walla, WA 99362

R. W. Wallace

89 Waldron Street

Richland, WA 99352

J. Watson

County Extension Agent

1121 Dudley Avenue

Prosser, WA 99350

L. Wendell

Wind Energy Consultants

2328 Enterprise Drive

Richland, WA 99352

R. N. Wing

727 Airway Avenue

Lewiston, ID 83501

L. Zeiher

405 South Dayton

Kennewick, WA 99336

W. Ziebell

Prosser School District

823 Park Avenue

Prosser, WA 99350
No. of

Copies

ONSITE

3 DOE Richland Operations Office
J. B. Hall
A5-15
D. C. Ward
A. $5-15$
Public Reading Rm
$\mathrm{H} 2-53$

5 Bechtel Hanford, Inc.

R. L. Biggerstaff $\quad \mathrm{HO}-02$

K. A. Gano HO-23

A. L. Johnson H0-23

C. J. Kemp S3-20

T. J. Rodovsky X9-08

CH2M Hill Hanford Group, Inc.

K. E. Cook H9-02

COGEMA Engineering Corporation

S. I. Ryburn S3-90

DynCorp Tri-Cities Services, Inc.

T. L. Ostrander S6-16

2 Fluor Hanford, Inc.

K. D. Johnson X3-65

J. W. Strege L6-82

3 Fluor Federal Services

T. E. Arndt

G3-17

P. G. Beaudet

S8-07

L. A. Gaddis

H5-57

Lockheed Martin Hanford Company

D. J. Washenfelder

H6-62

Distr.5 
No. of

Copies

Northwest Instrument Systems, Incorporated

E. C. Ladd

H6-62

Protection Technology Hanford

R. T. Toyooka

G3-43

U.S. Environmental Protection Agency

D. R. Einan

B5-01

4 Waste Management Federal Services of Hanford

E. G. Allen

T4-55

N. K. Myers

T3-28

J.R. Rosser

T4-03

R.W. Whitlock

T4-04

3 Waste Management Technical Services, Inc.

R. B. Barmettlor

L6-04

A. R. Johnson

$\mathrm{H} 1-13$

R. C. Roos

H1-11

139 Pacific Northwest National Laboratory

O. B. Abbey

K9-30

T.P. Ackerman

K9-90

O. P. Amacker

$\mathrm{K} 8-58$

D. L. Anderson

K9-72

G. L. Andrews

$\mathrm{K} 8-84$

W. R. Barchet

K9-30

J. C. Barnard

K9-30

D. B. Barnett

$\mathrm{K} 6-81$

L. E. Bisping

K6-75

W. J. Bjorklund

P7-72

K. W. Burk (10)
No. of

Copies

L. L. Cadwell

K6-84

R. E. Clayton K9-33

W. C. Cliff K6-48

T. S. Cress K9-38

G. W. Dennis K6-75

J. C. Doran K9-30

J. G. Droppo K6-80

M. J. Fayer K9-33

D. Felmy K9-33

H. D. Freeman P8-38

G. W. Gee K9-33

E. R. Gerk P7-63

B. M. Gillespie K6-96

C. S. Glantz K9-30

G. L. Gower P7-79

D. L. Hadley K5-16

D. R. Haffner K7-97

C. W. Hall P7-57

S. W. Heaberlin K8-31

D. J. Hoitink (75) K9-30

M. G. Horstman K4-10

D. G. Horton K6-81

R. R. Kirkham K9-33

N. R. Larson K9-55

N. S. Laulainen K9-30

R. N. Lee P7-07

J.P. McKinley K3-61

L. A. Monroe K1-53

T. L. Page K9-18

J. V. Ramsdell K9-30

K. Rhoads K3-54

M. R. Sackschewsky K6-84

W. J. Shaw K9-30

S. F. Snyder K3-54

G. M. Stokes K9-95

A. L. Ward K9-33

C. D. Whiteman K9-30

Technical Library P8-55

Information Release Office (7) K1-06 